\title{
Solar Chimney Turbine Performance
}

\author{
By \\ ANTHONY JOHN GANNON
}

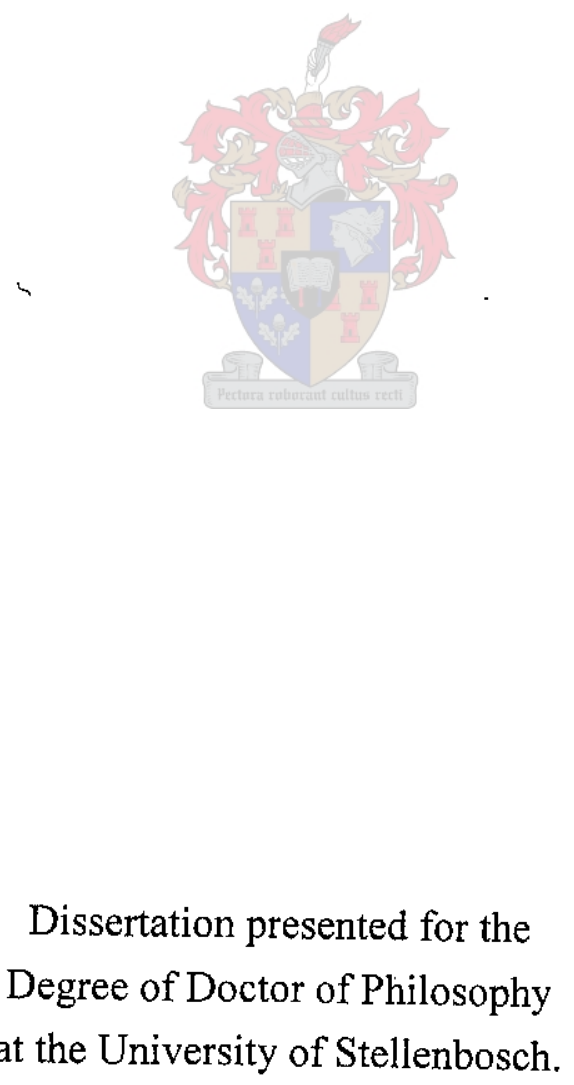

Promoter: Professor T.W. von Backström

March 2002 


\section{Declaration}

I, the undersigned, hereby declare that the work contained in this dissertation is my own original work and that I have not previously in its entirety or in part submitted it at any university for a degree.

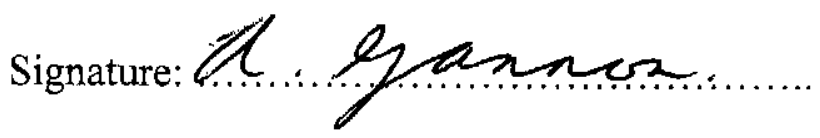
Date: $08-02-2002$ 
This project investigates the performance of solar chimney power plant turbines. A solar chimney power plant consists of a tall chimney surrounded by a transparent deck or solar collector. The sun heats the air in the collector through the greenhouse effect. A turbine extracts energy from the hot air rising up the chimney. An investigation of the requirements and operation of such turbines is needed.

Correct matching of the turbine to the plant requires the determination of the turbine operational range and other requirements. An air-standard cycle analysis is extended to include component and system losses. Simple steady-state and transient collector models are added to take into account the coupling effect of the collector air temperature rise and mass flow rate on the turbine operation. The predicted turbine operational range for a representative day shows that the expected pressure drop in a full-scale solar chimney turbine is significantly higher than has previously been predicted.

A turbine design method is developed and used to design a turbine for the representative day. The methods can easily be extended to include more operating points for a full year of operation. A turbine layout is suggested that uses the chimney support pillars as inlet guide vanes (IGVs). These introduce pre-whirl to the turbine and reduce the amount of exit whirl thus decreasing the kinetic energy at the turbine exit. Non-radial inlet guide vanes add to the torsional stiffness of the chimney base. A matrix throughflow method is used to design the radial to axial duct between the IGVs and rotor. The turbine blade profiles are simulated using a surface-vortex method. This is coupled to an optimisation scheme that minimises both the chord length and maximum flow velocity of the profile to reduce blade drag.

An experimental program investigates the performance of the turbine. Volume flow, pressure drop, torque and speed are measured on a scale model turbine to map the turbine performance over a wide range. The velocity and pressure profiles are measured at two design points to investigate the flow through the turbine in more detail. These are compared to the design predictions and used to improve the design method. The experiments show that the design of a solar chimney turbine with a total-to-total efficiency of $85 \%-90 \%$ and total-to-static efficiency of $75 \%-80 \%$ is possible. Analysis of the experimental results shows that the turbine efficiency can be improved. 


\section{OPSOMMING}

Hierdie projek ondersoek die gedrag van sonskoorsteen kragstasie turbines. 'n Sonskoorsteen kragstasie bestaan uit 'n hoë skoorsteen omring deur 'n deursigtige dak of son kollektor. Die son verhit die lug in die kollektor deur die kweekhuis effek. 'n Turbine onttrek energie uit die warm lug wat in die skoorsteen opstyg. 'n Ondersoek van die vereistes gestel aan, en werking van so 'n turbine is nodig.

Korrekte aanpassing van die turbine by die aanleg vereis die bepaling van die turbine se werkgebied en ander vereistes. 'n Standaard lugkringloop ontleding word uitgebrei om komponenten stelselverliese in ag te neem. Eenvoudige, bestendige en transiënte kollektor modelle word bygevoeg om die interaksie van die lug se temperatuurstyging en massavloeitempo te ondersoek. Die voorspelde werkgebied vir 'n verteenwoordigende dag toon dat die verwagte drukval vir ' $n$ volskaal sonskoorsteen merkbaar hoër is as voorheen voorspel.

'n Turbine ontwerpmetode is ontwikkel en gebruik in die ontwerp van 'n turbine vir die verteenwoordigende dag. Die metodes kan ook uitgebrei word om meer werkspunte wat oor ' $n$ hele jaar strek in te sluit. 'n Turbine uitleg, wat die skoorsteen se steunpilare as inlaatleilemme gebruik, word voorgestel. Die inlaatleilemme veroorsaak vloei voor-rotasie wat verminderde uitlaat-rotasie tot gevolg het en dus die kinetiese energie by die turbine uilaat verminder. Nie-radiale inlaatleilemme maak die skoorsteen basis torsioneel stywer. 'n Matriksdeurvloeimetode word gebruik in die ontwerp van die radiaal-tot-aksiale kanaal tussen die inlaatleileme en rotor. Die lemprofiele is gesimuleer deur middel van 'n oppervlakwerwel metode. Dit word gekoppel aan 'n optimeringskema waar die koordlengte en die maksimum snelheid geminimeer word om lemsleur te verminder.

'n Eksperimentele program ondersoek die vertoning van die turbine. Volumevloei, drukval, wringkrag en spoed word op 'n skaalmodel van die turbine gemeet om die vertoning oor 'n wye werksgebied vas te stel. Die snelheid- en drukprofiele word by twee ontwerpspunte gemeet om die vloei deur die turbine deegliker te ondersoek. Hierdie profiele is met die ontwerpvoorspellings vergelyk om die metodes vir ontwerp te verbeter. Die eksperimente toon dat die ontwerp van 'n sonskoorsteenturbine met 'n totaal-tot-totaal benuttingsgraad van $85 \%$ - $90 \%$ en totaal-tot-statiese benuttingsgraad van $75 \%$ - $80 \%$ moontlik is. 'n Ondersoek van die eksperimentele resultate bewys dat die turbine se benuttingsgraad verbeter kan word. 


\section{TABLE OF CONTENTS}

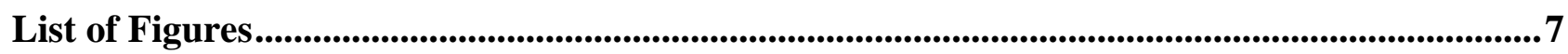

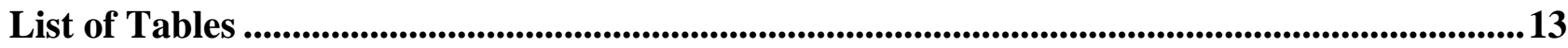

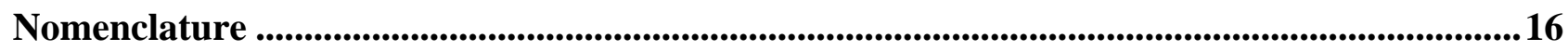

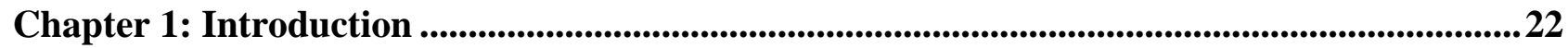

Background to Solar Powered Energy Sources ................................................................... 23

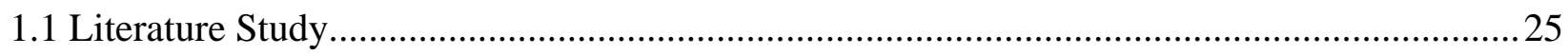

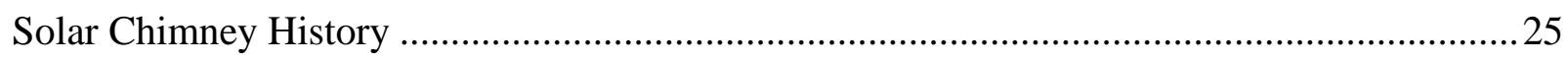

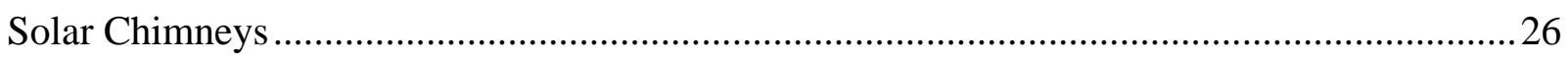

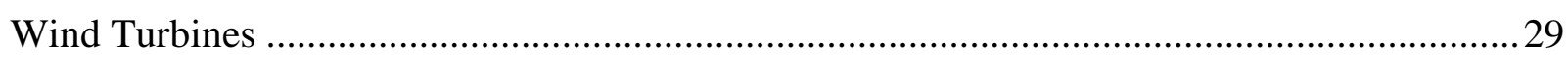

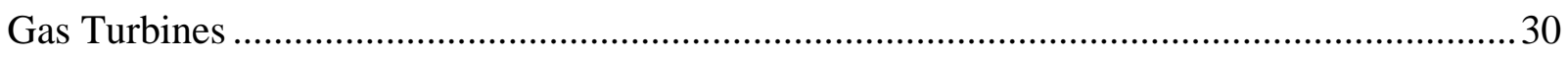

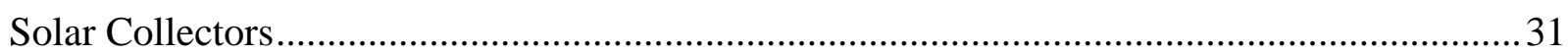

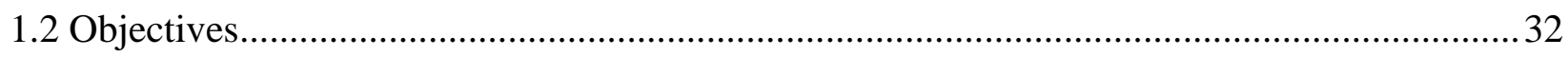

Chapter 2: Cycle Analysis of Solar Chimney ................................................................................34

2.1 Simple Thermodynamic Analysis of Solar Chimney …...................................................... 34

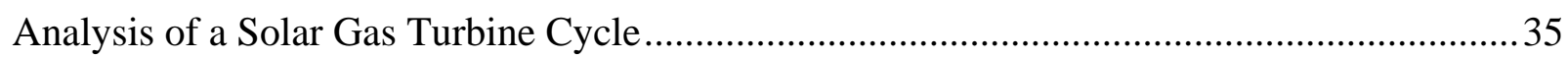

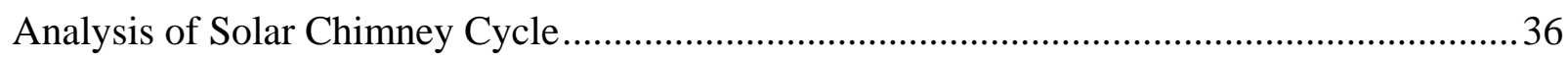

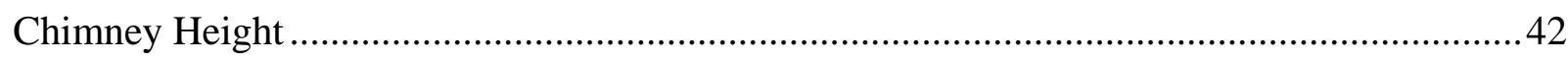

2.2 Cycle Analysis with Constant Chimney Height and Kinetic Energy Losses ........................43

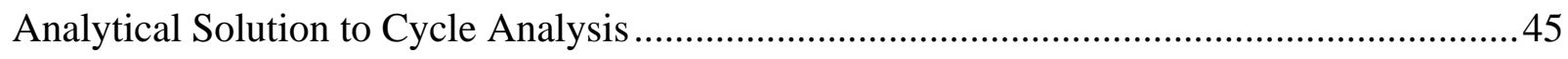

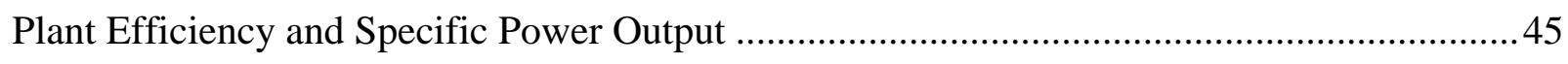

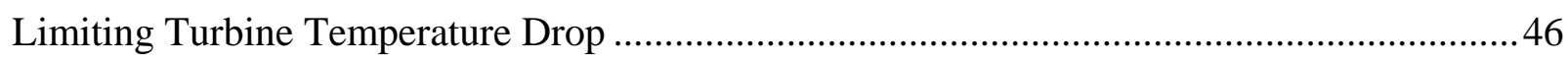

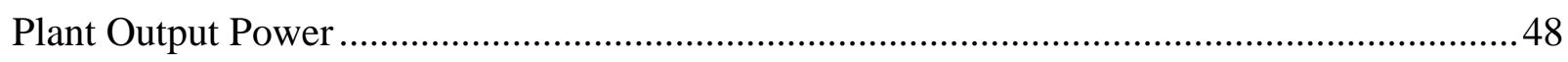

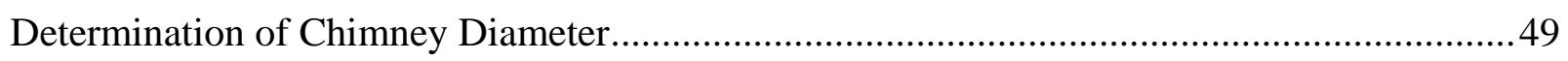

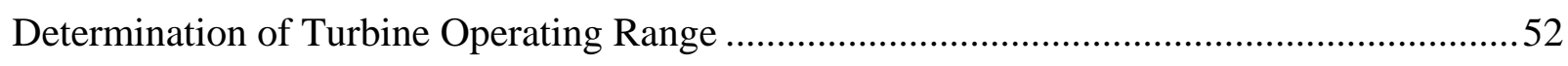

2.3 Cycle Analysis: Steady State Collector Model .................................................................. 54

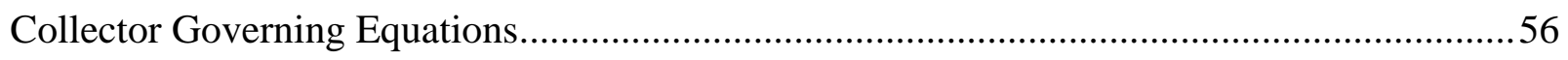

Operating Range Prediction Using Steady-State Model......................................................61

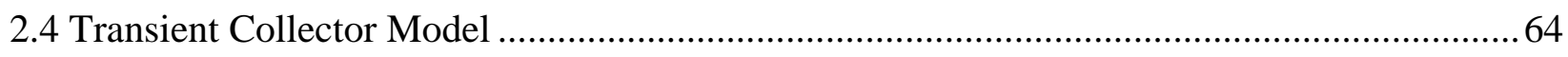

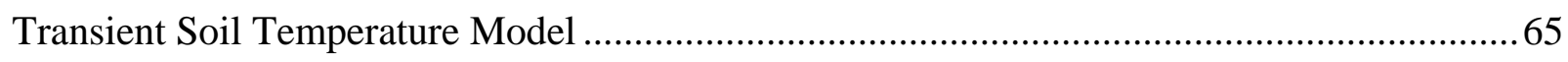

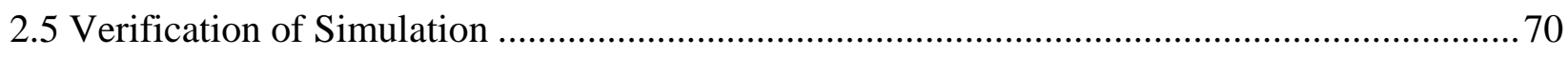

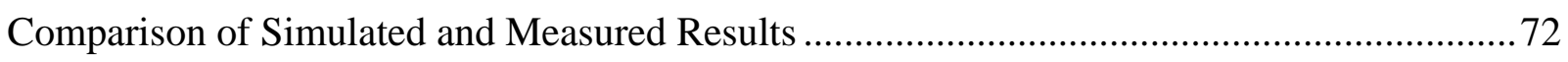




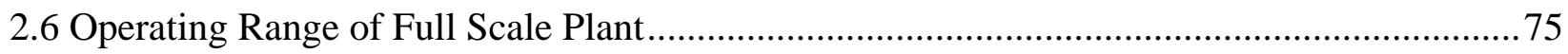

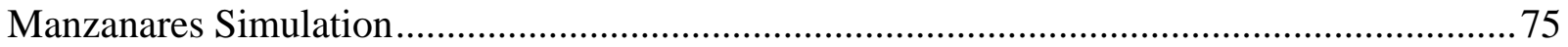

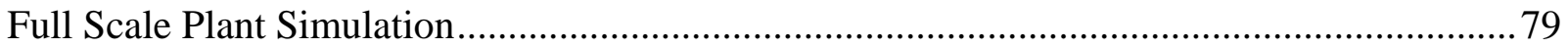

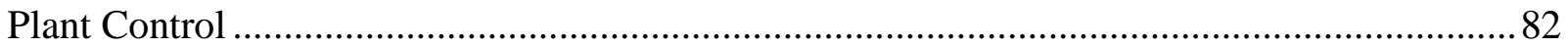

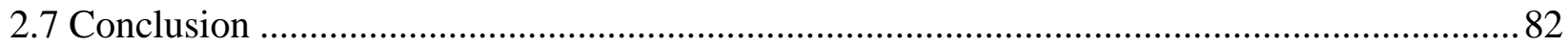

Chapter 3: Solar Chimney Turbine Design and Layout .............................................................83

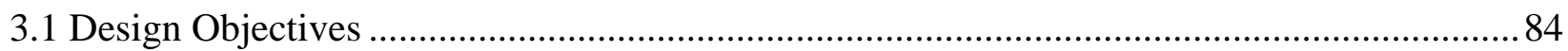

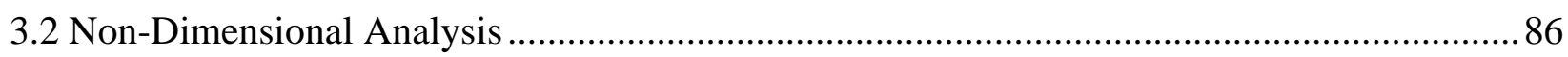

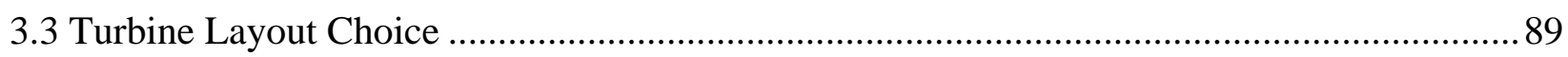

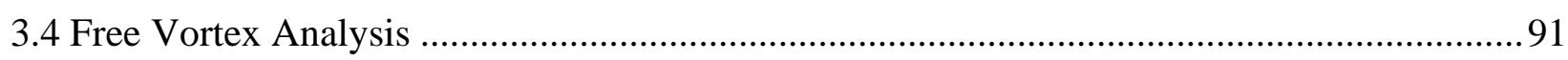

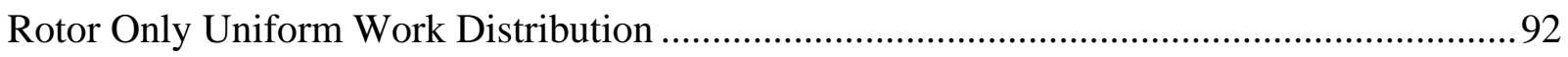

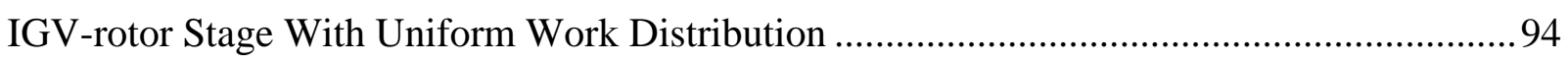

IGV-rotor Stage with Non-uniform Work Distribution ....................................................94

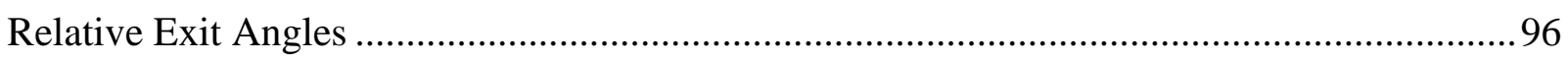

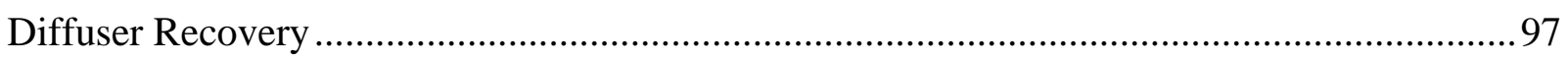

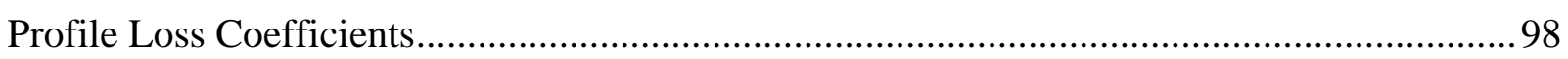

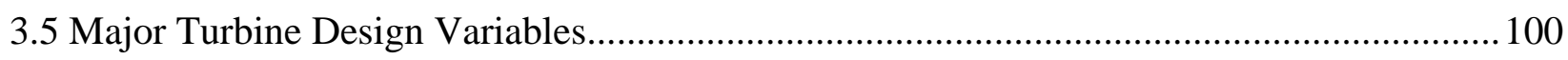

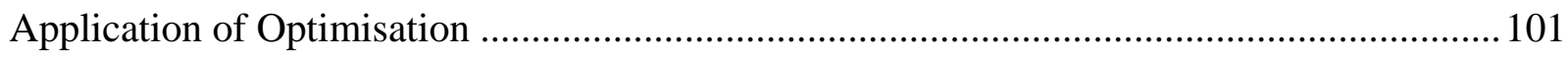

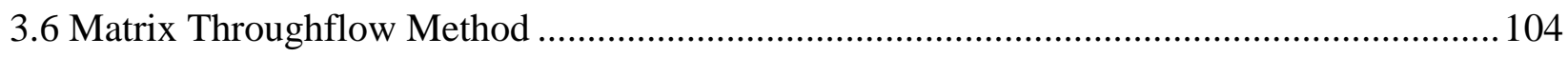

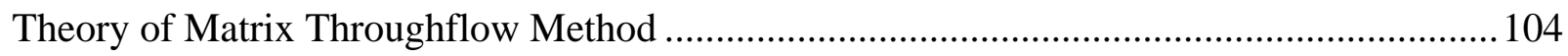

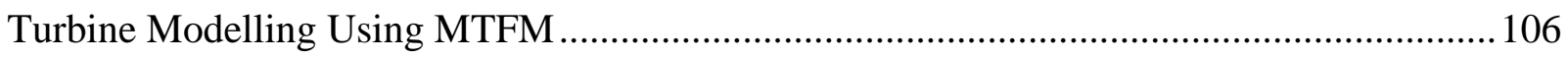

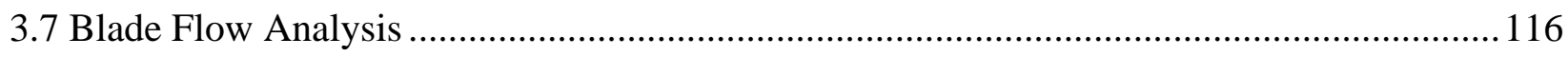

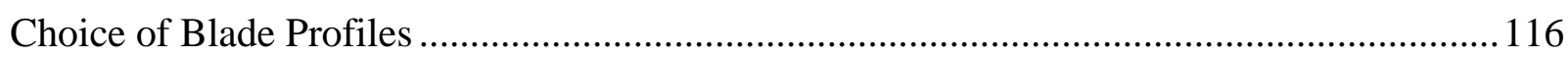

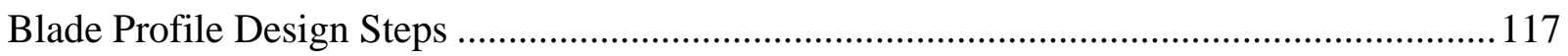

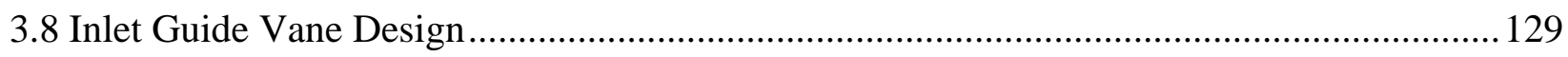

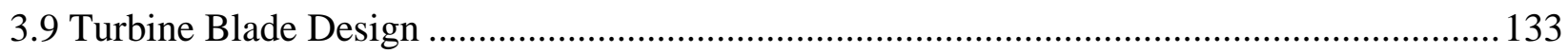

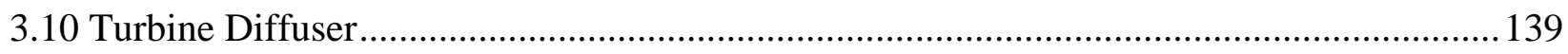

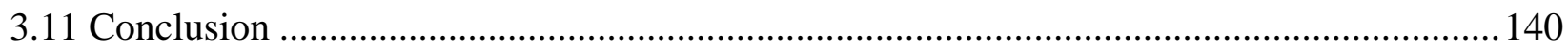

Chapter 4: Experimental Apparatus Design and Operation..............................................142

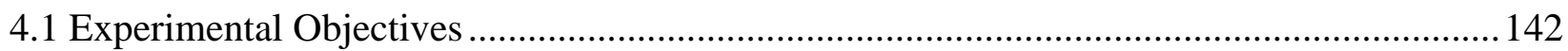

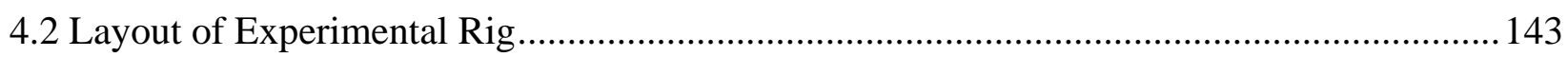

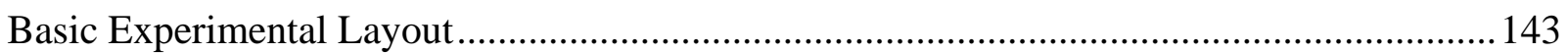

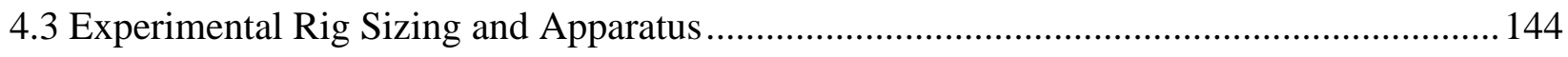

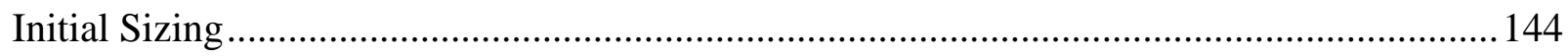




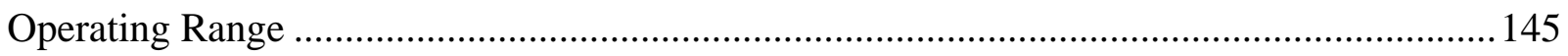

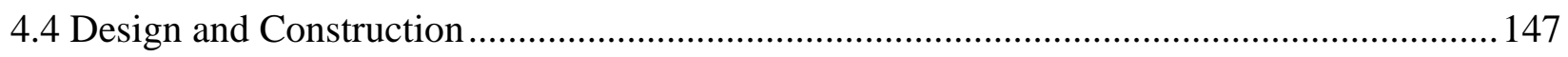

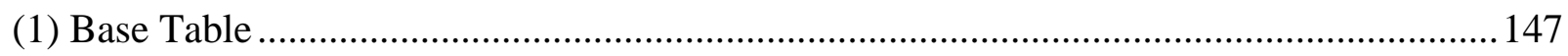

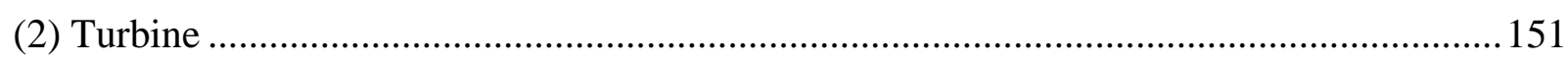

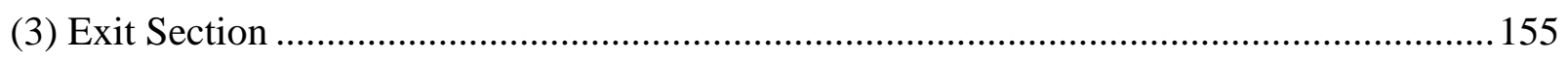

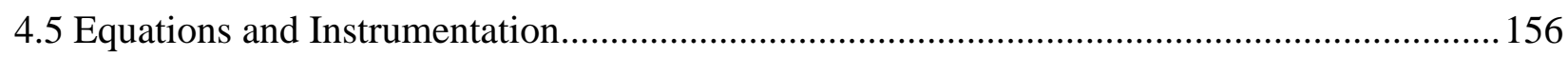

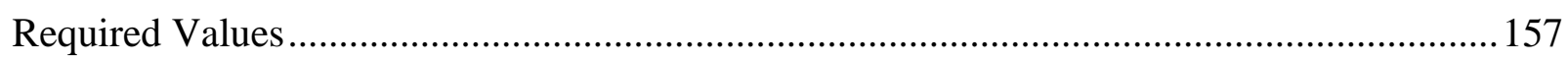

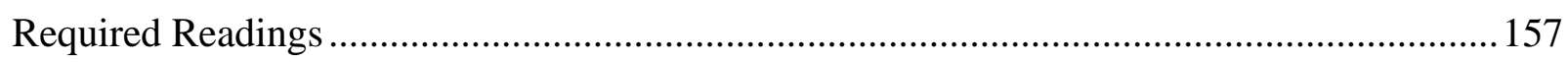

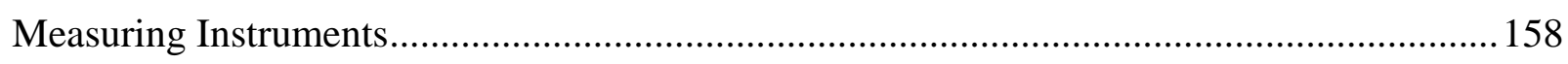

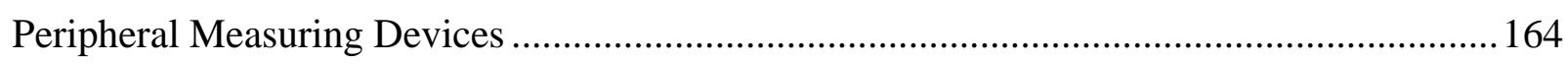

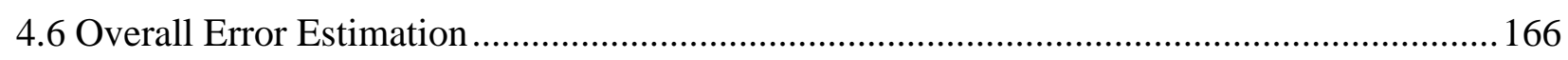

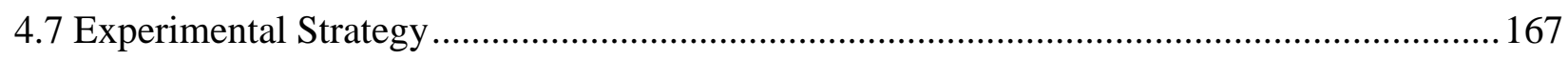

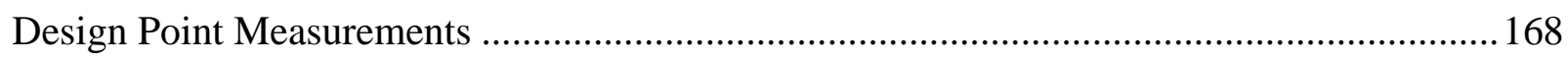

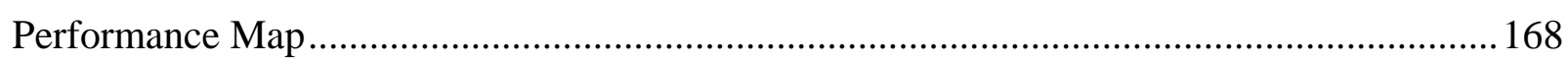

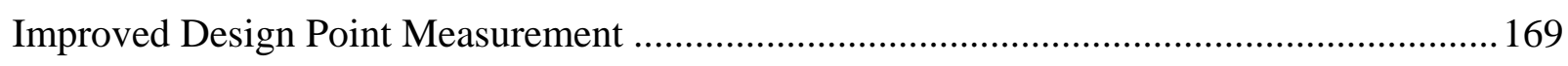

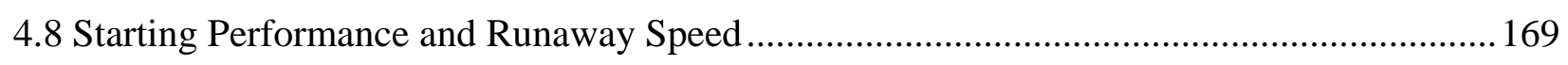

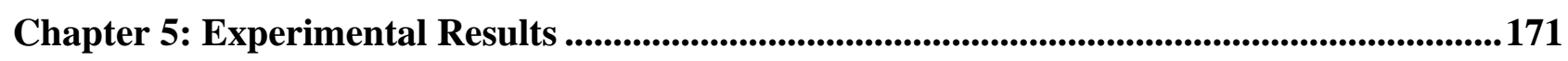

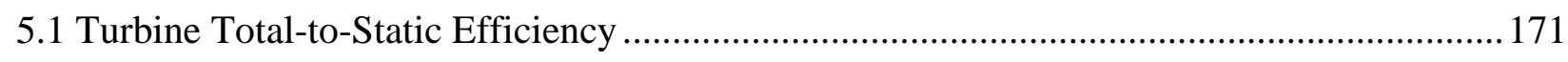

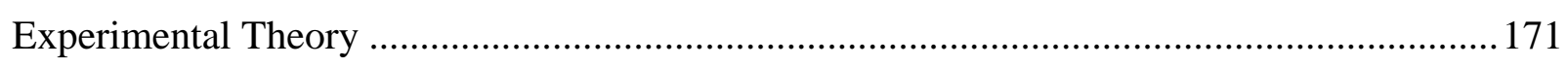

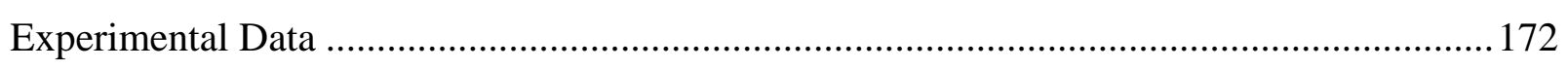

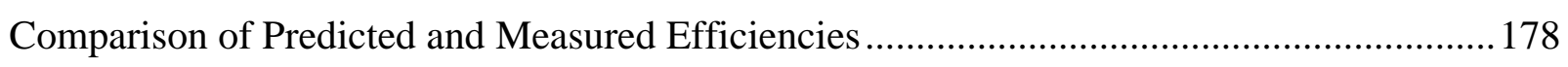

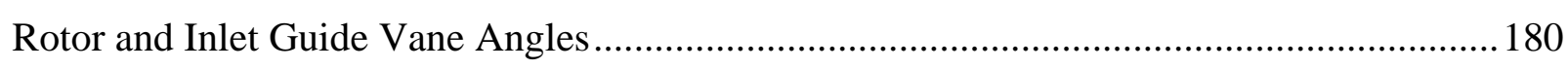

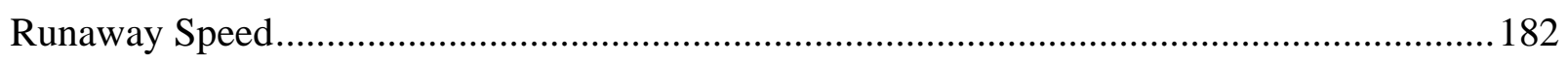

Assessment and Improvements to the Free Vortex Analysis................................................182

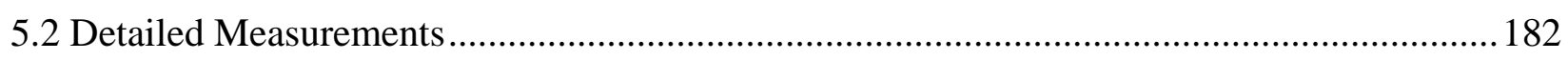

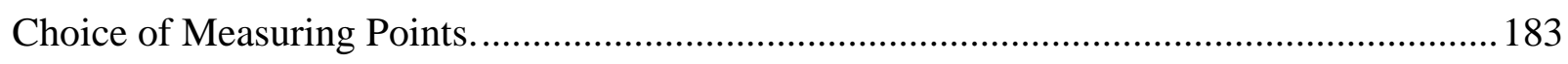

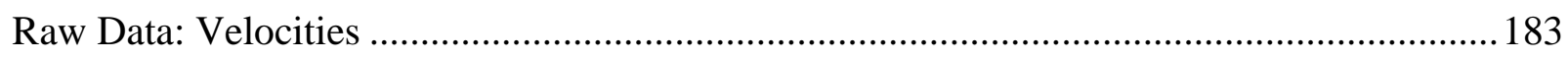

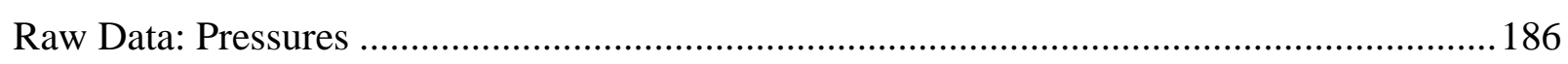

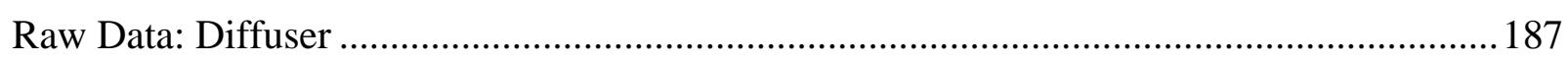

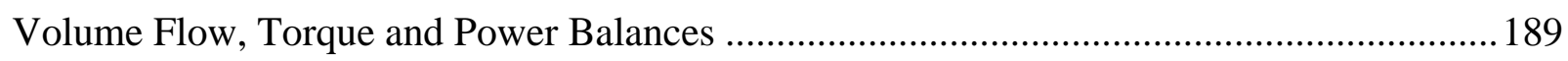

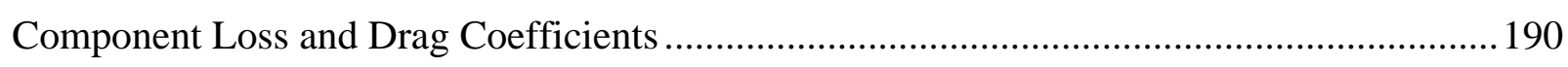

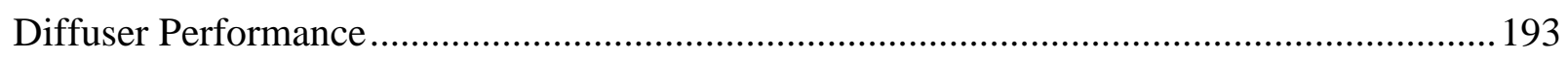

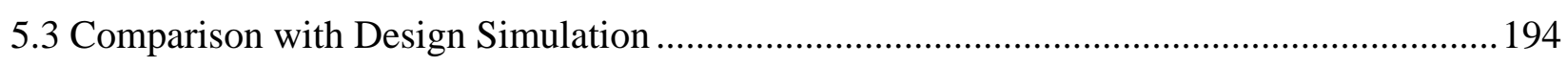

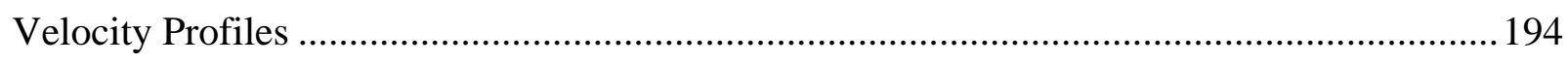

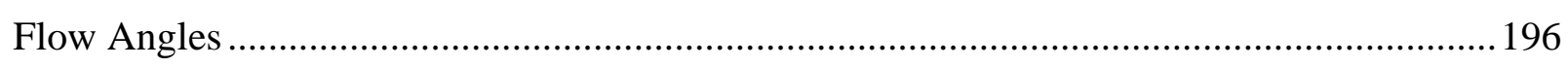




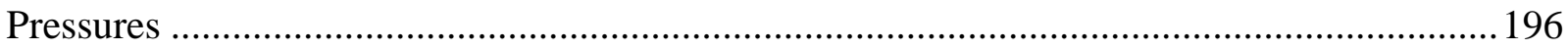

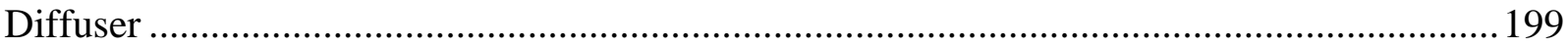

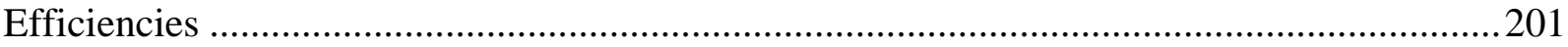

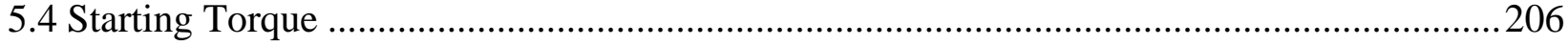

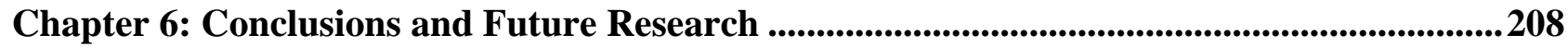

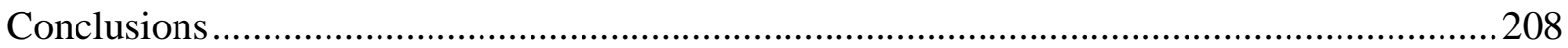

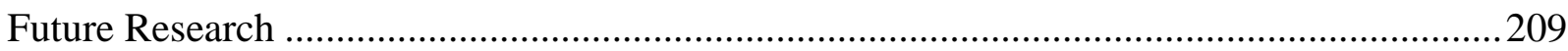

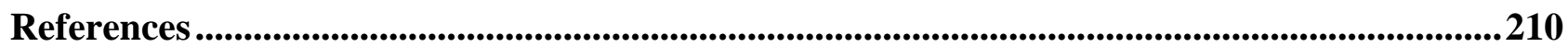

Appendix A: Sample Values from Solar Chimney Calculations.................................................215

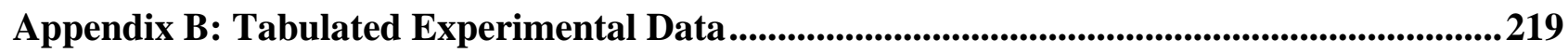

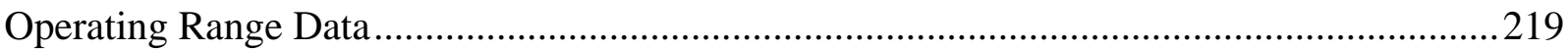

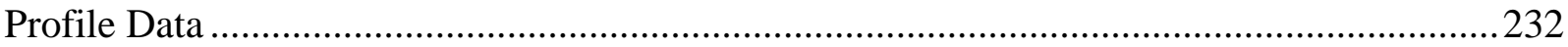

Appendix C: Turbine Efficiency Sample Calculation............................................................236

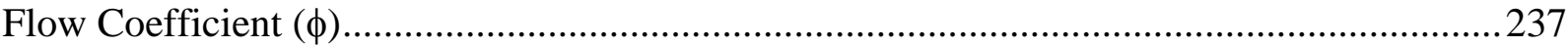

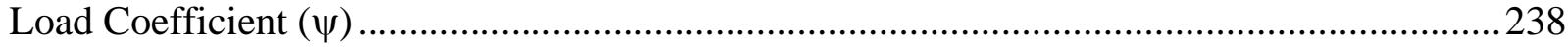

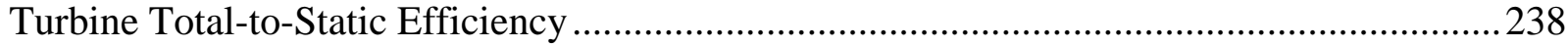

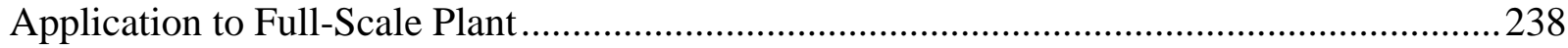

Appendix D: Profile Data Calculations............................................................................................2239

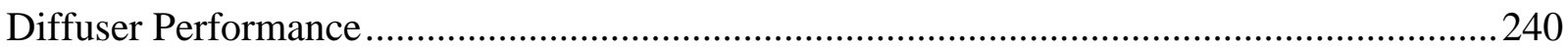




\section{LIST OF FIGURES}

Figure 1.1 Solar chimney power plant schematic

Figure 1.2. World energy consumption and sources of energy for 1989 and 1998 (US Department of Energy Web Site).

Figure 1.3 Spit of Leonardo da Vinci driven by rising hot air. 26

Figure 1.4. Power output records of experimental plant in Manzanares, Spain. 26

Figure 1.5. Demonstration solar collector (Pasumarthi \& Sherif 1997, 1998) .............................27

Figure 1.6. Power output represented along lines of constant collector temperature rise ..............28

Figure 1.7. Pressure drop along lines of constant collector temperature rise .28

Figure 2.1. Temperature-entropy (T-s) diagram for gas turbine air standard cycle......................35

Figure 2.2. Solar chimney schematic showing nomenclature ...................................................37

Figure 2.3. Temperature-entropy (T-s) diagram for solar chimney air standard cycle...................37

Figure 2.4. Specific power as a function of chimney height [m] and collector temperature rise $[\mathrm{K}]$ with efficiency [\%] overlaid.

Figure 2.5. Turbine pressure drop $[\mathrm{Pa}]$ as a function of chimney height $[\mathrm{m}]$ and collector temperature rise $[\mathrm{K}]$.

Figure 2.6. Required mass flow [tonnes/s] as a function of chimney height [m] and collector temperature rise [K] for $200 \mathrm{MW}$ design power output.

Figure 2.7. Temperature-entropy (T-s) diagram for solar chimney air standard cycle with kinetic energy loss at chimney exit.

Figure 2.8. Temperature-entropy (T-s) diagram for solar chimney air standard cycle for maximum turbine temperature drop.

Figure 2.9. Power / unit area $\left[\mathrm{W} / \mathrm{m}^{2}\right]$ for $1500 \mathrm{~m}$ chimney along lines of constant temperature rise $\Delta \mathrm{T}_{23}[\mathrm{~K}]$.

Figure 2.10. Required solar chimney diameter for $1500 \mathrm{~m}$ chimney along lines of constant temperature rise $\Delta \mathrm{T}_{23}[\mathrm{~K}]$ to obtain design power output of $200 \mathrm{MW}\left(\eta_{\text {turb }}=80 \%, \mathrm{k}=\right.$ 1).

Figure 2.11. Power output [MW] for chimney of height $1500 \mathrm{~m}$ and diameter $160 \mathrm{~m}$, along lines of constant temperature rise $\Delta \mathrm{T}_{23}[\mathrm{~K}]\left(\eta_{\text {turb }}=80 \%, \mathrm{k}=1\right)$.

Figure 2.12. Plant efficiency [\%] for chimney of height $1500 \mathrm{~m}$ and diameter $160 \mathrm{~m}$, along lines of constant temperature rise $\Delta \mathrm{T}_{23}[\mathrm{~K}]\left(\eta_{\text {turb }}=80 \%, \mathrm{k}=1\right)$.

Figure 2.13. Turbine pressure drop [Pa] for chimney of height $1500 \mathrm{~m}$ and diameter $160 \mathrm{~m}$, along lines of constant temperature rise $\Delta \mathrm{T}_{23}[\mathrm{~K}]\left(\eta_{\text {turb }}=80 \%, \mathrm{k}=1\right)$.

Figure 2.14. Top view of solar collector showing dimensioning and position of control volume. ..55

Figure 2.15. Detail of control volume of solar collector showing nomenclature for temperatures, heat, mass and radiation flow. 
Figure 2.16. Temperature rise [K] of ground, air, and glass surfaces through solar collector for

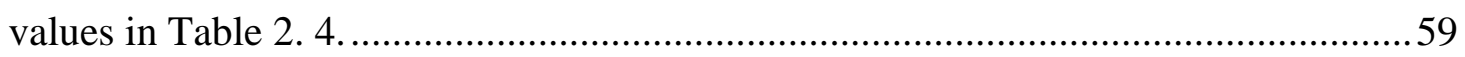

Figure 2.17. Heat flows $\left[\mathrm{W} / \mathrm{m}^{2}\right]$ in solar collector for values in Table 2. 4...................................59

Figure 2.18. Solar collector temperature rise [K] for varying mass flow [tonnes/s] along lines of constant inlet radiation $\left[\mathrm{W} / \mathrm{m}^{2}\right]$.

Figure 2.19. Solar collector efficiency [\%] for varying mass flow [tonnes/s] along lines of constant inlet radiation $\left[\mathrm{W} / \mathrm{m}^{2}\right]$.

Figure 2.20. Power output [MW] for chimney of height $1500 \mathrm{~m}$ and diameter $160 \mathrm{~m}$, along lines of constant inlet radiation $\left[\mathrm{W} / \mathrm{m}^{2}\right]\left(\eta_{\text {turb }}=80 \%, \mathrm{k}=1\right)$.

Figure 2.21. Turbine pressure drop [Pa] for chimney of height $1500 \mathrm{~m}$ and diameter $160 \mathrm{~m}$, along lines of constant inlet radiation $\left[\mathrm{W} / \mathrm{m}^{2}\right]\left(\eta_{\text {turb }}=80 \%, \mathrm{k}=1\right)$.

Figure 2.22. Thermal efficiency [\%] for chimney of height $1500 \mathrm{~m}$ and diameter $160 \mathrm{~m}$, along lines of constant inlet radiation $\left[\mathrm{W} / \mathrm{m}^{2}\right]\left(\eta_{\text {turb }}=80 \%, \mathrm{k}=1\right)$.

Figure 2.23. Overall plant efficiency [\%] for chimney of height $1500 \mathrm{~m}$ and diameter $160 \mathrm{~m}$, along lines of constant inlet radiation $\left[\mathrm{W} / \mathrm{m}^{2}\right]\left(\eta_{\text {turb }}=80 \%, \mathrm{k}=1\right)$.

Figure 2.24. Daily temperature variation in soil as function of soil depth....................................65

Figure 2.25. Discretisation scheme for transient soil model. .......................................................66

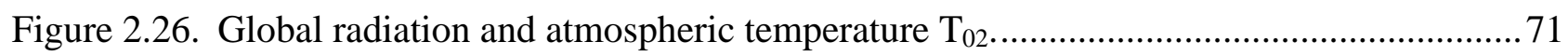

Figure 2.27. Range of soil heat conductivities in collector as a function of soil depth. ..................71

Figure 2.28. Collector temperature rise and chimney velocity in experimental plant....................72

Figure 2.29. Comparison of experimental air heat gain and simulated results..............................73

Figure 2.30. Comparison of experimental and simulated plant power output................................74

Figure 2.31. Pressure differences from experimental plant, Haaf (1984)......................................74

Figure 2.32. Predicted maximum power output from Manzanares size plant. ............................... 76

Figure 2.33. Predicted turbine pressure drop for maximum power output for Manzanares sized

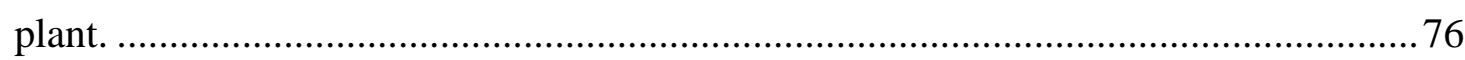

Figure 2.34. Predicted mass flow for maximum power output for Manzanares sized plant.............77

Figure 2.35. Predicted heat addition to air in solar collector for maximum power output for

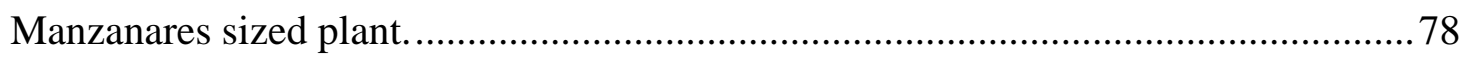

Figure 2.36. Predicted temperature rise $\Delta \mathrm{T}_{23}$ in solar collector for maximum power output for

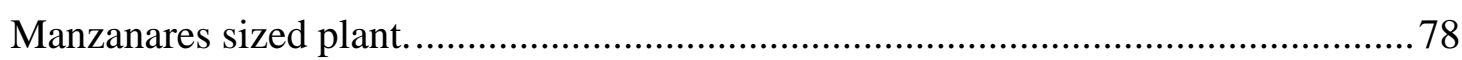

Figure 2.37. Full-scale plant maximum power output for 4 and $6 \mathrm{~km}$ diameter solar collector.......80

Figure 2.38. Full scale plant pressure drop for $4 \mathrm{~km}$ and $6 \mathrm{~km}$ diameter solar collector at maximum power output.

Figure 2.39. Full scale plant mass flow for $4 \mathrm{~km}$ and $6 \mathrm{~km}$ diameter solar collector at maximum power output. .....

Figure 2.40. Full scale plant collector temperature rise $\Delta \mathrm{T}_{23}$ for $4 \mathrm{~km}$ and $6 \mathrm{~km}$ collectors with Manzanares results superimposed. 
Figure 3.1. Predicted turbine pressure drop over a 24 hour period. .87

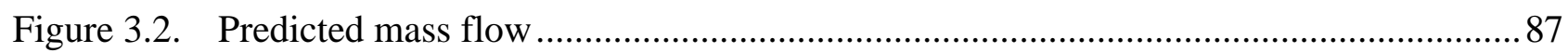

Figure 3.3. Specific diameter vs. specific speed diagram for single stage turbines operating with compressible fluids. Cases (1)-(3) superimposed on figure. .88

Figure 3.4. Schematic of proposed turbine layout with inlet guide vanes adding pre-swirl...........91

Figure 3.5. Velocity triangle diagram of solar chimney turbine near tip region...........................92

Figure 3.6. Schematic of linear function of work distribution across turbine blade......................95

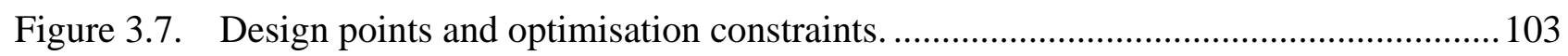

Figure 3.8. Solar chimney turbine layout with regions used in grid generation shown................108

Figure 3.9. Quasi-orthogonal grid used in matrix though-flow analysis. .................................108

Figure 3.10. Exit flow angles, $\beta_{2}$, for free vortex distribution and constant section IGV..............110

Figure 3.11. Meridional and tangential velocity profiles through inlet guide vanes....................110

Figure 3.12. Velocity profiles at inlet and exit of turbine for low and high pressure efficient

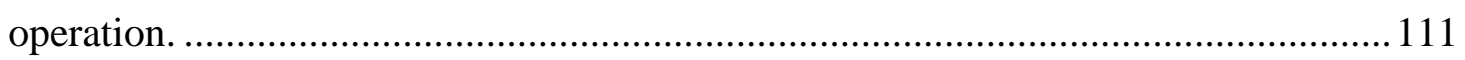

Figure 3.13. Camber angles of turbine blades for various design parameters compared to the free vortex analysis.

Figure 3.14. Turbine gas inlet and outlet angles for straight inlet guide vanes and non-uniform work being performed over the blade span.....

Figure 3.15. Pressure contours [Pa] relative to inlet stagnation pressure for high and low pressure

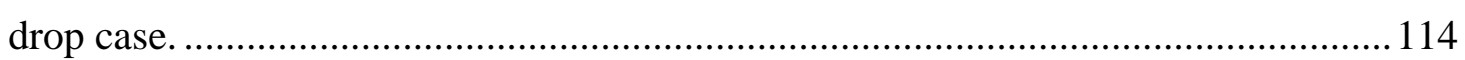

Figure 3.16 Coefficient of pressures on floor of transition section.............................................115

Figure 3.17. Coefficient of pressures on floor of transition section............................................115

Figure 3.18. Initial design point pitch/chord ratio.................................................................... 118

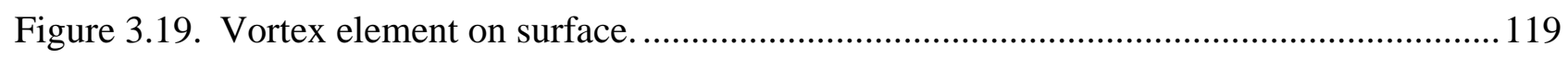

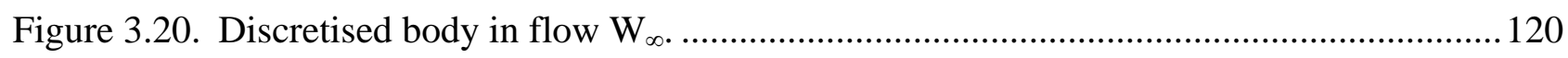

Figure 3.21. Trailing edge flow for the Kutta-Joukowski condition...........................................121

Figure 3.22. Comparison of surface vortex analysis with Abbott and Von Doenhoff for NACA 0010-35 profile.

Figure 3.23. (a) Turbomachinery cascade, (b) equivalent infinite array of point vortices and (c) nomenclature for velocity components.

Figure 3.24. Conformal transformation of rectilinear cascade in Cartesian (x,y) plane to radial cascade in cylindrical $(r, \theta)$ plane.

Figure 3.25. Laminar and turbulent separation point predictions from application of Stratford criterion for flow over NACA 0010-35 profile. .................................................... 127

Figure 3.26. Blade profile nomenclature............................................................................. 130

Figure 3.27. Detailed inlet guide vane blade profiles at extreme stagger angles..........................131

Figure 3.28. Inlet guide vane radial cascade at extreme stagger angles...................................... 132

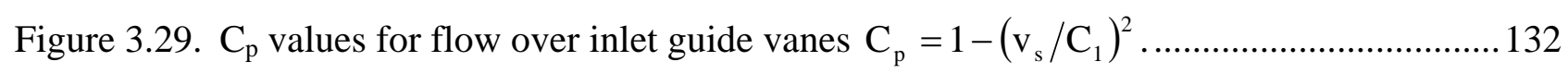


Figure 3.30. Turbine blade profile 4, showing pitch chord spacing. .........................................135

Figure 3.31. Blade profiles at each calculation station. ..........................................................136

Figure 3.32. Pressure distribution at blade midpoint, station 4, for high and low pressure case....137

Figure 3.33. Pressure distributions at each blade station for case (1) ......................................... 138

Figure 3.34. Pressure distributions at each blade station for case (2) ........................................ 138

Figure 3.35. Turbine diffuser (true aspect ratio) showing expansion angle..................................139

Figure 3.36. Section and top view of solar chimney turbine. ................................................... 140

Figure 4.1 Schematic of solar chimney turbine experimental rig. ........................................... 144

Figure 4.2. Performance characteristics of suction fan on experimental rig.............................. 146

Figure 4.3. (1) Base table schematic showing basic components...........................................148

Figure 4.4. Outer table segment showing construction........................................................ 148

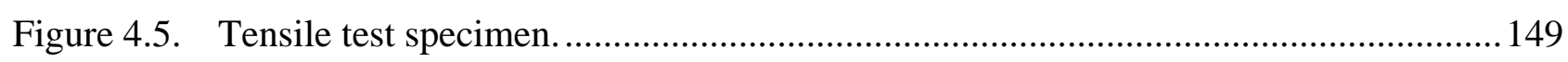

Figure 4.6. Moulded inlet guide vanes in position around central cone. ................................... 150

Figure 4.7. Upper ring showing the ducting leading into the diffuser. .....................................150

Figure 4.8. Inlet ducting leading into inlet guide vanes....................................................... 151

Figure 4.9. (2) Scale turbine model schematic showing basic components................................152

Figure 4.10. Turbine hub showing spherical shape and grooves for adjustable stagger angles. ....153

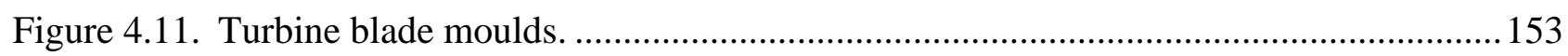

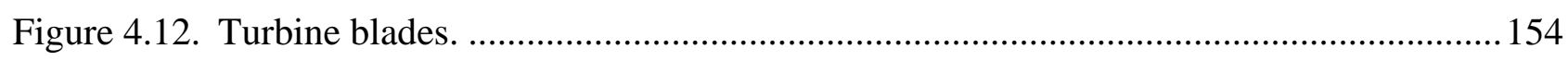

Figure 4.13. Generator coupled to variable speed drive with brake resistor................................. 154

Figure 4.14. Turbine exit piping schematic showing support structure.......................................155

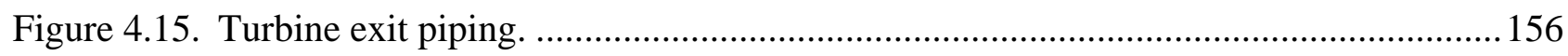

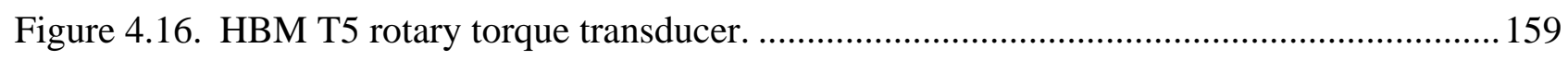

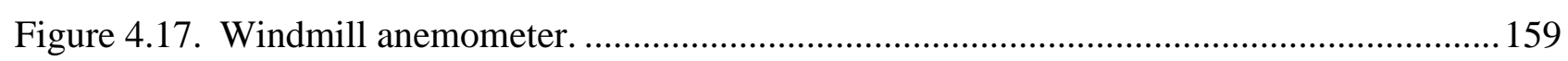

Figure 4.18. Windmill anemometer calibration curve for measuring velocity.............................160

Figure 4.19. Pressure tapping points on turbine exit pipe....................................................... 161

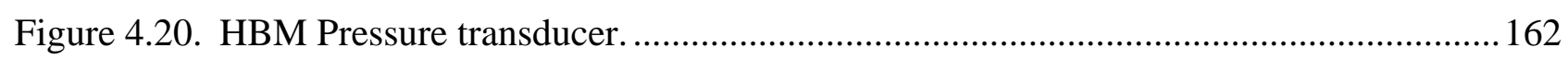

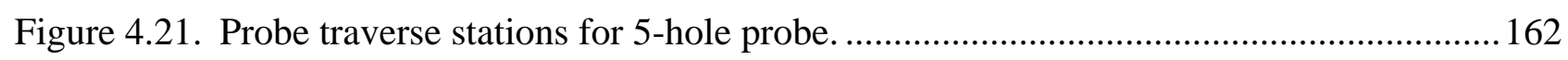

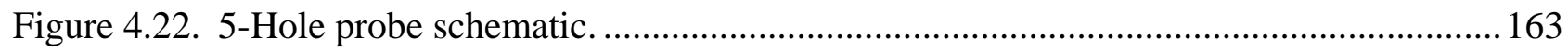

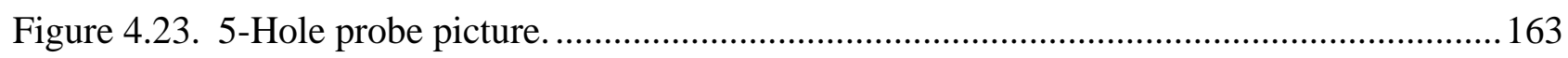

Figure 4.24. 5-Hole probe with piping to be connected to pressure transducers. ..........................163

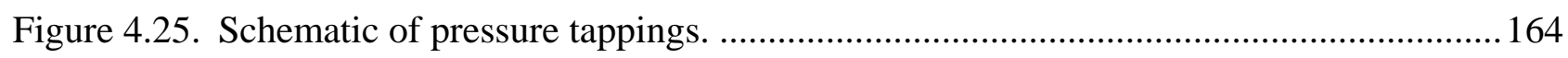

Figure 4.26. Picture of pressure tapping points and connecting pipes........................................164

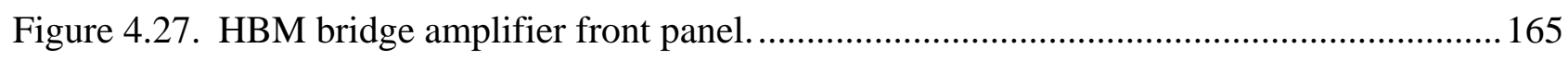

Figure 4.28. HBM bridge amplifier back showing input and output plugs. ................................ 165

Figure 4.29. Pressure switching box front panel.................................................................... 165

Figure 4.30. Pressure switching box back panel with multiple inputs........................................ 165 
Figure 5.1. Efficiency (\%) vs flow coefficient $(\phi)$ at $75.2^{0}$ stagger angle for IGV-flap angles of $-45^{0},-30^{0},-20^{0},-10^{0}, 0^{0},+10^{0},+20^{0},+30^{0},+45^{0}$.

Figure 5.2. Load coefficient, $(\psi)$ vs flow coefficient $(\phi)$ at $75.2^{0}$ stagger angle for IGV-flap angles of $-45^{0},-30^{0},-20^{0},-10^{0}, 0^{0},+10^{0},+20^{0},+30^{0},+45^{0} \ldots$

Figure 5.3. Efficiency (\%) vs flow coefficient $(\phi)$ for all rotor tip stagger angles....................... 175

Figure 5.4. Load coefficient, $(\psi)$ vs flow coefficient $(\phi)$ for all rotor stagger angles...................175

Figure 5.5. Experimentally derived total-to-static efficiencies [\%] against load, $\psi$, and flow, $\phi$, coefficients for optimal IGV angle.

Figure 5.6. Experimental total-to-static efficiency contours [\%] versus load, $\psi$, and flow, $\phi$, coefficients (for design IGV flap angle of $0^{0}$ ).

Figure 5.7. Predicted efficiency contours used in turbine design. 179

Figure 5.8. Predicted efficiency contours with Soderberg loss model. 179

Figure 5.9. IGV flap angles for maximum efficiency. 181

Figure 5.10. Rotor tip stagger angles for maximum efficiency. 181

Figure 5.11. Experimentally derived total-to-static efficiencies [\%] against load, $\psi$, and flow, $\phi$, coefficients (for design IGV flap angle of $0^{0}$ ).

Figure 5.12. Velocity profiles for low-pressure drop (case 1). 185

Figure 5.13. Velocity profiles for high-pressure design point (case 2). 185

Figure 5.14. Total and static pressures for low-pressure design point (case 1). 186

Figure 5.15. Total and static pressures for high-pressure design point (case 2). 186

Figure 5.16. Total and static pressures one pipe diameter downstream of the diffuser. 188

Figure 5.17. Velocities one diameter downstream of diffuser. 188

Figure 5.18. Loss coefficients for the rotor and inlet section. 191

Figure 5.19. Coefficients of Lift, $\mathrm{C}_{\mathrm{L} \infty}$, and Drag, $\mathrm{C}_{\mathrm{D} \infty}$.

Figure 5.20. Comparison of experimental with original MTFM results for $100 \%$ rotor total-to-total efficiency.

Figure 5.21. Comparison of experimental results with MTFM results corrected for rotor efficiency.

Figure 5.22. Comparison of relative rotor inlet and exit angles. 197

Figure 5.23. Comparison of gas deflection angles...... 197

Figure 5.24. Comparison of static and total pressure profiles at turbine inlet and outlet (lowpressure drop). 198

Figure 5.25. Comparison of static and total pressure profiles at turbine inlet and outlet (highpressure case).

Figure 5.26. Comparison of static and total pressure drop over the turbine. 199

Figure 5.27. Comparison of axial and whirl velocities after the diffuser. 200

Figure 5.28. Comparison of static and total pressure profiles after the diffuser. 200

Figure 5.29. Experimental layout showing various stations used to calculate efficiency.............. 201 
Figure 5.30. Graphical comparison of turbine efficiencies (low pressure drop case 1)................205

Figure 5.31. Graphical comparison of turbine efficiencies (high pressure drop case 2)...............205

Figure C 1. Source data for (Insert cross reference) figure 5.5................................................2 236 


\section{LIST OF TABLES}

Table 1.1. Advantages and disadvantages of the solar chimney technology.

Table 2.1 Standard conditions at collector inlet for solar chimney...... 40

Table 2.2 Values of solar chimney efficiency as derived from equation (2.20). (Figure 2.4) ......40

Table 2.3 Values used in calculations with kinetic energy losses............................................49

Table 2.4. Symbols and numerical values used for sample solar collector calculation. ................58

Table 2.5. Comparison of turbine mass flow rate and pressure drop at intersection of maximum

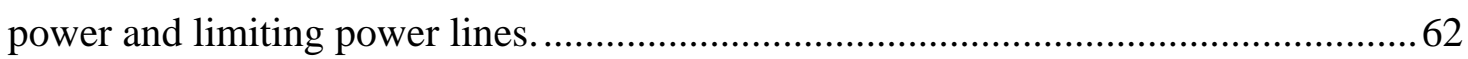

Table 2.6. Summary of solution methods used in solar collector simulation. ..............................70

Table 2.7 Material properties for experimental solar chimney plant, Manzanares, Spain............72

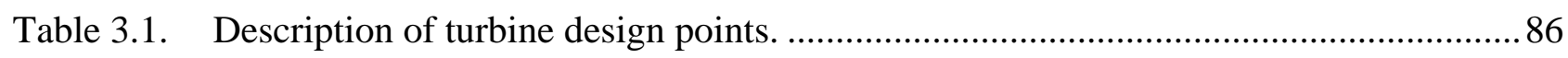

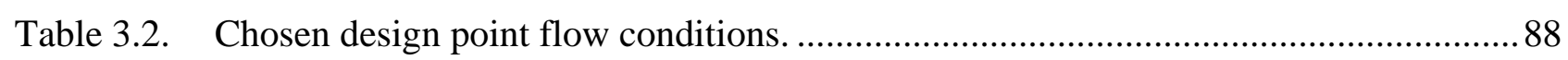

Table 3.3. Comparison of specific power for wind, solar chimney and gas turbines. ..................90

Table 3.4. Initial turbine and inlet guide vane dimensions relative to chimney......................... 102

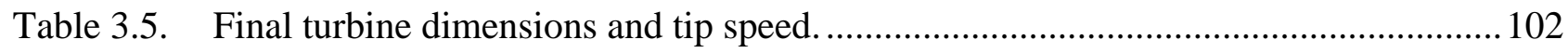

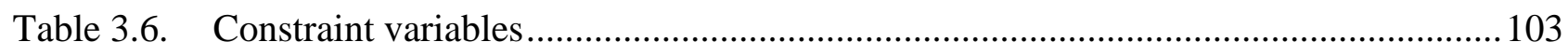

Table 3.7. Tables of operating efficiencies and flow angles for all design modes. .....................103

Table 3.8. Numerical comparison of surface vortex analysis with Abbott and Von Doenhoff for

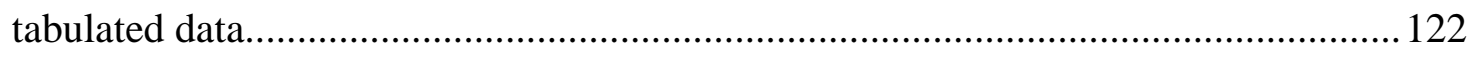

Table 3.9. Laminar-separation-point prediction by two methods. ............................................. 128

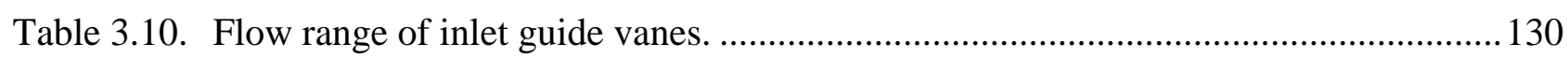

Table 3.11. Blade profile geometry of modified NACA 4-digit profile for IGVs.......................131

Table 3.12. Cascade geometry for radial layout of IGV's. ...................................................... 131

Table 3.13. Size constraints applied to experimental blade profiles ............................................. 134

Table 3.14. Relative inlet and outlet flow angles of turbine blades. .............................................135

Table 3.15. Blade profile geometry of modified NACA 4-digit profiles for turbine blades for low

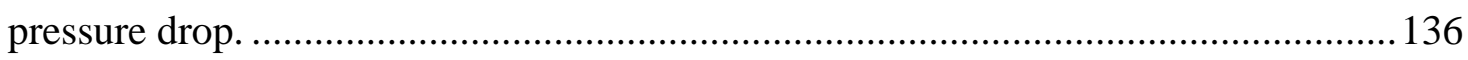

Table 4.1. Initial turbine and inlet guide vane dimensions relative to chimney........................145

Table 4.2. Experimental rig operational parameters. ............................................................. 146

Table 4.3. Windmill anemometer operating characteristics ................................................. 160

Table 4.4. Nominal and maximum errors for operational mode (1), Table 4.2 .........................167

Table 4.5. IGV and turbine blade stagger angle changes for experimental readings..................169

Table 5.1. Rotor blade stagger angles at tip measured from the axial direction. .......................173 
Table 5.2. Inlet guide vane adjustment about the design angle. .173

Table 5.3. Experimental total-to-static efficiencies measured at design points. 176

Table 5.4. Comparison of experimental and predicted total-to-static efficiencies at the design points. 178

Table 5.5. Comparison of volume flow rates from windmill anemometer with 5-hole probe.....189

Table 5.6. Comparison of torque and power from torque transducer and speed-reader with 5-hole probe. 190

Table 5.7. Average turbine loss coefficients. 192

Table 5.8. Average turbine rotor lift and drag coefficients. 193

Table 5.9. $\quad \alpha$ values at a) turbine exit/diffuser entrance and b) one pipe diameter downstream of the diffuser exit for total and axial velocity components. 193

Table 5.10. Diffuser efficiency and pressure recovery 194

Table 5.11. Summary of full-scale turbine data. 194

Table 5.12 Comparison of rotor efficiencies (no loss, NL)......................................................204

Table 5.13. Stage efficiency at rotor exit. 204

Table 5.14. Comparison of efficiencies after diffuser. 204

Table 5.15. Full-scale plant efficiencies with longer diffuser. 204

Table 5.16. Flow and load coefficient for $100 \%$ and $200 \%$ of case (1) torque at turbine start......207

Table 6.1. Summary of solar chimney turbine measured and predicted efficiencies. 208

Table A.1 Specific power output $\mathrm{P}_{2}$ * for solar chimney using equation (2.22). (Figure 2.4)......215

Table A.2 Turbine pressure drop [Pa] for solar chimney using equation (2.30). (Figure 2.5) ....215

Table A.3 Required mass flow [tonnes] for design power output of 200MW using equation (2.31). (Figure 2.6)

Table A.4 Power / unit area $\left[\mathrm{W} / \mathrm{m}^{2}\right]$ and exit velocity $[\mathrm{m} / \mathrm{s}]$ for maximum power and choking conditions for chimney height $=1500 \mathrm{~m}, \eta_{\text {turb }}=80 \%$ and $\mathrm{k}=1$. (Equation (2.43), Figure 2.9)

Table A.5 Required Diameter [m] to obtain design power at max power condition for chimney height $=1500 \mathrm{~m}, \eta_{\text {turb }}=80 \%$ and $\mathrm{k}=1$. (Equation (2.62), Figure 2. 10) .....

Table A.6 Power [MW], exit velocity [m/s], mass flow [tonnes/s] for maximum power, choking and limiting condition for chimney height $=1500 \mathrm{~m}$, diam $=160 \mathrm{~m} \eta_{\text {turb }}=80 \%, \mathrm{k}=1$ and $\alpha=1.0575$. (Equation (2.43), Figure 2.11, Figure 2.12, Figure 2.13)

Table A.7 Sample values for cycle analysis with solar collector included. Power [MW], exit velocity $[\mathrm{m} / \mathrm{s}]$, mass flow [tonnes/s] for maximum power, choking and limiting condition for chimney height $=1500 \mathrm{~m}$, diam $=160 \mathrm{~m} \eta_{\text {turb }}=80 \%, \mathrm{k}=1$ and $\alpha=$ 1.0575. (Figure 2.18, Figure 2.20, Figure 2.22, Figure 2.23 and Figure 2.21) 218 
Table C.1. Numerical values of data for sample calculation point.

Table D.1 Experimental turbine performance details............................................................2239

Table D.2 Full-scale turbine performance details...................................................................240

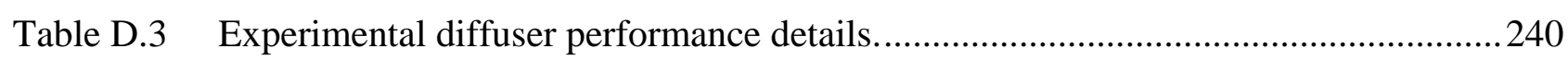

Table D.4 Full-scale diffuser performance details. ................................................................. 241

Table D.5 Turbine flow power $\mathrm{P}_{\text {flow }}$ calculation values. ........................................................241 


\section{NOMENCLATURE}

SYMBOL

A

AR

a

B

$\mathrm{Bi}$

Bir

b

C

$\mathrm{C}_{\mathrm{p}}$

$\mathrm{C}_{\mathrm{pD}}$

C

$\mathrm{C}_{\mathrm{p}}$

D

$\mathrm{D}_{\mathrm{s}}$

e

F

Fo

G

g

$\mathrm{H}$

$\mathrm{H}_{\mathrm{T}}$

$\mathrm{H}_{\text {turb }}$

$\mathrm{h}$

$\mathrm{I}_{\text {nose }}$

K
Description

Area

Area Ratio

Hub-to-tip turbine temperature drop ratio

Intermediate constant in quadratic equation

Experimental error estimate

Biot number (finite difference form)

Radiative Biot number

Axial chord length

Intermediate constant in quadratic equation

Mean-to-tip turbine temperature drop ratio

Absolute velocity

Coefficient of pressure

Diffuser pressure recovery coefficient

Blade chord length

Compression temperature ratio

Constant used in free-vortex analysis

Intermediate constant in quadratic equation

Specific heat capacity

Diameter

Specific diameter

Emissivity

Experimental error estimation variable

Fourier number (finite difference form)

Solar radiation

Gravitational constant

Blade height

Chimney height

Height of turbine off the ground

Enthalpy

Heat transfer coefficient

Profile nose radius

Dimensionless coupling coefficient

Extinction coefficient

Stratford separation criteria constant $\mathrm{m}$

\section{Units}

$\mathrm{m}^{2}$

$-$

$-$

$\mathrm{m} / \mathrm{s}$

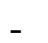

$-$

m

$-$

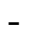

$\mathrm{J} / \mathrm{kg} \mathrm{K}$

$\mathrm{m}$

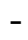

-

$-$

-

$\mathrm{W} / \mathrm{m}^{2}$

$\mathrm{m} / \mathrm{s}^{2}$

$\mathrm{m}$

$\mathrm{m}$

$\mathrm{m}$

$\mathrm{J} / \mathrm{kg}$

$\mathrm{W} / \mathrm{m}^{2} \cdot \mathrm{K}$

$-$

$-$

$1 / \mathrm{m}$ 
$\mathrm{k}$

L

M

$\dot{\mathrm{m}}$

$\mathrm{m}_{\text {ord }}$

$\mathrm{m}_{\mathrm{t} \max }$

$\mathrm{N}$

$\mathrm{N}_{\mathrm{s}}$

n

$\mathrm{P}$

$\mathrm{p}$

pord

Q

$\mathrm{R}_{\mathrm{C}}$

Re

$\mathrm{R}_{\mathrm{T}}$

r

S

$\mathrm{T}$

t

$\mathrm{t}_{\text {max }}$
$\mathrm{U}_{\infty}$
$\mathrm{V}$
$\mathrm{V}_{\infty}$
$\mathrm{W}$
$\mathrm{X}$

$\mathrm{x}$
Coupling coefficient

$1 / \mathrm{m}$

Thermal conductivity

$\mathrm{W} / \mathrm{m} \mathrm{K}$

Kinetic energy loss coefficient

Torque

$\mathrm{Nm}$

Mass flow rate

Profile maximum ordinate (camber)

Position of profile maximum thickness

Number of blades in radial cascade

Specific speed

Power law profile exponent

Refractive index

Power

Pressure

Position of profile maximum ordinate (camber)

Air heat gain

Volume flow rate

Collector canopy radius

Reynolds number

Chimney radius

Cycle pressure ratio

Radius from chimney axis

Reflectivity coefficient

Blade surface element length

Entropy

Temperature

Cycle temperature ratio

Cascade pitch

Time

Profile maximum thickness

Aerofoil freestream velocity (y-component)

Relative velocity

Aerofoil freestream velocity (x-component)

Freestream velocity

Blade surface element start point x co-ordinate

Minimisation goal function

Vertical depth below soil surface

Blade surface element midpoint x co-ordinate

Minimisation variables

Profile co-ordinate, chord direction
W

$\mathrm{Pa}$

$\%$

W

$\mathrm{m}^{3} / \mathrm{s}$

$\mathrm{m}$

$\mathrm{m}$

$-$

$\mathrm{m}$

$\mathrm{m}$

$\mathrm{J} / \mathrm{kg}$

$\mathrm{K}$

m

$\mathrm{S}$

$\%$

$\mathrm{m} / \mathrm{s}$

$\mathrm{m} / \mathrm{s}$

$\mathrm{m} / \mathrm{s}$

$\mathrm{m} / \mathrm{s}$

m

m

m

$\mathrm{kg} / \mathrm{s}$

$\%$

$\%$

$\%$

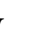

$\mathrm{m}$

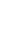

.

m

m 
Blade surface element start point y co-ordinate m

\section{GREEK}

$S Y M B O L$

\section{Description}

\section{Units}

$\alpha$

Absolute flow angle

rad

Absorbtance

Kinetic energy correction factor

Thermal diffusivity

$\mathrm{m}^{2} / \mathrm{s}$

$\alpha_{\infty}$

Profile freestream angle

rad

$\beta$

Relative flow angle

rad

$\beta_{\infty}$

Blade surface element angle

rad

$\Delta$

Change in quantity

$\phi$

Flow coefficienct

Latitude

deg

$\phi_{\mathrm{d}}$

Solar declination

deg

$\Gamma$

Circulation strength around aerofoil

Specific heat ratio of a gas (air $=1.4$ )

$\gamma$

Surface vortex strength

$\eta$

Efficiency

$\Lambda$

Swirl fraction

Blade surface velocity

$\mathrm{m} / \mathrm{s}$

Hub-tip ratio

Kinematic viscosity

$\mathrm{m}^{2} / \mathrm{s}$

Angle of radiation

$\mathrm{rad}$

Density

$\mathrm{kg} / \mathrm{m}^{3}$

Stefan-Boltzmann constant

$\mathrm{W} / \mathrm{m}^{2} \cdot \mathrm{K}^{4}$

Transmissivity

Rotor rotational speed

$\mathrm{rad} / \mathrm{s}$

Hour angle

deg

Load coefficient

Stream function value

Loss coefficient 


\section{SUBSCRIPT}

"

$\|$

$\perp$

$\infty$

1

$1^{\prime}$

2

3

3te

4

a

atm

available

chim

col

D

diff

exp

gli

glo

hub

i

ideal

IGV

in

1

lam

lift

loss

M

\section{Description}

Ideal process

Ideal process in chimney

Parallel

Perpendicular

Freestream

Cascade inlet

Inlet guide vane inlet

Solar gas turbine compressor inlet

Solar chimney atmospheric inlet

Cascade exit

Turbine rotor inlet

Solar collector inlet

Turbine rotor exit

Solar collector exit

Turbine exit

Chimney exit

Absorbtive

axial

Atmospheric property

Power available

Chimney

Collector

Diffuser

Diffuse

Expansion

Collector cover lower surface

Collector cover upper surface

Rotor hub

Collector inlet or exit value

Ideal or reversible process

Inlet guide vane exit

Collector cover inlet transmissivity values

Profile trailing edge

Stratford laminar separation criteria

Power required to lift air in chimney

Loss term

Number of surface vortex elements 
m

m'

$\max$

n

o

out

$\mathrm{P}$

$\mathrm{P}+1$

R

r

required

$\mathrm{S}$

S

sec

shaft

soil

surf

strat

te

tip

trans

ts

turb

turb lim

u

V

Z

$\theta$

Discretisation numbering for soil, control volume centre Meridional velocity

Surface vortex element number

Discretisation numbering for soil, control volume interface Maximum value

Surface vortex element number

Collector outer or inlet value

Stagnation quantities

Collector cover outlet transmissivity values

Previous time step

Current time step

Rotor

Radiative

Reflective

Power required from plant

Stator or IGV (stationary blade row)

Blade surface

Static quantity

Secondary losses

Turbine shaft

Soil properties under collector cover

Collector soil surface

Stratford separation criterion

Trailing edge

Rotor blade tip

Transient

Total-to-static

Turbine

Stratford turbulent separation criterion

Limiting turbine temperature or pressure drop

Profile surface element velocity $\mathrm{x}$-component

Profile surface element velocity y-component

Axial or in direction of chimney flow

Tangential

\section{SUPERSCRIPT Description}

Normalised quantity 
Solar chimney atmospheric inlet

Mean value

Indicates angle referenced in degrees

\section{ABBREVIATIONS}

ADDA Analogue-to-Digital Digital-to-Analogue

HTF Heat transfer fluid

IGV Inlet Guide Vanes

KE Kinetic Energy

MTFM Matrix Throughflow Method

PC Personal Computer 


\section{CHAPTER 1: INTRODUCTION}

The main objective of the thesis is to investigate the performance of solar chimney power plant turbines. The operation of a solar chimney power plant is based on a simple principle: when air is heated by the greenhouse effect under the large glass solar collector, this less dense hot air rises up a chimney at the centre of the collector. At the base of the chimney is the turbine driving a generator (Figure 1.1). The only operational solar chimney power plant built was an experimental plant in Manzanares, Spain (Haaf et al. 1983). This proved that the concept works. Plans for further plants are on a far greater scale and the turbine pressure drop is expected to be significantly higher. The large base pillars that are required to support potentially the highest man-made structures built also impose constraints on the turbine design. There is a need to investigate this class of turbine, the effect of the base pillars and the radial to vertical flow duct at the chimney base.

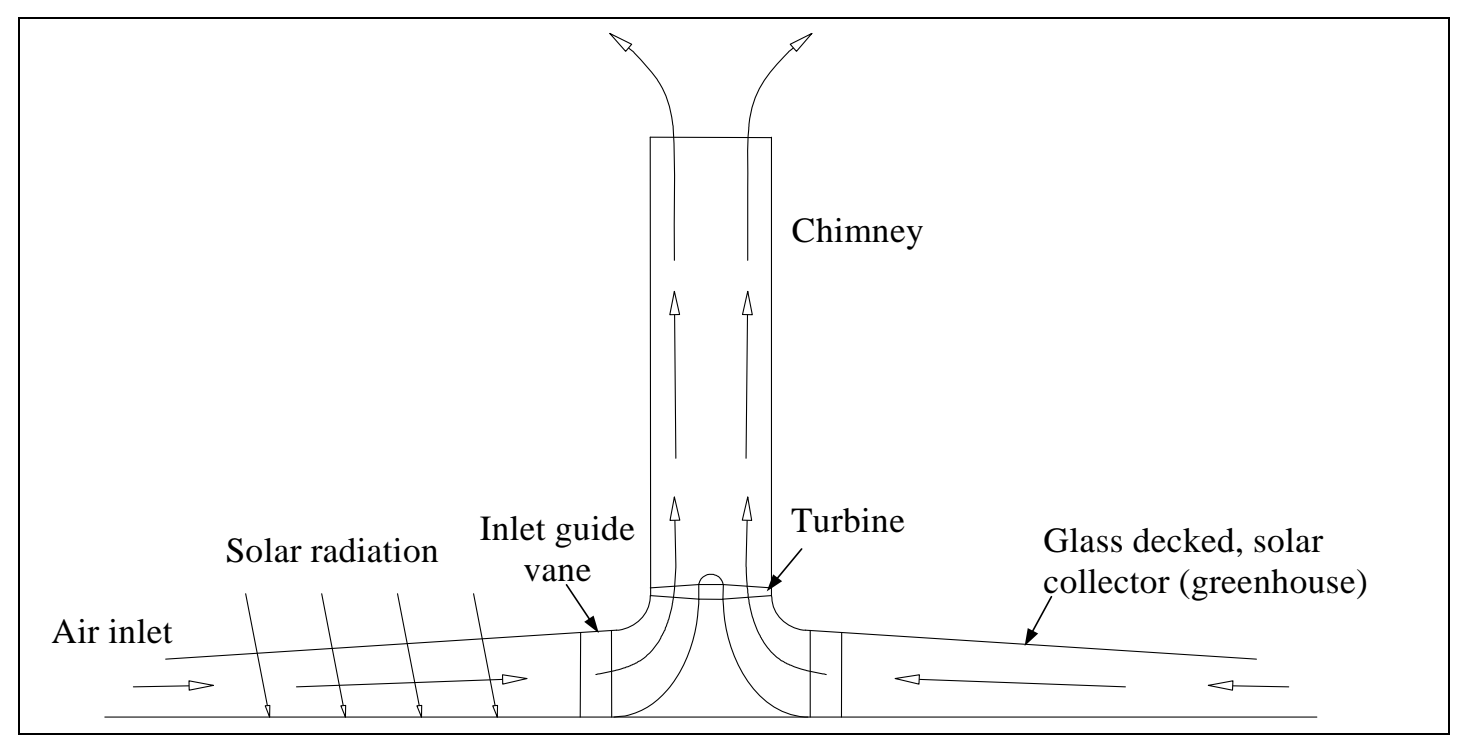

Figure 1.1 Solar chimney power plant schematic

Solar Chimney Power Plant Analysis: Since the Manzanares plant there has been no further construction of operational plants. A full-scale solar chimney is a capital-intensive undertaking and before building one a good understanding of plant operation is required. The analyses that have been performed have tended to simulate the plant operation at a particular operating point. To design the turbine effectively its operating region must be defined. At the outset of the present project the turbine operating range had not been well defined and so it was necessary to perform a simulation of the plant. This was done for a single day of operation (Von Backström and Gannon 2000). Since this time more detailed and comprehensive simulations have been performed (Kröger and Buys 2001).

Turbine Design: The turbine of the solar chimney is an important component of the plant as it extracts the energy from the air and transmits it to the generator. It has significant influence on the 
plant as the turbine pressure drop and plant mass flow rate are coupled. The turbine must operate efficiently and be correctly matched to the system to ensure proper plant operation. Designing a turbine for an incorrect operating point may result in unpredictable plant operation. Phenomena such as stall may occur resulting in a sudden decrease in the turbine pressure-drop. The raw data showing pressure drop, volume flow rate and power output allowed rudimentary turbine efficiencies to be calculated for the Manzanares plant (Haaf 1984). The turbine efficiency based on these readings was found to be lower than predicted. This is thought to be due to the turbine operating away from its design region. The need exists to demonstrate that a suitable turbine can be built that can operate at a high efficiency in the required design range for a full-scale plant.

\section{Background to Solar Powered Energy Sources}

Figure 1.2 shows that energy consumption in the ten years from 1989 to 1998 has increased significantly and there seems no reason for it to decrease in the future. Renewable energy still only accounts for a tiny fraction of overall power generation. An increase in the interest of renewable energy sources has seen a number of designs and concepts created to try and harness the various renewable sources such as solar and wind. These factors make research into this field potentially lucrative, apart from the environmental benefits. Figure 1.2 also shows the trends in energy consumption and the various energy sources over a decade. The potential for growth in renewable energy is large. Renewable energy sources are attractive because of their small environmental impact and potential to generate cheap power in the long term.

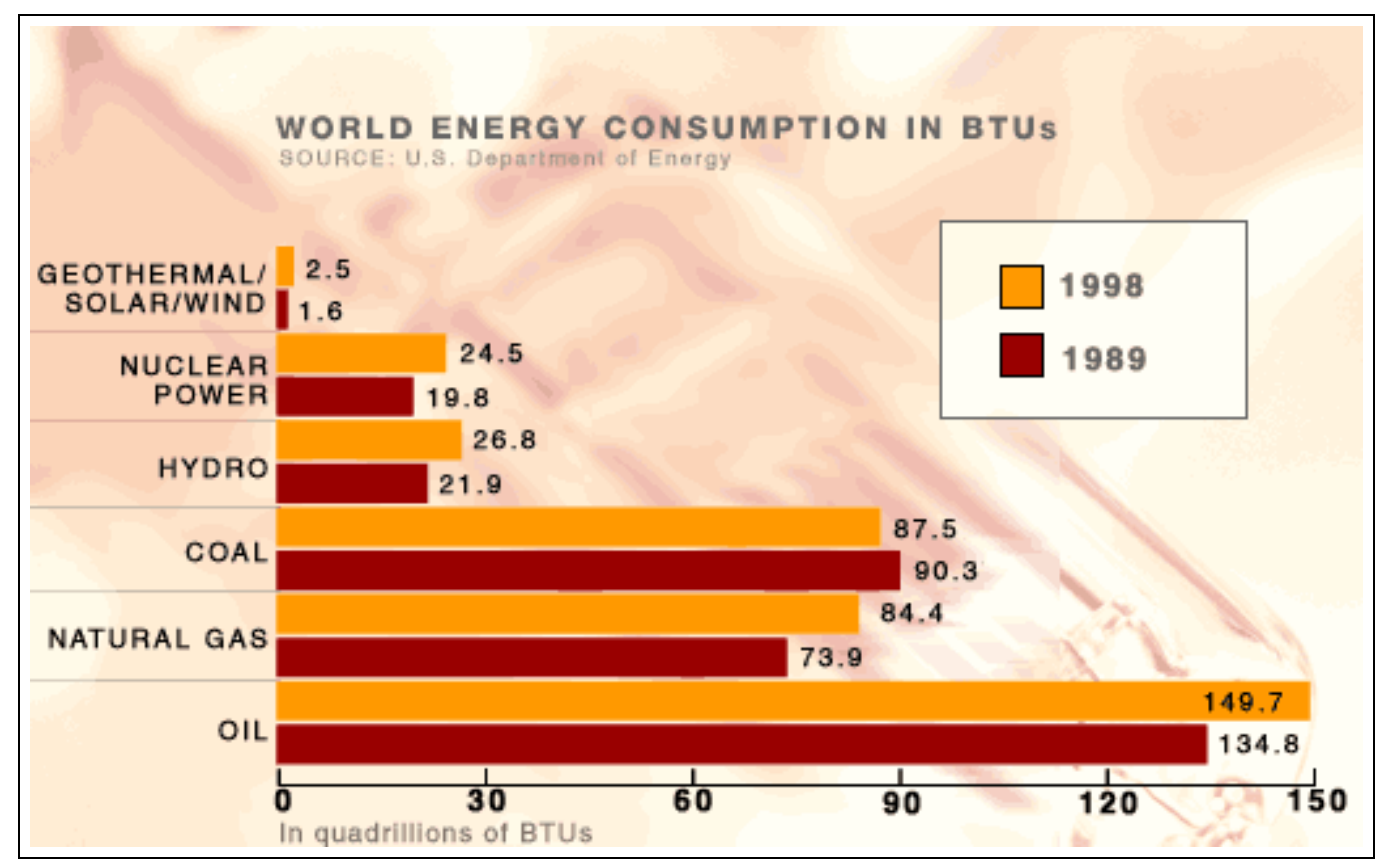

Figure 1.2. World energy consumption and sources of energy for 1989 and 1998 (US Department of Energy Web Site). 
There are a number of different methods of generating power from solar radiation. It is useful to investigate these briefly and compare them to the solar chimney. The comparison given here is largely based on the work by Trieb et al. (1997) supplemented with additional knowledge gained by studying the solar chimney plant. The main solar technologies that are being investigated on a large scale are listed along with their primary characteristics below.

Parabolic Trough Solar Electric Generating System (SEGS): The solar receiver consists of rows of reflective parabolic troughs. Along the focal line of these troughs are black absorber tubes that contain either a synthetic oil or water. In the case of oil it is used to heat water in a separate heat exchanger. In the case of water, steam is created directly and used to drive a turbine to create electrical power. They can be built in a modular fashion in a power range of 30-150 MW.

Central Receiver Power Plants: In this type of plant a large field of two axis tracked mirrors (heliostats) concentrate direct beam radiation onto a central receiver, mounted on the top of a tower. A number of absorber concepts have been tested: direct steam generating tubular receivers, open volumetric air receiver, molten salt tubular or film receiver and others. Usually a normal steam cycle is connected to the system for the electricity generation. Heat storage can be included in the system to reduce the effect of solar fluctuations. The molten salt concept is especially well suited to this.

Solar Chimney Power Plant: This concept uses both the diffuse and direct incoming solar radiation. Heat storage in the ground is inherent to the solar collector and it could be vastly improved through the use of water bags. The small temperature gradients found in the solar chimney make heat storage effective as heat losses to the environment are low.

Dish-Stirling Systems: This type of plant makes use of direct beam radiation that is focused using a paraboloidal dish reflector that is tracked on two axes. The heat absorber is usually a tube- or heat-pipe-absorber that is placed at the focal point of the dish reflector. The Stirling engine is an externally heated reciprocating piston engine with working fluids of either hydrogen or helium.

Solar Pond Power Plants: The naturally occurring phenomenon of a salt gradient in ponds allows hot water to rest on the bottom. High temperature water is able to dissolve more salt. The density of the liquid increases with the salt concentration. This results in a higher density and temperature stable layer in the bottom of the ponds. A black absorbing surface is placed on the pond bottom and temperatures here can reach $90^{\circ} \mathrm{C}$ without convection losses. A fluid with a boiling point of less than $100^{\circ} \mathrm{C}$ is used to generate power in a separate cycle. Significant energy storage is possible in salt gradient ponds.

Photovoltaic Power Plant: This is probably one of the most commonly known methods of solar electricity generation. These semiconductor devices have the ability to convert sunlight into direct 
current. They can be coupled in series and parallel to generate high voltages and powers. Energy storage is only possible using batteries.

Summary: The following table is taken from Trieb et al. (1997) with some changes. It summarises the advantages and disadvantages of the solar chimney power plant generation scheme. For more detail consult Trieb et al. (1997). They summarise the advantages and disadvantages of the various solar power generation schemes. making for simpler comparison between them. The following table summarises their views on the solar chimney.

Table 1.1. Advantages and disadvantages of the solar chimney technology.

\begin{tabular}{|c|c|}
\hline Advantages & Disadvantages \\
\hline \multicolumn{2}{|l|}{ Solar Chimney Power Plant } \\
\hline $\begin{array}{l}\text { - The glass collector uses diffuse and } \\
\text { beam radiation. } \\
\text { - The soil under the collector acts as } \\
\text { heat storage, avoiding sharp } \\
\text { fluctuations and allowing power } \\
\text { supply after sunset. } \\
\text { - Easily available and low cost materials } \\
\text { - for construction. } \\
\text { - Simple fully automatic operation. } \\
\text { - } \text { Po water requirements. } \\
\text { storage in collector to extend operating } \\
\text { hours. }\end{array}$ & $\begin{array}{l}\text { - Low thermodynamic efficiency. } \\
\text { - Hybridization not possible. } \\
\text { - Large, completely flat areas required for the } \\
\text { collector. } \\
\text { - Large material requirements for the chimney and } \\
\text { for the collector. } \\
\text { - Very high chimneys necessary for high power } \\
\text { output (e.g. 750m for a 30MW plant). } \\
\text { - Cosine losses high for low solar angles. }\end{array}$ \\
\hline
\end{tabular}

\subsection{LITERATURE STUDY}

The following literature survey focuses on the solar chimney but also includes other renewable energy sources and axial flow turbine designs. The solar chimney papers give insight into how far the field is developed and how previous research has focused on the overall plant analysis. It can be expected that the solar chimney will undergo a similar process of improvement as other renewable energy sources such as wind and hydroelectric power.

\section{Solar Chimney History}

The development of the solar chimney is presented briefly in Haaf et al. (1983) and Schlaich (1995). Haaf refers to an earlier study published by Simon (1976). Haaf points out that the concept is not new but was used by Leonardo da Vinci to design a spit that was driven by the air rising through the chimney (Figure 1.3). 
Haaf (1984) presents the results of the plant commissioned and operating in Manzanares, Spain. This plant proved that the concept of the solar chimney did work and operated reliably from 19821989 (Schlaich 1995).

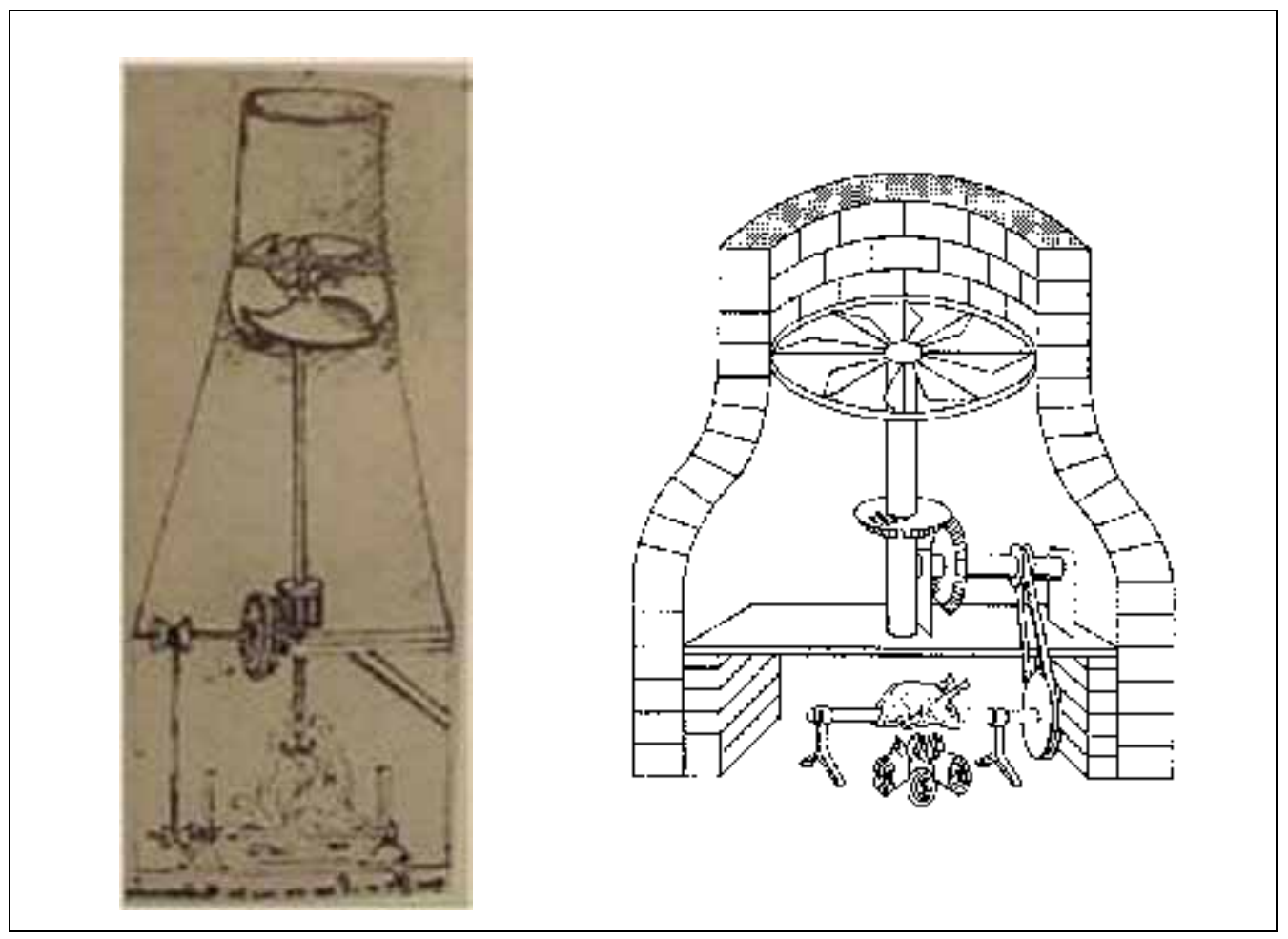

Figure 1.3 Spit of Leonardo da Vinci driven by rising hot air.

\section{Solar Chimneys}

In the open literature early references to solar chimneys are by Haaf et al. (1983) who dealt with the principle and construction of the plant in Manzanares, Spain. A second paper (Haaf 1984) dealt with the preliminary test results from the plant. Inspection of the available experimental data from the Manzanares plant raises some questions about the plant operation. In Haaf (1984) plant performance is presented for a day of operation.
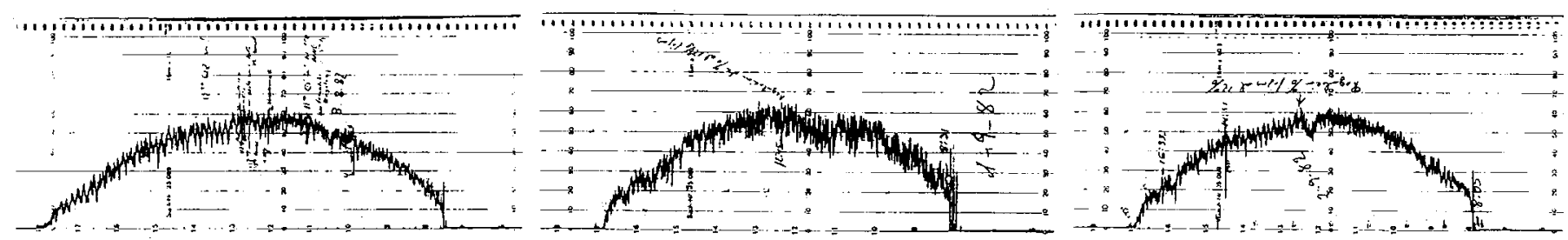

Figure 1.4. Power output records of experimental plant in Manzanares, Spain.

Figure 1.4 is taken directly from Haaf (1984) and cyclic fluctuations in the power output can be seen. The corresponding solar radiation for the central figure is presented later in the thesis and is relatively smooth. The plant design power was also quoted as being $50 \mathrm{~kW}$ while the maximum output power was $37 \mathrm{~kW}$. These aspects are covered in greater detail later. 
Since Haaf (1984) there have been sporadic publications dealing with the analysis of solar chimneys. Mullet (1987) investigates the design, performance and efficiency of a solar chimney. More recent research by Pasumarthi \& Sherif $(1997,1998)$ present the construction and testing of a small scale chimney. The testing focused on the heat transfer performance of the collector and methods to try and improve this.

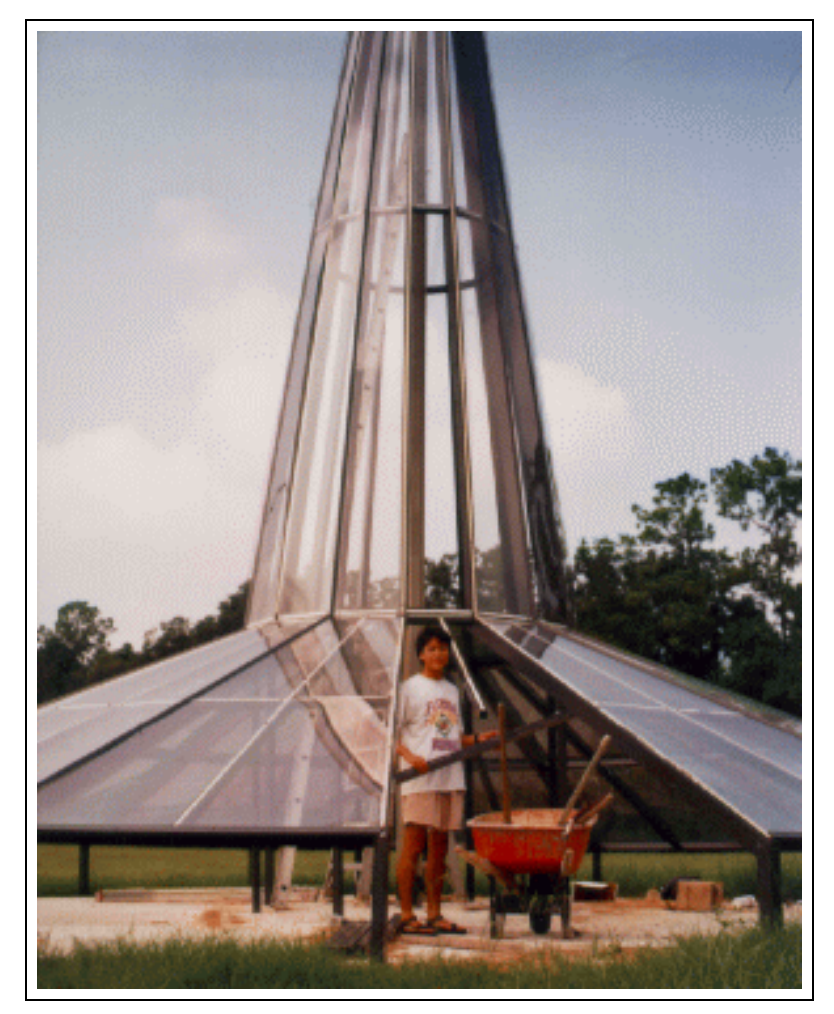

Figure 1.5. Demonstration solar collector (Pasumarthi \& Sherif 1997, 1998)

The overall plant analysis has been performed using two approaches, the first based on balancing the pressures through the plant. The first of these methods is presented by Haaf et al. (1983) and in the booklet by Schlaich (1996). This provides a useful background to the solar chimney and the project in Manzanares, Spain. The use of heat storage in water stored in bags or pipes is suggested. This would allow excess daytime energy to be stored to and released later for extended operation. The book does highlight one of the underlying assumptions borrowed from gas turbine theory.

Most existing analysis methods predict the solar chimney output for a given collector temperature rise within reasonable accuracy. Problems begin to arise, however, when the plant operating range is predicted. A gas turbine approach is often used in the presentation of the plant data (Schlaich 1996). What is meant is that power output is plotted along lines of constant collector temperature rise, as is common practice in gas turbine texts. The next assumption is usually to assume that the plant will operate along the maximum power line shown in Figure 1.6 with the turbine pressure drop equal to $2 / 3$ of the zero flow pressure drop (Figure 1.7). In doing this it is assumed that the collector temperature rise is independent of the mass flow. This is not the case as the temperature 
rise of the collector air and mass flow rate are coupled. If the inlet radiation $\mathrm{G}$ is assumed constant for a few minutes, the heat flow equation $G=\dot{m} c_{p} \Delta T_{23}$ shows that a decrease in the mass flow rate $\dot{\mathrm{m}}$ will increase the temperature rise $\Delta \mathrm{T}_{23}$ and an increase in mass flow will decrease it.

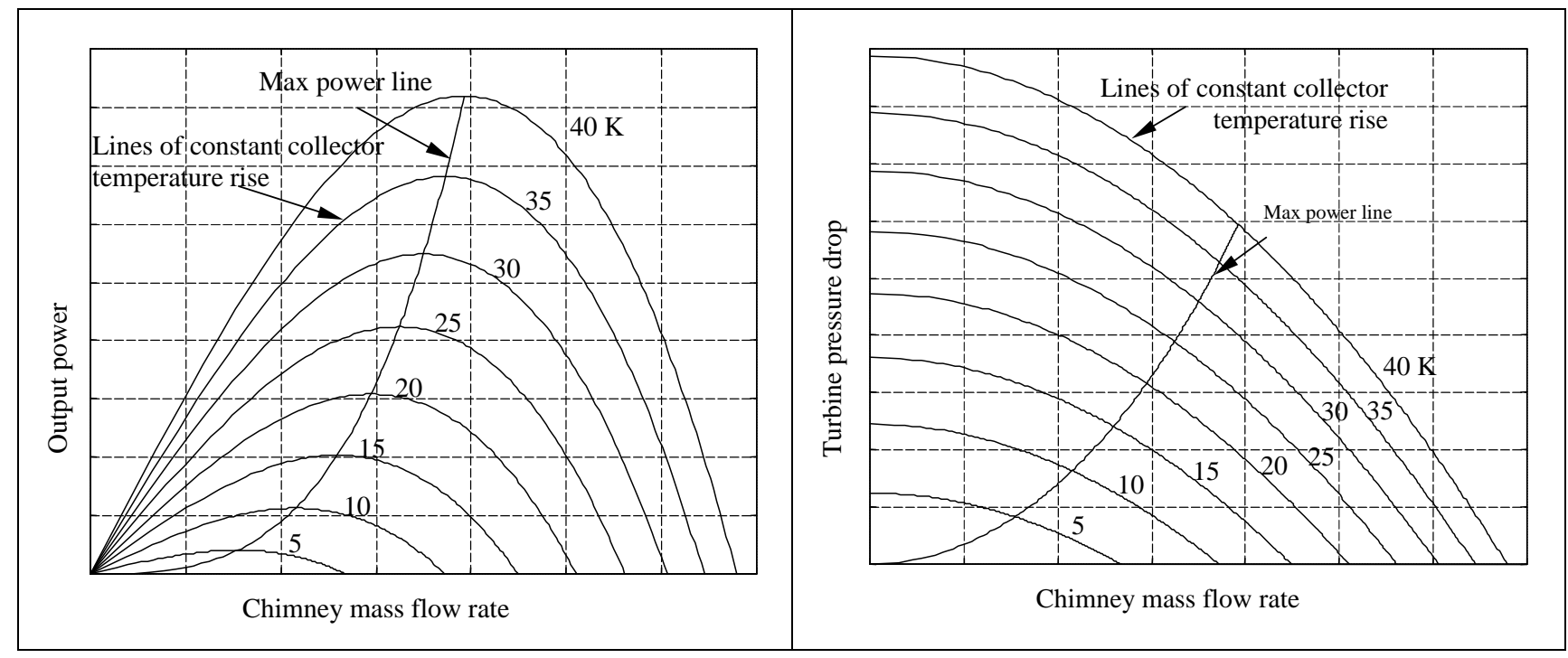

Figure 1.6. Power output represented along lines of constant collector temperature rise
Figure 1.7. Pressure drop along lines of constant collector temperature rise

The second analysis approach applies a fundamental air standard cycle and was pioneered by Von Backström \& Gannon (2000). This was used to find certain optimum operating points and also to find the required mass flows for various configurations of chimney height and gas temperatures. The cycle analysis has been extended to include system losses and a transient collector model by Gannon and Von Backström (2000). Kröger and Buys (2001) present a detailed plant analysis also with a transient collector to predict the maximum powers for a one year operational cycle.

Recent publications have started to focus in on the different areas of solar chimney operation: overall plant analysis (Von Backström \& Gannon 2000, Gannon \& Von Backström 2000) and compressible chimney flow (Von Backström \& Gannon 2000, Von Backström 2000). A proposed method of chimney construction is presented by Schlaich (1999) and the effect this may have on the chimney aerodynamics by Von Backström et al. (2002). Analysis of the chimney to calculate the driving potential and the effects of humidity are presented by Kröger and Blaine (1999). Von Backström and Gannon (2000) investigated the effects of compressibility of the flow through the chimney. Control of the plant and maximisation of the power output is presented in Gannon \& Von Backström (2002).

The concept of a solar collector to heat air is not unique to solar chimneys and there is literature freely available dealing with their simulation and operation. Kröger and Buys (1999) present work in their paper specific to the solar chimney collector. Maad and Belghith (1994) investigate improving the heat transfer rate of passive solar systems such as Trombe-walls and solar chimneys through the use of grids inserted in the flow to increase the level of turbulence. 
The turbine design for the Manzanares plant is presented in a report by Wortmann et al. (1981) based on the plant analyses available at that time. Von Backström (1998) used a fundamental approach to the turbine design through the investigation of the dimensionless diameter and speed. A useful publication that compares the different types of solar power plants is found in Trieb et al. (1997).

\section{Wind Turbines}

While the solar chimney is still at an early stage of development, wind turbines are already in operation and connected to the main grid in many countries. It is a mature technology and, like the solar chimney, uses a turbine. It was initially hoped that some of the knowledge of the operation and design of modern wind turbines could be applied to the solar chimney. In the comparison presented earlier, it can be seen, however, that solar chimney turbines have a much higher energy extraction per unit flow and are more akin to gas turbines.

The most useful guides to wind turbines have been two reviews of the technologies. At the outset to the present project a comprehensive guide to the current state of technology in wind turbines is presented in the British Wind Energy Conference (BWEA) (Hunter 1997). The publication is useful as it contains a number of theoretical analyses of wind turbines and the aerodynamics of the blade profiles and limiting operating conditions. A guide to the limiting axial velocity ratio (AVR) or, to use gas turbine terminology, the relative exit flow angle is presented. The types of blade profiles common to wind turbines such as the custom designed SERI and NACA 4 and 6 digit profiles are also presented. These provide useful starting points for the design of solar chimney turbines. Solar chimney turbines have a greater pressure drop than wind turbines and require more blades but not as many as gas turbines. It was found in the literature that wind turbine blades can be treated accurately as individual aerofoils. This is not the case in the solar chimney turbine due to the higher blade solidity (blade chord to pitch ratio).

As in wind turbine design there are certain practical aspects that need to be overcome to produce electricity with a solar chimney. Renewable energy is transient in its nature and the turbines must be able to handle these transient effects. It is expected that the solar chimney power plant will be connected to the main grid. For large generators $(>1 \mathrm{MW})$ it is necessary for them to run at a constant speed to be coupled to the power grid. At this early stage it was obvious that the turbine would have to run at a constant rotational speed.

One of the factors that may be relevant to the turbine design is the coupling of the generator to the electricity grid. In this regard the advice of an engineer involved in a pump-storage hydroelectric scheme was sought (Paul Nel 1999). In this communication certain practical aspects of coupling the generator to the grid were discussed, steady-state operation is usually stable once the turbine is running at a constant speed. Synchronisation of the generator with the grid requires that the speed 
and phase of the turbine are correct and this needs to be done by mechanical means such as adjusting the turbine blade angles.

Other environmental issues are those of aesthetics and noise. With a structure as large as a solar chimney, hiding it is almost impossible and so good site placement will be important. Noise issues, on the other hand, will probably not be as important as the edge of the plant is far from the turbine itself. These aspects, while important, were not covered in this project.

A more recent review of the technology is presented in Ackermann \& Söder (2000), showing that wind turbines are making significant inroads into power generation. Relevant to the solar chimney is the size of the new wind turbines with $1.5 \mathrm{MW}$ units with diameters of $70 \mathrm{~m}$ having been constructed and $4 \mathrm{MW} 88 \mathrm{~m}$ diameter plants being planned. This is of a similar diameter to that expected of the solar chimney turbine. It does demonstrate that very large turbines can be built. It must be remembered, however, that the loading on the solar chimney turbine is much higher than for a wind turbine.

There is far more literature available on wind turbines but only that which is directly relevant to the solar chimney is mentioned here. Rigid design procedures for turbines are better laid out in gas turbine texts.

\section{Gas Turbines}

Gas turbine engine technology is a field of intense and active research and has been so for a number of decades. The technology is mature but significant progress is still being made in almost all fields, for example materials and aerodynamics. More recent publications tend to be focused on more specific areas of turbomachinery development. Certain areas of gas turbine research are mature and the original papers are often the best source of information. The matrix throughflow method is an established 2-D inviscid analysis method. A formulation of the method by Bosman \& Marsh (1974) allows both axial and radial flow to be simulated. Two discretisation schemes that can be used to implement the method are Greyvenstein (1981) for the main grid and Harms et al. (1996) for finding local flow properties. A practical guide to the implementation of these methods is presented in Gannon (1996).

The blades required for the solar chimney turbine are of low solidity when compared to gas-turbine turbomachinery but they cannot be treated accurately as single blades like those of wind turbines. A problem with low solidity cascades as found in fans and in the solar chimney turbine is that little data exists to determine the deviation or influence of the blades on one another. Deviation in low solidity blades is significant and methods more accurate than extensions of empirical data are needed to accurately predict the flow. Lewis $(1991,1996)$ presents a surface vortex or Martensen method that is extended to cascade flow. It is an inviscid method suitable for subsonic accelerating 
flows. A boundary layer separation prediction method based on that of Stratford (1959) was used in conjunction with the surface vortex method.

The textbooks of Cohen et al. (1996) and Sayers (1990) provide useful design information such as the limiting relative exit angle and methods for choosing the initial number of blades and their aspect ratios. Granger (1988) provides useful information on the testing of a model turbine.

In many parts of the design processes the above analysis methods were coupled with optimisation methods by making use of the power of modern personal computers. The text of Rao (1996) provides a useful and practical guide to these methods. Almost all of the design was performed using the program MATLAB (1996). Optimisation is now commonly used in turbomachine design, either in overall cycle improvements or in specific areas such as producing low drag profiles. Such an approach is described in Howell et al. (2001) where high lift aft-loaded profiles were developed to reduce the number of blades in a low-pressure turbine.

Other references include the Diffuser Data Book (1975) for the diffuser design, Abbot \& Von Doenhoff (1959) for the blade geometry data for the NACA profiles and Ullman (1992) as a guide to the design process.

\section{Solar Collectors}

Solar collectors for heating air or for purposes of drying are already used and various methods for their modelling are available. A useful mathematical model and solution procedure for calculating the heat addition and temperature rise through a collector is presented by Ong (1995). This approach was used for the present analysis. A similar method for a high performance collector is presented by Mohamad (1997). Use is made of a porous collector covering and a counter flow collector to increase the collector efficiency. These improvements are possible additions to the solar chimney collector.

The ground beneath the solar collector required a transient model to take into account energy storage. The basic method used was obtained from the text of Incropera \& Witt (1990). Information on glass properties and the simulation of it with incoming solar radiation can be found in Duffy \& Beckman (1991). Private communications with Kröger (1999) and Harms (1999) were held in connection with the practical implementation of the above methods with further details given in the texts. Some material properties of the plant at Manzanares were obtained from the report of Schlaich (1995) but these were all very close to the typical values given in most texts. 


\subsection{OBJECTIVES}

The main objective of the thesis is to investigate the performance capabilities and limitations of solar chimney turbines. The following questions will be investigated:

- Is it possible to design a highly efficient turbine for large solar power plants?

- Can a successful compromise be found between the two functions of the radial flow inlet guide vanes of the turbine, namely: i) providing the optimum amount of pre-swirl and ii) supporting the chimney walls?

- Will a relatively small radius of curvature of the collector-top to the turbine-outer-wall induce flow separation?

- What level of turbine efficiency can be expected?

- How wide will the turbine operating range be?

- Will the turbine be self-starting?

- What will the runaway speed be?

- What is the most suitable design methodology to use?

The proposed research is outlined in the following paragraphs,

Cycle Analysis: An air standard thermodynamic analysis of the solar chimney cycle is presented. Such analyses assume ideal gas properties and no losses in the components. This has the great benefit of predicting what the limiting efficiencies of a solar chimney power plant are. It is impossible to perform better than the ideal thermodynamic cycle. Using the ideal cycle analysis as a base system, losses such as the turbine efficiency, frictional and exit kinetic energy losses need to be included. This is to ensure that the predicted efficiencies will be physically realistic. Even with the inclusion of only kinetic energy losses at the chimney exit a crude estimation of the plant operating range can be made. At some stage the kinetic energy losses will become greater than the plant power output thereby limiting the operating range.

Steady State Analysis: The solar collector is an integral part of the solar chimney yet many analyses have not taken its behaviour into account. A steady state analysis tends to predict too high temperature rises in the solar collector as the absorption of energy by the ground is not taken into account. It does, however, demonstrate the basic coupling of the temperature rise and mass flow rate in the collector. Presenting the plant power output along lines of constant solar radiation helps in illustrating this relationship graphically.

Transient Analysis: While the development of a steady-state collector model is simple, it is physically unrealistic as the assumption is made that the sun shines with a constant intensity indefinitely. There is also the effect of thermal storage in the ground under the collector that will affect the temperature rise. To properly account for these two factors, a transient analysis of the collector needs to be included in the solar chimney analysis. 
In the full-scale plant there may be a requirement to limit the plant power output. To purchase a generator that can operate at the plant peak power for a few minutes at midday is not economical. A generator that can operate at its rated power for a number of hours during the day will be chosen. There are a number of ways of limiting the plant power at midday. Some control methods are suggested.

Design Requirements: The solar chimney turbine has constraints on its geometry as it must operate at the base of the chimney. Large generators usually have to operate at constant speed and are locked into the frequency of the electricity grid. This of course means that the turbine rotational speed will be constant. The main design requirement of the turbine is that it operates efficiently at the required design points. Choosing or designing the correct type of turbine is important to ensure that the turbine will operate correctly. The turbine should be self-starting.

Design Scope: The current project investigated a single turbine that could be easily incorporated in the chimney base. A concept whereby the chimney base supports are used as inlet guide vanes to introduce pre-whirl into the turbine is investigated. This decreases the turbine exit whirl and increases the total-to-static efficiency. Non-radial chimney supports improve the torsional stiffness of the chimney base. The current project focused on the aerodynamic and geometric design of the turbine. The practical problems of building the full-scale turbine were not investigated.

With a more accurate determination of the turbine operating region in place, the second part of the project involved demonstrating that a high efficiency turbine could be designed. The first part of this phase involved an initial design process where the basic turbine layout was chosen. A rigorous design approach was used to evaluate a number of possible designs and ensure that the best one was chosen.

The eventual design was for a single axial turbine using a concept whereby the chimney supports act as inlet guide vanes (IGV). This was in an attempt to increase the turbine efficiency by reducing the exit whirl velocity. It had the added benefit of stiffening the base of the chimney.

Experimental Program: In the experimental plant in Manzanares, Spain (Haaf et al. 1983, Haaf 1984) basic turbine data of pressure drop, volume flow rate and power outputs were measured. Detailed velocity profiles were not measured as this would have been difficult on a large turbine. Building a test rig and scale model of the turbine allows the performance to be measured in detail without the operational constraints of a working plant being imposed. The results allow insight into the turbine operation to be gained as well as further design improvement. The starting torque and self-starting characteristics are also to be investigated. 


\section{CHAPTER 2: CyCle ANALYSis OF SOlAR CHIMNEY}

The aim of this chapter is to define the operating range of a full-size solar chimney plant to allow a turbine to be designed that is correctly matched to the required pressure drop and volume flow range. It also attempts to present some insights into the operation of the solar chimney. The basic principle and operation of the solar chimney is well understood. What is important is to understand the operating characteristics of the plant and quantify them. In this section it is simply assumed that a turbine can be built that can extract the correct power efficiently.

The analysis of the chimney begins with a simple air standard cycle analysis developed by Von Backström and Gannon (1998b). It is useful as it determines the upper performance limits of the ideal solar chimney plant. It is presented here for clarity. In this project the cycle analysis is extended to include system losses to obtain accurate predictions of the plant power output. A simple steady-state collector model is included in the simulation as this allows some insight into the solar chimney operation to be gained. A transient soil model is then added to allow the plant to be simulated as it would operate in the environment. The experimental plant built in Manzanares, Spain (Haaf et al. 1983), is simulated and the results are compared to verify the accuracy of the model. The final step is the simulation of a full-scale plant to determine the operating range of the turbine. Operation over a representative day's operation is simulated. The possibility of having to limit the power output of the plant to avoid generator damage is also covered briefly.

\subsection{Simple THERMOdYNAMIC ANALYSIS OF SOLAR CHIMNEY}

As a first step in calculating the operating range of the solar chimney turbine, it is useful to perform a thermodynamic cycle analysis. Schlaich (1995) and Haaf et al. (1983) have performed some simple cycle analyses for the solar chimney but as noted by Von Backström and Gannon (1998b) and Gannon and Von Backström (2000) a complete standard air cycle analysis had not been performed. They are relatively simple to perform and can be done without a detailed working knowledge of the components of a plant. They are useful in finding the relationships between the main operational variables and from the analysis it will be seen that the two most important variables in terms of power output in the solar chimney are the chimney height and the solar collector temperature rise.

Other objectives achieved in performing the cycle analysis were to determine if there were any optimum operating points and the thermal efficiency of the solar chimney. The assumptions used in the analysis are given by Von Backström and Gannon (1998b) and are as follows:

- The mass flow through the system is constant.

- Compression and expansion processes are adiabatic and reversible (isentropic). 
- The change in kinetic energy of the air between the inlet and exit of each component is negligible.

- The working fluid is a constant composition perfect gas throughout, with constant specific heat.

\section{Analysis of a Solar Gas Turbine Cycle}

It is a useful exercise to perform a gas turbine analysis of the solar chimney power plant as the cycle is fundamentally the same. There are however some practical differences in the operation of the plant, the first being that in a gas turbine the inlet and exit conditions are identical. In the solar chimney the atmosphere not only cools the exhaust air but also recompresses it back to the inlet air temperature and pressure as is descends from chimney top altitude to collector inlet altitude.

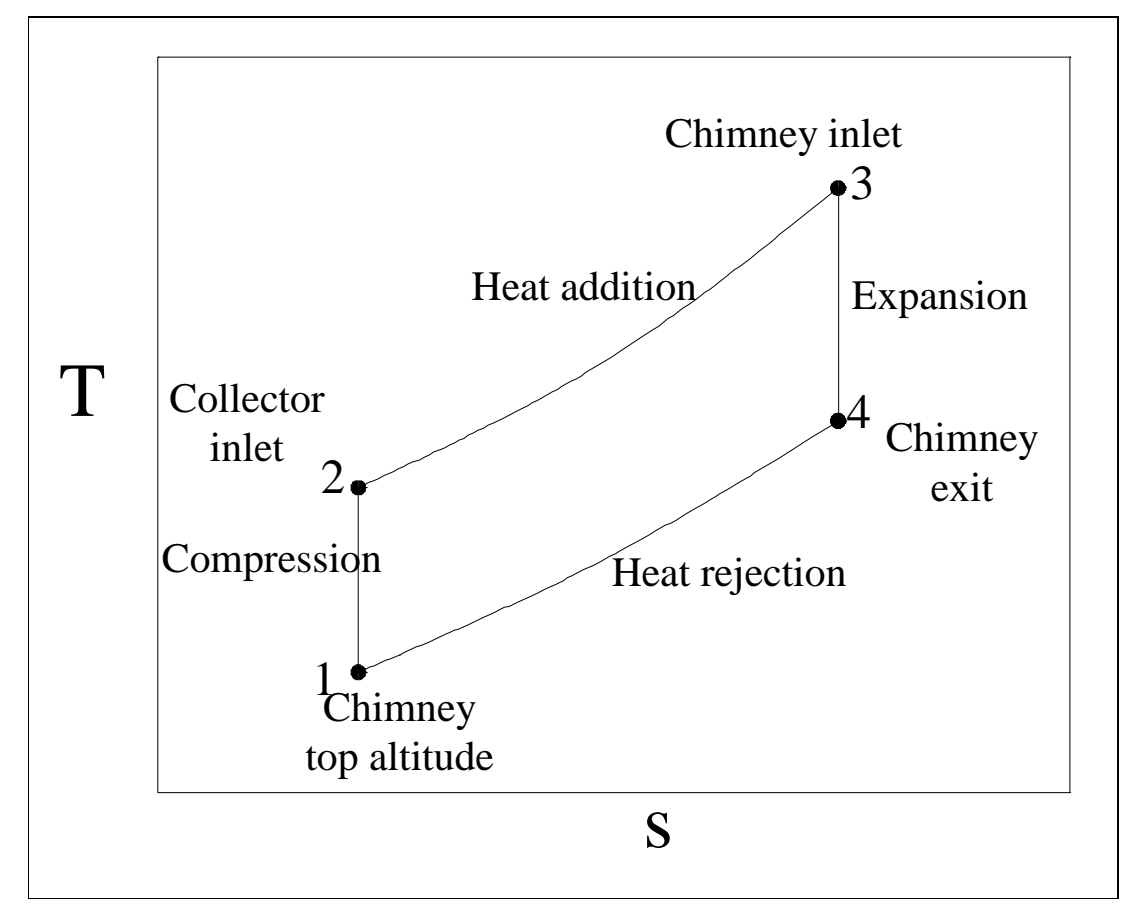

Figure 2.1. Temperature-entropy (T-s) diagram for gas turbine air standard cycle.

The T-s diagram for a simple gas turbine cycle is shown in the above figure. The analysis is taken from Cohen et al. (1996) and Archer and Saarlas (1996). The following derivation follows that of Von Backström and Gannon (1998b).

The cycle pressure ratio is defined as,

$$
\mathrm{r}=\mathrm{p}_{2} / \mathrm{p}_{1}=\mathrm{p}_{3} / \mathrm{p}_{4}
$$

and the compression temperature ratio,

$$
\begin{aligned}
\mathrm{c} & =\mathrm{T}_{2} / \mathrm{T}_{1}=\mathrm{T}_{3} / \mathrm{T}_{4} \\
& =\mathrm{r}^{(\gamma-1) / \gamma}
\end{aligned}
$$


The efficiency is the turbine output power divided by the solar energy transferred to the air moving through the collector.

$$
\eta=\frac{\text { Shaft power out }}{\text { Thermal power in }}
$$

The solar energy transferred to the air is,

$$
\Delta \mathrm{P}_{23}=\dot{\mathrm{m}} \mathrm{c}_{\mathrm{p}}\left(\mathrm{T}_{3}-\mathrm{T}_{2}\right)
$$

The shaft power out is:

$$
\mathrm{P}_{\text {shaft }}=\dot{\mathrm{m}} \mathrm{c}_{\mathrm{p}}\left(\mathrm{T}_{3}-\mathrm{T}_{4}\right)-\dot{\mathrm{m}} \mathrm{c}_{\mathrm{p}}\left(\mathrm{T}_{2}-\mathrm{T}_{1}\right)
$$

Thus the cycle efficiency can be written as,

$$
\begin{aligned}
\eta & =1-\frac{1}{c} \\
& =1-\frac{1}{r^{(\gamma-1) / \gamma}}
\end{aligned}
$$

From the above it can be seen that the efficiency is only dependent on the pressure ratio and not on the temperature ratio. In gas turbines the flow variables are often normalised to the inlet condition $T_{1}$. The specific power is a function of the cycle temperature ratio $t_{13}$ where $\Delta T_{23}$ is the temperature rise in the combustor and $T_{2}$ is the compressor exit temperature. The cycle temperature ratio is defined as:

$$
\begin{aligned}
\mathrm{t}_{13} & =\mathrm{T}_{3} / \mathrm{T}_{1} \\
& =\left(\mathrm{T}_{2}+\Delta \mathrm{T}_{23}\right) / \mathrm{T}_{1}
\end{aligned}
$$

The specific power is normalised with $\mathrm{T}_{1}$,

$$
\begin{aligned}
\mathrm{P}_{1}^{*} & =\frac{\mathrm{P}_{\text {shaft }}}{\mathrm{mc}_{\mathrm{p}} \mathrm{T}_{1}}=\mathrm{t}_{13}\left(1-\frac{1}{\mathrm{c}}\right)-(\mathrm{c}-1) \\
& =(\mathrm{c}-1)\left\{\left(\frac{\mathrm{t}_{13}}{\mathrm{c}}\right)-1\right\}
\end{aligned}
$$

While the plant efficiency is only a function of pressure ratio, it can be seen from the above equation that the specific power is also dependent on the temperature increase in the solar collector.

\section{Analysis of Solar Chimney Cycle}

The analysis of the solar chimney cycle is very similar to that of the gas turbine, but for the solar chimney reference to the temperature $T_{1}$ at the chimney top altitude will be avoided. It is better to use the collector inlet temperature $T_{2}$ as this can be easily measured. The temperature $T_{1}{ }^{\prime}$ is defined 
as the intersection of the isentropic line through point 2 and the constant pressure line through point 4.

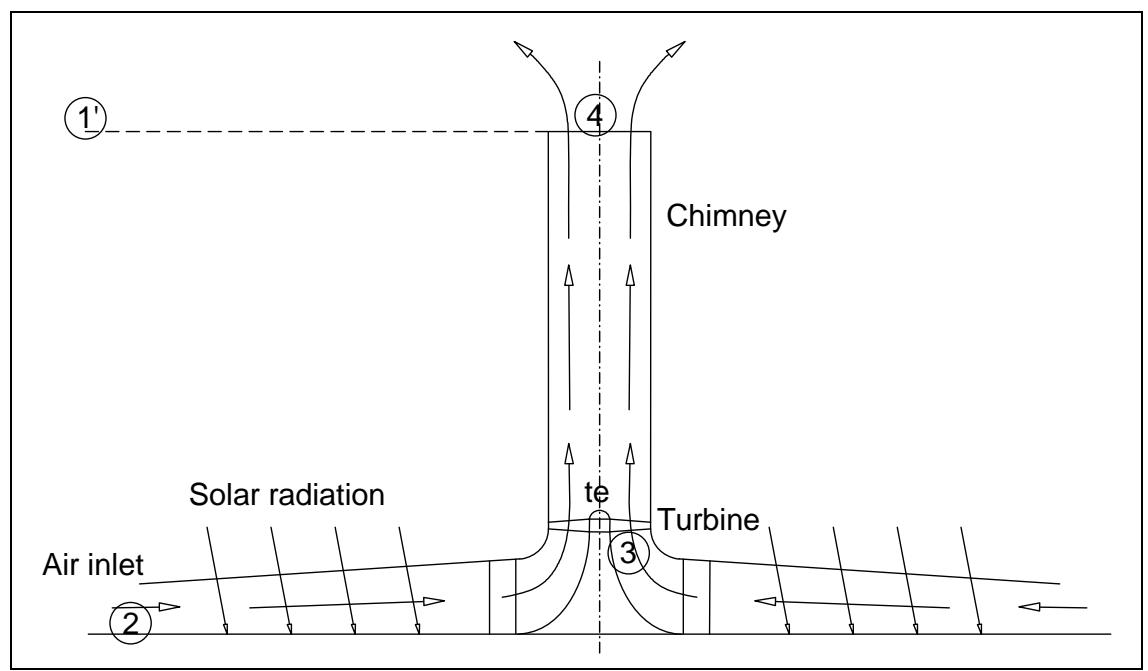

Figure 2.2. Solar chimney schematic showing nomenclature.

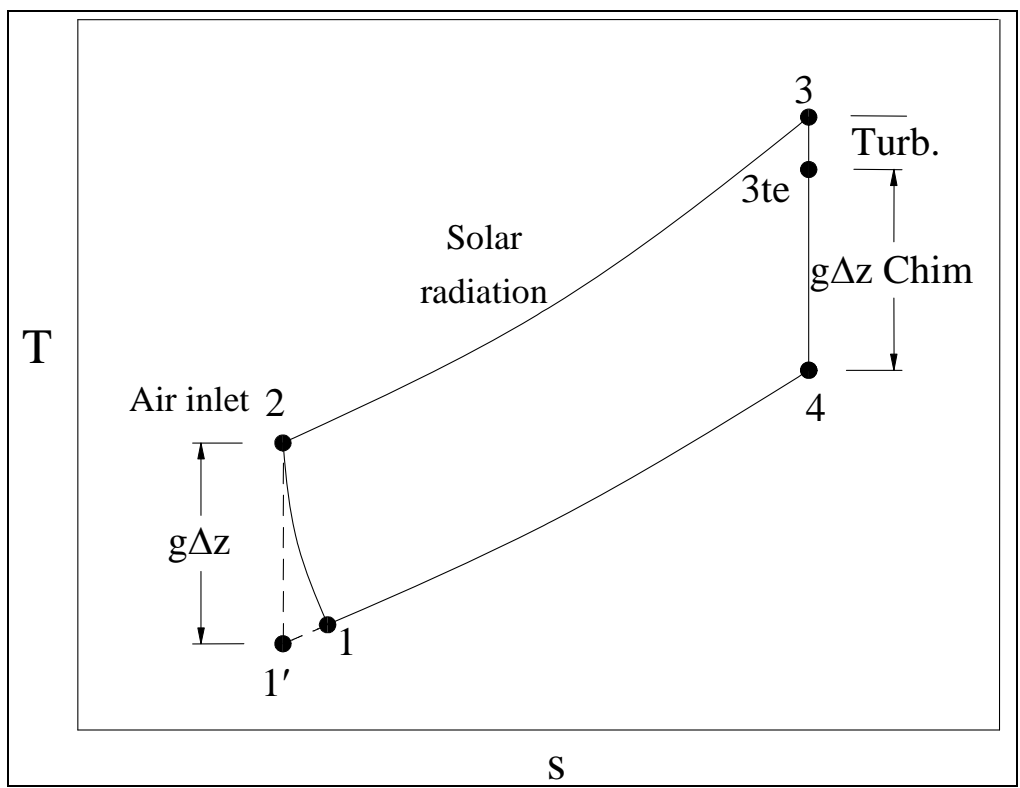

Figure 2.3. Temperature-entropy (T-s) diagram for solar chimney air standard cycle.

In an ideal gas turbine cycle where there are no irreversible processes all the power could be extracted as the gas expands from $\mathrm{p}_{3}$ to $\mathrm{p}_{4}$ to obtain the shaft power $\dot{\mathrm{m}}_{\mathrm{p}}\left(\mathrm{T}_{3}-\mathrm{T}_{4}\right)$. In the case of the solar chimney the power required to lift the air up the chimney must be taken into account. The total power available is,

$$
\mathrm{P}_{\text {available }}=\dot{\mathrm{m}} \mathrm{c}_{\mathrm{p}}\left(\mathrm{T}_{3}-\mathrm{T}_{4}\right)
$$

The power required to lift the air up the chimney,

$$
\mathrm{P}_{\text {lift }}=\dot{\mathrm{m} g} \Delta \mathrm{z}
$$


An internal enthalpy reduction provides the power for this:

$$
\Delta \mathrm{h}=\mathrm{g} \Delta \mathrm{z}
$$

The value of $\Delta \mathrm{h}$ can also be equated to the amount of enthalpy gained after the air from the chimney has cooled to $\mathrm{T}_{1}$ ' and descends again through the atmosphere.

Internal enthalpy gain provided by the air descending:

$$
\begin{aligned}
\Delta \mathrm{h} & =\mathrm{g} \Delta \mathrm{z} \\
& =\mathrm{C}_{\mathrm{p}}\left(\mathrm{T}_{2}-\mathrm{T}_{1}^{\prime}\right)
\end{aligned}
$$

Thus the shaft power out becomes,

$$
\mathrm{P}_{\text {shaft }}=\dot{\mathrm{mc}}_{\mathrm{p}}\left(\mathrm{T}_{3}-\mathrm{T}_{4}\right)-\dot{\mathrm{m}} \mathrm{c}_{\mathrm{p}}\left(\mathrm{T}_{2}-\mathrm{T}_{1}^{\prime}\right)
$$

While the temperature $T_{1}{ }^{\prime}$ is an imaginary temperature, it allows us to assume an isentropic compression in agreement with the air standard assumptions. Although the gas turbine and solar chimney T-s cycle diagrams are similar, there are some practical differences in the operation of the two.

In the gas turbine, work is extracted from the turbine as shaft work and transferred to the compressor where it is reintroduced. This is not practically possible in the solar chimney as the collector would have to be under pressure, a task that would be difficult, and the size and scale of the solar chimney would make the transfer of power from the turbine to the compressor difficult. Apart from this the power transferred would be much larger than the actual power generated by the plant. In the solar chimney an internal exchange between the enthalpy and geopotential energy takes place in the chimney and the process is reversed in the external environment. The derivation proceeds in a similar way. As in equation (2.3), the thermal efficiency was given in (2.3).

$$
\eta=\frac{\text { Shaft power out }}{\text { Thermal power in }}
$$

and the solar power input is,

$$
\Delta \mathrm{P}_{23}=\dot{\mathrm{mc}}_{\mathrm{p}}\left(\mathrm{T}_{3}-\mathrm{T}_{2}\right)
$$

The pressure ratio is defined as the collector exit pressure over the chimney exit pressure,

$$
\mathrm{r}=\mathrm{p}_{2} / \mathrm{p}_{1}=\mathrm{p}_{3} / \mathrm{p}_{4}
$$

and the temperature ratio:

$$
\begin{aligned}
\mathrm{C} & =\mathrm{T}_{2} / \mathrm{T}_{1}^{\prime}=\mathrm{T}_{3} / \mathrm{T}_{4} \\
& =\mathrm{r}^{(\gamma-1) / \gamma}
\end{aligned}
$$


The solar chimney efficiency is then the same as for the gas turbine.

$$
\begin{aligned}
\eta & =1-\frac{1}{\mathrm{c}} \\
& =\left(\mathrm{T}_{2}-\mathrm{T}_{1}^{\prime}\right) / \mathrm{T}_{2}
\end{aligned}
$$

This can be modified using the expression equating the temperature drop with the potential energy,

$$
\mathrm{c}_{\mathrm{p}}\left(\mathrm{T}_{2}-\mathrm{T}_{1}^{\prime}\right)=\mathrm{g} \Delta \mathrm{z}
$$

to obtain:

$$
\eta=\frac{g \Delta z}{c_{p} T_{2}}
$$

As can be seen the efficiency is proportional to the chimney height, inversely proportional to collector inlet temperature and independent of the collector temperature rise. As one observer remarked, the chimney efficiency is cast in concrete from the outset. To calculate the specific power the cycle temperature ratio is defined as,

$$
\mathrm{t}_{13}=\frac{\mathrm{T}_{3}}{\mathrm{~T}_{1}^{\prime}}
$$

and this gives a specific power normalised to $\mathrm{T}_{2}$.

$$
\begin{aligned}
\mathrm{P}_{2}^{*} & =\frac{\mathrm{P}}{\dot{\mathrm{mc}_{\mathrm{p}} \mathrm{T}_{2}}} \\
& =\left[\dot{\mathrm{m}}_{\mathrm{p}}\left(\mathrm{T}_{3}-\mathrm{T}_{4}\right)-\dot{\mathrm{m}} \mathrm{c}_{\mathrm{p}}\left(\mathrm{T}_{2}-\mathrm{T}_{1}^{\prime}\right)\right] /\left(\dot{\mathrm{mc}}_{\mathrm{p}} \mathrm{T}_{2}\right) \\
& =\left(\mathrm{T}_{3}-\mathrm{T}_{4}\right) / \mathrm{T}_{2}-\left(\mathrm{T}_{2}-\mathrm{T}_{1}^{\prime}\right) / \mathrm{T}_{2} \\
& =\left(\mathrm{t}_{13} / \mathrm{c}\right)(1-1 / \mathrm{c})-(1-1 / \mathrm{c}) \\
& =\left(\mathrm{t}_{13} / \mathrm{c}-1\right)(1-1 / \mathrm{c})
\end{aligned}
$$

Using equation (2.20) and the following expression,

$$
\frac{\mathrm{t}_{13}}{\mathrm{c}}=\left(1+\frac{\Delta \mathrm{T}_{23}}{\mathrm{~T}_{2}}\right)
$$

the specific power $\mathrm{P}_{2}^{*}$ can be written as,

$$
\mathrm{P}_{2}^{*}=\left\{\frac{\mathrm{g} \Delta \mathrm{z}}{\mathrm{C}_{\mathrm{p}} \mathrm{T}_{2}}\right\}\left\{\frac{\Delta \mathrm{T}_{23}}{\mathrm{~T}_{2}}\right\}
$$

Equation (2.24) shows that the normalised specific power is proportional to chimney height and collector temperature rise and inversely proportional to the square of collector inlet temperature $\mathrm{T}_{2}$. This trend is shown graphically in Figure 2.4 using the standard conditions shown in Table 2.1. These values will be used in all calculations unless stated otherwise and are representative of a potential site where the plant may be built. The efficiency as a function of chimney height is also superimposed on the graph and sample values from all graphs can be found in appendix A. 
Assuming the chimney height and collector temperature rise are known, it is possible to calculate the required mass flow using equation (2.22). Figure 2.4 shows that the ideal plant efficiency is only a function of chimney height. A plant with a 1500m high chimney will have a maximum efficiency of $4.83 \%$.

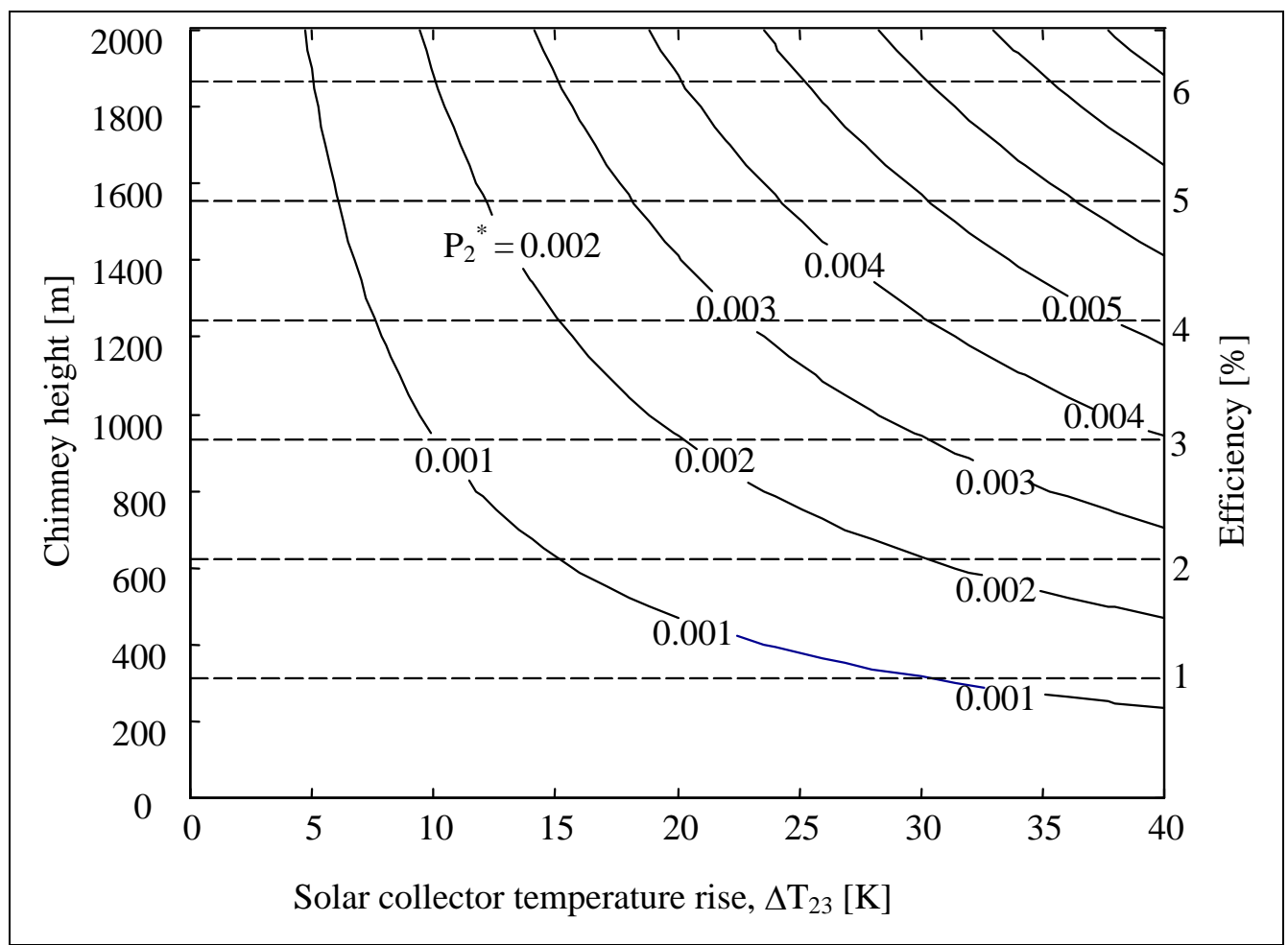

Figure 2.4. Specific power as a function of chimney height [m] and collector temperature rise [K] with efficiency [\%] overlaid.

Table 2.1 Standard conditions at collector inlet for solar chimney.

\begin{tabular}{|l|l|l|l|}
\hline Collector inlet Temperature $\left(\mathrm{T}_{2}\right)$ & $303.2 \mathrm{~K}$ & Collector inlet pressure $\left(\mathrm{p}_{2}\right)$ & $90000 \mathrm{~Pa}$ \\
\hline
\end{tabular}

Table 2.2 Values of solar chimney efficiency as derived from equation (2.20). (Figure 2.4)

\begin{tabular}{|l|l|l|l|l|}
\hline Chimney height $\Delta \mathrm{z}[\mathrm{m}]$. & 500 & 1000 & 1500 & 2000 \\
\hline Chimney efficiency [\%]. & 1.61 & 3.22 & 4.83 & 6.44 \\
\hline
\end{tabular}

Continuing with the above analysis the pressure drop over the turbine for the ideal cycle can be calculated. It is simpler, however, to use the specific power $\mathrm{P}_{3}{ }^{*}$ normalised to the turbine inlet temperature $\mathrm{T}_{3}$.

$$
\begin{aligned}
& \mathrm{P}_{3}^{*}=\frac{\mathrm{P}}{\dot{\mathrm{mc}_{\mathrm{p}} \mathrm{T}_{3}}} \\
& =\left[\dot{\mathrm{m}}_{\mathrm{p}}\left(\mathrm{T}_{3}-\mathrm{T}_{4}\right)-\dot{\mathrm{m}} \mathrm{c}_{\mathrm{p}}\left(\mathrm{T}_{2}-\mathrm{T}_{1}^{\prime}\right)\right] /\left(\dot{\mathrm{mc}}_{\mathrm{p}} \mathrm{T}_{3}\right) \\
& =\left(\mathrm{T}_{3}-\mathrm{T}_{4}\right) / \mathrm{T}_{3}-\left(\mathrm{T}_{2}-\mathrm{T}_{1}^{\prime}\right) / \mathrm{T}_{3} \\
& =\left(\mathrm{t}_{13} / \mathrm{c}\right)(1-1 / \mathrm{c})-(1-1 / \mathrm{c}) \\
& =(1-1 / \mathrm{c})\left(1-\mathrm{c} / \mathrm{t}_{13}\right)
\end{aligned}
$$


As done previously $\mathrm{T}_{1}$ ' is factored out of the equation to obtain the following,

$$
\mathrm{P}_{3}^{*}=\left\{\frac{\mathrm{g} \Delta \mathrm{z}}{\mathrm{C}_{\mathrm{p}} \mathrm{T}_{2}}\right\}\left\{\frac{\Delta \mathrm{T}_{23}}{\mathrm{~T}_{2}+\Delta \mathrm{T}_{23}}\right\}
$$

The power from the turbine normalised to $\mathrm{T}_{3}$ is,

$$
\mathrm{P}_{3}^{*}=\frac{\dot{\mathrm{m}} \mathrm{c}_{\mathrm{p}}\left(\mathrm{T}_{3}-\mathrm{T}_{3 \mathrm{te}}\right)}{\mathrm{mc}_{\mathrm{p}} \mathrm{T}_{3}}=1-\frac{\mathrm{T}_{3 \mathrm{e}}}{\mathrm{T}_{3}} .
$$

By equating (2.26) and (2.27) the turbine temperature ratio is,

$$
\frac{\mathrm{T}_{3 \text { te }}}{\mathrm{T}_{3}}=1-\left\{\frac{\mathrm{g} \Delta \mathrm{z}}{\mathrm{c}_{\mathrm{p}} \mathrm{T}_{2}}\right\}\left\{\frac{\Delta \mathrm{T}_{23}}{\mathrm{~T}_{2}+\Delta \mathrm{T}_{23}}\right\}
$$

Once the temperature ratio across the turbine has been found, the ideal gas relationship is used to find the pressure ratio and from this the pressure drop is as follows,

$$
\frac{\mathrm{p}_{3 \mathrm{te}}}{\mathrm{p}_{3}}=\left(\frac{\mathrm{T}_{3 \mathrm{te}}}{\mathrm{T}_{3}}\right)^{\frac{\gamma}{\gamma-1}}
$$

Substituting (2.28) into (2.29) gives the turbine pressure drop.

$$
\Delta \mathrm{p}_{\text {turb }}=\mathrm{p}_{2}\left\{1-\left[1-\left\{\frac{\mathrm{g} \Delta \mathrm{z}}{\mathrm{c}_{\mathrm{p}} \mathrm{T}_{2}}\right\}\left\{\frac{\Delta \mathrm{T}_{23}}{\mathrm{~T}_{2}+\Delta \mathrm{T}_{23}}\right\}\right]^{\frac{\gamma}{\gamma-1}}\right\}
$$

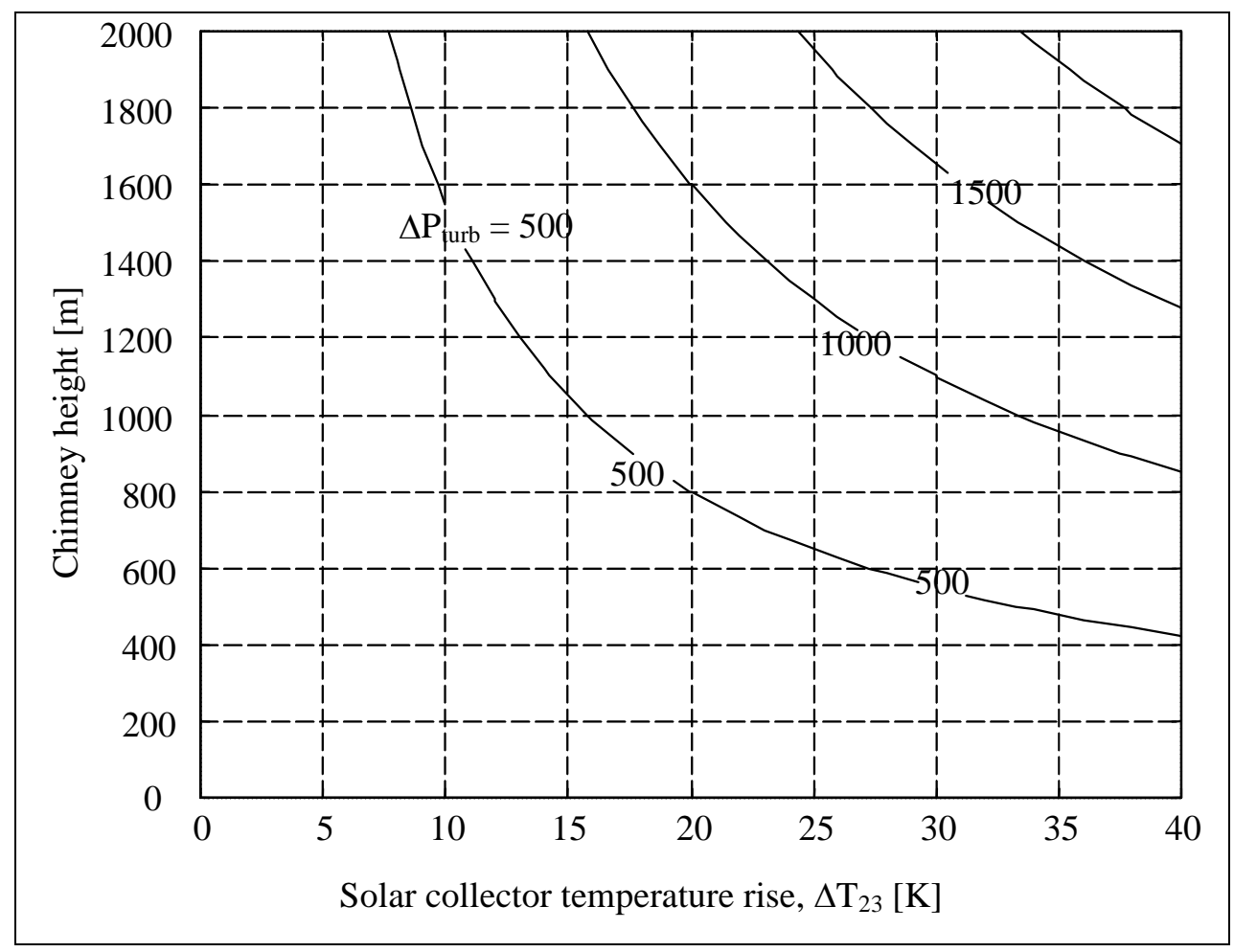

Figure 2.5. Turbine pressure drop [Pa] as a function of chimney height [m] and collector temperature rise $[\mathrm{K}]$. 
Figure 2.5 shows how the available turbine pressure drop changes for various values of chimney height and collector temperature while some sample values are found in appendix A.

The last value that will be calculated from the ideal cycle analysis is the required mass flow for a certain power output based on chimney height and collector temperature rise. The power output of $200 \mathrm{MW}$ is one that has been agreed upon as the design output of the full-scale plant. The results are shown graphically in Figure 2.6 and sample values are given in appendix A.

$$
\dot{\mathrm{m}}=\frac{\mathrm{P}_{\text {required }}}{\mathrm{P}_{2}^{*} \mathrm{c}_{\mathrm{p}} \mathrm{T}_{2}}
$$

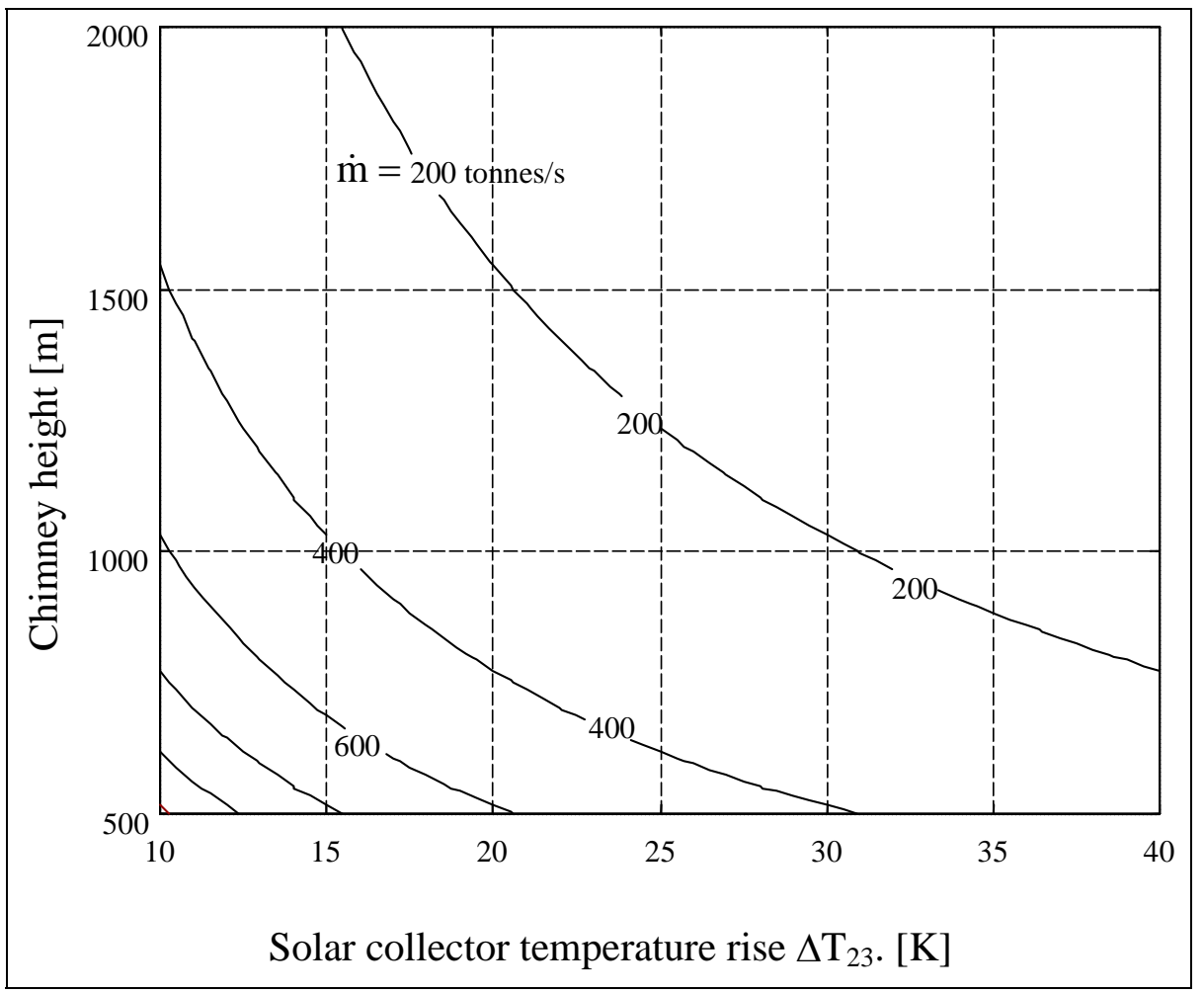

Figure 2.6. Required mass flow [tonnes/s] as a function of chimney height [m] and collector temperature rise [K] for $200 \mathrm{MW}$ design power output.

\section{Chimney Height}

From the simple cycle analysis it is possible to see that making the chimney as high as possible is favourable as it increases the efficiency and specific power while reducing the required mass flow, regardless of the solar collector temperature rise. Thus the constraints on the chimney height come not from the cycle analysis but rather from a structural and cost viewpoint. At the time of writing, a chimney height of $\mathbf{1 5 0 0} \mathbf{~ m}$ had been chosen as being feasible and will be used in the further calculations. The following section investigates the effects of kinetic energy losses on the solar chimney. 


\subsection{CyCle ANALYSIS WITH CONSTANT CHIMnEy HeIGHT AND KineTIC ENERGY LOSSES}

In the case of the solar chimney, the kinetic energy of the air that exits the chimney is lost. While the velocities in the chimney are not expected to be high, the available pressure drop over the turbine is of the order of $1000 \mathrm{~Pa}$ and so even low velocities could result in a dynamic pressure loss that would result in a significant power reduction. The effects of turbine efficiency and chimney losses are also included in the equations. In the sections that follow, the nomenclature is modified to include the kinetic energy terms to give stagnation values, e.g. $\mathrm{T}_{0}$. This is so that the stagnation and static terms at the chimney exit can be represented and compared to the ideal analysis.

The assumptions used in the analysis will now include:

- All kinetic energy is lost at the chimney exit as the construction of a diffuser at the top of the chimney would be impractical.

- The total pressure under the solar collector remains constant.

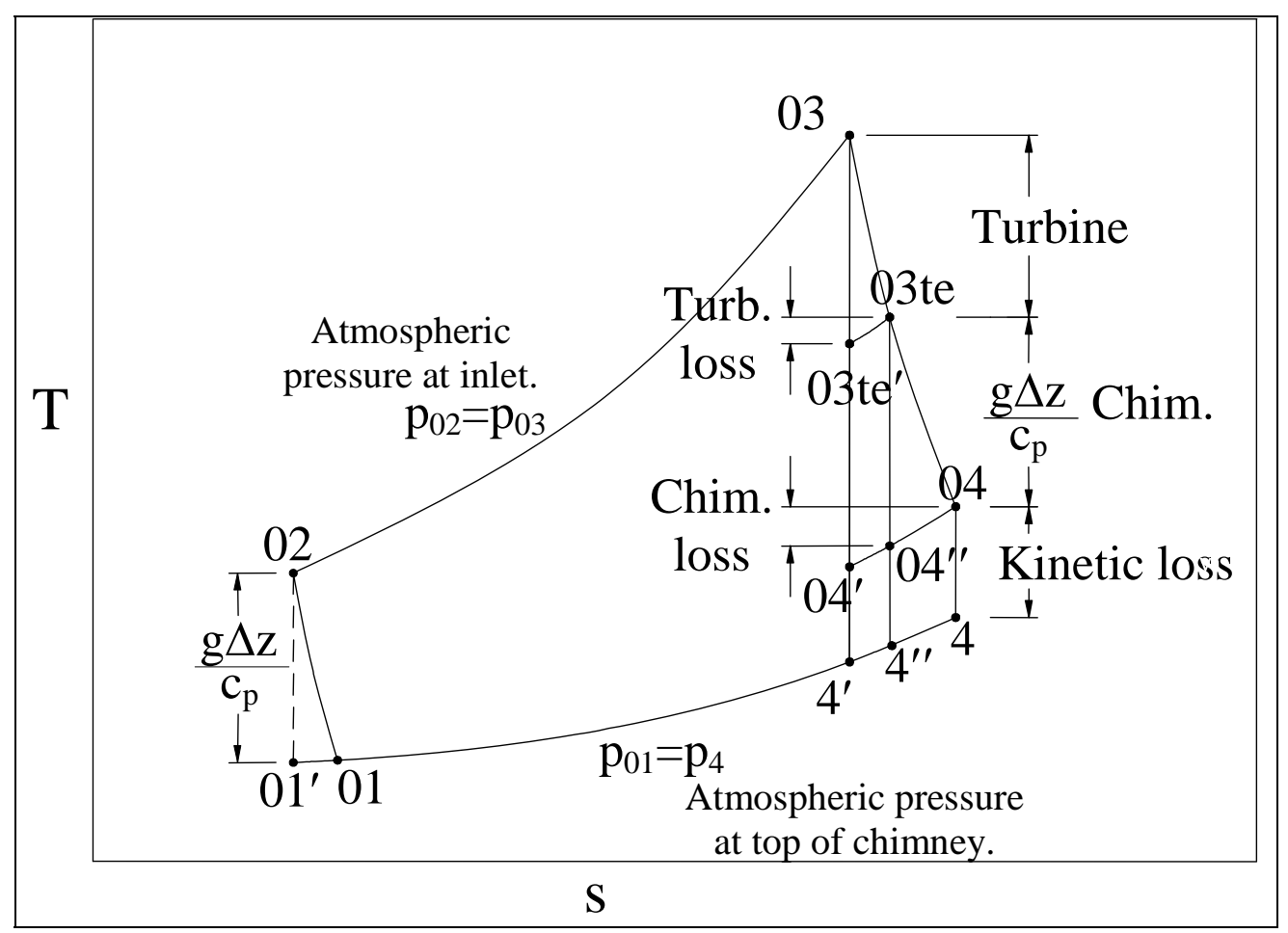

Figure 2.7. Temperature-entropy (T-s) diagram for solar chimney air standard cycle with kinetic energy loss at chimney exit.

To begin the analysis, a more detailed temperature entropy diagram needs to be constructed and the various efficiencies defined. The lengths in Figure 2.7 are purposely not drawn to scale as the temperature drop over the chimney is far larger than across the turbine or than the exit kinetic energy temperature drop, thus making the labelling difficult. Figure 2.7 shows the three main stages 
of the expansion process, namely the turbine, chimney and the kinetic energy loss at the exit. The operation of each component in the expansion part of the cycle will be discussed as well as the loss coefficients associated with each.

The turbine is important not only in that it produces the power but also because the mass flow of the plant is affected by the pressure drop across the turbine. It may also be possible to control the plant power output by increasing the turbine pressure drop. In the cycle analysis the turbine totalto-total efficiency is used as the kinetic energy is treated separately at the exit. It must be pointed out that the exit kinetic energy in the solar chimney power plant is not useful and considered a loss. In the case of a propulsion system like a gas turbine the exit kinetic energy produces useful thrust.

$$
\eta_{\text {turb }}=\frac{\left(\mathrm{T}_{03}-\mathrm{T}_{03 \mathrm{te}}\right)}{\left(\mathrm{T}_{03}-\mathrm{T}_{03 \mathrm{e}}^{\prime}\right)}
$$

The chimney creates the pressure difference that drives the entire plant but a certain amount of work is required to lift the air up the chimney. As mentioned in the previous section, it results in an enthalpy reduction of $\Delta \mathrm{h}_{\mathrm{o}}=\mathrm{g} \Delta \mathrm{z}=\mathrm{c}_{\mathrm{p}}\left(\mathrm{T}_{03 \mathrm{te}}-\mathrm{T}_{04}\right)=\mathrm{c}_{\mathrm{p}}\left(\mathrm{T}_{02}-\mathrm{T}_{01}{ }^{\prime}\right)$ but apart from this additional work is required to overcome the internal friction of the chimney. This loss can be due to the friction on the walls of the chimney but this is thought to be small as found in the analysis by Stephan et al. (1995). The main loss will probably be due to the internal bracing wires used in the construction of the chimney. A convenient way of representing these losses is to define a chimney efficiency, $\eta_{\text {chim, }}$ related to the exit kinetic energy. It was found in practice to be easier to use the chimney exit conditions rather than entry as an analytical solution to the problem could be found, as is shown later. The chimney efficiency is defined as,

$$
\mathrm{T}_{04}-\mathrm{T}_{04^{\prime \prime}}=\eta_{\text {chim }}\left(\mathrm{T}_{04}-\mathrm{T}_{4}\right)
$$

The last part of the expansion involves the exit kinetic energy (KE) and observing in general that the velocity profile will not be uniform it may be expressed as follows,

$$
\mathrm{KE}=\dot{\mathrm{m}}_{\mathrm{p}}\left(\mathrm{T}_{04}-\mathrm{T}_{4}\right)=\int\left(\rho \mathrm{C}_{\mathrm{z} 4}\right) \frac{1}{2} \mathrm{C}_{\mathrm{z} 4}^{2} \mathrm{dA}
$$

In practice it is much more useful to be able to perform the cycle analysis with a mean velocity, but to do this a constant, $\alpha$, is needed to relate the kinetic energy of a uniform and non-uniform velocity profile. This constant can be varied depending on the exit velocity profile,

$$
\mathrm{KE}=\dot{\mathrm{mc}} \mathrm{p}_{\mathrm{p}}\left(\mathrm{T}_{04}-\mathrm{T}_{4}\right)=\alpha\left(\rho \overline{\mathrm{C}_{\mathrm{z} 4}}\right) \frac{1}{2} \overline{\mathrm{C}_{\mathrm{z} 4}^{2}} \mathrm{~A}
$$

where $\alpha$ is defined,

$$
\alpha=\frac{\int\left(\rho C_{z 4}\right) \frac{1}{2} C_{z 4}^{2} d A}{\left(\rho \overline{C_{z 4}}\right) \frac{1}{2} \overline{C_{z 4}^{2}} A}
$$

A last definition that is used to simplify the analysis is the expansion efficiency.

$$
\eta_{\exp }=\left(\mathrm{T}_{03}-\mathrm{T}_{4}\right) /\left(\mathrm{T}_{03}-\mathrm{T}_{4}^{\prime}\right)
$$


This covers the entire expansion from $\mathrm{T}_{03}$ to $\mathrm{T}_{4}{ }^{\prime}$. It is used to simplify the writing of the main equations and eventually to aid in the determination of the chimney exit conditions.

\section{Analytical Solution to Cycle Analysis}

With the addition of the various loss coefficients, the thermodynamic analysis is now more complex but it is still possible to find a useful analytical solution. An overview of the analysis is given to clarify the derivation.

The plant efficiency and specific power output can be written in a similar form to that of the ideal cycle analysis in equations (2.20) and (2.24) but with the additional terms of the expansion efficiency and exit kinetic energy.

The solar chimney turbine has a similar type of operating range to a hydroelectric turbine. There are two extreme conditions that will result in a zero power output. The first is when there is no pressure drop across the turbine where system losses will eventually limit the mass flow rate. The second would be if the turbine pressure drop became high enough to block the flow completely. To calculate the plant power output, a turbine pressure drop must be chosen between these two extremes. Once a pressure drop is chosen the power output for this pressure drop can be calculated. Finding the maximum power output requires an iterative solution.

The basic steps of calculating the plant power output are :

1) choose a turbine temperature drop,

2) calculate the exit temperature $T_{4}$,

3) calculate the exit density,

4) calculate the mean exit velocity and then the power output.

The calculation of the maximum power output is an iterative process as the turbine temperature drop has to be chosen in order to calculate the power output. The theory is presented in the following paragraphs.

\section{Plant Efficiency and Specific Power Output}

The plant efficiency defined in equations (2.3) and (2.14) can be re-written using the process presented in Figure 2.7 as follows,

$$
\begin{aligned}
\eta & =\frac{\text { Shaft power out }}{\text { Thermal power in }} \\
& =\frac{\eta_{\text {exp }}\left(\mathrm{T}_{03}-\mathrm{T}_{4}^{\prime}\right)-\left(\mathrm{T}_{03 \mathrm{te}}-\mathrm{T}_{04}\right)-\left(\mathrm{T}_{04}-\mathrm{T}_{4}\right)}{\left(\mathrm{T}_{03}-\mathrm{T}_{02}\right)}
\end{aligned}
$$


A more insightful form using the following definitions and relationships,

$$
\begin{gathered}
\mathrm{c}=\mathrm{T}_{02} / \mathrm{T}_{01}^{\prime}=\mathrm{T}_{03} / \mathrm{T}_{4}^{\prime} \\
=\mathrm{r}^{(\gamma-1) / \gamma} \\
\mathrm{T}_{03}-\mathrm{T}_{02}=\Delta \mathrm{T}_{23} \\
\mathrm{~T}_{03 \mathrm{te}}-\mathrm{T}_{04}=\frac{\mathrm{g} \Delta \mathrm{z}}{\mathrm{c}_{\mathrm{p}}}
\end{gathered}
$$

and using equations (2.20) and (2.35) results in the following definition of efficiency,

$$
\eta=\left(\frac{g \Delta z}{c_{p} T_{02}}\right)\left[\frac{\eta_{\exp }\left(T_{02}+\Delta T_{23}\right)-T_{02}}{\Delta T_{23}}\right]-\alpha \frac{\overline{C_{z 4}^{2}}}{2 c_{p} \Delta T_{23}}
$$

The specific power is written in a similar form in terms of the component efficiencies and the basic variables of inlet temperature $\mathrm{T}_{02}$, collector temperature rise, $\Delta \mathrm{T}_{23}$ and chimney height $\Delta \mathrm{z}$.

$$
\begin{aligned}
\mathrm{P}_{2}^{*} & =\frac{\mathrm{P}}{\dot{\mathrm{m}}_{\mathrm{p}} \mathrm{T}_{02}} \\
& =\frac{\eta_{\exp }\left(\mathrm{T}_{03}-\mathrm{T}_{4}^{\prime}\right)-\left(\mathrm{T}_{02}-\mathrm{T}_{01}^{\prime}\right)-\left(\mathrm{T}_{04}-\mathrm{T}_{4}\right)}{\left(\dot{\left.\mathrm{m} \mathrm{c}_{\mathrm{p}} \mathrm{T}_{02}\right)}\right.} \\
& =\left(\frac{\mathrm{g} \Delta \mathrm{z}}{\mathrm{c}_{\mathrm{p}} \mathrm{T}_{02}}\right)\left(\frac{\eta_{\exp }\left(\mathrm{T}_{02}+\Delta \mathrm{T}_{23}\right)-\mathrm{T}_{02}}{\mathrm{~T}_{02}}\right)-\alpha \frac{\overline{\mathrm{C}_{\mathrm{z} 4}^{2}}}{2 \mathrm{c}_{\mathrm{p}} \mathrm{T}_{02}}
\end{aligned}
$$

It should be noted that when the expansion efficiency $\eta_{\exp }=1$ and the exit velocity $C_{z 4}$ is zero, the equations above revert to equations (2.20) and (2.24) respectively from the simple thermodynamic analysis.

\section{Limiting Turbine Temperature Drop}

As explained earlier, a useful step in the analysis is to calculate the limiting turbine pressure drop $\Delta \mathrm{T}_{\text {turb lim. }}$. The temperature entropy diagram from Figure 2.7 is simplified to show this specific operating condition when the mass flow is negligible (Figure 2.8). The temperature drop term $\left(\mathrm{T}_{03}{ }^{-}\right.$ $\left.\mathrm{T}_{03 \mathrm{te}}{ }^{\prime}\right)$ is defined in terms of the chimney height $\Delta \mathrm{z}$, collector inlet temperature $\mathrm{T}_{2}$, temperature rise $\Delta \mathrm{T}_{23}$ and turbine efficiency. Only the basic steps and results will be given as the intermediate steps are not complicated but involve some algebra. The derivation begins with the following,

$$
\begin{aligned}
\mathrm{T}_{03}-\mathrm{T}_{03 \mathrm{te}}^{\prime} & =\frac{1}{\eta_{\text {turb }}}\left(\mathrm{T}_{03}-\mathrm{T}_{03 \mathrm{te}}\right) \\
\Delta \mathrm{T}_{\text {turb lim }} & =\frac{1}{\eta_{\text {turb }}}\left[\left(\mathrm{T}_{03}-\mathrm{T}_{4}\right)-\left(\mathrm{T}_{03 \mathrm{te}}-\mathrm{T}_{4}\right)\right]
\end{aligned}
$$


and then making the following substitutions,

$$
\begin{aligned}
\mathrm{T}_{4} & =\mathrm{T}_{03 \text { te }} \frac{\mathrm{T}_{4}^{\prime}}{\mathrm{T}_{03 \text { te }}^{\prime}} \\
\mathrm{T}_{03 \text { te }} & =\mathrm{T}_{03}-\eta_{\text {turb }}\left(\mathrm{T}_{03}-\mathrm{T}_{03 \text { te }}^{\prime}\right)=\mathrm{T}_{03}-\eta_{\text {turb }}\left(\Delta \mathrm{T}_{\text {turb lim }}\right) \\
\mathrm{T}_{03 \text { te }}^{\prime} & =\mathrm{T}_{03}-\left(\mathrm{T}_{03}-\mathrm{T}_{03 \text { te }}^{\prime}\right)=\mathrm{T}_{03}-\Delta \mathrm{T}_{\text {turb lim }} \\
\mathrm{T}_{03 \text { te }}-\mathrm{T}_{4} & =\frac{\mathrm{g} \Delta \mathrm{z}}{\mathrm{c}_{\mathrm{p}}}
\end{aligned}
$$

the following implicit expression for the limiting turbine temperature drop is found.

$$
\Delta \mathrm{T}_{\text {turblim }}=\frac{1}{\eta_{\text {turb }}}\left[\left(\mathrm{T}_{03}-\mathrm{T}_{4}^{\prime} \frac{\mathrm{T}_{03}-\eta_{\text {turb }} \Delta \mathrm{T}_{\text {turblim }}}{\mathrm{T}_{03}-\Delta \mathrm{T}_{\text {turblim }}}\right)-\frac{\mathrm{g} \Delta \mathrm{z}}{\mathrm{c}_{\mathrm{p}}}\right]
$$

Using equations (2.39), (2.18) and (2.20) gives the following quadratic equation,

$$
\begin{aligned}
& 0=\mathrm{a} \Delta \mathrm{T}_{\text {turblim }}^{2}+\mathrm{b} \Delta \mathrm{T}_{\text {turblim }}+\mathrm{c} \\
& 0=\eta_{\text {turb }} \Delta \mathrm{T}_{\text {turblim }}^{2}+\left[\frac{\mathrm{g} \Delta \mathrm{z}}{\mathrm{c}_{\mathrm{p}}}\left(1-\eta_{\text {turb }} \frac{\mathrm{T}_{03}}{\mathrm{~T}_{02}}\right)-\mathrm{T}_{03}\right] \Delta \mathrm{T}_{\text {turblim }}+\mathrm{T}_{03} \frac{\mathrm{g} \Delta \mathrm{z}}{\mathrm{c}_{\mathrm{p}}}\left(\frac{\mathrm{T}_{03}}{\mathrm{~T}_{02}}-1\right)
\end{aligned}
$$

After inspection of the terms the physically realistic solution is,

$$
\Delta \mathrm{T}_{\text {turblim }}=\frac{-\mathrm{b}-\sqrt{\mathrm{b}^{2}-4 \mathrm{ac}}}{2 \mathrm{a}}
$$

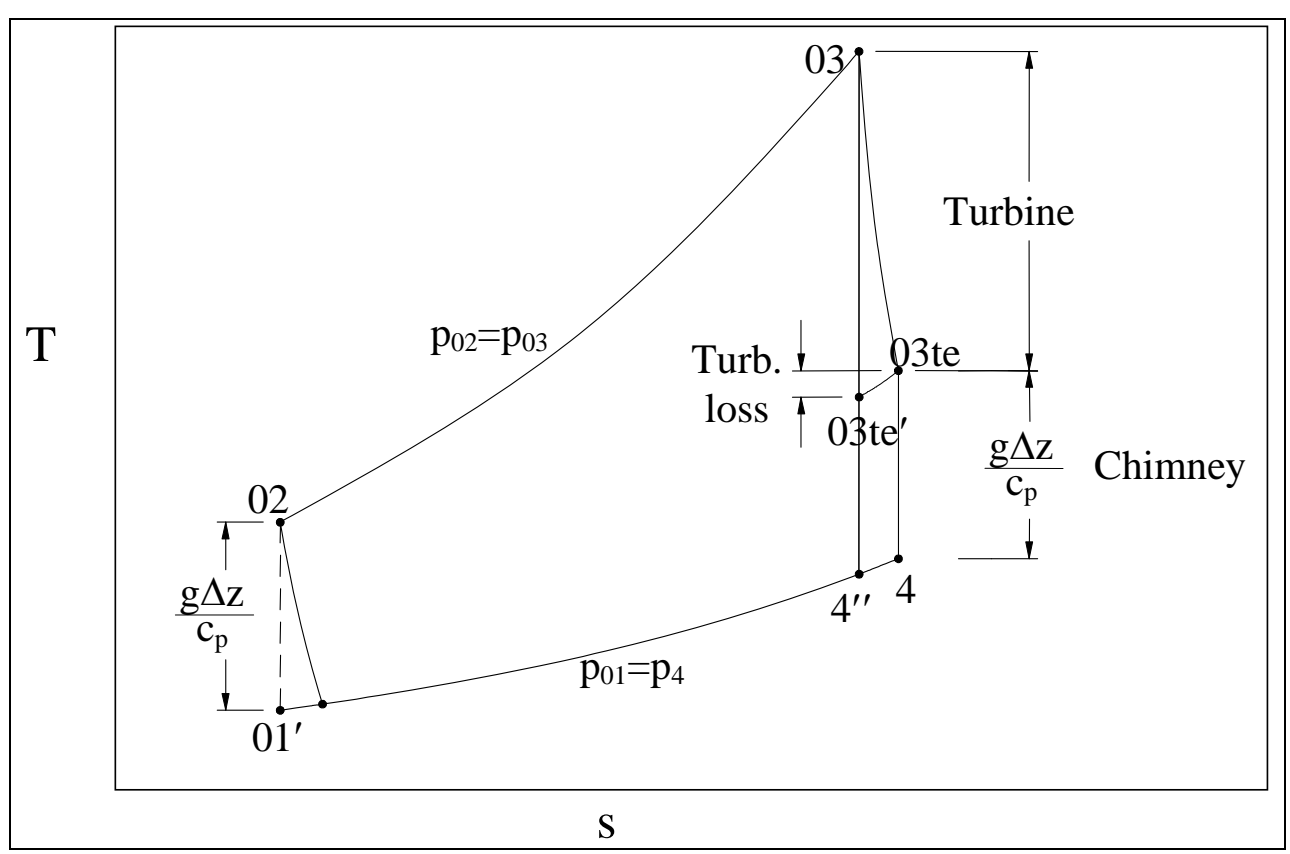

Figure 2.8. Temperature-entropy ( $T$-s) diagram for solar chimney air standard cycle for maximum turbine temperature drop. 


\section{Plant Output Power}

The final step of the analysis is to find the output power of the solar chimney. This requires the outlet temperature $\mathrm{T}_{4}$ to be written in the usual terms of the chimney height $\Delta \mathrm{z}$, collector inlet temperature $T_{2}$, temperature rise $\Delta T_{23}$ and turbine efficiency. Two additional variables are now also included, the ideal turbine temperature drop $\Delta \mathrm{T}_{\text {turb }}=\left(\mathrm{T}_{03}-\mathrm{T}_{03 \mathrm{te}}\right)$ and the chimney efficiency $\eta_{\text {chim. }}$.

Referring back to Figure 2.7 the analysis proceeds as follows,

$$
\begin{aligned}
& \frac{\mathrm{T}_{4}}{\mathrm{~T}_{04}}=\frac{\mathrm{T}_{4}^{\prime}}{\mathrm{T}_{04}^{\prime}} \Rightarrow \mathrm{T}_{4}=\mathrm{T}_{04} \frac{\mathrm{T}_{4}^{\prime}}{\mathrm{T}_{04}^{\prime}} \\
& \frac{\mathrm{T}_{04}^{\prime}}{\mathrm{T}_{03 \mathrm{te}}^{\prime}}=\frac{\mathrm{T}_{04}^{\prime \prime}}{\mathrm{T}_{03 \mathrm{te}}} \Rightarrow \mathrm{T}_{04}^{\prime}=\mathrm{T}_{03 \mathrm{te}}^{\prime} \frac{\mathrm{T}_{04}^{\prime \prime}}{\mathrm{T}_{03 \text { te }}} \\
& \mathrm{T}_{04}^{\prime \prime}=\mathrm{T}_{04}-\eta_{\text {chim }}\left(\mathrm{T}_{04}-\mathrm{T}_{4}\right)
\end{aligned}
$$

Substituting equations (2.50) and (2.51) into (2.49), an implicit expression for $\mathrm{T}_{4}$ can be written and arranged into a quadratic form,

$$
\begin{aligned}
& \mathrm{T}_{4}=\frac{\mathrm{T}_{04} \mathrm{~T}_{4}^{\prime} \mathrm{T}_{03 \text { te }}}{\mathrm{T}_{03 \mathrm{te}}^{\prime}\left[\mathrm{T}_{04}-\mathrm{k}\left(\mathrm{T}_{04}-\mathrm{T}_{4}\right)\right]} \\
& \mathrm{aT}_{4}^{2}+\mathrm{bT}_{4}+\mathrm{c}=0 \\
& \eta_{\text {chim }} \mathrm{T}_{4}^{2}+\left(\mathrm{T}_{04}\left(1-\eta_{\text {chim }}\right)\right) \mathrm{T}_{4}-\frac{\mathrm{T}_{04} \mathrm{~T}_{4}^{\prime} \mathrm{T}_{03 \mathrm{te}}}{\mathrm{T}_{03 \mathrm{te}}^{\prime}}=0
\end{aligned}
$$

After inspection of the terms the physically realistic one is,

$$
\mathrm{T}_{4}=\frac{-\mathrm{b}+\sqrt{\mathrm{b}^{2}-4 \mathrm{ac}}}{2 \mathrm{a}}
$$

The terms of equation (2.53) can be represented in terms of the base variables mentioned above,

$$
\begin{aligned}
\mathrm{T}_{04} & =\left(\mathrm{T}_{02}+\Delta \mathrm{T}_{23}\right)-\eta_{\text {turb }} \Delta \mathrm{T}_{\text {turb }}-\frac{\mathrm{g} \Delta \mathrm{z}}{\mathrm{c}_{\mathrm{p}}} \\
\mathrm{T}_{4}^{\prime} & =\left(\mathrm{T}_{02}+\Delta \mathrm{T}_{23}\right)\left(\frac{\mathrm{c}_{\mathrm{p}} \mathrm{T}_{02}-\mathrm{g} \Delta \mathrm{z}}{\mathrm{c}_{\mathrm{p}} \mathrm{T}_{02}}\right) \\
\mathrm{T}_{03 \text { te }} & =\left(\mathrm{T}_{02}+\Delta \mathrm{T}_{23}\right)-\eta_{\text {turb }} \Delta \mathrm{T}_{\text {turb }} \\
\mathrm{T}_{\text {03te }}^{\prime} & =\left(\mathrm{T}_{02}+\Delta \mathrm{T}_{23}\right)-\Delta \mathrm{T}_{\text {turb }}
\end{aligned}
$$

From the exit temperature the mean exit velocity is,

$$
\overline{C_{z 4}}=\sqrt{\frac{2 c_{p}}{\alpha}\left(T_{04}-T_{4}\right)}
$$

To calculate the chimney mass flow, the exit density and thus pressure is required. Assuming an adiabatic lapse rate the chimney exit pressure $\mathrm{p}_{4}$ can be expressed as, 


$$
\begin{aligned}
& \frac{\mathrm{p}_{4}}{\mathrm{p}_{03}}=\frac{\mathrm{p}_{01}}{\mathrm{p}_{02}}=\left(\frac{\mathrm{T}_{01}}{\mathrm{~T}_{02}}\right)^{\frac{\gamma}{\gamma-1}} \\
& \Rightarrow \mathrm{p}_{4}=\mathrm{p}_{02}\left(\frac{\mathrm{T}_{02}-\frac{\mathrm{g} \Delta \mathrm{z}}{\mathrm{C}_{\mathrm{p}}}}{\mathrm{T}_{02}}\right)^{\frac{\gamma}{\gamma-1}} \\
& \rho_{4}=\frac{\mathrm{p}_{4}}{\mathrm{RT}_{4}}
\end{aligned}
$$

For each chimney diameter the mass flow is,

$$
\dot{\mathrm{m}}=\rho_{4} \mathrm{C}_{\mathrm{z} 4} \frac{\pi \mathrm{D}_{\text {chim }}^{2}}{4}
$$

and finally the power output can be calculated,

$$
\mathrm{P}=\dot{\mathrm{m}} \mathrm{c}_{\mathrm{p}} \mathrm{T}_{02} \mathrm{P}_{02}^{*}
$$

\section{Determination of Chimney Diameter}

In equation (2.59) it can be seen that the chimney diameter has been used but this has not been fixed. The first step in determining this is to calculate the power output per unit area of the chimney. From this the required chimney diameter to produce the design power output can be calculated. As in the previous section sample values from the figures are given in appendix A.

Table 2.3 Values used in calculations with kinetic energy losses.

\begin{tabular}{|l|l|l|l|}
\hline Chimney height $[\mathrm{m}]$ & 1500 & Total-to-total turbine efficiency [\%] & 80 \\
\hline Chimney constant k & 1 & Exit constant $\alpha(\mathrm{n}=5)$ & 1.1058 \\
\hline
\end{tabular}

Table 2.3 shows the values used in the initial analysis of the plant. The turbine efficiency is based on conservative total-to-static operating values of existing turbines. The chimney loss constant $\mathrm{k}$, is large but the chimney interior may have bracing wires that have high losses. The exit constant $\alpha$ is based on a power law velocity profile. While high, this value is similar to those found experimentally by Von Backström et al. (2002). Equation (2.61) presents the basic power law equation where the velocity across the pipe $\mathrm{C}_{\mathrm{z} 4}$ is a function of the maximum velocity at the pipe centre $\mathrm{C}_{\mathrm{z} 4 \max }$. $\mathrm{D}$ is the pipe diameter and $\mathrm{r}$ the distance from the pipe centre.

$$
\frac{C_{z 4}}{C_{z 4 \max }}=\left(\frac{D / 2-r}{D / 2}\right)^{\frac{1}{n}}
$$

Figure 2.9 shows that power per unit area of the chimney as a function of temperature rise and the exit chimney velocity. At this stage we assume that the maximum power is developed when the turbine pressure drop is 2/3 the no-flow pressure drop found in equation (2.48), Haaf (1984). This 
assumption is true for all systems with a constant potential pressure drop and a pressure loss proportional to the volume flow squared. Later in the analysis, the effect of the inclusion of the solar collector in the analysis shows that the pressure potential is dependent on the flow. The maximum power is actually developed at a higher pressure drop than 2/3 of the zero-flow potential.

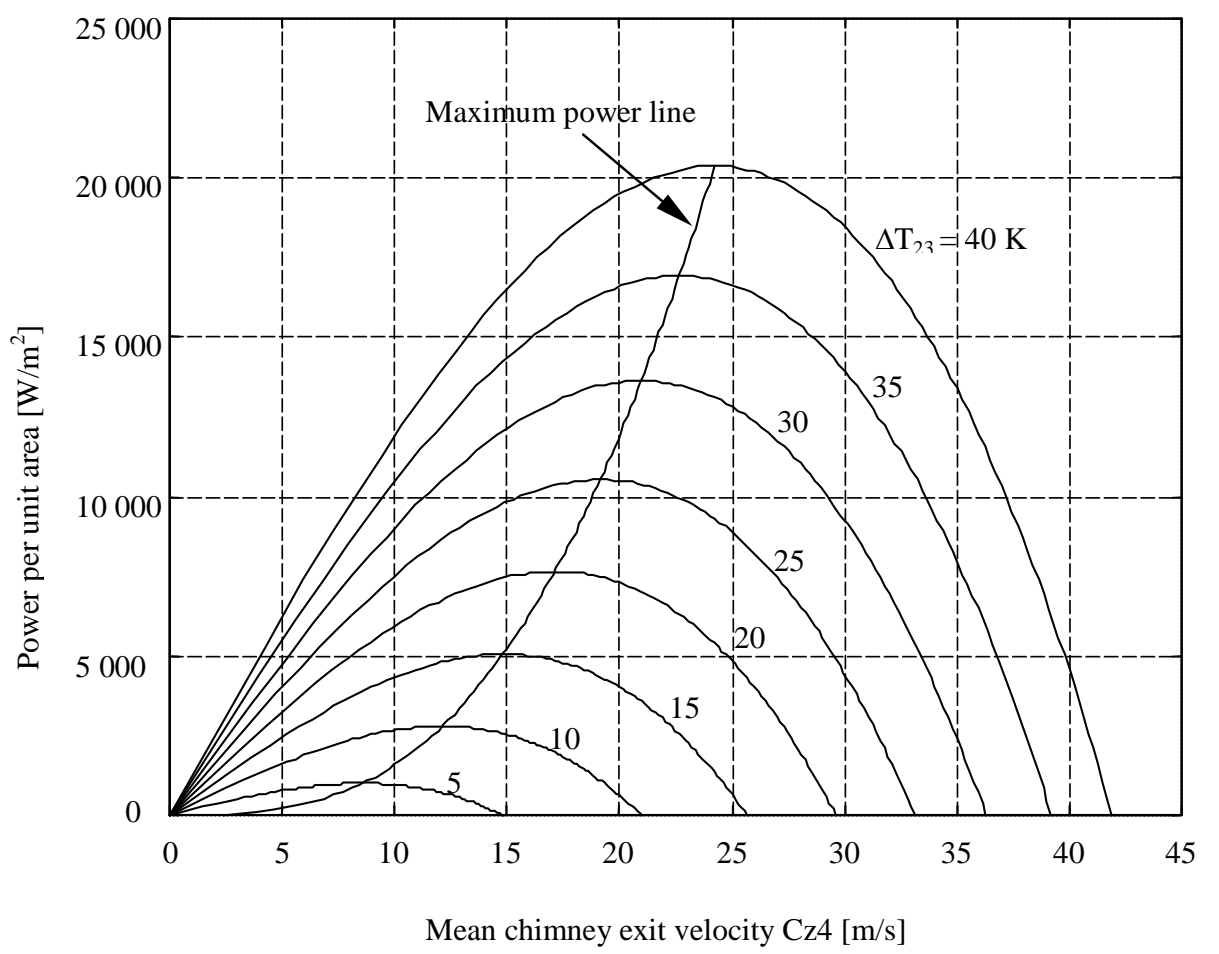

Figure 2.9. Power / unit area $\left[\mathrm{W} / \mathrm{m}^{2}\right]$ for $1500 \mathrm{~m}$ chimney along lines of constant temperature rise $\Delta \mathbf{T}_{23}[\mathrm{~K}]$.

Figure 2.10 can be used to calculate the required chimney diameter to develop a certain output power. The design power of $200 \mathrm{MW}$ will once again be used along with the following equation to calculate the required chimney diameter at various operating conditions.

$$
\text { Diameter }=\sqrt{\frac{4}{\pi} \frac{\text { Design power }}{\text { Power / Unit area }}}
$$

Figure 2.10 shows the relationship between the chimney diameter and exit velocity with a larger chimney diameter requiring a lower collector temperature rise. This is due to the reduced exit kinetic and chimney loss at lower velocities. For a given temperature rise increasing the exit velocity or mass flow initially decreases the required chimney diameter up to a point where it starts to increase once again. Other factors need to be considered such as the structural considerations of the height to base ratio of the chimney for it to be stable and the cost of building a large diameter chimney. At this stage of the design a diameter of $\mathbf{1 6 0} \mathbf{~ m}$ has been chosen and this value will be used in all further calculations. It can be seen in the above figure that it would require a minimum temperature rise of about $24 \mathrm{~K}$ and an exit velocity of approximately $19 \mathrm{~m} / \mathrm{s}$ to generate $200 \mathrm{MW}$ at this diameter. 


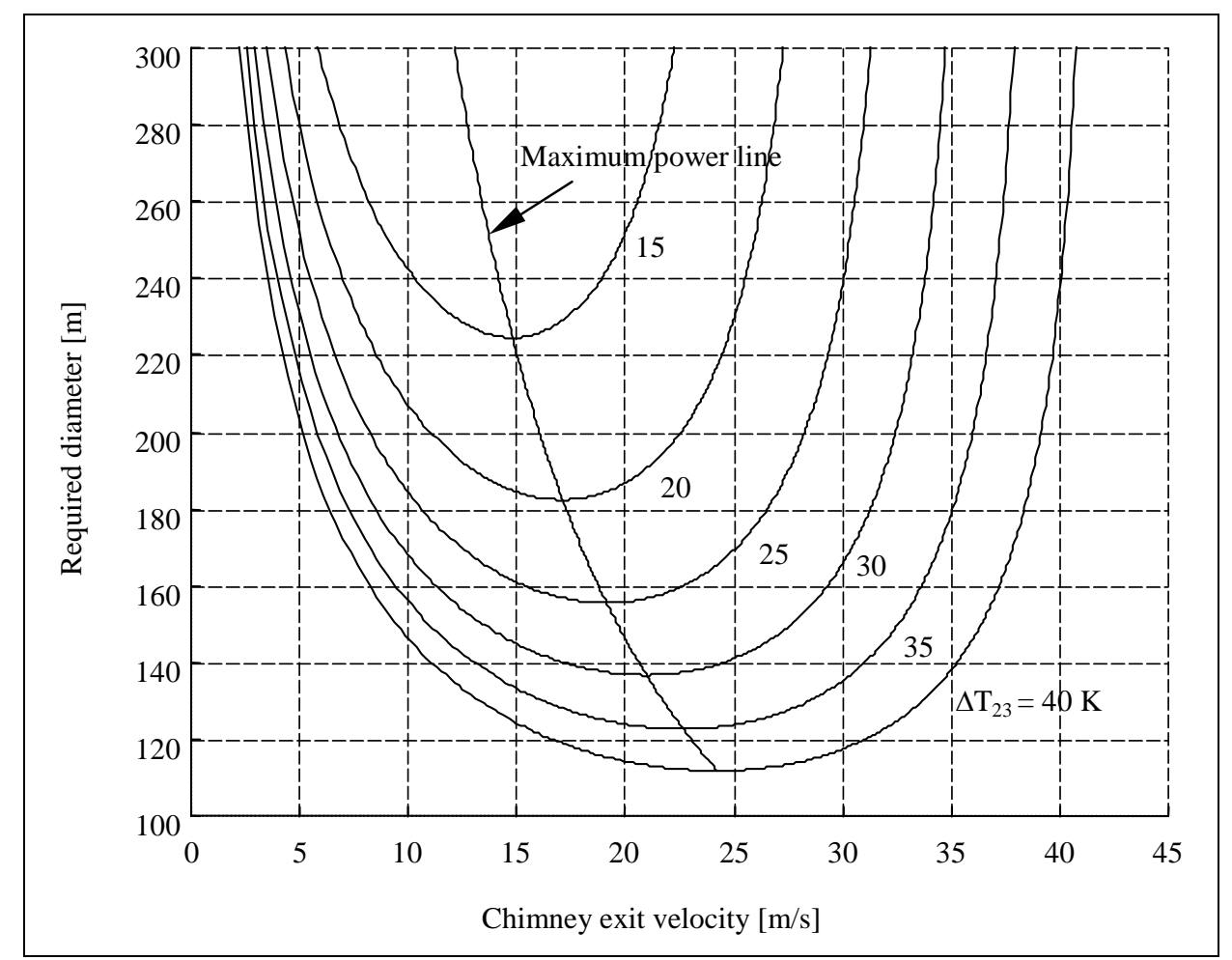

Figure 2.10. Required solar chimney diameter for $1500 \mathrm{~m}$ chimney along lines of constant temperature rise $\Delta T_{23}[K]$ to obtain design power output of $200 \mathrm{MW}\left(\eta_{\text {turb }}=80 \%, k=1\right)$.

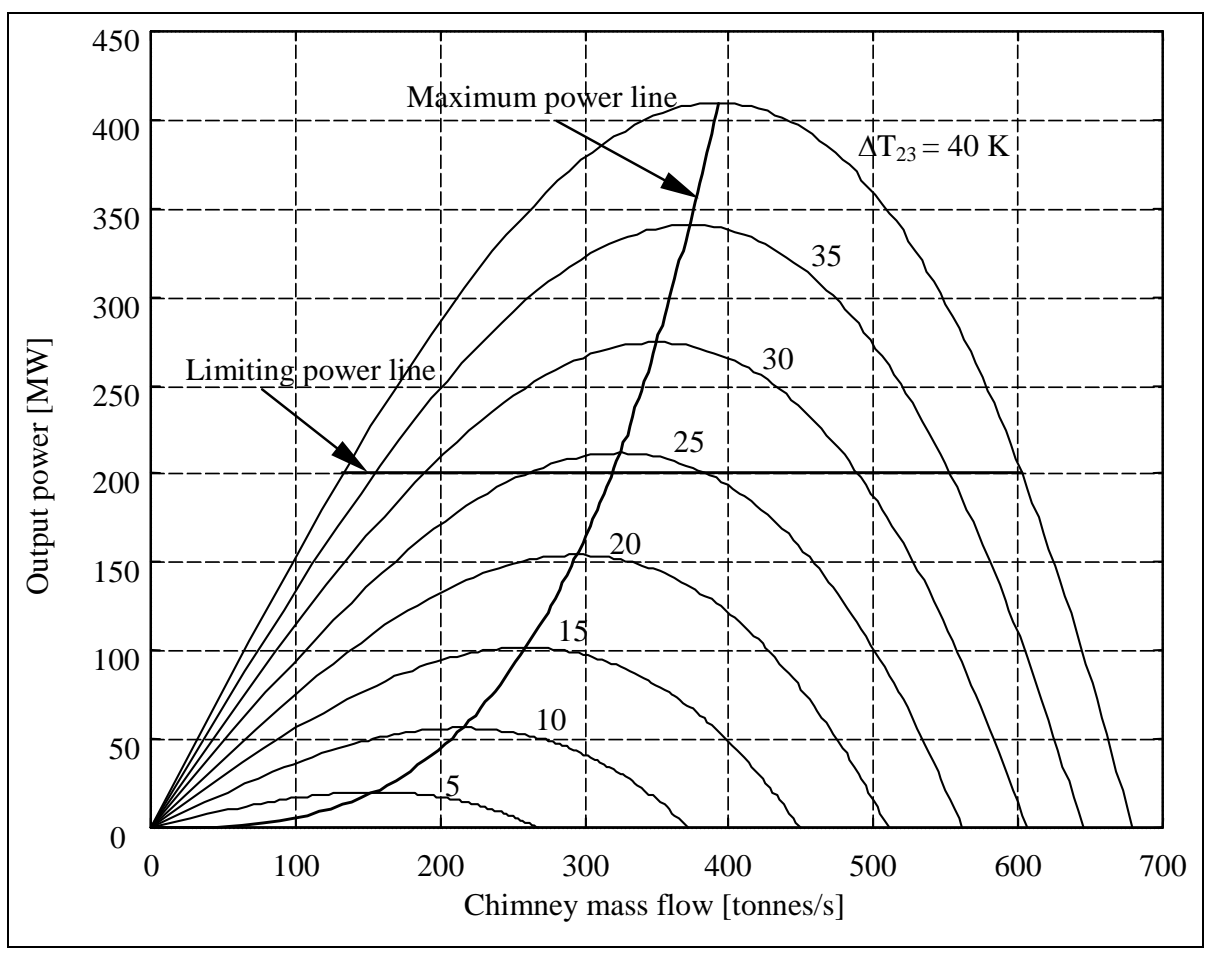

Figure 2.11. Power output [MW] for chimney of height $1500 \mathrm{~m}$ and diameter $160 \mathrm{~m}$, along lines of constant temperature rise $\Delta T_{23}[K]\left(\eta_{\text {turb }}=80 \%, k=1\right)$. 


\section{Determination of Turbine Operating Range}

Now that the geometry of the chimney is defined, the operating region of the turbine can be better investigated. With the chimney diameter known, equation (2.43) can be modified to give the actual power output vs. the mass flow (Figure 2.11).

It would seem reasonable to assume that at below peak power the turbine would operate along the maximum power line but to avoid damage to the generator, the turbine would not be able to deliver substantially more than $200 \mathrm{MW}$ for any extended period. There are two methods that could be used: the first is to increase the mass flow through the system, thus increasing the kinetic energy and friction losses while the second is to decrease the mass flow, thus allowing less work to be extracted from the turbine. Looking at the thermal efficiency (2.42) graph, Figure 2.12, it is possible to see that the option of decreasing the mass flow is best as plant efficiency improves for lower mass flow rates but drops for higher mass flow rates.

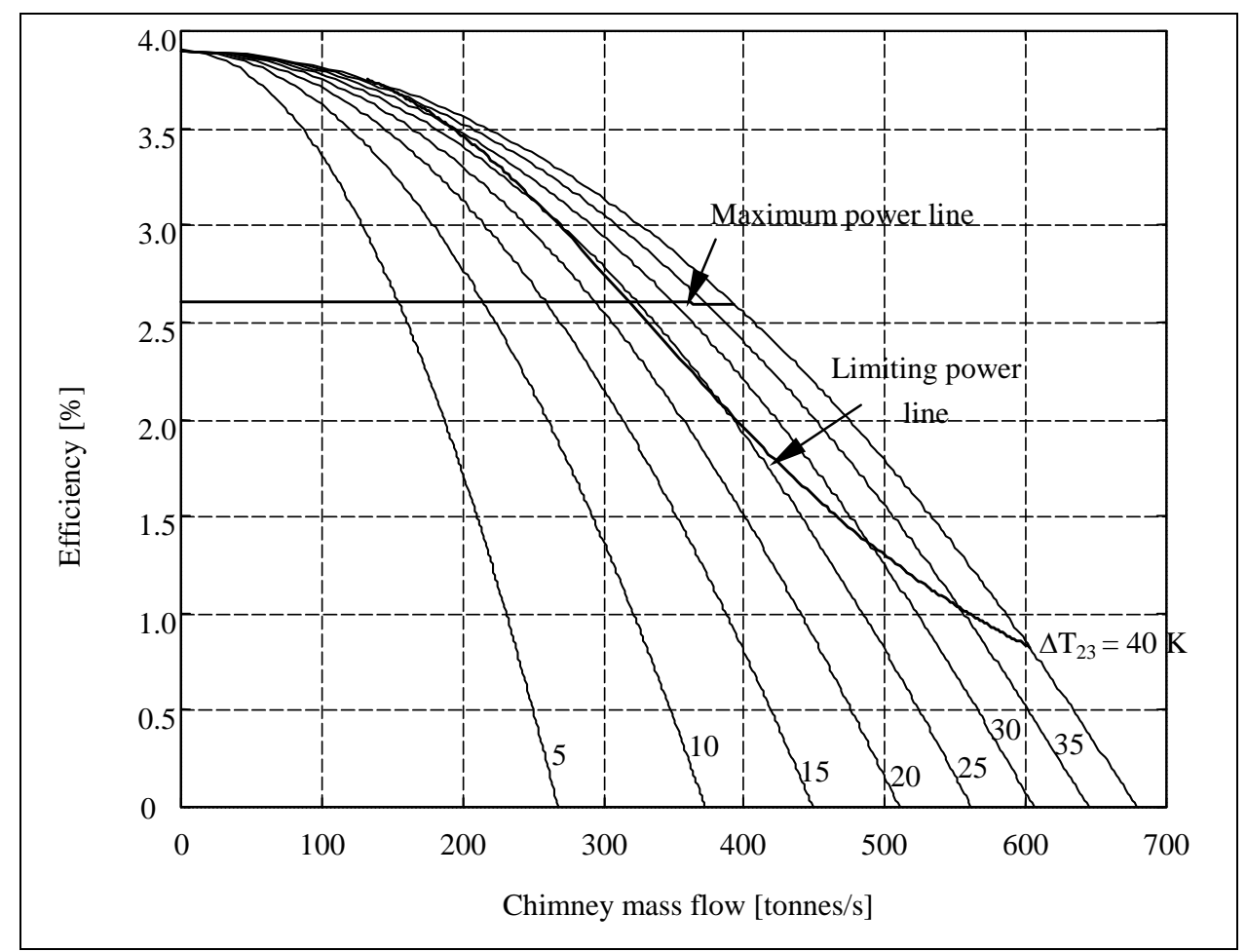

Figure 2.12. Plant efficiency [\%] for chimney of height $1500 \mathrm{~m}$ and diameter $160 \mathrm{~m}$, along lines of constant temperature rise $\Delta T_{23}[K]\left(\eta_{\text {turb }}=80 \%, k=1\right)$.

In the solar chimney the mass flow and turbine pressure drop are coupled- thus an increase in turbine pressure drop would decrease the mass flow and power output. Figure 2.13 shows how the turbine pressure drop is related to the mass flow. Until now the analysis has not taken into account the performance of the solar collector. This will be done in the next section but it should be borne in mind that the collector will not usually operate along lines of constant temperature. The option of 
decreasing the mass flow rate could help with the use of a thermal storage system for use at night but this will be discussed later.

Figure 2.11 shows the mass flow range of the turbine to be between 0 and 319 [tonnes / sec] but the total available pressure drop across the turbine still needs to be calculated. The temperature ratio across the turbine can be derived from the power equation.

$$
\begin{aligned}
\mathrm{P} & =\dot{\mathrm{m}} \mathrm{c}_{\mathrm{p}}\left(\mathrm{T}_{03}-\mathrm{T}_{03 \mathrm{te}}\right)=\left(\dot{\mathrm{mc}_{\mathrm{p}}} \mathrm{T}_{2} \mathrm{P}_{2}^{*}\right) \\
& =\dot{\mathrm{m}} \mathrm{c}_{\mathrm{p}} \eta_{\text {turb }}\left(\mathrm{T}_{03}-\mathrm{T}_{03 \mathrm{te}}^{\prime}\right)=\left(\dot{\mathrm{m}}_{\mathrm{p}} \mathrm{T}_{2} \mathrm{P}_{2}^{*}\right)
\end{aligned}
$$

rearranging

$$
\frac{\mathrm{T}_{03 \text { te }}^{\prime}}{\mathrm{T}_{03}}=1-\frac{\mathrm{T}_{02}}{\mathrm{~T}_{02}+\Delta \mathrm{T}_{23}} \frac{\mathrm{P}_{2}^{*}}{\eta_{\text {turb }}}
$$

Modifying equation (2.29) and substituting (2.63) gives the pressure drop across the turbine when kinetic energy loss is taken into account.

$$
\Delta \mathrm{p}_{\text {turb }}=\mathrm{p}_{2}\left\{1-\left[1-\left(\frac{\mathrm{T}_{02}}{\mathrm{~T}_{02}+\Delta \mathrm{T}_{23}}\right)\left(\frac{\mathrm{P}_{2}^{*}}{\eta_{\text {turb }}}\right)\right]^{\frac{\gamma}{\gamma-1}}\right\}
$$

Figure 2.13 shows that the turbine will have to operate over a fairly large pressure range, from 0 to $749 \mathrm{~Pa}$ along the line of maximum power and up to $1700 \mathrm{~Pa}$ for a collector temperature rise of $40 \mathrm{~K}$. This figure is often found in gas turbine texts and shows that the maximum power is obtained when the turbine pressure drop is $2 / 3$ of the maximum available. In section 2.3 and 2.4 where the collector characteristics are added this is shown not to be the optimum design point for the turbine.

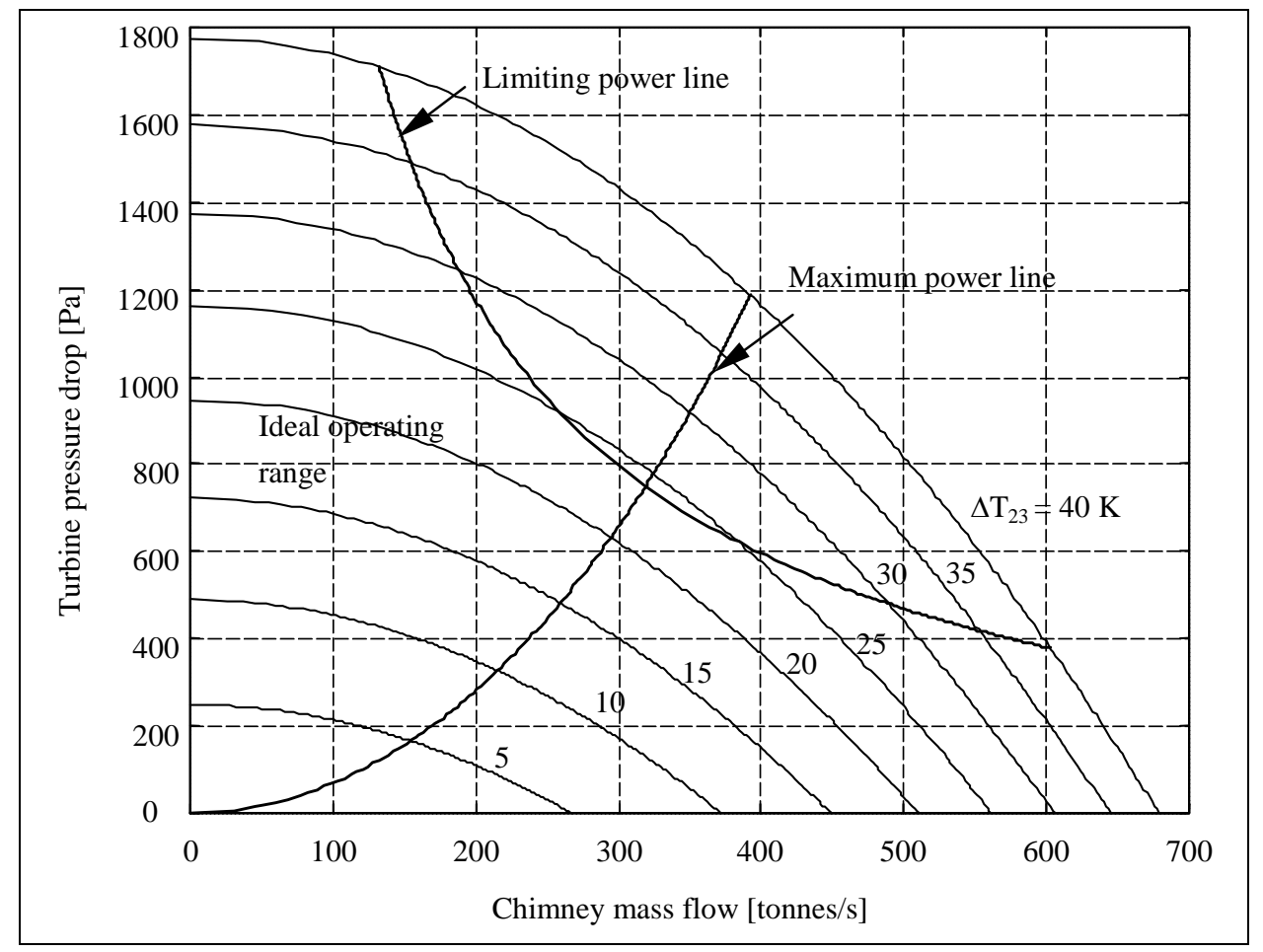

Figure 2.13. Turbine pressure drop [Pa] for chimney of height $1500 \mathrm{~m}$ and diameter $160 \mathrm{~m}$, along lines of constant temperature rise $\Delta T_{23}[K]\left(\eta_{\text {turb }}=80 \%, k=1\right)$. 
At this stage the possible operating range of the turbine has been well defined by looking at the cycle analysis and including kinetic energy loss and turbine efficiency. As was mentioned there has been no attempt to include the operating characteristic of the solar collector but it can be expected that this will have an effect on the operation of the plant. The next section will determine more accurately the operating range of the solar chimney turbine through the inclusion of the solar collector.

\subsection{CyCle AnAlysis: STEAdy STATE COLlECTOR MODEL}

From the previous section, a well-defined set of equations is available to calculate a particular solar chimney's performance when the inlet conditions and collector temperature rise are known. Using these equations, it was possible to calculate certain limiting values of the solar chimney performance such as efficiency, power output for a given plant and turbine pressure drops and mass flow rates. In this section these limiting values will be more accurately determined through the inclusion of the solar collector performance. The steady-state model tends to overestimate the collector temperature rise especially for low mass flow rates and for this reason a transient model was developed. However, the results from the steady-state analysis provide some useful insights into the plant operation and are thus presented here. In the development of the simple steady-state model for the solar collector the following assumptions are made:

- The conditions are steady state.

- There is no evaporation in the collector.

- The vertical temperature gradient of the air under the glass roof is zero.

- Heat transfer to the air in the collector is only by convection at the top and bottom collector surfaces.

The reason for these assumptions is to simplify the analysis and find the limiting conditions at which the turbine operates. Simulation of the solar collector at steady-state conditions would give the maximum temperature rise for a given inlet solar radiation. The solar collector model is developed using existing methods of heat transfer analysis to perform the cycle analysis with the collector characteristics included. The model was developed using the texts of Incopera and De Witt (1990), Holman (1992) and Sears et al. (1987). The following figures show the model used to simulate the solar collector, 


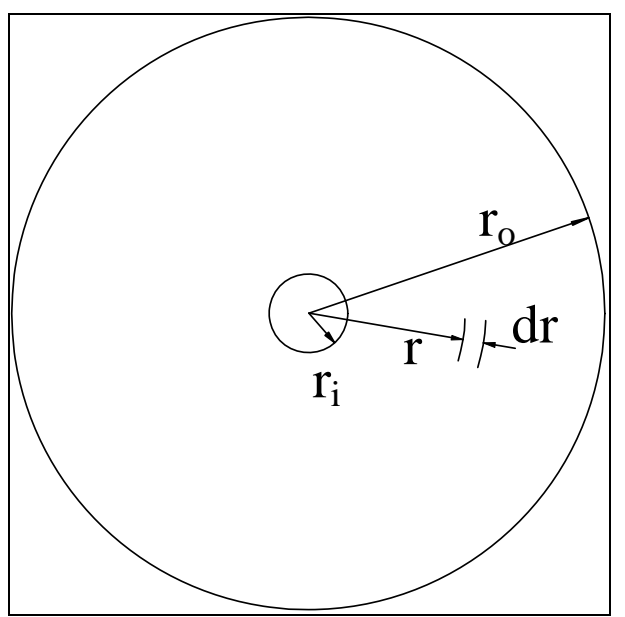

Figure 2.14. Top view of solar collector showing dimensioning and position of control volume.

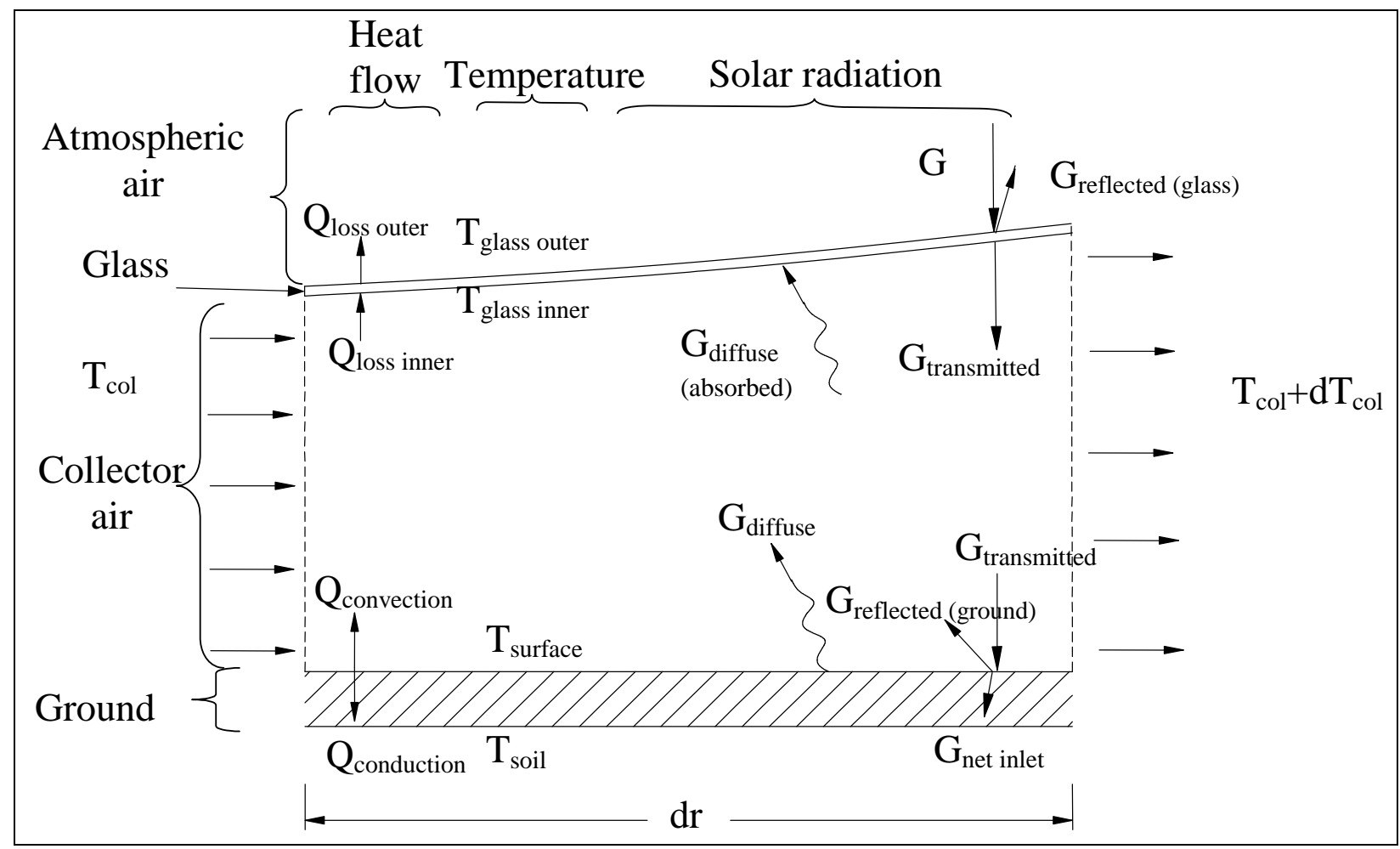

Figure 2.15. Detail of control volume of solar collector showing nomenclature for temperatures, heat, mass and radiation flow.

The simplest analysis of the collector is to assume that all incoming solar radiation is used to heat the collector air as in equation (2.65) below. This gives insight into the coupling between massflow and temperature rise.

$$
\mathrm{G}=\dot{\mathrm{mc}}_{\mathrm{p}} \Delta \mathrm{T}_{23}
$$

If $\mathrm{G}$ remains constant then a higher mass flow will result in a lower temperature rise and vice versa. It is for this reason that using the maximum power line of Figure 2.11 to define the operating range of the solar chimney turbine is inaccurate. In a gas turbine engine the turbine inlet temperature is 
normally kept constant and constrained by the thermal strengths of the first row of turbine blades. This is the reason for this type of figure but it is not applicable to solar chimney operation.

\section{Collector Governing Equations}

Equation (2.65) assumes that the air moving through the collector absorbs all the incoming solar radiation. Obviously this is not the case and only part of the incoming radiation is absorbed. Some is absorbed by the soil and some lost back to the environment. As stated before, it is assumed that the air is heated by convection as it moves through the solar collector. Figure 2.15 shows a radial control volume of the solar collector as the air moves through it. The differential equation for temperature rise of the collector air as it moves through the control volume is given by,

$$
\frac{\mathrm{dT}_{\text {col }}}{\mathrm{dr}}=\frac{2 \pi \mathrm{r}}{\dot{\mathrm{m}_{\mathrm{p}}}}\left(\mathrm{h}_{\text {surf }}\left(\mathrm{T}_{\text {surf }}-\mathrm{T}_{\text {col }}\right)+\mathrm{h}_{\text {gli }}\left(\mathrm{T}_{\text {col }}-\mathrm{T}_{\text {gli }}\right)\right)
$$

The above equation shows that the incremental temperature rise is inversely proportional to the mass flow and proportional to the temperature difference at the lower and upper collector surfaces. The above equation is a first order differential equation and can be solved numerically using a Runge-Kutta numerical integration scheme. The collector is divided into a series of control volumes and the calculation begins at the collector inlet with the atmospheric temperature. The incremental temperature rise in each successive control volume is calculated to eventually calculate the overall temperature rise of the collector. This method has the advantage of being computationally cheap. This is because the calculation of the temperature rise requires just one sweep through the collector.

Additional equations are required to calculate the soil surface, $\mathrm{T}_{\text {surf }}$ and inner glass, $\mathrm{T}_{\text {gli }}$ temperatures. The temperature of the glass outer (top) surface $T_{\text {glo }}$ is also required. In each control volume an up-winding scheme is used to determine the collector air temperature. This assumes that the temperature of the collector air in a certain control volume is constant and equal to the temperature at the exit of the previous control volume. For example the temperature in the first control volume is assumed to be the same as the atmospheric temperature $T_{a t m}$. This assumption was thought to be reasonable as the temperature change between each collector control volume is very small.

To set up three energy balance equations and solve for $\mathrm{T}_{\text {surf }}$, $\mathrm{T}_{\text {gli }}$ and $\mathrm{T}_{\text {glo }}$ certain assumptions are made about the ground and collector cover. They are as follows:

The ground beneath the collector

- The temperature at some depth below the surface is constant.

- There is no radial conduction.

- The soil specific heat cpsoil and density are constant. 


\section{The collector cover material}

- Reflected radiation by the cover and ground is lost back to the environment.

- All radiation absorbed by the cover material is done so on the surface.

- There is no internal reflection in the cover material.

- The emissivity of the cover material is assumed to be equal to unity.

- The sky temperature is assumed to be the same as the atmospheric temperature.

For the steady state simulation it is assumed that the temperature at some distance below the surface is constant and that there is steady-state conduction occurring in the ground. As mentioned earlier, this will lead to higher estimates of the collector temperature rise especially for low volume flow rates.

The collector model is kept as basic as possible to simplify its calculation. The basic assumption is that all short wave radiation passes through the collector. For long wave or infrared radiation, it is assumed that the collector is so opaque that the radiation is absorbed at the surface or only penetrates a small distance into it. The three energy balance equations are presented next.

1. Soil Surface. At this position in the collector the heat gain is by the solar radiation transmitted by the collector cover. Heat loss is by reflection of some of this transmitted radiation, conduction of heat into the ground, convection of heat into the collector air and diffuse radiation being transmitted and absorbed by the collector cover. The following equation presents the energy balance at the soil surface.

$$
\underbrace{\tau_{\text {in }} \mathrm{e}_{\text {surf }} \mathrm{G}}_{\text {Net inlet radiation }}=\underbrace{\mathrm{h}_{\text {surf }}\left(\mathrm{T}_{\text {surf }}-\mathrm{T}_{\text {col }}\right)}_{\text {Convection }}+\underbrace{\frac{\mathrm{k}_{\text {soil }}}{\mathrm{L}_{\text {soil }}}\left(\mathrm{T}_{\text {surf }}-\mathrm{T}_{\text {soil }}\right)}_{\text {Conduction }}+\underbrace{\left(1-\tau_{\text {out }}\right) \mathrm{e}_{\text {surf }} \sigma\left(\mathrm{T}_{\text {surf }}^{4}-\mathrm{T}_{\text {gli }}^{4}\right)}_{\text {Diffuse radiation }}
$$

2. Lower Collector Surface. At the lower surface heat is absorbed by convection from the collector air and diffuse radiation from the ground. Heat is lost through conduction to the upper surface.

$$
\underbrace{\mathrm{h}_{\text {glo }}\left(\mathrm{T}_{\text {glo }}-\mathrm{T}_{\text {atm }}\right)}_{\text {convection }}=\underbrace{\frac{\mathrm{k}_{\text {gla }}}{\mathrm{L}_{\text {gla }}}\left(\mathrm{T}_{\text {gli }}-\mathrm{T}_{\text {glo }}\right)}_{\text {conduction }}+\underbrace{\left(1-\tau_{\text {out }}\right) \mathrm{e}_{\text {soil }} \sigma\left(\mathrm{T}_{\text {surf }}^{4}-\mathrm{T}_{\text {gli }}^{4}\right)}_{\text {diffuse radiation }}
$$

3. Upper Collector Surface. At the upper surface heat is gained through conduction from the lower surface and a certain amount of solar radiation absorbed. Heat is lost to the atmosphere by convection and emitted radiation.

$$
\underbrace{\mathrm{h}_{\text {glo }}\left(\mathrm{T}_{\text {glo }}-\mathrm{T}_{\text {atm }}\right)}_{\text {convection }}+\underbrace{\mathrm{e}_{\text {glass }} \sigma\left(\mathrm{T}_{\text {glo }}^{4}-\mathrm{T}_{\text {atm }}^{4}\right)}_{\text {emitted radiation }}=\underbrace{\frac{\mathrm{k}_{\text {gla }}}{\mathrm{L}_{\text {gla }}}\left(\mathrm{T}_{\text {gli }}-\mathrm{T}_{\text {glo }}\right)}_{\text {conduction }}+\underbrace{\mathrm{G}\left(1-\tau_{\text {in }}\right)}_{\text {absorbed solar radiation }}
$$


As a first step, a single operating point of the solar collector is simulated and the various temperatures and heat flows through the solar collector plotted. The various material constants are taken from Schlaich et al. (1995) and atmospheric constants from Preston-Whyte et al. (1989). The lower ground temperature and depth are based on the measurements at the experimental plant (Haaf 1984). The values used in the sample calculation are given in the following table.

Table 2.4. Symbols and numerical values used for sample solar collector calculation.

\begin{tabular}{|l|l|l|l|}
\hline Description & Symbol & Unit & Value \\
\hline Mass flow rate & $(\dot{\mathrm{m}})$ & {$[$ tonnes/s] } & 200 \\
\hline Glass transmissivity, visible light range. & $\left(\tau_{\text {in }}\right)$ & & 0.85 \\
\hline Glass transmissivity, infrared range. & $\left(\tau_{\text {out }}\right)$ & & 0.00018 \\
\hline Ground emissivity & $\left(e_{\text {surf }}\right)$ & & 0.9 \\
\hline Ground conductivity & $\left(k_{\text {ground }}\right)$ & {$[\mathrm{W} / \mathrm{mK}]$} & 0.6 \\
\hline Glass conductivity & $\left(k_{\text {glass }}\right)$ & {$[\mathrm{W} / \mathrm{mK}]$} & 0.9 \\
\hline Outer radius & $\left(r_{o}\right)$ & {$[\mathrm{m}]$} & 2000 \\
\hline Convective heat transfer coeff. & $\left(h_{\text {gli }}, h_{\text {glo }}, h_{\text {gro }}\right)$ & {$\left[\mathrm{W} / \mathrm{m}^{2} \mathrm{~K}\right]$} & 5 \\
\hline Inlet radiation & $\mathrm{G}_{\text {in }}$ & {$\left[\mathrm{W} / \mathrm{m}^{2}\right]$} & 800 \\
\hline Collector inlet temperature & $\mathrm{T}_{\text {o2 }}$ & {$[\mathrm{K}]$} & 303.2 \\
\hline Collector inlet pressure & $\mathrm{P}_{\mathrm{o} 2}$ & {$[\mathrm{~Pa}]$} & 90000 \\
\hline Ground storage temp & $\left(T_{\text {soil }}\right)$ & {$[\mathrm{K}]$} & 283.2 \\
\hline Thermal storage depth & $\left(L_{\text {soil }}\right)$ & {$[\mathrm{m}]$} & 0.8 \\
\hline Glass thickness & $\left(L_{\text {glass }}\right)$ & {$[\mathrm{m}]$} & 0.004 \\
\hline Inner radius & $\left(r_{i}\right)$ & {$[\mathrm{m}]$} & 160 \\
\hline
\end{tabular}

The subscripts are as follows,

\begin{tabular}{|l|l|l|l|l|l|l|l|}
\hline $\begin{array}{l}\text { Soil } \\
\text { properties }\end{array}$ & (soil) & $\begin{array}{l}\text { Ground } \\
\text { surface }\end{array}$ & (surf) & $\begin{array}{l}\text { Glass inner } \\
\text { surface }\end{array}$ & (gli) & $\begin{array}{l}\text { Glass outer } \\
\text { surface }\end{array}$ & (glo) \\
\hline Inlet & (in) & Outlet & (out) & \multicolumn{1}{|l}{} \\
\hline
\end{tabular}

The thermal efficiency of the solar collector is defined as the heat delivered to the collector air over the total solar radiation in

$$
\eta_{\text {col }}=\frac{\dot{m c_{p}}\left(\Delta T_{23}\right)}{G A_{c o l}}
$$

Figure 2.16 shows the air temperature rise $\left(\mathrm{T}_{\mathrm{col}}-\mathrm{T}_{\mathrm{o} 2}\right)$ in the solar collector as it moves from right to left under the glass. The fastest temperature rise of the air is at the outer radius. The heat flows between components are shown in Figure 2.17 where the magnitudes of the losses can be seen. The overall efficiency of $\mathbf{6 2 . 5} \%$ and the magnitude of losses into the ground compared well with the values given in Schlaich et al. (1995). The steady-state assumption tends to make the calculated 
temperatures higher than would be expected. In the real plant there will always be a time lag between the incoming solar radiation and heat being transferred to the air as some of the heat is absorbed by the ground.

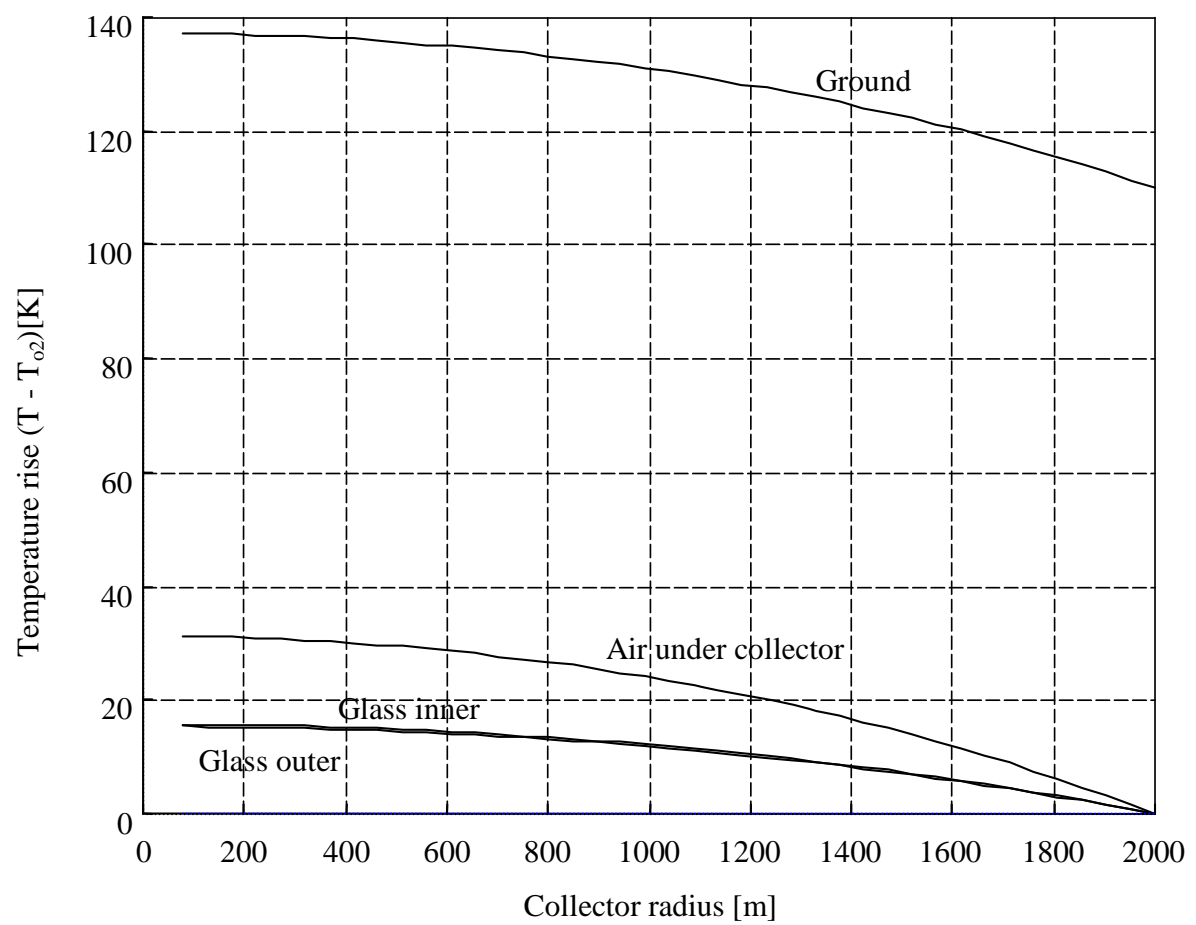

Figure 2.16. Temperature rise [K] of ground, air, and glass surfaces through solar collector for values in Table 2.4 .

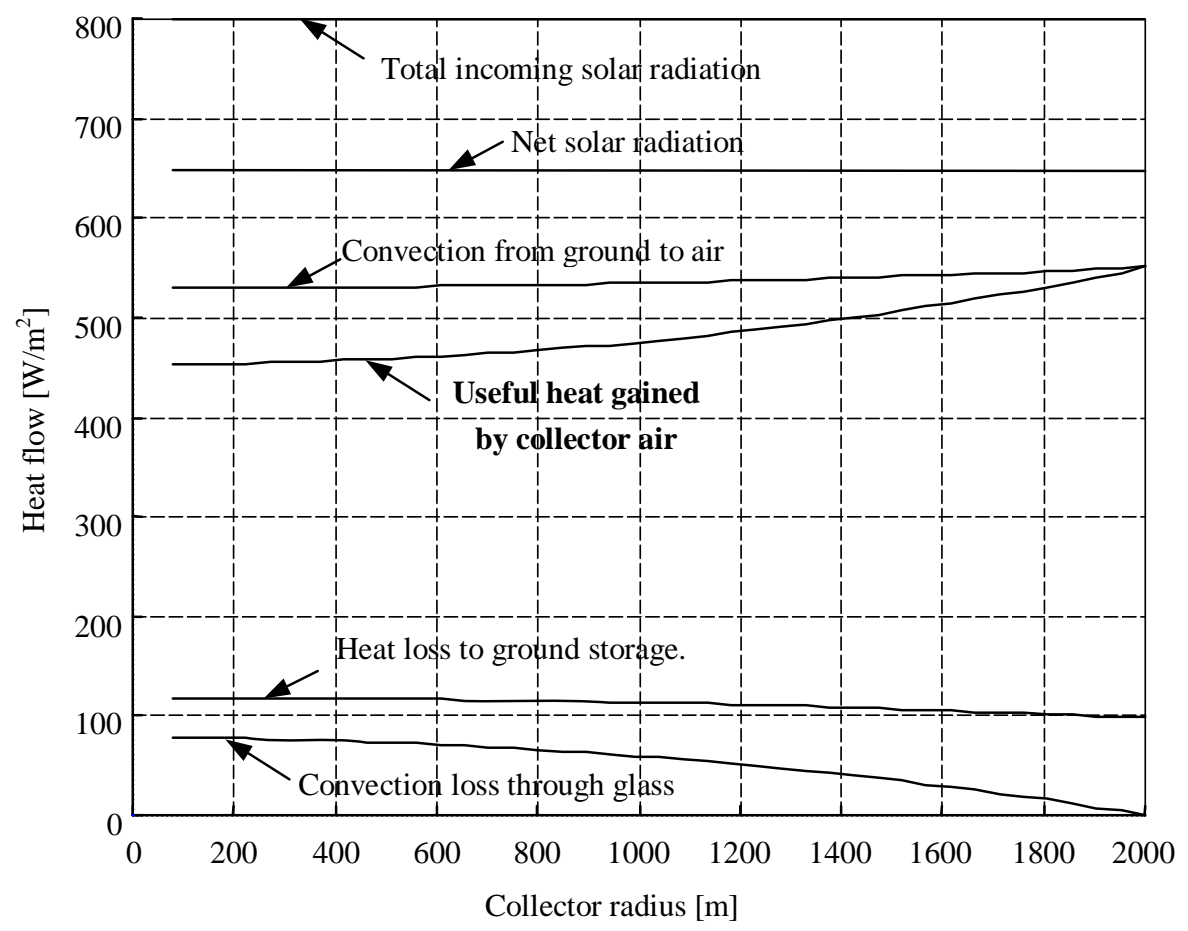

Figure 2.17. Heat flows $\left[\mathrm{W} / \mathrm{m}^{2}\right]$ in solar collector for values in Table 2.4. 


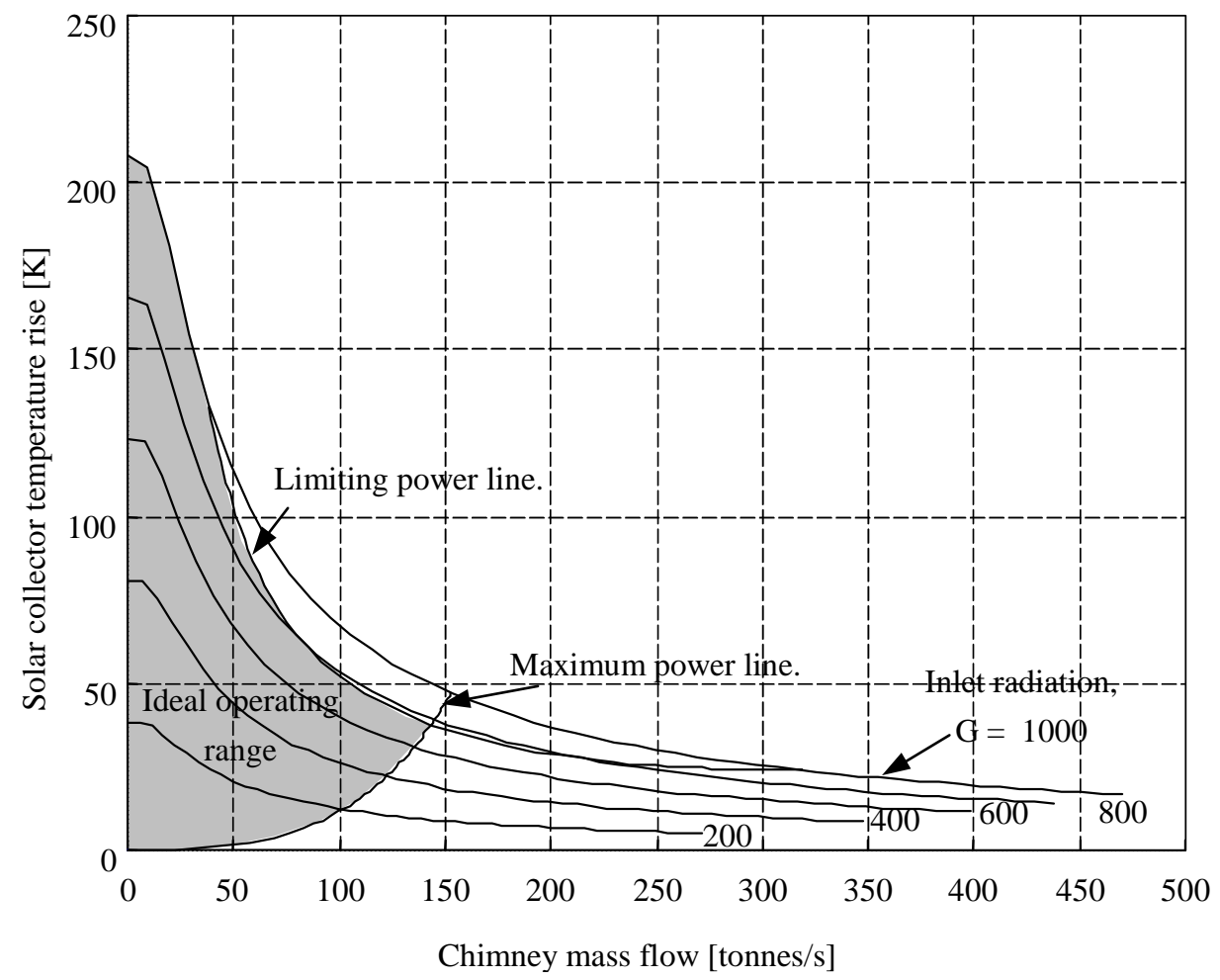

Figure 2.18. Solar collector temperature rise [K] for varying mass flow [tonnes/s] along lines of constant inlet radiation $\left[\mathrm{W} / \mathrm{m}^{2}\right]$.

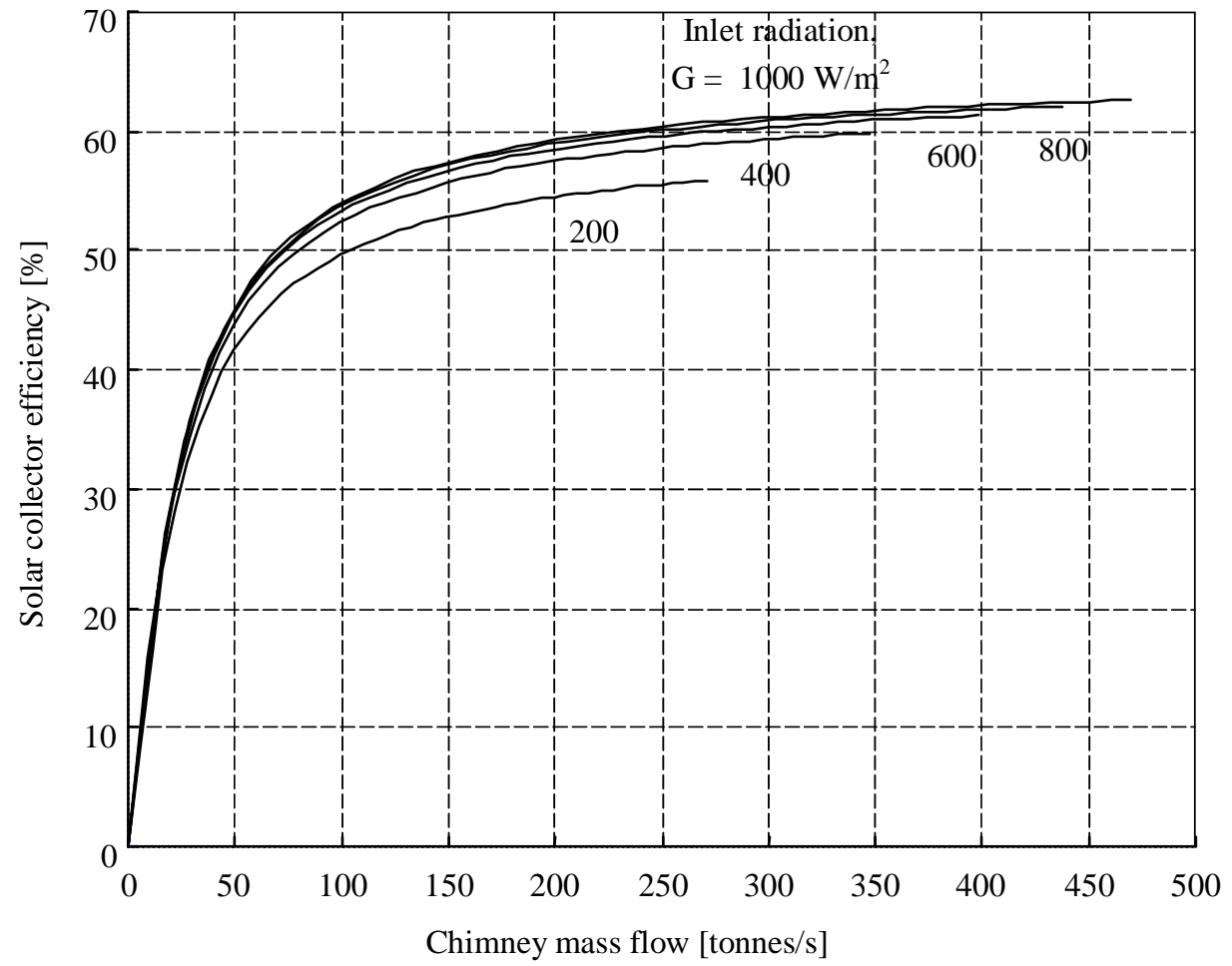

Figure 2.19. Solar collector efficiency [\%] for varying mass flow [tonnes/s] along lines of constant inlet radiation $\left[\mathrm{W} / \mathrm{m}^{2}\right]$. 


\section{Operating Range Prediction Using Steady-State Model}

Figure 2.18 shows the results of entire range of steady-state calculations of the solar collector. The calculations were performed for inlet solar radiation values ranging from $200 \mathrm{~W} / \mathrm{m}^{2}$ to $1000 \mathrm{~W} / \mathrm{m}^{2}$. The mass flows were adjusted from zero to the choking value of the plant where the turbine pressure drop is zero. The increase of the temperature rise for a decrease in the mass flow can be clearly seen. Figure 2.19 shows the corresponding efficiencies with the efficiency increasing as the mass flow increases.

In Figure 2.11 the solar chimney power output was plotted along lines of constant collector temperature rise. While there is no error in presenting the data this way, it is not correct to assume that the plant will operate along the line of maximum power presented in that figure. As mentioned before, the heat addition to the solar chimney is governed by the incoming solar radiation. For short time periods, say a few minutes, the solar radiation could be considered constant and plant output plotted along lines of constant solar radiation. Figure 2.20 presents the plant power output along lines of constant solar radiation. The predicted maximum power line is at a lower mass flow rate than predicted previously.

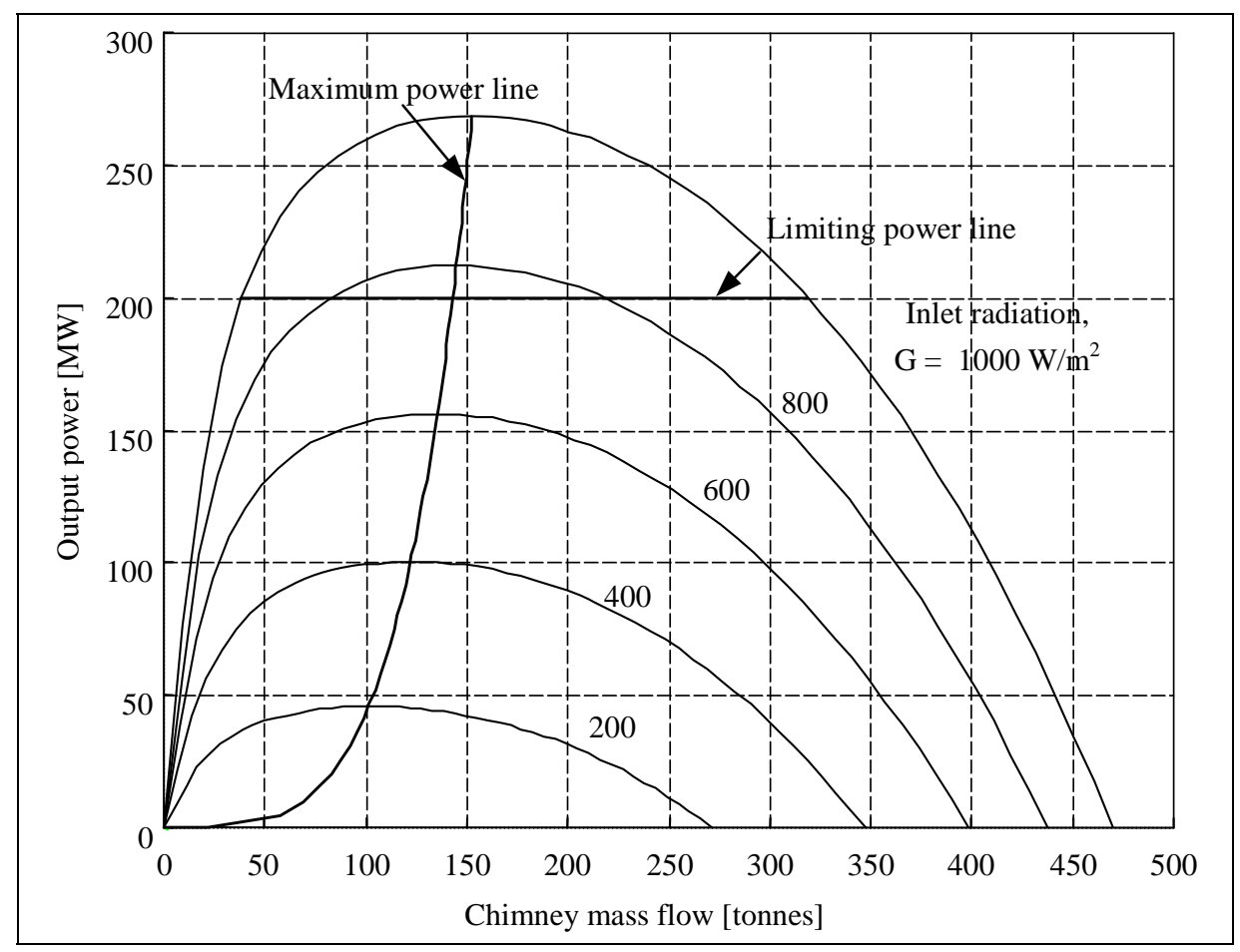

Figure 2.20. Power output [MW] for chimney of height $1500 \mathrm{~m}$ and diameter $160 \mathrm{~m}$, along lines of constant inlet radiation $\left[\mathrm{W} / \mathrm{m}^{2}\right]\left(\eta_{\text {turb }}=\mathbf{8 0} \%, \mathrm{k}=1\right)$.

To achieve the same maximum power at a lower mass flow rate, there must be a corresponding increase in the turbine pressure drop. Figure 2.21 shows that the expected pressure drop over the turbine is higher than that predicted in Figure 2.13. Table 2.5 compares the predicted mass flows 
and pressure drops at the intersection of the maximum and limiting power lines obtained from the two figures. There is a large difference between the two operating points and a turbine designed for either one would not work efficiently at the other operating point.

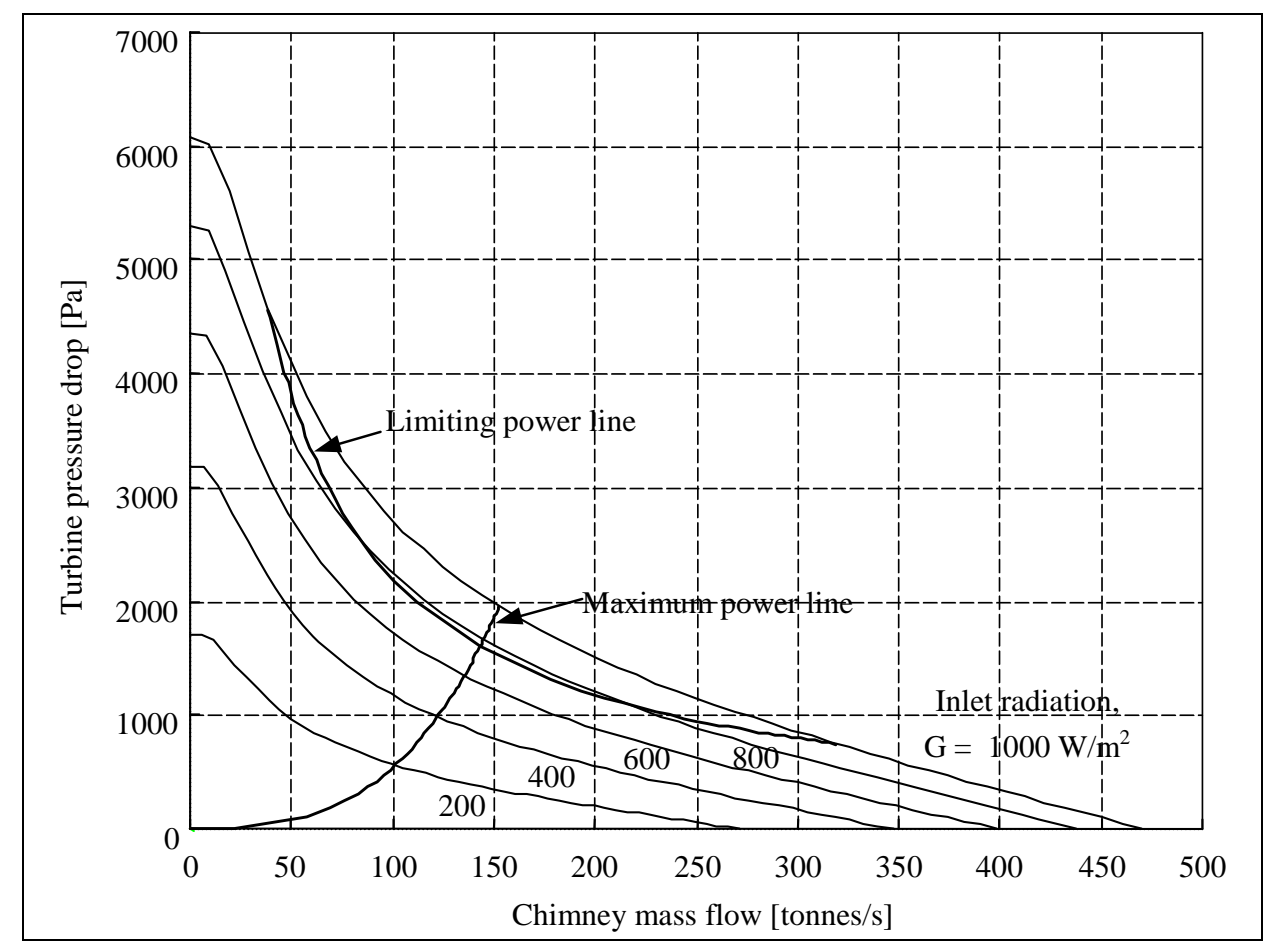

Figure 2.21. Turbine pressure drop [Pa] for chimney of height $1500 \mathrm{~m}$ and diameter $160 \mathrm{~m}$, along lines of constant inlet radiation $\left[\mathrm{W} / \mathrm{m}^{2}\right]\left(\eta_{\text {turb }}=80 \%, k=1\right)$.

Table 2.5. Comparison of turbine mass flow rate and pressure drop at intersection of maximum power and limiting power lines.

\begin{tabular}{|r|c|c|}
\cline { 2 - 3 } \multicolumn{1}{c|}{} & $\begin{array}{c}\text { Lines of constant radiation } \\
{\left[\mathrm{W} / \mathrm{m}^{2}\right]}\end{array}$ & $\begin{array}{c}\text { Lines of constant temperature } \\
{[\mathrm{K}]}\end{array}$ \\
\hline $\begin{array}{r}\text { Solar collector } \\
\text { temperature rise, } \Delta \mathrm{T}_{23}\end{array}$ & $37.4[\mathrm{~K}]$ & $24.01[\mathrm{~K}]$ \\
\hline $\begin{array}{r}\text { Turbine pressure } \\
\text { drop, } \Delta \mathrm{P}_{\text {turb }}\end{array}$ & $1597[\mathrm{~Pa}]$ & $749[\mathrm{~Pa}]$ \\
\hline Mass flow, $\dot{\mathrm{m}}$ & $143100[\mathrm{~kg} / \mathrm{s}]$ & $318900[\mathrm{~kg} / \mathrm{s}]$ \\
\hline
\end{tabular}

The turbine for the experimental plant in Manzanares was designed using a graph similar to Figure 2.13 (Schlaich 1995). It is thought that this may be the cause of some of the operational characteristics of the Manzanares plant. The following reasoning is based on the design variation between the two different single stage turbines that would have to be built for each of the cases shown in Table 2.5. In general a turbine that is designed to operate at a certain high pressure drop but then operates at a lower one with a higher mass flow rate will have a low total-to-static efficiency. The turbine will have too many blades and the flow area will be too small, resulting in 
high friction and exit kinetic energy loss. The opposite case of a low-pressure turbine subjected to a high-pressure drop may result in the turbine stalling especially in a turbine with few blades. In the case of the solar chimney, this would result in the mass flow increasing and the collector temperature rise decreasing, reducing pressure potential bringing the system back to a point where the turbine would be able to operate. This process of stalling and unstalling would then be cyclic and it is thought that this may be the cause of the fluctuating power output observed in the Manzanares plant (Haaf 1984). As has been emphasised a number of times, it is important to match the turbine to the plant properly. In the case of the solar chimney, it may be better to have a turbine that is capable of a slightly higher pressure drop than the maximum predicted. A slight loss in efficiency in a full-scale plant would be preferable to supplying a fluctuating power output in the megawatt range.

The overall plant efficiency, $\eta_{\text {plant}}$, is a product of the solar collector efficiency (Figure 2.19) and thermal chimney efficiencies (Figure 2.22) defined in equations (2.38) and (2.70) respectively. It can be seen that the maximum efficiency corresponds to the position of maximum power which is to be expected (Figure 2.20). One other property of the plant that can be seen is that there is a fairly large region from $\mathbf{1 0 0}$ - $\mathbf{2 0 0}$ tonnes/s where the plant efficiency remains fairly constant (Figure 2.23). This will have benefits in the control of the plant as it will not be too sensitive to changes in the pressure drop over the turbine. The efficiency of the plant also rises very steeply up to an inlet radiation of $\mathbf{2 0 0} \mathrm{W} / \mathrm{m}^{2}$ but does not change by more than $\mathbf{0 . 3 5}$ percentage points after this (about $15 \%$ of the peak values). The numerical values are listed in Appendix A.

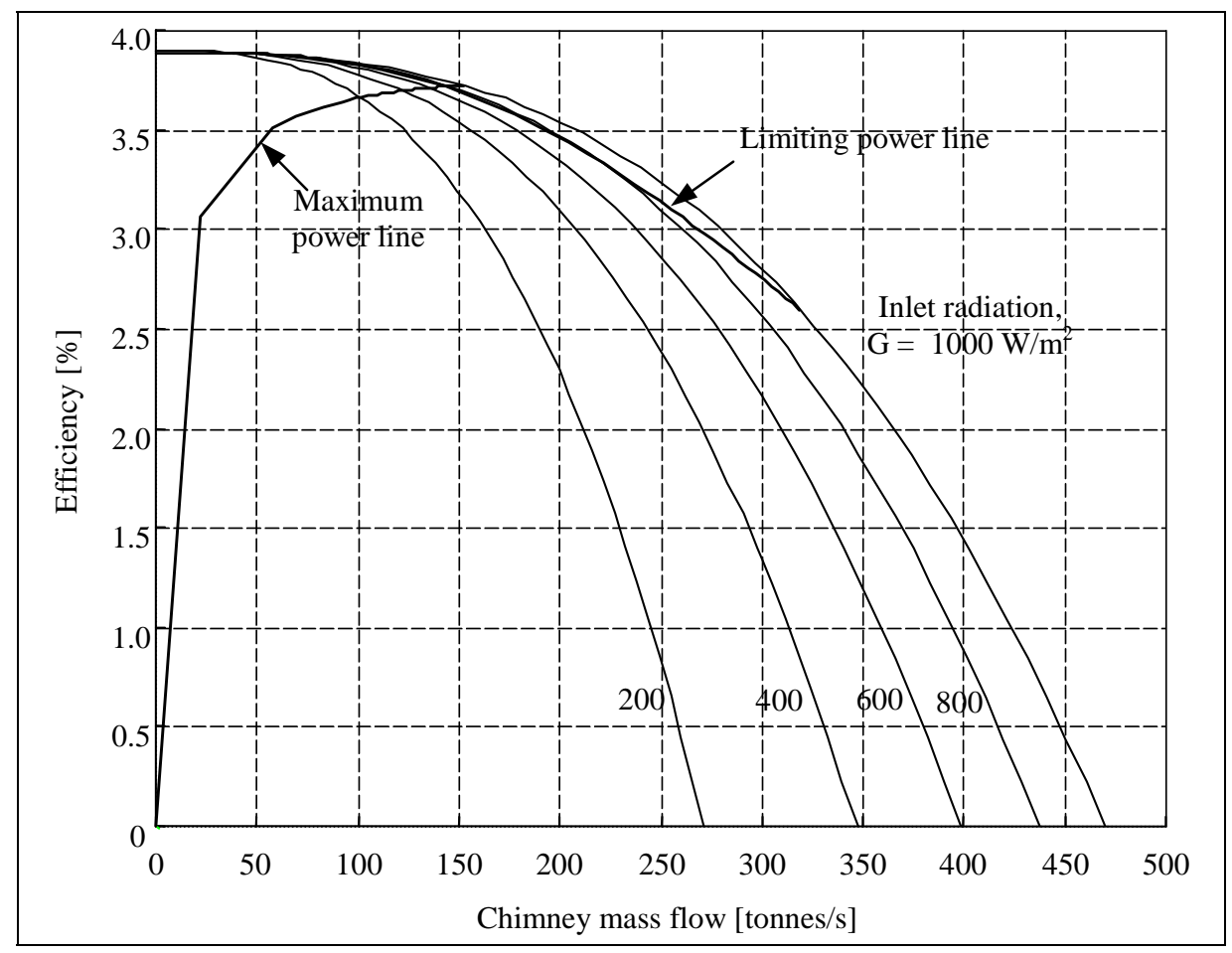

Figure 2.22. Thermal efficiency [\%] for chimney of height $1500 \mathrm{~m}$ and diameter $160 \mathrm{~m}$, along lines of constant inlet radiation $\left[\mathrm{W} / \mathrm{m}^{2}\right]\left(\eta_{\mathrm{turb}}=\mathbf{8 0} \%, \mathbf{k}=\mathbf{1}\right)$. 


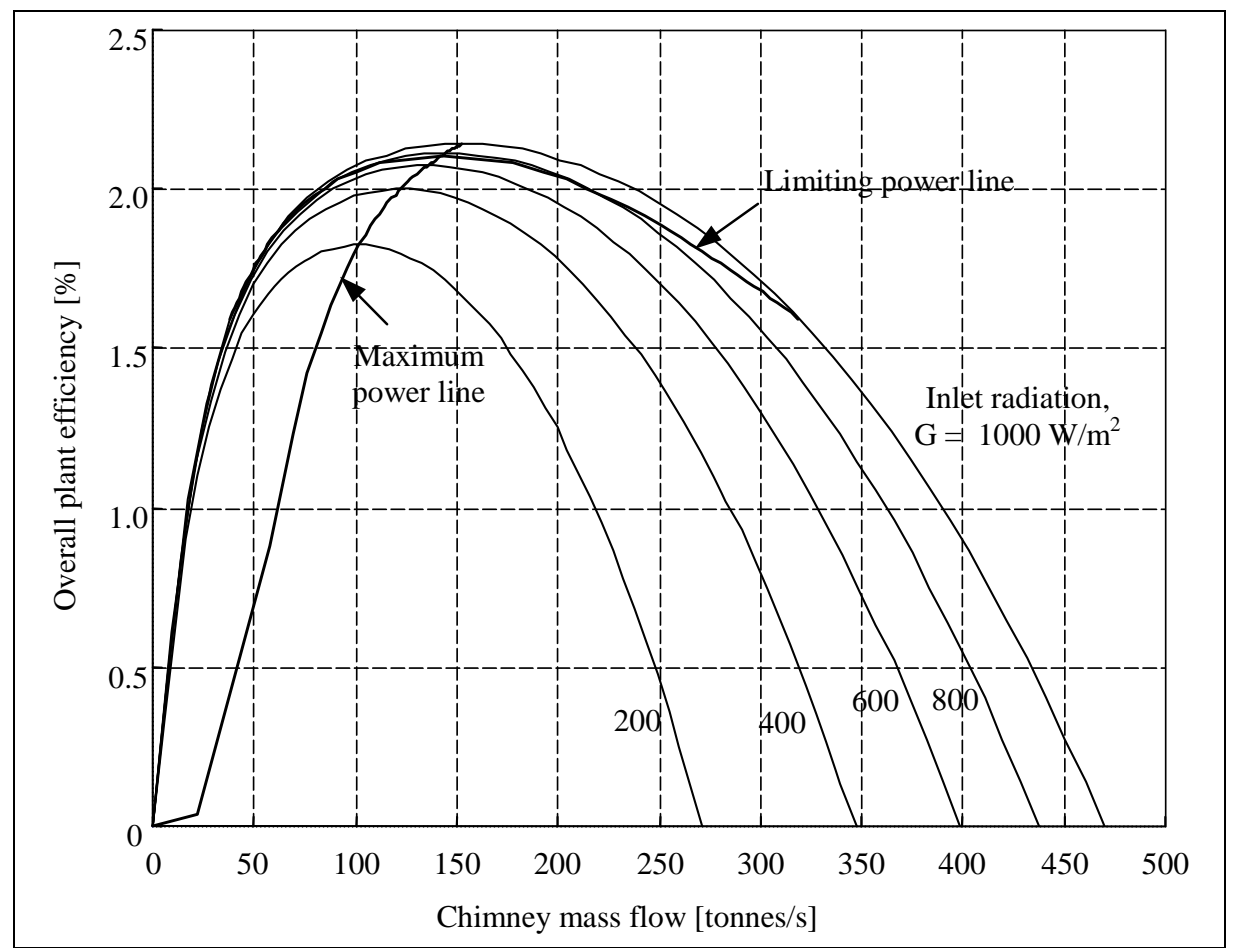

Figure 2.23. Overall plant efficiency [\%] for chimney of height $1500 \mathrm{~m}$ and diameter $160 \mathrm{~m}$, along lines of constant inlet radiation $\left[\mathrm{W} / \mathrm{m}^{2}\right]\left(\eta_{\text {turb }}=80 \%, k=1\right)$.

\subsection{Transient COLLECTOR MODEL}

The last addition to the solar chimney simulation in this project will be to include the transient effect that the soil beneath the collector has on the operation of the plant. The inclusion of the steady-state collector model has a significant effect on the prediction of the plant operating range. It will be seen that the predicted turbine pressure drops that result from the steady-state analysis are high. The transient pressures predicted tend to be lower than the steady-state ones but still higher than those predicted in Figure 2.13. The steady-state analysis demonstrated the effect of the coupling of the collector temperature rise and mass flow rate. The transient analysis will investigate the effect of the soil absorbing some of the incoming solar radiation in the morning and midday and then releasing it later in the afternoon and evening.

The plant power output prediction remains unchanged for a given collector temperature rise. This is possible as the transient response of the air will be quicker than the thermal response of the soil. Compared to the soil transient effects, the air flow is essentially steady-state. Jumping ahead to Figure 2.39 for the $6 \mathrm{~km}$ collector, there is an approximately 20\% decrease in the mass flow over a 20 minute period. Performing a simplified transient analysis on the air in the chimney shows that the pressure change over the turbine to account for this transient effect will be about $5 \mathrm{~Pa}$. This is small compared to the turbine pressure drop of about $1000 \mathrm{~Pa}$. While there is considerably more air in the collector, it is moving more slowly than the air in the chimney. It is also assumed in this 
analysis that the operation of the solar chimney will not involve sudden transient changes, meaning that the mass flow changes slowly over the day allowing the steady-state assumption to be used.

The calculation of the temperature rise of the air as it moves through the collector is almost the same as described in the previous section. Two modifications are required to introduce a simple transient model. In equation (2.67) the surface temperature, $\mathrm{T}_{\text {surf }}$, is now also dependent on the temperature distribution of the lower soil layers. The second modification is to include the effect of the angle of the incoming solar radiation. This has an effect on the transmittance property of the cover material. At low incidence angles very little solar radiation is transmitted through the cover material.

\section{Transient Soil Temperature Model}

In the steady-state model for the soil, it is assumed that there is a linear temperature gradient through the soil between the surface and the soil at a certain depth. In normal ground this is not the case with the top layer's temperature varying the most as it is exposed to solar radiation in the day and then losing its heat in the night. The layers below have less variation and tend to lag behind the day temperatures due to the low conductivity of the soil. At some distance below the top layer the temperature remains almost constant over a daily period. Figure 2.24 below is used to illustrate this effect with an artificial situation of the midnight starting temperature distribution in the soil assumed constant (the units are intentionally left out).

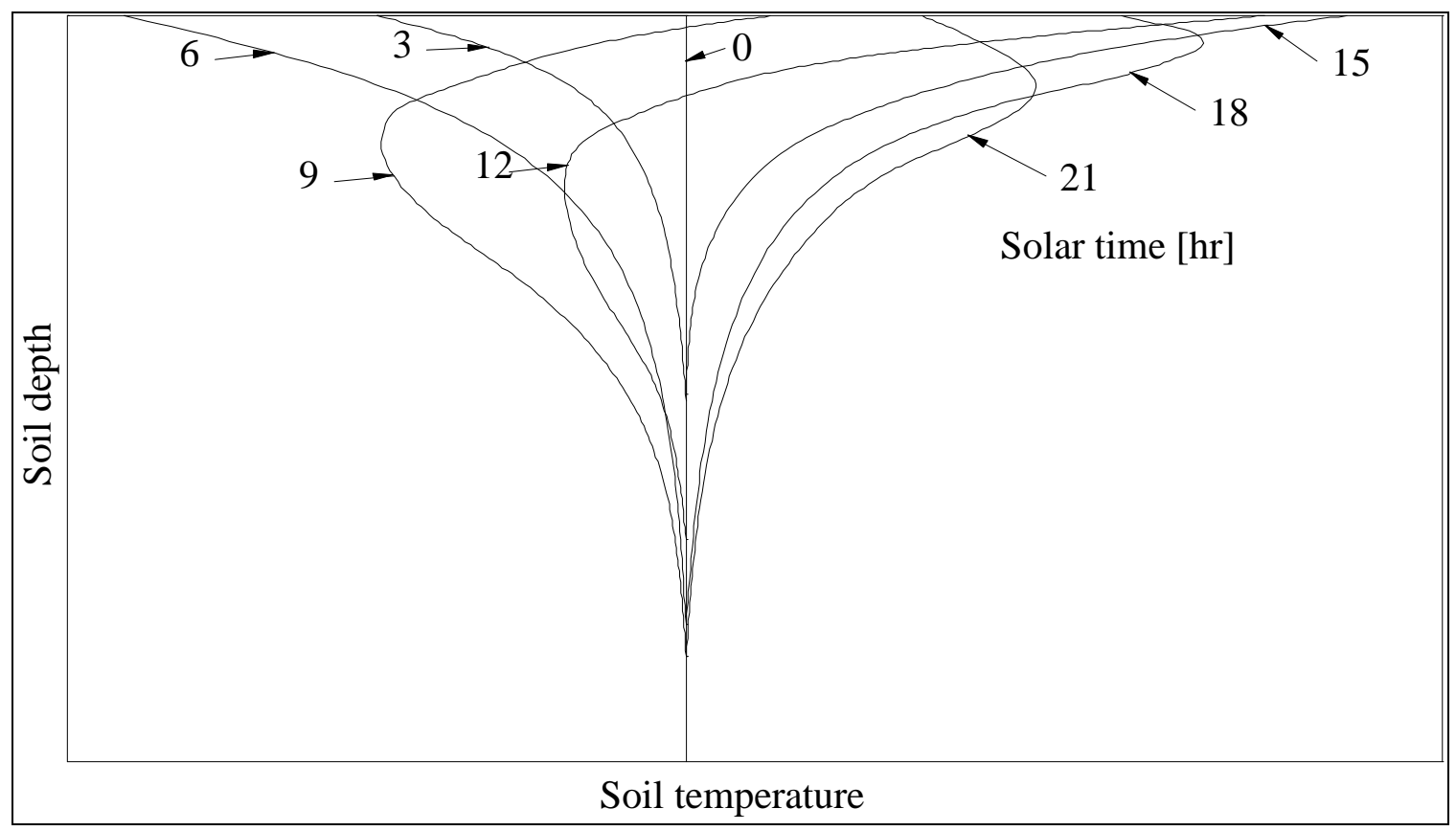

Figure 2.24. Daily temperature variation in soil as function of soil depth.

In the solar collector model it is assumed that there is no radial conduction in the soil. Only vertical heat transfer takes place, meaning the soil within each control volume of the solar collector can be 
treated separately. An implicit finite difference method is applied to the soil in each control volume, Incropera and De Witt (1990), p 292. The implicit scheme is slightly more difficult to implement computationally than in explicit scheme but it does have the advantage of being unconditionally stable for any time step. The soil in the control volume is divided into a number of equally spaced vertical layers where it is assumed that the temperature gradient in each is linear.

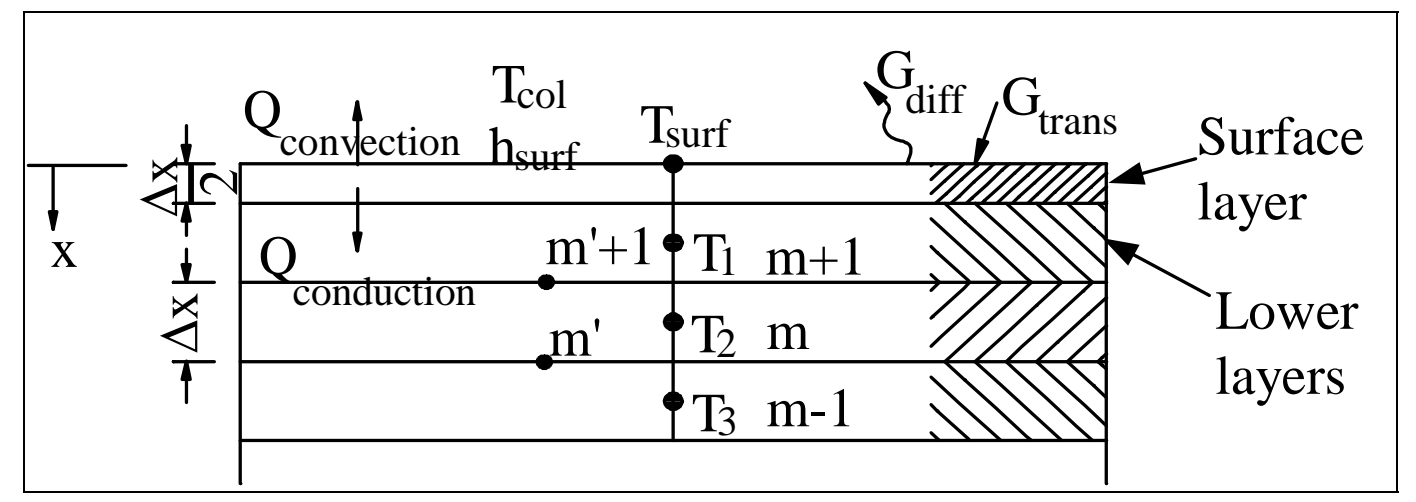

Figure 2.25. Discretisation scheme for transient soil model.

There are two governing equations for the above model, one applied to the ground below the surface where conduction is the only heat transfer mechanism and the other at the surface where radiative and convective heat transfer takes place.

\section{Discretisation Scheme for Lower Soil Layers}

The governing equation for the lower layers of soil is the heat equation for one-dimensional transient conduction,

$$
\frac{\partial^{2} \mathrm{~T}}{\partial \mathrm{x}^{2}}=\frac{1}{\alpha} \frac{\partial \mathrm{T}}{\partial \mathrm{t}}
$$

where $\alpha=$ thermal diffusivity,

$$
\alpha=\frac{\mathrm{k}_{\text {soil }}}{\rho_{\text {soil }} \mathrm{C}_{\mathrm{psoil}}}
$$

Using the finite difference form of the Fourier equation as follows,

$$
\text { Fo }=\frac{\alpha \Delta t}{(\Delta x)^{2}}
$$

an implicit, discrete form of the one-dimensional heat equation, (2.72), can be written relating the temperatures at a future time step, $\mathrm{T}^{\mathrm{P}+1}$, in terms of the previous time and neighbouring soil layers,

$$
\left(1+2 \mathrm{Fo}_{0}\right) \mathrm{T}_{\mathrm{m}}^{\mathrm{P}+1}-\mathrm{Fo}_{\mathrm{o}}\left(\mathrm{T}_{\mathrm{m}-1}^{\mathrm{P}+1}+\mathrm{T}_{\mathrm{m}+1}^{\mathrm{P}+1}\right)=\mathrm{T}_{\mathrm{m}}^{\mathrm{P}}
$$


As the conductivity in soil generally changes with the depth, $\mathrm{k}_{\text {soil }}$ (eq (2.71)), will change for each control volume. In calculating its value from the interface values, the harmonic mean must be used to obtain the correct value (Patankar, 1980).

$$
\mathrm{k}_{\mathrm{m}}=\frac{2 \mathrm{k}_{\mathrm{m}^{\prime}}, \mathrm{k}_{\mathrm{m}^{\prime}+1}}{\mathrm{k}_{\mathrm{m}^{\prime}}+\mathrm{k}_{\mathrm{m}^{\prime}+1}}
$$

\section{Discretisation Scheme for Surface}

The surface layer of soil in the solar collector has three heat transfer mechanisms. The first is radiation, the incoming solar radiation and the radiative loss due to the ground heating up. The second is convection to the air above the ground, which heats the air in the solar collector and the third is conduction to the lower soil layers. The one-dimensional heat equation with additional heat terms that include the radiative and convective heat transfer mechanisms are added.

$$
\underbrace{\frac{\partial^{2} \mathrm{~T}}{\partial \mathrm{x}^{2}}}_{\begin{array}{c}
\text { conductive } \\
\text { term }
\end{array}}+\underbrace{\frac{\mathrm{G}_{\text {net inlet }}}{\mathrm{k}_{\text {soil }}}}_{\text {Inlet radiation }}-\underbrace{\frac{\mathrm{G}_{\text {loss }}}{\mathrm{k}_{\text {soil }}}}_{\text {Loss radiation }}-\underbrace{\frac{\mathrm{h}\left(\mathrm{T}_{\text {surf }}-\mathrm{T}_{\text {col }}\right)}{\mathrm{k}_{\text {soil }}}}_{\text {Loss convection }}=\underbrace{\frac{1}{\alpha} \frac{\partial \mathrm{T}}{\partial \mathrm{t}}}_{\begin{array}{c}
\text { transient } \\
\text { term }
\end{array}}
$$

This is discretised,

$$
\begin{gathered}
\underbrace{\mathrm{k}_{\text {soil }} \frac{\mathrm{T}_{1}^{\mathrm{P}+1}-\mathrm{T}_{\text {surf }}^{\mathrm{P}+1}}{\Delta \mathrm{x}}}_{\begin{array}{c}
\text { conductive } \\
\text { term }
\end{array}}+\underbrace{\mathrm{G}_{\text {net inlet }}}_{\text {Inlet radiation }}-\underbrace{\mathrm{e}_{\text {surf }} \sigma\left[\left(\mathrm{T}_{\text {surf }}^{\mathrm{P}+1}\right)^{4}-\mathrm{T}_{\text {gli }}^{4}\right.}_{\text {Loss radiation }}]-\underbrace{\mathrm{h}\left(\mathrm{T}_{\text {surf }}^{\mathrm{P}+1}-\mathrm{T}_{\text {col }}\right)}_{\text {Loss convection }} \\
=\underbrace{\underbrace{}_{\text {soil }} \mathrm{C}_{\mathrm{p} \mathrm{soil}} \frac{\Delta \mathrm{x}}{2} \frac{\mathrm{T}_{\text {surf }}^{\mathrm{P}+1}-\mathrm{T}_{0}^{\mathrm{P}}}{\Delta \mathrm{t}}}_{\begin{array}{c}
\text { transient } \\
\text { term }
\end{array}}
\end{gathered}
$$

The above form is still not able to be implemented directly as the radiative loss terms must be linearised resulting in,

$$
\begin{gathered}
\underbrace{\mathrm{k}_{\text {soil }} \frac{\mathrm{T}_{1}^{\mathrm{P}+1}-\mathrm{T}_{\text {surf }}^{\mathrm{P}+1}}{\Delta \mathrm{x}}}_{\begin{array}{c}
\text { conductive } \\
\text { term }
\end{array}}+\underbrace{\mathrm{G}_{\text {net inlet }}}_{\text {Inlet radiation }}-\underbrace{\mathrm{h}_{\mathrm{r}}^{\mathrm{P}}\left[\mathrm{T}_{\text {surf }}^{\mathrm{P}+1}-\mathrm{T}_{\text {gli }}\right.}_{\text {Loss radiation }}]-\underbrace{\mathrm{h}\left(\mathrm{T}_{\text {surf }}^{\mathrm{P}+1}-\mathrm{T}_{\text {col }}\right)}_{\text {Loss convection }} \\
=\underbrace{\rho_{\text {soil }} \mathrm{C}_{\text {p soil }} \frac{\Delta \mathrm{x}}{2} \frac{\mathrm{T}_{\text {surf }}^{\mathrm{P}+1}-\mathrm{T}_{0}^{\mathrm{P}}}{\Delta \mathrm{t}}}_{\begin{array}{c}
\text { transient } \\
\text { term }
\end{array}}
\end{gathered}
$$

where $h_{r}$ is the linearised radiative heat transfer coefficient,

$$
\mathrm{h}_{\mathrm{r}}^{\mathrm{P}}=\mathrm{e}_{\text {surf }} \sigma\left(\mathrm{T}_{\text {surf }}^{\mathrm{P}}+\mathrm{T}_{\mathrm{gli}}\right)\left(\left(\mathrm{T}_{\text {surf }}^{\mathrm{P}}\right)^{2}+\left(\mathrm{T}_{\mathrm{gli}}\right)^{2}\right)
$$


The following finite difference forms of the Biot (Bi) and Fourier (Fo) numbers are defined,

$$
\begin{gathered}
\text { Bir }=\frac{h_{r}^{\mathrm{P}} \Delta \mathrm{x}}{\mathrm{k}_{\text {soil }}} \\
\mathrm{Bi}=\frac{\mathrm{h}_{\text {soil }} \Delta \mathrm{x}}{\mathrm{k}_{\text {soil }}} \\
\text { Fo }=\frac{\alpha \Delta \mathrm{t}}{(\Delta \mathrm{x})^{2}}
\end{gathered}
$$

The above terms are substituted into (2.77) and the temperature terms grouped to give the final implicit form of the heat transfer equation in terms of temperature,

$$
\begin{aligned}
& \mathrm{T}_{\text {surf }}^{\mathrm{P}+1}(1+2 \mathrm{Fo}+2 \mathrm{FoBi}+2 \mathrm{FoBir})-2 \mathrm{FoT}_{1}^{\mathrm{P}+1} \\
& =\mathrm{T}_{\text {surf }}^{\mathrm{P}}+2 \mathrm{FoBiT}_{\text {col }}+2 \mathrm{FoBirT}_{\text {gli }}+2 \mathrm{Fo} \frac{\Delta \mathrm{x}}{\mathrm{k}_{\text {soil }}} \mathrm{G}
\end{aligned}
$$

\section{Collector Glass Transmittance}

The amount of solar radiation that is transmitted through the collector glass over the daily cycle changes through the day. This is due to the angle of the incoming solar radiation changing and during the early morning and late afternoon much of the solar radiation is not transmitted (Duffie \& Beckman 1991). What is required is to find the transmittance $\tau_{\text {in }}$ as a function of the time of day. The first parameter that is required is the angle of the refracted beam when the solar radiation strikes the collector glass. This is obtained from the law of refraction, Sears et al. (1987),

$$
\begin{gathered}
\frac{\mathrm{n}_{\text {glass }}}{\mathrm{n}_{\mathrm{atm}}}=\frac{\sin \theta_{\text {atm }}}{\sin \theta_{\text {glass }}} \\
\Rightarrow \theta_{\text {glass }}=\mathrm{a} \sin \left(\frac{\mathrm{n}_{\text {atm }}}{\mathrm{n}_{\text {glass }}} \sin \theta_{\text {atm }}\right)
\end{gathered}
$$

where $\theta_{\text {atm }}$ is the angle of the incoming solar radiation and $n_{\text {atm }}$ and $n_{\text {glass }}$ are the refractive indices of the atmospheric air and glass respectively. Once the refraction angle is known the parallel, $\mathrm{r}_{\|}$, and perpendicular, $\mathrm{r}_{\perp}$, reflectivity coefficients are calculated,

$$
\begin{array}{r}
r_{\|}=\frac{\tan ^{2}\left(\theta_{\text {glass }}-\theta_{\text {atm }}\right)}{\tan ^{2}\left(\theta_{\text {glass }}+\theta_{\text {atm }}\right)} \\
r_{\perp}=\frac{\sin ^{2}\left(\theta_{\text {glass }}-\theta_{\text {atm }}\right)}{\sin ^{2}\left(\theta_{\text {glass }}+\theta_{\text {atm }}\right)}
\end{array}
$$

The reflective transmittance, $\tau_{\mathrm{r}}$, of a material is dependent on the reflectivity coefficients,

$$
\tau_{\mathrm{r}}=\frac{1}{2}\left(\frac{1-\mathrm{r}_{\|}}{1+\mathrm{r}_{\|}}+\frac{1-\mathrm{r}_{\perp}}{1+\mathrm{r}_{\perp}}\right)
$$


and the absorbtive transmittance, $\tau_{\mathrm{a}}$, is dependent on the extinction coefficient, $\mathrm{K}$, and material thickness, L,

$$
\tau_{\mathrm{a}}=\exp \left(-\frac{K L_{\text {glass }}}{\cos \theta_{\text {glass }}}\right)
$$

The fraction of solar radiation transmitted through the material, $\tau_{\perp}$, is

$$
\tau_{\perp}=\tau_{\mathrm{a}} \tau_{\mathrm{r}}
$$

The amount of energy absorbed by the glass is represented by the absorbtance, $\alpha$,

$$
\alpha=1-\tau_{\text {a }}
$$

The last variable that needs to be calculated is the angle of the incoming solar radiation, $\theta_{\text {atm, }}$ otherwise called the zenith angle and is the angle between the suns rays and the local vertical (Preston-Whyte \& Tyson (1989)). Three factors govern the zenith angle: the latitude, $\phi$, the angle of the sun north or south of the equator (solar declination), $\phi_{\mathrm{d}}$, and the time of day defined by the hour angle $\omega_{\mathrm{h}}$. The solar declination depends on the time of year and can be calculated from the following function where Date solar $_{1}$ is the solar date beginning with $1=1 \mathrm{Jan}$ and $365=31$ Dec,

$$
\phi_{\mathrm{d}}=23.45\left(\frac{2 \pi\left(284+\text { Date }_{\text {solar }}\right)}{365}\right)
$$

The hour angle, $\omega_{\mathrm{h}}$, is defined as zero at noon and increases by $15^{0}$ for every hour after noon. Using the above variables and substituting them in the following equation the zenith angle, $\theta_{1}$, can be calculated

$$
\cos \theta_{\mathrm{atm}}=\sin \phi \sin \phi_{\mathrm{d}}+\cos \phi \cos \phi_{\mathrm{d}} \cos \omega_{\mathrm{h}}
$$

\section{Calculation of New Temperatures}

The calculation of the temperature rise through the collector proceeds in exactly the same way as before using a Runge-Kutta numerical integration to solve equation (2.66). As before, the incremental temperature rise in each control volume is calculated.

The solution of the temperatures $T_{\text {surf }}, T_{\text {gli }}$ and $T_{\text {glo }}$ in each control volume uses the same three equations, (2.67)-(2.69), as before. In equation (2.67) the value of $\mathrm{L}_{\text {soil }}$ now becomes small enough that it can be assumed that the temperature gradient is linear within the top soil layer. In the simulation a value of $10 \mathrm{~mm}$ was used for the $\Delta \mathrm{x}$ shown in Figure $2.25\left(\mathrm{~L}_{\text {soil }}=5 \mathrm{~mm}\right)$. There was no discernible change in the results between a $20 \mathrm{~mm}$ and $10 \mathrm{~mm}$ increment but as the methods used are computationally cheap, the $10 \mathrm{~mm}$ step size was kept. The value of $\mathrm{T}_{\text {depth }}$ is dependent on the temperature of the lower soil layers at the previous time step and is calculated using the implicit 
method described in the previous section. The time step $\Delta \mathrm{t}$ used in equation was $10 \mathrm{~min}$. There was no change between 20min and 10min time steps.

As soil is not a very good heat conductor, it would take a number of days for the temperature of the lower soil layers to stabilise. In the simulation performed only one representative day was simulated. To obtain the correct soil temperature in the lower layers, the simulation was repeated using the results from the previous simulation until the lower soil layer temperature stabilised. Soil to a depth of $0.5 \mathrm{~m}$ was simulated. The following table summarises the methods and quantities used in the simulation of the solar collector.

Table 2.6. Summary of solution methods used in solar collector simulation.

\begin{tabular}{|c|c|c|c|c|c|}
\hline Eq. & Description & Solution method & Reason for use & $\begin{array}{l}\stackrel{N}{N} \\
\text { के } \\
\frac{0}{\infty}\end{array}$ & 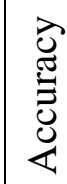 \\
\hline (2.66) & $\begin{array}{l}\text { Incremental } \\
\text { temperature rise in } \\
\text { each control volume }\end{array}$ & $\begin{array}{l}\text { Runge-Kutta: } \\
\text { numerical } \\
\text { integration }\end{array}$ & $\begin{array}{l}\text { Method is not iterative, } \\
\text { requires only one pass } \\
\text { through collector }\end{array}$ & $5 \mathrm{~m}$ & 吕 \\
\hline $\begin{array}{l}(2.67)- \\
(2.69)\end{array}$ & $\begin{array}{l}\text { Surface } \\
\text { temperatures in each } \\
\text { control volume }\end{array}$ & $\begin{array}{l}\text { Newton method for } \\
\text { solving non-linear } \\
\text { simultaneous eqn }\end{array}$ & $\begin{array}{l}\text { As only three variables } \\
\text { are solved for an iterative } \\
\text { scheme it is still quick }\end{array}$ & NA & $\begin{array}{c}x \\
m \\
d \\
-1\end{array}$ \\
\hline $\begin{array}{l}(2.73) \& \\
(2.80)\end{array}$ & $\begin{array}{l}\text { Implicit transient } \\
\text { model for soil. }\end{array}$ & Matrix reduction & $\begin{array}{l}\text { Method is } \\
\text { unconditionally stable, } \\
\text { allows large time steps }\end{array}$ & $\begin{aligned} \Delta \mathrm{x} & =10 \mathrm{~mm} \\
\Delta \mathrm{t} & =10 \mathrm{~min}\end{aligned}$ & $\frac{x}{2}$ \\
\hline
\end{tabular}

The solar collector model was not intended to be state of the art but rather used well-established and existing heat transfer analysis methods. The most complicated part of the model is probably the transient soil simulation where the implicit rather than explicit scheme was used. The main reason for doing this was to make the method computationally more efficient. It will be seen in the following sections that the transient simulation of the plant, especially to find the maximum power output, requires the collector model to be used often. The initial aim was to set up a simulation of the plant that is simple to perform and understand. With judicious use of analytical solutions combined with numerical methods where convenient, a plant simulation has been set up.

\subsection{VERIFICATION OF SIMULATION}

In order to verify the solar chimney simulation it is necessary to compare the results with those obtained experimentally. For this purpose a simulation of the plant built in Manzanares, Spain, is performed and the results compared with data for a representative day, 02-09-1982, of plant operation, Haaf (1984). The plant in Manzanares had a number of different materials in the 
collector covering material and the ground conductivity varied greatly. This made it difficult to determine the exact properties. Figure 2.26 and Figure 2.27 are taken directly from Haaf (1984).

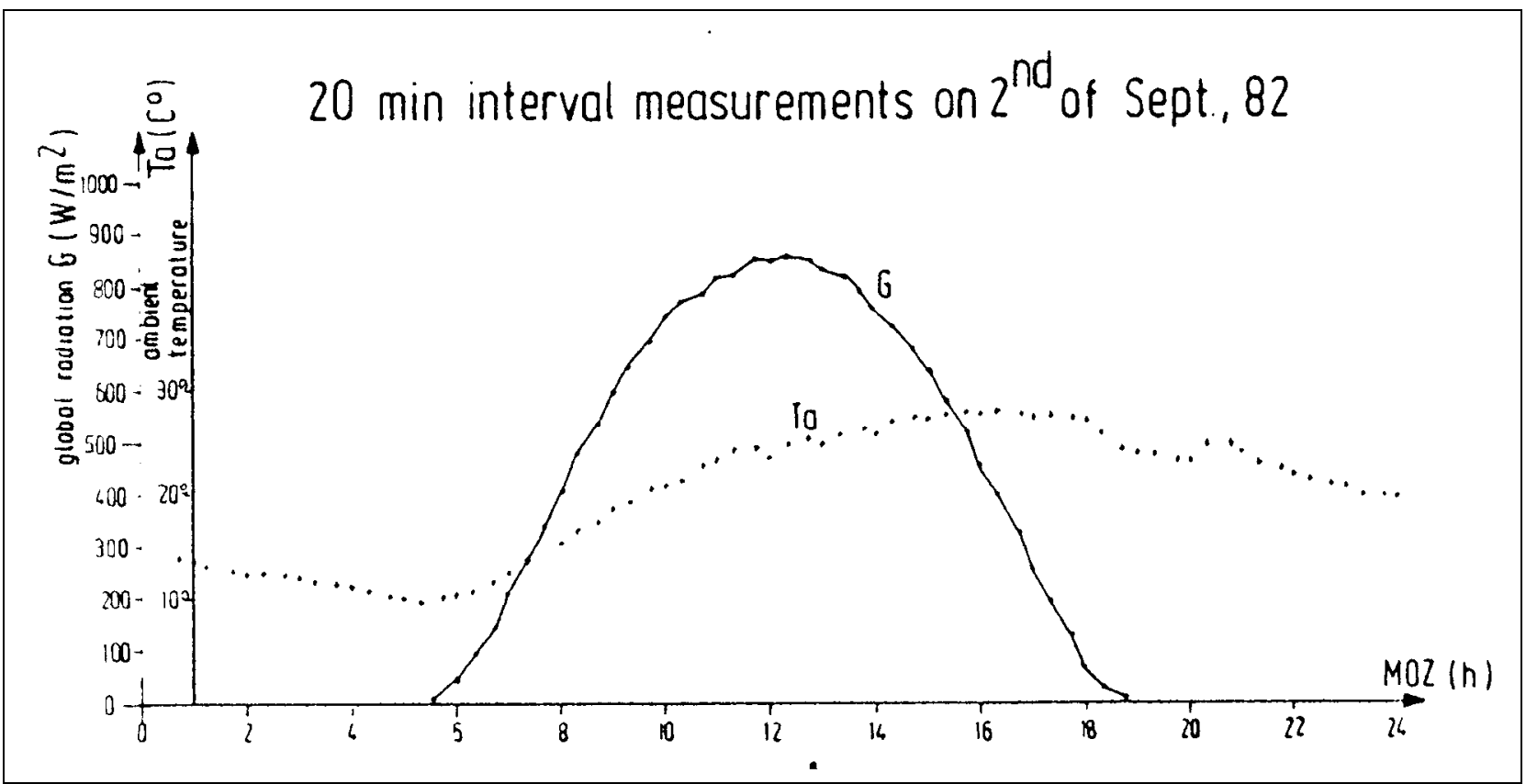

Figure 2.26. Global radiation and atmospheric temperature $\mathbf{T}_{\mathbf{0 2}}$.

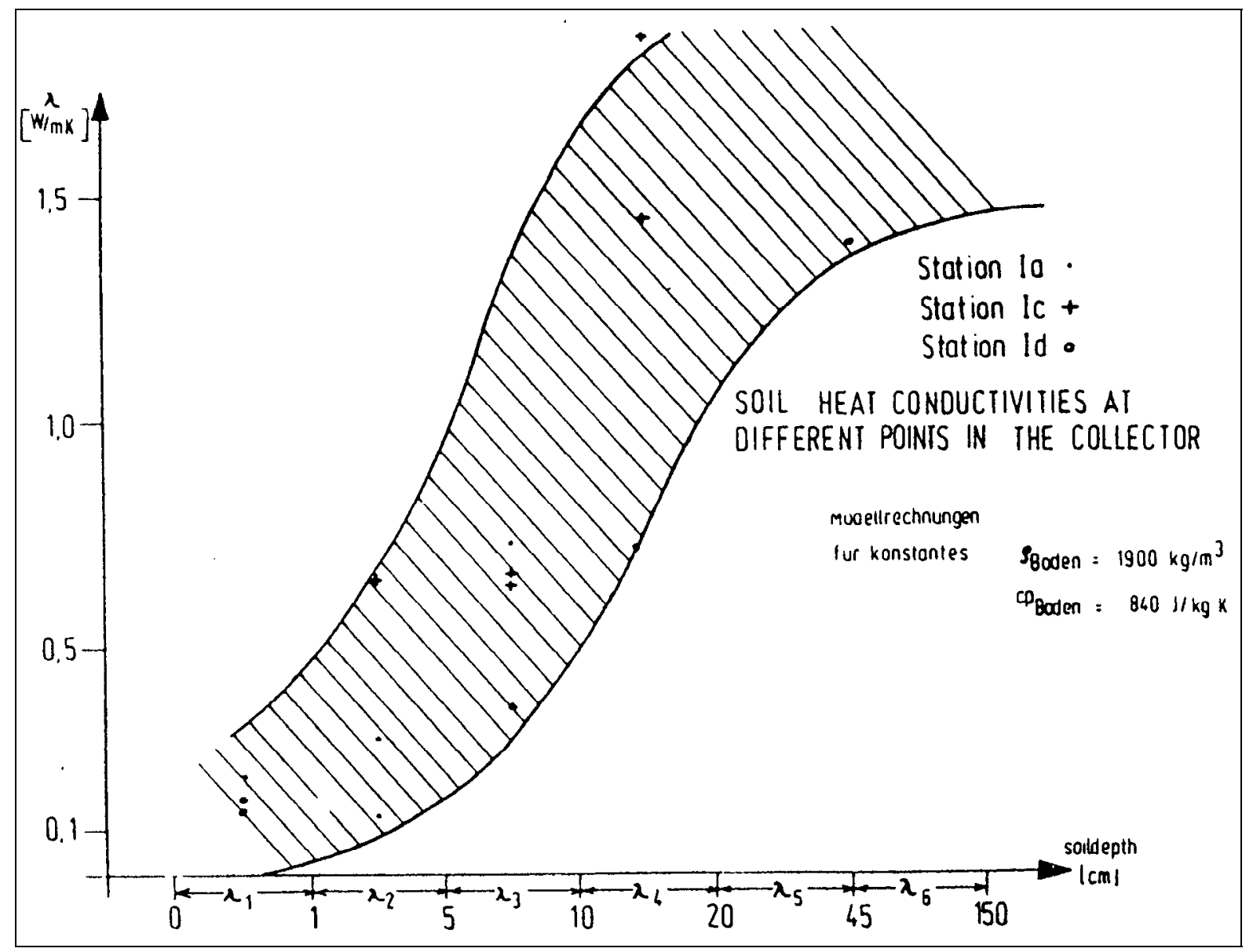

Figure 2.27. Range of soil heat conductivities in collector as a function of soil depth. 
Figure 2.26 shows the environmental data for 2 September 1982 in Manzanares, Spain. Figure 2.27 shows the range of conductivities found under the solar collector at various stations. In the transient simulation the conductivities used at each soil depth were taken from a mean line traced through the range of conductivities presented in Figure 2.27. Table 2.7 shows the property values used for the experimental plant simulation of Manzanares (the subscript 'glass' is used for the cover material).

Table 2.7 Material properties for experimental solar chimney plant, Manzanares, Spain.

\begin{tabular}{|l|l|l|l|}
\hline Description & Symbol & Unit & Value \\
\hline Ground emissivity & $\left(\mathrm{e}_{\text {surf }}\right)$ & & 0.65 \\
\hline Convective heat transfer coeff. & $\left(\mathrm{h}_{\text {gli }}, \mathrm{h}_{\text {glo }}, \mathrm{h}_{\text {gro }}\right)$ & {$\left[\mathrm{W} / \mathrm{m}^{2} \mathrm{~K}\right]$} & 5 \\
\hline Inlet \& Outlet height & $\left(\mathrm{H}_{\text {outlet }}\right)\left(\mathrm{H}_{\text {inlet }}\right)$ & {$[\mathrm{m}]$} & 1.85 \\
\hline Perspex conductivity & $\left(\mathrm{k}_{\text {glass }}\right)$ & {$[\mathrm{W} / \mathrm{mK}]$} & 0.4 \\
\hline Extinction coefficient, & $(\mathrm{K})$ & & 32 \\
\hline Cover thickness & $\left(\mathrm{L}_{\text {glass }}\right)$ & {$[\mathrm{m}]$} & 0.0001 \\
\hline Collector inner radius & $\left(\mathrm{r}_{\mathrm{i}}\right)$ & {$[\mathrm{m}]$} & 15 \\
\hline Collector outer radius & $\left(\mathrm{r}_{\mathrm{o}}\right)$ & {$[\mathrm{m}]$} & 144 \\
\hline Perspex transmissivity, infrared range. & $\left(\tau_{\text {out }}\right)$ & & 0.07 \\
\hline
\end{tabular}

\section{Comparison of Simulated and Measured Results}

To verify the solar chimney model, the Manzanares plant is simulated using the experimentally measured mass flow rate. This allows the measured and predicted plant performance to be compared. This can be calculated using the chimney area and updraught velocity published in the figure below,

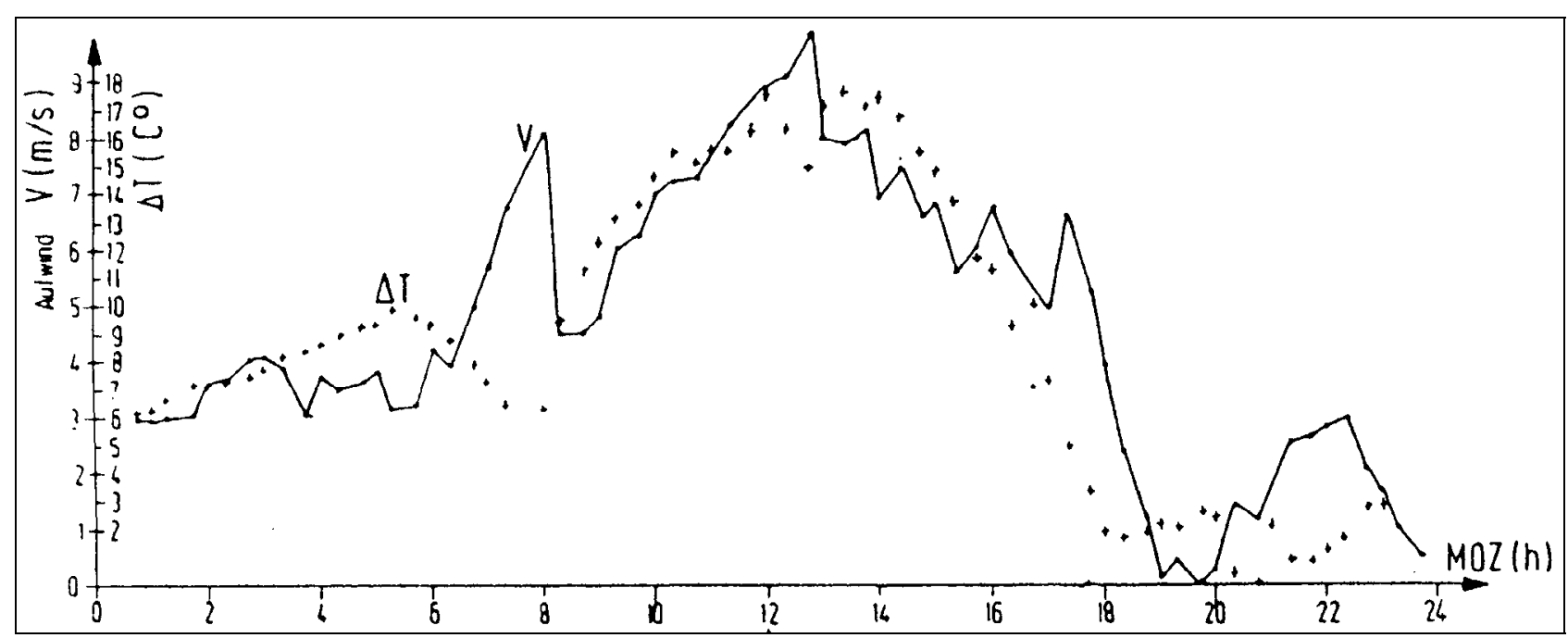

Figure 2.28. Collector temperature rise and chimney velocity in experimental plant. 
Figure 2.29 shows the amount of heat gained by the air moving through the solar collector as measured in the Manzanares plant. The data is obtained from Figure 2.28 above and uses the standard equation relating the heat gain of the air to the mass flow and temperature rise,

$$
\mathrm{Q}=\dot{\mathrm{mc}}_{\mathrm{p}} \Delta \mathrm{T}
$$

The simulated results tend to over predict the heat addition in the early morning and late afternoon while under predicting the midday heat addition. This can be due to a number of factors such as incorrect conductivity values for the soil or transmissivity values for the cover. The values calculated for the evening are inaccurate probably due to the low mass flow rates where a small error in any one of the constants such as heat transfer coefficient will have a large effect on the amount of heat gained by the air.

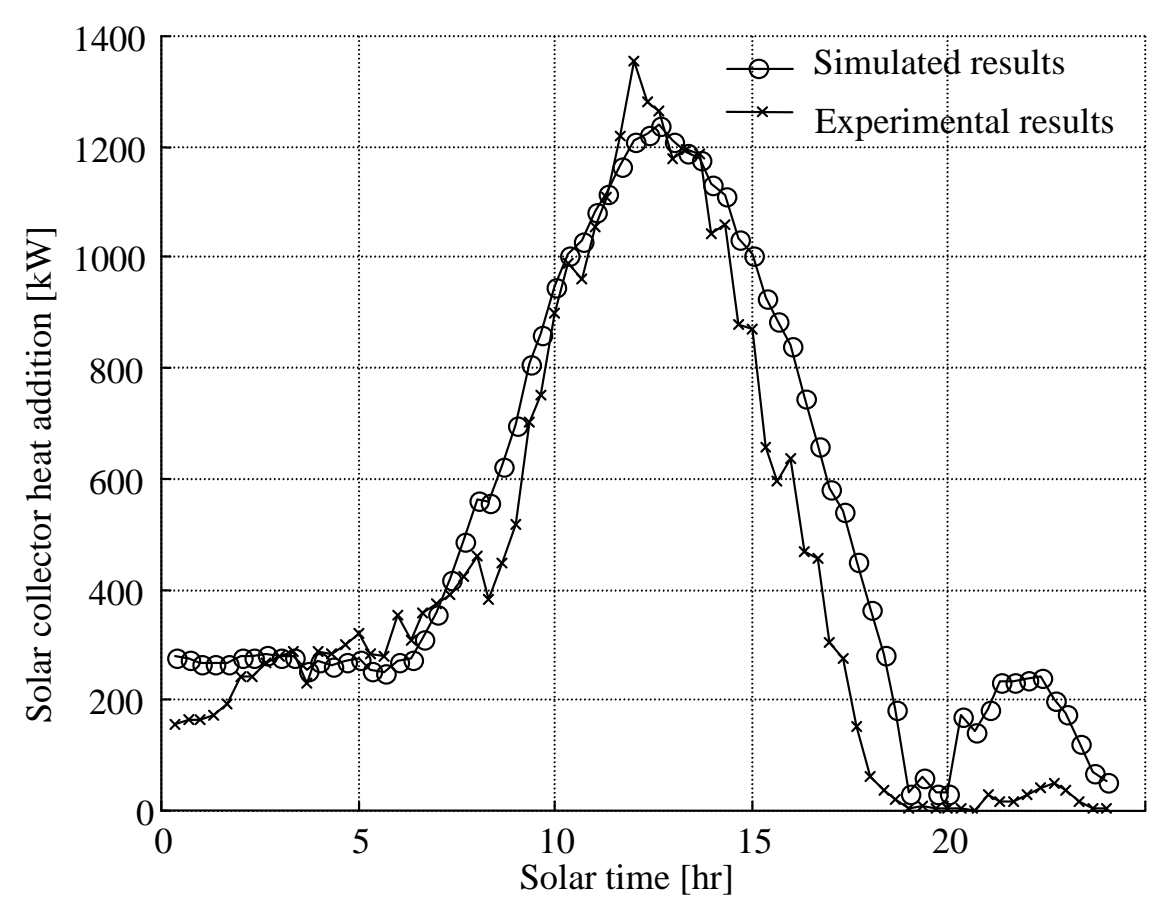

Figure 2.29. Comparison of experimental air heat gain and simulated results.

Calculating the collector temperature rise the plant power output can then be predicted and in Figure 2.30 the simulated results are superimposed over the experimental. The initial prediction of the power output was too high by a factor of almost 2 . Investigation of the published results showed that the design of the efficiency of the turbine, 76\% Haaf (1984), was not being reached. The power output (Figure 2.30) of the turbine was published along with the turbine pressure drop and volume flow rate (Figure 2.28, Haaf (1984)). Using these three values, it is possible to calculate the turbine efficiency for the Manzanares plant using,

$$
\eta_{\text {turb }}=\frac{\mathrm{P}_{\text {turb }}}{\mathrm{P}_{\text {ideal }}}=\frac{\mathrm{P}_{\text {turb }}}{\mathrm{Q} \Delta \mathrm{P}_{\text {turb }}}
$$




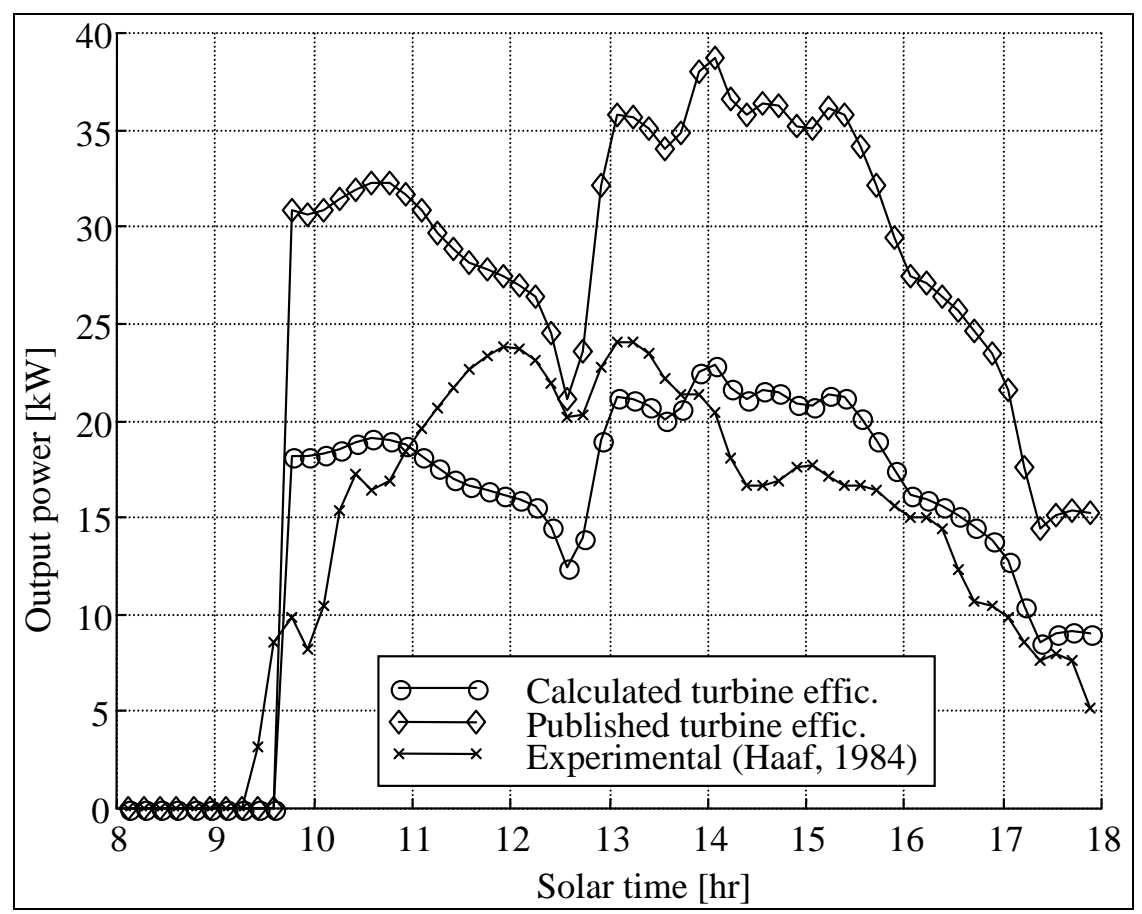

Figure 2.30. Comparison of experimental and simulated plant power output.

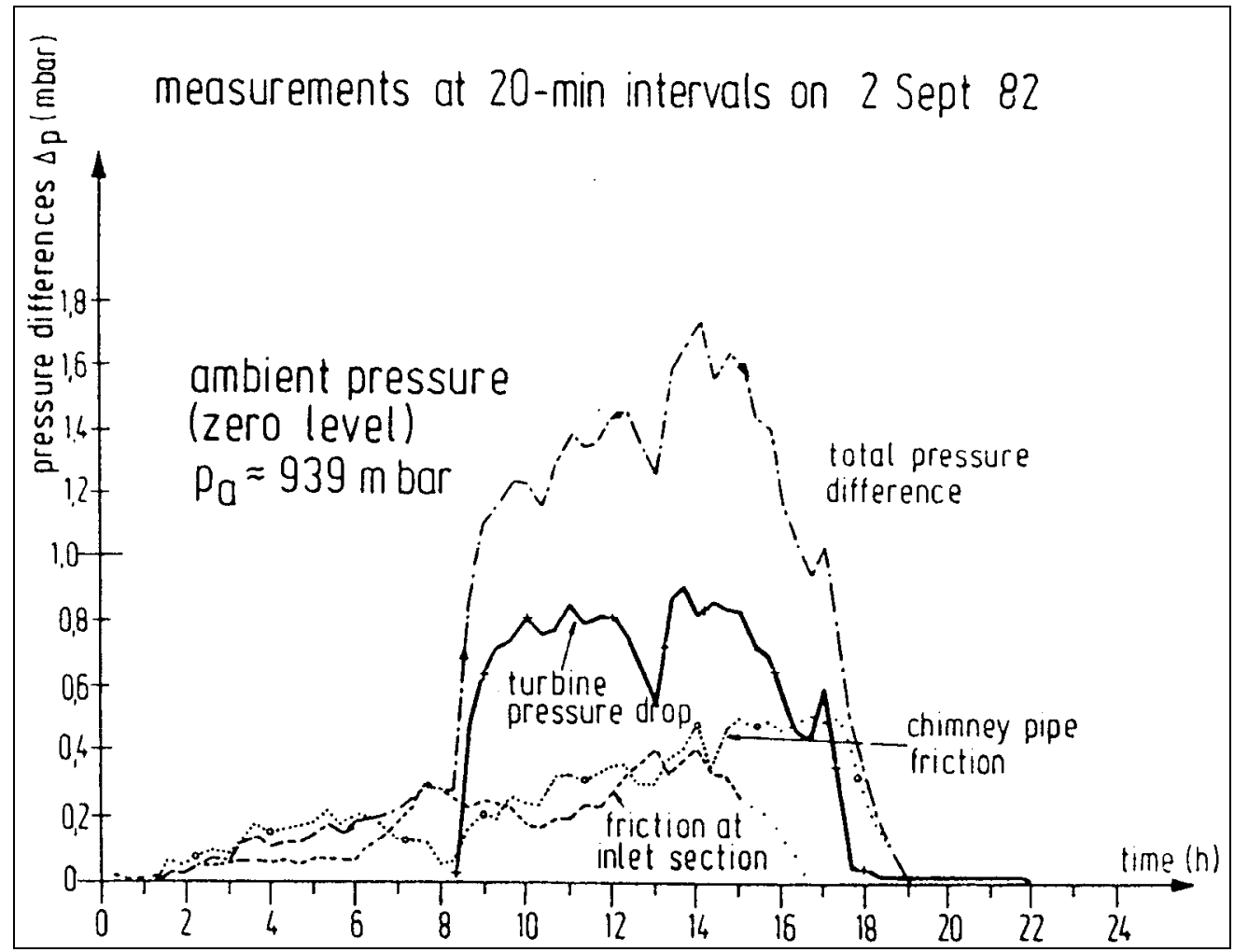

Figure 2.31. Pressure differences from experimental plant, Haaf (1984).

Using the calculated efficiencies in calculating the plant output power, the prediction is closer to the experimental results. The same trends as in the heat addition to the solar collector can be seen in the predicted plant power output. The morning and late afternoon power is over predicted by the 
simulation while the midday values are under predicted. The turbine operation is characterised by a sudden pressure drop at midday resulting in an increased mass flow and decreased power output. It is suggested that the turbine might have stalled at this point, the increase in mass flow and lower pressure drop would result in the turbine un-stalling. This would explain the sudden dip in power. While the power prediction of the model is not very accurate, the purpose of the exercise is to define the operating range of the turbine. At present the transient model does predict the range of operation of the plant well. It has also highlighted the importance of including the solar collector in any simulation.

\subsection{Operating Range of Full Scale Plant}

The simulation model is now developed to a point where the operating range of a full-scale plant can be predicted with reasonable accuracy. The first step in finding the turbine-operating region will be to simulate the experimental plant in Manzanares using the plant dimensions but using a turbine pressure drop that will provide the maximum power. The Manzanares turbine was designed using a figure similar to Figure 2.11 where it is implied that the collector and plant operate along lines of constant temperature rise, Schwarz \& Knauss (1981). This approach is similar to that used in designing gas turbine turbines. In the solar chimney the temperature in the collector is controlled by the amount of incoming radiation G. Performing a steady state simulation to calculate the plant power output along lines of constant inlet radiation, Figure 2.20 results in a rather different operating range being predicted with lower mass flows and higher pressure drops over the turbine. This is due to the coupling of the mass flow and temperature rise as given in equation (2.89). The transient analysis is intended to be most representative of what actually occurs in the solar chimney plant. The development of the plant model and the need to look at the solar chimney turbine operation in a different way to that of a gas turbine and as a function of incoming radiation is presented in Gannon \& Von Backström (2000).

The present simulation is modified to find the maximum power output at each time step. To do this the pressure drop at each time step is adjusted until the maximum power output is found. This process is repeated at each time step during the simulated day except when the limiting power is reached. Here the pressure drop is increased until the power is reduced to the limiting value, $50 \mathrm{~kW}$ in the case of the Manzanares plant and $200 \mathrm{MW}$ for the full-scale plant.

\section{Manzanares Simulation}

A simulation of the Manzanares plant is performed with the only change being made in the turbine. It is assumed that its total-to-static efficiency is now $80 \%$ and that it can provide the pressure drop required to give the maximum power output at any time of the day. The other change to the simulation is the introduction of a limiting power of $50 \mathrm{~kW}$. This was the original design power of the experimental plant. This is done because in the real plant the power output will be limited by 
the size of the generator and an effective method of controlling the plant power, as mentioned earlier, is to increase the pressure drop over the turbine and thus decrease the mass flow. This also has the added advantage of increasing the temperature in the collector and increasing the amount of heat stored in the ground or any storage device due to the higher temperature gradient.

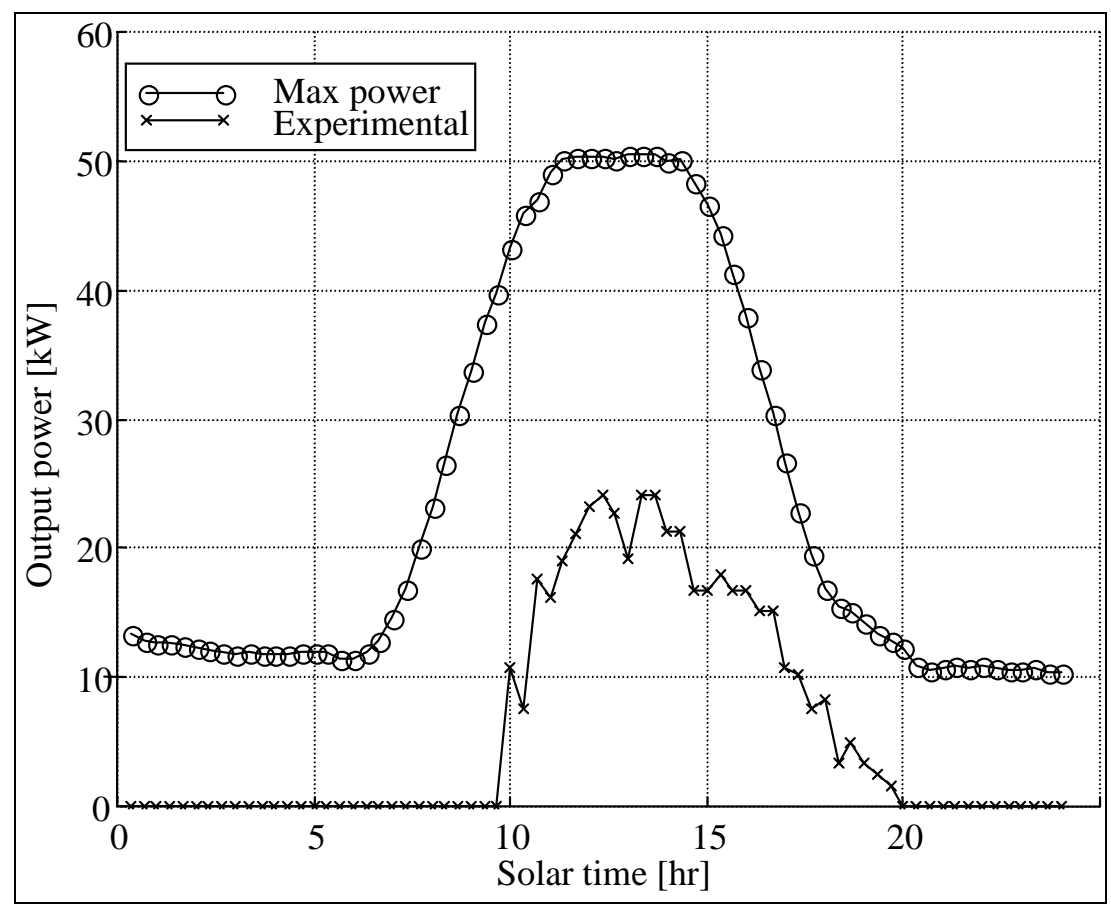

Figure 2.32. Predicted maximum power output from Manzanares size plant.

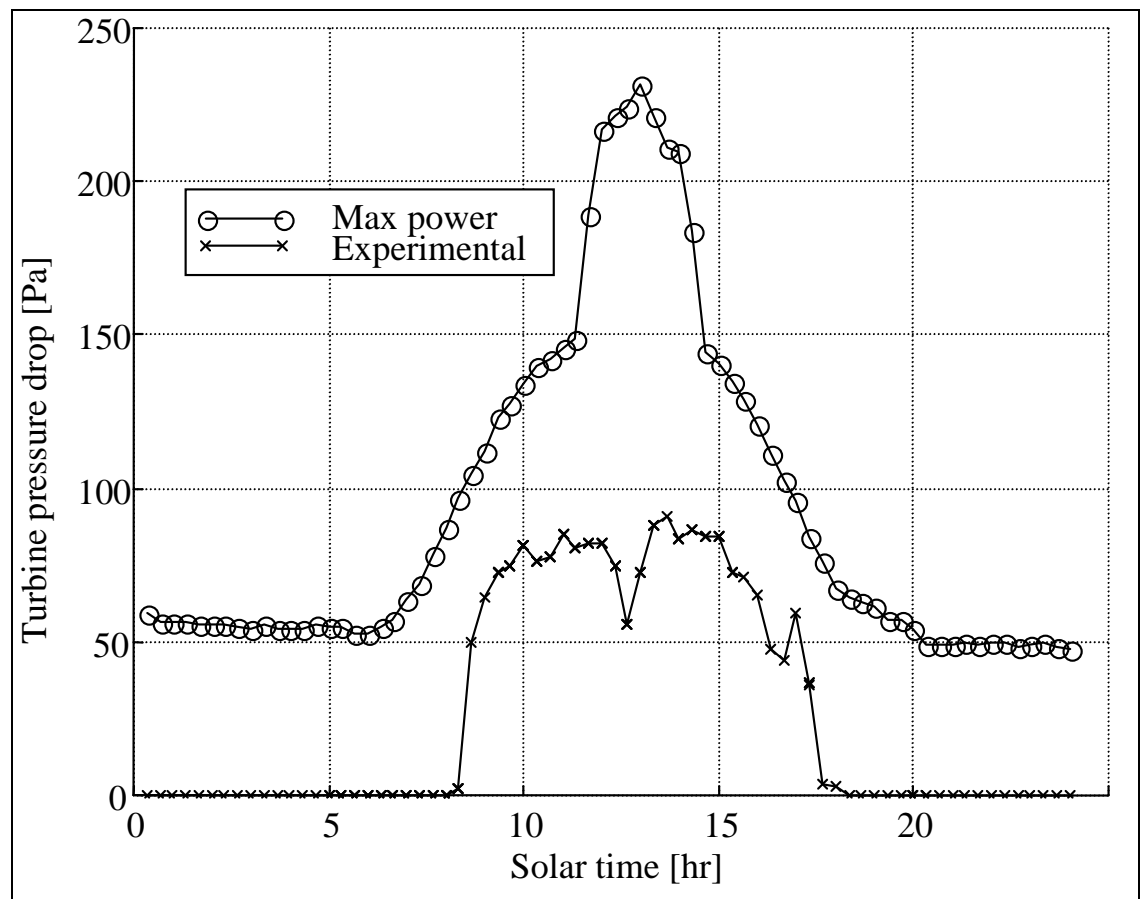

Figure 2.33. Predicted turbine pressure drop for maximum power output for Manzanares sized plant. 
In Figure 2.32-Figure 2.36 the experimental measurements are superimposed so that they can be compared to the predicted maximum power output. Figure 2.32 shows the predicted maximum power output possible by using a higher turbine pressure drop. The limiting power is reached for just over 2 hours around the solar noon.

The pressure drop to obtain the maximum power is shown in Figure 2.33 and it is clear that a far higher pressure drop is required over the turbine than was available in the experimental plant. The experimental plant turbine seemed to stall when the pressure drop reached more than $90 \mathrm{~Pa}$. A turbine that operated at a higher pressure drop, say 50 to $150 \mathrm{~Pa}$, efficiently may have been able to extract more power from the Manzanares plant. This analysis aims to define what is required from the solar chimney turbine and will later be applied to a full-scale plant.

With a higher pressure drop the mass flow decreases significantly as shown in Figure 2.34. The effect of the limiting pressure drop can be seen near midday when the mass flow decreases significantly to limit the plant power output.

Figure 2.35 shows the amount of heat added to the air as it passes through the solar collector for the predicted maximum power condition. What is most significant here is that the heat addition is not significantly different to that shown in Figure 2.29 except for the limiting power region where it is less due to most of the heat being either stored in the soil or lost to the environment. Slightly more heat is added to the air in the late afternoon due to the heat stored in the soil being released. There is obviously scope for improvements in the method of heat storage such as in water contained in pipes or bags but for the present analysis it will be assumed that only soil is under the collector.

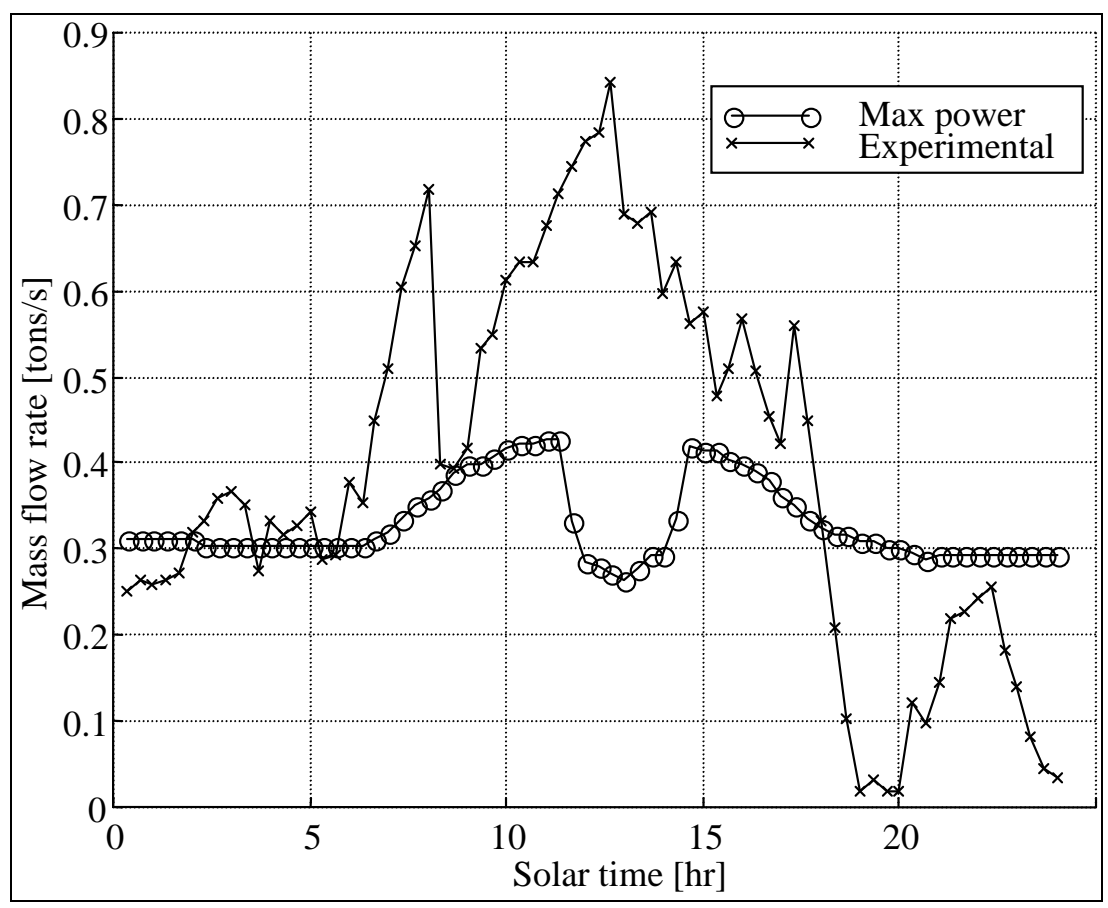

Figure 2.34. Predicted mass flow for maximum power output for Manzanares sized plant. 


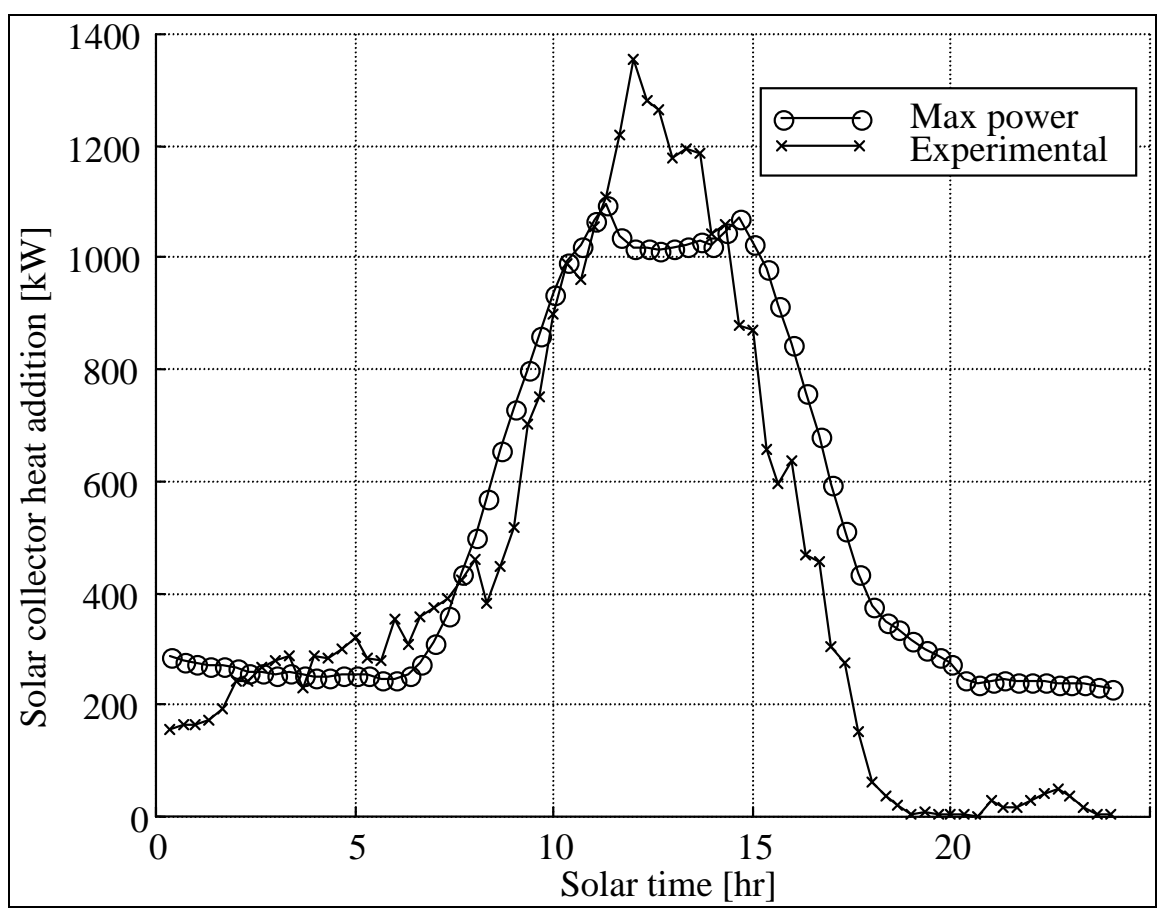

Figure 2.35. Predicted heat addition to air in solar collector for maximum power output for Manzanares sized plant.

Reducing the mass flow increases the temperature rise in the solar collector for the same inlet radiation as shown in Figure 2.36. In the middle of the day the temperature rise increases with the decrease in mass flow rate as the power output is limited.

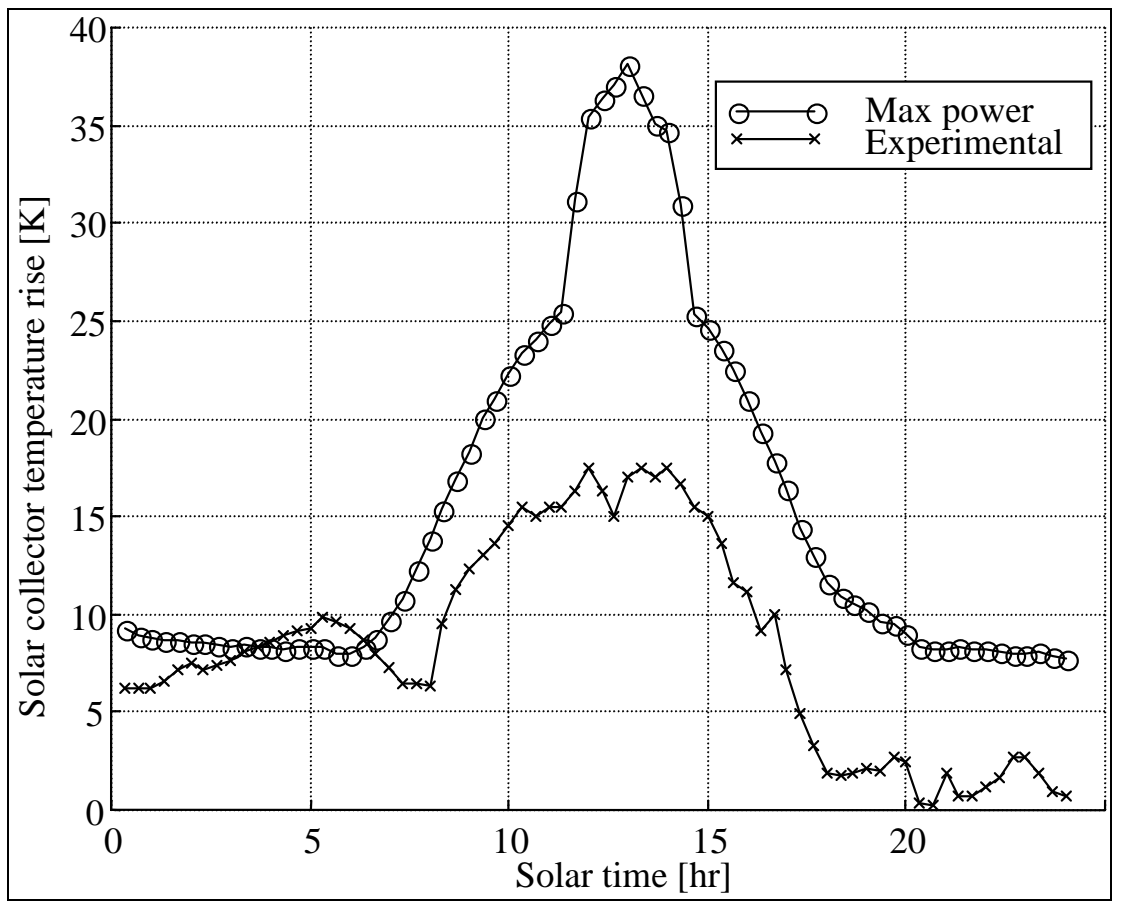

Figure 2.36. Predicted temperature rise $\Delta T_{23}$ in solar collector for maximum power output for Manzanares sized plant. 


\section{Full Scale Plant Simulation}

The next step in the transient analysis is to apply the existing model to a full-scale plant. It was decided to use the environmental conditions of the Manzanares plant as this meant the simulation could be simply modified to simulate a full-scale plant. The initial simulation of the full-scale plant used a $4 \mathrm{~km}$ diameter collector but it was found that this did not produce the design power of $200 \mathrm{MW}$. As the main aim of the analysis is to try and determine the operating region of the solar chimney turbine, it was decided to increase the collector radius to $6 \mathrm{~km}$ to ensure that the design power was achieved. It is generally more successful to decrease an existing turbine's pressure drop than to try and increase it. Simulating a $6 \mathrm{~km}$ diameter collector will ensure that the turbine is designed for a wide operating region. In the results that follow the plant operating characteristics for both the $4 \mathrm{~km}$ and $6 \mathrm{~km}$ diameter collector are presented. The plant dimensions and properties are the same as those presented in Table 2.4 with the plant being simulated for the same day as the published data from Haaf (1984) the data being 2-September-1982.

Figure 2.37 shows the effect of increasing the collector area. At $4 \mathrm{~km}$ diameter the design power is not reached. Increasing the diameter to $6 \mathrm{~km}$ ensures that the plant operates at its design power for approximately 4 hours of the day. It can be seen that the operation at the design power setting is not symmetrical around the solar noon but skewed towards the afternoon. This is due to the lag effects of the soil absorbing heat in the morning and releasing it in the afternoon. The power increase is due to the $6 \mathrm{~km}$ collector increasing the area by a factor of 2.25 over the $4 \mathrm{~km}$ collector.

Figure 2.38 shows the pressure drop for obtaining the maximum output power for the $4 \mathrm{~km}$ diameter collector and the $6 \mathrm{~km}$ collector. The trend for the $6 \mathrm{~km}$ collector turbine operation is very similar to that shown in Figure 2.33 for the Manzanares plant operating at a higher pressure drop. When at a lower power setting (200 MW) than the design power, the turbine must operate as efficiently as possible to maximise the plant output power. Once the plant reaches the limiting power conditions, it is advantageous if the turbine can operate efficiently as this means that more heat can be stored in the collector. What is most important, though, is that the turbine can operate at the high pressure drop and not stall.

The effect of the turbine limiting the plant power can be seen in Figure 2.39 with the mass flow rate dropping significantly. In Figure 2.40 the experimental temperature rises in the collector from the plant in Manzanares, Spain are superimposed. The temperature rises in the full scale plant will not be significantly higher than those in Manzanares except when the plant power is limited. 


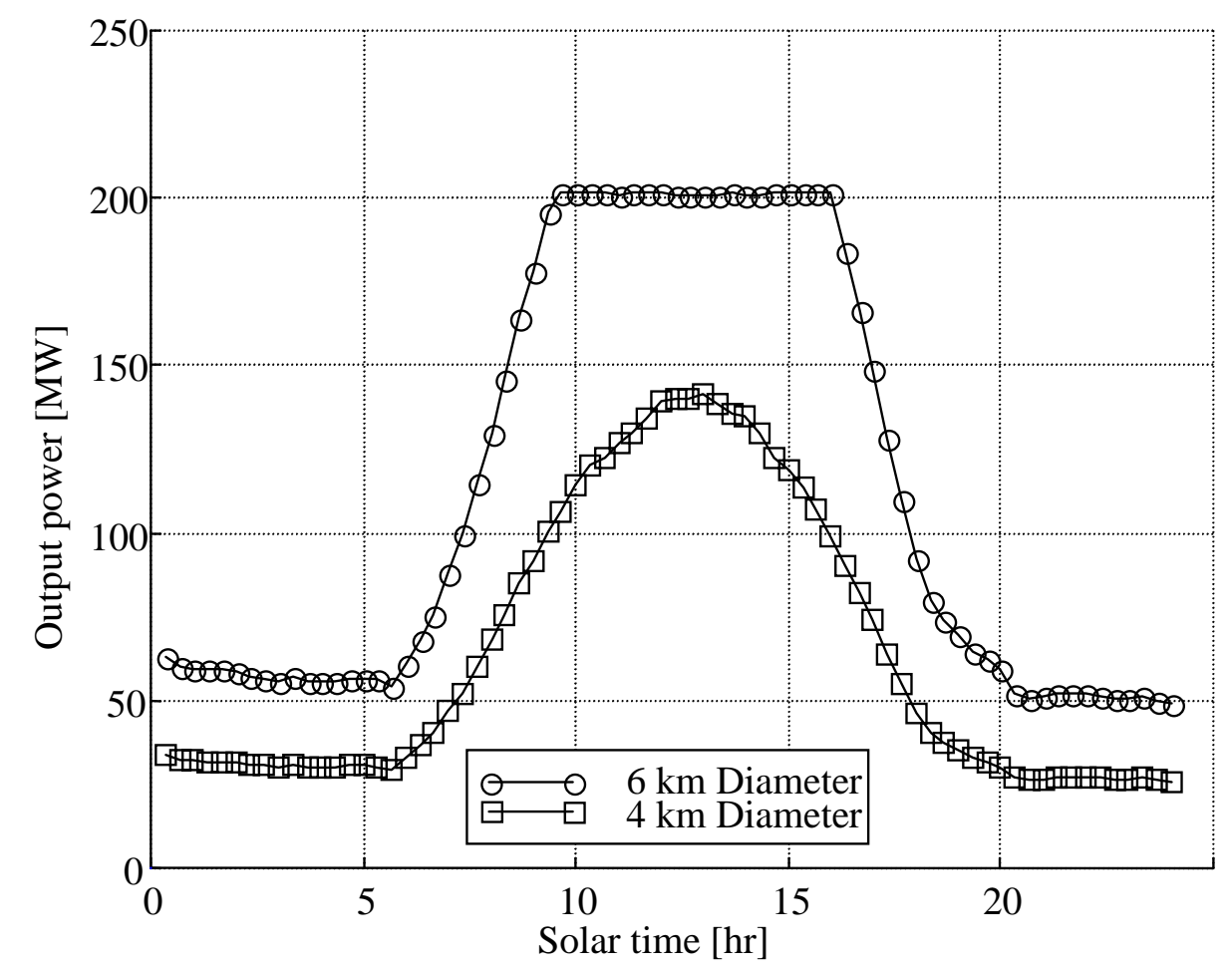

Figure 2.37. Full-scale plant maximum power output for 4 and 6 km diameter solar collector.

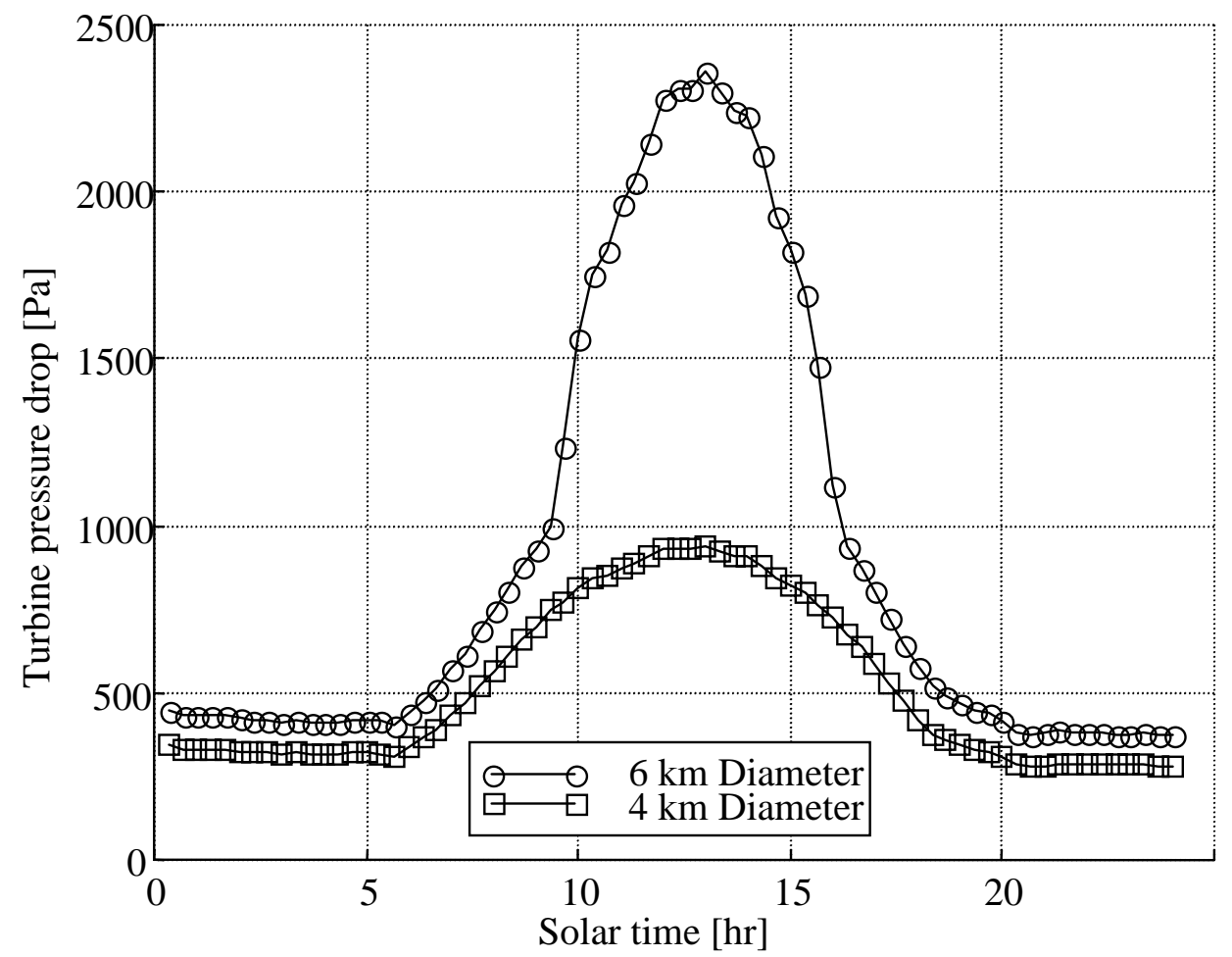

Figure 2.38. Full scale plant pressure drop for $4 \mathrm{~km}$ and $6 \mathrm{~km}$ diameter solar collector at maximum power output. 


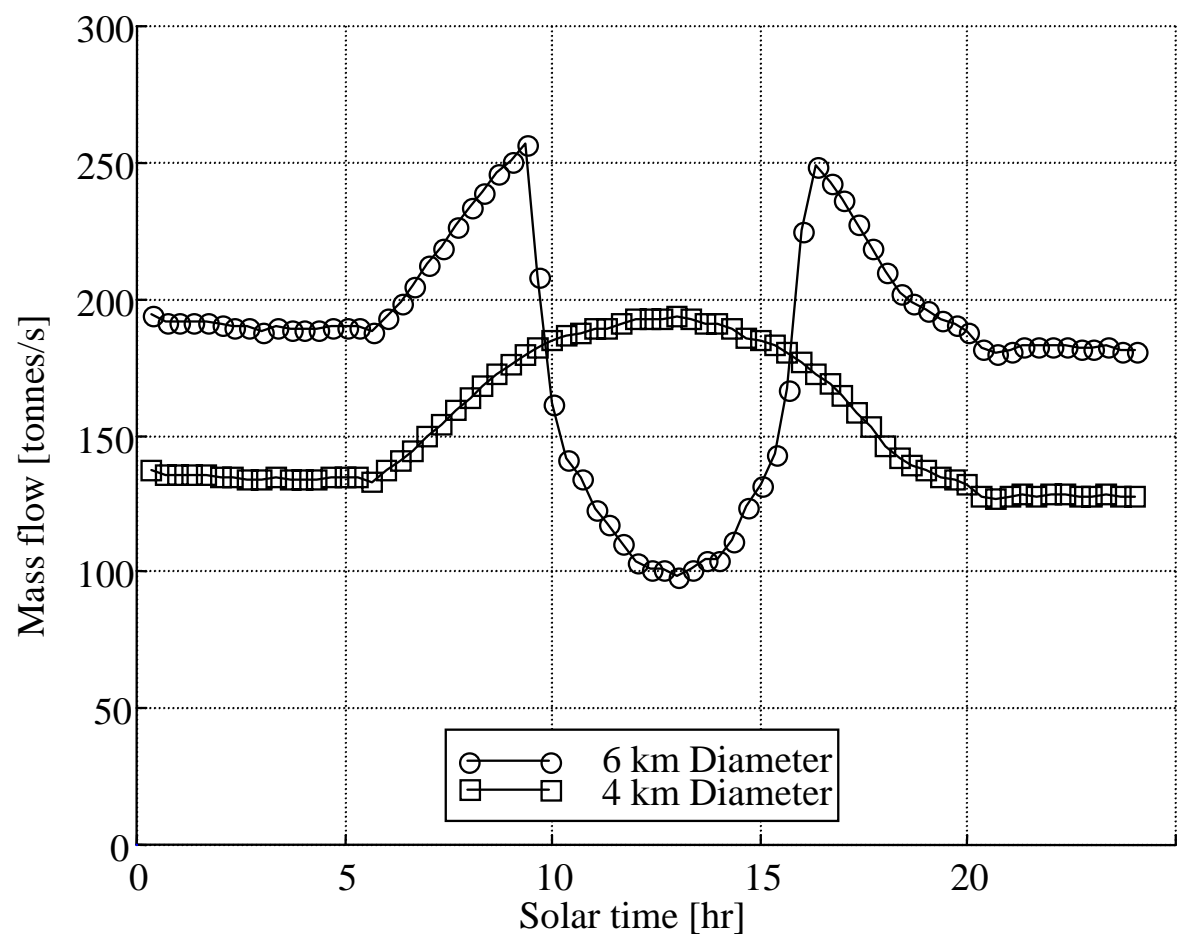

Figure 2.39. Full scale plant mass flow for $4 \mathrm{~km}$ and $6 \mathrm{~km}$ diameter solar collector at maximum power output.

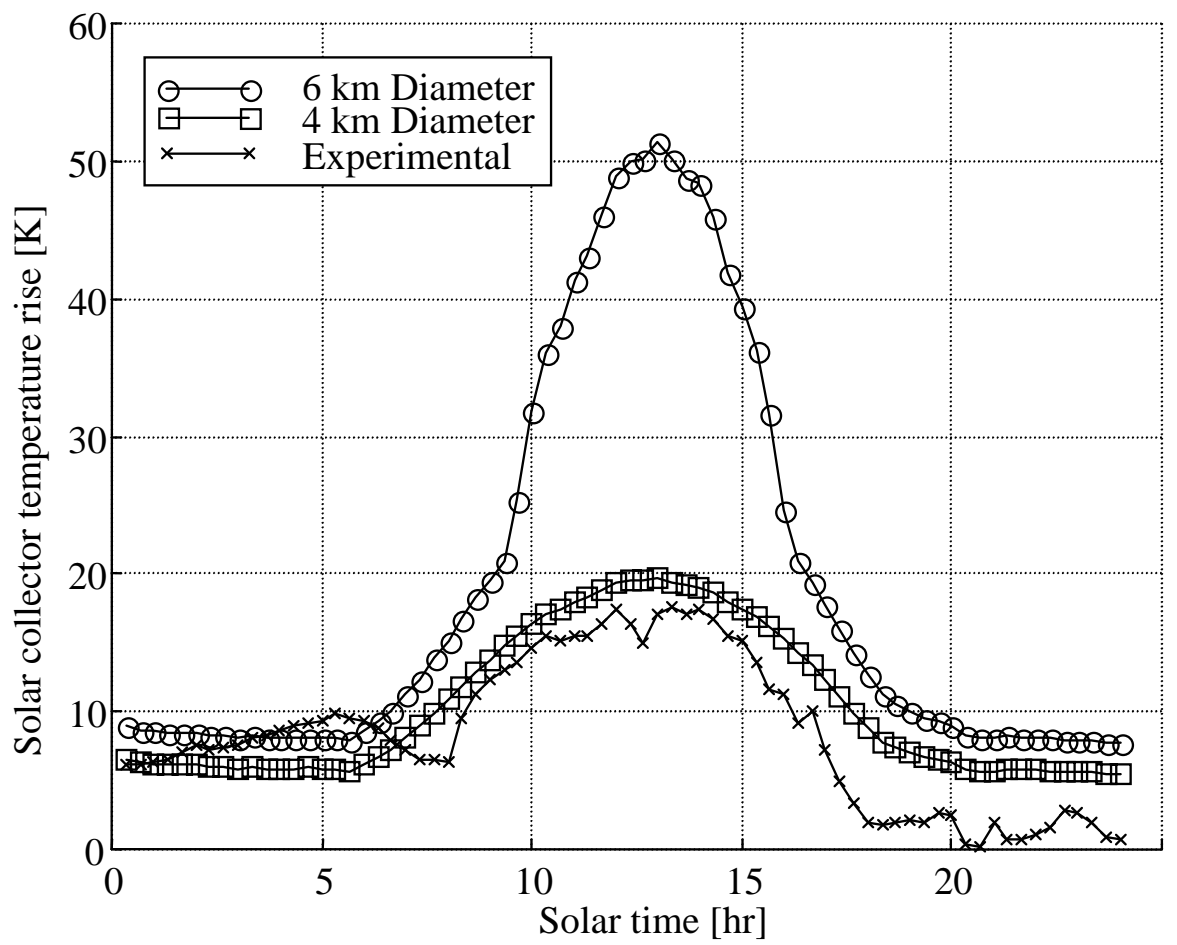

Figure 2.40. Full scale plant collector temperature rise $\Delta T_{23}$ for $4 \mathrm{~km}$ and $6 \mathrm{~km}$ collectors with Manzanares results superimposed. 


\section{Plant Control}

Some comments need to be made about the plant control in the light of the turbine experimental results and new concepts in new plant control. It was initially thought that it would be simple to control the plant by increasing the turbine pressure drop. The experimental results showed that attempting to do this would mean the turbine would operate in a stalled condition. This would result in large fluctuating loads on the blade. It has been pointed out that while in a small experimental turbine this is not a problem, it could be catastrophic in a large plant.

The objectives that need to be achieved in controlling the plant output power are to limit the power output while attempting to store the excess solar energy by some method in the collector. To do this only enough energy must be released into the flow to drive the turbine at the design power. A far more elegant approach to this problem is to allow cool air to be bled into the collector at some point near the turbine. This will keep the mass flow through most of the collector low while allowing the turbine to operate closer to its design operating range (Gannon \& Von Backström 2002).

\subsection{CONCLUSION}

The present chapter has outlined the development of the model used to simulate the solar chimney operation. The simple cycle analysis allowed the limiting plant efficiencies to be found and demonstrated the effect of the chimney height and collector temperature rise on the plant operation. The inclusion of plant losses allowed more realistic plant output powers to be calculated for a given collector temperature rise. Inclusion of the solar collector showed that the solar chimney turbine will operate at a higher pressure drop and lower mass flow than originally calculated. The steadystate collector model allowed insight into the plant operation to be gained such as controlling the plant power output near the middle of the day. The transient model was developed to more accurately determine the turbine operating range.

The solar chimney simulation used here is simple but was intended to be able to investigate the operating characteristics of the plant. What has been found is that the solar chimney turbine will operate at a lower mass flow rate and higher pressure drop than predicted with simple analysis methods. Similar results were found subsequently by Kröger and Buys (2001) where a more detailed transient analysis was also performed and the maximum power output sought. The results from the plant simulation are now used in the following chapter to design a suitable turbine. The eventual turbine design will be seen to have higher solidity blades over existing designs to be able to handle the pressure drop. 


\section{CHAPTER 3: SOLAR CHIMNEY TURBINE DESIGN AND LAYOUT}

Presented in the current chapter are details of the design process of the solar chimney turbine. In the previous chapter the required operating region of the turbine was defined from a simulation of a full-scale plant for one day of operation. From this data three critical points are defined but the design process presented here could easily be extended to as many operating points as required.

As a first step a turbine layout needs to be chosen and to help in this process the design objectives are listed and discussed. To aid in the choice of layout, a non-dimensional analysis is performed using the specific speed and diameter to see what existing designs could be used. The various layouts are described and the merits of each discussed. A single turbine with inlet guide vanes (IGV) was the one eventually chosen as being the most suitable. This layout allows for effective control of the exit kinetic energy thus increasing the turbine efficiency. The pre-whirl introduced by the IGV reduces the amount of exit whirl from the turbine. The IGV can also act as the supports for the chimney and their offset angle over radial supports stiffens the base structure.

Once the turbine layout has been decided upon, a free vortex analysis is performed to find a first approximation of the plant performance. The analysis also demonstrates the effect that the inlet guide vanes have in increasing the turbine efficiency. From this the plant diameter, hub-tip ratio and rotational speed can be found for the most efficient design possible over the required operating range. In the design of the turbine rather conservative constraints were applied. A relative rotor exit flow angle of $80^{\circ}$ for efficient operation and $85^{\circ}$ for power control and IGV exit flow angle of $20^{\circ}$ were assumed. This resulted in a potentially lower efficiency but was done to ensure that the first turbine design operated properly.

With the overall sizes fixed the next step of the analysis is to perform an axi-symmetric simulation of the turbine. A Matrix Throughflow Method (MTFM) was used for this purpose, a formulation proposed by Bosman and Marsh (1974), as this was able to handle the radial inflow and axial outflow that are found in the solar chimney turbine. The MTFM allows the gas inflow and outflow angles from the turbine to be found. The values were slightly different from the free vortex prediction as discussed later.

The final part of the design process is the design of the blades themselves. For this purpose a surface vortex method was used to perform the cascade analysis to try and accurately predict the deflection of the flow so that the correct stagger and camber angles were chosen. Most empirical correlations for flow deflection are not accurate for the flat stagger angles and low solidity blades found in the current type of turbine.

The blade analysis procedure was coupled to an optimisation method to minimise both the chord length and the maximum surface velocity to reduce the profile losses as much as possible. In the 
full-scale plant any reduction in the dimensions of the turbine would simplify the construction. The full-scale turbine will obviously be much larger than the experimental model built to test the performance and so differences in Reynolds number effects have to be taken into account. It is for this reason that the inlet guide vanes were designed, so that the flow would still remain attached for laminar flow while for the turbine blades, a turbulent separation criterion was used. This is covered in more detail in the section covering the design of the inlet guide vanes. The eventual turbine has 12 rotor blades, $18 \mathrm{IGV}$, a hub-tip ratio of 0.4 and is expected to run at a tip speed of $88.6 \mathrm{~m} / \mathrm{s}$.

\subsection{DESIGN OBJECTIVES}

The design process starts with a listing of the design objectives, an approach suggested by Ullman (1992). This leads to a more ordered design process. The aim is to achieve a solution to as many of the objectives with the basic design. Once the basic design has been chosen the flexibility to satisfy the design objectives becomes more difficult. A list of the main design objectives of the turbine is given below, these are then discussed in more detail afterwards.

- High efficiency over wide-operating range.

- Ability to operate at high pressure drops to control the plant mass flow.

- Must operate at a constant speed for synchronisation with the electricity grid.

- A simple robust design.

- Comply with the geometric constraints of the chimney.

- Reliable operation with low maintenance over a prolonged period.

- Self starting or at least require very small forces to start it.

- Speed control to ensure damage is not done in the case of a runaway condition.

High Efficiency Over a Wide-Operating Range

A high turbine efficiency is required as the plant overall efficiency is low as discussed in the previous chapter. High efficiencies are most important at the lower power settings found in the early mornings and late afternoons into the evening when the plant will be operating at some fraction of its peak design output. In the middle of the day the plant power output may have to be limited, depending on the solar collector size as outlined in the previous chapter. Therefore, high efficiencies here are not as critical. It would still be desirable to keep the efficiency as high as possible as this would result in more energy being stored in the collector.

In most single rotor turbomachines the largest proportion of the losses come from the two components of the exit kinetic energy, the tangential and axial velocity. In the design of the turbine the exit kinetic must be minimised over the operating range. Other losses are minimised by keeping the velocities through the machine as low as possible. 
The cycle investigation performed in the previous chapter showed that near midday more power could be developed than the generator capacity. One method of limiting the power output that was suggested was to throttle the plant by increasing the turbine pressure drop. The lower mass flow rate would result in higher temperatures in the collector that would increase the effectiveness of any thermal storage system. As discussed in the previous chapter, other methods of plant control have been suggested.

\section{Constant Speed Operation}

For large generators it is usual to use a synchronous generator whose speed is governed by the number of pole pairs and once running is locked into the frequency of the local grid. This means that the rotational speed of the turbine is constant and thus speed cannot be used as a means to control the turbine and other methods need to be investigated.

\section{Simple Robust Design}

The entire aim of the solar chimney is to be a simple electricity generating plant (Schlaich 1995), and this philosophy should be applied to the turbine. An attempt will be made to create an efficient turbine of simple layout and construction and rather use detailed and rigorous analysis to optimise the design and obtain high efficiencies.

\section{Chimney Geometric Constraints}

The chimney of the plant is extremely high and will need a stable base while still allowing free flow of air through the turbine. It would also be advantageous to have the turbine as low as possible in the chimney to make its construction simpler.

\section{Low Maintenance, Reliable Operation}

The solar chimney has high capital costs and must have low running costs to be viable. The small plant built at Manzanares demonstrated that this was possible and so in the large plant the turbine must be able to run with little or no maintenance for prolonged periods. The sheer size of the turbine required will also mean that once it is built it would not be desirable to have to undergo major adjustment or repair work often.

\section{Starting}

Ideally the turbine should be self-starting and then once it is running at the correct speed it could be connected to the grid. If this is not possible then a very small force must be used to get it started 
using the generator as a motor. The least amount of energy possible must be used in this process. It is also beneficial if as many power plants as possible in a grid are self starting.

\section{Runaway Speed Limitation}

In the case of sudden load-loss, such as generator failure, the turbine will almost invariably start to speed up as there is no torque. The design of the turbine must be such that this can be controlled and not fail in the process.

\subsection{NON-DIMENSIONAL ANALYSIS}

Non-dimensional analysis allows a new turbine to be investigated without having detailed knowledge of the design. It can also be useful in choosing a design, as the non-dimensional values can be compared to those of existing machines and a similar one chosen. In the previous chapter it was simply assumed that a turbine could be designed to operate in the desired range. Based on this assumption the volume flow rates and pressure drops to extract the maximum power from the plant were calculated. The turbine must now be designed to be able to extract power at these volume flows and pressure drops. Two operational modes are defined, part load and design load of 200 MW for the full-scale plant.

Three design points that cover the operational range are defined, two for part load and one at the design load. As mentioned earlier, the number of design points can easily be extended to include an entire year's operation. The three design points are shown in Figure 3.1 and Figure 3.2 taken from chapter 2. Table 3.1 summarises the design cases in comparative terms. They are referred to as cases 1, 2 and 3. The turbine design is optimised for case (1) and case (2). Case (3) was only taken into account in the free-vortex analysis as there is still debate about what is the best method of plant control.

Table 3.1. Description of turbine design points.

\begin{tabular}{|l|l|l|l|l|}
\hline & Description & Pressure drop & Mass flow & Efficiency \\
\hline Case 1) & Early morning operation & low & medium & high \\
\hline Case 2) & Mid morning operation & medium & high & high \\
\hline Case 3) & Midday operation, power limitation & high & low & medium \\
\hline
\end{tabular}

The equations used for the definition of specific speed are those given by Balje (1981) and implemented by Von Backström (1998). The definition of specific speed is

$$
\mathrm{N}_{\mathrm{s}}=\frac{\omega \sqrt{\mathrm{Q}}}{\left(\Delta \mathrm{p}_{\mathrm{o}} / \rho\right)^{0.75}}
$$

and the specific diameter,

$$
\mathrm{D}_{\mathrm{s}}=\frac{\mathrm{D}\left(\Delta \mathrm{p}_{\mathrm{o}} / \rho\right)^{0.25}}{\sqrt{\mathrm{Q}}}
$$




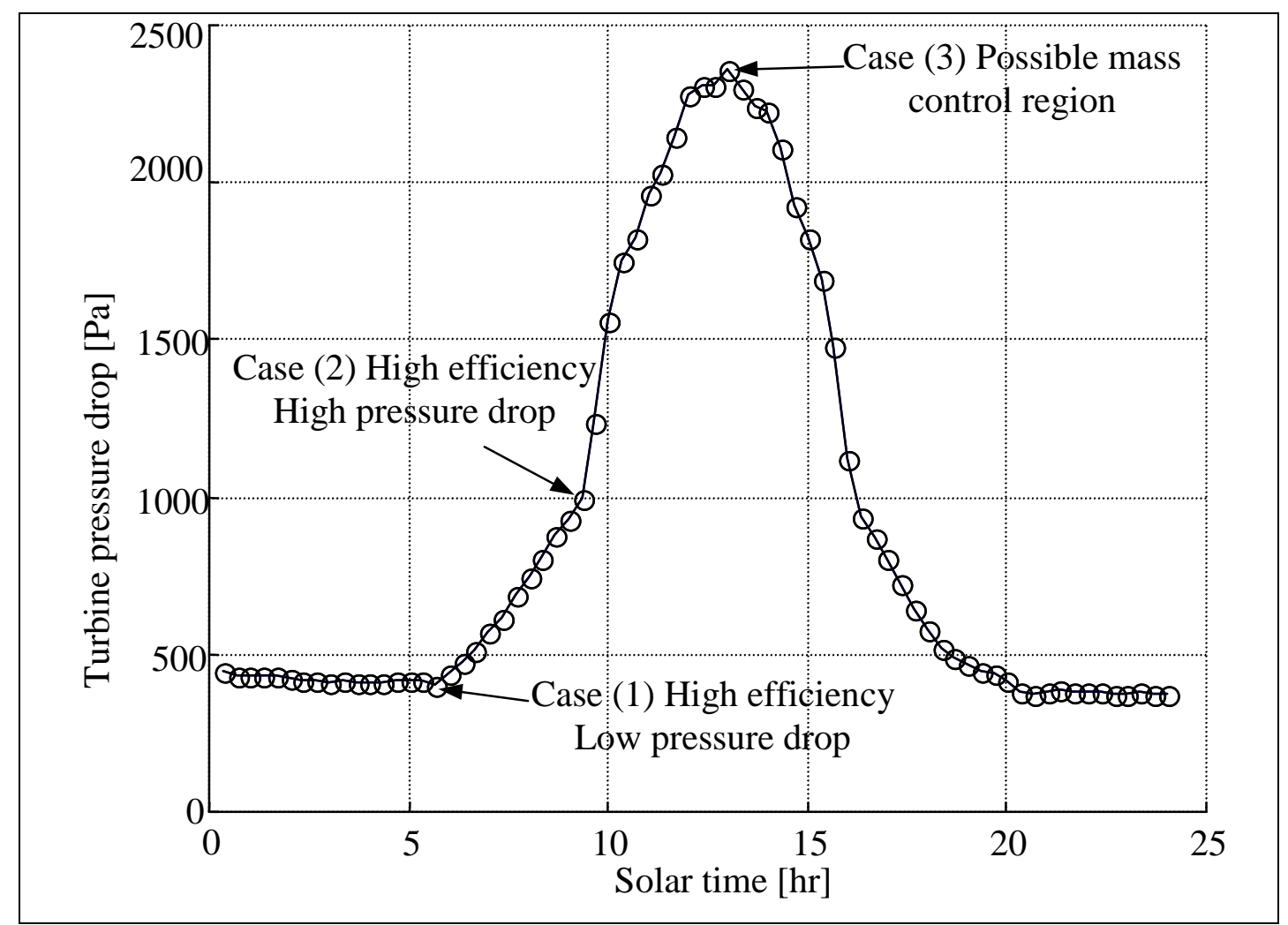

Figure 3.1. Predicted turbine pressure drop over a 24 hour period

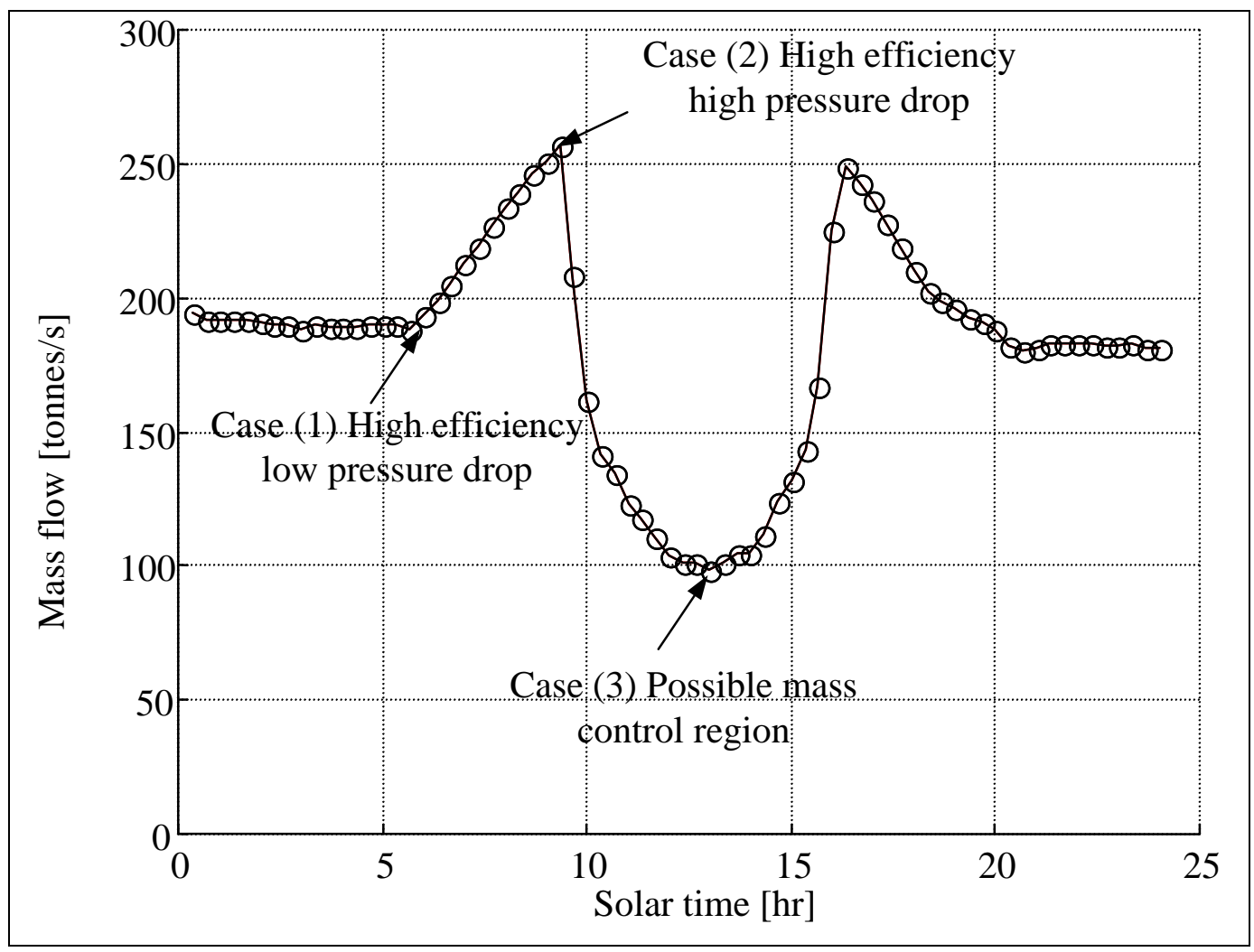

Figure 3.2. Predicted mass flow 
Obviously at the beginning of the design process certain design values need to be assumed. For the solar chimney turbine the tip speed was taken to be similar to those of modern wind turbines. As a start the turbine diameter is assumed to be a certain fraction of the chimney diameter. This was slightly different to the eventual design. The initial estimates are left as they were and presented in Table 3.2.

Table 3.2. Chosen design point flow conditions.

\begin{tabular}{|l|l|l|l|l|}
\hline \multicolumn{2}{|l|}{ Design point } & Case (1) & Case (2) & Case (3) \\
\hline Turbine Diam [m] & $\mathrm{D}_{\text {turb }}$ & 130 & & \\
\hline Tip speed [m/s] & $\mathrm{U}_{\text {tip }}$ & 100 & & \\
\hline Rotational speed [rad/s] & $\omega$ & 1.538 & & \\
\hline Power output [W] & $\mathrm{P}$ & $50.6 \times 10^{6}$ & $200 \times 10^{6}$ & $200 \times 10^{6}$ \\
\hline Mass flow [kg/s] & $\mathrm{m}$ & 180330 & 256480 & 98839 \\
\hline Volume flow [m $3 / \mathrm{s}]$ & $\mathrm{Q}$ & 168360 & 245150 & 105350 \\
\hline Pressure drop [Pa] & $\Delta \mathrm{p}_{\mathrm{o}}$ & 375 & 994 & 2623 \\
\hline Density [kg/m $\left.{ }^{3}\right]$ & $\rho$ & 1.0711 & 1.0462 & 0.9382 \\
\hline Specific speed & $\mathrm{N}_{\mathrm{s}}$ & 7.7981 & 4.4507 & 1.4048 \\
\hline Specific diameter & $\mathrm{D}_{\mathrm{s}}$ & 1.3706 & 1.4577 & 2.8372 \\
\hline
\end{tabular}

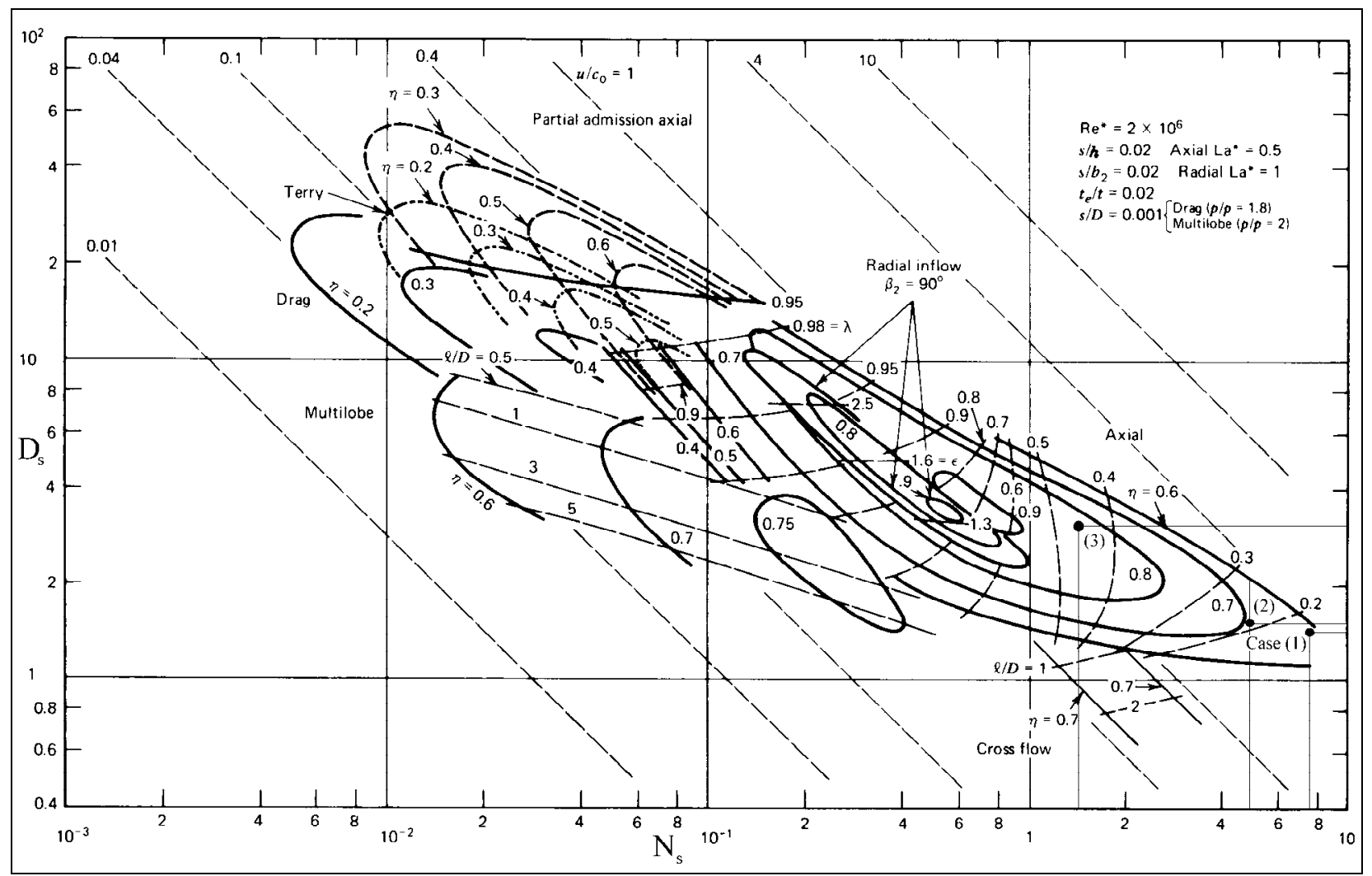

Figure 3.3. Specific diameter vs. specific speed diagram for single stage turbines operating with compressible fluids. Cases (1)-(3) superimposed on figure. 
Figure 3.3 is taken from Balje (1981) and shows the performance of various single stage turbines over a range of specific speeds and diameters for compressible fluids. The predicted specific speed and diameter of the solar chimney turbine is superimposed on the figure. The superimposed points fall firmly in the region of axial turbines albeit for low efficiencies. This prompted the consideration of using IGV to increase the expected values of efficiencies.

\subsection{TURBINE LAYOUT CHOICE}

Calculating the approximate specific speed and diameter of the turbine from the previous analysis, it would seem that an axial turbine would be the most sensible choice based on the figure of Balje (1981). It was also decided early on in the design process to keep to conventional turbine designs. This is in keeping with the rest of the solar chimney plant being as simple as possible. Other types of turbines may certainly be possible for a first time design of a full-scale turbine. The aim was to first demonstrate a working turbine.

\section{Comparison with Existing Turbines}

In designing a new type of turbine it is best to compare it to existing types so that design information that may be useful can be obtained. The solar chimney turbine is unique in terms of its large size but it can be compared using non-dimensional analysis with wind and gas turbines. Both of these types of turbines have been widely researched and there is extensive data available. The data used here for the solar chimney turbine is obtained from the present project but early estimated values gave the same order results.

To demonstrate where the solar chimney turbine is positioned in terms of non-dimensional and normalised power output, it is compared to wind and gas-turbine turbines. The non-dimensional power is defined by

$$
\overline{\mathrm{P}}=\frac{\mathrm{Q} \Delta \mathrm{p}}{\rho \omega^{3} \mathrm{D}^{5}}
$$

Another useful non-dimensional parameter is the normalised power output. It is the ratio of the power extracted from the flow through the system to the power available and is defined by

$$
\mathrm{P}^{*}=\frac{\mathrm{Q} \Delta \mathrm{p}}{\dot{\mathrm{m} \mathrm{c}_{\mathrm{p}} \mathrm{T}}}
$$

Table 3.3 compares the three turbine types being discussed. The wind turbine data is taken from Von Backström (1998a) for the Growian 1 as this is one of the largest built. The gas turbine data is taken from a representative example presented in Cohen et al. (1996). 
Table 3.3. Comparison of specific power for wind, solar chimney and gas turbines.

\begin{tabular}{|c|c|c|c|c|c|}
\hline & & & Wind turbine & Solar chimney & Gas turbine \\
\hline Vol. flow rate & $\mathrm{Q}$ & {$\left[\mathrm{m}^{3} / \mathrm{s}\right]$} & 62300 & 245148 & 15.58 \\
\hline Pressure drop & $\Delta \mathrm{p}$ & {$[\mathrm{Pa}]$} & 53.2 & 994 & 188863 \\
\hline Inlet density & $\rho$ & {$\left[\mathrm{kg} / \mathrm{m}^{3}\right]$} & 1.2 & 1.05 & 1.28 \\
\hline Rot. speed & $\omega$ & {$[\mathrm{rad} / \mathrm{s}]$} & 1.94 & 1.37 & 1571 \\
\hline Diameter & $\mathrm{D}$ & [m] & 100.4 & 127.86 & 0.5011 \\
\hline Gas constant & $c_{p}$ & [J/kg.K] & 1005 & 1005 & 1148 \\
\hline Inlet temperature & $\mathrm{T}$ & {$[\mathrm{K}]$} & 288 & 313 & 1100 \\
\hline Non-dimensional power & & & $3.72 \times 10^{-5}$ & $2.55 \times 10^{-3}$ & $1.87 \times 10^{-2}$ \\
\hline Normalised power & & & $1.39 \times 10^{-4}$ & $2.47 \times 10^{-3}$ & $1.46 \times 10^{-1}$ \\
\hline
\end{tabular}

The non-dimensional power of the solar chimney turbine is an order of magnitude smaller than the gas turbine but two orders larger than the wind turbine. This would point to the solar chimney turbine being geometrically similar to a gas turbine. The normalised power of the solar chimney turbine is an order of magnitude larger than that of the wind turbine, but two orders of magnitude smaller than that of a gas turbine.

\section{Turbine Choice}

For the solar chimney there are two methods of using an axial turbine: the first is a single large turbine and the second a cluster arrangement of a number of smaller turbines. Various cluster arrangements have been investigated by Schlaich et al. (1995) and these do have the advantage of allowing the plant to carry on running if one of the turbines break down. They would also be simpler to build and maintain as there would not be the associated structural difficulties of a single large turbine. In this arrangement some method of blocking a malfunctioning turbine is needed, as the flow would tend to flow through the holes with the least pressure drop.

The main advantage of a large single turbine is that the exit kinetic energy can be more easily minimised without worrying about the interference of other turbines close by. For the single turbine layout there is also the ability to modify the chimney supports to act as inlet guide vanes (IGV's). The reason they are not used in wind turbines is that the pressure drops are not that high and the increase in efficiency is not worth the added complexity of having to incorporate inlet guide vanes. In the solar chimney the modification of the chimney base supports would be simple. The pre-swirl must be of the opposite rotation to the swirl leaving the turbine, as this would reduce the net outlet swirl thus raising the total-to-static efficiency of the turbine. It is proposed that the inlet guide vanes have adjustable trailing sections. This would be simple to do in practice as the entire blade would not rotate. It was thought that the adjustable IGV would allow a high efficiency over wide operating range to be maintained. 


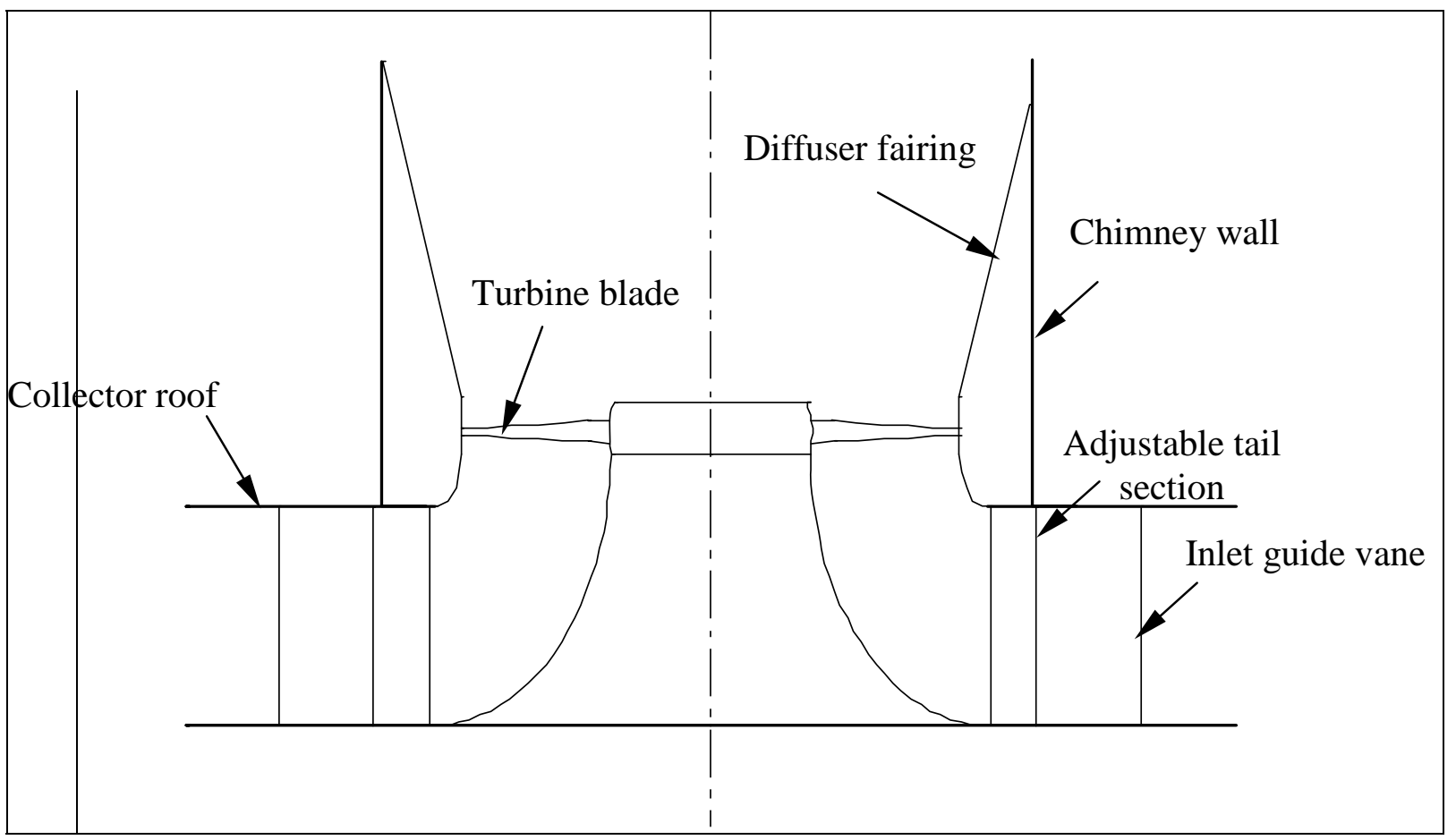

Figure 3.4. Schematic of proposed turbine layout with inlet guide vanes adding pre-swirl.

The above figure shows the proposed single turbine layout for the solar chimney with the inlet guide vanes producing pre-swirl before the turbine rotor. In the following section a free vortex analysis is performed and it can be demonstrated that the efficiency is higher when inlet guide vanes are included in the design.

\subsection{FREE VORTEX ANALYSIS}

To gain some insight into the operation of the turbine a free vortex analysis is applied to show the effect of the main variables such as the tip speed and diameter. The free vortex analysis assumed that whirl velocity is inversely proportional to radius at all stations between blade rows. Using the free-vortex analysis, the turbine tip speed, diameter, hub-tip ratio and inlet guide vane and turbine height will be determined. Certain constraints are introduced into the theory such as the maximum turning possible by the IGV. These will be discussed where they are applied.

The free-vortex theory is presented before applying it to the turbine design. To start the free vortex theory for a rotor-only-turbine is presented. This basic analysis is then extended to include the IGV that introduces pre-swirl and a non-uniform pressure drop along the blade length. Finally the effect of the diffuser and blade losses are added. The free vortex analysis can be used in the preliminary calculation of the relative flow angles in the rotor blades. Figure 3.5 shows a typical velocity triangle diagram for the solar chimney turbine. The velocities and angles have been drawn to scale and are representative of the flow at the turbine tip. An IGV-Turbine stage is shown in the figure showing the effect of adding pre-swirl. 


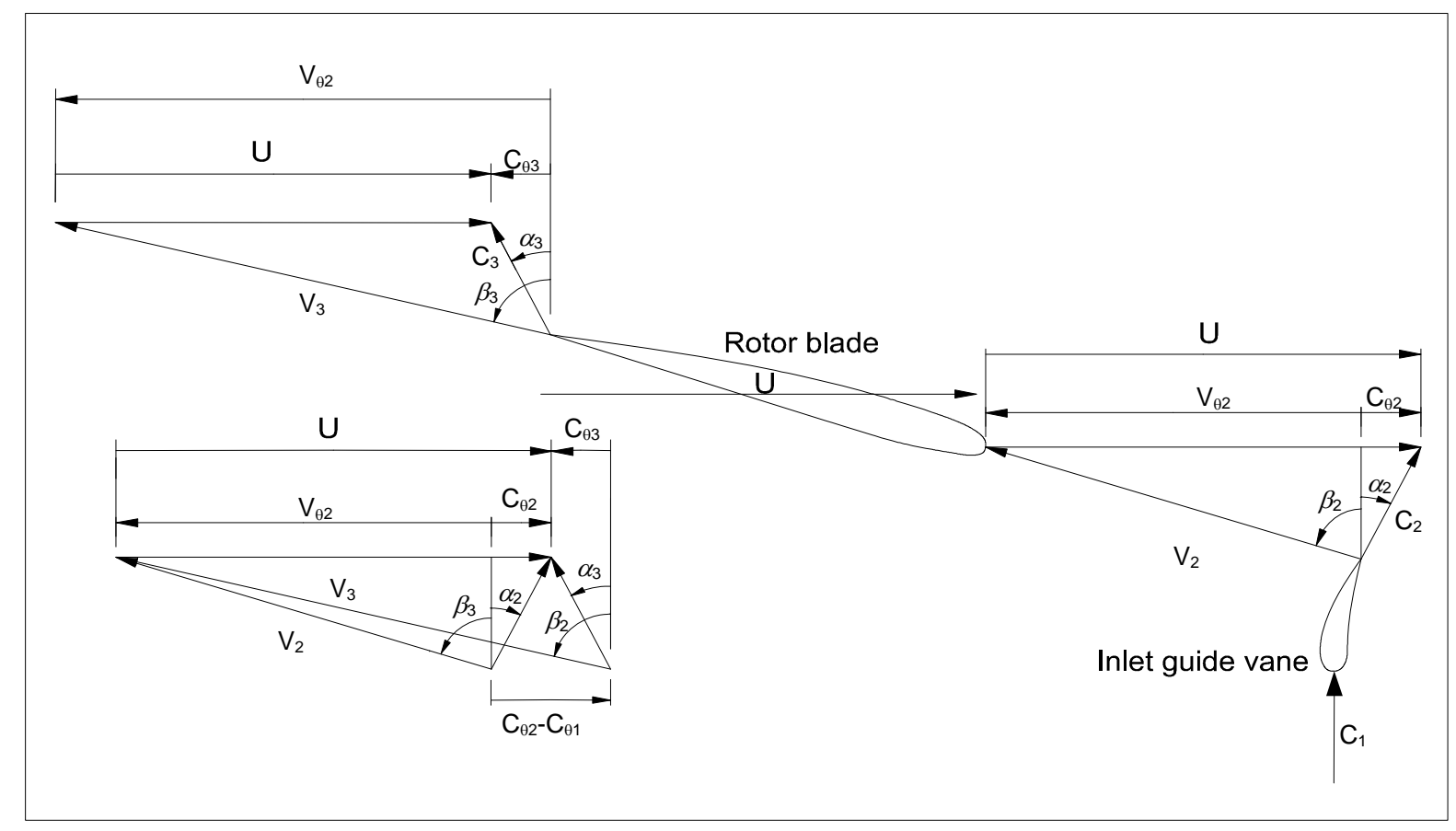

Figure 3.5. Velocity triangle diagram of solar chimney turbine near tip region.

Throughout the analysis the turbine temperature instead of pressure drop will be used, as this is far simpler to work with when calculating the required gas turning angles. Two non-dimensional constants are defined: the flow coefficient $(\phi)$, and the load coefficient $(\psi)$, using the definitions of Cohen et al. (1996) (p 274).

$$
\begin{aligned}
& \phi=\frac{\mathrm{C}_{\mathrm{z}}}{\mathrm{U}_{\text {tip }}} \\
& \psi=\frac{2 \mathrm{C}_{\mathrm{p}} \Delta \mathrm{T}_{\text {turb }}}{\mathrm{U}_{\text {tip }}^{2}}
\end{aligned}
$$

\section{Rotor Only Uniform Work Distribution}

In the free vortex analysis actuator discs replace the blades and all flow changes occur within these, meaning that there is no radial flow in the free stream. The flow is assumed to be incompressible, which means that the axial velocity remains constant unless there is an area change through the turbomachine. The axial velocity profile is also assumed to be constant across the section. The turbine total-to-static efficiency is first defined and can be rewritten in terms of the kinetic energy losses,

$$
\begin{aligned}
\eta_{\text {turb ts }} & =\frac{\Delta \mathrm{T}_{\text {turb }}}{\Delta \mathrm{T}_{\text {turb }}+\mathrm{C}_{3}^{2} / 2 \mathrm{c}_{\mathrm{p}}}=\frac{1}{1+\mathrm{C}_{3}^{2} /\left(2 \Delta \mathrm{T}_{\text {turb }} \mathrm{C}_{\mathrm{p}}\right)} \\
& =\frac{1}{1+\mathrm{L}_{\mathrm{a}}+\mathrm{L}_{\theta}}
\end{aligned}
$$


These loss components $L_{a}$ and $L_{\theta}$ are now defined in terms of the flow $(\phi)$ and load $(\psi)$ coefficient, starting by looking at the total kinetic energy loss at the exit and assuming $\mathrm{C}_{\mathrm{r}}$ is small:

$$
\mathrm{KE}_{\text {turb }}=\frac{1}{2} \int_{\mathrm{r}_{\mathrm{rub}}}^{\mathrm{r}_{\text {tip }}} \rho \mathrm{C}_{\mathrm{z} 3}\left(\mathrm{C}_{\mathrm{z} 3}^{2}+\mathrm{C}_{\theta 3}^{2}\right) 2 \pi \mathrm{rdr}
$$

This is normalised as follows,

$$
\begin{aligned}
\mathrm{KE}_{\text {turb }}^{*} & =\frac{\mathrm{KE}_{\text {turb }}}{\dot{\mathrm{m}}_{\mathrm{p}} \Delta \mathrm{T}_{\text {turb }}} \\
= & \frac{\frac{1}{2} \int_{\mathrm{r}_{\text {tub }}}^{\mathrm{r}_{\text {tip }}} \rho \mathrm{C}_{\mathrm{z3}}\left(\mathrm{C}_{\mathrm{z} 3}^{2}+\mathrm{C}_{\theta 3}^{2}\right) 2 \pi r d r}{\mathrm{c}_{\mathrm{p}} \Delta \mathrm{T}_{\text {turb }} \int_{\mathrm{r}_{\text {tub }}}^{\mathrm{r}_{\text {tip }}} \rho \mathrm{C}_{\mathrm{z} 3} 2 \pi \mathrm{rdr}}
\end{aligned}
$$

and then separated into the axial and tangential loss components (in general $C_{z 3} \neq C_{\theta 3}$ ),

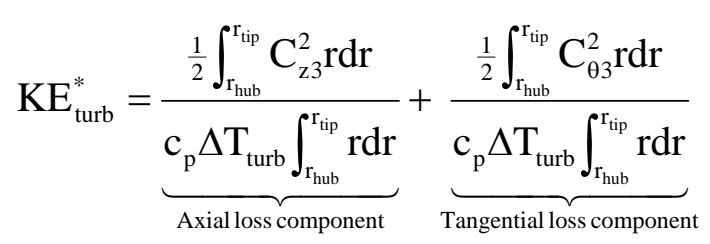

Integrating the axial loss component first and substituting the flow $(\phi)$ and load $(\psi)$ coefficients, the following expression is derived,

$$
\left(\mathrm{KE}_{\text {turb }}^{*}\right)_{\text {axial }}=\mathrm{L}_{\mathrm{a}}=\frac{\mathrm{C}_{\mathrm{z} 3}^{2}}{2 \mathrm{c}_{\mathrm{p}} \Delta \mathrm{T}_{\text {turb }}}=\frac{\phi^{2}}{\psi}
$$

The tangential loss term is slightly more complex as a relation between the exit swirl and turbine temperature drop is needed. For a constant work distribution and no inlet guide vanes and using the Euler turbomachinery equation,

$$
\mathrm{c}_{\mathrm{p}} \Delta \mathrm{T}_{\text {turb }}=\omega\left[\left(\mathrm{rC}_{\theta 2}\right)-\left(\mathrm{rC}_{\theta 3}\right)\right]
$$

and for the rotor-only-case, there is no inlet swirl so $\mathrm{C}_{\theta 3}$ can be written as,

$$
\mathrm{C}_{\theta 3}=\frac{\mathrm{C}_{\mathrm{p}} \Delta \mathrm{T}_{\text {turb }}}{\mathrm{r} \omega}
$$

This can be substituted into the tangential loss term and with some simple cancellation of terms the integral shown needs to be evaluated.

$$
\begin{aligned}
\left(\mathrm{KE}_{\text {turb }}^{*}\right)_{\text {tan gential }} & =\mathrm{L}_{\theta}=\frac{\frac{1}{2} \mathrm{C}_{\mathrm{p}} \Delta \mathrm{T}_{\text {turb }} \int_{\mathrm{r}_{\text {hub }}}^{\mathrm{r}_{\text {tip }}} \frac{1}{\mathrm{r}} \mathrm{dr}}{\omega^{2} \int_{\mathrm{r}_{\text {hub }}}^{\mathrm{r}_{\text {tip }}} \mathrm{rdr}} \\
& =\frac{\mathrm{c}_{\mathrm{p}} \Delta \mathrm{T}_{\text {turb }}}{\omega^{2}} \frac{\ln \frac{\mathrm{r}_{\text {tip }}}{\mathrm{r}_{\text {hub }}}}{\left(\mathrm{r}_{\text {tip }}^{2}-\mathrm{r}_{\text {hub }}^{2}\right)}
\end{aligned}
$$


Once again substituting the flow $(\phi)$ and load $(\psi)$ coefficients and the hub-tip ratio $v=\mathrm{r}_{\text {hub }} / \mathrm{r}_{\text {hub }}$, the axial loss term is written as (Von Backström et al. 1996),

$$
\mathrm{L}_{\theta}=\frac{\psi}{2} \frac{\ln \frac{1}{v}}{\left(1-v^{2}\right)}
$$

It is now possible to find the turbine efficiency due to kinetic loss only with the few terms required from equations (3.5) and (3.6).

\section{IGV-rotor Stage With Uniform Work Distribution}

The next part of the analysis is to add the effect of the inlet guide vanes. The same amount of work must be done across the turbine but now the inlet swirl is non zero. A parameter that defines how much swirl is added is the swirl fraction $\Lambda$. This defines the amount of swirl remaining after the rotor as a fraction of the amount remaining when there is no IGV. Thus for a rotor-only-stage $\Lambda=$ 1 , while for a stage where all swirl that the inlet guide vanes introduce is removed by the turbine, $\Lambda$ $=0$. The advantage of such a stage is that the exit flow is purely axial thus reducing the exit kinetic energy and increasing the total-to-static efficiency.

As before, the Euler turbomachinery equation is used and has the same form as in (3.12) as the same amount work is required. The exit swirl is reduced depending on the swirl fraction,

$$
\begin{aligned}
\left(\mathrm{rC}_{\theta 3}\right) & =\Lambda \frac{\mathrm{C}_{\mathrm{p}} \Delta \mathrm{T}_{\text {turb }}}{\omega} \\
\mathrm{C}_{\theta 3} & =\Lambda \frac{\mathrm{C}_{\mathrm{p}} \Delta \mathrm{T}_{\text {turb }}}{\mathrm{r} \omega}
\end{aligned}
$$

and this can be seen to be the same as equation (3.13) except for the addition of the constant $\Lambda$. The axial loss term remains exactly the same as the flow is still free-vortex and has a uniform axial velocity profile while the tangential loss term is multiplied by the $\Lambda^{2}$ term.

$$
\mathrm{L}_{\theta}=\Lambda^{2} \frac{\psi}{2} \frac{\ln \frac{1}{v}}{\left(1-v^{2}\right)}
$$

\section{IGV-rotor Stage with Non-uniform Work Distribution}

While it is usual to deal with blades with a uniform temperature drop, it is sometimes desirable to design blades with non-uniform distributions. In the current project the tip temperature drop was decreased in an attempt to increase the rotational speed of the turbine. There is a limit to the relative exit flow angle $\beta_{3}$ and thus to the rotational speed. Reducing the amount of work at the turbine tip where $\beta_{3}$ is normally highest, means less flow deflection and a reduced value of $\beta_{3}$. The rotational speed of the turbine can then be increased. The assumption of a uniform axial velocity profile is 
then not accurate as the turbine exit flow will no longer have a free vortex distribution. To predict the axial velocities more accurately a two-dimensional analysis is performed later. There are many different possible non-uniform work distributions but for this analysis a linear function was chosen to have a workable analytical solution. The function shape is shown schematically in Figure 3.6.

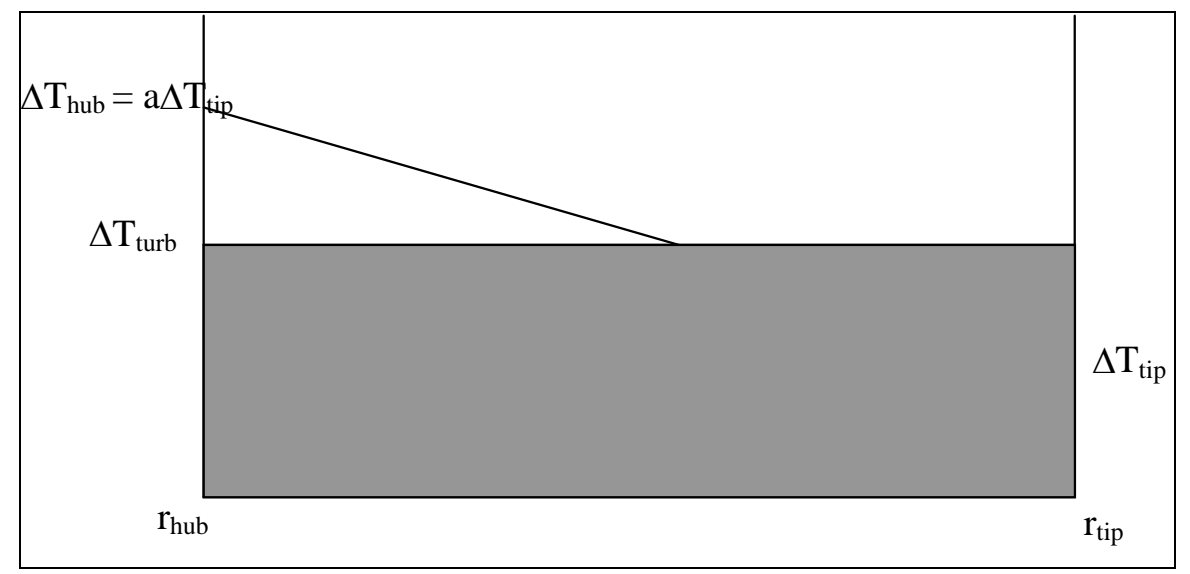

Figure 3.6. Schematic of linear function of work distribution across turbine blade.

When a non-uniform work distribution is chosen, the new distribution must have the same work

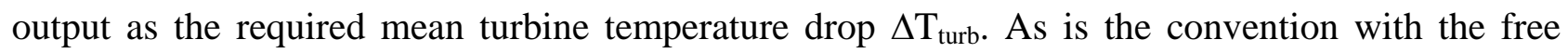
vortex analysis the tip temperature drop is chosen as the reference and a ratio, a, of tip, $\Delta \mathrm{T}_{\text {tip }}$ to hub. $\Delta \mathrm{T}_{\text {hub }}$ temperature drop is defined as,

$$
\mathrm{a}=\frac{\Delta \mathrm{T}_{\text {hub }}}{\Delta \mathrm{T}_{\text {tip }}}
$$

Using the term, a, the temperature drop at a given radius is as follows,

$$
\Delta \mathrm{T}=\Delta \mathrm{T}_{\text {tip }}\left(1-\frac{(1-\mathrm{a})}{(1-\mathrm{v})}\left(1-\frac{\mathrm{r}}{\mathrm{r}_{\text {tip }}}\right)\right)
$$

To calculate the total power from the turbine, an integration across the blade is performed and this must give the same power output as that from the mean temperature drop, $\Delta T_{\text {turb}}$,

$$
\begin{aligned}
\text { Power } & =\dot{\mathrm{mc}_{\mathrm{p}}} \Delta \mathrm{T}_{\text {turb }}=2 \pi \rho \mathrm{C}_{\mathrm{z} 3} \mathrm{c}_{\mathrm{p}} \Delta \mathrm{T}_{\text {turb }} \int_{\mathrm{r}_{\text {thub }}}^{\mathrm{r}_{\text {tip }}} r d r \\
& =2 \pi \rho \mathrm{C}_{\mathrm{z3}} \mathrm{c}_{\mathrm{p}} \int_{\mathrm{r}_{\text {hub }}}^{\mathrm{r}_{\text {tip }}} \Delta \mathrm{Trdr}
\end{aligned}
$$

Equating the quotient of the above equation to unity and substituting the equation for the linear temperature distribution $\Delta \mathrm{T}$ across the blade, a relationship between $\Delta \mathrm{T}_{\text {turb }}$ and $\Delta \mathrm{T}_{\text {tip }}$ based on the ratio, a, can be found, 


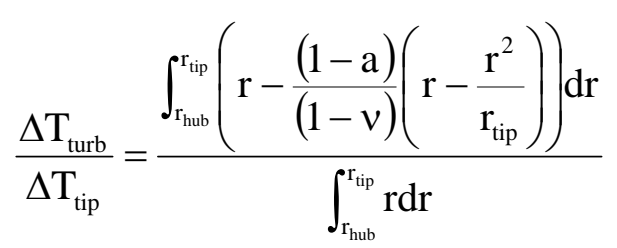

Integrating this and simplifying the ratio of $\Delta \mathrm{T}_{\text {turb }} / \Delta \mathrm{T}_{\text {tip }}$,

$$
\frac{\Delta \mathrm{T}_{\text {turb }}}{\Delta \mathrm{T}_{\text {tip }}}=\mathrm{b}=1-\left[\left(\frac{1-\mathrm{a}}{1-v}\right)\left\{1-\frac{2}{3}\left(\frac{1+v+v^{2}}{1+v}\right)\right\}\right]
$$

The above expression must now be substituted into the tangential loss expression (3.17), $\Delta \mathrm{T}_{\text {turb }}=\mathrm{b} \Delta \mathrm{T}_{\text {tip }}$,

$$
\left(\mathrm{KE}_{\text {turb }}^{*}\right)_{\text {tan gential }}=\mathrm{L}_{\theta}=\Lambda^{2} \frac{\frac{1}{2} \mathrm{C}_{\mathrm{p}} \int_{\int_{\text {rub }}}^{\mathrm{r}_{\text {tip }}} \frac{\left(\mathrm{b} \Delta \mathrm{T}_{\text {tip }}\right)^{2}}{\mathrm{r}} \mathrm{dr}}{\omega^{2} \Delta \mathrm{T}_{\text {turb }} \int_{\Gamma_{\text {rub }}}^{\mathrm{r}_{\text {rip }}} \mathrm{rdr}}
$$

The numerator term is integrated and it can be shown that the tangential loss term $\mathrm{L}_{\theta}$ will be unchanged,

$$
\begin{aligned}
& \int_{\text {rinb }}^{\mathrm{r}_{\text {tip }}} \frac{\left(\mathrm{b} \Delta \mathrm{T}_{\text {tip }}\right)^{2}}{\mathrm{r}} \mathrm{dr}=\Delta \mathrm{T}_{\text {tip }}^{2} \int_{\mathrm{r}_{\text {rub }}}^{\mathrm{r}_{\text {tip }}} \frac{\mathrm{b}^{2}}{\mathrm{r}} \mathrm{dr} \\
& =\mathrm{b}^{2} \Delta \mathrm{T}_{\text {tip }}^{2} \ln \left(\frac{1}{v}\right) \\
& =\Delta \mathrm{T}_{\text {turb }}^{2} \ln \left(\frac{1}{v}\right)
\end{aligned}
$$

\section{Relative Exit Angles}

During the optimisation the relative gas exit angles from the turbine blades are required and these can be calculated from the free-vortex analysis. The absolute gas exit angle is derived from the Euler turbomachinery equation by looking first at the blade tip,

$$
\mathrm{C}_{\mathrm{p}} \Delta \mathrm{T}_{\mathrm{o}}=\mathrm{U}_{\text {tip }}\left(\mathrm{C}_{\theta 2}-\mathrm{C}_{\theta 3}\right)
$$

and recalling that the swirl introduced by the IGV is given as follows,

$$
\mathrm{C}_{\theta 2}=(1-\Lambda) \frac{\mathrm{C}_{\mathrm{p}} \Delta \mathrm{T}_{\text {turb }}}{\mathrm{U}_{\text {tip }}}
$$

Substituting (3.26) into (3.25) the absolute gas flow exit whirl velocity $\mathrm{C}_{\theta 3}$ is,

$$
\mathrm{C}_{\theta 3}=\frac{\mathrm{C}_{\mathrm{p}} \Delta \mathrm{T}_{\text {turb }}}{\mathrm{U}_{\text {tip }}}(\Lambda+1 / \mathrm{b}-1)
$$


The relative exit angle can be obtained by vector addition (refer to Figure 3.5),

$$
\begin{aligned}
\beta_{3 \text { tip }} & =a \tan \left(\frac{C_{\theta 3 \text { tip }}+U_{\text {tip }}}{C_{z}}\right) \\
& =a \tan \left(\frac{(\Lambda+1 / b-1) \psi+2}{2 \phi}\right)
\end{aligned}
$$

\section{Diffuser Recovery}

As the turbine diameter is less than that of the chimney, a diffuser is placed after the turbine in an attempt to recover some of the kinetic energy from the turbine to improve the turbine efficiency. Equation (3.7) is modified with the addition of the diffuser loss coefficient. The area ratio (AR) is defined at the ratio of the diffuser exit area over the turbine exit area (Japikse \& Baines 1997).

$$
\mathrm{AR}=\frac{\mathrm{D}_{\text {chim }}^{2}}{\mathrm{D}_{\text {turb }}^{2}}\left(1-v^{2}\right)
$$

leading to the ideal pressure recovery based on the inlet axial velocity of,

$$
\mathrm{C}_{\mathrm{pD}, \text { ideal }}=1-1 / \mathrm{AR}^{2}
$$

A real diffuser has a certain operating efficiency or diffuser effectiveness $\eta_{D}$. The diffused loss coefficient $\mathrm{K}$ is defined using $\eta_{\mathrm{D}}$,

$$
K_{D}=\left(1-\eta_{D}\right) C_{p D, \text { ideal }}
$$

The actual diffuser recovery coefficient is then,

$$
\mathrm{C}_{\mathrm{pD}}=\mathrm{C}_{\mathrm{pD} \text {,ideal }}-\mathrm{K}_{\mathrm{D}}
$$

The axial loss is split into two terms, the pressure recovery $\left(1-\mathrm{C}_{\mathrm{p}, \text { ideal }}\right)$ and diffuser loss term $\mathrm{K}_{\mathrm{D}}$,

$$
\eta_{\text {turb ts }}=\frac{1}{1+\left(1-\mathrm{C}_{\mathrm{p} \text {,ideal }}\right) \mathrm{L}_{\mathrm{a}}+\mathrm{K}_{\mathrm{D}} \mathrm{L}_{\mathrm{a}}+\mathrm{L}_{\theta}}
$$

The axial loss term, $\mathrm{L}_{\mathrm{a}}$ in the above equation, is split into two terms as the axial loss term after the diffuser, $\left(1-\mathrm{C}_{\mathrm{p} \text {,ideal }}\right) \mathrm{L}_{\mathrm{a}}$ is discarded in the optimisation. This is done as the axial loss has already been accounted for at the chimney exit in the cycle analysis. The total-to-static efficiency used in the optimisation scheme is,

$$
\eta_{\text {turb ts }}=\frac{1}{1+K_{D} L_{a}+L_{\theta}}
$$

In retrospect, it would have been better to include all the loss terms in the optimisation. Even though the axial losses have been taken into account at the chimney exit, there is no detrimental 
effect in minimising the overall kinetic energy at the diffuser exit before it enters the chimney. This is simple to implement and would result in a less distorted axial velocity profile entering the chimney.

\section{Profile-Loss Coefficients}

The final terms to be included in this free vortex analysis are the losses due to profiles losses. These are as a direct result of viscous and frictional effects of the flow over the inlet guide vanes and rotor blades. It should be noted that the loss coefficients were not used in the initial design calculations. It is expected that the loss coefficients would lower the efficiency. The experimental results showed that their inclusion has a significant effect on choosing the optimum design point. This is discussed in more detail with the experimental results. In brief, the fact that the rotor tip speed is so high relative to the other velocities means that the losses are almost constant. This means that it affects the efficiency at the low power setting more than at the design power.

The subscript $\mathrm{S}$ is used for the inlet guide vane loss coefficient and in most texts it is referred to as the stator loss coefficient but more generally it refers to a stationary blade row. The efficiency equation has the IGV loss, $\mathrm{L}_{\mathrm{S}}$ and rotor loss, $\mathrm{L}_{\mathrm{R}}$ terms added,

$$
\eta_{\text {turb ts }}=\frac{1}{1+\mathrm{K}_{\mathrm{D}} \mathrm{L}_{\mathrm{a}}+\mathrm{L}_{\theta}+\mathrm{L}_{\mathrm{s}}+\mathrm{L}_{\mathrm{R}}}
$$

Two loss coefficient terms are normally defined in terms of a pressure or total temperature loss relative to the exit blade velocity. These are based on the exit velocities, $C_{2}$ for the stator and $V_{3}$ for the rotor shown in Figure 3.5.

$$
\begin{aligned}
& \zeta_{\mathrm{S}}=\frac{\Delta \mathrm{p}_{\text {loss }}}{\frac{1}{2} \rho \mathrm{C}_{2}^{2}} \approx \frac{\mathrm{C}_{\mathrm{p}} \Delta \mathrm{T}_{\text {loss }}}{\frac{1}{2} \mathrm{C}_{2}^{2}} \text { (IGV loss coefficient) } \\
& \zeta_{\mathrm{R}}=\frac{\Delta \mathrm{p}_{\text {loss }}}{\frac{1}{2} \rho \mathrm{V}_{3}^{2}} \approx \frac{\mathrm{C}_{\mathrm{p}} \Delta \mathrm{T}_{\text {loss }}}{\frac{1}{2} \mathrm{~V}_{3}^{2}} \text { (rotor loss coefficient) }
\end{aligned}
$$

The derivation of the IGV loss coefficient, $L_{s}$, is first performed and as before the total loss term is normalised as follows,

$$
\begin{aligned}
& \mathrm{L}_{\mathrm{s}}= \frac{\Delta \mathrm{T}_{\text {loss }}}{\dot{\mathrm{m}_{\mathrm{p}} \Delta \mathrm{T}_{\text {turb }}}} \\
&=\frac{\frac{1}{2} \int_{\mathrm{r}_{\text {tub }}}^{\mathrm{r}_{\text {tip }}} \rho \mathrm{C}_{\mathrm{z}}\left(\mathrm{C}_{2}^{2}\right) \zeta_{\mathrm{s}} 2 \pi \mathrm{rdr}}{\mathrm{c}_{\mathrm{p}} \Delta \mathrm{T}_{\text {turb }} \int_{\mathrm{r}_{\text {hub }}}^{\mathrm{r}_{\text {tip }}} \rho \mathrm{C}_{\mathrm{z}} 2 \pi \mathrm{rdr}}
\end{aligned}
$$

Due to the radial inflow through the IGVs in the solar chimney the exit velocity $C_{2}$ is constant resulting in $\mathrm{L}_{\mathrm{s}}$ being simplified to the following, 


$$
\begin{aligned}
\mathrm{L}_{\mathrm{s}} & =\frac{\frac{1}{2} \mathrm{C}_{2}^{2} \zeta_{\mathrm{s}}}{\mathrm{C}_{\mathrm{p}} \Delta \mathrm{T}_{\text {turb }}}=\frac{\frac{1}{2}\left(\mathrm{C}_{\mathrm{z}}^{2}+\mathrm{C}_{\theta 2}^{2}\right) \zeta_{\mathrm{s}}}{\mathrm{C}_{\mathrm{p}} \Delta \mathrm{T}_{\text {turb }}} \\
& =\left[\frac{\phi^{2}}{\psi}+\frac{1}{4}(\Lambda-1)^{2} \psi\right] \zeta_{\mathrm{s}}
\end{aligned}
$$

Equation (3.38) is only true for an axial throughflow machine with the IGVs placed axially upstream of the rotor blades. It can be simply modified to the radial configuration of the solar chimney turbine. This is achieved by multiplying the first term by the turbine-IGV area ratio and the second term by the ratio of the turbine tip radius and IGV exit radius with the results shown in

$$
\mathrm{L}_{\mathrm{s}}=\left[\left(\frac{\mathrm{A}_{\text {turb }}}{\mathrm{A}_{\mathrm{IGV}}}\right)^{2} \frac{\phi^{2}}{\psi}+\frac{1}{4}\left(\frac{\mathrm{r}_{\text {turb }}}{\mathrm{r}_{\mathrm{IGV}}}\right)^{2}(\Lambda-1)^{2} \psi\right] \zeta_{\mathrm{s}}
$$

The calculation of the normalised rotor loss, $L_{R}$, is more complicated than for the IGV loss, $L_{S}$, due to the added effect of the rotation of the blades. $L_{R}$ can be written as an integral equation similar to (3.48) based on the rotor relative exit velocity

$$
\begin{aligned}
\mathrm{L}_{\mathrm{R}}= & \frac{\Delta \mathrm{T}_{\text {loss }}}{\dot{\mathrm{m}_{\mathrm{p}} \Delta \mathrm{T}_{\text {turb }}}} \\
= & \frac{\frac{1}{2} \int_{\mathrm{r}_{\text {hub }}}^{\mathrm{r}_{\text {tip }}} \rho \mathrm{C}_{\mathrm{z}}\left(\mathrm{V}_{3}^{2}\right) \zeta_{\mathrm{R}} 2 \pi \mathrm{rdr}}{\mathrm{c}_{\mathrm{p}} \Delta \mathrm{T}_{\text {turb }} \int_{\mathrm{r}_{\text {hub }}}^{\mathrm{r}_{\text {tip }}} \rho \mathrm{C}_{\mathrm{z}} 2 \pi \mathrm{rdr}}
\end{aligned}
$$

$\mathrm{V}_{3}$ can be written in terms of the blade speed, $\mathrm{U}$ and the absolute exit tangential, $\mathrm{C}_{\theta 3}$, and axial, $\mathrm{C}_{\mathrm{z} 3}$, velocities. This is obtained from the velocity triangles shown in Figure 3.5.

$$
\begin{aligned}
& \mathrm{L}_{\mathrm{R}}=\frac{\Delta \mathrm{T}_{\text {loss }}}{\dot{\mathrm{mc}}_{\mathrm{p}} \Delta \mathrm{T}_{\text {turb }}} \\
& =\frac{\frac{1}{2} \zeta_{\mathrm{R}} \int_{\mathrm{r}_{\text {hub }}}^{\mathrm{r}_{\mathrm{tip}}} \rho \mathrm{C}_{\mathrm{z}}\left(\mathrm{C}_{\mathrm{z}}^{2}+\mathrm{C}_{\theta 3}^{2}+\mathrm{U}^{2}+2 \mathrm{UC}_{\theta 3}\right) 2 \pi \mathrm{rdr}}{\mathrm{C}_{\mathrm{p}} \Delta \mathrm{T}_{\text {turb }} \int_{\mathrm{r}_{\text {hub }}}^{\mathrm{r}_{\mathrm{tip}}} \rho \mathrm{C}_{\mathrm{z}} 2 \pi \mathrm{rdr}}
\end{aligned}
$$

The above integral is best evaluated by splitting it into four separate equations and treating each velocity component separately. Immediately it can be seen that the first two terms involving the axial velocity $\mathrm{C}_{\mathrm{z} 3}$ and whirl $\mathrm{C}_{\theta 3}$ can be treated in the same way as equation (3.10).

$$
\begin{aligned}
\mathrm{L}_{\mathrm{RC}_{z}}+\mathrm{L}_{\mathrm{RC}_{\theta}} & =\frac{\frac{1}{2} \zeta_{\mathrm{R}} \int_{\mathrm{r}_{\text {hub }}}^{\mathrm{r}_{\text {tip }}}\left(\mathrm{C}_{\theta 3}^{2}\right) \mathrm{rdr}}{\mathrm{C}_{\mathrm{p}} \Delta \mathrm{T}_{\text {turb }} \int_{\mathrm{r}_{\text {rub }}}^{\mathrm{r}_{\text {tip }}} r d r}+\frac{\frac{1}{2} \zeta_{\mathrm{R}} \int_{\mathrm{r}_{\text {hub }}}^{\mathrm{r}_{\text {tip }}}\left(\mathrm{C}_{\mathrm{z}}^{2}\right) \mathrm{rdr}}{\mathrm{C}_{\mathrm{p}} \Delta \mathrm{T}_{\text {turb }} \int_{\mathrm{r}_{\mathrm{rub}}}^{\mathrm{r}_{\text {tip }}} \mathrm{rdr}} \\
& =\zeta_{\mathrm{R}}\left[\mathrm{L}_{\mathrm{a}}+\mathrm{L}_{\theta}\right]
\end{aligned}
$$

The $3^{\text {rd }}$ and $4^{\text {th }}$ term involving the blade speed $\mathrm{U}^{2}$ and product term $2 \mathrm{UC}_{\theta 3}$ simplify to the following terms, 


$$
\begin{aligned}
& \mathrm{L}_{\mathrm{RU}}= \frac{\frac{1}{2} \zeta_{\mathrm{R}} \int_{\mathrm{r}_{\text {hub }}}^{\mathrm{r}_{\text {tip }}}\left(\mathrm{U}^{2}\right) \mathrm{rdr}}{\mathrm{c}_{\mathrm{p}} \Delta \mathrm{T}_{\text {turb }} \int_{\mathrm{r}_{\text {hub }}}^{\mathrm{r}_{\text {tip }}} \mathrm{rdr}}=\zeta_{\mathrm{R}} \frac{1}{3 \psi}\left[1+v+v^{2}\right] \\
& \mathrm{L}_{\mathrm{R} 2 \mathrm{C}_{\theta} \mathrm{U}}=\frac{\frac{1}{2} \zeta_{\mathrm{R}} \int_{\mathrm{r}_{\text {hub }}}^{\mathrm{r}_{\text {tip }}}\left(2 \mathrm{UC}_{\theta 3}\right) \mathrm{rdr}}{\mathrm{c}_{\mathrm{p}} \Delta \mathrm{T}_{\text {turb }} \int_{\mathrm{r}_{\text {hub }}}^{\mathrm{r}_{\text {tip }}} \mathrm{rdr}}=\zeta_{\mathrm{R}} \Lambda
\end{aligned}
$$

Bringing the four terms together gives the rotor loss coefficient as follows,

$$
\mathrm{L}_{\mathrm{R}}=\zeta_{\mathrm{R}}\left[\mathrm{L}_{\mathrm{a}}+\mathrm{L}_{\theta}+\frac{1}{3 \psi}\left(1+v+v^{2}\right)+\Lambda\right]
$$

The Soderberg loss correlation, Lewis (1996), was used to analyse the effect of profile losses on the performance of the turbine. It is based on a constant loss increasing with an increase in the flow deflection over the blade row. It is proportional to the reciprocal of the blade row aspect ratio $\mathrm{H} / \mathrm{b}$, where $b$ is the axial chord (chord $x \cos ($ stagger)). The method defined two loss coefficients $\zeta$ that accounts for profile loss only and $\zeta_{\text {sec }}$ that accounts for other secondary losses.

$$
\begin{aligned}
\zeta & =0.025\left(1+\left(\frac{\varepsilon^{\mathrm{o}}}{90}\right)^{2}\right) \\
\zeta_{\mathrm{sec}} & =3.2\left(\frac{\mathrm{b}}{\mathrm{H}}\right) \zeta
\end{aligned}
$$

\subsection{MAJOR TURBINE DESIGN VARIABLES}

Now that the turbine operating range and turbine type has been chosen the major design variables need to be fixed. They are the hub-tip ratio, turbine diameter and height, blade tip speed and inlet guide vane height. Each is discussed briefly with any limitations that may apply.

\section{Hub-tip Ratio}

A compromise between minimising the axial loss (a small hub) and the tangential loss (a large hub) normally governs the hub-tip ratio size. In the solar chimney size, is also a factor and it would be impractical to have too large a hub in the centre.

\section{Turbine Diameter}

Much the same approach applies to the turbine diameter in that exit kinetic energy losses must be minimised but the blades must not be too long as they would become difficult to build. 


\section{Blade Tip Speed}

For high efficiencies a high blade rotational speed is required as this reduces the tangential kinetic energy loss as shown in equation (3.14) where the higher $\omega$ is, the smaller the loss term. The tip speed is limited by various factors. Firstly, the blade strength, the centripetal acceleration, cannot be higher than a certain amount or the blades may fail. Aerodynamic constraints apply because if the relative velocity increases too much, losses become high. At certain conditions compressibility problems may occur when parts of the relative flow reach Mach 1, but this is unlikely except at runaway conditions. The factor that has the dominating effect in limiting the tip speed is the relative exit angle $\beta_{3}$ shown in Figure 3.5. If this becomes too high, the profile losses increase significantly, and for any given set of flow conditions $\beta_{3}$ increases with the tip speed. A figure from Japikse and Baines (1997), pp 6-28, shows that the losses remain constant until $80^{\circ}$. Above this value the losses increase significantly and so $80^{\circ}$ was used as a limit for the efficient operating region. A value of up to $85^{\circ}$ is possible but with high losses. It is used as the limiting constraint for case (3). Wind turbines often operate with even higher relative outlet angles (Spera 1995). As the pressure drop for the solar chimney turbine is expected to be about ten times that of a wind turbine the gas turbine data was used.

\section{Inlet Guide Vane Height}

The inlet guide vanes would be an integral part of the plant construction and would support the chimney of the plant. From a structural viewpoint they should be made as low and thick as possible without constricting the flow. Short IGV's would increase the velocity at the base of the chimney as the inlet area would be smaller but this would allow more pre-whirl to be added. The reason for this is that there is a limit as to the angle the blades can turn the flow. When looking at Figure 3.5, an increase in the inlet and outlet velocities due to reduced IGV area would increase $C_{1}$ and $C_{2}$ leading to an increased $\mathrm{C}_{\theta 2}$ for the same flow turning angle. The advantage of high inlet guide vanes is that there would be a large flow contraction in the duct section, resulting in an accelerating flow that would be resistant to separation on the outer wall.

\section{Application of Optimisation}

When an optimisation is performed, one variable is usually minimised by changing a number of variables, sometimes subject to certain constraints. The optimisation method used was the simplex algorithm and is well described by Rao (1996). The program MATLAB (1996) was used and its simplex algorithm was modified to be able to apply constraints. The simplex algorithm is a very stable optimisation scheme and while there are faster methods many of these do not converge when the functions that are being optimised are not smooth while the simplex algorithm handles discontinuities quite easily. 
In the solar chimney turbine the total-to-static efficiency of equation (3.34) was maximised for cases (1) and (2). For case (3) the efficiency was not maximised but a constraint was placed on the relative exit flow angle from the rotor $\beta_{3}$. The optimisation variables were the turbine diameter, IGV turning angle, hub-tip ratio and blade tip speed. Constraints that were applied were a limit on the turning angle possible by the inlet guide vanes and a maximum value on the relative exit flow angle $\beta_{3}$. The efficiencies were calculated using the free vortex analysis outlined above for a given set of design variables. The constraints are listed in Table 3.6.

The ratio of IGV and turbine height to the turbine diameter was fixed. The ratio was chosen to ensure that the flow accelerated in the duct section and did not separate from the roof as it exits the IGV and turns into the rotor. A matrix throughflow method (MTFM) was used to calculate the velocity profile in this duct section with the results presented later.

Table 3.4. Initial turbine and inlet guide vane dimensions relative to chimney.

\begin{tabular}{|l|l|l|l|}
\hline & $\mathrm{D}_{\text {turb }}$ & $\mathrm{H}_{\mathrm{IGV}}$ & $\mathrm{H}_{\text {turb }}$ \\
\hline Fraction of turbine diameter & 0.9 & 0.4 & 0.5 \\
\hline
\end{tabular}

A non-uniform temperature drop with $\mathrm{a}=1.2$ (equation (3.18)) was chosen in an attempt to increase the turbine rotational speed. The rotor relative inlet angle, $\beta_{2}$, is not significantly affected by the temperature drop distribution along the blade and so reducing the tip temperature drop reduces the relative outlet angle, $\beta_{3}$. If the maximum of $\beta_{3}$ is limited, inspection of Figure 3.5 shows that a higher tip speed $U_{\text {tip }}$ is only possible if the amount of flow turning and hence temperature drop is decreased. As is found in the MTFM and then later in the experimental results the pre-swirl introduced by the IGV results in the flow velocity at the blade tip being higher than at the hub. This meant that a higher temperature drop at the tip was not as necessary as was thought. The one advantage is that the blade stress is reduced as the tip load and bending moments are decreased.

Table 3.5 presents the results of the optimisation with the turbine dimensions expressed as a fraction of the chimney diameter as well as the actual sizes. The rather high hub-tip ratio was a concession made in an attempt to have high pressure drops over the turbine.

Table 3.5. Final turbine dimensions and tip speed.

\begin{tabular}{|l|l|l|l|l|l|l|}
\hline & $\mathrm{D}_{\text {chim }}$ & $\mathrm{D}_{\text {turb }}$ & $\mathrm{D}_{\text {hub }}$ & $\mathrm{H}_{\text {IGV }}$ & $\mathrm{H}_{\text {turb }}$ & $\mathrm{U}_{\text {tip }}$ \\
\hline Fraction of chimney diameter & 1 & 0.7991 & $0.4 \mathrm{D}_{\text {turb }}$ & 0.355 & 0.444 & \\
\hline Full scale plant value $[\mathrm{m}]$ & 160 & 127.9 & 51.1 & 56.83 & 71.03 & $88.6{\mathrm{~m} . \mathrm{s}^{-1}}^{-1}$ \\
\hline
\end{tabular}


Table 3.6 lists the constraint variables used in the optimisation.

Table 3.6. Constraint variables

\begin{tabular}{|l|l|l|}
\hline Relative turbine gas exit angle, cases (1) and (2). & $\beta_{\text {3tip }}$ [degrees] & $80^{0}$ \\
\hline Relative turbine gas exit angle, case (3) & $\beta_{\text {3tip }}$ [degrees] & $85^{0}$ \\
\hline Inlet guide vane exit angle & $\alpha_{2}$ [degrees] & $20^{0}$ \\
\hline
\end{tabular}

Figure 3.7 attempts to graphically represent the optimisation results. The effect of the optimisation constraints can quite clearly be seen. The two operating points cases (1) and (2) are indicated on the figure and can be seen to be limited by the relative exit angles of the IGV and rotor.

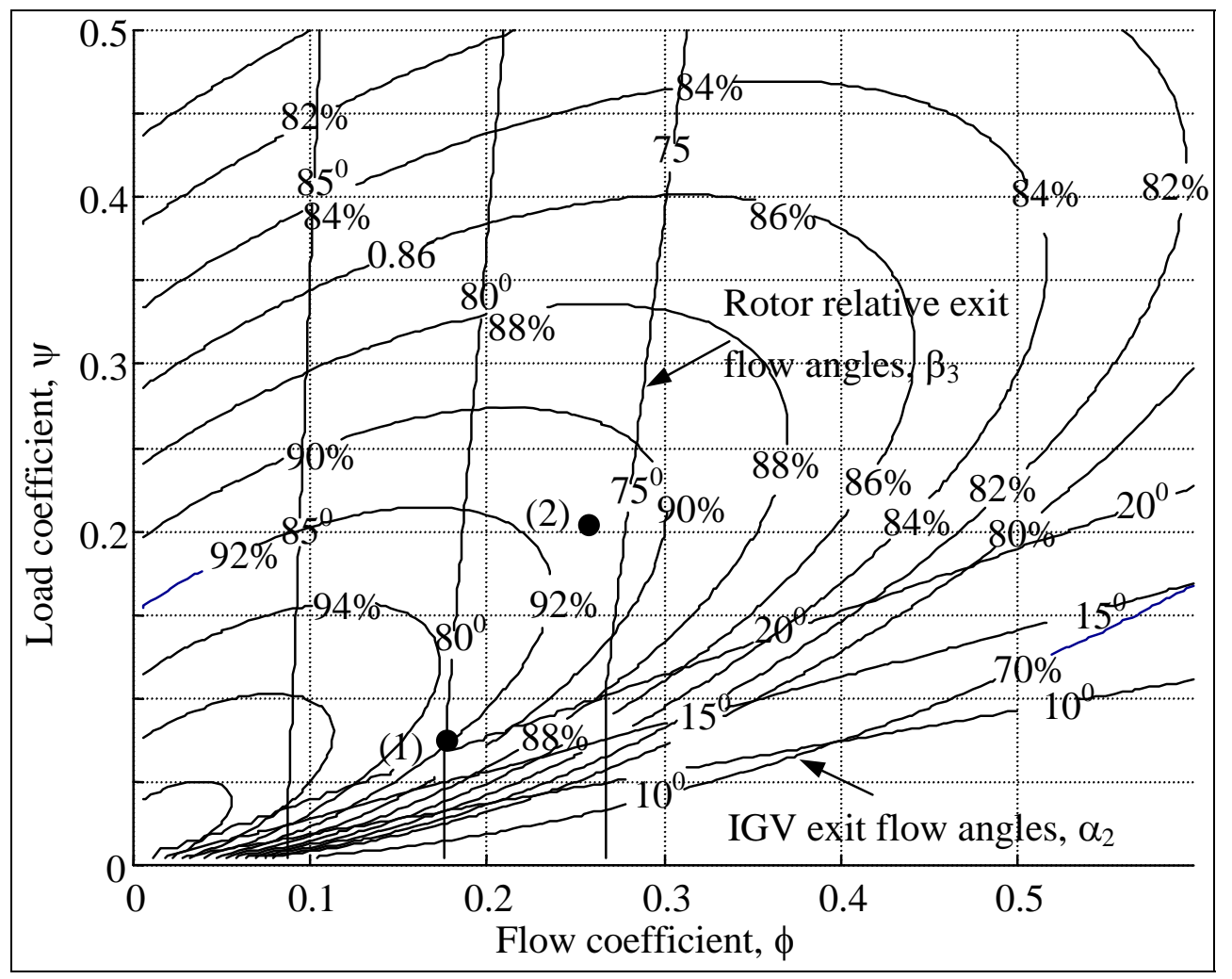

Figure 3.7. Design points and optimisation constraints.

Table 3.7 shows the predicted relative flow angles and the total-to-static efficiency calculated using equation (3.34). In all three cases the maximum amount of inlet swirl was needed, showing that some method of introducing more inlet swirl was required. The limitation on $\beta_{3}$ in case (1) governed the other two cases.

Table 3.7. Tables of operating efficiencies and flow angles for all design modes.

\begin{tabular}{|l|l|l|l|}
\hline \multicolumn{4}{|l|}{ Case (1) High efficiency, low pressure } \\
\hline$\alpha_{2}$ [degrees] & $\beta_{2 \text { tip }}$ [degrees] & $\beta_{3 \text { tip }}$ [degrees] & $\eta_{\text {turb }}[\%]$ \\
\hline $20^{0}$ & $79.67^{0}$ & $80.00^{0}$ & 91.84 \\
\hline
\end{tabular}




\begin{tabular}{|l|l|l|l|}
\hline \multicolumn{4}{|l|}{ Case (2) High efficiency, high pressure } \\
\hline$\alpha_{2}$ [degrees] & $\beta_{2 \text { tip }}$ [degrees] & $\beta_{3 \text { tip }}$ [degrees] & $\eta_{\text {turb }}[\%]$ \\
\hline $20^{0}$ & $74.90^{0}$ & $76.17^{0}$ & 91.24 \\
\hline
\end{tabular}

\begin{tabular}{|l|l|l|l|}
\hline \multicolumn{4}{|l|}{ Case (3) Mass control region } \\
\hline$\alpha_{2}$ [degrees] & $\beta_{2 \text { tip }}$ [degrees] & $\beta_{3 \text { tip }}$ [degrees] & $\eta_{\text {turb }}[\%]$ \\
\hline $20^{0}$ & $83.58^{0}$ & $84.83^{0}$ & 79.60 \\
\hline
\end{tabular}

\subsection{MATRiX ThROUghfLOW METHOD}

The Matrix Throughflow Method (MTFM) is a two-dimensional analysis tool that is useful in the design phase of turbomachinery development. It simulates the machine as an axi-symmetric duct with the blade rows represented by actuator discs or volumes. The free vortex method above assumed that all flow changes occurred within the blade row while in a real machine the velocity profiles change in the space ahead and behind the blades as well. The MTFM takes this into account and its main use in this analysis is to calculate the correct gas inlet and outlet angles to produce the correct amount of work from the turbine. In the next design step, profile shapes are designed based on these gas inflow and outflow angles. Using the MTFM method, the flow in the duct is also analysed to check and see that there are no adverse pressure gradients as the flow turns into the turbine as this could lead to separation at parts of the wall.

Only a basic explanation of the theory involved in the matrix throughflow method will be given as it is well documented and fairly widely used. The particular method used and its implementation will be covered in more detail. The use of the MTFM in simulating the turbine is covered and the methods used in representing the blade rows and aspects such as the non-uniform work distribution mentioned above. The results from the analysis are shown and their use in the next part of the design process, the physical blade design, explained.

\section{Theory of Matrix Throughflow Method}

The term matrix throughflow method is used to describe a class of inviscid flow analysis models used in the simulation of, usually, turbomachines. They differ to the streamline curvature (SCM) and streamline throughflow methods (STFM) where the position of individual streamlines through the machine is calculated. Matrix throughflow methods employ a fixed grid to represent the machine being simulated and calculate the stream function value at each point and find the streamline positions through the machine by interpolation of values from the grid. They are generally more stable than the throughflow methods but use more memory, although on modern PCs this is not usually a problem. Davis and Millar (1975) provide a good overview of both 
methods and compare the performance of the two. The MTFM simplifies the momentum equations written in cylindrical co-ordinates using the following assumptions:

- Inviscid flow, the fluid has zero or negligible viscosity.

- Axi-symmetric flow, all gradients in the tangential direction are zero.

- Flow changes occur in actuator discs: blade rows are represented by actuator discs.

- Loss free flow- for this analysis, no loss models are included.

The above assumptions do not lead to large inaccuracies in the MTFM when it is used for the purposes of design as it is usual to try and design for the lowest possible loss. When simulating an existing machine operating at off-design conditions, it is necessary to improve the analysis method.

The simplification of the momentum equations is slightly different for the various matrix throughflow methods, depending on the intended application. In the case of the solar chimney, the method needs to be able to handle radial inflow from the solar collector and axial outflow from the turbine. For this application, the formulation given by Bosman and Marsh (1974) is suitable as the equations are written in term of meridional velocity so that if either the radial or axial velocity is zero, the equations are still valid. When the meridional velocity is zero (both axial and radial components) then the Bosman and Marsh (1974) formulation also becomes invalid but in the design phase this is not usually a problem as the machine would be modified if any stagnation points were found. The simplified momentum equation can be combined to form the following,

$$
\begin{aligned}
& \mathrm{C}_{\mathrm{m}}^{2}\left[\frac{\partial \mathrm{C}_{\mathrm{r}}}{\partial \mathrm{z}}-\frac{\partial \mathrm{C}_{\mathrm{z}}}{\partial \mathrm{r}}\right]=\left[\mathrm{C}_{\mathrm{r}} \frac{\partial \mathrm{h}_{\mathrm{o}}}{\partial \mathrm{z}}-\mathrm{C}_{\mathrm{z}} \frac{\partial \mathrm{h}_{\mathrm{o}}}{\partial \mathrm{r}}\right]- \\
& \mathrm{T}\left[\mathrm{C}_{\mathrm{r}} \frac{\partial \mathrm{s}}{\partial \mathrm{z}}-\mathrm{C}_{\mathrm{z}} \frac{\partial \mathrm{s}}{\partial \mathrm{r}}\right]-\frac{\mathrm{C}_{\theta}}{\mathrm{r}}\left[\mathrm{C}_{\mathrm{r}} \frac{\partial}{\partial \mathrm{z}}\left(\mathrm{rC}_{\theta}\right)-\mathrm{C}_{\mathrm{z}} \frac{\partial}{\partial \mathrm{r}}\left(\mathrm{rC}_{\theta}\right)\right]
\end{aligned}
$$

The stream function (3.47) that satisfies the continuity equation (3.48) is defined as follows,

$$
\begin{aligned}
& \frac{\partial \psi}{\partial r}=\rho r C_{z} \quad \frac{\partial \psi}{\partial z}=-\rho r C_{r} \\
& \frac{\partial}{\partial z}\left(\rho r C_{r}\right)-\frac{\partial}{\partial z}\left(\rho r C_{z}\right)=0
\end{aligned}
$$

Substituting this into the momentum equation expression (3.46) leads to,

$$
\begin{array}{r}
\frac{\partial^{2} \psi}{\partial \mathrm{r}^{2}}-\frac{\partial^{2} \psi}{\partial \mathrm{z}^{2}}=\mathrm{C}_{\mathrm{z}} \frac{\partial}{\partial \mathrm{r}}(\rho \mathrm{r})-\mathrm{C}_{\mathrm{r}} \frac{\partial}{\partial \mathrm{z}}(\rho \mathrm{r})+(\rho \mathrm{r})^{2} \frac{\mathrm{dh}_{\mathrm{o}}}{\mathrm{d} \psi}- \\
\frac{\rho \mathrm{rT}}{\mathrm{C}_{\mathrm{m}}^{2}}\left[\mathrm{C}_{\mathrm{z}} \frac{\partial \mathrm{s}}{\partial \mathrm{r}}-\mathrm{C}_{\mathrm{r}} \frac{\partial \mathrm{s}}{\partial \mathrm{z}}\right]+\frac{\rho \mathrm{C}_{\theta}}{\mathrm{C}_{\mathrm{m}}^{2}}\left[\mathrm{C}_{\mathrm{z}} \frac{\partial}{\partial \mathrm{r}}\left(\mathrm{rC}_{\theta}\right)-\mathrm{C}_{\mathrm{r}} \frac{\partial}{\partial \mathrm{z}}\left(\mathrm{rC}_{\theta}\right)\right]
\end{array}
$$

Simplifying for incompressible loss free flow, a shorter expression is found,

$$
\frac{\partial^{2} \psi}{\partial \mathrm{r}^{2}}-\frac{\partial^{2} \psi}{\partial \mathrm{z}^{2}}=\rho \mathrm{C}_{\mathrm{z}}+(\rho \mathrm{r})^{2} \frac{\mathrm{dh}_{\mathrm{o}}}{\mathrm{d} \psi}+\frac{\rho \mathrm{C}_{\theta}}{\mathrm{C}_{\mathrm{m}}^{2}}\left[\mathrm{C}_{\mathrm{z}} \frac{\partial}{\partial \mathrm{r}}\left(\mathrm{rC}_{\theta}\right)-\mathrm{C}_{\mathrm{r}} \frac{\partial}{\partial \mathrm{z}}\left(\mathrm{rC}_{\theta}\right)\right]
$$


The above equation is discretised using the method outlined by Greyvenstein (1981) for an orthogonal grid. When simulating the solar chimney turbine, it is necessary to use a quasiorthogonal grid while still using the above scheme. This is achieved by using the interpolation method of Harms (1995, 1996) that allows values on a local orthogonal grid from the quasiorthogonal grid to be calculated using a simple linear interpolation method. In Gannon (1996), this approach is used in an almost identical form in the application of the streamline through flow method (STFM) and detail of the implementation is given.

Once the experimental program was complete, blade blockage and non-uniform flow turning was also implemented in the MTFM. This improved the agreement between the experimental and simulated results. In the initial simulation, it was assumed that the blades turned the flow linearly across their span. Investigation of the blade flow analysis shows that this was not the case and that most of the turning took place over the first half of the blade. This is implemented by inserting the correct value of $\mathrm{rC}_{\theta}$ in the source terms for each grid point. Blade blockage takes into account the physical area that the blades occupy. The blockage term, b, is added to the stream function definition,

$$
\frac{\partial \psi}{\partial \mathrm{r}}=\mathrm{b} \rho \mathrm{rC}_{\mathrm{z}} \quad \frac{\partial \psi}{\partial \mathrm{z}}=-\mathrm{b} \rho \mathrm{rC}_{\mathrm{r}}
$$

Some terms left out of equation (3.49) need to be included in the implementation of the blockage terms. The new formulation is as follows,

$$
\begin{aligned}
& \frac{\partial^{2} \psi}{\partial \mathrm{r}^{2}}-\frac{\partial^{2} \psi}{\partial \mathrm{z}^{2}}=\mathrm{C}_{\mathrm{z}} \frac{\partial}{\partial \mathrm{r}}(\mathrm{b} \rho \mathrm{r})-\mathrm{C}_{\mathrm{r}} \frac{\partial}{\partial \mathrm{z}}(\mathrm{b} \rho \mathrm{r})+(\mathrm{b} \rho \mathrm{r})^{2} \frac{\mathrm{dh}_{\mathrm{o}}}{\mathrm{d} \psi} \\
&+\frac{\rho b \mathrm{C}_{\theta}}{\mathrm{C}_{\mathrm{m}}^{2}}\left[\mathrm{C}_{\mathrm{z}} \frac{\partial}{\partial \mathrm{r}}\left(\mathrm{rC}_{\theta}\right)-\mathrm{C}_{\mathrm{r}} \frac{\partial}{\partial \mathrm{z}}\left(\mathrm{rC}_{\theta}\right)\right]
\end{aligned}
$$

The boundary conditions required are the duct shape, bounding stream function, $\psi$, values and the change in flow conditions over the inlet guide vanes and turbine blades. These are discussed in the following section where the MTFM is implemented in the simulation of the turbine.

\section{Turbine Modelling Using MTFM}

The modelling of the solar chimney turbine begins with the construction of the MTFM grid to which the governing equations are applied. The blade rows are then represented within the grid. The IGVs are assumed to have constant profile sections as they will be used to support the chimney. This means that the exit flow angle will be constant. The required pressure drop governs the flow through the rotor blades.

The implementation of the individual blade rows is not explained in more detail. The differences between the MTFM and free-vortex analysis will also be highlighted. As has been mentioned, the main purpose of the MTFM is to find the relative inlet and outlet flow angles of the rotor blades. It 
is also used to analyse the flow in the duct between the IGV and turbine rotor to try and ensure that a positive pressure gradient is maintained and that separation is avoided.

\section{Grid Generation}

For the set-up of the grid in the solar chimney turbine a grid generator was used that produces grids for axi-symmetric duct flow with blade rows. The machine is divided into regions, the exit to one being the entrance to the next and certain of the regions can be specified as bladed, with either inlet guide vane or turbine blades. A section of one side of the solar chimney turbine with the various regions is shown in Figure 3.8.

The layout shows the experimental set up used and its relation to the full-size chimney. In the experimental rig the diffuser does not expand as much as expected in the full-scale plant. It was decided to perform the simulation using the test rig dimensions to make the comparison between the design and measured results more relevant. The larger diffuser expansion would increase the total-to-static efficiency but would not have a significant effect on the flow through the turbine.

When performing the simulation for the first time, the axial chord length of the blades is not accurately known. Reasonable estimates can be made about their size using some basic turbine design guides (Cohen et al. 1996). Once the blades were designed, the modified dimensions were used in the MTFM simulation and it was found that the changes were small. In Chapter 5 experimental observations lead to other effects being implemented in the MTFM. Furthermore, all results presented in the current chapter are from the original design process using the correct turbine blades dimensions.

Figure 3.9 shows a coarse quasi-orthogonal grid used in the simulation. The eventual grid used where the solution became grid independent had a grid density eight times finer than the one shown. The central section after the turbine has been left going straight in the MTFM analysis while in the real turbine the hub would end quite abruptly. The reason for this is that being an inviscid method the MTFM would not be able to predict this flow region properly as the flow would almost certainly separate. Simulating a straight region allows the flow through the turbine to be predicted. The diffuser efficiency is eventually measured experimentally and this efficiency can be applied to a diffuser that expands more than the experimental one. 


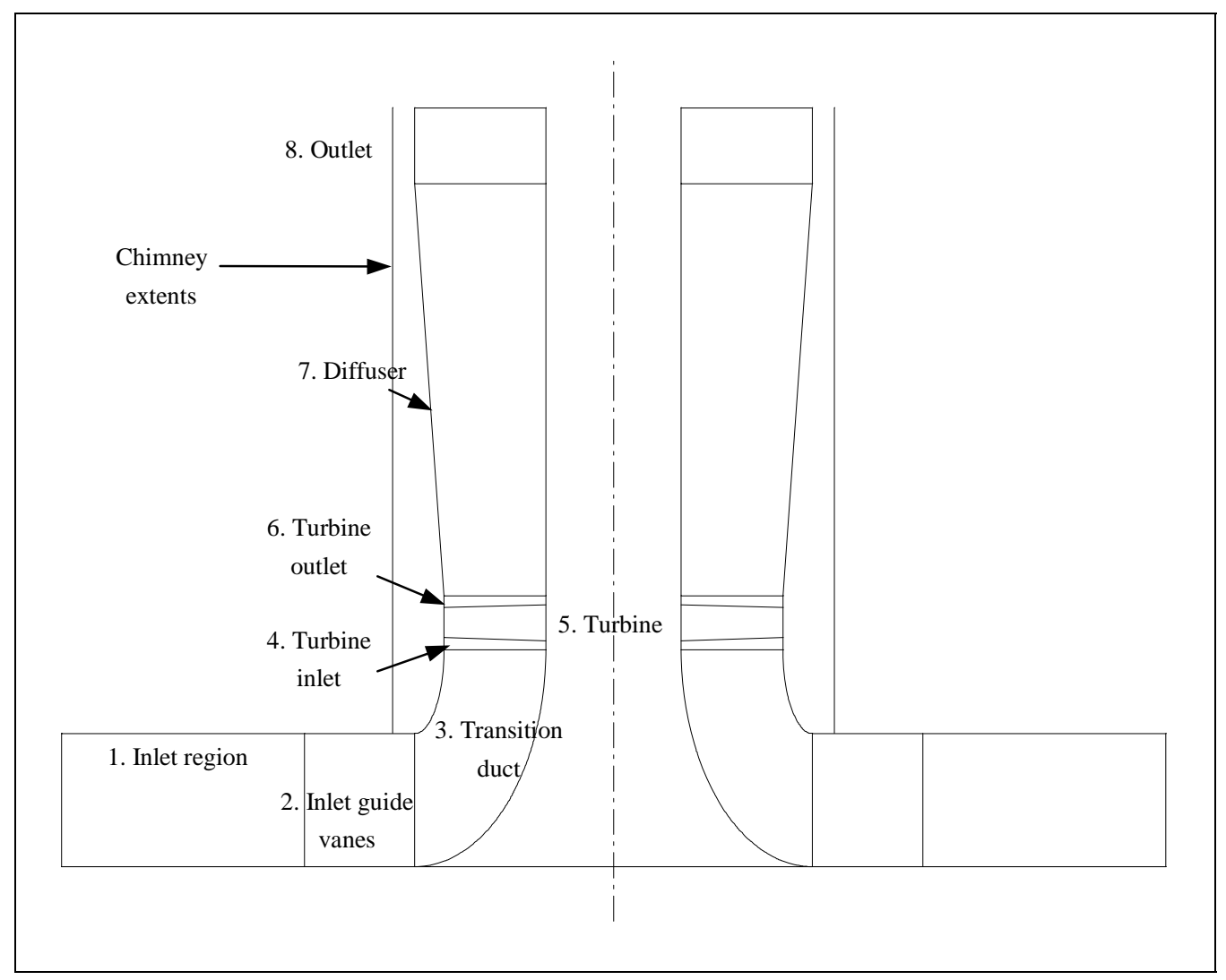

Figure 3.8. Solar chimney turbine layout with regions used in grid generation shown.

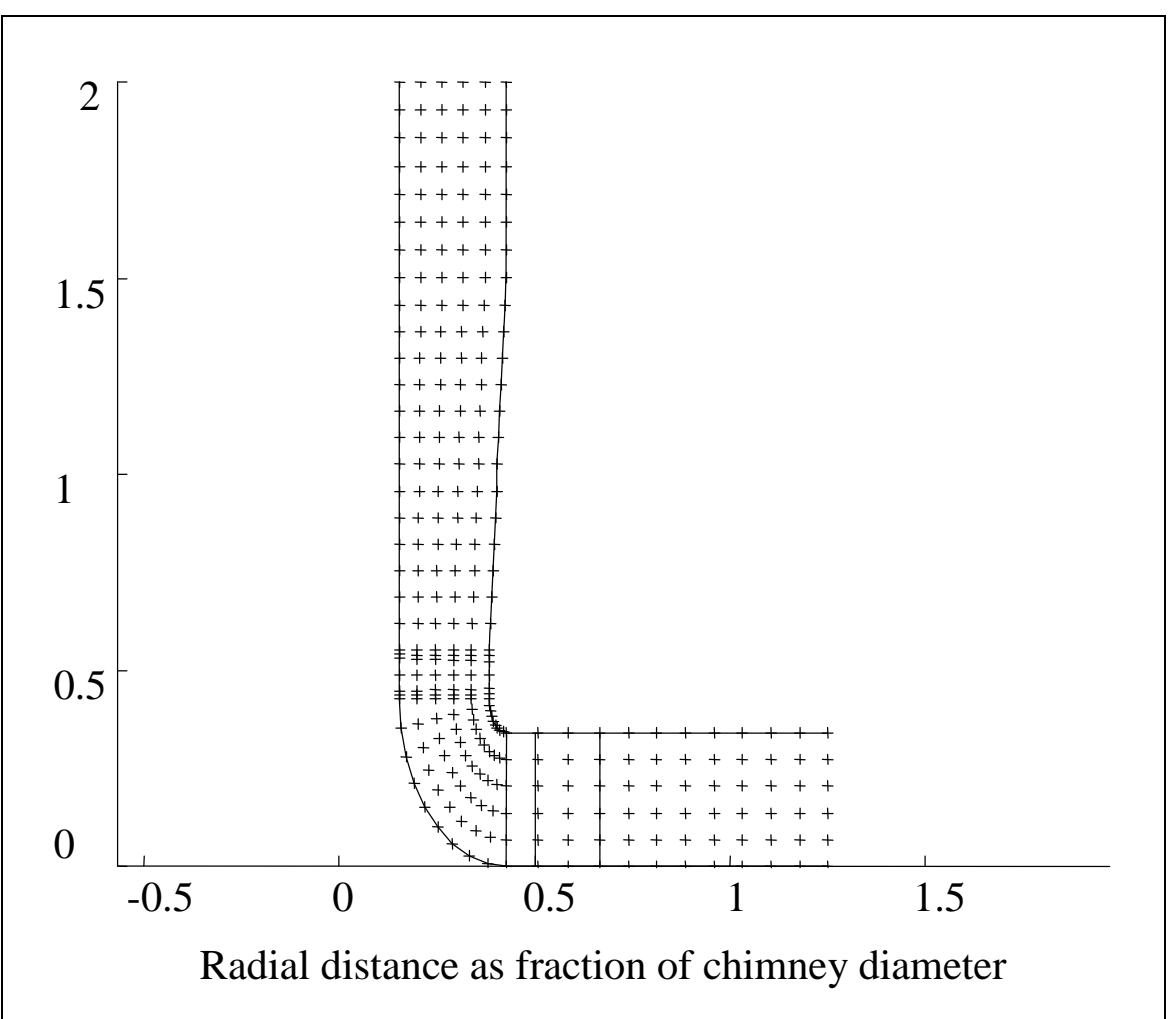

Figure 3.9. Quasi-orthogonal grid used in matrix thoughflow analysis. 


\section{Inlet Guide Vanes}

The inlet guide vanes add pre-swirl before the flow enters the turbine blades but do no work as they are stationary. To model the inlet guide vanes, the swirl component $\mathrm{C}_{\theta 2}$ after the inlet guide vanes must be specified and used in equation (3.50). The enthalpy $h_{0}$ will not change across the inlet guide vanes as no work is done. Initially a free vortex flow distribution through the inlet guide vanes was assumed. The value of the exit angular momentum could be obtained by using equation (3.16). This calculates the amount of exit whirl from the turbine, and recalling for $\Lambda=1$ the machine consists of a rotor only, while for $\Lambda=0$ all the swirl introduced by the inlet guide vanes is removed by the rotor. Modification of this equation leads to the following expression for the angular momentum leaving the IGV,

$$
\left(\mathrm{rC}_{\theta 2}\right)=(1-\Lambda) \frac{\mathrm{C}_{\mathrm{p}} \Delta \mathrm{T}}{\omega}
$$

It was desired that the IGV be of a constant section to make their construction simpler and more effective as supports for the chimney. This means that the exit flow angle from the IGV is the same over the entire blade span. In the free vortex analysis, it was assumed that the value of $\mathrm{rC}_{\theta 2}$ exiting the IGV was constant. This implies a uniform axial velocity over the IGV. Due to the streamlines starting to shift upwards before the duct this is not the case in practice. The MTFM allows a constant exit angle to be simulated and the effect of the velocity profiles into the turbine rotor to be predicted. Figure 3.10 shows what the required exit angles along the IGV span would be to obtain a free vortex distribution. This would require the construction of $57 \mathrm{~m}$ high chimney supports with twist, a challenging task.

Figure 3.11 shows the difference between the assumed free vortex flow distribution and a constant flow angle resulting from a straight IGV. A free vortex distribution would require an IGV with twist (Figure 3.10). The axial velocity profiles do not change as much as the whirl velocity values at the IGV exit. What must be remembered is that as the flow moves in radially, the conservation of angular momentum results in the whirl velocity increasing. An accurate prediction of the flow at the IGV exit is important in predicting the correct flow angles at the rotor inlet. 


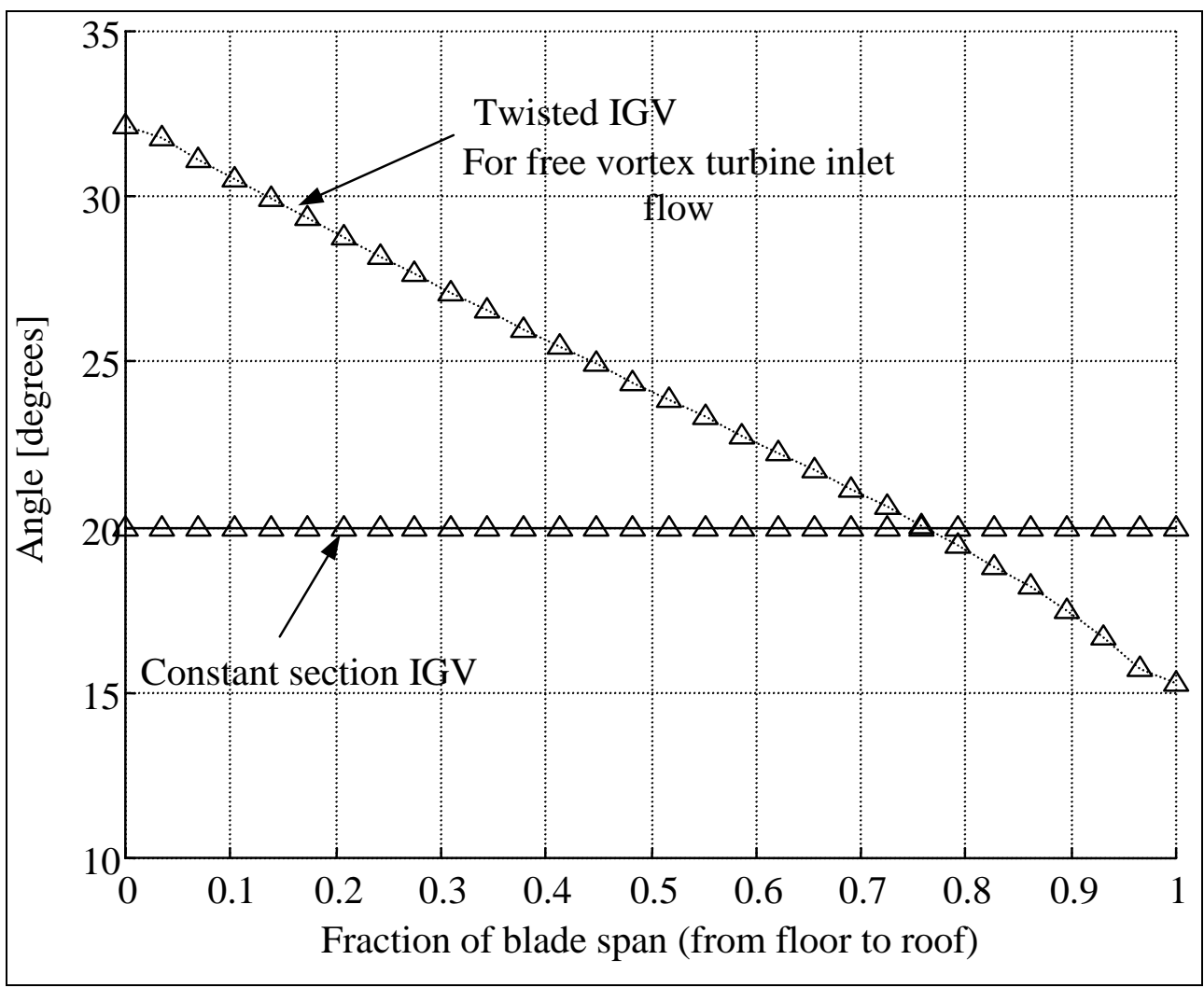

Figure 3.10. Exit flow angles, $\beta_{2}$, for free vortex distribution and constant section IGV.

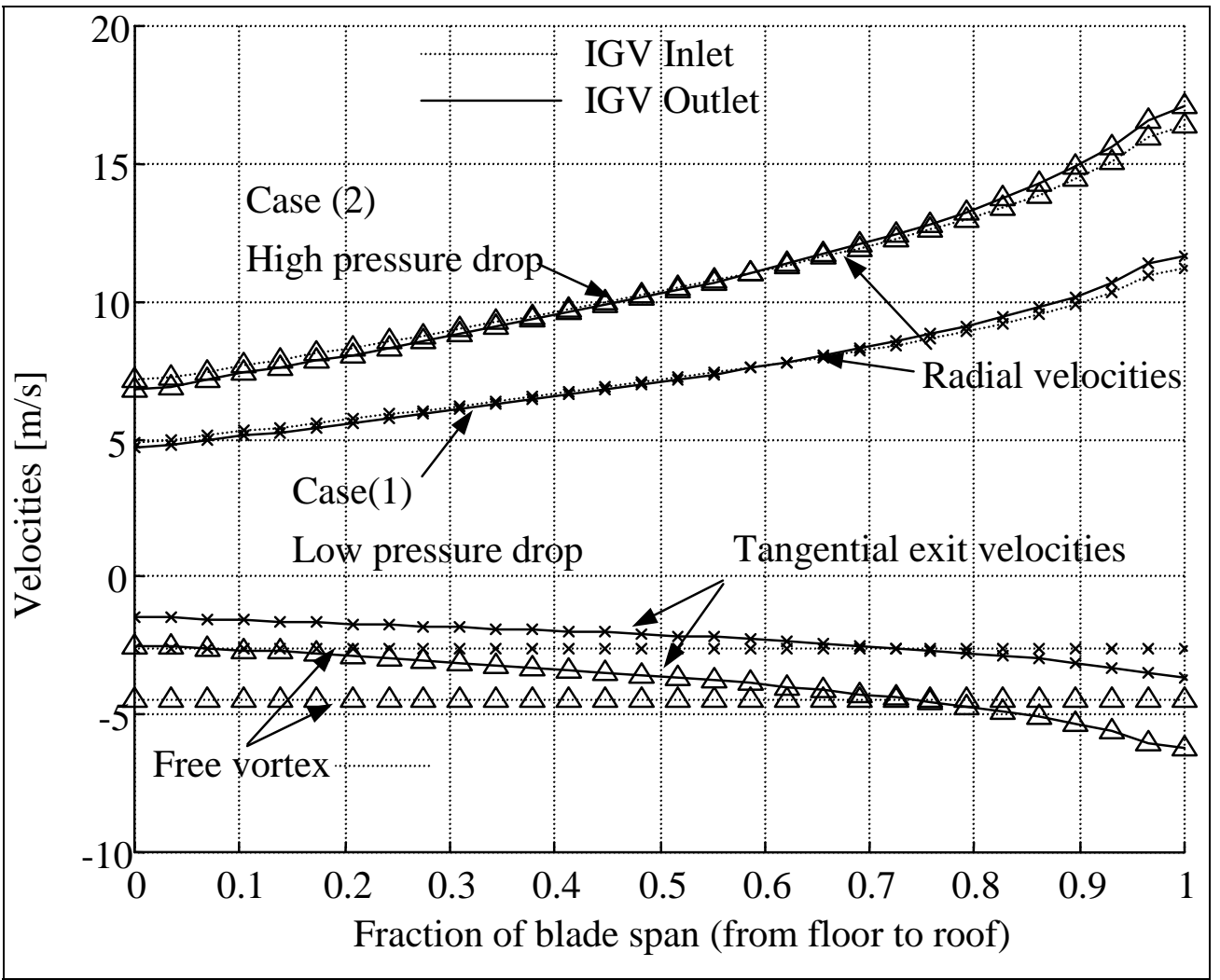

Figure 3.11. Meridional and tangential velocity profiles through inlet guide vanes. 
The practical implementation of the constant exit angle requires that the angular momentum values required in the source terms of equation (3.50) need to be updated for each iteration. This is because the axial velocity changes at each iteration and this in turn means that the swirl component must change to keep the same exit angle.

\section{Turbine Blades}

The turbine blades extract energy from the flow by turning the flow a certain amount, resulting in an enthalpy drop across them. From the flow exiting the inlet guide vanes, the inlet flow angle into the turbine across its blade span is known. The amount of flow deflection and thus the exit flow angle can be calculated from the Euler turbomachinery equation, (3.12) as the turbine temperature drop is known. For non-uniform work distributions equations (3.19) and (3.22) are used to find the temperature drop at each station along the blade and these are used to find the required exit angle for a given inlet angle.

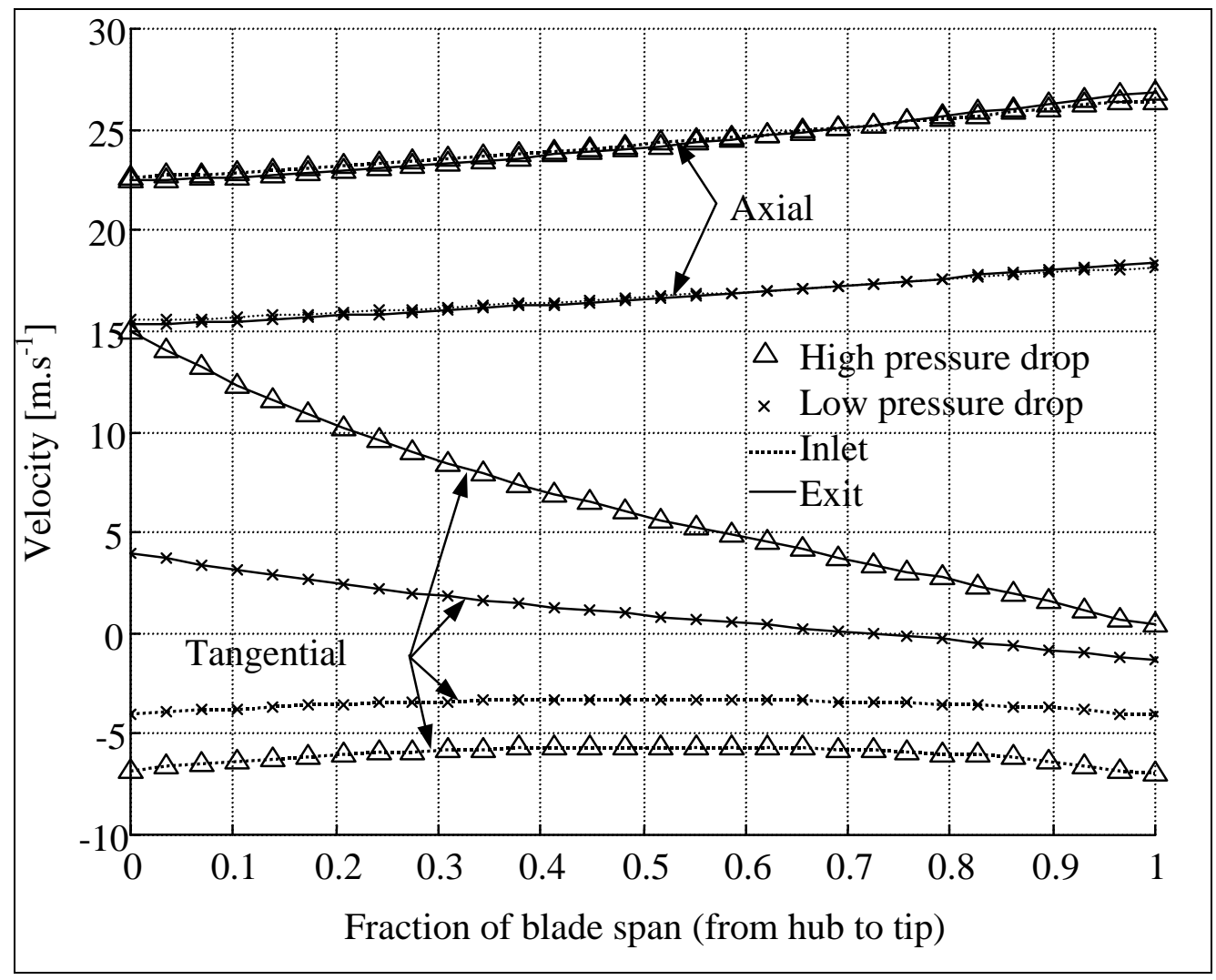

Figure 3.12. Velocity profiles at inlet and exit of turbine for low and high pressure efficient operation.

The above figure shows two of the velocity components, axial and tangential, at the entry and exit to the turbine. Both the low and high pressure cases are plotted on the same figure so that the differences in the flow conditions can be seen. The radial velocity is not shown in the figure as it is very small but is taken into account when designing the blade profiles. The axial velocity is lower at the hub than at the tip. At the hub, the flow decelerates slightly over the blade while accelerating 
at the tip. The tangential velocity has the greatest change across the turbine due to the energy being extracted from the flow. The pre-swirl can be seen to be in a different direction to the exit-swirl due to the inlet guide vanes. Without this the magnitude of exit swirl would be higher thus increasing the exit losses. For the high-pressure case even more pre-swirl could be added to the flow to improve the efficiency. The current IGVs were also designed to give zero swirl if turned radially so that the effect of zero inlet swirl could be investigated. Later in the experimental program, it was realised that there are no benefits in having no inlet whirl. Future IGV designs should be able to produce much higher swirl angles.

An assumption made in the free-vortex analysis of the non-uniform work distribution was that the axial velocity was constant. Figure 3.12 shows that this is not true and means that the work distribution is slightly different to that predicted by the free-vortex analysis. To ensure that the overall turbine temperature drop is correct the total work is calculated by integrating the incremental work along the turbine blade. The integrated value is compared to the required value and adjusted to ensure that the correct amount of work is extracted. The adjustment was in the order of $0.5 \%$.

\section{Predicted Gas Angles}

The main reason for performing the MTFM analysis of the turbine is to predict the required flow angles across the turbine and the IGV passages. It must be emphasised that these are the required gas flow angles- the blade design process to achieve these flow angles has yet to be performed. It is also necessary to perform a number of design iterations as the initial analysis did not take into account limiting factors such as the maximum amount of turning that the inlet guide vanes could perform. This could only be calculated by analysing the blade flow and then using these results as the whirl angle constraint for the IGV. The results from the final design iteration are presented here.

Camber angles for three different cases are shown in Figure 3.13 and once again for the high and low pressure flow conditions. Two turbine layouts simulated using the MTFM are compared to the free vortex distribution, the first being a free vortex distribution. It can be seen that there is a slight difference in the camber angle prediction, of the order of 0.3 degrees. This is due to the various assumptions made in the free vortex analysis. The second case is where the inlet guide vanes are straight and there is a non-uniform work distribution across the blade. It can be seen that the overall camber angle is less. This is due to the higher rotational speed possible when doing less work at the tip and resulting in an overall drop in blade loading.

Figure 3.14 shows the inlet and outlet gas angles for the non-uniform work distribution turbine compared to the free vortex prediction. From this figure and the previous one, it can be seen that the flow angles differ quite significantly from the free-vortex analysis prediction. This is why it is important to perform a MTFM analysis on the turbine to take the effects of the duct and shortcomings of the free-vortex analysis into account. 


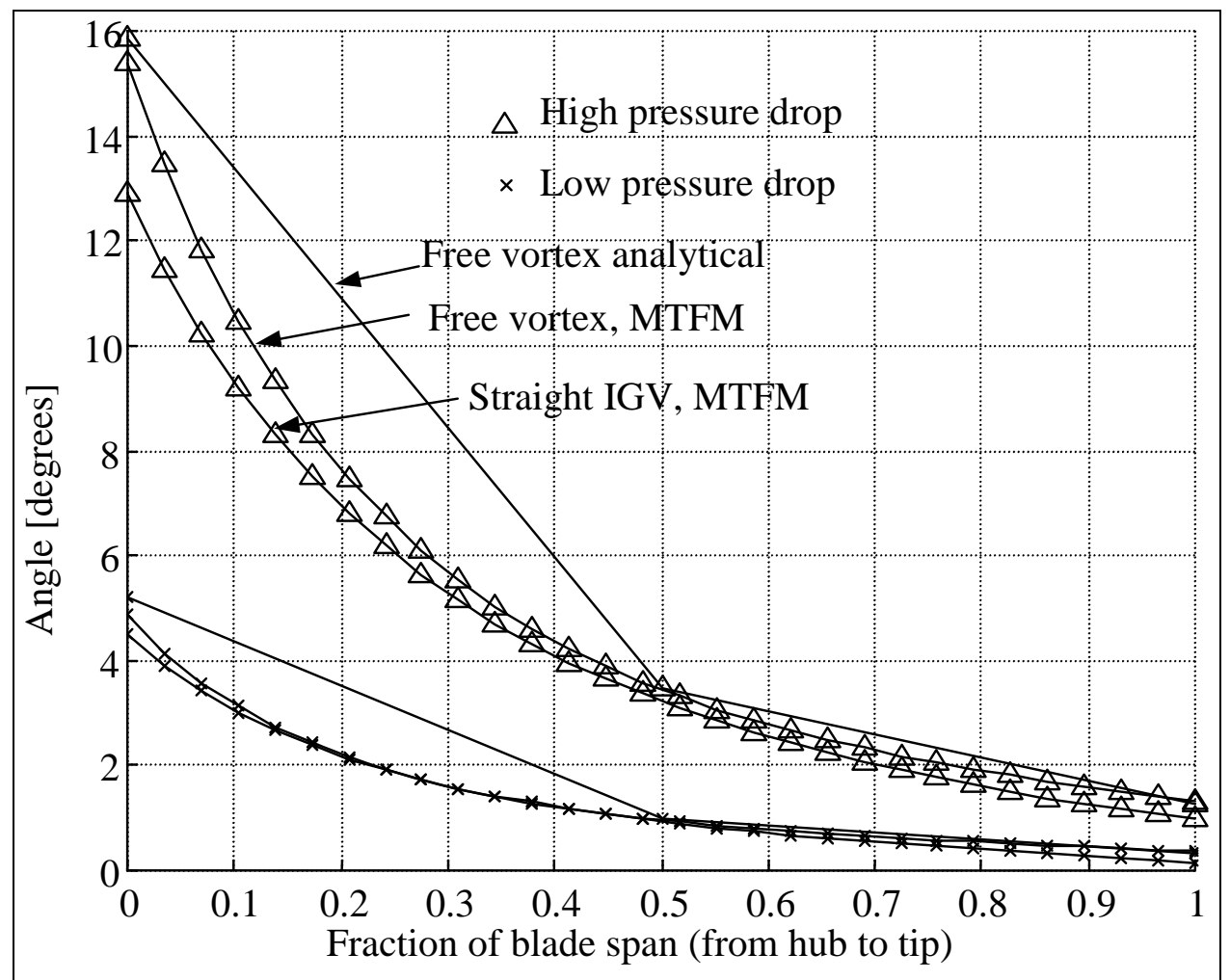

Figure 3.13. Camber angles of turbine blades for various design parameters compared to the free vortex analysis.

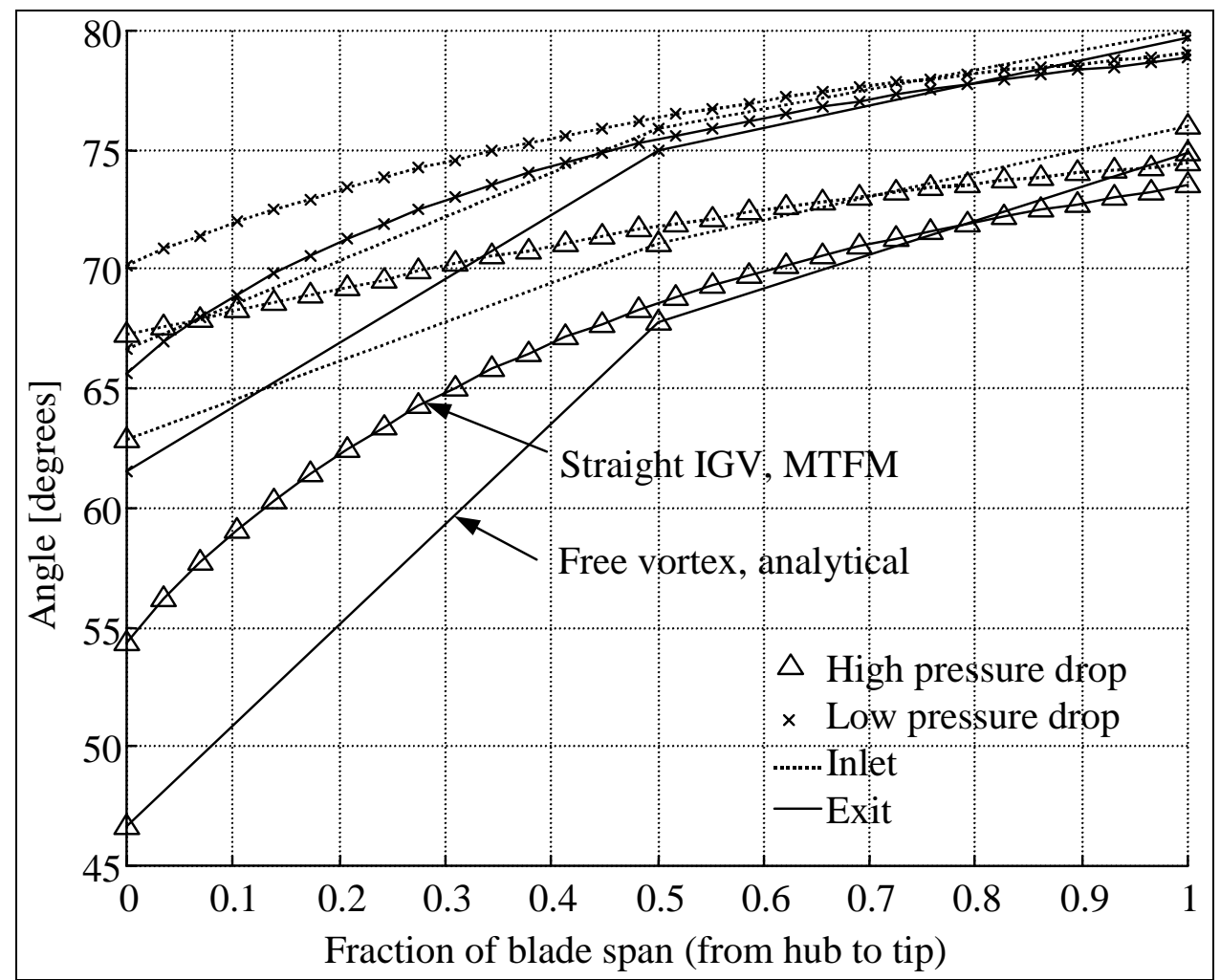

Figure 3.14. Turbine gas inlet and outlet angles for straight inlet guide vanes and nonuniform work being performed over the blade span. 


\section{Duct Flow}

It is important that in the transition duct from radial to axial, the flow does not separate. The place most likely for the flow to separate is on the upper surface. It was thought that it was possible to make the pressure gradient on the top surface favourable along the entire section from the inlet guide vane exit to the turbine blade entry. This was done by ensuring that the contraction from the IGVs to the turbine blades was large, ensuring the flow was always accelerating. For the transition ducting shape, as shown in Figure 3.8, a simple ellipse was used as this is simple to work with and gives a smooth transition between the blade rows.

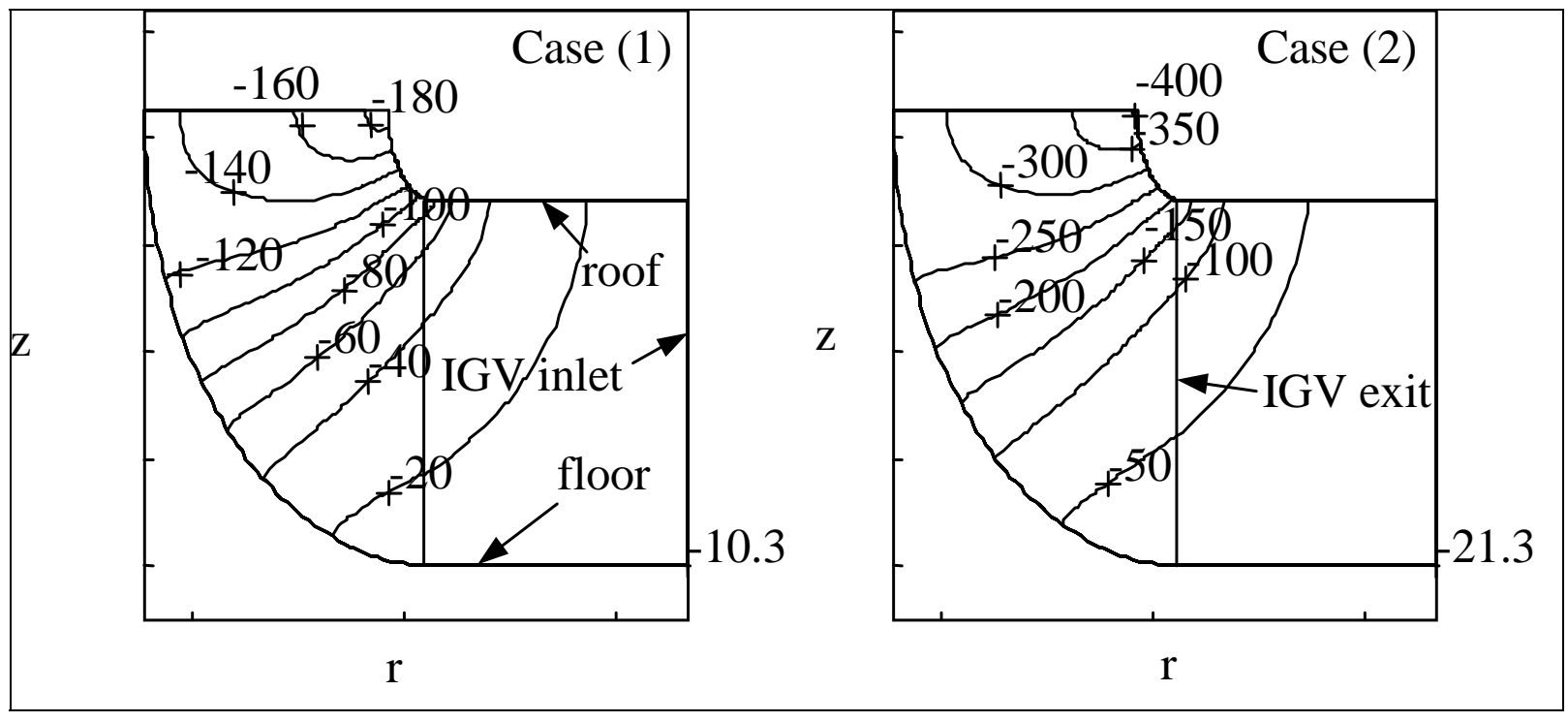

Figure 3.15. Pressure contours [Pa] relative to inlet stagnation pressure for high and low pressure drop case.

The above figures contain two contour plots of the pressure drop through the turbine section for the high and low pressure drops in the high efficiency modes. It can be seen that the pressure decreases steadily through the section for both cases due to the flow accelerating. Figure 3.16 and Figure 3.17 show the coefficient of pressure relative to the chimney velocity for the floor and roof respectively. The negative $C_{p}$ value is used so that an increase in the figure represents an increase in velocity. The flow along the floor can be seen to decelerate slightly before exiting the inlet guide vanes and then accelerate along the central cone and it seems unlikely that separation will occur here. The flow along the roof accelerates along the entire length of the channel from the IGV exit to the turbine inlet. With no adverse pressure gradient predicted this will avoid flow separation in this region. 


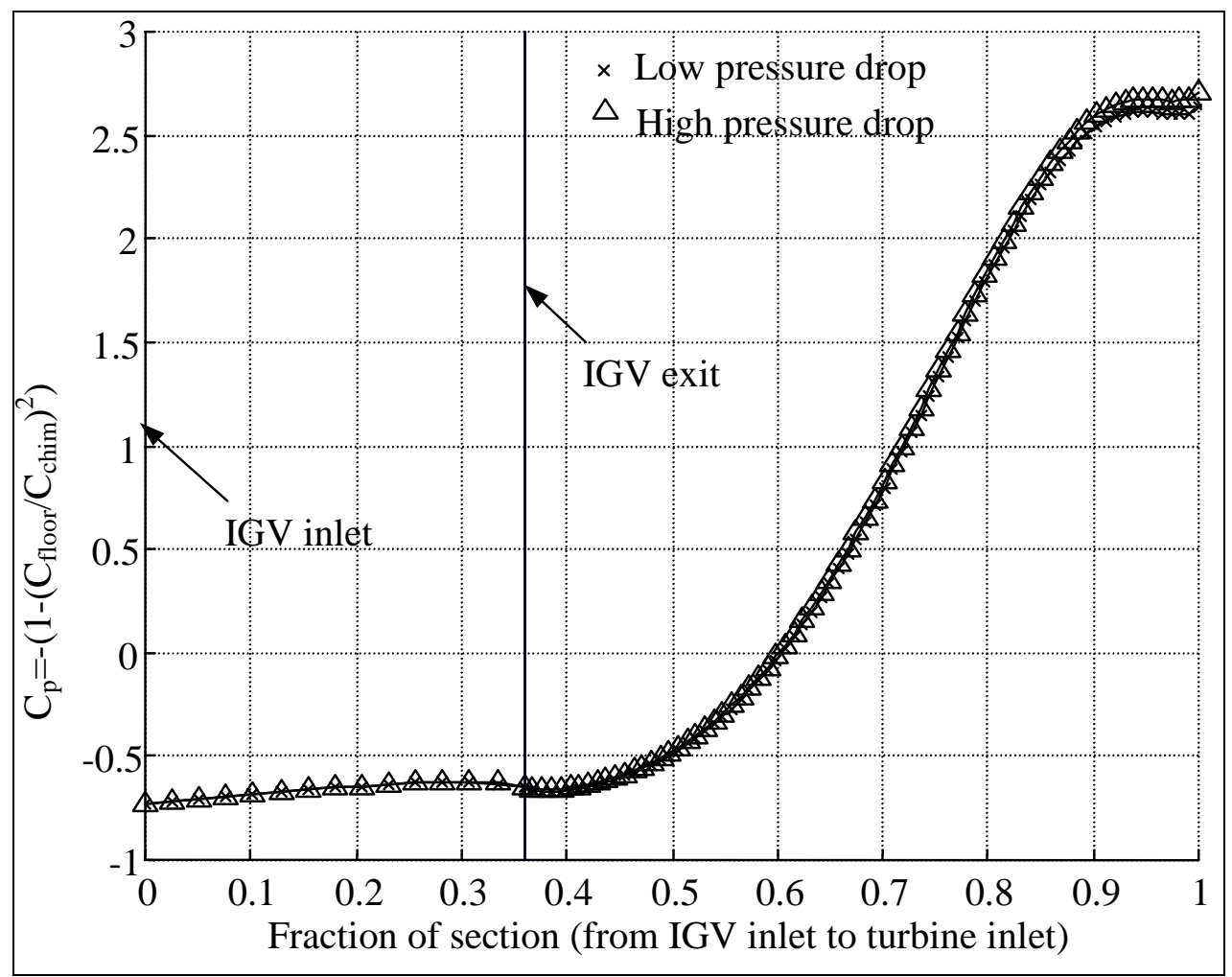

Figure 3.16 Coefficient of pressures on floor of transition section.

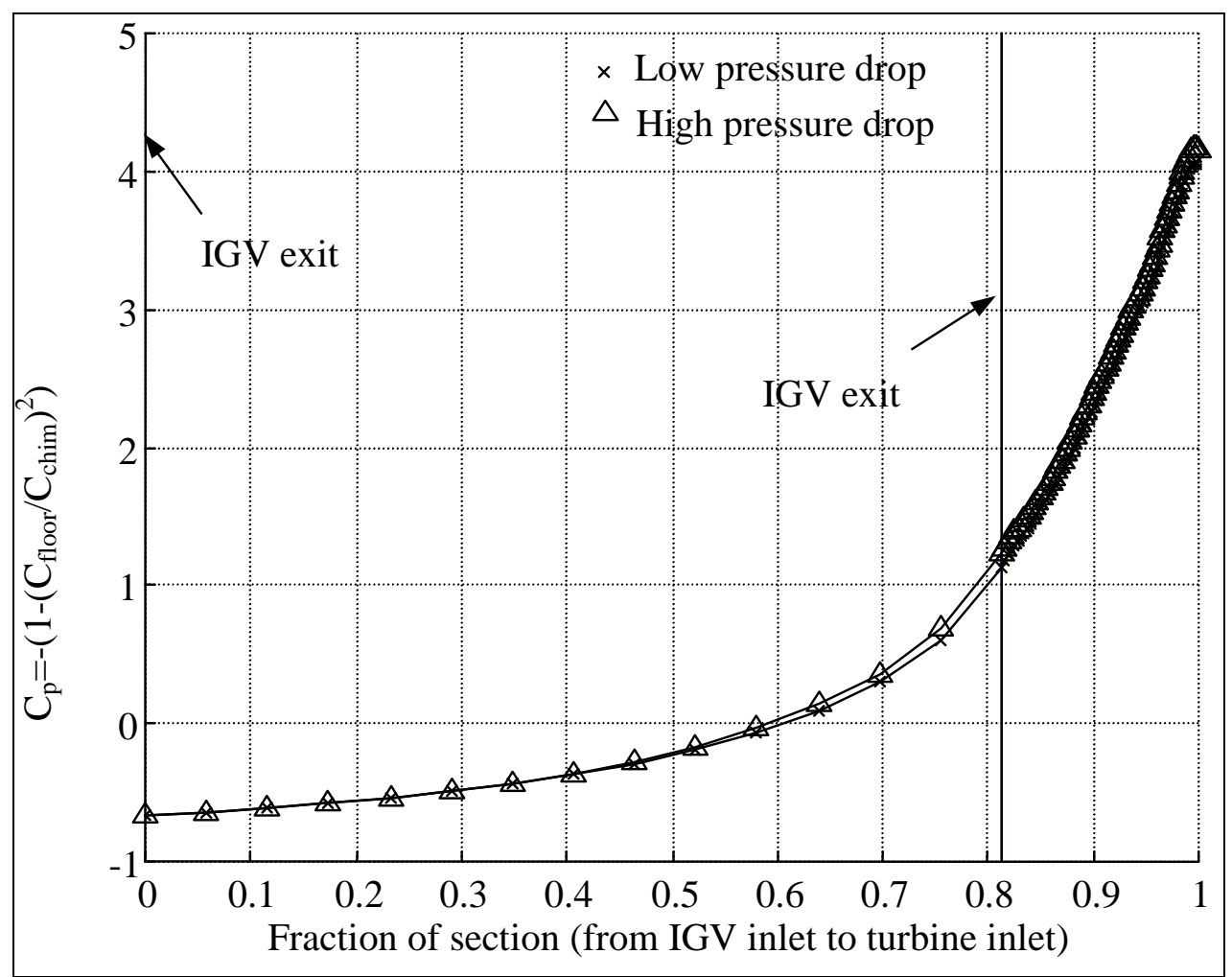

Figure 3.17. Coefficient of pressures on floor of transition section. 


\subsection{BLADE FLOW ANALYSIS}

The purpose of the blades in a turbomachine is to turn the flow through some required angle with minimum losses. In the case of inlet guide vanes this leads to the flow just being deflected while in the turbine blades work is done by the flow. In the matrix throughflow (MTFM) analysis performed in the previous section the flow deflection was modelled through the use of actuator discs, in this section the blade profiles required to turn the flow through the required angles are developed.

The layout of the section begins with the choice of the blade profile shape family followed by the design steps followed in the profile development. This includes the flow analysis of the blade and cascades as well as the method used for separation prediction. A brief outline of the simplex optimisation scheme used is also presented. The following section deals with the application of these methods in the actual blade design with the inlet guide vane design dealt with first, as they are simpler being of constant section and static. The turbine blades are more complex as the section must change along the blade span. In both cases certain concessions had to be made to accommodate the size of the experimental model.

\section{Choice of Blade Profiles}

Early in the design process it was decided to use existing aerofoil profiles for the inlet guide vanes and turbine blades. The main reason for this is that as the solar chimney turbine is a new type, every attempt is made to keep the design variables to a minimum. For existing profiles, experimental data exists for the drag and lift coefficient and they have been demonstrated to work in other applications already. This approach has been taken in wind turbines where existing aeroplane wing profiles were used for early designs before dedicated profiles were developed, Spera (1995) (p 293). The author points out that there is 'no unambiguous procedure for choosing a wind turbine profile but that most profiles are chosen based on past experience.' This is true in many types of turbomachines especially new types. It is often desirable to use existing profiles in new types of machines as their performance is known.

It also seemed sensible to compare the solar chimney turbine with existing wind turbines. The solar chimney turbine operates at higher pressures than a wind turbine but still in the incompressible flow range and at comparatively low throughflow velocities and the sizes of the two are also similar. The 3 main types of profiles used in wind turbines are the NACA 4 digit series, 6 series and custom designed SERI series. The 6 series and SERI profiles have been used more successfully in wind turbine designs although the 4 digit series is still used in many modern designs. The 4 digit series does not have a stable $\mathrm{C}_{\mathrm{L}}$ value near stall which is not desirable for stall controlled blades. Their performance also tends to deteriorate faster when the sections are made thicker than for the other profiles. In the solar chimney turbine the flow is controlled both by adjustable inlet guide 
vanes and adjustable turbine blades allowing the flow to be well matched to the profile design region.

Eventually it was decided to use standard NACA profiles with data obtained from Abbot and Von Doenhoff (1959), where much of the geometric and aerodynamic data for these profiles is presented. The NACA profiles consist of a thickness distribution wrapped around a chord line to give the resulting blade shape. What is very useful about them is that the blade form can be controlled with just a few variables. More details of these will be given later. Initially the NACA 4digit series profiles were used but later it was decided to use modified NACA 4-digit series as these have very high lift/drag ratios. They do not have a large operating region but as mentioned before it should be possible to control the flow direction through the turbine very well. The camber line used throughout was the NACA 4-digit distribution as it is more suitable for cascade applications than the 5-digit camber line. The 5-digit camber line has its position of maximum camber far forward and is useful for individual aerofoils but in a cascade the position of maximum camber often needs to be moved far back.

\section{Blade Profile Design Steps}

The design process starts with an initial layout of the blade where initial values for blade numbers, pitch/chord and aspect ratios are given (Figure 3.18). The next phase of the process is to find the exact blade shape and pitch chord ratios that are able to turn the flow through the required angle. This is done using an optimisation technique where the maximum velocity is minimised while ensuring that the flow does not separate before the end of the blade. Integrated with this optimisation are the constraints applied by the small size of the experimental rig.

The theory of the design and analysis will first be covered starting with the method used to find the basic layout. Following that will be the surface vortex analysis used to predict the velocity on the blade surface and outlet angles of the turbine and inlet guide vane cascades. The Stratford (1959) separation method is used to check whether the flow separates on the blade and the way the method is implemented is presented. Finally the simplex optimisation method used will be briefly covered and following the presentation of the theory the application to the turbine and inlet guide vanes will be presented in the next section.

\section{Initial Layout}

The initial layout of the inlet guide vanes and turbine rotor blades was found using a method outlined by Cohen et al. (1996). This text does apply to gas turbines but it was simpler to extend this method to the solar chimney turbine than wind turbine design methods as aerodynamically a three bladed wind turbine already has too many blades, with the numbers chosen to balance the machine dynamically, Spera (1995) (p 109). By using existing data from previous machines the likelihood of obtaining a suitable design is increased and the time spent optimising is also reduced. 
As a first step the pitch/chord, (t/c), ratio was chosen, based on the relative inlet and exit gas angles from Figure 3.18 taken directly from Cohen et al. (1996) (p 297).

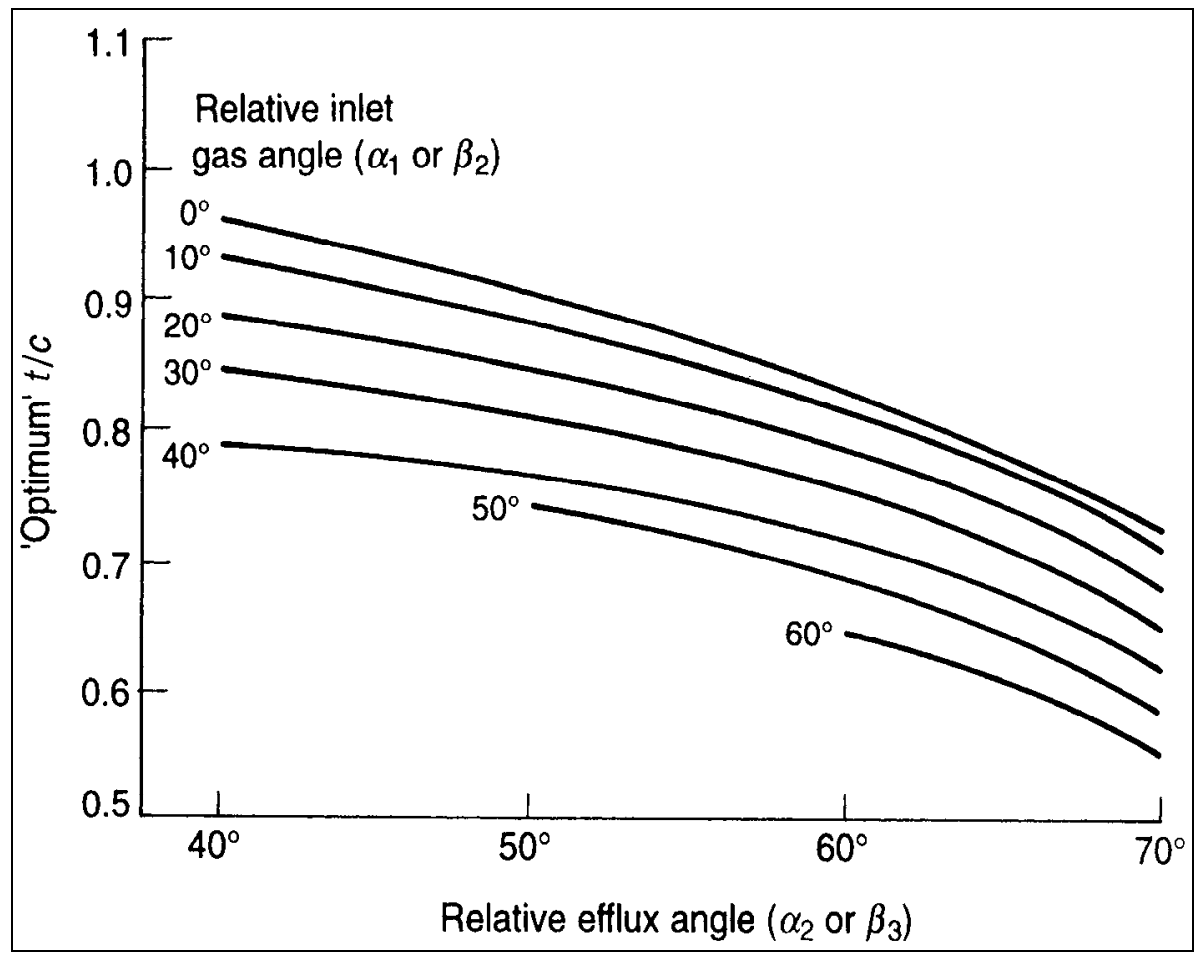

Figure 3.18. Initial design point pitch/chord ratio.

The other initial choice is the blade aspect ratio, H/c, (blade height/blade chord) and here Cohen et al. (1996) recommend values of 1 to 4 with a value of between 3 and 4 recommended as ideal. Long slender blades can lead to strength and vibrational problems while secondary flow losses can become significant in blades with long chords.

\section{Surface Vorticity Modelling}

In the development of solar chimney turbine inlet guide vanes and turbine blades, use is made of surface vorticity modelling to simulate the blade profiles and more importantly the blade cascades. This may seem a rather complex way of choosing the blade forms as in gas turbines there are a number of empirical methods for choosing the stagger and camber angles of cascades. The problem is that there is a lack of data for widely spaced blades where deviation angles (the difference between the exit blade angle and the gas flow angle) can be large. In wind turbines the blades are often treated as individual aerofoils and in the small experimental plant built in Manzanares, Spain this approach was used for the turbine, Wortmann (1981) (p 15). For the large scale plant the pressure drops expected are high enough for this approach not to be accurate and so it was decided to simulate the actual cascades in order to be able to predict the flow accurately.

The surface vorticity method used is well described in Lewis $(1991,1996)$ but a basic outline of the method will be presented here. It is presented here without much of the detailed theory but it can be 
applied from this text. As a first step, a single aerofoil is analysed, then this analysis is extended to a cascade and finally to a radial cascade.

The method presented is sometimes called the Martensen method, and a brief outline of the method is quoted from Lewis (1996),

1. The body surface is covered by a surface vorticity sheet of initially unknown strength $\gamma(\mathrm{s})$.

2. A surface boundary condition $v_{\mathrm{si}}=0$ is imposed on the inner surface of the sheet. This is stated in the form of an integral equation to be derived in the next section.

3. The integral equation is solved for a selection of discrete surface vortex elements at surface locations $\mathrm{s}_{\mathrm{n}}$, resulting in the required $\gamma\left(\mathrm{s}_{\mathrm{n}}\right)$ values.

4. The local surface velocity $v_{\mathrm{sn}}$ follows directly from $\gamma(\mathrm{s})=v_{\mathrm{so}}=v_{\mathrm{s}}$ since $v_{\mathrm{sn}}=\gamma\left(\mathrm{s}_{\mathrm{n}}\right)$.

Here $\gamma\left(s_{n}\right)$ is the surface vorticity sheet strength for a small element ds and $v_{\mathrm{sn}}$ is the surface velocity at that point. Physically an infinitely thin vortex sheet is placed on the surface of the body corresponding to an infinitely thin boundary layer or inviscid flow. The figure below is taken from Lewis, showing the vortex sheet on the body surface and the zero velocity condition on the body surface.

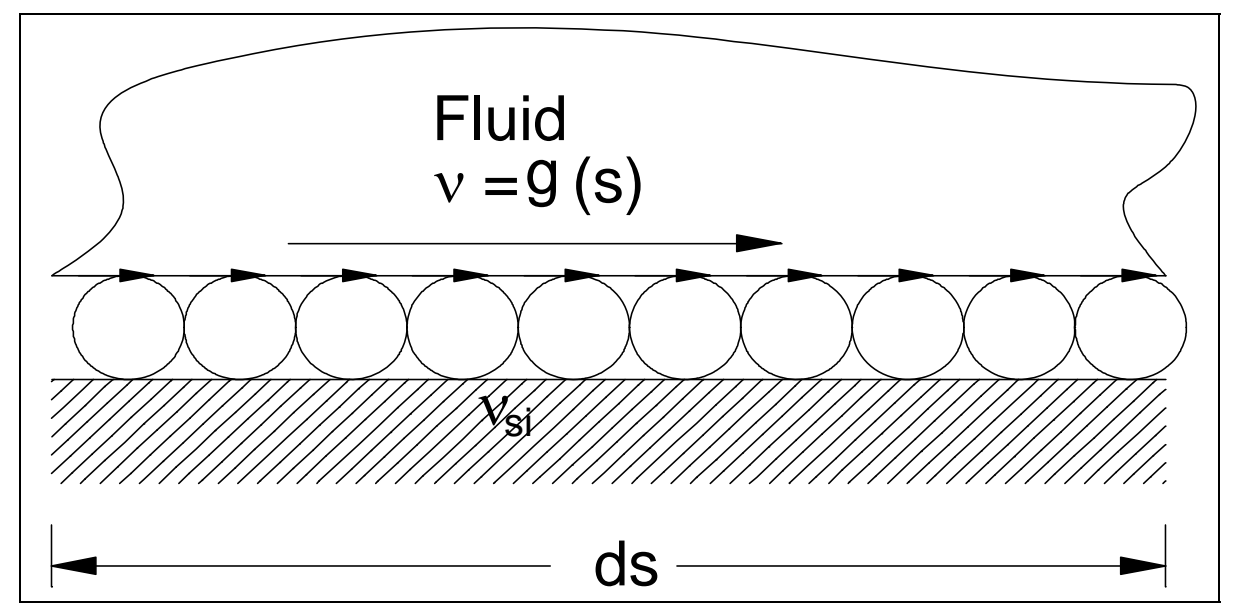

Figure 3.19. Vortex element on surface.

The method can best be understood by looking at the discretised form of the equation. A continuous body immersed in a flow $\mathrm{W}_{\infty}$ is replaced by $\mathrm{M}$ discrete elements. The continuous vortex sheet is replaced by finite length vortex elements of strength $\gamma\left(s_{n}\right) \Delta s_{n}$ where the vortex strengths must satisfy the condition that $v_{\mathrm{si}}=0$ (wall velocity is zero). The equation written in continuous form that satisfies this is,

$$
-\frac{1}{2} \gamma\left(\mathrm{s}_{\mathrm{m}}\right)+\oint \mathrm{k}\left(\mathrm{s}_{\mathrm{m}}, \mathrm{s}_{\mathrm{n}}\right) \gamma\left(\mathrm{s}_{\mathrm{m}}\right) \mathrm{ds} \mathrm{s}_{\mathrm{n}}+\mathrm{W}_{\infty}\left(\cos \alpha_{\infty} \cos \beta_{\mathrm{m}}+\sin \alpha_{\infty} \sin \beta_{\mathrm{m}}\right)=0
$$

with the coupling coefficient $\mathrm{k}\left(\mathrm{s}_{\mathrm{m}}, \mathrm{s}_{\mathrm{n}}\right)$ linking points $\mathrm{s}_{\mathrm{m}}$ and $\mathrm{s}_{\mathrm{n}}$ given by, 


$$
\mathrm{k}\left(\mathrm{s}_{\mathrm{m}}, \mathrm{s}_{\mathrm{n}}\right)=\frac{1}{2 \pi}\left\{\frac{\left(\mathrm{y}_{\mathrm{m}}-\mathrm{y}_{\mathrm{n}}\right) \cos \beta_{\mathrm{m}}-\left(\mathrm{x}_{\mathrm{m}}-\mathrm{x}_{\mathrm{n}}\right) \sin \beta_{\mathrm{m}}}{\left(\mathrm{x}_{\mathrm{m}}-\mathrm{x}_{\mathrm{n}}\right)^{2}+\left(\mathrm{y}_{\mathrm{m}}-\mathrm{y}_{\mathrm{n}}\right)^{2}}\right\}
$$

\section{Application to a Single Profile}

The following figure shows the discretisation of a continuous body with a freestream velocity of $\mathrm{W}_{\infty}$ showing the influence of element $\mathrm{m}$ on $\mathrm{n}$.

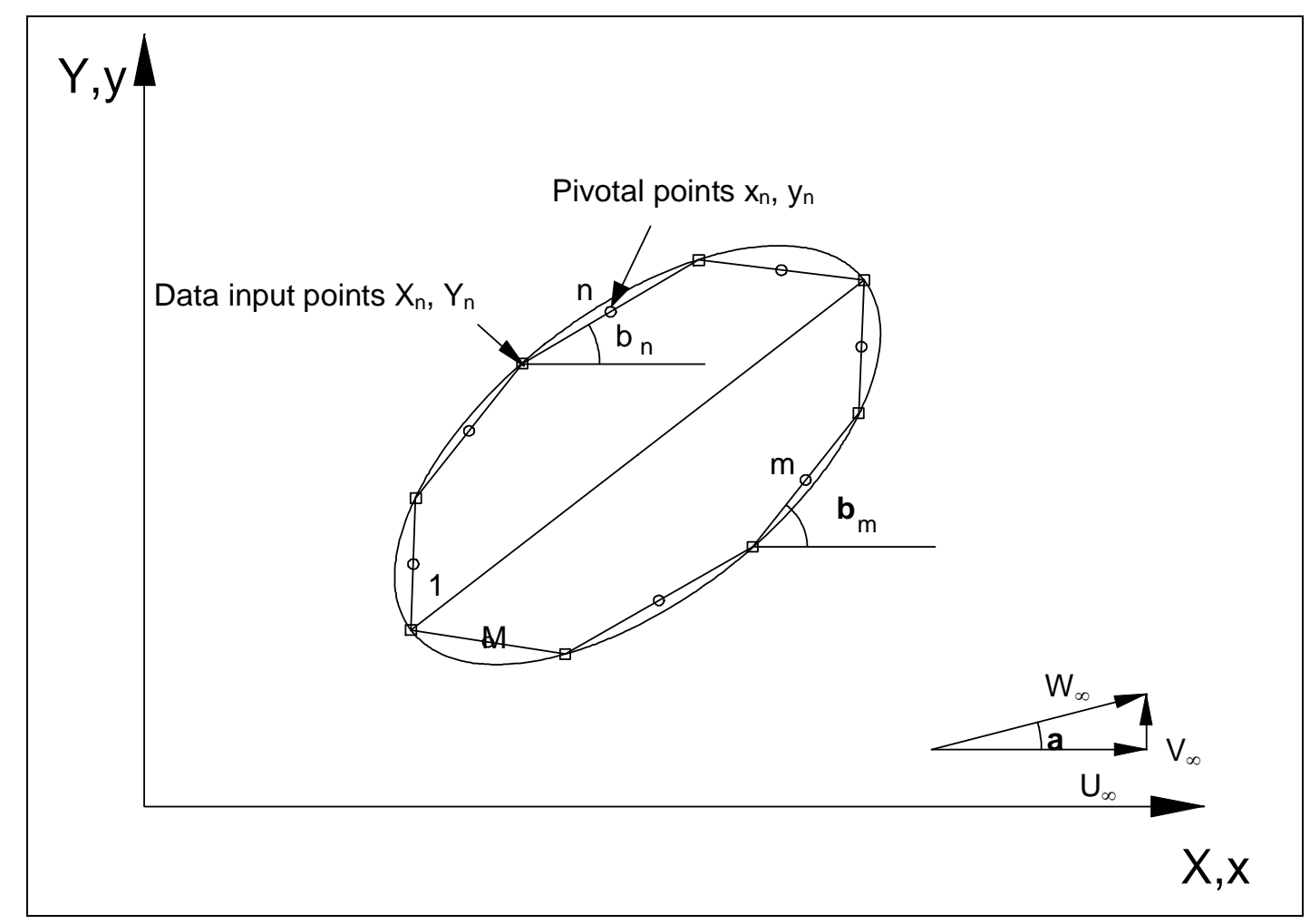

Figure 3.20. Discretised body in flow $W_{\infty}$.

Equation (3.54) transforms into the linear equation,

$$
\sum_{\mathrm{n}=1}^{\mathrm{M}} \mathrm{K}\left(\mathrm{s}_{\mathrm{m}}, \mathrm{s}_{\mathrm{n}}\right) \gamma\left(\mathrm{s}_{\mathrm{n}}\right)=-\mathrm{U}_{\infty} \cos \beta_{\mathrm{m}}--\mathrm{V}_{\infty} \sin \beta_{\mathrm{m}}
$$

and the coupling coefficient,

$$
\mathrm{K}\left(\mathrm{s}_{\mathrm{m}}, \mathrm{s}_{\mathrm{n}}\right)=\mathrm{k}\left(\mathrm{s}_{\mathrm{m}}, \mathrm{s}_{\mathrm{n}}\right) \Delta \mathrm{s}_{\mathrm{n}}=\frac{\Delta \mathrm{s}_{\mathrm{n}}}{2 \pi}\left\{\frac{\left(\mathrm{y}_{\mathrm{m}}-\mathrm{y}_{\mathrm{n}}\right) \cos \beta_{\mathrm{m}}-\left(\mathrm{x}_{\mathrm{m}}-\mathrm{x}_{\mathrm{n}}\right) \sin \beta_{\mathrm{m}}}{\left(\mathrm{x}_{\mathrm{m}}-\mathrm{x}_{\mathrm{n}}\right)^{2}+\left(\mathrm{y}_{\mathrm{m}}-\mathrm{y}_{\mathrm{n}}\right)^{2}}\right\}
$$

leading to $M$ linear equation for $M$ unknown $\gamma\left(s_{n}\right)$ values. The self coupling term $K\left(s_{m}, s_{m}\right)$ using L'Hopital's rule resulting in,

$$
\mathrm{K}\left(\mathrm{s}_{\mathrm{m}}, \mathrm{s}_{\mathrm{m}}\right)=\mathrm{k}\left(\mathrm{s}_{\mathrm{m}}, \mathrm{s}_{\mathrm{m}}\right) \Delta \mathrm{s}_{\mathrm{m}} \approx-\frac{\Delta \beta_{\mathrm{m}}}{4 \pi}
$$


These are then formulated in the usual way, where $\mathrm{K}_{\mathrm{mn}} \equiv \mathrm{K}\left(\mathrm{s}_{\mathrm{m}}, \mathrm{s}_{\mathrm{n}}\right)$

$$
\left(\begin{array}{ccccc}
\mathrm{K}_{11} & \mathrm{~K}_{12} & \mathrm{~K}_{13} & \cdots & \mathrm{K}_{1 \mathrm{M}} \\
\mathrm{K}_{21} & \mathrm{~K}_{22} & \mathrm{~K}_{23} & \cdots & \mathrm{K}_{2 \mathrm{M}} \\
\mathrm{K}_{31} & \mathrm{~K}_{32} & \mathrm{~K}_{33} & \cdots & \mathrm{K}_{3 \mathrm{M}} \\
\vdots & \vdots & \vdots & \ddots & \vdots \\
\mathrm{K}_{\mathrm{M} 1} & \mathrm{~K}_{\mathrm{M} 2} & \mathrm{~K}_{\mathrm{M} 1} & \cdots & \mathrm{K}_{\mathrm{MM}} \\
& \text { ( Coupling coefficient matrix) }
\end{array}\right)\left(\begin{array}{c}
\gamma\left(\mathrm{s}_{1}\right) \\
\gamma\left(\mathrm{s}_{2}\right) \\
\gamma\left(\mathrm{s}_{3}\right) \\
\vdots \\
\gamma\left(\mathrm{s}_{\mathrm{M}}\right) \\
\left(\begin{array}{l}
\text { Vorticity } \\
\text { Vector }
\end{array}\right)
\end{array}\right)=\left(\begin{array}{c}
\text { rhs }_{1} \\
\text { rhs }_{2} \\
\text { rhs }_{3} \\
\vdots \\
\text { rhs } \\
\left(\begin{array}{c}
\text { rhs } \\
\text { vector }
\end{array}\right)
\end{array}\right)
$$

The terms in equations (3.56) to (3.59), some of which are obtained by inspection of Figure 3.20, are,

$$
\begin{aligned}
\text { rhs }_{\mathrm{n}} & =-\mathrm{U}_{\infty} \cos \beta_{\mathrm{n}}-\mathrm{V}_{\infty} \sin \beta_{\mathrm{n}} \\
\Delta \mathrm{s}_{\mathrm{n}} & =\sqrt{\left(\mathrm{X}_{\mathrm{n}+1}-\mathrm{X}_{\mathrm{n}}\right)^{2}+\left(\mathrm{Y}_{\mathrm{n}+1}-\mathrm{Y}_{\mathrm{n}}\right)^{2}} \\
\mathrm{X}_{\mathrm{n}} & =\frac{1}{2}\left(\mathrm{X}_{\mathrm{n}}+\mathrm{X}_{\mathrm{n}+1}\right), \mathrm{y}_{\mathrm{n}}=\frac{1}{2}\left(\mathrm{Y}_{\mathrm{n}}+\mathrm{Y}_{\mathrm{n}+1}\right), \\
\beta_{\mathrm{n}} & =\arctan \left(\frac{\mathrm{y}_{\mathrm{n}+1}-\mathrm{y}_{\mathrm{n}}}{\mathrm{X}_{\mathrm{n}+1}-\mathrm{X}_{\mathrm{n}}}\right) \\
\mathrm{K}\left(\mathrm{s}_{\mathrm{m}}, \mathrm{s}_{\mathrm{m}}\right) & \approx-\frac{1}{2}-\frac{\beta_{\mathrm{n}+1}-\beta_{\mathrm{n}+1}}{8 \pi}
\end{aligned}
$$

Due to numerical inaccuracies, the bound circulation of the body is not zero and a correction of the back diagonal is required ensuring that the bound circulation is zero.

$$
\mathrm{K}_{\mathrm{a}, \mathrm{b}}\left(\begin{array}{c}
\mathrm{a}=1 . . \mathrm{M} \\
\mathrm{b}=\mathrm{M} . .1
\end{array}\right)=\frac{1}{\Delta \mathrm{s}_{\mathrm{a}}}\left\{\sum_{\mathrm{c}=1}^{\mathrm{M}} \mathrm{K}_{\mathrm{c}, \mathrm{b}} \Delta \mathrm{s}_{\mathrm{c}}: \text { where } \mathrm{c} \neq \mathrm{a}\right\}
$$

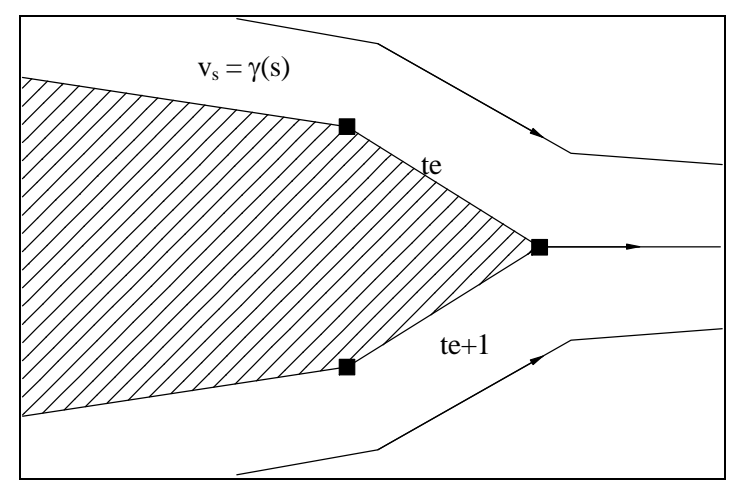

Figure 3.21. Trailing edge flow for the Kutta-Joukowski condition

For a body to create lift, it must have a bound vorticity or circulation. While it is difficult to specify this directly, it can be implied by the Kutta-Joukowski condition. This states that the velocities at the trailing edge must be equal or in the case of the vorticities of equal strength but opposite sign as illustrated in Figure 3.21. 
To solve for the flow around a lifting aerofoil, Lewis $(1991,1996)$ proposes a method where three sets of unit equations are solved. The equations are for unit velocities $U_{\infty}=1, V_{\infty}=1$ and unit circulation $\Gamma=1$ resulting in the following sets of equations that can be solved simultaneously, (3.59)

$$
\begin{array}{ll}
\sum_{n=1}^{M} K\left(s_{m}, s_{n}\right) \gamma_{u}\left(s_{n}\right)=-\cos \beta_{m} & \text { for } U_{\infty}=1, V_{\infty}=0 \\
\sum_{n=1}^{M} K\left(s_{m}, s_{n}\right) \gamma_{v}\left(s_{n}\right)=-\sin \beta_{m} & \text { for } U_{\infty}=0, V_{\infty}=1 \\
\sum_{n=1}^{M} K\left(s_{m}, s_{n}\right) \gamma_{\Gamma}\left(s_{n}\right)=1 & \text { for } \Gamma=1
\end{array}
$$

Through a simple substitution of a dummy equation, the Kutta-Joukowski condition can be implemented. The te ${ }^{\text {th }}$ (trailing edge) equation is replaced with,

$$
\begin{aligned}
& \gamma_{\mathrm{u}}\left(\mathrm{s}_{\mathrm{te}}\right)+\gamma_{\mathrm{u}}\left(\mathrm{s}_{\mathrm{te}+1}\right)=0 \\
& \gamma_{\mathrm{v}}\left(\mathrm{s}_{\mathrm{te}}\right)+\gamma_{\mathrm{v}}\left(\mathrm{s}_{\mathrm{te}+1}\right)=0 \\
& \gamma_{\Gamma}\left(\mathrm{s}_{\mathrm{te}}\right)+\gamma_{\Gamma}\left(\mathrm{s}_{\mathrm{te}+1}\right)=0
\end{aligned}
$$

The final step of the analysis is to calculate the surface velocities and circulation strength. The surface velocities are simply obtained by multiplying the unit solution with the actual inlet velocity magnitude in each direction.

$$
\mathrm{v}_{\mathrm{s}}=\mathrm{U}_{\infty} \gamma_{\mathrm{u}}+\mathrm{V}_{\infty} \gamma_{\mathrm{v}}
$$

From this the total circulation strength is calculated as,

$$
\Gamma=\sum_{\mathrm{n}=1}^{\mathrm{M}} \mathrm{v}_{\mathrm{sn}} \Delta \mathrm{s}_{\mathrm{n}}
$$

A modified NACA 4 digit profile is simulated using the above method and compared to results from Abbot and Von Doenhoff (1959). The continuous line is the results from the surface vortex analysis while the $\mathrm{x}$ marks are obtained from tabulated data. The agreement is very close as can also be seen in the table that follows. The grid used had 100 points around the perimeter of the blade, which is the grid spacing used for the optimisation of the blade profiles. A finer grid does improve the results but is not suitable when many profiles have to be simulated for design.

Table 3.8. Numerical comparison of surface vortex analysis with Abbott and Von Doenhoff for tabulated data.

\begin{tabular}{|l|c|c|c|c|c|c|c|c|c|}
\hline $\mathrm{x} / \mathrm{l}$ & 0.1 & 0.2 & 0.3 & 0.4 & 0.5 & 0.6 & 0.7 & 0.8 & 0.9 \\
\hline Abott $\left(\mathrm{v}_{\mathrm{s}} / \mathrm{W}_{\infty}\right)^{2}$ & 1.141 & 1.194 & 1.214 & 1.229 & 1.235 & 1.240 & 1.227 & 1.176 & 1.046 \\
\hline Surf vort $\left(\mathrm{v}_{\mathrm{s}} / \mathrm{W}_{\infty}\right)^{2}$ & 1.141 & 1.190 & 1.215 & 1.229 & 1.236 & 1.242 & 1.229 & 1.176 & 1.050 \\
\hline
\end{tabular}




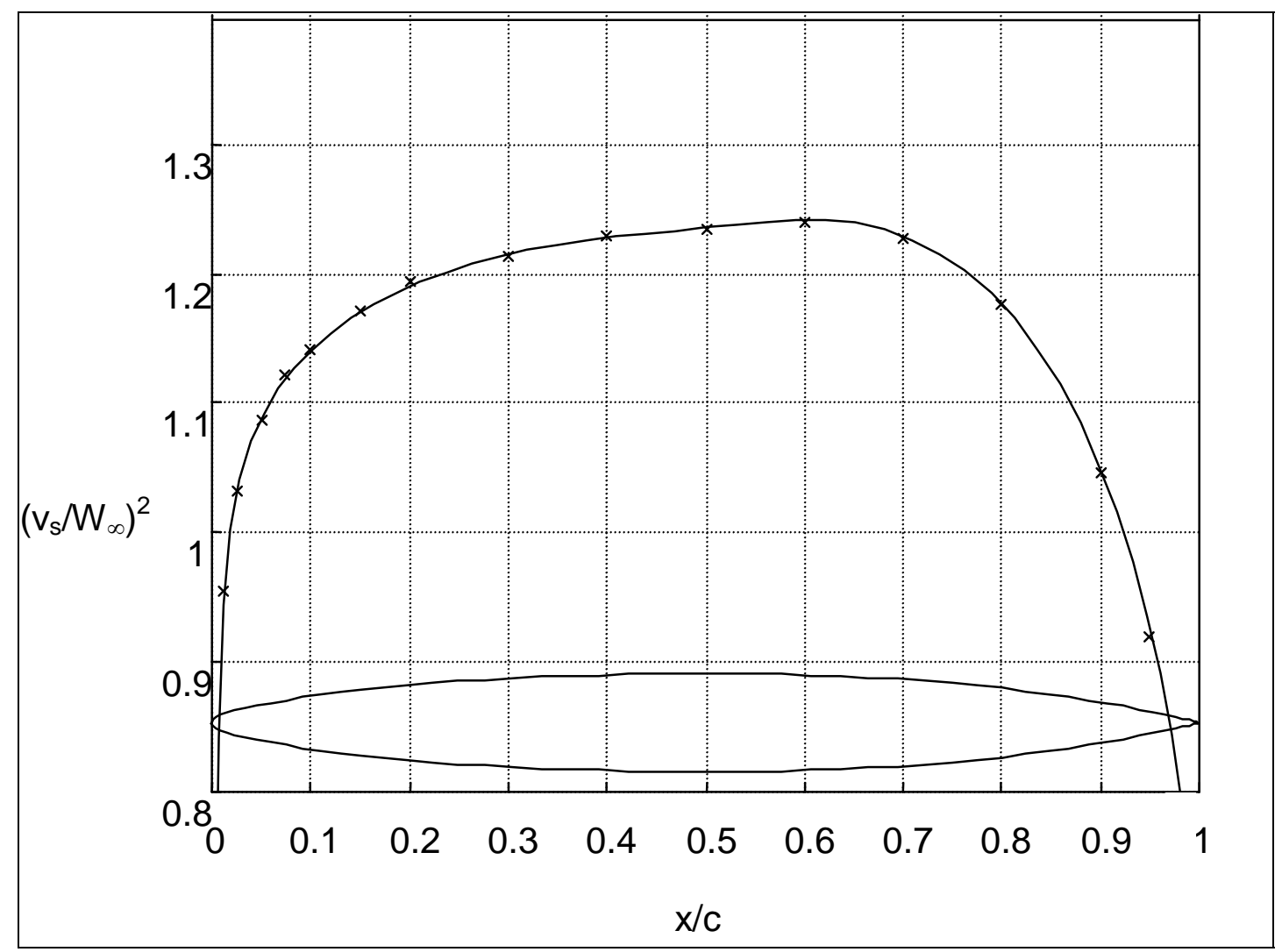

Figure 3.22. Comparison of surface vortex analysis with Abbott and Von Doenhoff for NACA 0010-35 profile.

Application to a Cascade

Analysis of a single aerofoil can quite simply be extended to deal with a blade cascade used in a turbomachine. The individual blades now form part of an infinite cascade that can be represented as an infinite line of point vortices having the same pitch spacing, $t$, as the cascade blades.

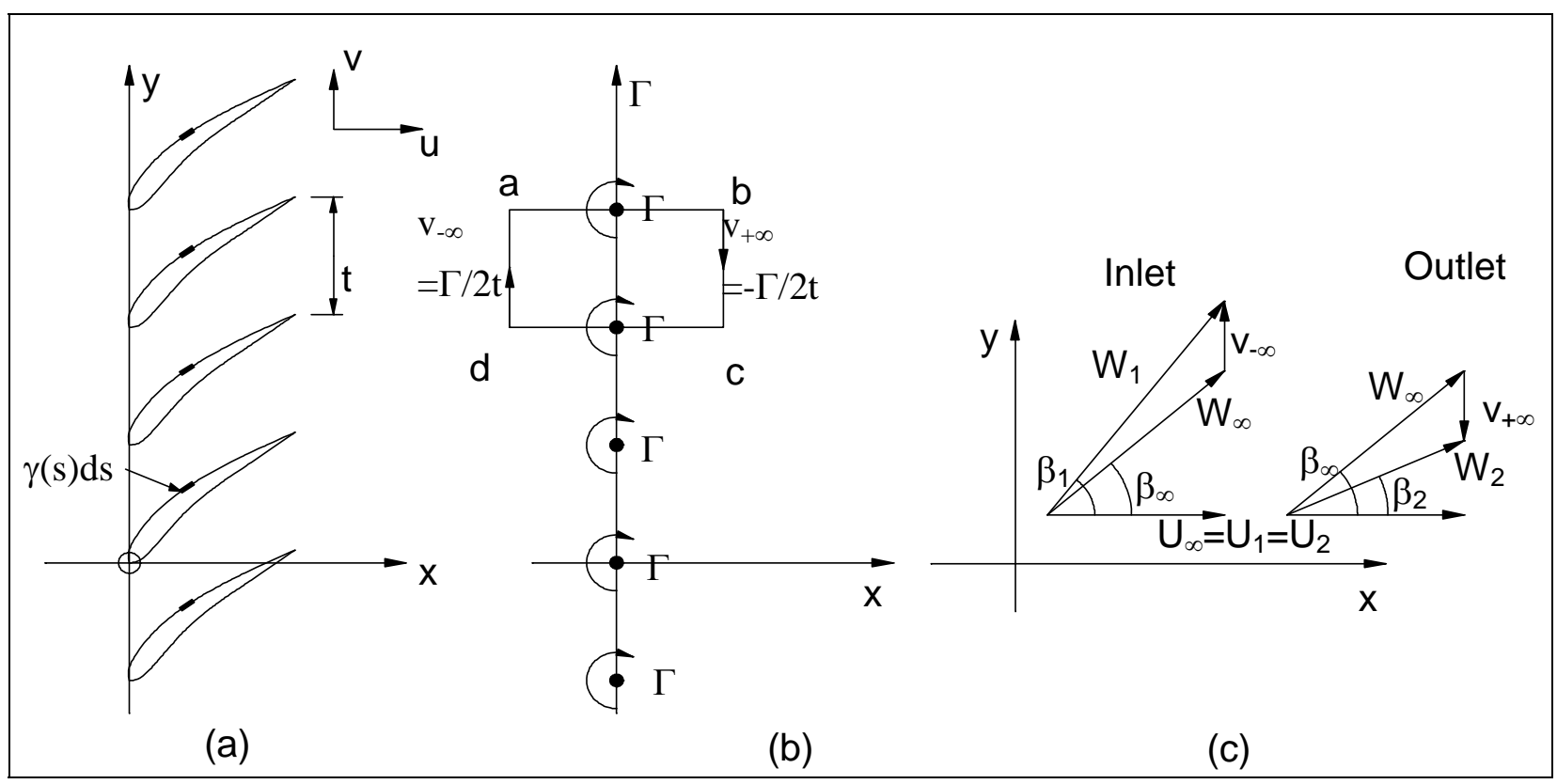

Figure 3.23. (a) Turbomachinery cascade, (b) equivalent infinite array of point vortices and (c) nomenclature for velocity components. 
The uniform stream $\mathrm{W}_{\infty}$ is now a theoretical value used in the analysis where $\mathrm{W}_{1}$ and $\mathrm{W}_{2}$ are the inlet and outlet velocities of the cascade. $\mathrm{K}$, the coupling coefficient, is the only term to change in the above analysis.

$$
\mathrm{K}\left(\mathrm{s}_{\mathrm{m}}, \mathrm{s}_{\mathrm{n}}\right)=\frac{\Delta \mathrm{s}_{\mathrm{n}}}{2 \pi}\left\{\frac{\sin \left\{\frac{2 \pi}{\mathrm{t}}\left(\mathrm{y}_{\mathrm{m}}-\mathrm{y}_{\mathrm{n}}\right)\right\} \cos \beta_{\mathrm{m}}-\sinh \left\{\frac{2 \pi}{\mathrm{t}}\left(\mathrm{x}_{\mathrm{m}}-\mathrm{x}_{\mathrm{n}}\right)\right\} \sin \beta_{\mathrm{m}}}{\cosh \left\{\frac{2 \pi}{\mathrm{t}}\left(\mathrm{x}_{\mathrm{m}}-\mathrm{x}_{\mathrm{n}}\right)\right\}+\cos \left\{\frac{2 \pi}{\mathrm{t}}\left(\mathrm{y}_{\mathrm{m}}-\mathrm{y}_{\mathrm{n}}\right)\right\}}\right\}
$$

Replacing of equation (3.57) with (3.66) is the only change to the analysis. The surface velocity is still calculated using equation (3.64). The exit flow angle from the cascade, $\beta_{2}$, is needed for use in predicting the amount of flow turning in the cascade. The calculation of $\beta_{2}$ requires the unit circulation components $\Gamma_{\mathrm{u}}$ and $\Gamma_{\mathrm{v}}$ in the $\mathrm{x}$ and $\mathrm{y}$ directions respectively, defined by,

$$
\begin{aligned}
\Gamma_{\mathrm{u}} & =\sum_{\mathrm{n}=1}^{\mathrm{M}} \gamma_{\mathrm{u}} \Delta \mathrm{s}_{\mathrm{n}} \\
\Gamma_{\mathrm{v}} & =\sum_{\mathrm{n}=1}^{\mathrm{M}} \gamma_{\mathrm{v}} \Delta \mathrm{s}_{\mathrm{n}}
\end{aligned}
$$

The exit angle can then be calculated as follows,

$$
\beta_{2}=\arctan \left[\left(\frac{1-\frac{\Gamma_{\mathrm{v}}}{2 \mathrm{t}}}{1+\frac{\Gamma_{\mathrm{v}}}{2 \mathrm{t}}}\right) \tan \beta_{1}-\left(\frac{2}{1+\frac{\Gamma_{\mathrm{v}}}{2 \mathrm{t}}}\right) \frac{\Gamma_{\mathrm{u}}}{2 \mathrm{t}}\right]
$$

\section{Application to Axial and Radial Cascades}

To be useful, the above analysis must be applied to the radial and axial cascades found in the solar chimney turbine and inlet guide vanes. Lewis (1991) presents the transformation of a general mixed flow cascade of which axial and radial cascades are special cases. The axial case is simple, being equivalent to wrapping the Cartesian problem onto a cylinder.

The transformation to radial co-ordinates is illustrated above, where the blade profile is transformed from the cartesian, $(\mathrm{x}, \mathrm{y})$, co-ordinate system to the radial, $(\mathrm{r}, \theta)$, co-ordinate system. The geometric transformation is very simple,

$$
\begin{aligned}
& r=\exp (x) \\
& \theta=y
\end{aligned}
$$

When simulating a radial cascade, the inlet and outlet radii will generally be known as well as the number of blades. It is important that the correct cartesian cascade is simulated, for this the pitch and chord length of the cartesian cascade must be calculated. 
The pitch, t, can be calculated from the number of blades in the radial cascade,

$$
\mathrm{t}=\frac{2 \pi}{\mathrm{N}}
$$

while the chord length, c, is dependent on both the inlet and outlet radii.

$$
c=\log _{\mathrm{e}}\left(\frac{\mathrm{r}_{1}}{\mathrm{r}_{2}}\right)
$$

The blade surface velocity on the radial cascade is simply obtained from the cartesian cascade by dividing by the radius,

$$
v_{\mathrm{sr}}=\frac{v_{\mathrm{s}}}{\mathrm{r}}
$$

The inlet and exit angles, $\beta_{1}$ and $\beta_{2}$, are identical in both co-ordinate systems at the blade inlet and exit.
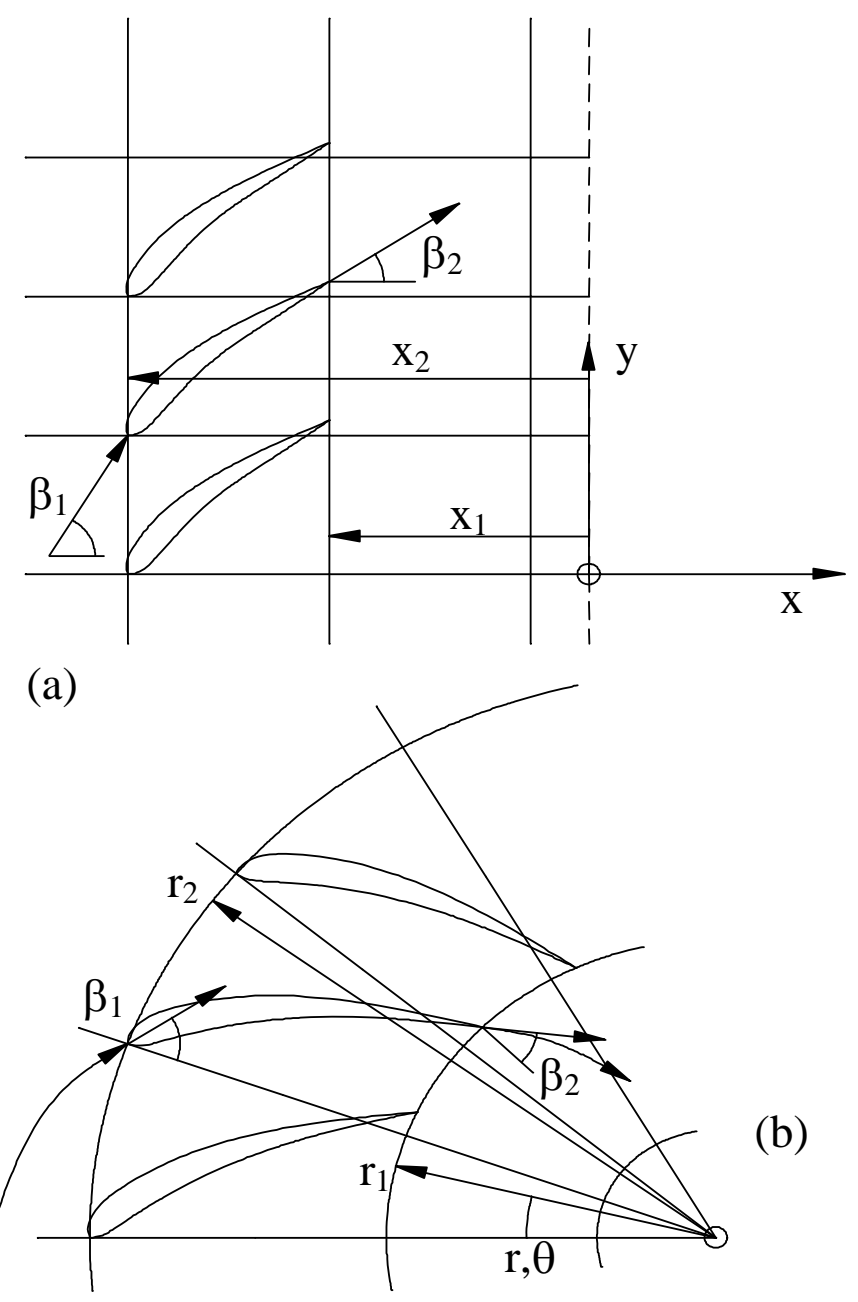

(b)

Figure 3.24. Conformal transformation of rectilinear cascade in Cartesian $(x, y)$ plane to radial cascade in cylindrical $(r, \theta)$ plane. 


\section{Separation Prediction}

To design the blade cascades to operate over their design range correctly, the flow must not separate. A disadvantage of the surface vortex method of analysis is that as a potential flow method it disregards boundary layers and thus it is necessary to use another method to predict whether separation will occur. While there are a number of separation prediction methods, the Stratford (1959) method was chosen. As pointed out by White (1991), 'the Stratford method is a one shot separation prediction method' requiring no intermediate steps in predicting the separation point. It is quite simple to use and is outlined by Benadé (1986), who showed a practical way of implementing the method.

The Stratford method can predict the separation point for both laminar and turbulent flows using two slightly different equations. The Stratford method predicts how far after the point of maximum velocity separation will occur. To start, the following pressure coefficient based on the maximum velocity is defined,

$$
\mathrm{C}_{\mathrm{p} \text { Strat }}=\frac{\mathrm{p}-\mathrm{p}_{\max }}{\frac{1}{2} \rho v_{\mathrm{s} \max }^{2}}=1-\frac{v_{\mathrm{s}}^{2}}{v_{\mathrm{s} \max }^{2}}
$$

This coefficient of pressure, $C_{p}$ Strat must not be confused with the more traditional one based on the freestream velocity $\mathrm{W}_{\infty}$. Laminar separation occurs at a distance, $\mathrm{x}_{\text {lam }}$, after the position of maximum velocity when the following equation, White (1991), is satisfied,

$$
\mathrm{x}_{\text {lam }}^{2} \mathrm{C}_{\mathrm{p} \text { Strat }}\left(\frac{\mathrm{dC}_{\mathrm{p} \text { Strat }}}{\mathrm{dx}_{\text {lam }}}\right)^{2} \approx 0.0104
$$

For turbulent flow, the Stratford separation criterion is also dependent on the Reynolds number and at values of $10^{6}$ the separation distance, $\mathrm{x}_{\mathrm{turb}}$ is,

$$
\mathrm{C}_{\mathrm{p}}\left(\mathrm{x}_{\text {turb }} \frac{\mathrm{dC}_{\mathrm{p} \text { Strat }}}{\mathrm{dx}_{\text {turb }}}\right)^{\frac{1}{2}}=\mathrm{K}\left\{\left(10^{-6} \mathrm{Re}\right)^{1 / 10}\right\}
$$

where

$$
K=0.39 \text { for } \frac{d^{2} C_{p \text { Strat }}}{d x_{\text {turb }}^{2}} \geq 0 \text { and } K=0.35 \text { for } \frac{d^{2} C_{p \text { Strat }}}{d x_{\text {turb }}^{2}}<0
$$

As the method is applied within an optimisation method where it will be used a number of times it was decided to solve the above equations analytically. To do this, a curve is fitted to the decelerating part of the flow and then this equation substituted into equation (3.74) or (3.75) depending on whether the laminar or turbulent separation point is required. For NACA 4-digit and modified 4-digit profiles, the decelerating parts of the profiles is always either linear or of parabolic 
form, thus these two curve fits were used to predict the laminar separation point. White (1991) provides some solutions to these for the laminar case and the implementation was verified against these. A single case is implemented on the flow profile predicted in Figure 3.22 where it can be seen that the flow starts to decelerate from $60 \%$ of the chord. The linear and quadratic equations fitted to the flow are of the following form, where a and b are constants to ensure that the overall slope is correct,

$$
\begin{aligned}
& \mathrm{v}_{\mathrm{s}}=1-\mathrm{a}(\mathrm{x} / \mathrm{c}) \\
& \mathrm{v}_{\mathrm{s}}=1-\mathrm{b}(\mathrm{x} / \mathrm{c})^{2}
\end{aligned}
$$

Figure 3.25 shows three velocity profiles and the predicted laminar and turbulent separation point. The separation points are based on the parabolic curve fit, even though it does not appear to lie very close to the actual profile. The justification for using this prediction is that it is conservative.

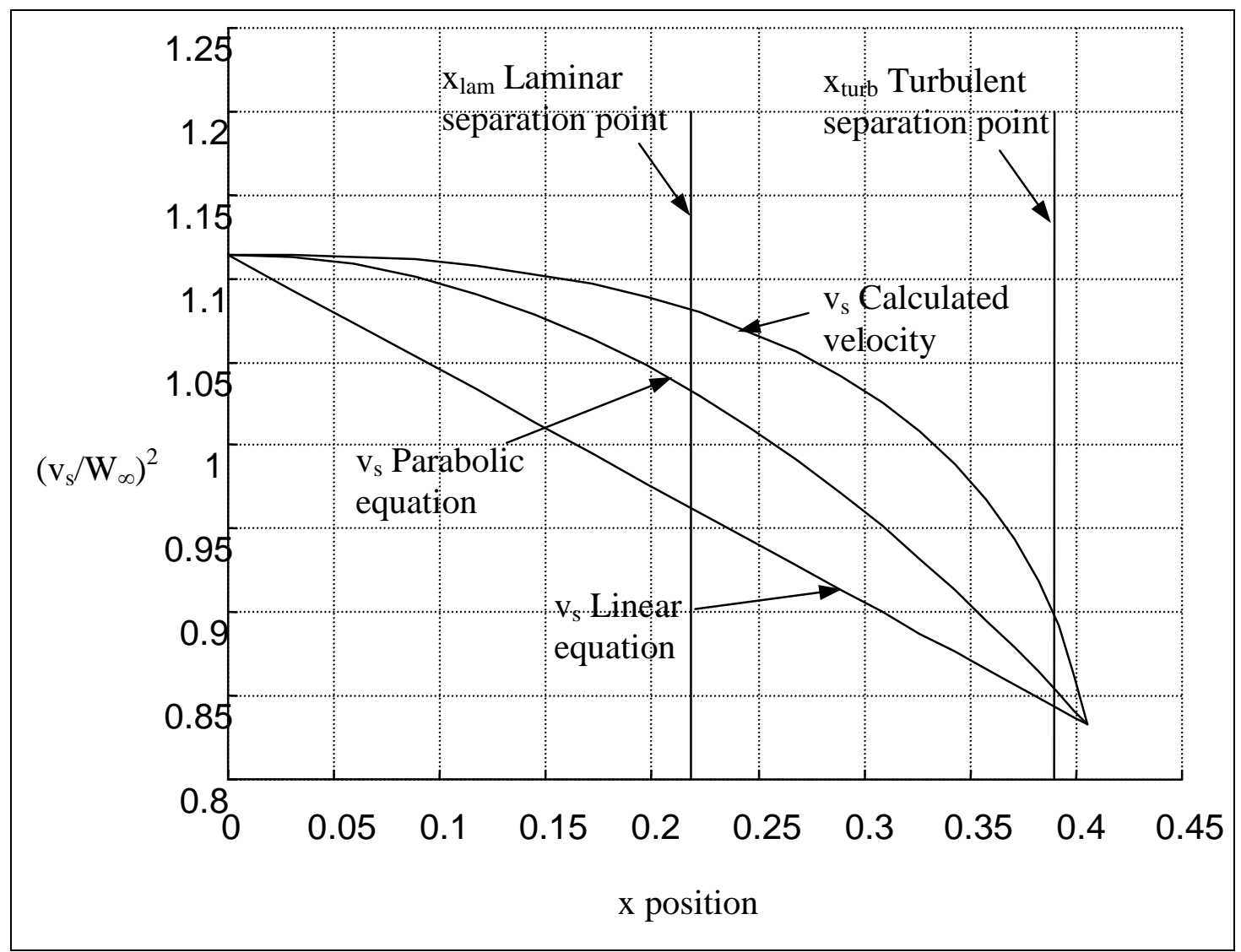

\section{Figure 3.25. Laminar and turbulent separation point predictions from application of Stratford criterion for flow over NACA 0010-35 profile.}

Higher order polynomial curve fits or flows having a similar form to the calculated velocity have separation points farther downstream as given by White (1991) where up to $6^{\text {th }}$ order polynomials are solved. The proposed approach, however, keeps the analysis simple and as it is conservative should ensure that if the method predicts that the flow will not separate then in practice it should 
not either. Table 3.9 is taken from White (1991), p 274, and compares different laminar separation point methods; it can be clearly seen that the flow described by higher order polynomials separate further downstream after the maximum velocity.

Table 3.9. Laminar-separation-point prediction by two methods.

\begin{tabular}{|l|l|l|l|l|l|}
\hline Velocity & \multicolumn{1}{|c|}{ Exact } & \multicolumn{2}{c|}{ Thwaites } & \multicolumn{2}{c|}{ Stratford } \\
\hline $\mathrm{U}(\mathrm{x})$ & $\mathrm{x}_{\text {sep }}$ & $\mathrm{x}_{\text {sep }}$ & Error, \% & $\mathrm{x}_{\text {sep }}$ & Error, \% \\
\hline $1-\mathrm{x}$ & 0.120 & 0.123 & +2.5 & 0.122 & +1.7 \\
\hline $1-\mathrm{x}^{2}$ & 0.271 & 0.268 & -1.1 & 0.271 & 0 \\
\hline $1-\mathrm{x}^{4}$ & 0.462 & 0.449 & -2.8 & 0.461 & -0.2 \\
\hline $1-\mathrm{x}^{8}$ & 0.640 & 0.621 & -3.0 & 0.639 & -0.2 \\
\hline
\end{tabular}

Simplex Algorithm for Optimisation

The optimisation method used for the design of the blade profiles is presented by Rao (1996). Using that reference, an optimisation scheme can be described as,

$$
\text { Find } X=\left\{\begin{array}{l}
x_{1} \\
x_{2} \\
\vdots \\
x_{n}
\end{array}\right\} \text { which minimizes } f(X)
$$

subject to the constraints

$$
\begin{array}{lcc}
g_{j}(X) \leq 0, & j=1,2, \ldots, m & \text { inequality constraint } \\
l_{j}(X)=0, & j=1,2, \ldots, p & \text { equality constraint }
\end{array}
$$

$\mathrm{X}$ is an $\mathrm{n}$-dimensional vector called the design vector, $\mathrm{f}(\mathrm{X})$ the objective function, and $\mathrm{g}_{\mathrm{j}}(\mathrm{X})$ and $l_{j}(X)$ are known as inequality and equality constraints respectively. The variables and constraints used for the profiles will be listed in the following sub-section where all the methods described are applied to the blade designs. The simplex algorithm is implemented using the MATLAB computer program's optimisation toolbox and the m-file fmins.m. This has been modified to be able to include constraints, as these are needed to apply the separation criterion.

The simplex algorithm was chosen for two main reasons, the first being its stability as an optimisation method. There are faster methods (requiring less intermediate steps) but they are not always stable where the simplex method tends to be very robust and handles any discontinuous functions with ease. The second is that the simplex algorithm does not require any function gradients to be specified and as the outputs from the surface vortex method used in the optimisation are discrete, this is an advantage. 


\subsection{INLET GUIDE VANE DESIGN}

To recap, the purpose of the inlet guide vanes, IGVs, is to add pre-swirl to the flow before it enters the turbine blades. They must also support the mass of the chimney so it is important that they are also strong structurally. The initial design point calculated from Cohen et al. (1996), called for 12 blades with a pitch/chord ratio of approximately 1.5. This resulted in rather long flow passages but as the flow into the inlet guide vanes is almost purely radial and accelerating the secondary flows would be very small.

\section{Reynolds Number Investigation}

It is at this point that the first concession was made to the small scale of the experimental rig constructed to test the turbine design. Due to the flow velocity into the turbine being very small and the flow also accelerating, there is a very good chance that the flow over the inlet guide vanes would be laminar, so for this reason a laminar separation criterion was used for the IGVs. On the real plant, the flow is likely to be turbulent due to the large size and therefore high Reynolds numbers. It was felt, however, that it was very important that the IGVs operate correctly, as an important part of the experimental investigation is to check their effectiveness. It is also important to verify the overall design method and so if the design and analysis methods can accurately predict the flow on a small-scale model then there is more certainty that the results can be scaled up to the large plant. From Figure 3.11 and using air at standard atmospheric conditions, the Reynolds number can be calculated,

$$
\begin{aligned}
\text { Re } & =\frac{C_{l} L}{v} \\
& =\frac{17.1 \times 0.226}{1.5 \times 10^{-5}} \\
& =2.58 \times 10^{5}
\end{aligned}
$$

In White (1991), (p 395), turbulence is found to occur on a flat plate at Reynolds number values of $2 \times 10^{5}$, meaning the flow over the IGVs will be predominantly laminar and in an accelerating flow the transition Reynolds number is usually higher.

\section{Optimisation Problem}

$f(X)$, the objective function to be minimised in the blade profile design is the maximum velocity, as by keeping this as low as possible it is hoped that the drag will be minimised, thus increasing the efficiency. The constraint function is that the separation length $\mathrm{x}_{\mathrm{lam}}$ is greater than the distance from the position of maximum velocity to the trailing edge. Practically, this means that the flow must not separate before the trailing edge of the blade. The design vector, $\mathrm{X}$, consists of the following components: 
- $\mathrm{X}_{1}=\mathrm{m}_{\text {ord }}$

Maximum ordinate of mean line expressed as a fraction chord.

- $\mathrm{X}_{2}=\mathrm{p}_{\text {ord }}$ Chordwise position of maximum ordinate.

- $\mathrm{X}_{3}=\mathrm{t}_{\mathrm{max}}$ Maximum thickness as a fraction of chord.

- $\mathrm{X}_{4}=\mathrm{m}_{\mathrm{t} \max }$ Position of maximum thickness as a fraction of chord.

- $\mathrm{X}_{5}=\mathrm{I}_{\text {nose }}$ Nose radius where 6 is the standard NACA 4-digit size.

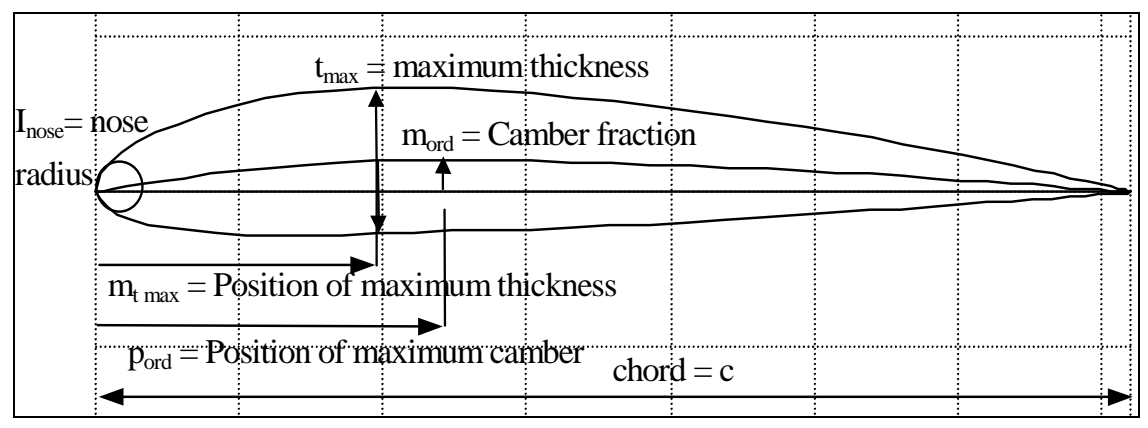

Figure 3.26. Blade profile nomenclature.

Figure 3.26 shows the nomenclature usage in the profile. The design inlet and exit flow range and design stagger angles of the IGV profiles are presented in Table 3.10 below. The blade design allowed for any exit flow angle between $0^{0}$ and $22.5^{0}$.

Table 3.10. Flow range of inlet guide vanes.

\begin{tabular}{|l|l|l|l|}
\hline & $\alpha_{1}$ (inlet) & $\alpha_{2}$ (outlet) & Stagger angle \\
\hline minimum & $0^{0}$ & $0^{0}$ & -3.35 \\
\hline maximum & $0^{0}$ & $22.5^{0}$ & 18.06 \\
\hline
\end{tabular}

\section{Implementation of Optimisation and Results}

The surface vortex method is used to analyse the flow over the profile in the cartesian co-ordinate system and these results are then transformed into the radial system. This radial result is then investigated using the Stratford separation criterion to check if the flow remains attached. The profile is then modified by the simplex optimisation method and the loop repeated until a profile with the minimum surface velocity in the radial co-ordinate system is obtained. Outside of this loop there is another one that adjusts the pitch/chord ratio to the maximum value where the flow will remain attached. This keeps the blade size as small as possible and while this is not so important in the inlet guide vanes as they are static, it is extremely important in the turbine blades.

The above calculation was performed and due to the laminar criterion used it was found that the blade aspect ratio was very low resulting in long flow passages. While secondary losses are not expected to be high, it was decided to increase the blade number from 12 to 18 and this resulted in shorter flow passages and higher aspect ratio blades. 


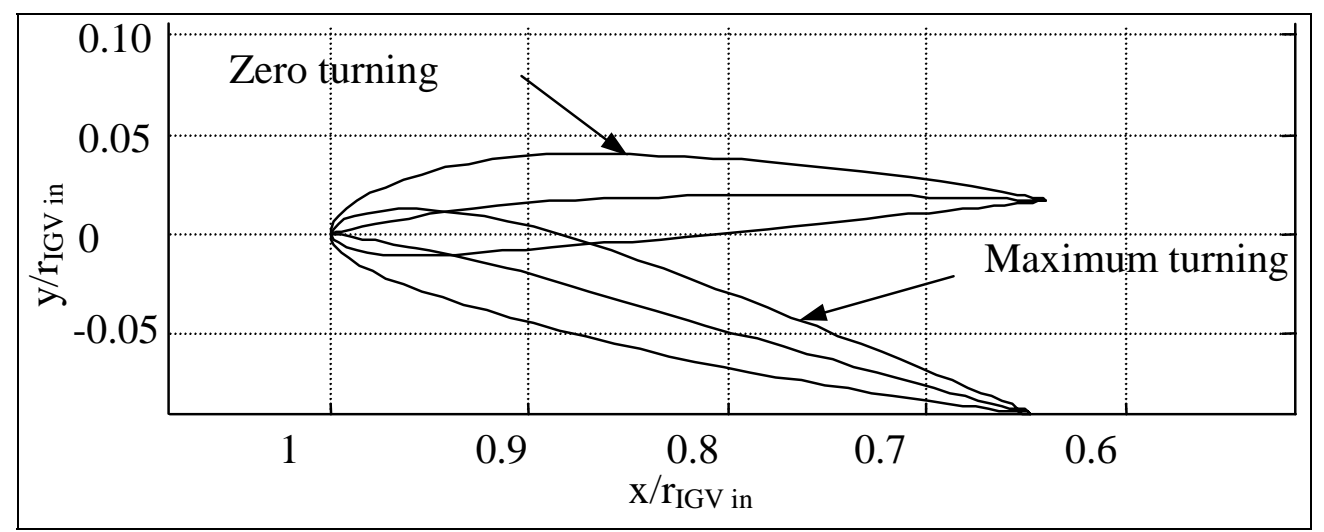

Figure 3.27. Detailed inlet guide vane blade profiles at extreme stagger angles.

The final blade form is shown in Figure 3.26. Figure 3.27 shows the IGV stagger angles for $0^{0}$ and $22.5^{0}$ turning. Figure 3.28 shows the complete cascade. A unit outer radius is used to simplify the analysis. As the surface vortex method is an inviscid method, the results can be scaled to a different size cascade. In the following tables, the dimensions of the final blade design are given, where a detailed explanation of the meaning is given in Abbot and Von Doenhoff (1959).

Table 3.11. Blade profile geometry of modified NACA 4-digit profile for IGVs.

\begin{tabular}{|l|l|l|}
\hline $\mathrm{X}$ (Design vector) & Value & Description \\
\hline $\mathrm{m}_{\text {ord }}$ & $2.64 \%$ & Maximum camber as a fraction of chord \\
\hline $\mathrm{p}_{\text {ord }}$ & $29.85 \%$ & Position of maximum camber as a fraction of chord \\
\hline $\mathrm{t}_{\max }$ & $11.70 \%$ & Maximum thickness as a fraction of chord \\
\hline $\mathrm{m}_{\mathrm{t} \max }$ & $28.58 \%$ & Position of maximum thickness as a fraction of chord \\
\hline $\mathrm{I}_{\text {nose }}$ & 5.47 & Nose radius relative to NACA 4-digit profile. $(6=$ standard) \\
\hline
\end{tabular}

Table 3.12. Cascade geometry for radial layout of IGV's.

\begin{tabular}{|l|l|l|}
\hline IGV exit rad/ inlet rad $\left(\mathrm{r}_{\mathrm{i}} / \mathrm{r}_{\mathrm{o}}\right)$ & pitch/chord $(\mathrm{t} / \mathrm{c})$ & aspect ratio $(\mathrm{H} / \mathrm{c})$ \\
\hline 0.64 & 0.35 & 1.42 \\
\hline
\end{tabular}

Figure 3.29 shows the negative $C_{p}$ values as this is the usual convention as a higher velocity then corresponds to a higher $\mathrm{C}_{\mathrm{p}}$ value. Both the zero turning and maximum turning of $22.5^{0}$ top and bottom surface pressure distributions are shown. For the $0^{0}$ turning the blade is not highly loaded with the largest adverse pressure gradient occurring on the pressure surface (bottom) near the leading edge and the suction surface (top) having an accelerating flow over almost its entire length. At the maximum turning angle, the pressure surface has the accelerating flow while the suction surface has an almost constant pressure distribution with the flow only diffusing near the trailing edge. The flow should not separate for both these extreme cases, even if the flow is laminar. The reason for not wanting the flow to ever separate is to attempt to keep the profile drag as low as possible. 


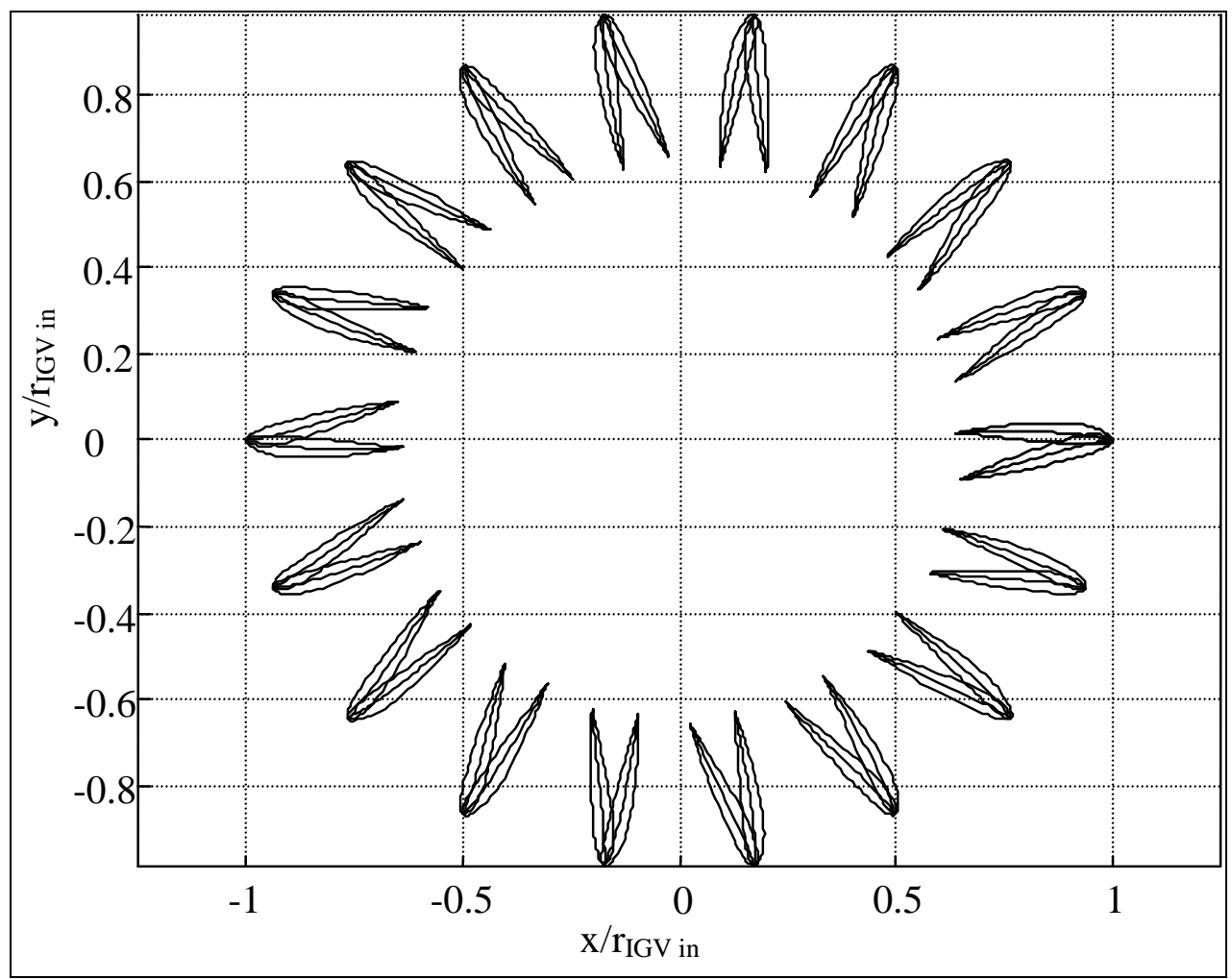

Figure 3.28. Inlet guide vane radial cascade at extreme stagger angles.

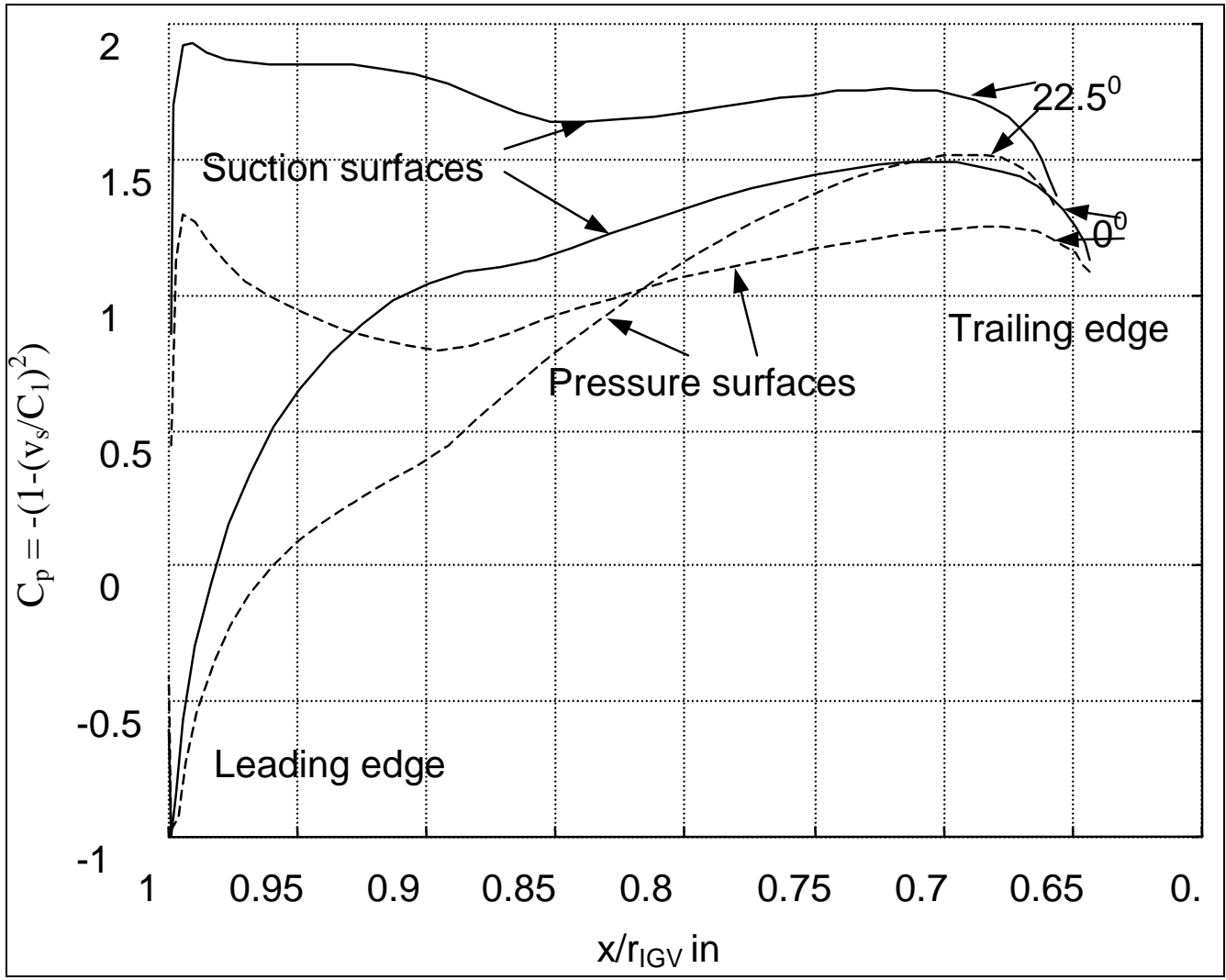

Figure 3.29. $C_{\mathbf{p}}$ values for flow over inlet guide vanes $C_{p}=1-\left(v_{s} / C_{1}\right)^{2}$. 


\subsection{TURBine BLADE DESIGN}

Design of the turbine blades is now possible as all the theory is in place. As with the IGVs, a brief summary of the function of the turbine blades is useful. The turbine blades extract energy from the flow and deliver the useful power output of the solar chimney. Optimisation and design of the turbine blades is more complex than that of the inlet guide vanes as for efficient operation the blade profile must change from root to tip. The design criteria of the turbine profile is to be able to turn the flow the required amount without stalling and with minimum drag.

\section{Reynolds Number Investigation}

As with the inlet guide vanes, an initial number of blades was chosen, in this case 12 with a pitch/chord ratio of 1 at the blade root decreasing towards the blade tip. In the turbine blades the relative flow velocity is much higher due to the rotation of the turbine. The blade root has the slowest relative velocity and this is where the Reynolds number analysis is applied. Figure 3.5 and Figure 3.12 show the definition of the velocity $V_{2}$ and its magnitude respectively. In the inlet guide vane analysis the highest velocity was used to demonstrate that the flow would always be laminar while for the turbine the critical value is the low one thus the low pressure drop flow velocities are used. The distance to the point where the flow is expected to be turbulent $\left(\operatorname{Re}=2 \times 10^{5}\right)$ is calculated at the blade root and tip.

$$
\begin{aligned}
\operatorname{Re} & =\frac{\mathrm{V}_{2} \mathrm{~L}}{v} \\
\mathrm{~L}_{\text {root }} & =\frac{v \mathrm{Re}}{\mathrm{V}_{2}}=\frac{v \mathrm{Re}}{\sqrt{\mathrm{C}_{\mathrm{z}}^{2}+\mathrm{U}^{2}}} \\
& =\frac{1.5 \times 10^{-5} \times 2 \times 10^{5}}{\sqrt{15^{2}+(0.4 \times 88.6)^{2}}} \\
& =0.078 \mathrm{~m} \\
\mathrm{~L}_{\text {tip }} & =\frac{1.5 \times 10^{-5} \times 2 \times 10^{5}}{\sqrt{18^{2}+88.6^{2}}} \\
& =0.033 \mathrm{~m}
\end{aligned}
$$

In the following section it will be seen that these positions are actually near to the trailing edge of the blade and it may be necessary to place a trip wire on the blade surface just before the flow starts to decelerate to ensure that it is turbulent. The use of trip wires is outlined in White (1991), (p 386), and a very small wire, in the order of half the boundary layer thickness is needed to ensure that the flow becomes turbulent. At this stage, it seems that the blades will stall for case 3 and its associated low volume flow rate. The experimental program showed that this prediction was correct. 


\section{Optimisation Problem}

The optimisation problem for the turbine blades is very similar to that of the inlet guide vanes except that now the profile must change from the blade root to tip. Seven stations were eventually chosen from the blade root to tip as beyond five stations the change to the blade design was very little. At each station the objective function $f(X)$ was the same as before- to try and keep the maximum velocity as low as possible to try and minimise the profile drag. The design vector, $\mathrm{X}$, is repeated here and consists of the following components:

- $\mathrm{X}_{1}=\mathrm{m}_{\text {ord }}$

- $\mathrm{X}_{2}=$ pord

- $\mathrm{X}_{3}=\mathrm{t}_{\mathrm{max}}$

- $\mathrm{X}_{4}=\mathrm{m}_{\mathrm{t} \max }$

- $\mathrm{X}_{5}=\mathrm{I}_{\text {nose }}$
Maximum ordinate of mean line expressed as a fraction chord.

Chordwise position of maximum ordinate.

Maximum thickness as a fraction of chord.

Position of maximum thickness as a fraction of chord.

Nose radius where 6 is the standard NACA 4-digit size.

As with the inlet guide vanes, the turbine blade stagger angle will be adjustable for different flow conditions. Obviously the entire blade must turn as a unit. It cannot twist, and so a compromise must be made by choosing one stagger adjustment angle for the entire blade. The method chosen eventually was to find the most highly aerodynamically loaded part of the blade, to find the required stagger adjustment angle at this station and then use this adjustment for the other stations. As the other stations are not as highly loaded, they can operate slightly outside their ideal operating region. In practice, certain blade stations turned the flow slightly more than was required but this was never more than a fraction of a degree.

As with the inlet guide vanes, the main constraint applied was that the flow must not separate before the trailing edge of the blade. In the turbine blades the turbulent separation criterion was used, not the laminar, meaning that the blades could be more highly loaded. A concession that was made for the small size of the experimental model was the minimum blade thickness as direct scaling of a full-size turbine blade would have resulted in unrealistically thin trailing edges and blade tip sections. These would have been difficult to manufacture at model scale and would have been extremely fragile. The size constraints are,

Table 3.13. Size constraints applied to experimental blade profiles

\begin{tabular}{|l|l|}
\hline Section & Minimum Thickness \\
\hline Trailing edge & $1 \mathrm{~mm}$ \\
\hline Tip profile maximum thickness & $4 \mathrm{~mm}$ \\
\hline Root profile maximum thickness & $12 \mathrm{~mm}$ \\
\hline
\end{tabular}

In addition to the size constraints, the thickness must always increase towards the root and the pitch/chord ratio decrease for strength considerations. 


\section{Implementation of Optimisation}

A similar optimisation scheme to that used to design the IGV profile is used for the rotor blade. In the case of the rotor blade the optimisation needs to be applied at a number of stations along the chord. An added complication is the rotor blade design is introduced by the stagger angle of the rotor blade changing for cases (1) and (2). Obviously the rotor blade will be rigid and twist as a unit. This means that one of the design stations must be chosen to govern the overall stagger angle adjustment. To find this one the optimisation was initially performed at seven stations while assuming that the stagger angle adjustment could be different at each. The largest stagger angle adjustment was chosen as the optimisation reapplied to all other stations. Station 4, where the numbering is taken from root to tip, has the largest stagger adjustment angle. The gas inlet and outlet angles at the seven stations, obtained from Figure 3.14 for case (1) and (2) are as follows.

Table 3.14. Relative inlet and outlet flow angles of turbine blades.

\begin{tabular}{|l|l|l|l|l|l|l|l|l|}
\hline & Station & 1 & 2 & 3 & 4 & 5 & 6 & 7 \\
\hline Case $(1)$ & Inlet $\left(\beta_{2}\right)$ & 65.4 & 70.3 & 73.3 & 75.4 & 76.9 & 78.0 & 79.0 \\
\hline & Outlet $\left(\beta_{3}\right)$ & 70.1 & 72.8 & 74.8 & 76.3 & 77.5 & 78.3 & 79.1 \\
\hline Case $(2)$ & Inlet $\left(\beta_{2}\right)$ & 54.2 & 61.1 & 65.5 & 68.5 & 70.7 & 72.3 & 73.6 \\
\hline & Outlet $\left(\beta_{3}\right)$ & 67.2 & 68.8 & 70.3 & 71.7 & 72.9 & 73.8 & 74.6 \\
\hline
\end{tabular}

Blade and Velocity Profiles

The optimisation result consists of seven profile shapes and the corresponding pitch/chord ratios for each station. Profile 4, the one governing the stagger adjustment angle, is shown below as a cartesian cascade layout before it is wrapped onto a cylinder. The relative gas outlet angle is shown as well as the blade outlet angle, the difference between the two being the deviation angle, calculated from the surface vortex method.

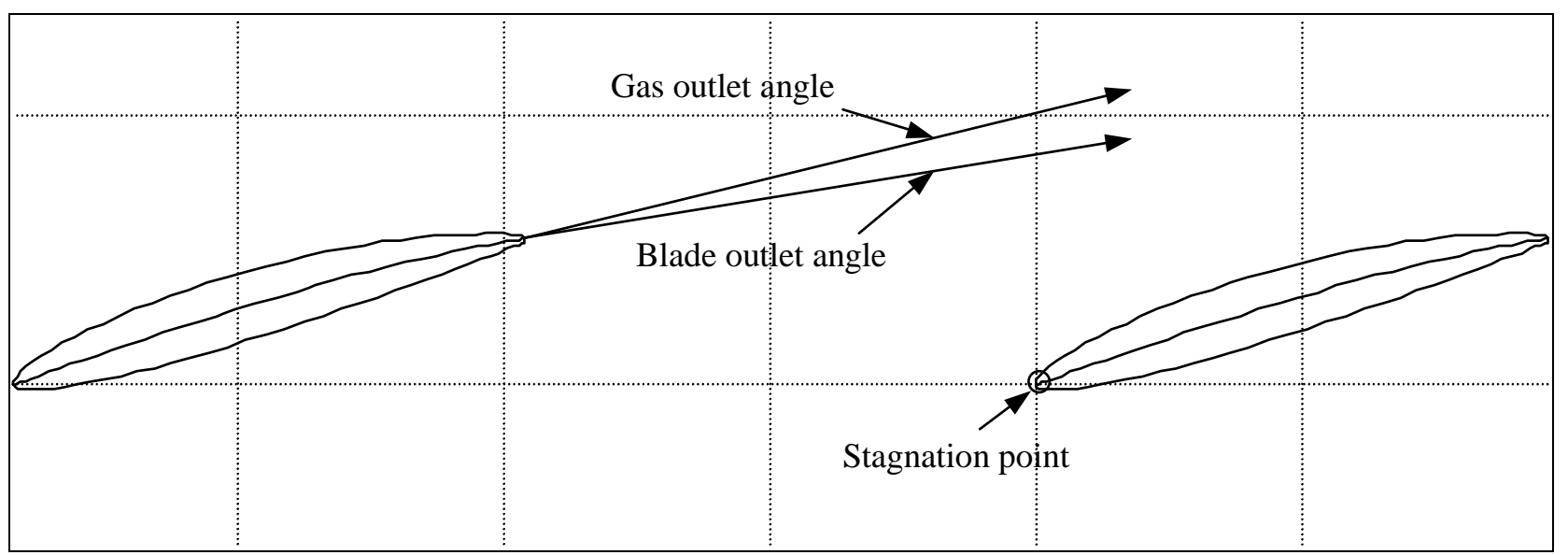

Figure 3.30. Turbine blade profile 4, showing pitch chord spacing. 
Table 3.15. Blade profile geometry of modified NACA 4-digit profiles for turbine blades for low pressure drop (case 1).

\begin{tabular}{|r|l|l|l|l|l|l|l|}
\hline $\begin{array}{l}\text { Station number } \Rightarrow \\
\mathrm{X} \text { (Design vector) } \Downarrow\end{array}$ & \multicolumn{1}{|c|}{1} & \multicolumn{1}{|c|}{2} & \multicolumn{1}{|c|}{3} & \multicolumn{1}{|c|}{4} & \multicolumn{1}{|c|}{5} & \multicolumn{1}{|c|}{7} \\
\hline $\mathrm{m}_{\text {ord }}[\%]$ & 3.75 & 3.55 & 3.35 & 2.28 & 2.50 & 2.67 & 2.59 \\
\hline $\mathrm{p}_{\text {ord }}[\%]$ & 51.1 & 59.7 & 60 & 60 & 56.4 & 55.3 & 55.0 \\
\hline $\mathrm{t}_{\max }[\%]$ & 17.4 & 15.2 & 13.3 & 12.3 & 12.1 & 11.05 & 9.57 \\
\hline $\mathrm{m}_{\mathrm{t} \max }[\%]$ & 32.2 & 31.4 & 35.8 & 42.4 & 45.5 & 49.2 & 39.4 \\
\hline $\mathrm{I}_{\text {nose }}$ & 3.0 & 3.5 & 3.4 & 3.5 & 3.1 & 2.8 & 3.6 \\
\hline Base stagger [deg] & 62.0 & 67.4 & 71.0 & 74.2 & 75.7 & 76.9 & 78.0 \\
\hline Pitch/chord & 1.03 & 1.30 & 1.57 & 1.92 & 2.52 & 3.34 & 4.50 \\
\hline
\end{tabular}

The modified NACA 4-digit geometry at each of the seven stations is shown above in Table 3.15 where the definitions are the same as given in Table 3.11. The stacking point of the blade was chosen as being about the position of maximum thickness and not the usual practice of using the geometric centre. This was to simplify construction of the scale model turbine blades and would not have a measurable effect on the aerodynamics. This was done for two reasons, the first being that it could be simpler to put reinforcing through the blade profiles at their thickest points. The second is that due to the blade geometry having its geometric centre far back stacking around this line could result in aerodynamic instability as it would lead to a positive pitching moment. The cartesian blade geometry from the optimisation is wrapped onto a cylinder for the turbine tip while at the hub, the profile is wrapped onto a sphere. The reason for this is that with a spherical hub the blade could be made to seal at any stagger angle.

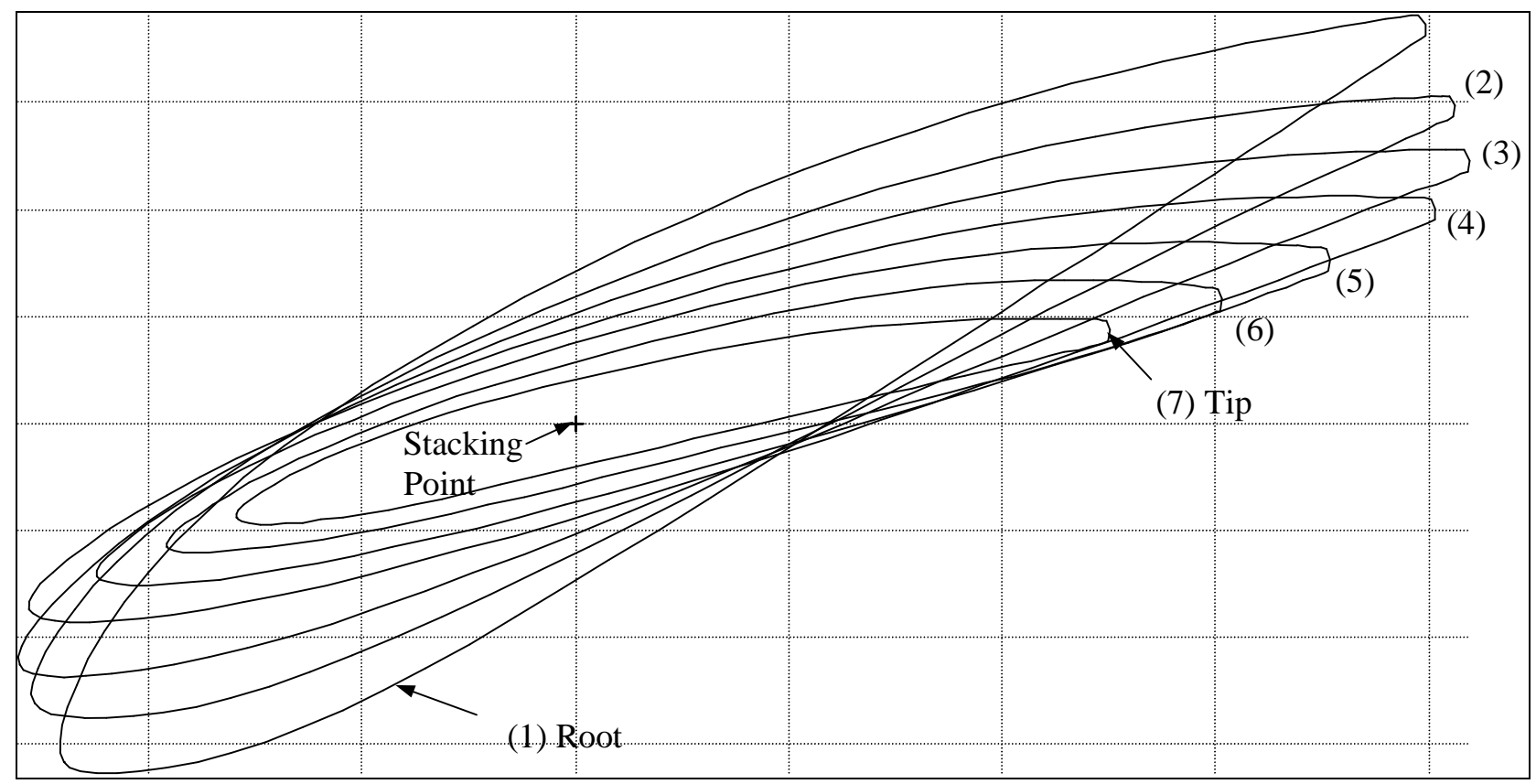

Figure 3.31. Blade profiles at each calculation station. 
The final wrapped geometry of the profiles is shown in Figure 3.31. Here the thick trailing edge can clearly be seen and while this is large in comparison with the blade profile in practice it is only $1 \mathrm{~mm}$. The blade profiles all have similar velocity profiles with the flow accelerating quickly and then staying constant before diffusing near the trailing edge of the blade for the high pressure drop case. For lower pressure drops the blade profile is not as highly loaded and the flow and the lower surface accelerating slightly more than in the high pressure case. In the design of the blades, the separation constraint is applied on both the top and bottom of the blades.

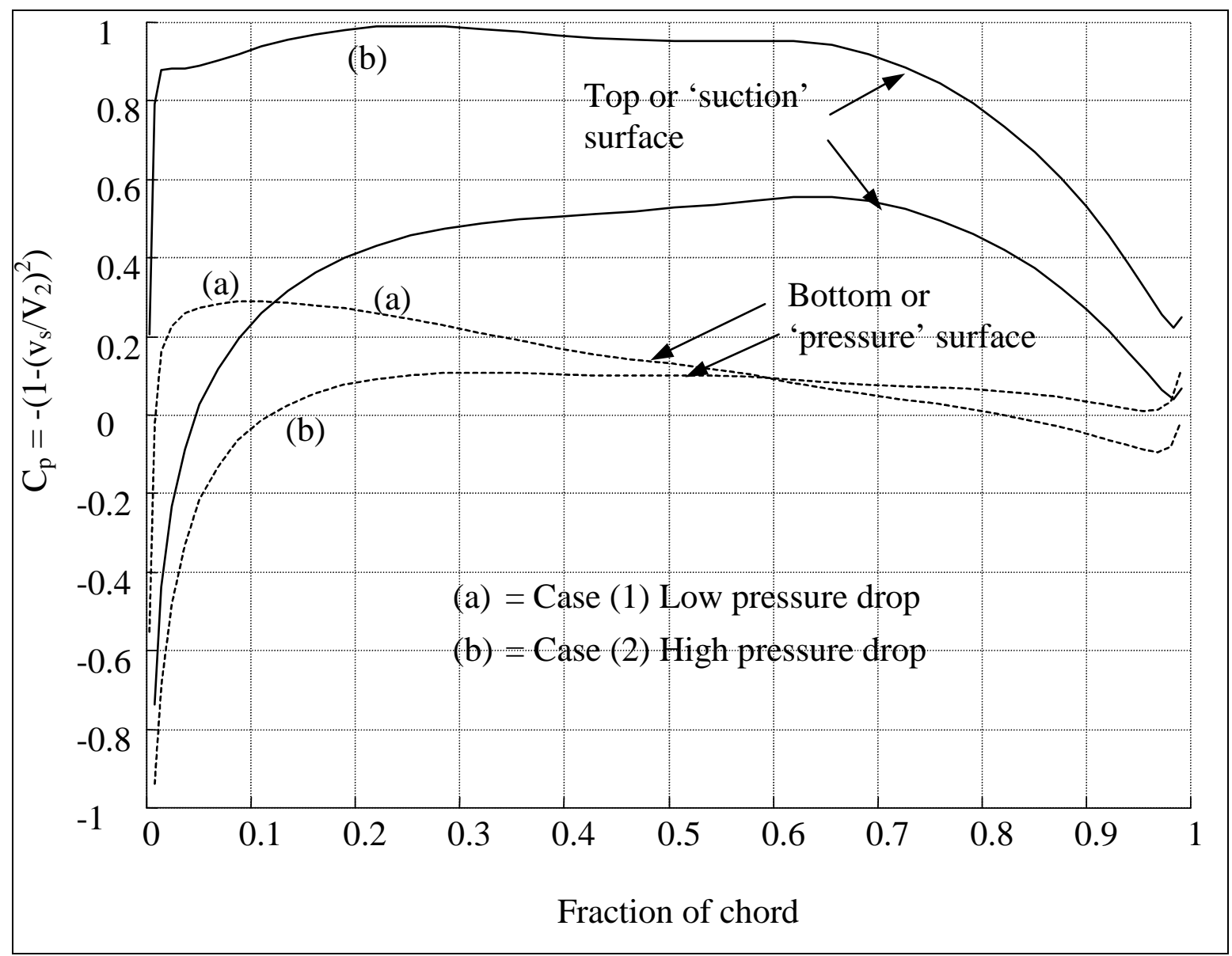

Figure 3.32. Pressure distribution at blade midpoint, station 4, for high and low pressure case.

Figure 3.32 shows the pressure distribution over the turbine blade midpoint for the high and low pressure drop cases. Looking first at the high pressure drop case on the top surface, the flow accelerates quickly, remains fairly constant until approximately $65 \%$ chord where it begins to diffuse towards the trailing edge. The blunt trailing edge of the profiles result in a meaningless result in the last point as the method assumed the flow remains attached. This is not the case and so the point is discarded The separation calculation is applied up to the sharp corner where it is assumed that the flow will separate. 


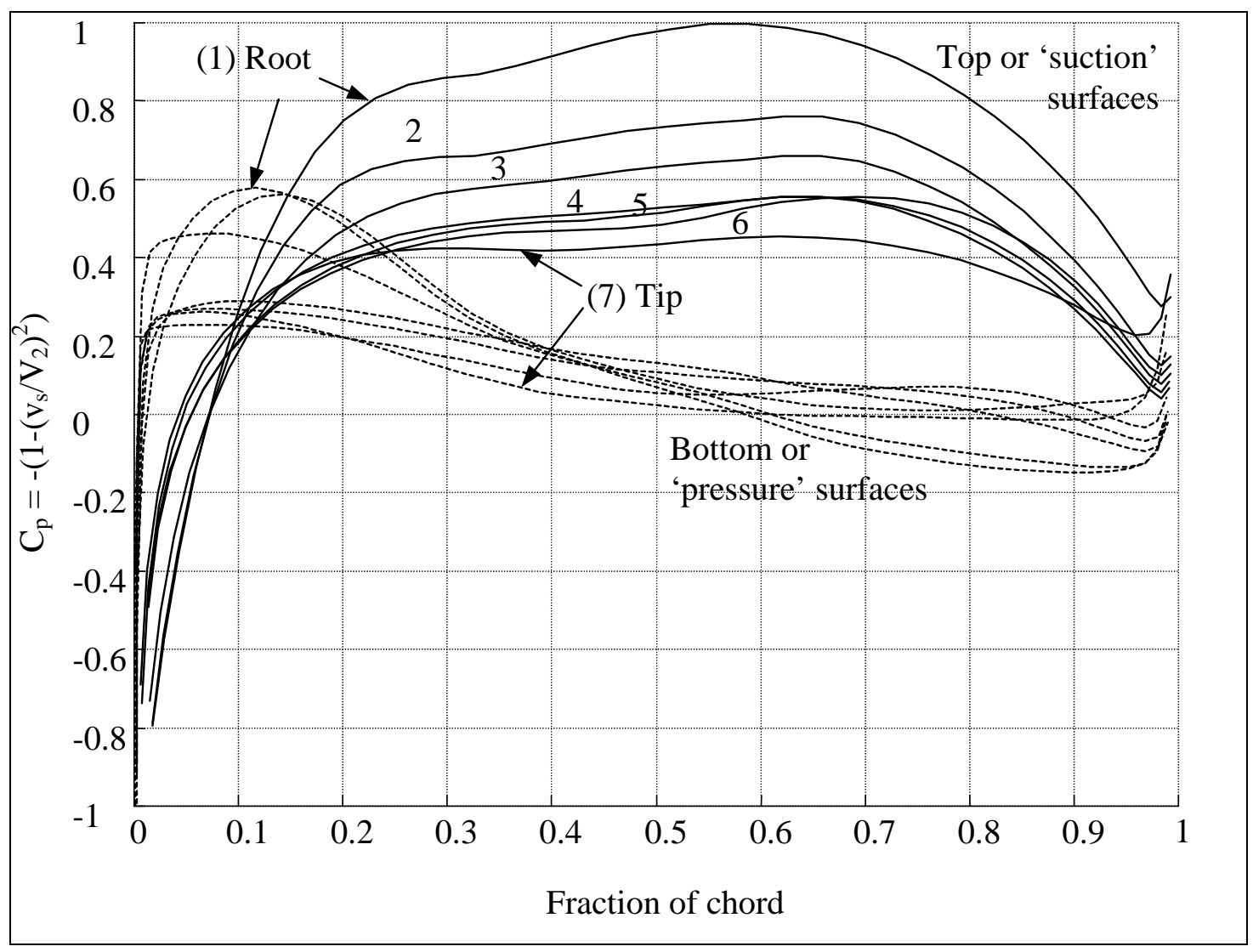

Figure 3.33. Pressure distributions at each blade station for case (1).

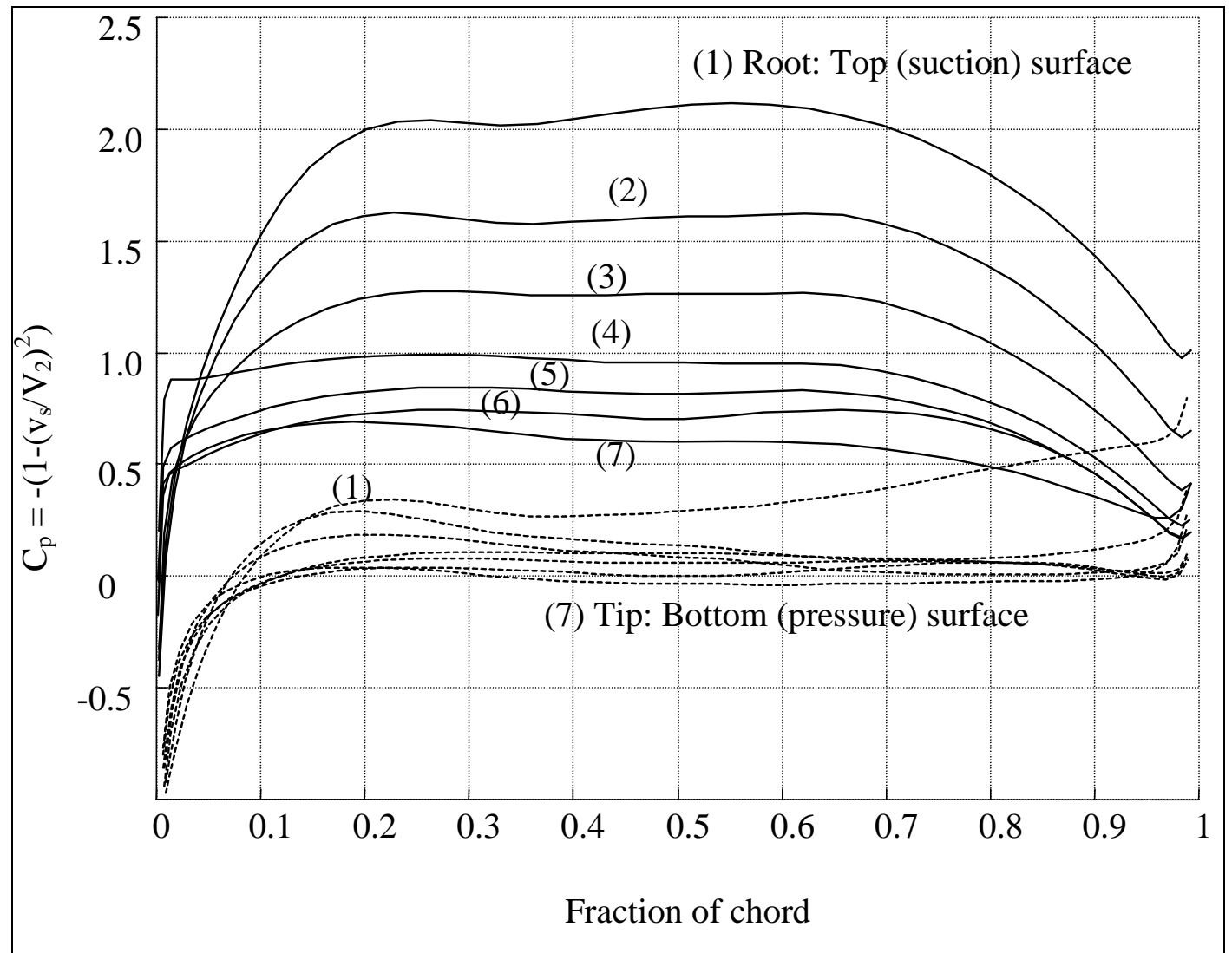

Figure 3.34. Pressure distributions at each blade station for case (2). 
Figure 3.33 and Figure 3.34 show the complete pressure distribution for all seven of the design stations for the low and high-pressure drop, case (1) and (2) respectively. For the low pressure case the top surface accelerates the flow over most of its length and then diffuses the flow over the last section. The bottom surface accelerates the flow more than for the high pressure case due to the inlet flow angle being slightly negative. Decreasing this angle would result in the flow over the top surface increasing in speed and increasing the overall drag.

The high pressure case has far lower pressures over the top surface and once again the flow profiles can be seen to belong to a 'family' with the velocity increasing towards the root. The flow description given for Figure 3.32 is repeated here with the flow accelerating rapidly over the nose and then having a fairly constant velocity until approximately $65 \%$ chord where it diffuses until the trailing edge. On the lower surface the flow accelerates and then remains constant until the trailing edge with the only exception at the lower surface near the root. Here the flow accelerates over the entire chord as this section is not as highly loaded as the rest of the blade due to the pitch/chord ratio being low near the root and thus the blade is easily able to turn the flow.

\section{Discussion}

Both the inlet guide vanes and turbine blades were designed using the same design criteria: being able to deflect the flow the required amount and with minimum drag. This was achieved by attempting to keep the velocities as low as possible. Existing profiles, modified NACA 4-digit series, were used as these have already been used in practice and as the solar chimney turbine is a new turbine application the number of new design variables should be kept to a minimum.

\subsection{TURBINE DIFFUSER}

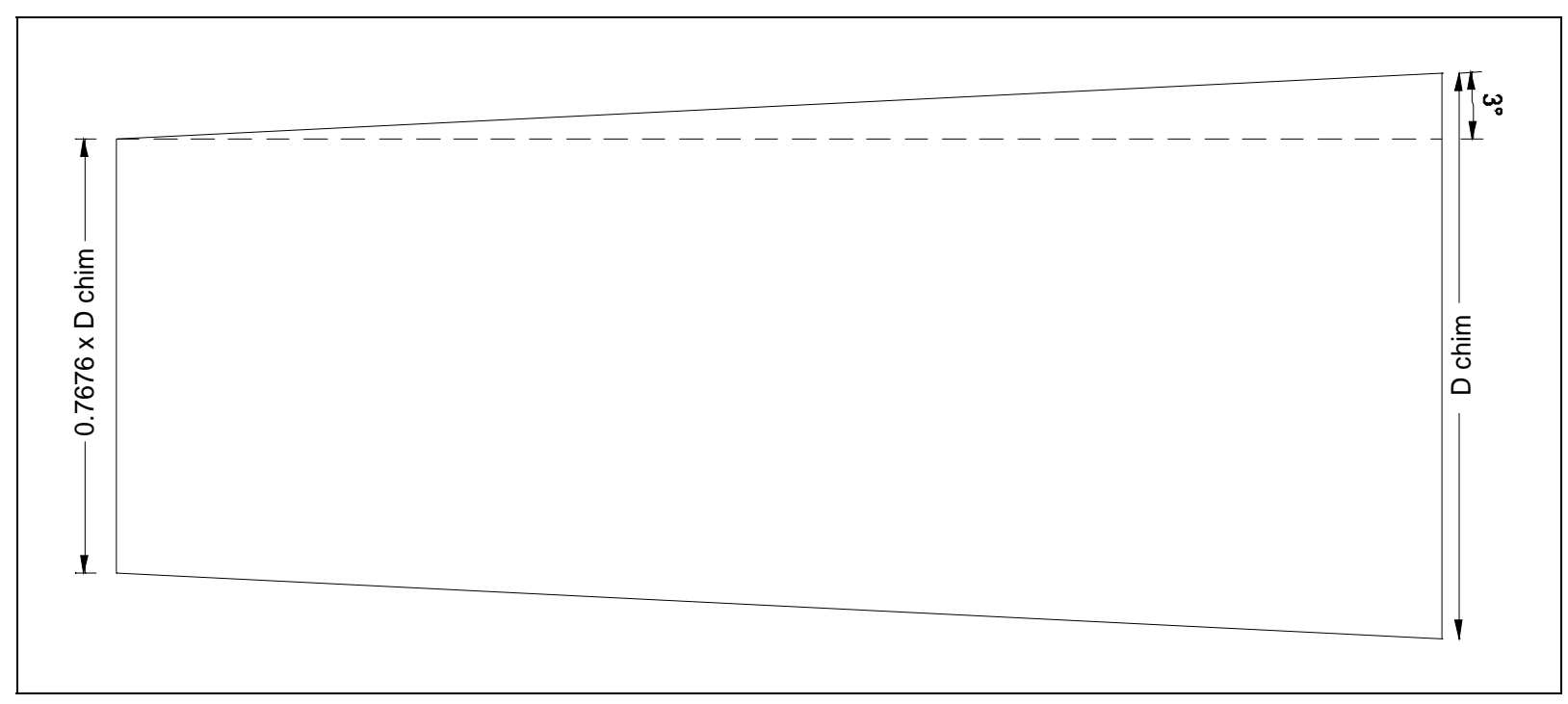

Figure 3.35. Turbine diffuser (true aspect ratio) showing expansion angle. 
As has been stated a number of times, decreasing the exit kinetic energy from the turbine is essential in obtaining a high efficiency turbine. Due to the turbine diameter being 0.7991 (Table 3.5) of the chimney diameter, the addition of a diffuser will allow a significant proportion of the turbine exit kinetic energy to be recovered. A very conservative approach was taken to the diffuser design to ensure that it worked correctly and as the chimney is extremely high the diffuser can be long. It is expected that with the exit swirl, high velocity at the turbine tip and shallow diffuser angle that the flow should not separate and significantly decrease the exit kinetic energy. The reference, Runstadler et al. (1975) was used to calculate the required diffuser length and angle. Figure 3.35 shows the diffuser section and its dimensions relative to the chimney diameter. A very low expansion angle, $3^{0}$ (included angle $=6^{0}$ ), has been used to ensure that the flow does not separate.

\subsection{CONCLUSION}

A schematic similar to Figure 3.4 is shown in Figure 3.36 but in this case the size of the components relative to the chimney diameter is correct. The numerical values of the various components relative to the chimney diameter can be found in Table 3.5.

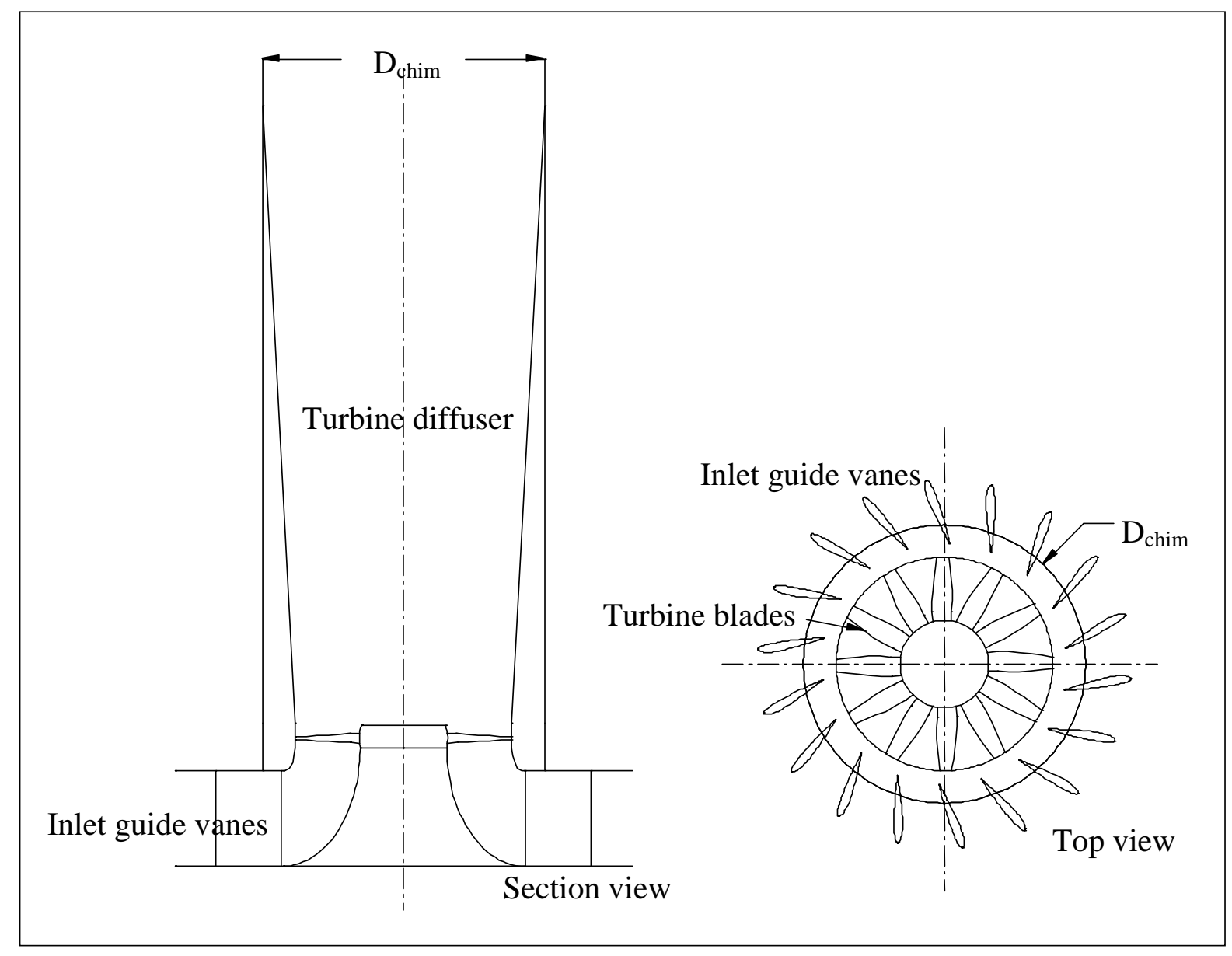

Figure 3.36. Section and top view of solar chimney turbine. 
This concludes the design process of the solar chimney turbine where a general and fundamental approach has been taken, as there are no existing turbines of similar size and operating point to compare to. To summarise the process, the turbine operating range was obtained from the cycle analysis followed by a free vortex analysis to obtain the overall plant dimensions. The Matrix Throughflow Method (MTFM) predicted the required gas flow angles for the inlet guide vanes and turbine blades. These angles were then used in conjunction with a surface vortex method to design the blade profiles. The next chapter covers the design of the experimental apparatus used to test a scale model of the turbine to verify the design method. 


\section{CHAPTER 4: EXPERIMENTAL APPARATUS DESIGN AND OPERATION}

In the previous chapter the design of the solar chimney turbine was covered. To check the design method and turbine design, it is necessary to verify the design experimentally. This chapter covers the development of the experimental apparatus used to verify the design.

The initial part of the chapter outlines the experimental objectives as these govern how the experiment is performed and run. Following this the conceptual design of the experimental layout is presented. Once these two tasks are completed the physical sizes of the components in the rig are defined. This is a compromise between a manageable size scale model while still being able to scale the results to the full-size plant.

The next section covers the practical aspects of the experimental installation beginning with the design and construction of the experimental set up followed by the instrumentation. The instrumentation is obviously important as the quality of the measurements determines how effective the experiment is.

The final sections deal with the experimental strategy used to determine the turbine performance over the entire operating range. From this the most effective operational set up is found. The starting characteristics of the turbine were also tested.

\subsection{EXPERIMENTAL OBJECTIVES}

The main objective of the experimental program is to demonstrate that the solar chimney turbine can operate efficiently over the required range of mass flows and pressure drops. The experimental program will be used to quantify the turbine performance as well as gain a better understanding of the turbine operation. The experimental results will also be able to find differences in predicted and real operation and allow further improvements of the turbine design.

The experimental rig will also be used for other projects to test new concepts and changes to the turbine design in future projects, so for this reason the rig must be robust and easily adjustable.

The objectives are:

- Demonstration that the solar chimney turbine operates effectively over the predicted design range.

- Quantification of the performance of the turbine over the design range and also performance outside of the design range.

- Verification of design performance prediction and comparison of simulated and experimental results.

- Verification of design assumptions and possible modification of these. 
- Use of results to improve turbine prediction model and suggestion of improvements to turbine design.

- Investigation of starting performance.

\subsection{LAYOUT OF EXPERIMENTAL RIG}

The experimental rig must obviously satisfy the design objectives listed above and allow for detailed investigation of the turbine performance. The concept of the solar chimney power plant has already been proven by the construction of a small working plant in Manzanares, Spain, as reported by Schlaich et al. (1995) that demonstrated that the solar chimney concept does work. For this reason, the present experimental rig only investigates the turbine performance. This allows just the turbine performance to be investigated without having other environmental variables influencing the results. A further reason is that small solar chimney power plants have very small pressure drops over the turbine meaning that the design would be significantly different to a full size one. In the experimental turbine rig it is possible to run the scale model at a similar tip speed and pressure drop as in the full-scale plant allowing the results to be more accurately scaled and applied to the full-scale plant.

\section{Basic Experimental Layout}

The experimental turbine layout consists of a short turbine inlet section, essentially the exit section of the solar collector, the turbine and ducting and part of the diffuser section. To drive the experimental turbine air, is sucked through it by a fan to simulate the effect of the chimney draught. Sucking air through the turbine also ensures that the airflow into the turbine is uniform while when blowing air some method of ensuring this would have to be devised. A similar test rig was constructed by Schlaich et al. (1995) to investigate the flow through various turbine layouts but without a working turbine in the model. He used mesh to simulate the pressure drop with the flow being sucked though the mesh. The present experimental rig adds a working turbine into the model.

Figure 4.1 is a schematic of the experimental rig showing the major components and is taken from the design drawings, so scale and proportions are correct. The air is sucked by a fan above the main table exhausting to the outside of the building thus avoiding re-circulating the laboratory air. Recirculating air can lead to a steady temperature increase in the lab from the fan power which is about $7.5 \mathrm{~kW}$. The possibility of swirling flows exiting the fan being ingested by the turbine is also avoided. The air enters the rig radially around the edges of the table and proceeds through the inlet guide vanes that add pre-swirl to the flow before it enters the turbine. Here the working scale model extracts energy from the flow. Once the flow exits from the turbine it enters the diffuser section where some the exit kinetic energy is recovered to increase the static pressure. This is the end of the experimental section, the ducting after this is designed to have minimum losses so that the fan is as effective as possible. The bend in the pipe contracts so that the flow accelerates through this, 
ensuring that the flow does not separate. After the fan there is a diffuser section to increase the volume flow rate through the system by recovering the exit kinetic energy from the fan.

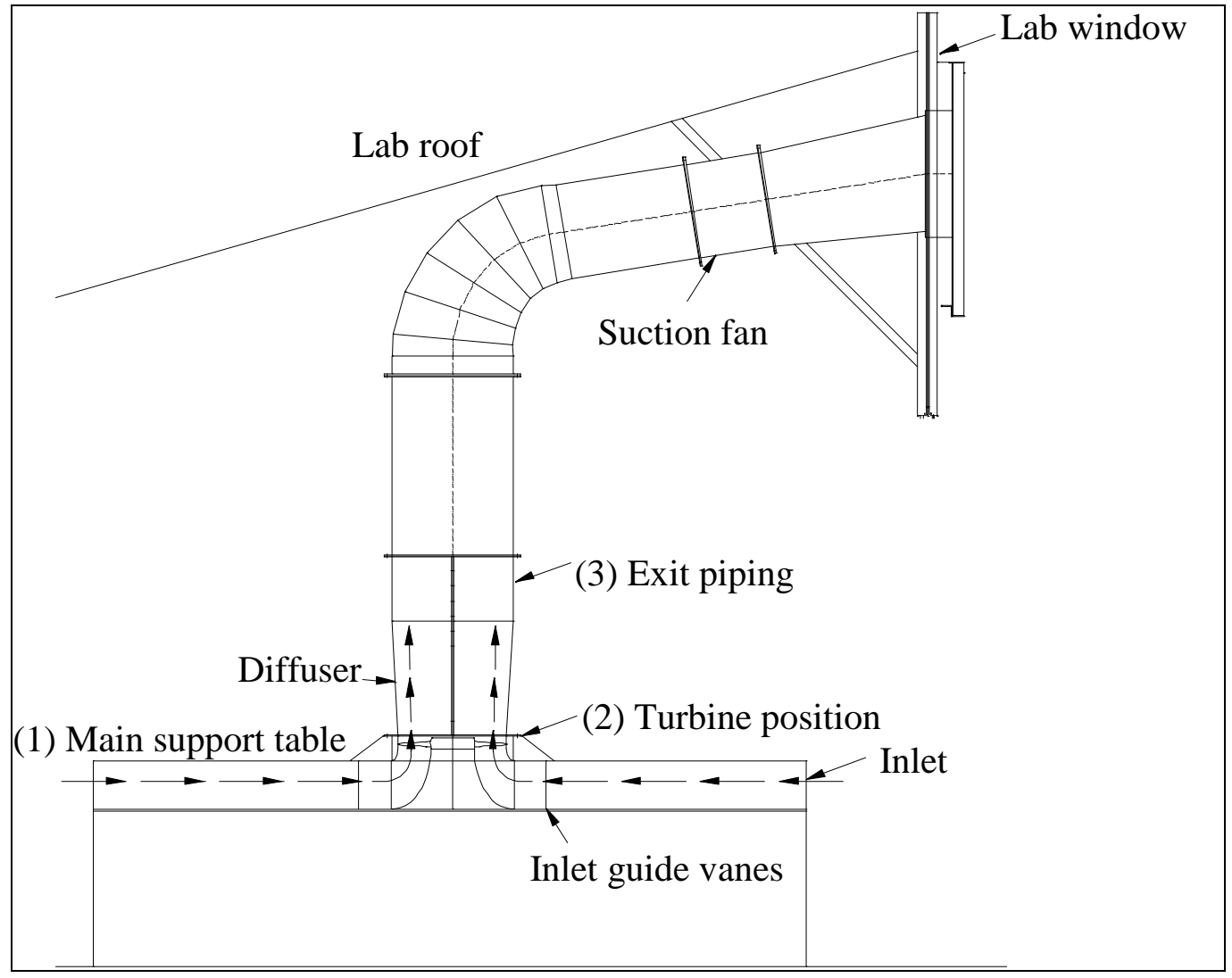

Figure 4.1 Schematic of solar chimney turbine experimental rig.

There is ample room under the table to allow for instrumentation from the turbine to measure the torque and turbine speed. Allowance also has to be made for absorption of the power from the turbine but this is dealt with in the next section.

\subsection{EXPERIMENTAL RIg SIZING AND APPARATUS}

In any scale model a decision has to be made on the eventual size of the model. Usually the closer the model size to the real plant the more representative the results. Obviously here there is a large difference in the scale model and real solar chimney turbine size. As with the turbine design itself a fairly systematic approach to the size of the model was taken. Not all the design iterations will be shown in detail as they are not important in the actual operation of the turbine, just the initial design points and the final design sizes will be given.

\section{Initial Sizing}

Early in the design process it was decided to use standard industrial duct sizes. The following four sizes were investigated: $630 \mathrm{~mm}, 800 \mathrm{~mm}, 1000 \mathrm{~mm}$ and $1250 \mathrm{~mm}$. The $1000 \mathrm{~mm}$ and $1250 \mathrm{~m}$ 
fan sizes were quickly discarded as the power requirements for the required volume flow rate at the relevant pressure drop was approximately $30 \mathrm{~kW}$. This meant that the fan motors could not be mounted in the pipe but externally and would drive the fan using pulleys. The $800-\mathrm{mm}$ fan could quite easily provide the volume flow rate but not the pressure drop as the maximum speed of this fan size was $1440 \mathrm{rpm}$. The $630 \mathrm{~mm}$ fan is able to operate at $2880 \mathrm{rpm}$ to provide the required pressure drop and volume flow. It was decided to keep the nominal pipe diameter $800 \mathrm{~mm}$ below the pipe bend (Figure 4.1) as this resulted in a larger scale model turbine that is simpler to work with.

In the previous chapter the initial design of the turbine was as follows (table repeated for clarity),

Table 4.1. Initial turbine and inlet guide vane dimensions relative to chimney.

\begin{tabular}{|r|c|c|c|c|c|}
\hline & $\mathrm{D}_{\text {chim }}$ & $\mathrm{D}_{\text {turb }}$ & $\mathrm{D}_{\text {hub }}$ & $\mathrm{H}_{\text {IGV }}$ & $\mathrm{H}_{\text {turb }}$ \\
\hline Fraction of chimney diameter & 1 & 0.9 & $0.4 \mathrm{D}_{\text {turb }}$ & 0.4 & 0.5 \\
\hline Actual size $[\mathrm{m}]$ & 160 & 144 & 57.6 & 64 & 80 \\
\hline
\end{tabular}

It was decided to build the rig to these dimensions. This resulted in the experimental turbine diffuser not expanding as much as the full-scale plant is expected to. The main reason this was done was to keep the turbine size as large as possible to make measuring more accurate. To be able to apply the measured turbine efficiency including the diffuser to the full scale plant the experimental diffuser efficiency is measured. This efficiency is then applied to the longer diffuser that has a higher pressure recovery. The only problem with this approach will be if the diffuser stalls. Due to the very conservative design used this is unlikely. The experimental results will be conservative when compared to the full-scale turbine. Recall that the full-scale turbine diameter is $0.7991 \mathrm{D}_{\text {chim }}$.

\section{Operating Range}

To find the required operating range of the experimental rig, the performance characteristics of the full-size turbine were scaled down to the dimensions of the rig, using the fan scaling laws.

$$
\begin{aligned}
\text { Power } \mathrm{P}_{\text {exp }} & =\mathrm{P}\left(\frac{\rho_{\text {exp }}}{\rho}\right)\left(\frac{\mathrm{rpm}_{\text {exp }}}{\mathrm{rpm}}\right)^{3}\left(\frac{\text { Diam }_{\text {exp }}}{\text { Diam }^{5}}\right)^{5} \\
\text { Pr essure } \mathrm{P}_{\text {exp }} & =\mathrm{P}\left(\frac{\rho_{\text {exp }}}{\rho}\right)\left(\frac{\mathrm{rpm}_{\text {exp }}}{\mathrm{rpm}}\right)^{2}\left(\frac{\text { Diam }_{\text {exp }}}{\operatorname{Diam}^{2}}\right)^{2} \\
\text { Vol flow } \mathrm{Q}_{\text {exp }} & =\mathrm{Q}\left(\frac{\mathrm{rpm} \text { exp }}{\mathrm{rpm}}\right)\left(\frac{\text { Diam }_{\text {exp }}}{\text { Diam }^{3}}\right)^{3}
\end{aligned}
$$

In the previous chapter three main operating points were defined and are repeated here. It must be recalled that for the cycle analysis the turbine total-to-static efficiency was assumed to be $80 \%$. This value is used in the design of the experimental set up to ensure that the suction fan will deliver 
the correct pressure and volume flow rate over the turbine. It is expected that the real turbine will have a higher efficiency and so deliver more power for the same pressure drop.

Table 4.2. Experimental rig operational parameters.

\begin{tabular}{|c|c|c|c|c|c|c|}
\hline & \multicolumn{3}{|c|}{ Full-size plant } & \multicolumn{3}{|c|}{ Experiment-size rig } \\
\hline $\begin{array}{l}\text { Op. mode } \Rightarrow \\
\text { Variable } \Downarrow\end{array}$ & Case (1) & Case (2) & Case (3) & Case (1) & Case (2) & Case (3) \\
\hline Diameter [m] & \multicolumn{3}{|l|}{127.86} & \multicolumn{3}{|l|}{0.72} \\
\hline Tip speed [m/s] & \multicolumn{3}{|l|}{88.63} & \multicolumn{3}{|l|}{44.99} \\
\hline Rot. speed [rpm] & \multicolumn{3}{|l|}{13.24} & \multicolumn{3}{|c|}{$1175\left(123.1\right.$ rad.s $\left.^{-1}\right)$} \\
\hline Torque [Nm] & $35.7 \times 10^{6}$ & $141 \times 10^{6}$ & $141 \times 10^{6}$ & 1.83 & 7.23 & 8.28 \\
\hline Density $\left[\mathrm{kg} / \mathrm{m}^{3}\right]$ & 1.07 & 1.05 & 0.94 & 1.2 & 1.2 & 1.2 \\
\hline Pressure drop [Pa] & 375 & 994 & 2623 & 105 & 285 & 839 \\
\hline Vol. flow $\left[\mathrm{m}^{3} / \mathrm{s}\right]$ & 168360 & 245150 & 105350 & 2.67 & 3.89 & 1.67 \\
\hline Power output [W] & $50.6 \times 10^{6}$ & $200 \times 10^{6}$ & $200 \times 10^{6}$ & 225 & 890 & 1019 \\
\hline
\end{tabular}

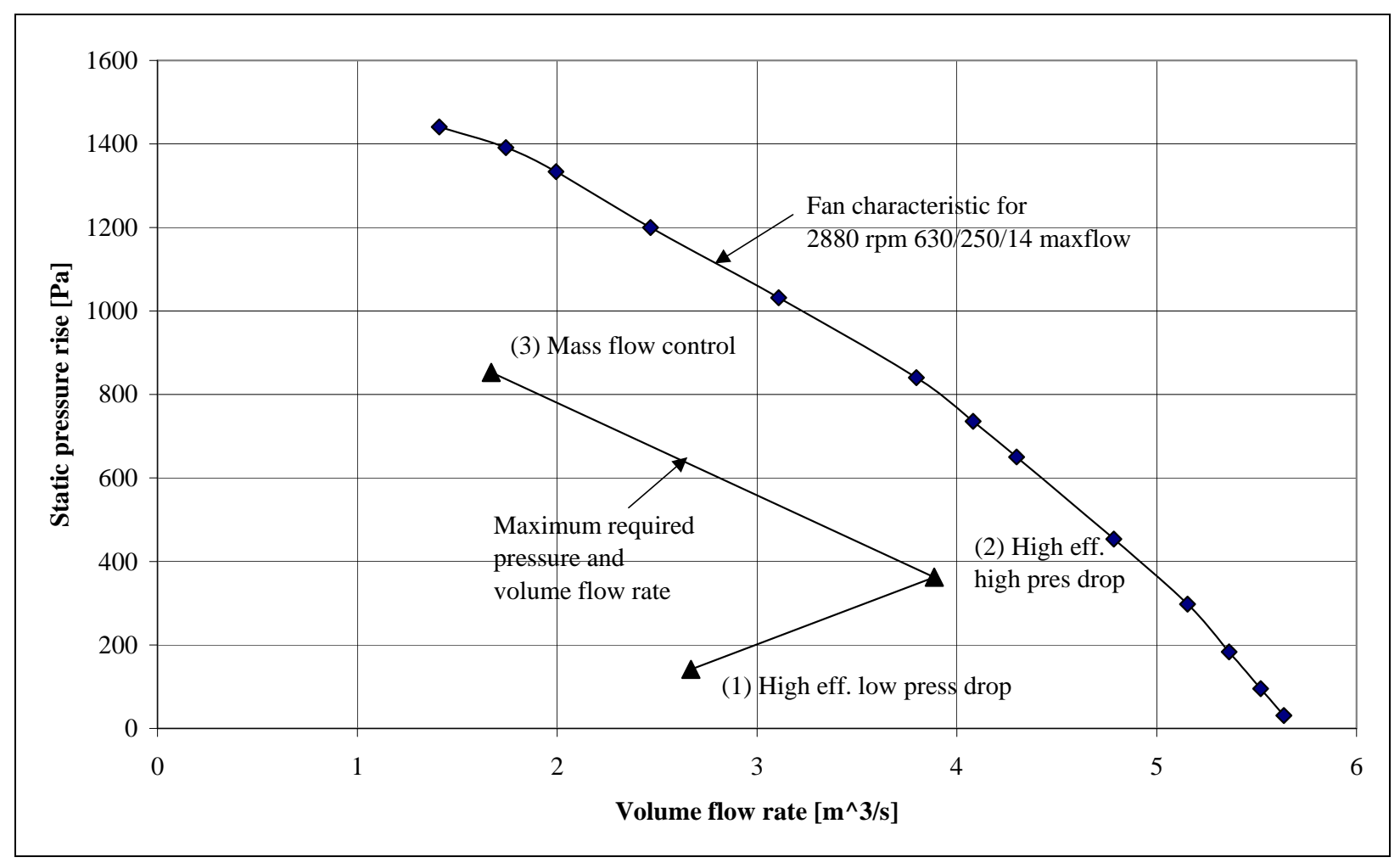

Figure 4.2. Performance characteristics of suction fan on experimental rig.

In Table 4.2 the scaling laws are applied to the full-scale plant to calculate what the required mass flows and pressure drops are in the experimental rig. The two main factors are the turbine rotational speed and diameter. In the experimental rig there was concern about the rotational speed of the turbine being too high and so the tip speed was reduced by a factor of 2 over the tip speed of the full scale turbine. The construction and allowable stress of the turbine blades is described in detail 
later. The air density does influence the operation of the experimental rig as in the real plant the air under the glass collector heats up and is less dense than the surroundings while in the experiment, atmospheric air is simply drawn through the turbine. This effect is most noticeable case 3 where the mass flow needs to be controlled as this is when the temperature under the glass collector would be highest with a corresponding low air density. The fan used to suck the air through the turbine must be able to provide the pressure drop at the required volume flow rate listed in the previous table. Both the required static pressure and dynamic pressure must be taken into account when calculating the fan pressure. Figure 4.2 shows the fan characteristic with the required operating range superimposed.

\subsection{DESIGN AND CONSTRUCTION}

The design of the experimental apparatus consists of a few modular items that are assembled to form the large unit. To begin a basic overview of the design is given that lists the main components and how they are assembled. This is to aid in the understanding of the individual parts while details of the operations of individual parts will be covered with each component. The design and construction of the basic rig is fairly simple and can be seen in the photographs. Major dimensions such as the inlet heights and lengths are given in the schematic drawings.

The overall rig consists of three main sections:

- the base table that supports the inlet of the turbine,

- the turbine ducting and turbine itself and

- the exit section after the turbine shown in Figure 4.1.

The base table, (1), provides the support of the lower and upper wooden decks that form the turbine inlet section. The base table surrounds the central smaller table that supports, (2), the turbine ducting and turbine with the instrumentation required to measure the turbine performance below. The exit section is supported from above through a system of I-beams and remains supported from above without having to rest on the table below. This allows the turbine diffuser to be taken out easily to gain quick access to the turbine for adjustment.

\section{(1) Base Table}

The base table consists of five main parts with a schematic assembly shown in Figure 4.3:

(1a) Lower frame, that supports the lower deck and surrounds the inner table (1b).

(1b) Inner table, into which the inlet guide vanes (1c) fit.

(1c) Inlet guide vanes that in turn support the upper ring (1d).

(1d) Upper ring, used to adjust the inlet guide vanes angle and support the upper frame (1e).

(1e) Upper frame from which the top decks hang.

(1f) Decking- wooden decking that forms the long inlet section of the turbine. 


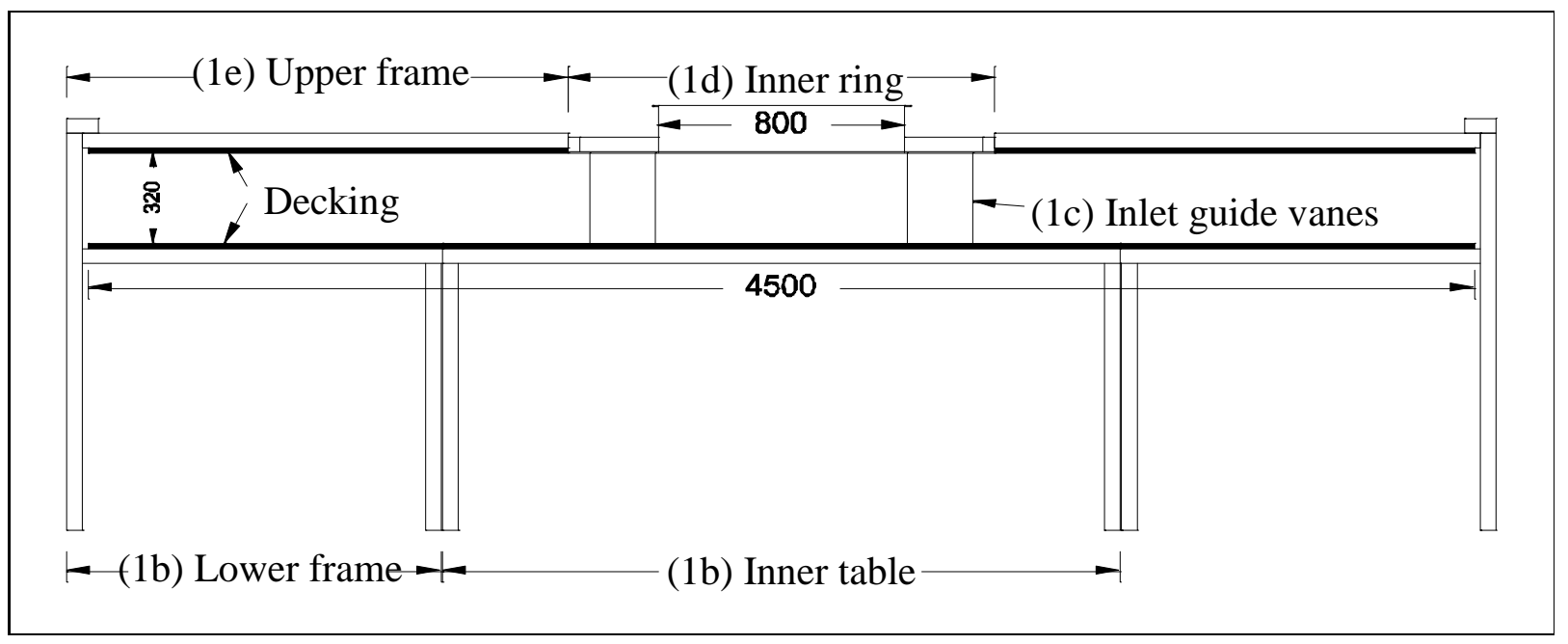

Figure 4.3. (1) Base table schematic showing basic components.

(1a) Lower Frame

The lower frame consists of three separate pieces that are fixed together to surround the inner table. The inlet surface is a smooth varnished wooden deck that is fixed to the lower and upper frame. To ensure that all the surfaces sit flush relative to each other, the table has adjustable feet for height adjustment. This also helps in the fitting of the lower table to the exit pipe. The construction of the frame is welded steel with the three components being bolted together for simple assembly and dismantling.

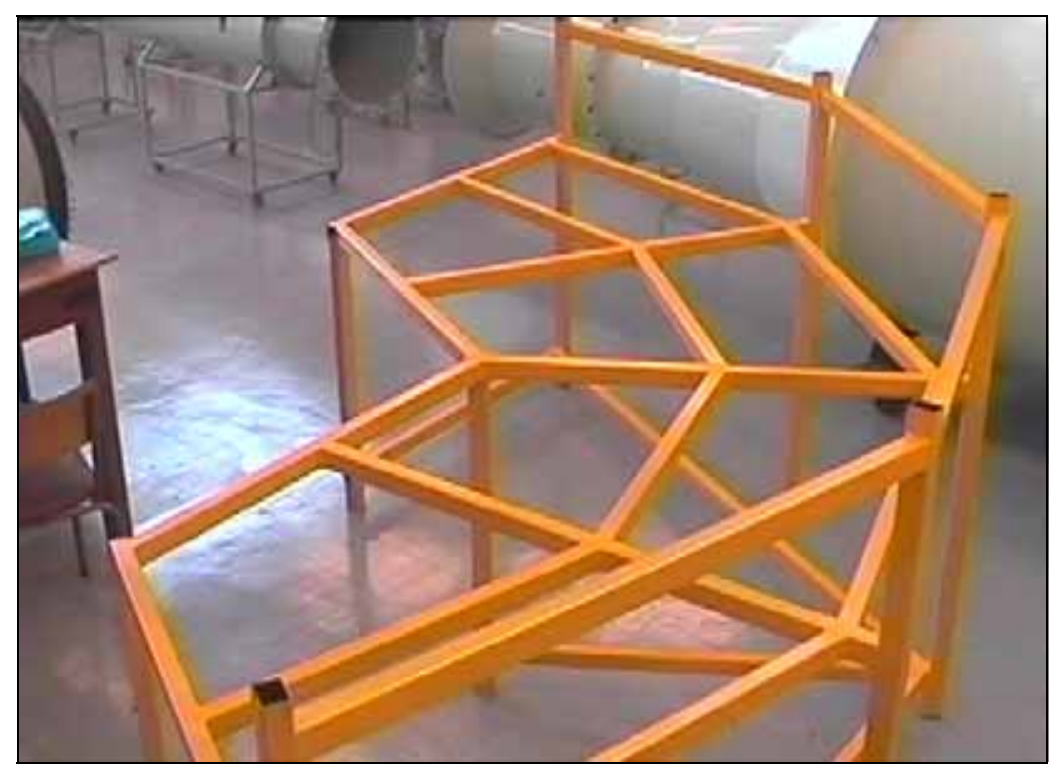

Figure 4.4. Outer table segment showing construction. 


\section{(1b) Inner Table}

The inner table construction is very similar to the construction of the outer frame with welded steel tube and a wooden deck. The wooden deck has an additional steel sheet over this to make it more durable as the inlet guide vanes stand on the table and support the upper frame and top decks. In addition to this, the turbine central cone and support shaft are fixed to the centre with the generator fixed below the table.

\section{(1c) Inlet Guide Vanes}

The main function of the inlet guide vanes is to add pre-swirl to the flow upstream of the turbine. Their primary function in the real plant is to support the chimney and in the scale model they support the inner ring upon which the upper frame rests. The construction of the blades required that the blade be the correct shape, structurally strong, and preferably manufactured economically. It was decided to use moulded blades with steel reinforcing rods within them for strength. The mould was cut out of aluminium on an NC milling machine and the blade material was a two component polyurethane with aluminium powder added. This was chosen as it was easily machinable for any post moulding work that needed to be performed.

A tensile test was performed on a specimen of the material and its ultimate strength was $30 \mathrm{MPa}$ with almost no plastic region, meaning the material properties stay constant until failure. For the inlet guide vane the material strength is not critical but for the turbine blades it is. This is covered in more detail in the turbine section.

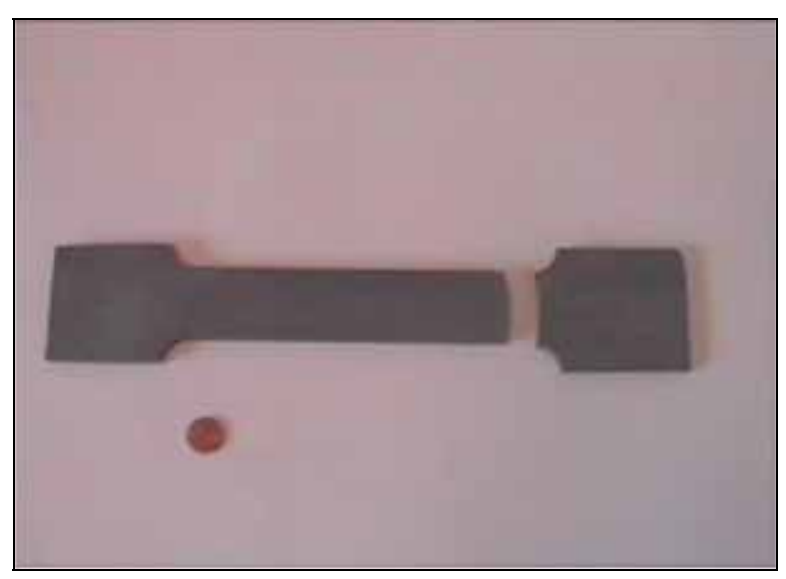

Figure 4.5. Tensile test specimen.

(1d) Upper Ring

The upper ring rests on the inlet guide vanes and supports the upper frame from which the upper deck hangs. The upper ducting ring, (2a), of the turbine is supported on the inside of the upper ring. The figure below shows the ring as seen from the bottom. The upper ducting ring that the turbine 
runs in can be seen in its centre. The centre table is hexagonal in shape with the outer frames fitting around this.

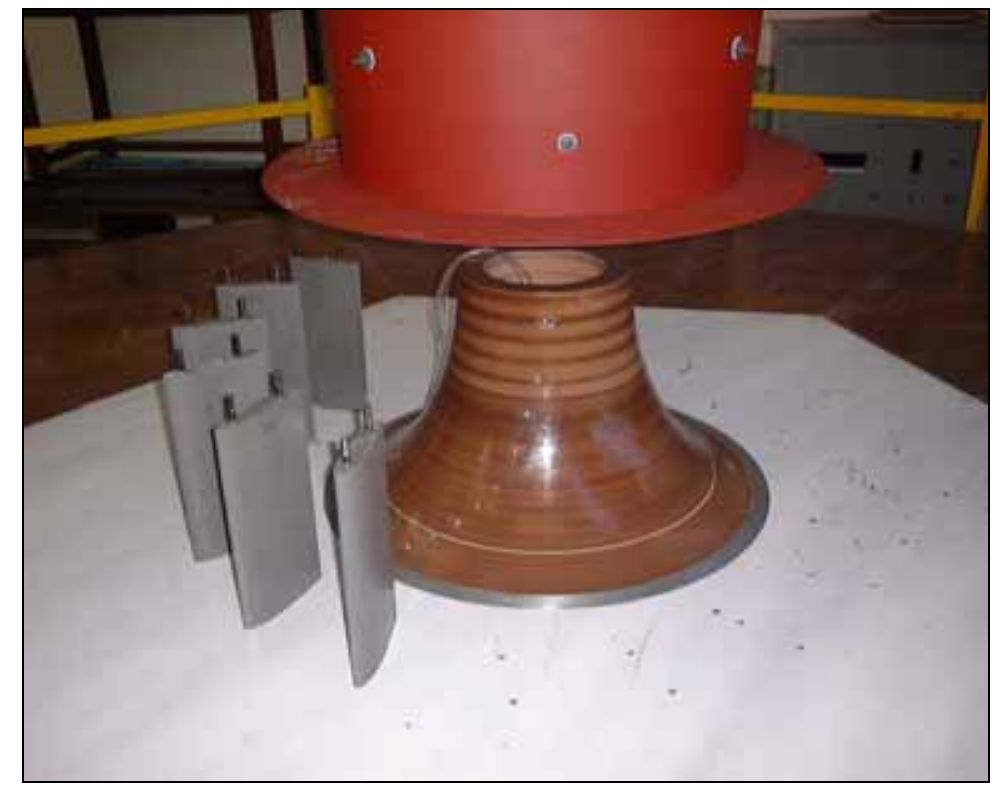

Figure 4.6. Moulded inlet guide vanes in position around central cone.

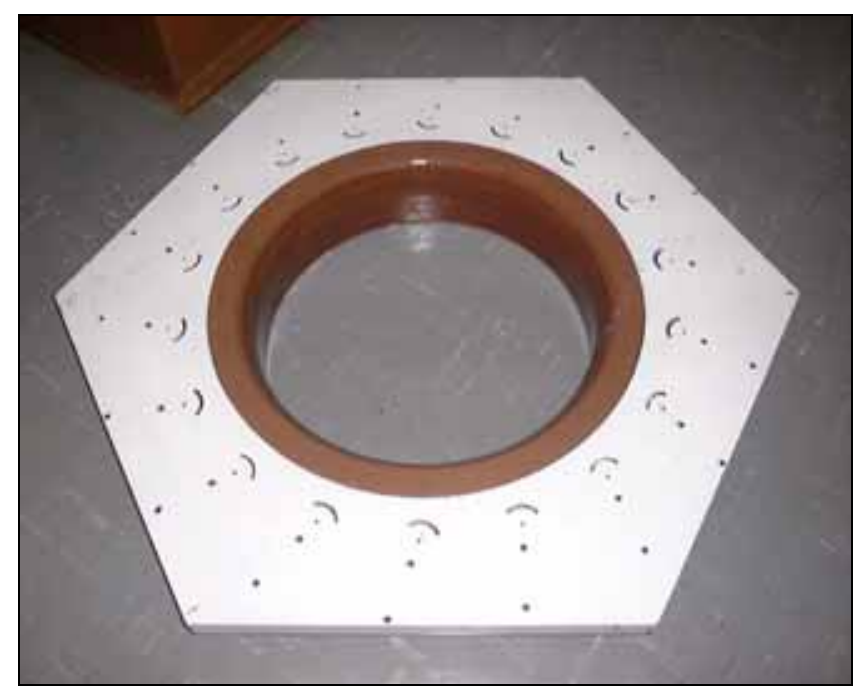

Figure 4.7. Upper ring showing the ducting leading into the diffuser.

(1e) Upper Frame

The upper frame's function is to support the top inlet decking that forms the long inlet duct. It also allows access to the inlet guide vanes as it can be walked upon. This speeds up the time taken to adjust the blade angles as the rig does not have to be taken apart to do this. 


\section{(1f)Decking}

The decking consists of varnished wooden plank to form a long inlet section for the flow to accelerate through before reaching the inlet guide vanes. As the flow is accelerating any distortions at the inlet to the rig should no longer be significant once the flow enters the IGVs. The outer edges of the deck are cut in a large circle and are also rounded to ensure that there is no separation of the flow at the inlet.

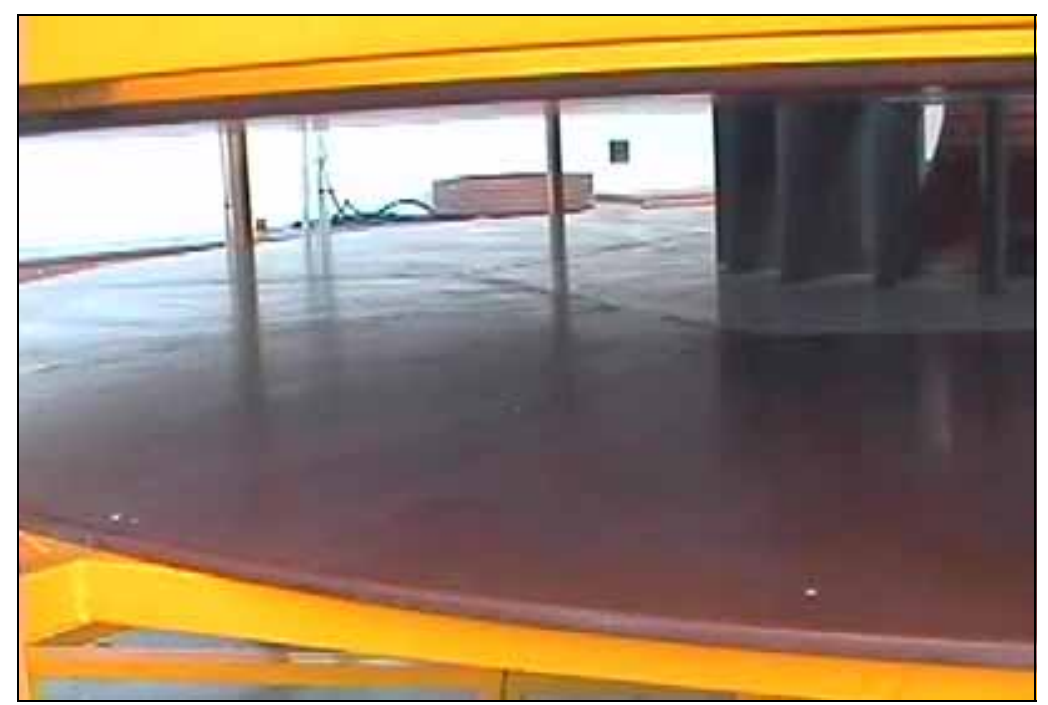

Figure 4.8. Inlet ducting leading into inlet guide vanes.

\section{(2) Turbine}

The turbine scale model with its instrumentation consists of seven main parts with the assembly schematic shown in Figure 4.9,

(2a) Upper ducting ring which fits inside the upper ring (1c).

(2b) Base cone that along with the upper ducting ring (2a) forms the turbine inlet duct.

(2c) Central shaft connecting the turbine with the instrumentation, (2f), and generator, (2g).

(2d) Turbine hub that rests on the central shaft, (2c), and supports the turbine blades, (2e).

(2e) Turbine blades.

(2f) Instrumentation, speed reader and torque transducer used to measure the turbine performance.

(2g) Generator, absorbs the power from the turbine.

\section{(2a \& 2b) Upper Ducting Ring and Base Cone}

The duct between the inlet guide vanes and turbine is formed by the upper ducting ring, (2a), and base cone, (2b). Their function is purely to guide the flow from the IGVs into the turbine blades and so their geometric shape is important. They are both made from layers of wood, glued together and then turned on a lathe to obtain the correct shape. Layers of varnish were applied and sanded to obtain a smooth finish. The cone in position between the inlet guide vanes can be seen in Figure 4.6. 


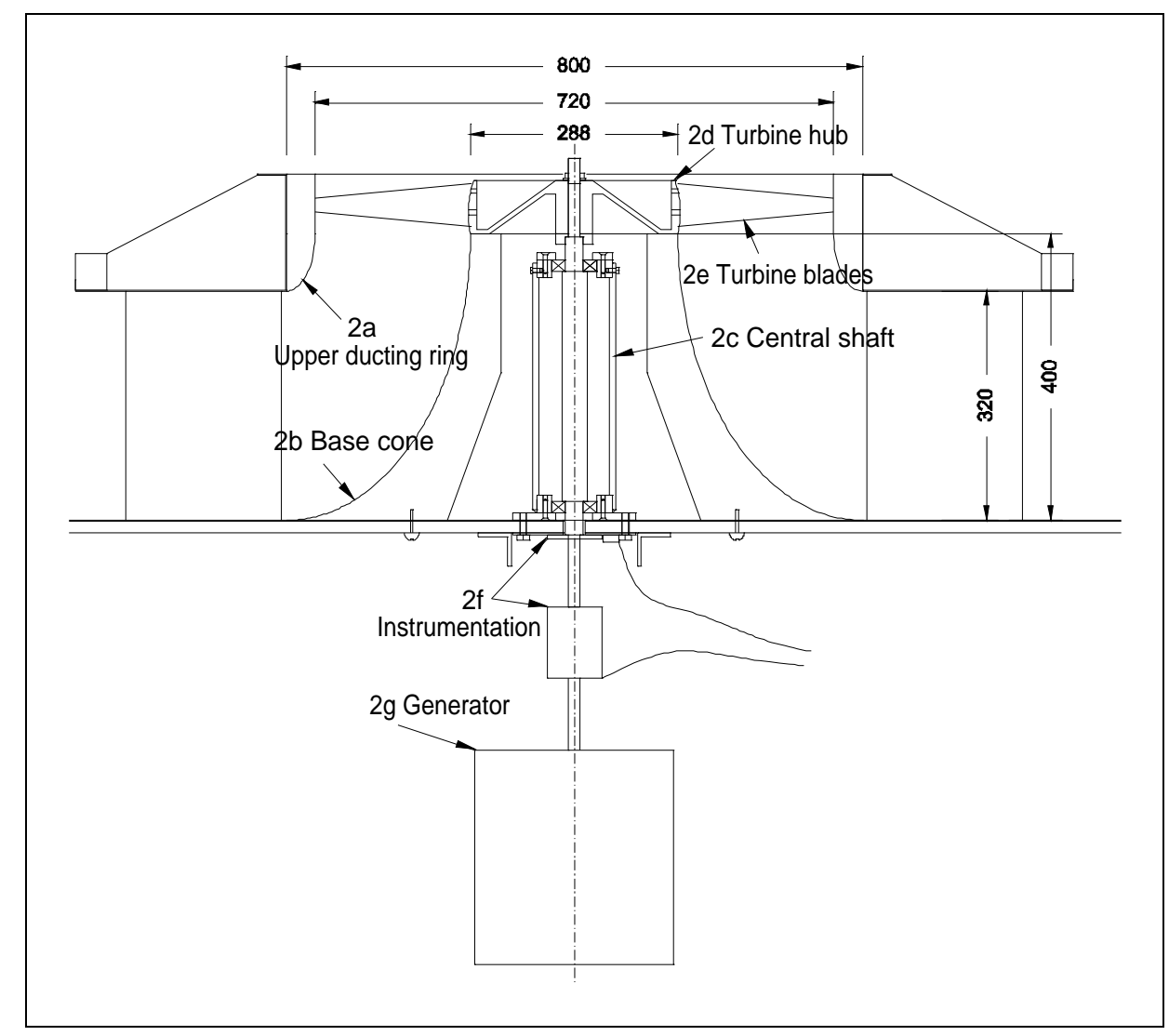

Figure 4.9. (2) Scale turbine model schematic showing basic components.

\section{(2c) Central Shaft}

The central shaft's primary function is to transmit the power from the turbine to the generator below. It runs on angular contact ball bearings as the main load is in the axial direction and this ensures that the friction is as low as possible and the outer pipe provides a stable base. The shaft design allows the turbine or the instrumentation to be removed separately without having to dismantle the entire arrangement.

\section{(2d) Turbine Hub}

The hub is machined from a solid piece of aluminium and rather than being cylindrical in shape is a section of a sphere. Adjustment of the stagger angle is made through the use of two rods in the turbine blade. With only one support rod there is always the possibility that the torque applied during tightening could twist the rod in the material thereby changing the reference angle and resulting in an incorrect stagger angle. With two rods there is only an axial load applied to each and the setting of the stagger angle is greatly simplified as the rods can be used for the reference points. The centre shape of the hub ensures that it is axial and torsionally stiff and also allows the turbine stagger angles to be changed without having to remove the hub from the shaft. The rod slots in the hub were sealed with masking tape during the experiments. 


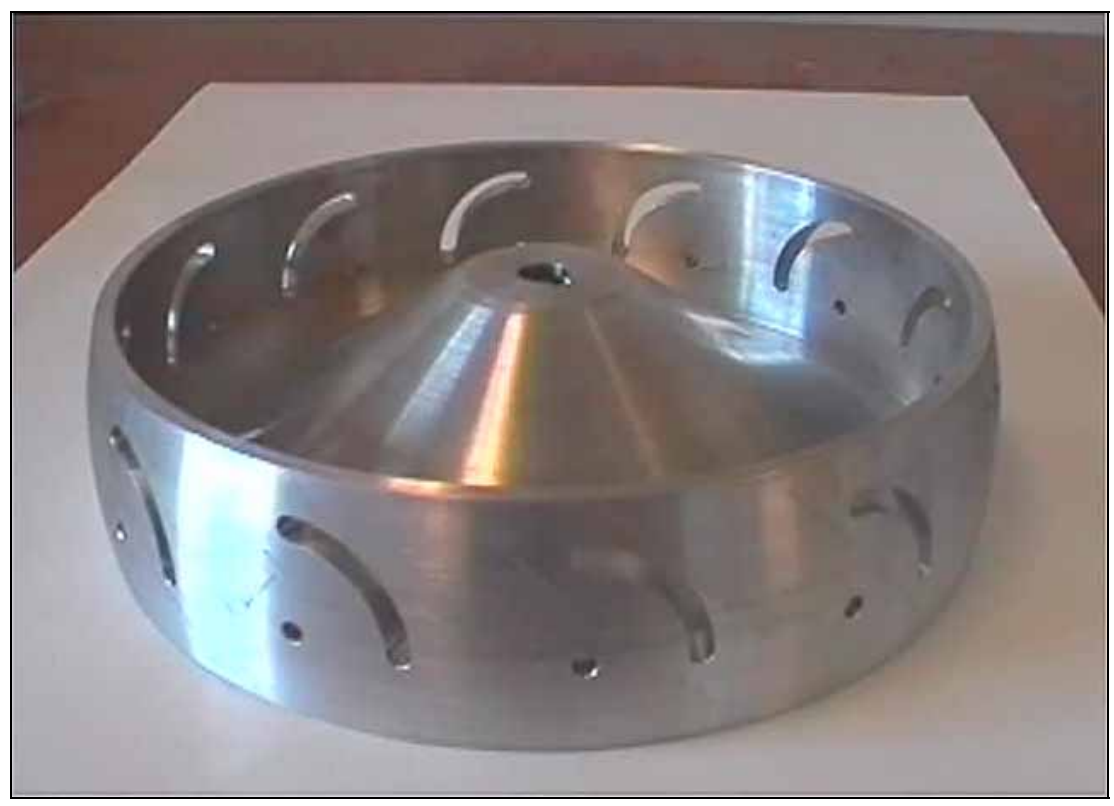

Figure 4.10. Turbine hub showing spherical shape and grooves for adjustable stagger angles.

\section{(2e) Turbine Blades}

The construction of the turbine blades is similar to that of the inlet guide vanes, a polyurethane with aluminium power. As mentioned when discussing the hub, two reinforcing rods are used and are used for setting the stagger angles. The moulds are more complex than for the inlet guide vanes due to the section changing over the blade length and the blade base being spherical to fit onto the hub. The pictures below (Figure 4.11 and Figure 4.12) show the moulds and turbine blades respectively. The main load on the turbine blades is bending due to upward force on the blades and results in a maximum stress of $5 \mathrm{MPa}$ at the experimental speed. The centrifugal stress is of the order of 2 MPa.

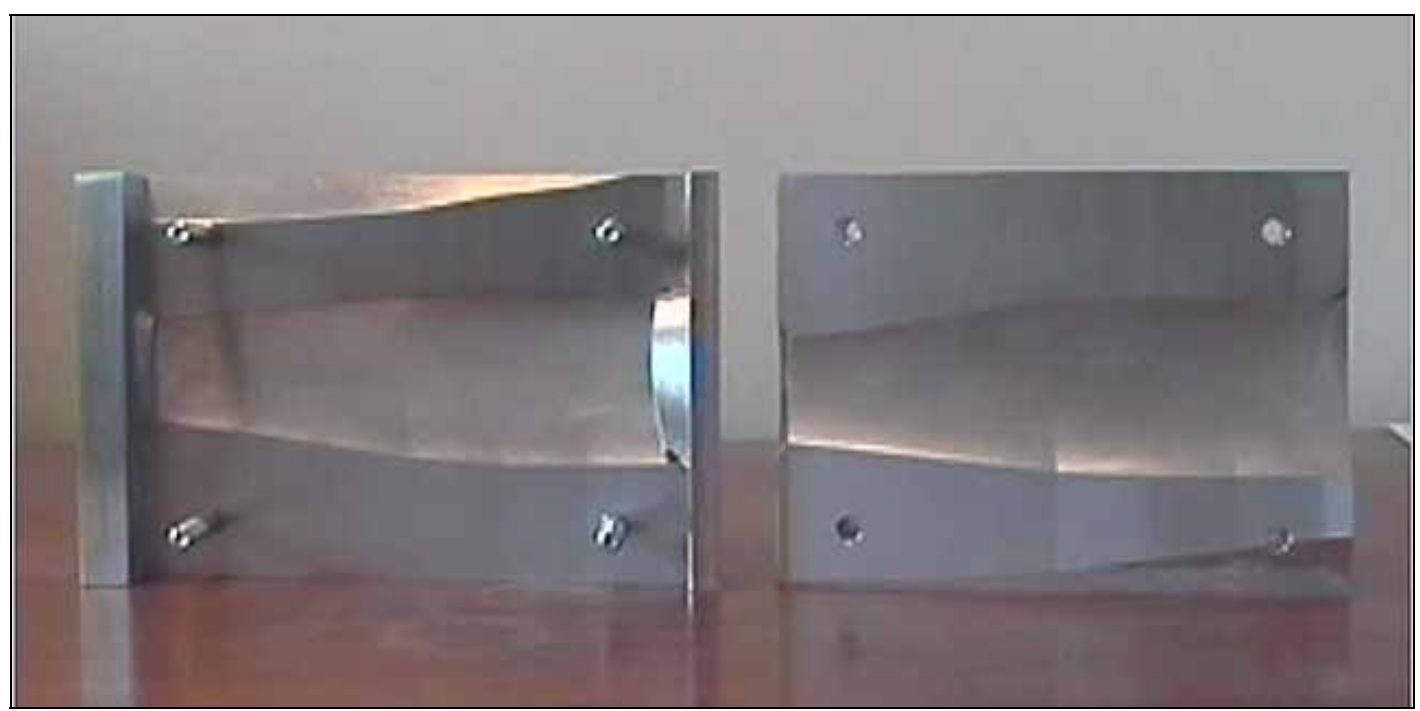

Figure 4.11. Turbine blade moulds. 


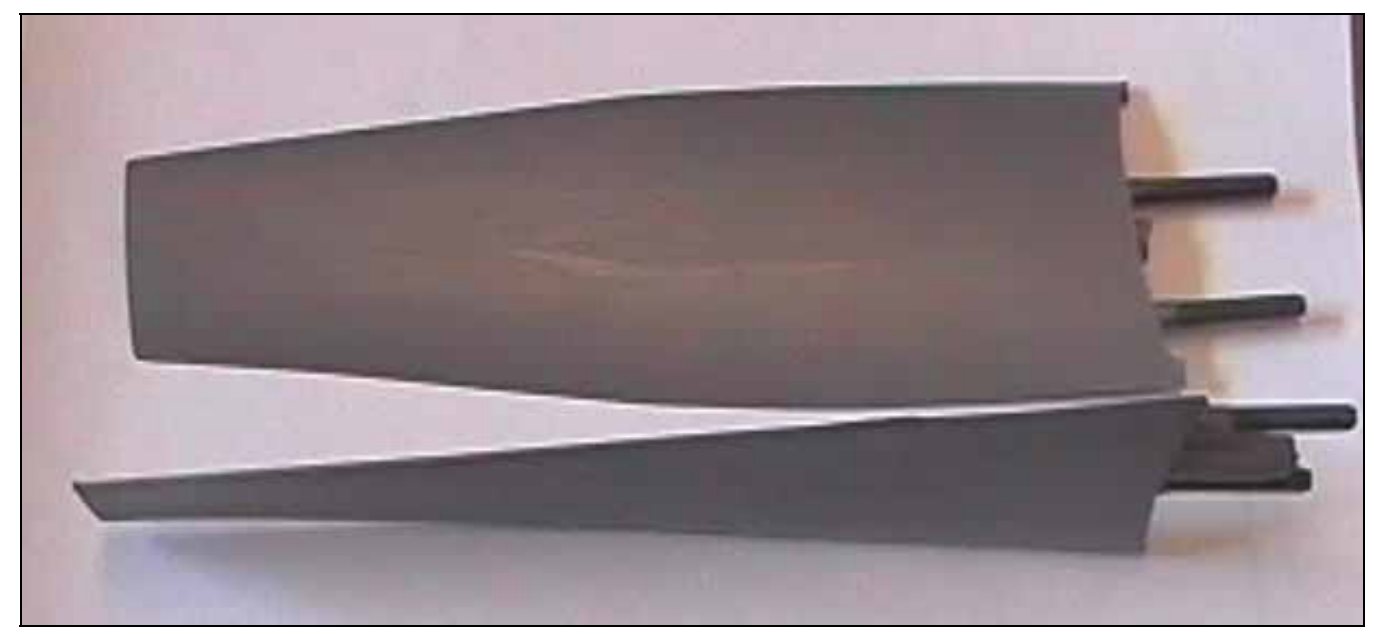

Figure 4.12. Turbine blades.

\section{(2f) Instrumentation}

To measure the turbine performance, a speed-reader to measure the rpm and a torque transducer are coupled to the lower end of the shaft. These two readings are used to calculate the turbine power. The two bearings in the shaft will have a certain amount of friction but this can be measured by running the shaft without the turbine and the friction loss added to the turbine output power. The speed reader is above the torque transducer as for runaway tests the torque transducer must be uncoupled allowing only the speed to be read. The details of the operation of the speed reader and torque transducer will be covered along with the other instrumentation.

\section{(2g) Generator}

To control the speed of the turbine and apply a torque, an induction motor is used as a generator. It is used purely as a brake and was chosen for its ease of use. The generator is connected to a variable speed drive with a brake resistor used to disperse the turbine power as heat.

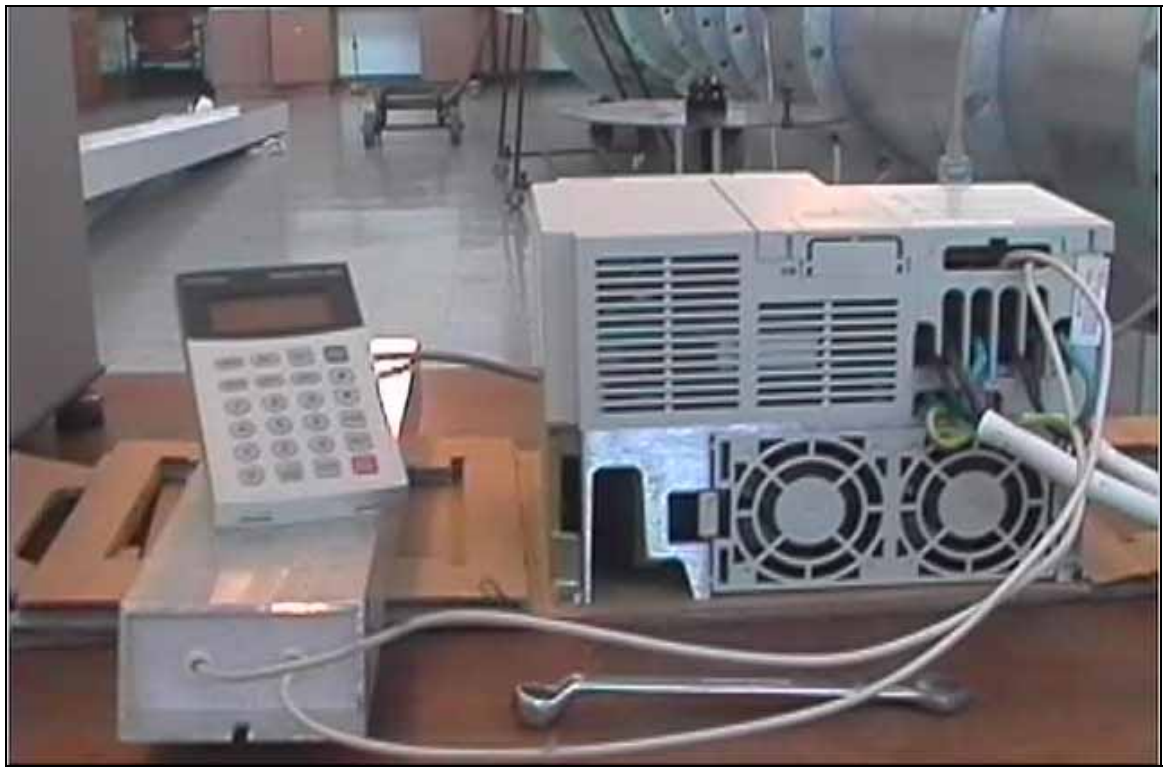

Figure 4.13. Generator coupled to variable speed drive with brake resistor. 


\section{(3) Exit Section}

The exit piping is supported by beams fixed to the building and consists of the following:

(3a) Turbine diffuser, expands from the turbine exit to the exit pipe (3b).

(3b) Exit pipe, a straight length of pipe in which the downstream pressure is measured.

(3c) Elbow bend, contracting bend to attempt to minimise losses in the corner.

(3d) Suction fan.

(3e) Fan diffuser, allows the fan to provide a higher pressure drop.

(3f) Louvers, allow adjustment of the fan pressure to be made.

A schematic of the outlet section is presented in Figure 4.14, showing the basic support structure allowing the diffuser to be removed while the top pipe remains above the table.

(3a) Turbine Diffuser

The diffuser on the experimental rig is shorter than the one that will be on the full-scale plant. It will result in some pressure recovery but not as much as on the real plant. Separate tests of longer diffusers will be carried out to see how well they perform and these results used to estimate the improvement in turbine performance with the full length diffuser.

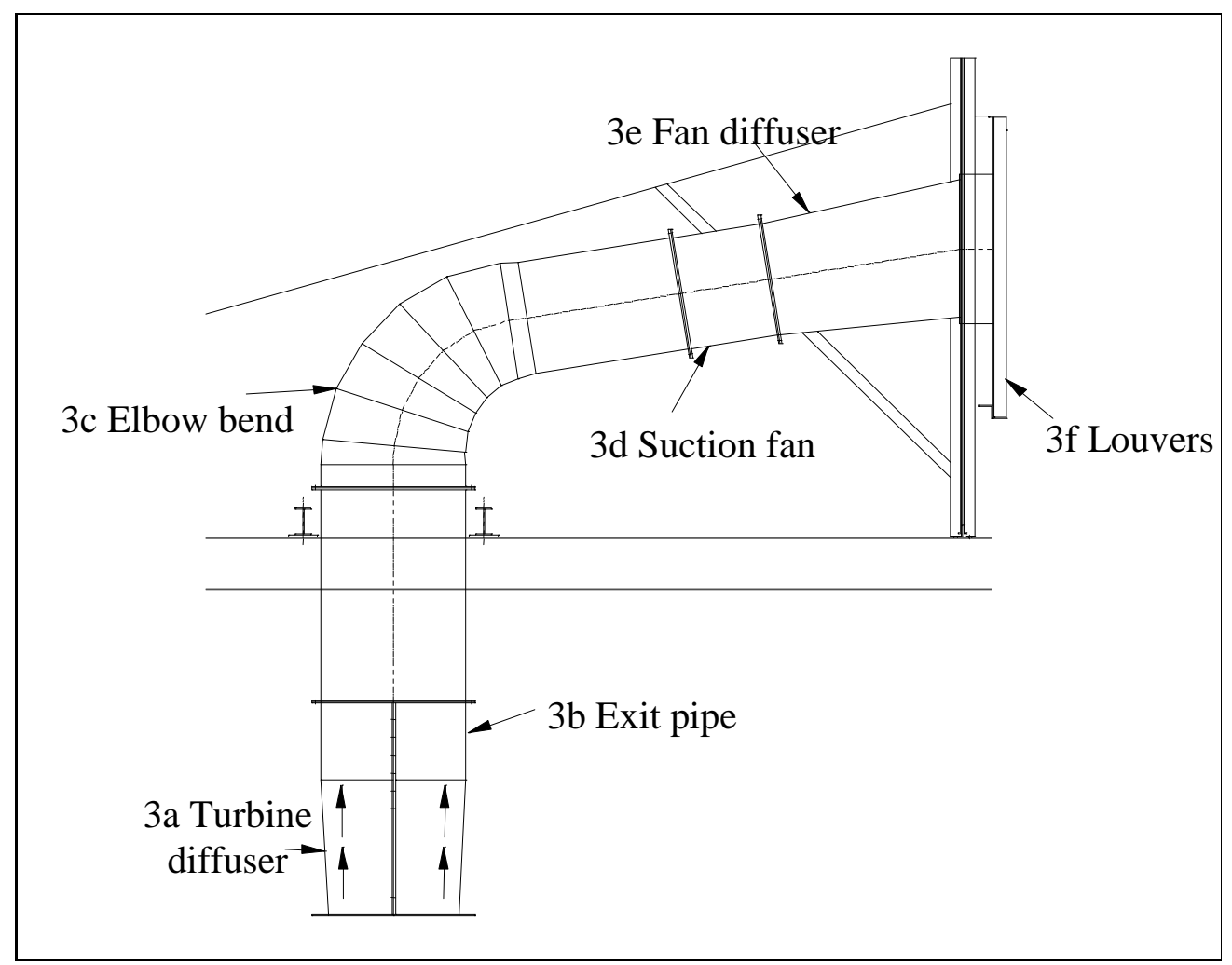

Figure 4.14. Turbine exit piping schematic showing support structure. 


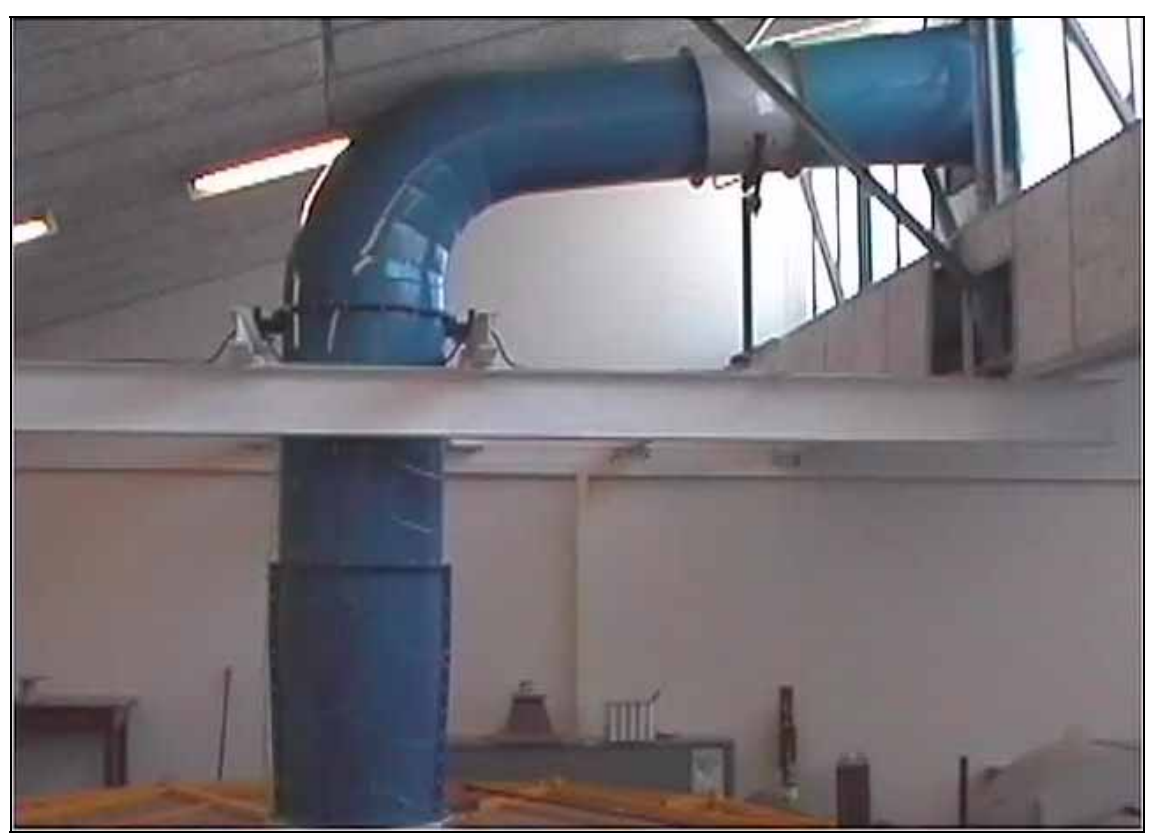

Figure 4.15. Turbine exit piping.

\section{(3b) Exit Pipe}

The exit pipe consists of a long straight section to allow any transient flow patterns to dissipate so that static pressure readings can be accurately taken. The performance of the turbine as an entire system is to be investigated, basically the efficiency is calculated as the actual power output due to the pressure drop from the inlet guide vanes up to the exit pipe. This is done to test the overall turbine efficiency as it is possible to design individual components of high efficiency but have to have an overall efficiency lower than expected.

\section{(3c, 3d, 3e \& 3f) Elbow bend, Suction Fan, Fan Diffuser \& Louvers.}

The fan is not directly involved with the experiment except to suck the flow through the turbine. This system has been designed to have as few losses as possible with the elbow bend contracting to accelerate the flow through the corner. A diffuser is placed after the fan to increase the volume flow rate through the turbine and the louvres used are able to open completely for when the maximum volume flow rate is required.

\subsection{EQUATIONS AND INSTRUMENTATION}

To quantify the performance of the solar chimney, from the experimental rig, certain physical properties have to be measured. This section describes the quantities that need to be measured and the instrumentation used to do this. The use of the measured results in calculating the turbine efficiency is also presented. 


\section{Required Values}

To calculate the performance of the solar chimney, the turbine efficiency has to be determined. In addition to this, the analysis and design methods have to be verified. To do this, the velocity profiles at various stations are measured directly and compared with the simulated results. Static pressure readings are also taken in the duct region between the inlet guide vanes and turbine blades. The following is a summary of what needs to be calculated:

- The turbine total-to-static efficiencies at various operating points and blade setting angles.

- Velocity and static pressure profiles to be compared with simulated results and accurately calculate the total-to-total and total-to-static efficiencies..

\section{Required Readings}

\section{Turbine Efficiency}

To calculate the turbine total-to-total and total-to-static efficiencies the following equations apply,

$$
\begin{array}{rlrl}
\eta_{\text {turb }} & =\frac{\mathrm{P}_{\text {turb }}}{\mathrm{P}_{\text {ideal }}} & \eta_{\text {turb ts }} & =\frac{\mathrm{P}_{\text {turb }}}{\mathrm{P}_{\text {ideal ts }}} \\
& =\frac{\omega \mathrm{M}_{\text {turb }}}{\mathrm{Q} \Delta \mathrm{p}_{\text {turb }}} & =\frac{\omega \mathrm{M}_{\text {turb }}}{\mathrm{Q} \Delta \mathrm{p}_{\text {turb ts }}}
\end{array}
$$

The turbine shaft output power, $P_{\text {turb}}$, is the product of the turbine rotational speed, $\omega$, and the torque, $\mathrm{M}_{\text {turb }}$. The ideal power is the product of the volume flow, $\mathrm{Q}$, and the pressure drop over the

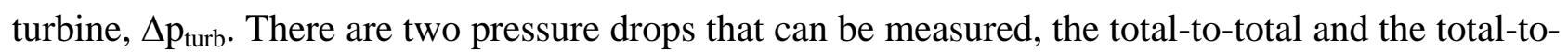
static. The total-to-static is the one used in the case of the solar chimney turbine and as mentioned the exit kinetic energy is not useful and is wasted unlike in a propulsion system where it is normal to use the total-to-total pressure drop in calculating the efficiency. The total-to-static efficiency can be calculated using the wall static pressure. This is simple to measure and in the case of the solar chimney turbine, where it is desirable to keep the exit kinetic energy as low as possible the total-tostatic efficiency is especially relevant.

The total-to-total efficiency is calculated later from the detailed measurements and allows other losses such and blade profile and inlet frictional losses to be measured. Minimising these will also increase the total-to-static efficiency.

The torque, shaft speed and static pressure drop can be measured directly while the volume flow rate is calculated using the flow velocity in the following equation,

$$
\begin{gathered}
\dot{\mathrm{m}}=\rho \mathrm{C}_{\text {in }} \mathrm{A}_{\text {in }}=\rho \mathrm{Q} \\
\Rightarrow \mathrm{Q}=\mathrm{C}_{\text {in }} \mathrm{A}_{\text {in }}
\end{gathered}
$$

The following quantities need to be measured:

- $\quad$ Turbine shaft speed, $\omega$. 
- Turbine torque, $\mathrm{M}_{\text {turb }}$.

- Velocity at IGV inlet, $\mathrm{C}_{\text {in }}$.

- $\quad$ Pressure drop over turbine, $\Delta \mathrm{p}$.

\section{Velocity Profiles}

In the turbine section of the solar chimney, the flow velocity is low enough to make the assumption of incompressible flow. Bernoulli's equation relating the stagnation and static pressure can be applied to the low velocities,

$$
\begin{aligned}
\mathrm{p}_{\mathrm{o}} & =\mathrm{p}_{\mathrm{s}}+\frac{1}{2} \rho \mathrm{C}^{2} \\
& =\mathrm{p}_{\mathrm{s}}+\frac{1}{2} \rho\left(\mathrm{C}_{\mathrm{r}}^{2}+\mathrm{C}_{\mathrm{z}}^{2}+\mathrm{C}_{\theta}^{2}\right)
\end{aligned}
$$

In the above equation there are five unknown values: the static and stagnation pressure and the three velocity components. All the values can be calculated by measuring five different pressure components at a point. This is possible through the use of a five-hole probe that has five holes in very close proximity and different orientations to the flow.

\section{Measuring Instruments}

The following section describes the measuring instruments used on the experimental rig. For each device, the make, method of measurement, operation and accuracy are covered.

\section{Turbine Shaft Speed, $\omega$.}

The turbine rotational speed is measured using an inductive pickup that is triggered by a small grub screw on the turbine shaft. The frequency output of the inductive pickup is converted to a voltage proportional to the frequency. A Turck MS 25-UI frequency to voltage converter is used. The maximum error of the inductive pickup is specified as $0.1 \%$ of the actual value.

Turbine Torque, $M_{\text {turb }}$.

The output torque of the turbine is measured directly through the use of a dynamic torque transducer. The make is a Hottinger Baldwin Messtechnik (HBM) rotary torque transducer, type T5. A shaft with strain gauges is coupled between the turbine and the generator and the strain on the shaft due to the torque is measured resulting in an output voltage that is proportional to the torque. The coupling to the strain gauges is made through slip rings rotating on the shaft. The transducer is tested in the factory of manufacture and the maximum measured error was $0.002 \%$ between the applied and measured torque at full deflection (50 Nm). To take into account the losses of the support bearings, the generator is used as a motor and the shaft run without the turbine attached. This must be added to the measured torque with the turbine running to calculate the actual turbine power output. 


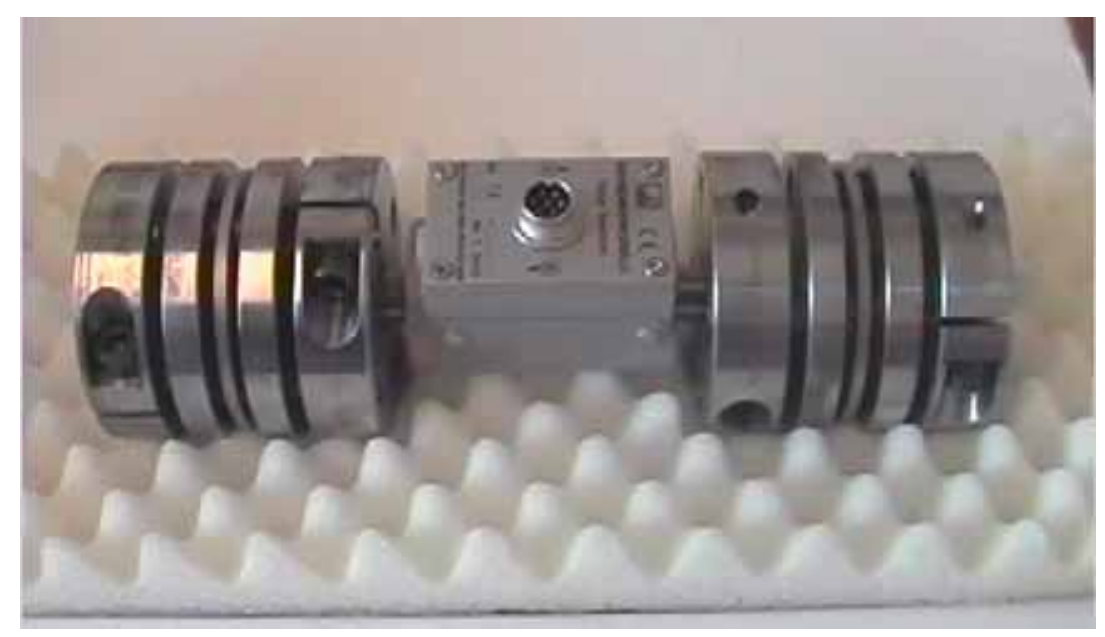

Figure 4.16. HBM T5 rotary torque transducer.

Volume Flow Rate, Q.

In many flow experiments the volume flow rate is usually determined by measuring the static pressure drop at the inlet. In the solar chimney the flow velocity even slightly upstream of the inlet guide vanes is very low resulting in a small static pressure drop relative to atmospheric pressure. The maximum velocity one IGV chord length upstream is expected to be approximately $5 \mathrm{~m} / \mathrm{s}$. This results in a small and difficult to measure static pressure drop. A windmill anemometer was used as these are able to measure low flow velocities accurately and are simple to use. When allowing a smoke trail to pass through the rotating blades, it remains almost undisturbed demonstrating that the overall flow is almost unaffected. Details of the anemometer's positioning and expected operating range are given in Table 4.3. Equation (4.3) is used to calculate the velocity and the volume flows are obtained from Table 4.2.

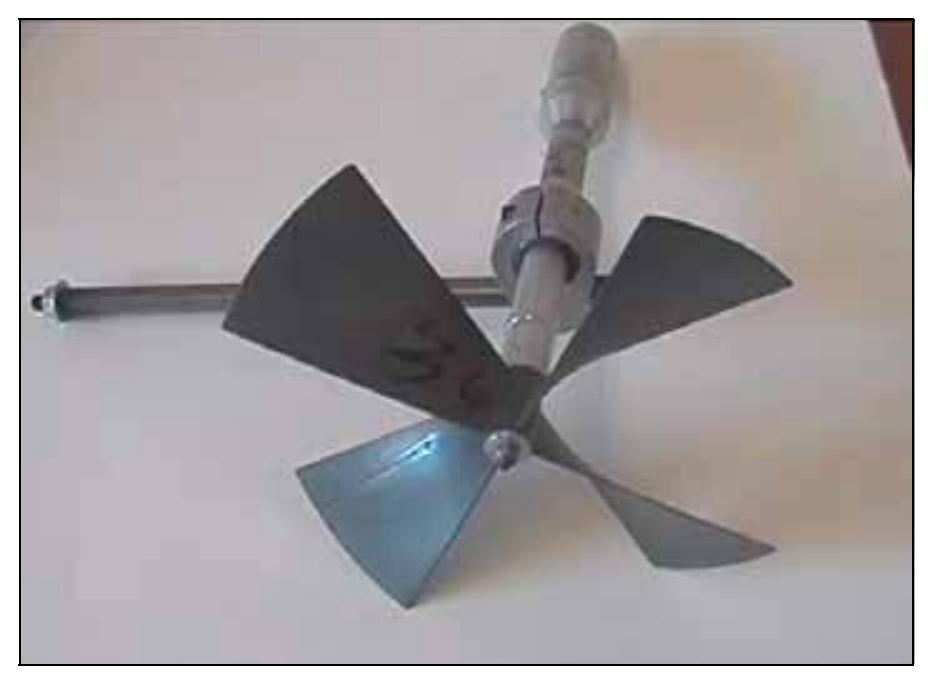

Figure 4.17. Windmill anemometer. 
Table 4.3. Windmill anemometer operating characteristics

\begin{tabular}{|l|l|l|l|}
\hline Description & Value & Flow condition & Velocity \\
\hline Radial position & $0.9 \mathrm{~m}$ & High eff, low pressure & $1.65 \mathrm{~m} . \mathrm{s}^{-1}$ \\
\hline Radial Area & $2 \pi \times 0.8 \times 0.32=1.810 \mathrm{~m}^{2}$ & Low eff, high pressure & $2.42 \mathrm{~m} . \mathrm{s}^{-1}$ \\
\hline & & Mass flow control & $1.04 \mathrm{~m} . \mathrm{s}^{-1}$ \\
\hline
\end{tabular}

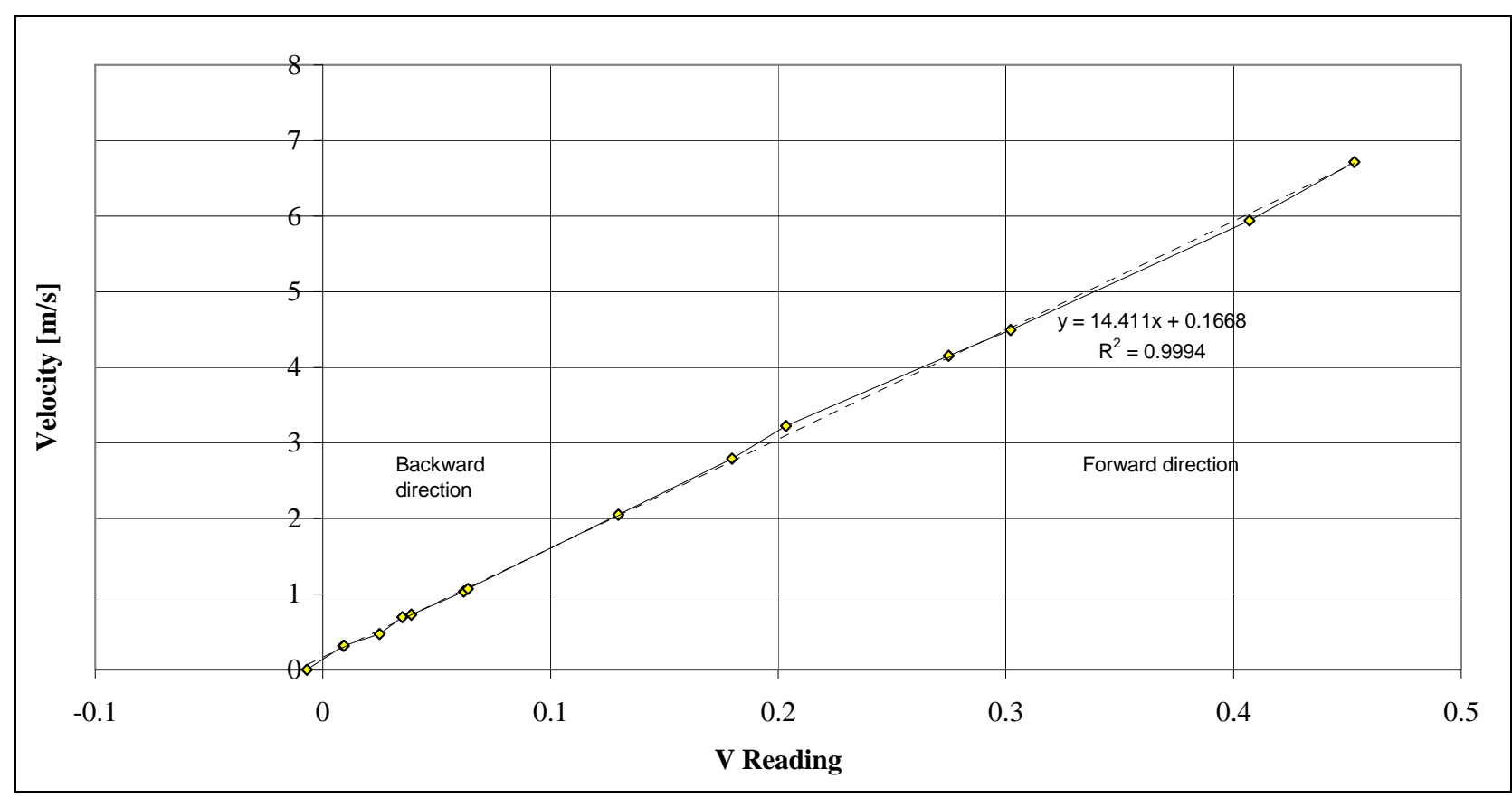

Figure 4.18. Windmill anemometer calibration curve for measuring velocity.

The calibration curve was determined experimentally using a small calibration wind tunnel where the flow is accurately measured through a small nozzle downstream of the larger tunnel in which the anemometer is placed.

Figure 4.18 shows the calibration curve obtained: there is a slight scatter around the fitted curve but the linear correlation is good. The average error between the correlation and the measured result is $2.0 \%$.

Turbine Pressure Drop, $\Delta p$.

When calculating the turbine efficiency, the losses in the inlet guide vanes, ducting and diffuser are also included resulting in a stage or overall turbine efficiency. The overall turbine efficiency is useful when analysing the entire solar chimney plant. The turbine can be treated as a single unit to simplify the simulation and understanding of the overall plant operation. This means in practice that the turbine pressure drop, $\Delta \mathrm{p}$, in equation (4.2) is the difference in pressure from the inlet of the IGVs to the diffuser exit. 
The pressure drop used is the total-to-static pressure drop as this also takes into account the kinetic energy losses at the diffuser exit. For the inlet, total pressure atmospheric pressure is used as the losses in the inlet section will be very small. The reason is that the inlet velocity is very low, coupled with the small losses found in accelerating flows. This results in a small inlet pressure loss especially in comparison with the overall turbine pressure drop. Trying to measure such a small pressure is very difficult and hence the decision to use the atmospheric pressure as the total inlet reference pressure.

The static pressure is measured 0.625 pipe diameters downstream of the end of the turbine diffuser to allow the flow to settle. The static pressure is measured on the pipe wall through a small hole that is flush with the pipe (Figure 4.19). It is important that there are no burs or protrusions at the entrance to the static pressure tappings as this would result in part of the stagnation pressure being measured.

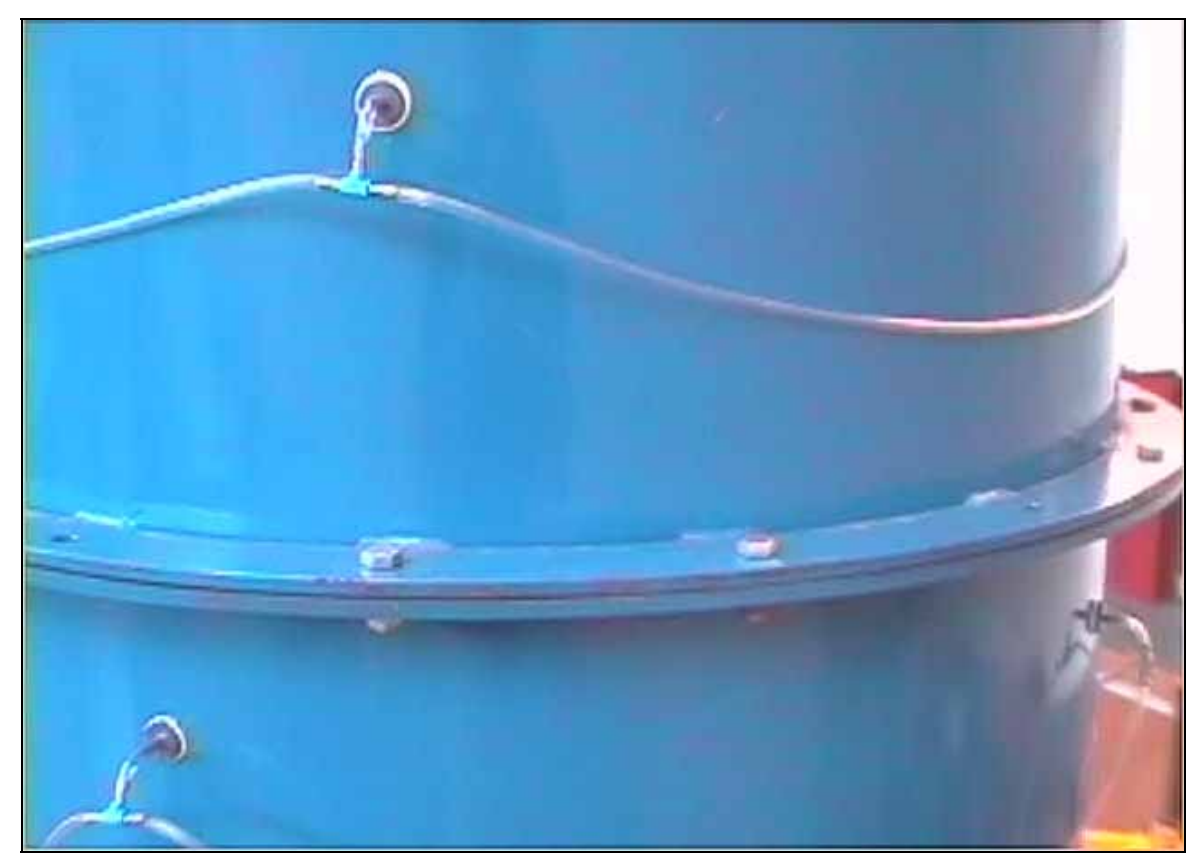

Figure 4.19. Pressure tapping points on turbine exit pipe.

The physical measuring of the pressure is performed with an HBM pressure transducer that is connected to the tapping via a pipe. At each radial static pressure station, there are three tapping points around the pipe to obtain the average static pressure. The pressure transducers are calibrated before each experiment, using a Betz water manometer. It has a resolution of $0.1 \mathrm{~mm}$ water or approximately $1 \mathrm{~Pa}$ and comparing this to the pressure drop values in Table 4.2 results in a maximum error of $0.95 \%$ for the low pressure drop case (105 Pa). The combined error (inlet and measurement errors) for the three operating modes of Table 4.2. are $1.32 \%, 0.64 \%$ and $0.14 \%$ for cases 1,2 and 3 respectively. 


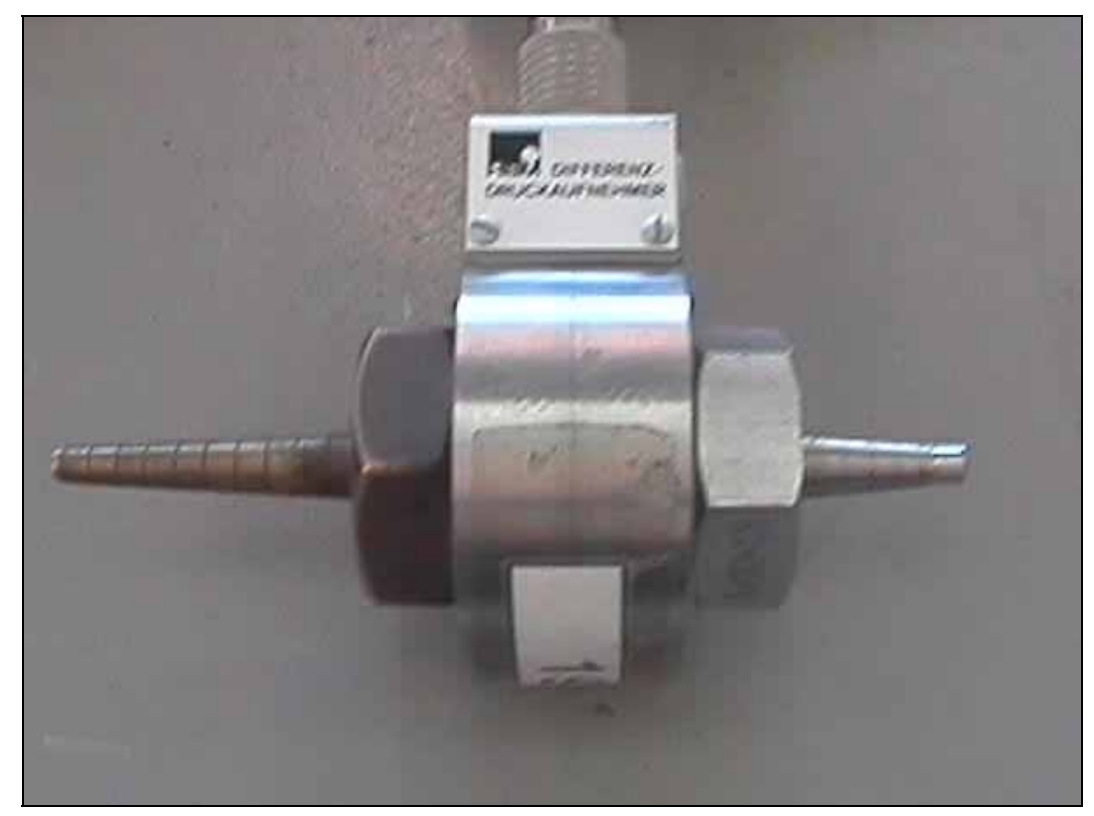

Figure 4.20. HBM Pressure transducer.

Velocity Profile.

The velocity profile just upstream and downstream of the turbine rotor is measured using a fivehole probe. Strohmaier (1997) presents the operation and calibration of the probe, and programs have been written by $\mathrm{Du}$ Toit (2000) to calculate the velocity components from the pressure measurements. The following figure shows where the velocity profiles probe traverse stations. The probe was traversed as close to the blade as possible without having it touch at the root.

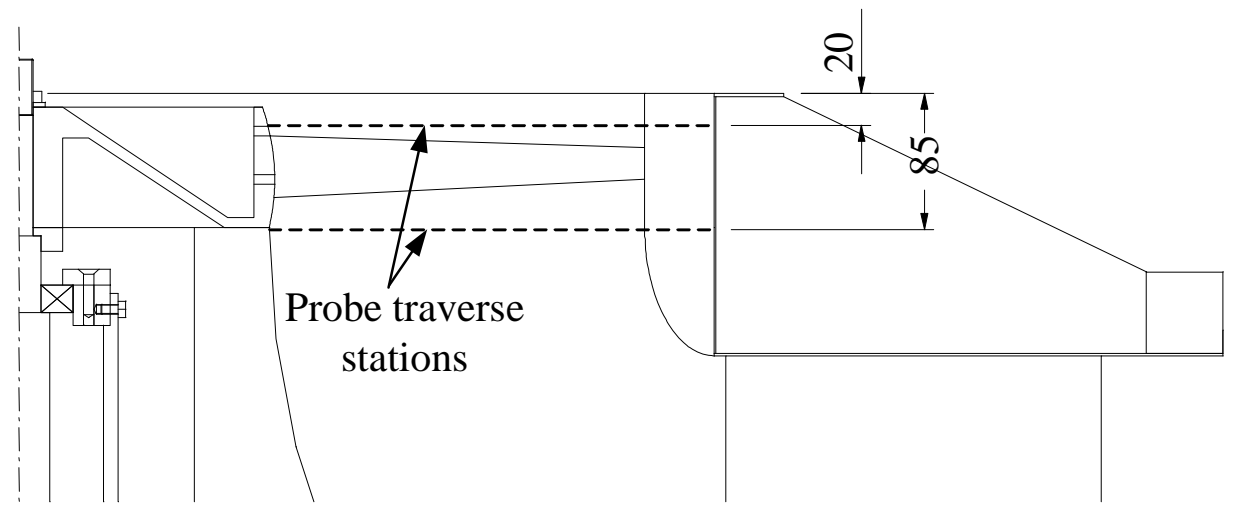

Figure 4.21. Probe traverse stations for five-hole probe. 
A schematic of the probe nozzle is shown in the following figure.

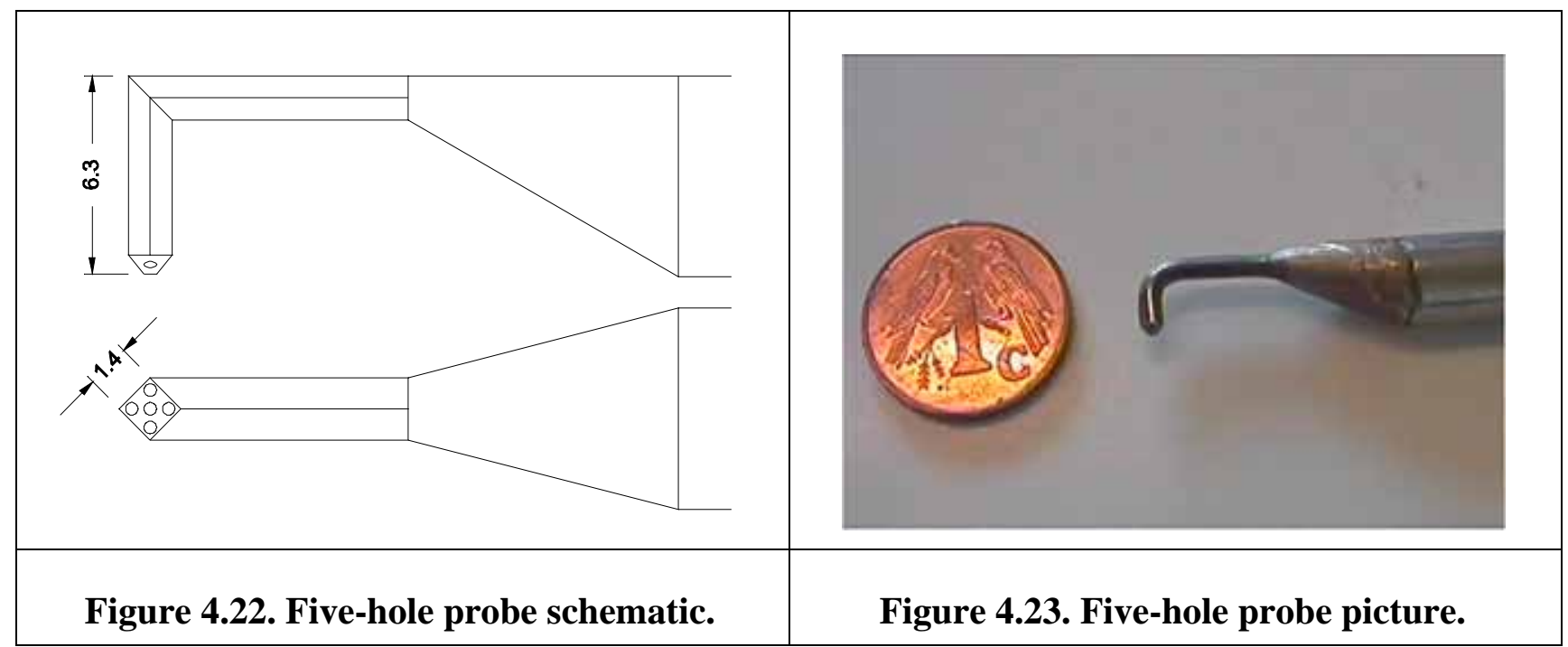

To use the probe effectively it is best to use five pressure transducers, each connected to one of the probe holes. This allows the pressure readings to be taken simultaneously, and speeds up the sampling process dramatically. Due to the holes and connecting pipes to the probes being very small, there is delay between the probe being moved into a new position and the pressure being measured by the probe changing as the air moves in or out of the pipes. If only one transducer is used, time has to be left between each of the 5 readings to ensure that the pressure has stabilised.

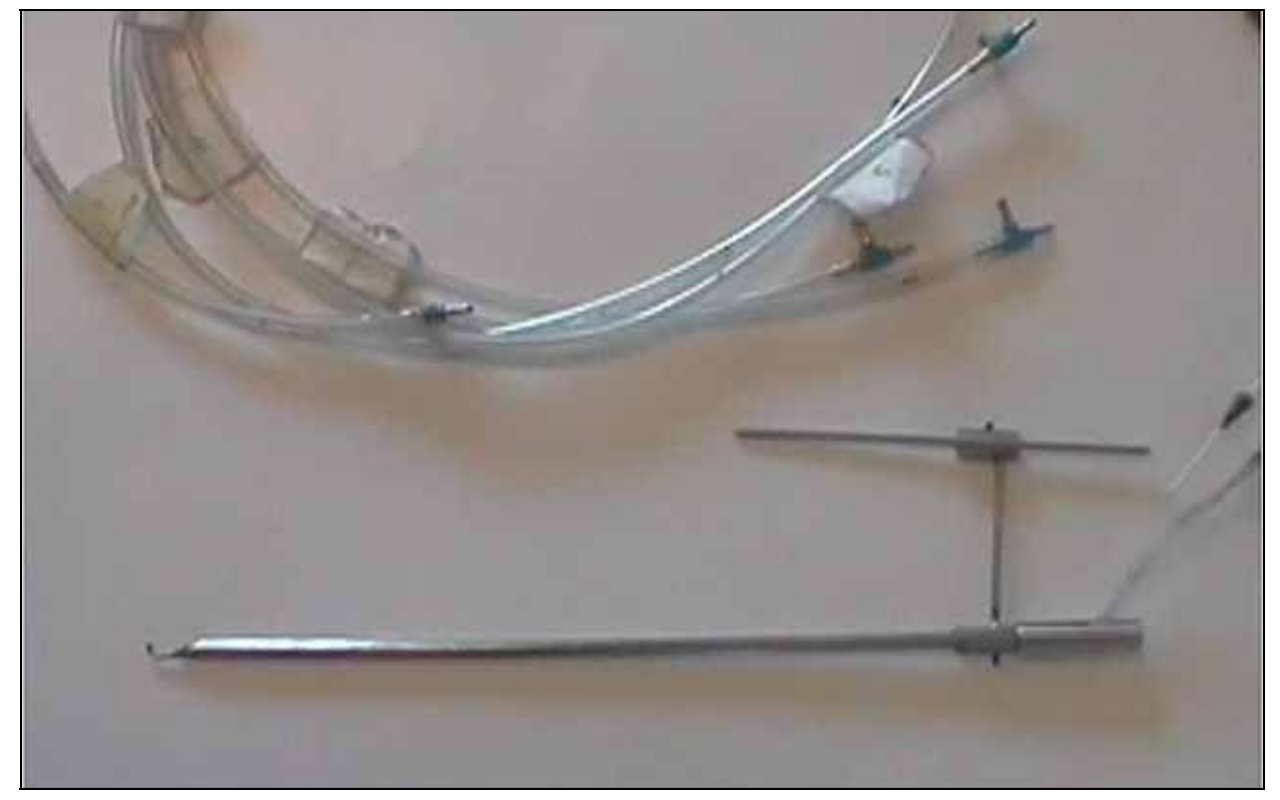

Figure 4.24. Five-hole probe with piping to be connected to pressure transducers.

To obtain the velocity profile at a particular station, the probe is carefully moved into the flow and to the inner wall to start. Here the first reading is taken and then in steps towards the outer wall. Performing the probe traverse from the outside in could lead to the probe being pushed into the hub and damaging it. To take a single reading, the probe is left in position until the pressures are 
stabilised, the readings taken and then the probe is moved slightly outward and the process repeated. In areas where the velocity gradient is low, fewer readings are taken and in places where it is high, the measuring stations are closer together.

\section{Wall Static Pressures}

The static pressure tappings on the diffuser and exit pipe have three holes around the pipe circumference at each radial station to obtain an average static pressure. The figures below show the position of the static measuring stations and the photo of the physical set up. The pipes from the static pressure station are connected to a pressure transducer and the pressure measured relative to atmospheric pressure. As there are many stations, a pressure switching box is used that allows many pressure tapping points to be connected to it with only one output to a single pressure transducer.

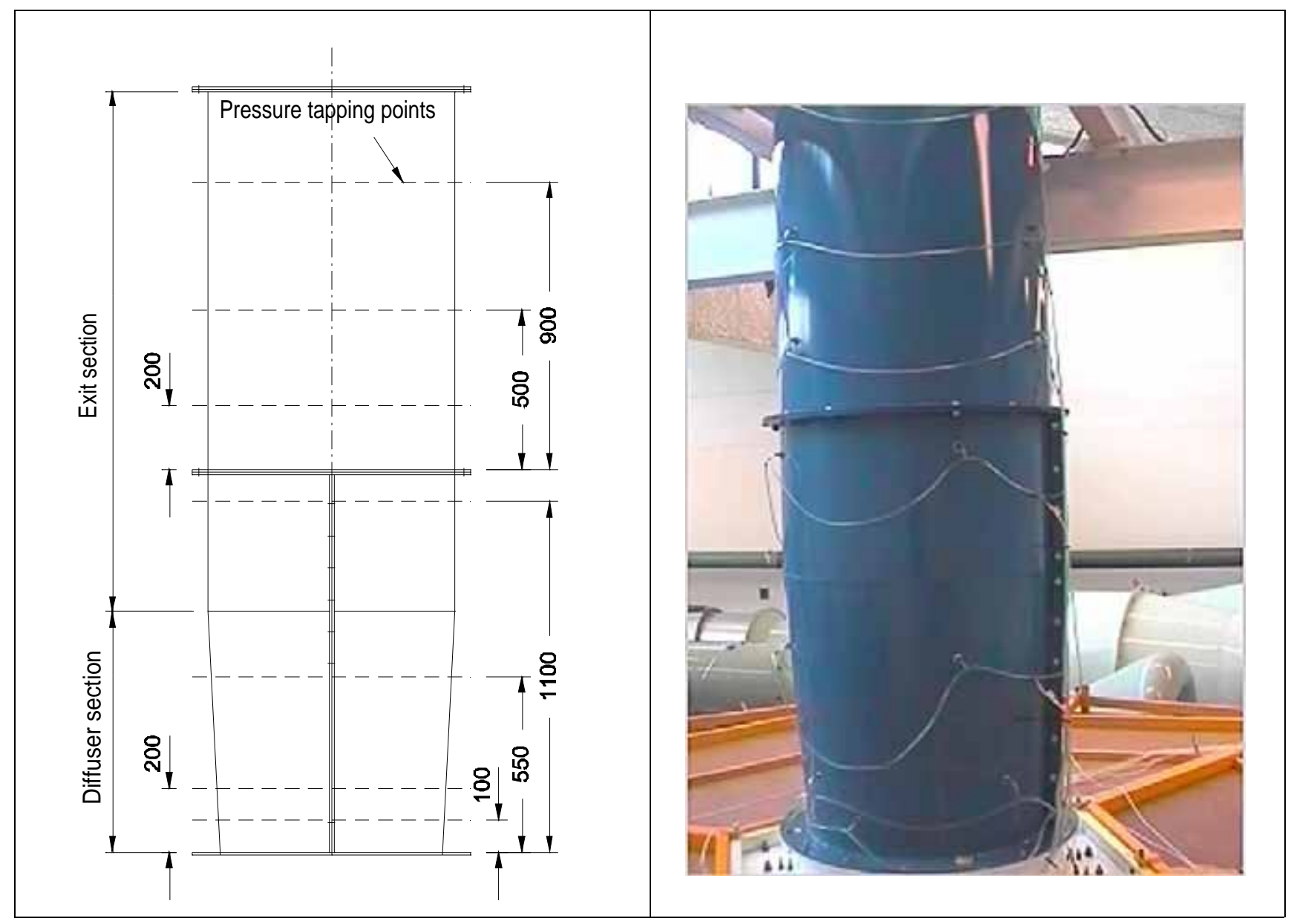

Figure 4.25. Schematic of pressure tappings.

Figure 4.26. Picture of pressure tapping points and connecting pipes.

\section{Peripheral Measuring Devices}

Some instruments that are used are not directly involved with the measuring but are essential or speed up obtaining the data. The bridge amplifier is required to amplify the very small output 
signals from the transducers to the useful range of $0-5 \mathrm{~V}$. A pressure switching box coupled to a Personal Computer (PC) allows all the static pressure readings to be read automatically as the PC both controls the box and logs the data (see PC section next page).

\section{Bridge Amplifier}

The bridge amplifier is an HBM DA24 with six channels, model KWS 3073, each connected to one of the relevant pressure or torque transducers. The input signal is amplified and displayed on the front digital display and sent to an ADDA card on the PC used for data logging.

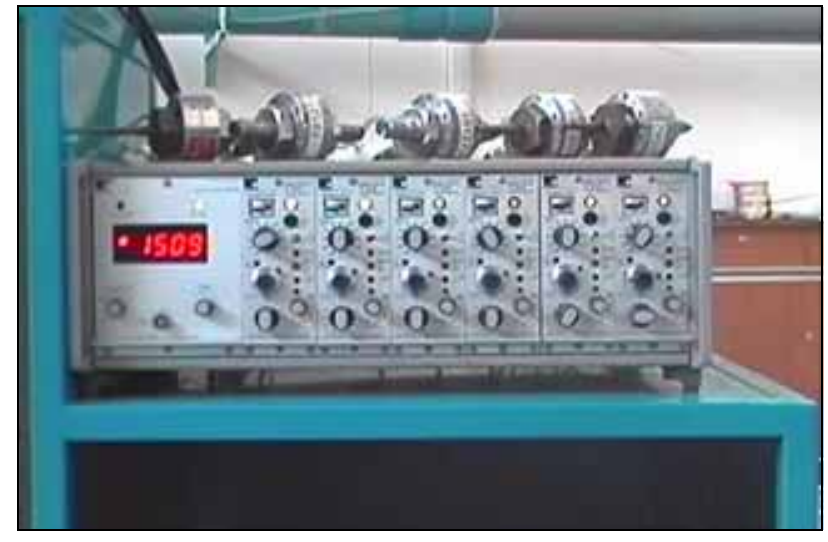

Figure 4.27. HBM bridge amplifier front panel.

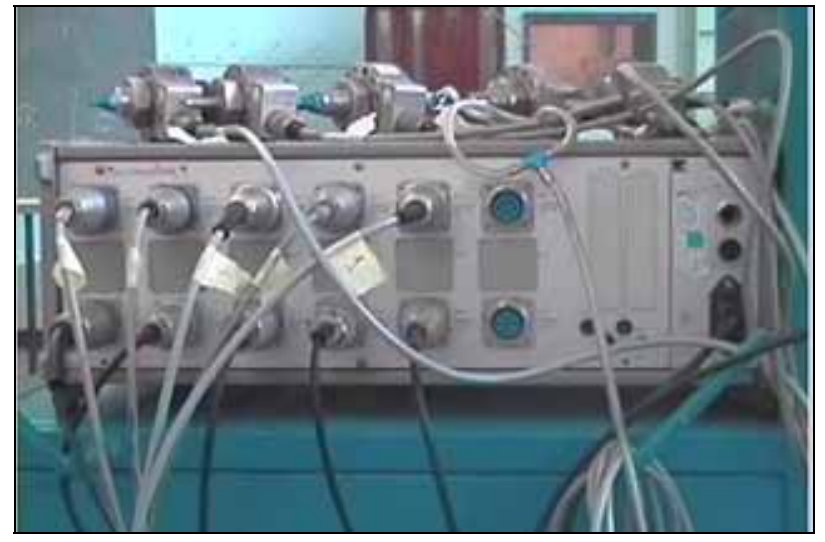

Figure 4.28. HBM bridge amplifier back showing input and output plugs.

\section{Pressure Switching Box}

The pressure switching box is a FCO 91 MkII selection box that has a number of pressure input channels (10 or 20). The selection of the channels can either be done manually or remotely using a PC as used in this project. The use of the box and the method of connecting it to a PC is described comprehensively by Strohmaier (1997).

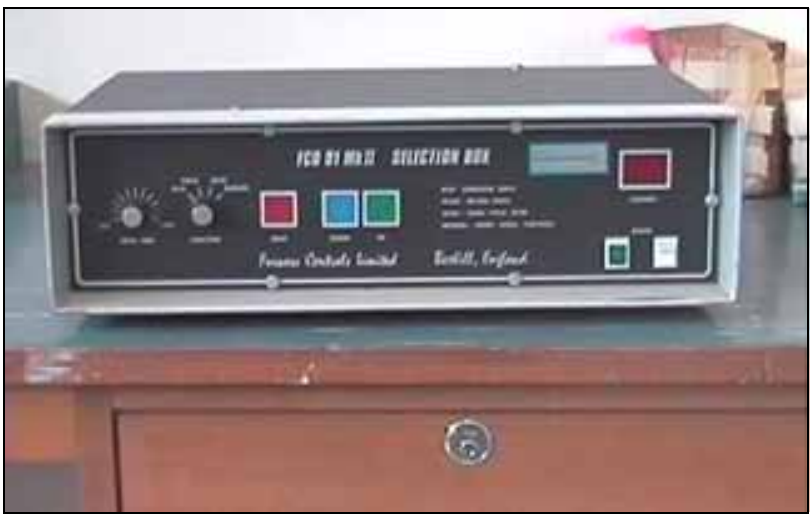

Figure 4.29. Pressure switching box front panel.

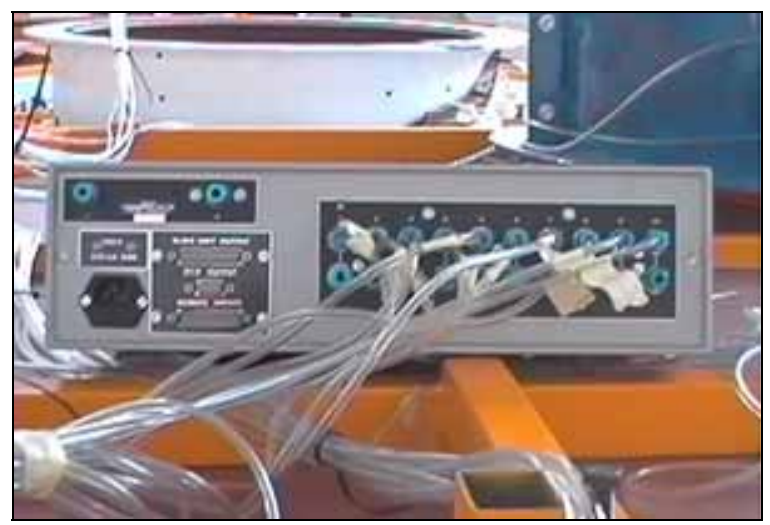

Figure 4.30. Pressure switching box back panel with multiple inputs. 


\section{Personal Computer}

The PC is a standard Pentium Celeron 400 with an Eagle technologies Analogue-to-Digital Digitalto-Analogue card (ADDA) added. The programs to control the switching box and sample data from the card are written in MATLAB and the data is processed immediately. Use of the PC greatly simplifies the process of measuring and acquisition of the data. To measure the data a sample of approximately 10 seconds at $3 \mathrm{kHz}$ is taken and the result averaged to eliminate any instantaneous fluctuations.

\subsection{OVERALL ERROR ESTIMATION}

It is necessary to be able to estimate how accurate the results of the experimental measurements are when using them to say compare with simulation and also future analysis of the solar chimney. In the sections above each of the instruments has a certain maximum error when measuring each property of the turbine. The method for estimating the overall error in measuring the turbine efficiency is outlined in Granger (1988) where a practical example of the application of the ISO method of error estimation is provided. If a function $F=f\left(X_{1}, X_{2}, X_{3}, \ldots\right)$ where $F$ is calculated and $\mathrm{X}_{\mathrm{i}}$ are measured then the error, $\mathrm{B}_{\mathrm{F}}$ in $\mathrm{F}$ is calculated from,

$$
\mathrm{B}_{\mathrm{F}}^{2}=\left(\frac{\delta \mathrm{F}}{\delta \mathrm{X}_{1}} \mathrm{~B}_{\mathrm{x}_{1}}\right)^{2}+\left(\frac{\delta \mathrm{F}}{\delta \mathrm{X}_{2}} \mathrm{~B}_{\mathrm{X}_{2}}\right)^{2}+\cdots
$$

Applying this to equation (4.2) for the turbine efficiency the error is,

$$
B_{\eta_{\text {turb }}}=\left[\left(\frac{\partial \eta_{\text {turb }}}{\partial \omega} B_{\omega}\right)^{2}+\left(\frac{\partial \eta_{\text {turb }}}{\partial M_{\text {turb }}} B_{M_{\text {turb }}}\right)^{2}+\left(\frac{\partial \eta_{\text {turb }}}{\partial Q} B_{Q}\right)^{2}+\left(\frac{\partial \eta_{\text {turb }}}{\partial \Delta \mathrm{p}_{\text {turb }}} B_{\Delta \mathrm{p}_{\text {turb }}}\right)^{2}\right]^{1 / 2}
$$

Substituting the partial differential equations derived from the turbine efficiency into the above equation the expression for the measured error is,

$$
\mathrm{B}_{\eta_{\text {turb }}}=\left[\left(\frac{\mathrm{M}_{\text {turb }} \mathrm{B}_{\omega}}{\mathrm{Q} \Delta \mathrm{p}_{\text {turb }}}\right)^{2}+\left(\frac{\omega \mathrm{B}_{\mathrm{M}_{\text {turb }}}}{\mathrm{Q} \Delta \mathrm{p}_{\text {turb }}}\right)^{2}+\left(-\frac{\omega \mathrm{M}_{\text {turb }} \mathrm{B}_{\mathrm{C}_{\text {in }}}}{\mathrm{Q}^{2} \Delta \mathrm{p}_{\text {turb }}}\right)^{2}+\left(-\frac{\omega \mathrm{M}_{\text {turb }} \mathrm{B}_{\Delta \mathrm{p}_{\text {turb }}}}{\mathrm{Q} \Delta \mathrm{p}_{\text {turb }}^{2}}\right)^{2}\right]^{1 / 2}
$$

Dividing the left hand by the turbine efficiency and the right hand side by the expression for turbine efficiency, (4.2) the above equation can be simplified to,

$$
\frac{\mathrm{B}_{\eta_{\text {turb }}}}{\eta}=\left[\left(\frac{\mathrm{B}_{\omega}}{\omega}\right)^{2}+\left(\frac{\mathrm{B}_{\mathrm{M}_{\text {turb }}}}{\mathrm{M}_{\text {turb }}}\right)^{2}+\left(-\frac{\mathrm{B}_{\mathrm{C}_{\text {in }}}}{\mathrm{Q}}\right)^{2}+\left(-\frac{\mathrm{B}_{\Delta \mathrm{p}_{\text {turb }}}}{\Delta \mathrm{p}_{\text {turb }}}\right)^{2}\right]^{1 / 2}
$$

The maximum error will occur at the lowest power setting of the turbine as the absolute errors remain constant while the measured values are at their smallest. In the following table the nominal value of the variable as well as the expected maximum errors are listed. 
Table 4.4. Nominal and maximum errors for operational mode (1), Table 4.2.

\begin{tabular}{|l|l|l|l|l|}
\hline Property & $\begin{array}{l}\text { Rotational speed } \\
\omega\end{array}$ & $\begin{array}{l}\text { Torque } \\
\mathrm{M}_{\text {turb }}\end{array}$ & $\begin{array}{l}\text { Volume flow rate } \\
\mathrm{Q}\end{array}$ & $\begin{array}{l}\text { Pressure drop } \\
\Delta \mathrm{p}_{\text {turb }}\end{array}$ \\
\hline Error & $0.1231\left[\mathrm{rad}_{\mathrm{r}} \mathrm{s}^{-1}\right]$ & $0.001[\mathrm{Nm}]$ & $0.0774\left[\mathrm{~m}^{3} / \mathrm{s}\right]$ & $1.39 \mathrm{~Pa}$ \\
\hline Nominal value & $123.1\left[\mathrm{rad}_{\mathrm{s}} \mathrm{s}^{-1}\right]$ & $1.83[\mathrm{Nm}]$ & $2.67\left[\mathrm{~m}^{3} / \mathrm{s}\right]$ & $105 \mathrm{~Pa}$ \\
\hline
\end{tabular}

Using the above values and an assumed turbine efficiency of $80 \%$, the maximum error in measuring the efficiency is

$$
\begin{aligned}
\mathrm{B}_{\eta_{\text {turb }}} & =0.8 \times\left[\left(\frac{0.1231}{123.1}\right)^{2}+\left(\frac{0.001}{1.83}\right)^{2}+\left(-\frac{0.0774}{2.67}\right)^{2}+\left(-\frac{1.39}{105}\right)^{2}\right]^{1 / 2} \\
& =0.00102 \\
& =0.102 \%
\end{aligned}
$$

The pressure loss at the inlet is, as mentioned before, very small. Assuming a conservative nozzle discharge coefficient, $C_{d \text { noz }}$ of 0.9 and using the highest volume flow rate from Table 4.2, the total pressure loss at the table inlet is very small. The table radius is $2.25 \mathrm{~m}$

$$
\begin{aligned}
\mathrm{P}_{\text {Oloss }} & =\left(1-\mathrm{C}_{\mathrm{d} \text { noz }}\right) \frac{1}{2} \rho \mathrm{C}^{2}=\left(1-\mathrm{C}_{\mathrm{d} \text { noz }}\right) \frac{1}{2} \rho\left(\mathrm{Q} / \mathrm{A}_{\text {inlet }}\right)^{2} \\
& =(1-0.9) \frac{1}{2} 1.2\left(\frac{3.89}{2 \pi \times 2.25 \times 0.32}\right)^{2} \\
& =0.05 \mathrm{~Pa}
\end{aligned}
$$

This is only $0.29 \%$ of the predicted turbine pressure drop for the highest mass flow case. It will be less for the others as the flow rate is less and, therefore, also the pressure loss. Trying to measure such a small pressure is very difficult, hence the decision to use the atmospheric pressure as the total inlet pressure.

\subsection{EXPERIMENTAL STRATEGY}

The experimental readings to be taken fall into two categories:

- Overall turbine performance measurements, simple to perform, allow many different configurations to be compared. Here the turbine torque and speed and static pressures are measured to calculate the overall turbine efficiency.

- Detailed flow measurements- flow profiles are measured upstream and downstream of the turbine allowing for comparison with simulated results. Using the complete velocity profile, it is also possible to more accurately calculate the turbine efficiency as the complete kinetic energy at the exit can be calculated.

The first method where only turbine static pressures, torque and speed are measured can be inaccurate as the static pressure at the pipe wall may be different to the average value. By 
measuring the velocity profile downstream of the turbine and in the exit pipe, the static pressure profiles can be determined and the exit kinetic energy calculated. Once the exit kinetic energy is known, it is possible to very accurately calculate the turbine efficiency. The only drawback of this approach is that it would be time consuming to measure the velocity profile at each flow condition. It is more effective to measure the overall efficiency at a number of operating points and find the optimum operating conditions. Detailed measurements will then be taken at these points and the efficiency accurately determined.

\section{Design Point Measurements}

The first set of experimental readings will be taken at the three design conditions listed in Table 4.2 at the relevant blade setting angles from the previous chapter. Both experimental methods will be used to measure the turbine efficiency to investigate the difference between the results. The results will also be compared with the simulated results to check the accuracy of the design analysis.

\section{Performance Map}

The second set of experimental readings will be to investigate the turbine performance over a wide operating range. The tests cover the entire range of inlet guide vane and turbine blade setting angles. At each combination of inlet guide vane and turbine stagger angle the efficiency over the widest possible flow range will be tested. The tests will begin with as low a volume flow rate as can be measured and then increased in steps until the power limitations of the turbine or of the suction fan are reached.

The table below shows the algorithm for changing the turbine and IGV stagger angles. The first readings will be performed at the high efficiency, low mass flow design point, (Table 4.2). The operational map will then be extended first above and then below this point as this will make it simpler to see if there is an optimum point. If the turbine performance is seen to degrade significantly when the blade angle is adjusted past a certain angle, no more changes will be taken beyond this as it is unlikely that the turbine will be operated at some low efficiency point. The IGV angles are adjusted over the operating range first as it is more difficult to change the stagger angles of the turbine. Once the optimum IGV angles have been bounded the stagger angle of the turbine blades are changed using the same method as for the IGV's, first increasing the angles and then later decreasing them. The incremental change to the turbine stagger angle is much lower than for the IGV's as they are more sensitive to changes in angle due to the stagger angle already being very high. By changing both the IGV and turbine stagger angles, the optimum design range can be bounded and the optimum points chosen from the large data base of data. 
Table 4.5. IGV and turbine blade stagger angle changes for experimental readings.

\begin{tabular}{|l|l|l|l|l|l|l|}
\hline $\begin{array}{l}\text { Turbine } \\
\text { stagger } \Downarrow\end{array}$ & $\begin{array}{l}\text { IGV } \\
\text { stagger } \Rightarrow\end{array}$ & a & b & c & d & e \\
\hline Design & Design $\left(0^{0}\right)$ & $+2.5^{0}$ & $-2.5^{0}$ & $+5.0^{0}$ & $-5.0^{0}$ & etc \\
\hline$+1^{0}$ & Design $\left(0^{0}\right)$ & $+2.5^{0}$ & $-2.5^{0}$ & $+5.0^{0}$ & $-5.0^{0}$ & etc \\
\hline$-1^{0}$ & Design $\left(0^{0}\right)$ & $+2.5^{0}$ & $-2.5^{0}$ & $+5.0^{0}$ & $-5.0^{0}$ & etc \\
\hline$+2^{0}$ & Design $\left(0^{0}\right)$ & $+2.5^{0}$ & $-2.5^{0}$ & $+5.0^{0}$ & $-5.0^{0}$ & etc \\
\hline etc & & & & & & \\
\hline
\end{tabular}

\section{Improved Design Point Measurement}

Once the design range has been mapped, the planned optimum design points and the measured ones can be compared. It is likely that there will be a difference as some of the assumed limiting conditions such as the maximum inlet swirl and turbine stagger angles are conservative. In practice it may be possible to introduce more inlet swirl or set the turbine blades at a higher stagger angle without increasing the profiles losses or stalling, thus increasing the turbine efficiency.

At the experimental optimum operating points, detailed flow measurements will be taken to accurately calculate the efficiency. These results will then be compared with the optimum design points and used to see why the differences occur and how the turbine design may be improved.

\subsection{Starting PERFormanCE AND RunAWAy SPEED}

\section{Starting Performance}

In the previous chapter it was mentioned that it is advantageous if the turbine is self-starting as this avoids having to use energy to get the plant running and adds to the simplicity of the solar chimney operation. To start easily the turbine must have a high torque at zero rotational speed and so in the experimental rig the turbine is clamped below the torque transducer, allowing the torque to be measured. While it is possible to adjust the IGV and turbine blades to have a very high starting torque, it is more desirable to have them set near their operating angles. This avoids requiring large blade adjustments when the plant is generating little power. The aim of these experiments will be to find blade-setting angles near the low-mass flow design point while giving sufficient torque to start the turbine.

\section{Runaway Speed}

A potential problem with the turbine is destructive runaway when there is a sudden no load condition such as generator failure. An emergency procedure needs to be outlined in the case of no load to either stop the turbine or ensure that it never reaches a speed where it can fail. The first step of this process is to see what the runaway speeds of the turbine at the various design points are. 
Here the turbine is uncoupled from the torque transducer and generator to give no load while still being attached to the speed reader. Even in the experimental turbine there is a chance of blade failure if the rotational speed is too high and so the mass flow is reduced and the runaway speed measured for this lower mass flow and then scaled up using the fan laws, Equation (4.1). As with the starting torque, the minimum change in either turbine or IGV stagger angle must be found to be able to limit the runaway speed to safe levels.

The results of these two operating points will be presented with the starting torque and runaway speeds as functions of the IGV and turbine stagger angles. In this way the data can be used when more is known about the full scale plant's operating characteristics. The plant may eventually operate for 24 hours a day, in which case the starting torque will not be important. The runaway speed will almost certainly be governed by the blade strength. 


\section{CHAPTER 5: EXPERIMENTAL RESULTS}

The results of the experimental measurements taken from the solar chimney turbine model are presented. The results are divided into two broad categories. The first measurements were the turbine overall performance over a wide operating range. Here only the turbine output power and wall static pressure drops were measured. Using this data, a performance map over a wide range of inlet guide vane and turbine rotor blade angles is presented. The second set of data consisted of detailed measurements of the velocity profiles up and downstream of the turbine and downstream of the diffuser. These velocity and pressure profiles were compared to the design point predictions obtained, using the matrix throughflow method.

\subsection{TURBINE TOTAL-TO-STATIC EFFICIENCY}

The theory used to calculate the turbine efficiency from the raw data is presented below. Details of the instrumentation and measuring points were covered in Chapter 4. The performance map obtained from the experimental data is then compared to the theoretical maps used in the design process outlined in Chapter 3.

\section{Experimental Theory}

Unless otherwise noted all experimental efficiencies presented are for the complete turbine stage. This includes the inlet, inlet guide vanes, rotor and diffuser. The turbine stage (inlet-IGV-rotordiffuser) total-to-static efficiency is calculated by comparing the actual power output with the theoretical power available,

$$
\begin{aligned}
\eta_{\text {turb }} & =\frac{\mathrm{P}_{\text {turb }}}{\mathrm{P}_{\text {ideal }}} \\
& =\frac{\omega \mathrm{M}_{\text {turb }}}{\mathrm{Q} \Delta \mathrm{p}_{\text {turb }}}
\end{aligned}
$$

The rotational speed, $\omega$, and torque, $\mathbf{M}_{\text {turb}}$, are measured directly on the turbine shaft. The volume flow rate, $\mathrm{Q}$, is calculated using the known area of the inlet and the air velocity measured using a windmill anemometer. The total-to-static pressure drop is the atmospheric pressure minus the exit static pressure. The exit static pressure is measured on the pipe wall, one diameter downstream from the diffuser exit. This total-to-static pressure drop includes the rotor, inlet guide vane and inlet pressure drop and was expected to give a conservative estimate of the turbine efficiency as all the losses associated with the inlet, IGV, rotor and diffuser are included. This is because the wall static pressure is measured relative to atmospheric or inlet pressure. The detailed velocity profiles presented later in this chapter show that for higher load coefficients the wall static pressures are 
higher than average. This can lead to slightly optimistic predictions of the turbine total-to-static efficiency.

To simplify the comparison of experimental readings to a full-scale solar chimney turbine, all results are plotted relative to the load coefficient $\psi$ and flow coefficient $\phi$. The definitions are the same as used in Chapter 3 but rearranging the equations makes the calculation of $\phi$ and $\psi$ from the experimental data more convenient. The flow coefficient is the mean throughflow velocity divided by turbine tip speed with the throughflow area being taken as the annular area between the casing and hub.

$$
\begin{aligned}
\phi & =\frac{\mathrm{C}_{\mathrm{z}}}{\mathrm{U}_{\text {tip }}} \\
& =\frac{4 \mathrm{Q} /\left(\pi \mathrm{D}_{\text {turb }}^{2}\left(1-v^{2}\right)\right)}{\mathrm{U}_{\text {tip }}}
\end{aligned}
$$

The load coefficient is usually calculated using the turbine total temperature or total pressure drop. It is difficult to measure these directly without taking the average of detailed measurements over the entire rotor span, so a simpler method is employed. Using the relationship between measured power output and total pressure drop and beginning with the definition of the load coefficient

$$
\psi=\frac{2 \mathrm{c}_{\mathrm{p}} \Delta \mathrm{T}_{\mathrm{o}}}{\mathrm{U}_{\mathrm{tip}}^{2}}
$$

the relation between power and total temperature drop is substituted,

$$
\mathrm{P}=\dot{\mathrm{mc}}_{\mathrm{p}} \Delta \mathrm{T}_{\mathrm{o}}
$$

and the load coefficient can be written as,

$$
\psi=\frac{2 \mathrm{P}}{\mathrm{U}_{\mathrm{tip}}^{2} \dot{\mathrm{m}}}
$$

Calculation of the mass flow requires the density, $\rho$, using measured values of atmospheric temperature and pressure. The volume flow rate, $\mathrm{Q}$, is measured using the windmill anemometer as mentioned above. The mass flow is calculated as follows,

$$
\dot{\mathrm{m}}=\rho \mathrm{Q}=\frac{\mathrm{P}_{\mathrm{atm}}}{\mathrm{R}_{\mathrm{atm}} \mathrm{T}_{\mathrm{atm}}} \mathrm{Q}
$$

\section{Experimental Data}

The turbine was tested over a range of rotor blade angles, including the two design tip stagger angles of $75.2^{0}$ and $78^{0}$ shown in bold in Table 5.1. Only one lower tip stagger angle $\left(72^{0}\right.$ ) was tested as the turbine is not expected to operate at higher volume flow rates. A range of higher tip stagger angles up to $96^{\circ}$ was tested to investigate the feasibility of using a high turbine pressure 
drop to control the plant power output. At stagger angles beyond $90^{\circ}$ the turbine blade tips are operating as fan blades attempting to pump flow back towards the collector.

The experimental IGVs were modified so that the trailing $30 \%$ of the blade is a flap so that the effect of changes in the inlet swirl could be measured in detail (Hoerner \& Borst 1975). For each rotor blade setting angle, the IGV flaps were adjusted through their full range from $-45^{0}$ to $+45^{0}$, showing a typical range of angles as in Table 5.2. At each particular setting of the rotor and IGV blades, the turbine performance was measured over wide operating range from zero power output, through to peak power and finally to a flow coefficient, $\phi$, of 0.6. This was near the limit of the experimental rig in terms of the power that could be absorbed from the turbine and the pressure drop that could be delivered by the extraction fan.

Table 5.1. Rotor blade stagger angles at tip measured from the axial direction.

\begin{tabular}{|l|l|l|l|l|l|l|l|l|}
\hline $72^{0}$ & $\mathbf{7 5 . 2}^{\mathbf{0}}$ (des) & $\mathbf{7 8}^{\mathbf{0}}$ (des) & $81^{0}$ & $84^{0}$ & $87^{0}$ & $90^{0}$ & $93^{0}$ & $96^{0}$ \\
\hline
\end{tabular}

Table 5.2. Inlet guide vane adjustment about the design angle.

\begin{tabular}{|l|l|l|l|l|l|l|l|l|}
\hline+45 & +30 & +20 & +10 & 0 (des) & -10 & -20 & -30 & -45 \\
\hline
\end{tabular}

A large amount of experimental data was generated from the tests (2929 points) and is presented graphically (Appendix C: Raw Data). Figure 5.1 and Figure 5.2 show the data for a single rotor blade stagger angle tested over the full range of IGV flap angles.

The total-to-static efficiency versus flow coefficient for a single rotor blade angle $\left(75.2^{0}\right)$ is plotted in Figure 5.1. Changing the IGV angle between $-45^{\circ}$ and $+45^{\circ}$, changes the flow coefficient by about 0.1 and the peak efficiency from about $80 \%$ to $65 \%$. The highest efficiency was obtained when more inlet swirl than the design value was added with a flap angle of $+20^{\circ}$.

Load coefficient versus flow coefficient is plotted in Figure 5.2 for the same rotor stagger angle of $75.2^{0}$ (Figure 5.1). For $\phi<0.26$ to 0.36 , the characteristics are represented well by steep straight lines but for higher $\psi$ values the slope decreases. The steep straight lines can be well predicted by the basic free vortex theory by making the assumption that the relative exit flow angles from the rotor blades and IGV remain constant (Von Backström \& Gannon 2001). When the flow is attached, the theoretical and experimental data agree well. When separation begins to occur, the blade stalls and the slope of the $\psi$ - $\phi$ lines deviates from the theory. 


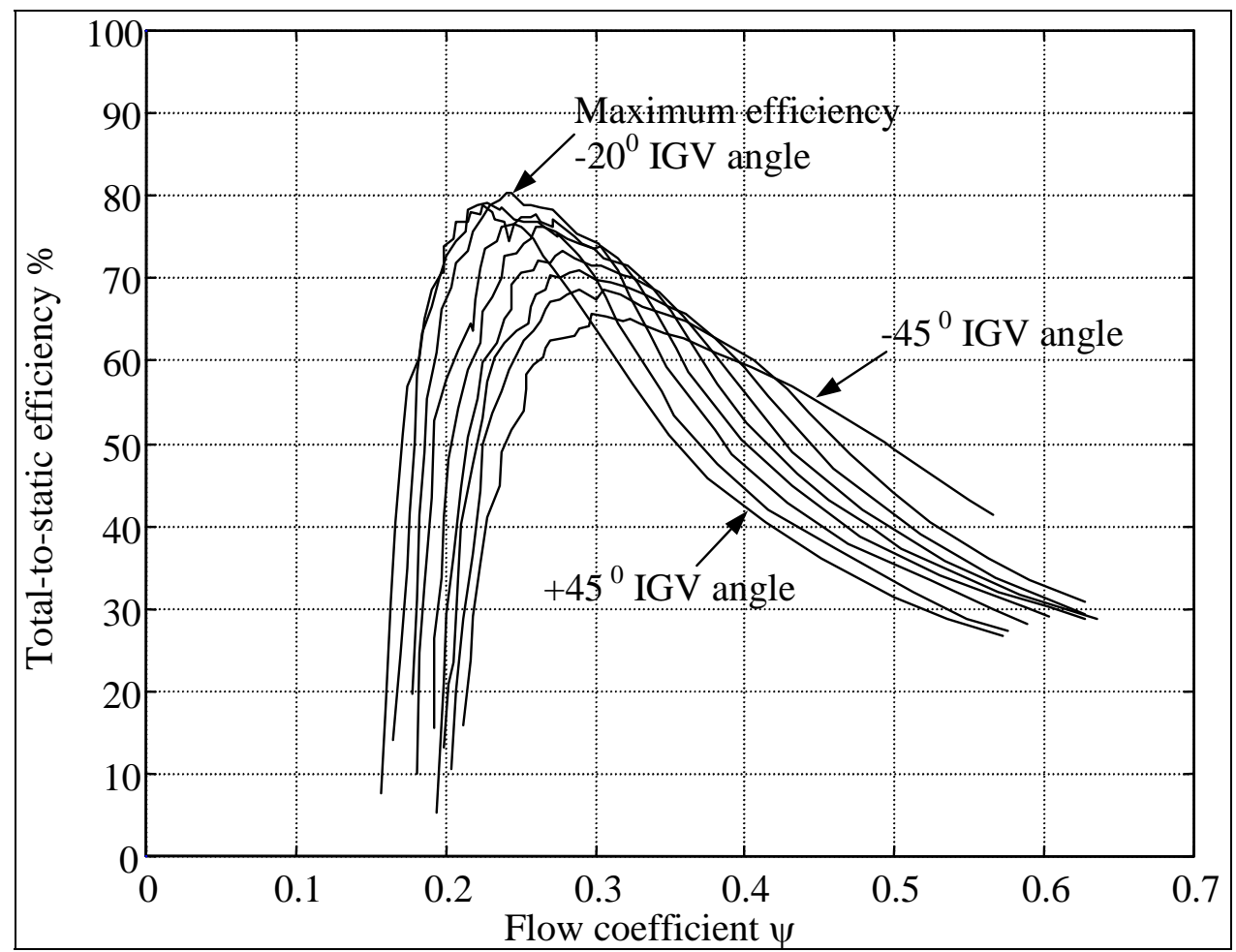

Figure 5.1. Efficiency (\%) vs flow coefficient $(\phi)$ at $75.2^{0}$ stagger angle for IGV-flap angles of $-45^{0},-30^{0},-20^{0},-10^{0}, 0^{0},+10^{0},+20^{0},+30^{0},+45^{0}$.

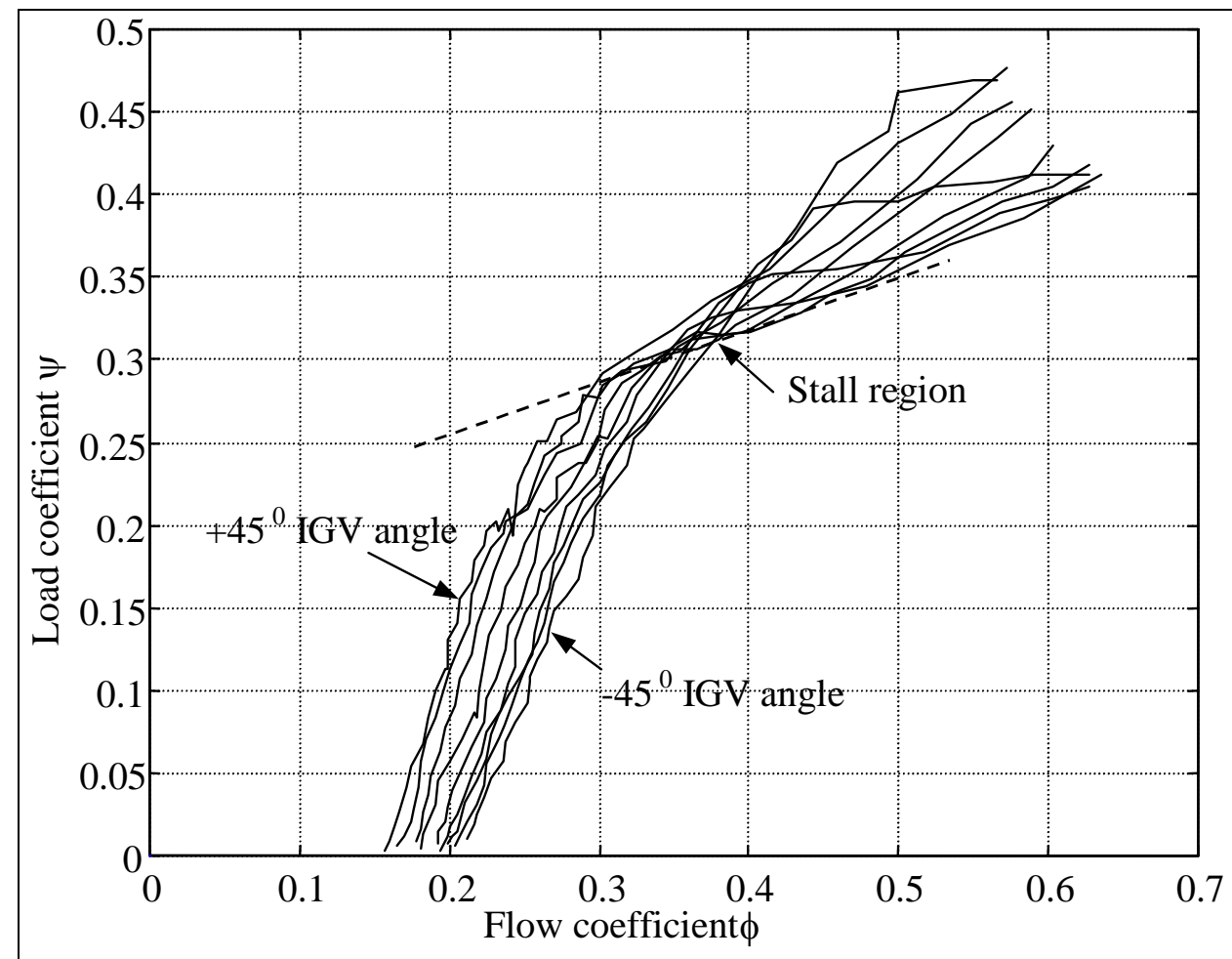

Figure 5.2. Load coefficient, $(\psi)$ vs flow coefficient $(\phi)$ at $75.2^{0}$ stagger angle for IGV-flap angles of $-45^{0},-30^{0},-20^{0},-10^{0}, 0^{0},+10^{0},+20^{0},+30^{0},+45^{0}$. 


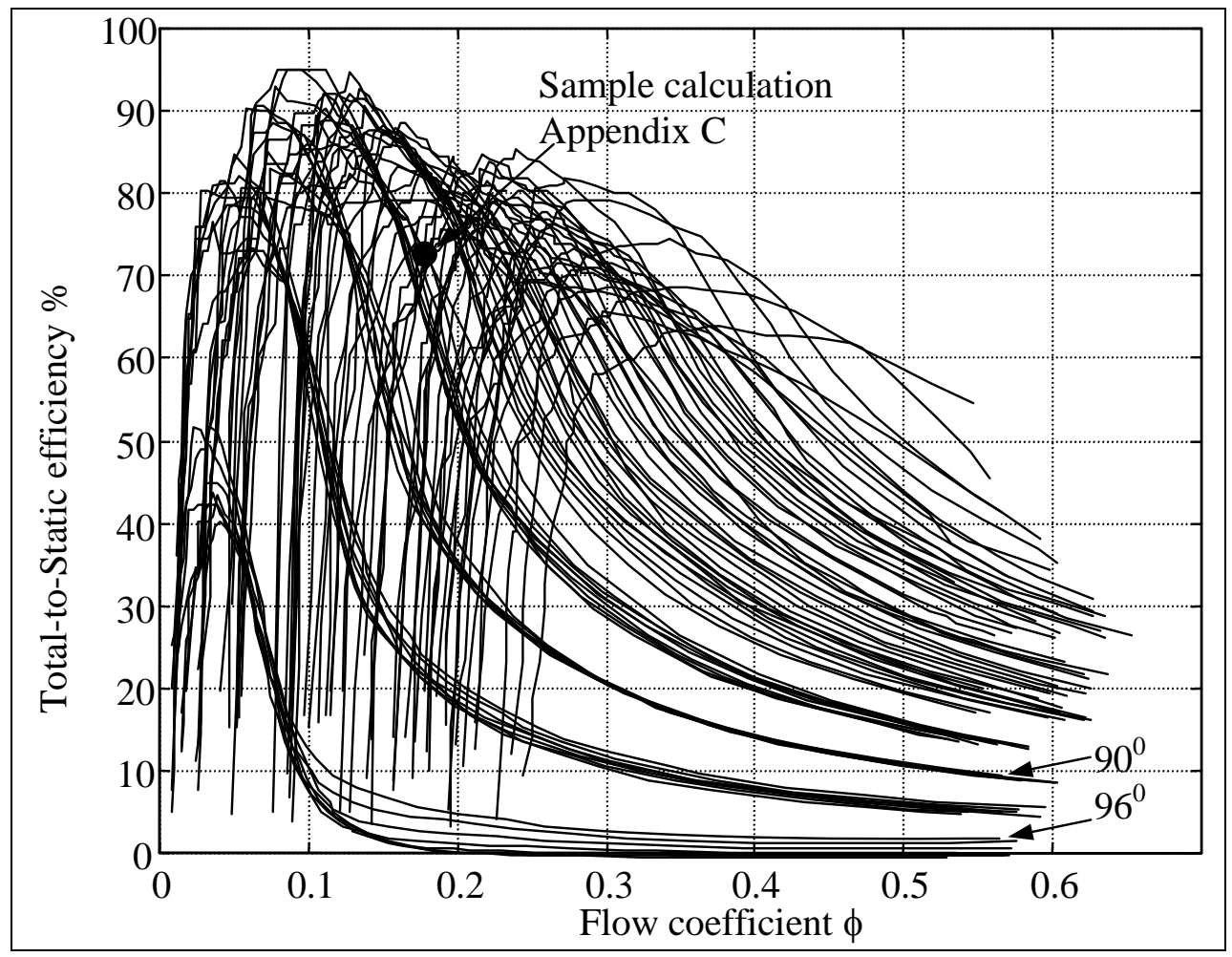

Figure 5.3. Efficiency (\%) vs flow coefficient $(\phi)$ for all rotor tip stagger angles.

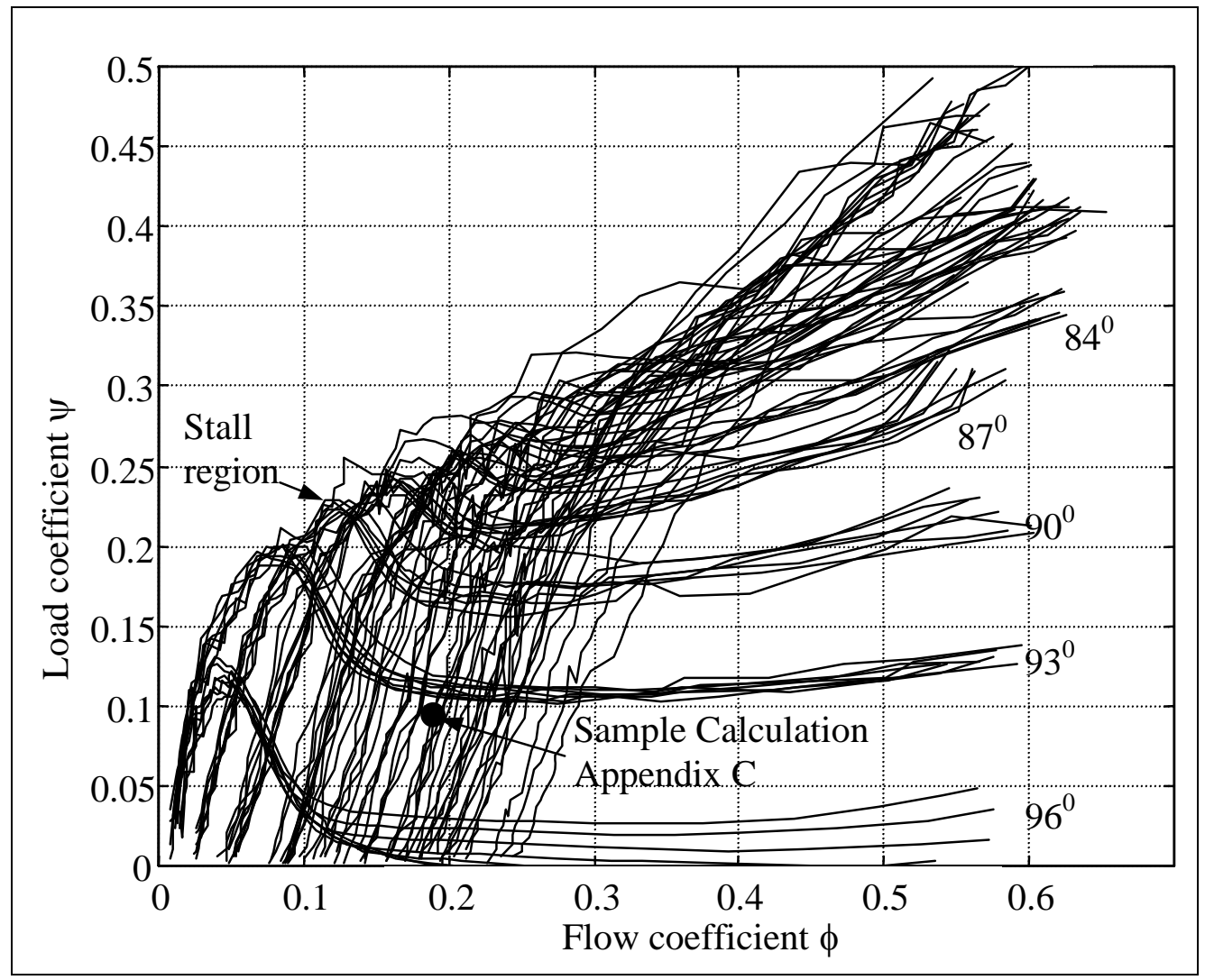

Figure 5.4. Load coefficient, $(\psi)$ vs flow coefficient $(\phi)$ for all rotor stagger angles. 
Figure 5.3 shows the full range of experimental efficiency data points taken. At very high rotor stagger angles there is definite banding of the results from a single rotor blade setting with the IGV angle only affecting the maximum efficiency but not the operating range. At lower rotor stagger angles the IGV setting angle affects the maximum efficiency and optimum operating point with the individual operating lines being more widely spread out.

In Figure 5.4 the entire range of load versus flow coefficient is plotted. The banding of the lines at rotor stagger angles close to $90^{\circ}$ can be seen. The stall region for the high stagger angles is also quite easily seen. What is also evident is that there is more than one possible operating point in terms of flow coefficient for certain values of load coefficient.

Using the data from Figure 5.3 and Figure 5.4 and discarding the low efficiency points, a contour plot of the highest measured total-to-static efficiencies is presented in Figure 5.5. With a performance map of the turbine available, it is now possible to investigate how well the design criteria have been met. At this stage of the experimental program, it could be seen that controlling the plant mass flow rate by increasing the turbine pressure drop is not possible. While it was possible to have a high-pressure drop at very high stagger angles $\left(90^{\circ}+\right)$, the efficiency drops considerably and the design power would not be extracted from the flow. The flow rate would then need to be increased and the initial intent of energy being stored in the collector would not be achieved. It would be better to use another control method and optimise the turbine for very efficient operation.

Table 5.3. Experimental total-to-static efficiencies measured at design points.

\begin{tabular}{|c|c|c|c|}
\hline & Flow coefficient $(\phi)$ & Load coefficient $(\psi)$ & Efficiency. $(\%)$ \\
\hline 1) Low Press & 0.1770 & 0.0742 & 70.6 \\
\hline 2) High Press & 0.2577 & 0.2018 & 82.8 \\
\hline
\end{tabular}

Recall from Chapter 3 that there were three design points chosen: case (1) low-pressure drop, case (2) high-pressure drop and case (3) intended for very high pressure drop to control the mass flow. Case (3) is unlikely to be used in practice for the reasons given above. The flow and load coefficients for cases (1) and (2) are presented in Table 5.3 and superimposed on Figure 5.7. Using the experimental data presented in this figure, the total-to-static efficiencies calculated using the wall static pressure are interpolated. They are given in column three of the table.

Figure 5.6 shows the maximum total-to-static efficiencies using the design IGV flap angle of $0^{0}$ giving a $22.5^{0}$ flow deflection. The initial design assumption that the inlet guide vanes would not be able to turn the flow more than this proved to be conservative. The Reynolds numbers were low and it was thought that the flow may be laminar and thus separate very easily. The experimental results show that more turning is possible and results in a higher overall efficiency. This is discussed in more detail later. 


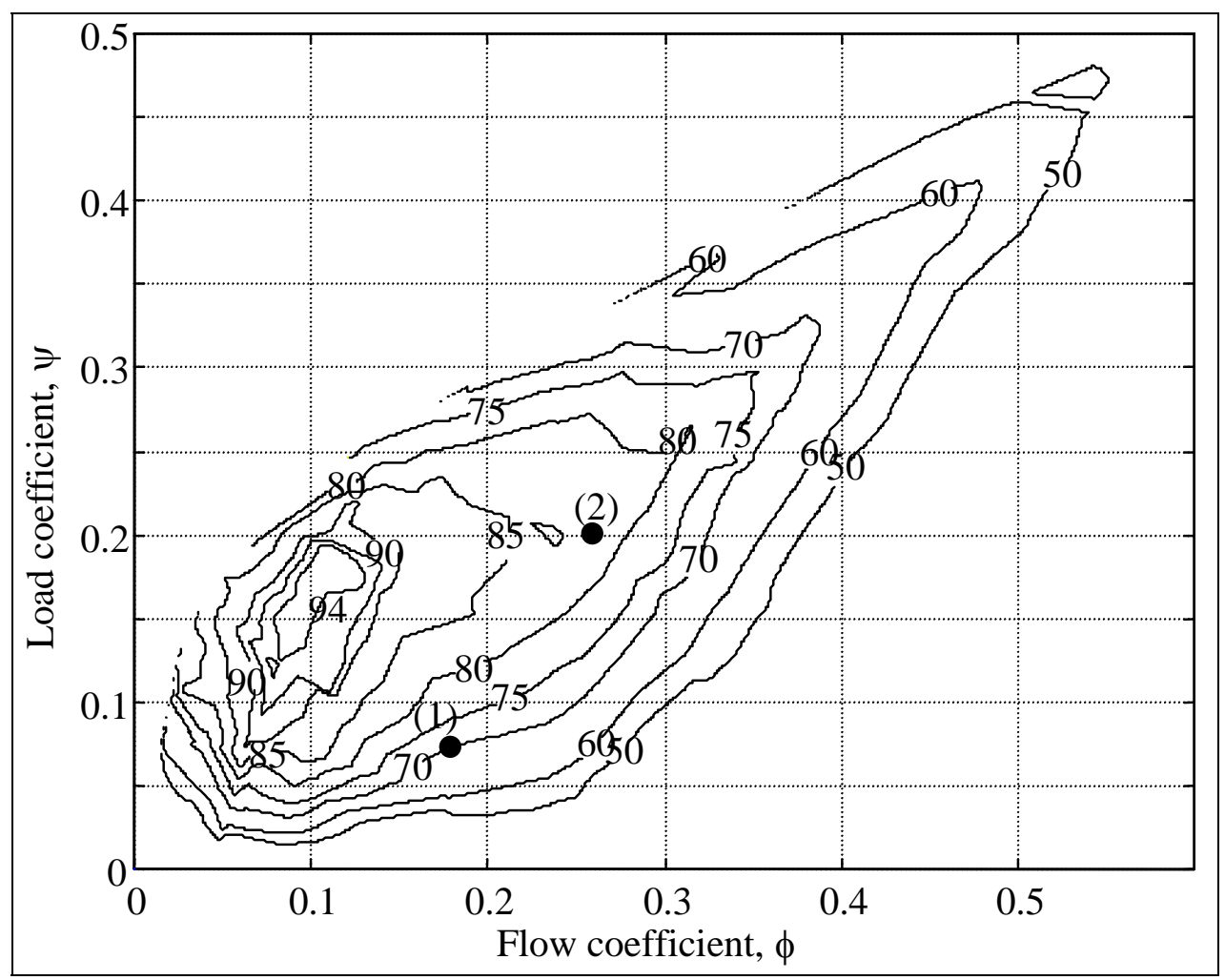

Figure 5.5. Experimentally derived total-to-static efficiencies [\%] against load, $\psi$, and flow, $\phi$, coefficients for optimal IGV angle.

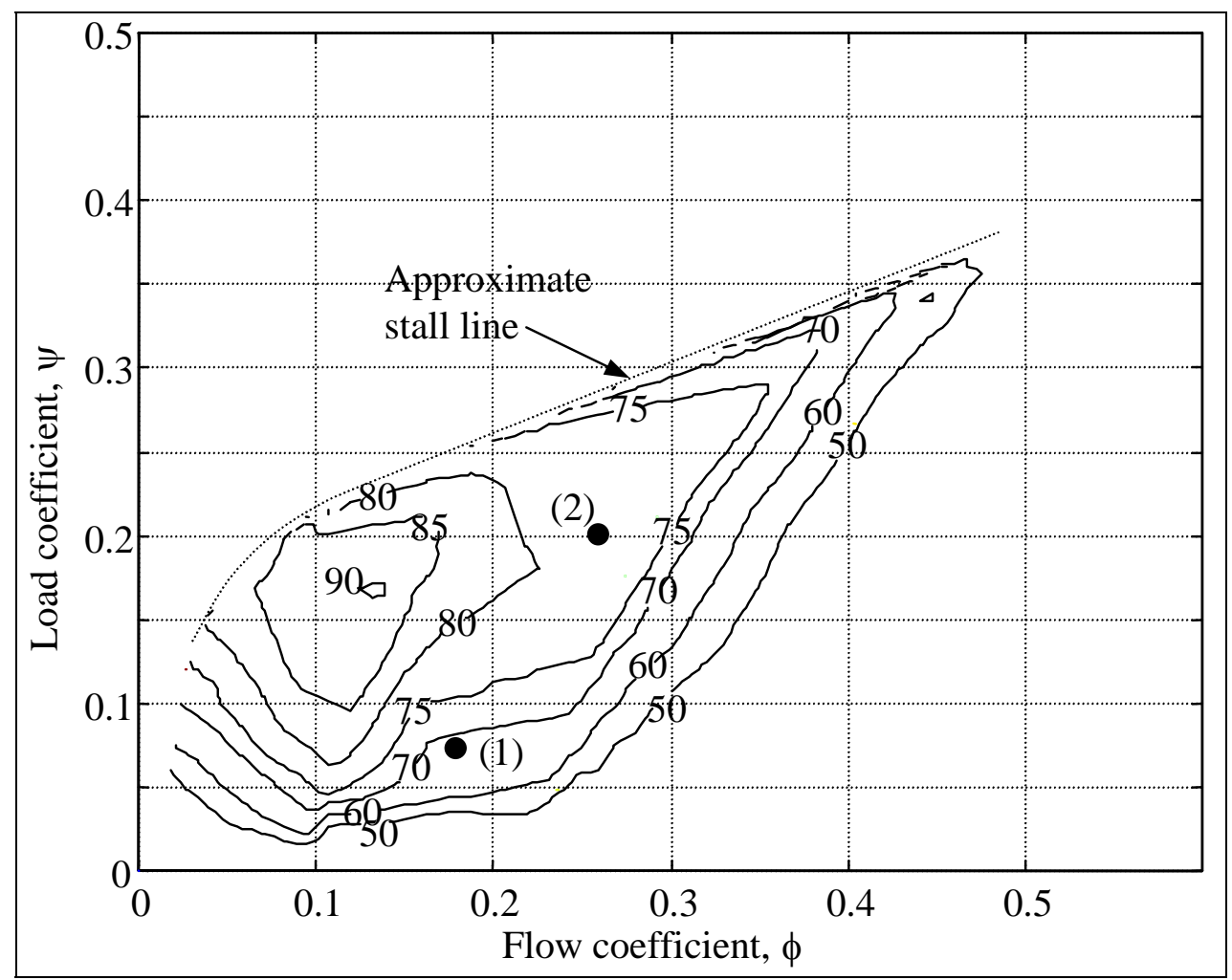

Figure 5.6. Experimental total-to-static efficiency contours [\%] versus load, $\psi$, and flow, $\phi$, coefficients (for design IGV flap angle of $0^{0}$ ). 


\section{Comparison of Predicted and Measured Efficiencies}

With a turbine efficiency map obtained experimentally, it is now possible to compare the design predictions with the working turbine. It must be remembered that at high $\psi$ values, when the swirl velocity is typically high, the wall static pressures tend to be higher than the average. This leads to the measured total-to-static efficiencies being optimistic.

During the turbine design process, the axial kinetic energy loss was not included in the optimisation as it was included in cycle analysis. When using the wall static pressure measured after the diffuser section, it is difficult to separate the axial and tangential kinetic energy losses. It is simpler to perform the design calculation with axial kinetic energy losses included and then compare this to the experimental values.

Figure 5.7 is similar to the one presented in Chapter 3 for the optimisation but with the axial kinetic energy loss added. The maximum turning allowed by the IGV was also increased to $22.5^{0}$ instead of the more conservative value of $20^{\circ}$ used for the initial design. Figure 5.7 should be compared to Figure 5.6 as both assume $22.5^{0}$ flow turning by the IGV. A first comparison between the two does not show good agreement between the efficiencies especially at low $\phi$ values. This is due to the lack of a loss model in the theoretical calculations.

Better agreement between the two is obtained when the Soderberg loss correlation is introduced into the free vortex analysis (Lewis, 1996). The theory behind this is presented in Chapter 3. Figure 5.8 shows how the theoretical efficiency decreases with the introduction of this loss model. The Soderberg model is too pessimistic, however, as shown in Table 5.4. The experimental and simulated efficiencies predicted using the free vortex method are compared.

Table 5.4. Comparison of experimental and predicted total-to-static efficiencies at the design points.

\begin{tabular}{|l|c|c|c|c|}
\hline & $\begin{array}{c}\text { Figure 5.5 } \\
\text { Experimental }\end{array}$ & $\begin{array}{c}\text { Figure 5.6 } \\
\text { Experimental 22.5 }\end{array}$ & $\begin{array}{c}\text { Figure 5.7 } \\
\text { Simulated ideal }\end{array}$ & $\begin{array}{c}\text { Figure 5.8 } \\
\text { Simulated loss model }\end{array}$ \\
\hline 1) Low press. & 70.6 & 69.0 & 81.9 & 66.6 \\
\hline 2) High press. & 82.8 & 78.2 & 83.3 & 74.9 \\
\hline
\end{tabular}




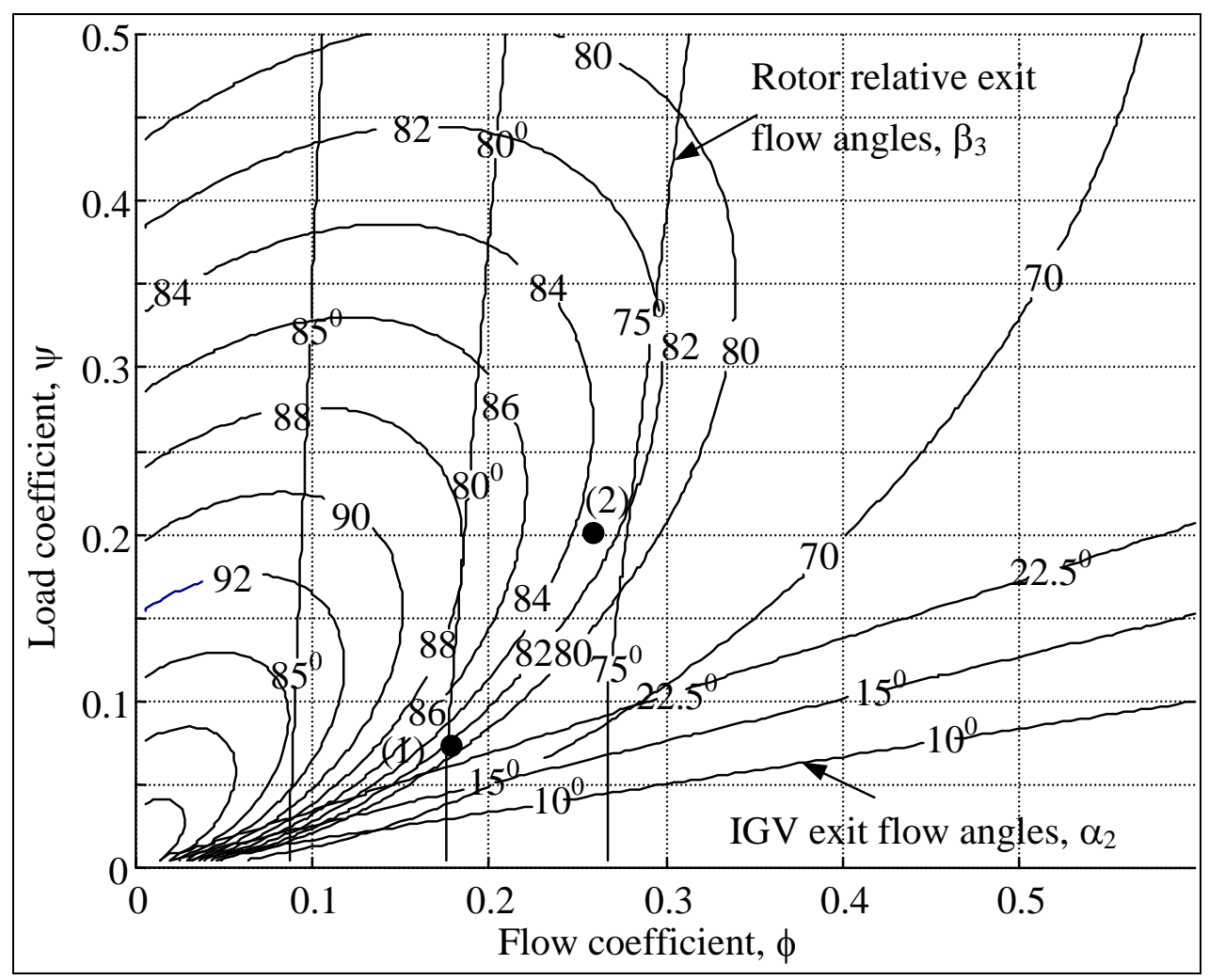

Figure 5.7. Predicted efficiency contours used in turbine design.

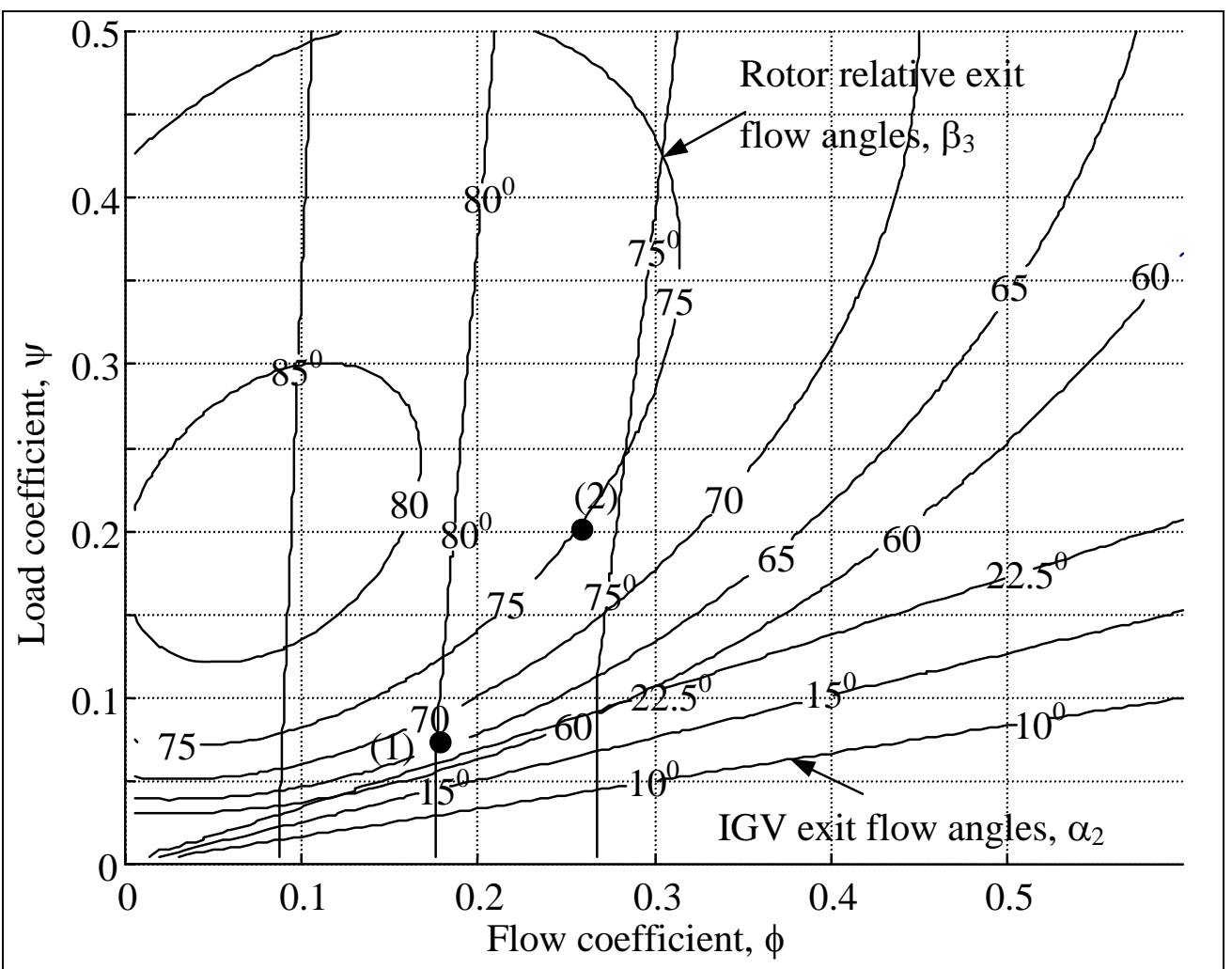

Figure 5.8. Predicted efficiency contours with Soderberg loss model. 
The most obvious difference between the experimental and theoretical efficiency contour plots is the effect of stall with the real turbine having a finite operating region. The comparison of efficiencies at the design points show that a loss model is needed to predict the correct efficiencies. In the second half of this chapter the detailed flow measurements taken over the rotor blade allow the blade loss coefficient to be calculated.

One of the most significant effects brought to light in the experimental program was the effect of the loss coefficient. While it is obvious that losses will lower the efficiency, it was found that they had a greater effect on the low-pressure drop case. This is not desirable, as it is more important to have an efficient turbine in the morning than near midday when there is surplus energy. The reason for the greater loss effect at low pressures is the high turbine tip speed relative to the throughflow velocity, and generator requirements of a turbine operating at a constant rotational velocity. Due to the high tip speed the main loss component in the system, the rotor loss, is almost constant over the entire operating range. Thus at lower power settings it has a greater effect on the efficiency. This fact is shown clearly in both the experimental data and simulation with the simple loss model included shown in Table 5.4.

\section{Rotor and Inlet Guide Vane Angles}

Using Figure 5.5 and superimposing the corresponding IGV flap angles used as contours, produces Figure 5.9. Recall that the trailing $30 \%$ of the IGV can be adjusted to increase or decrease the turbine pre-swirl, $\alpha_{2}$. Positive adjustment increases the amount of inlet swirl while negative decreases it. What is significant in the above figure is that the maximum efficiency at the two design points occurs with a higher IGV angle than the design value $\left(+20^{\circ}\right)$. According to the surface vortex method used in the blade design, this flap angle results in $\alpha_{2}=42^{0}$. Increasing the flap angle beyond this did not improve the efficiencies further as indicated by the large plateau of $+20^{\circ}$. Due to the low Reynolds numbers in the scale model, the flow may be separated in parts of the IGV at these high setting angles. This would lead to increased losses over the IGV but improves the overall efficiency by decreasing the exit kinetic energy.

Figure 5.10 superimposes the rotor tip stagger angles over the experimental efficiencies. In the design process the rotor relative exit flow angle, $\beta_{3}$, was limited to $80^{\circ}$. Even though there will be a deviation angle between the blade exit and flow direction, the figure shows that the highest

efficiency is obtained when $\beta_{3} \cong 87^{0}$. This is partly due to the axial velocity near the blade tip being higher than at the root resulting in a smaller $\beta_{3}$. Fundamental to the free vortex analysis is the assumption of a uniform axial velocity profile. Both the matrix throughflow method and detailed experimental results of the next section show that the axial velocity is higher at the tip than at the hub. 


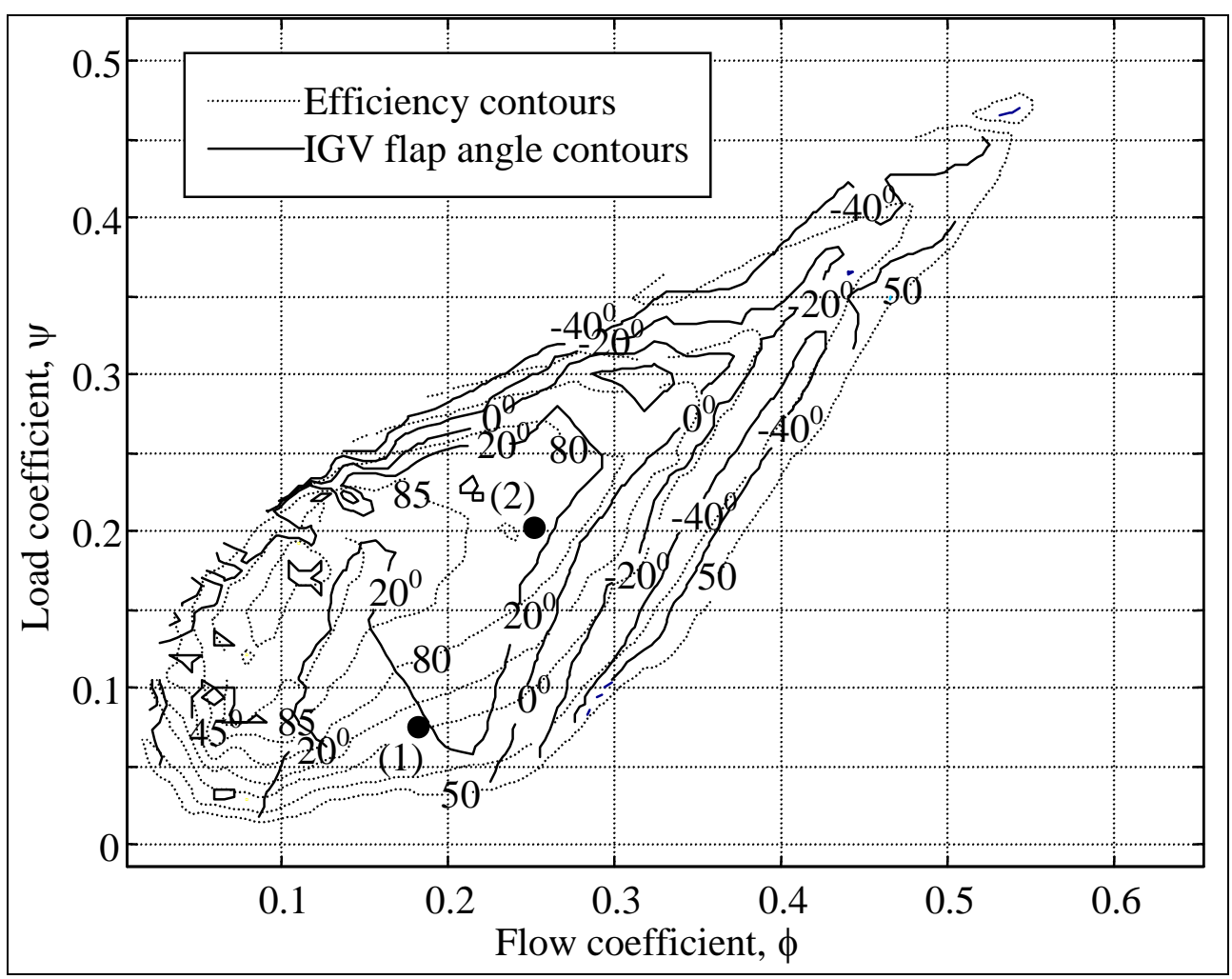

Figure 5.9. IGV flap angles for maximum efficiency.

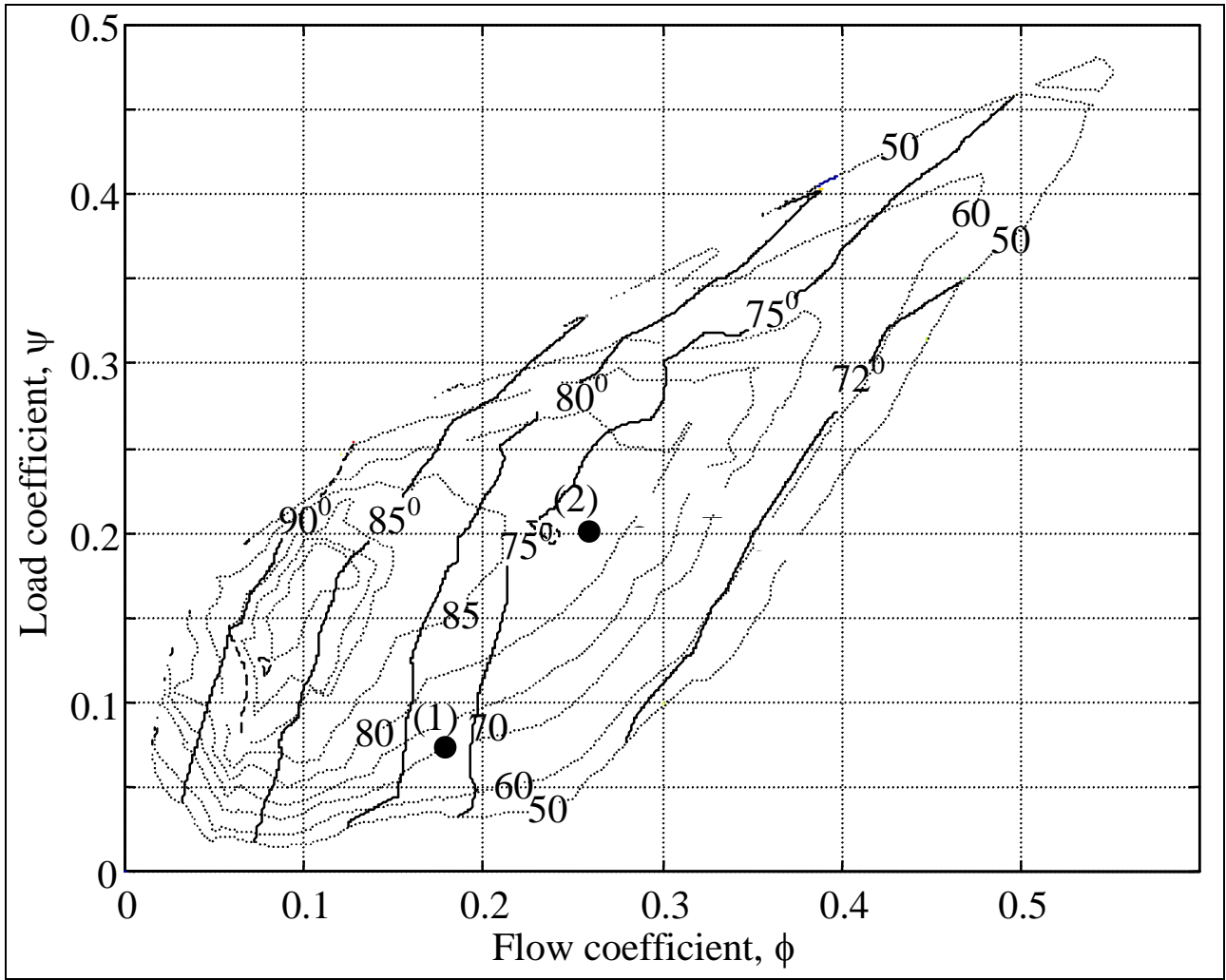

Figure 5.10. Rotor tip stagger angles for maximum efficiency. 


\section{Runaway Speed}

In the case of a sudden loss of generator load the turbine will run away. If the volume flow rate stays the same, the speed will stabilise when the inlet and exit velocity triangles are the same, i.e. no flow deflection. The runaway blade speed $U_{\text {runaway }}$ for a constant flow rate increases by the amount of flow deflection,

$$
\mathrm{U}_{\text {runaway }}=\mathrm{U}+\left(\mathrm{C}_{\theta 3}-\mathrm{C}_{\theta 2}\right)
$$

As the whirl velocity is much smaller than the tip speed, it would seem that the runaway condition is not serious. The main problem with the solar chimney is actual plant runaway- if the turbine pressure drops suddenly, the mass flow will increase significantly. A similar phenomenon was observed in the experimental program. If the load was removed from the turbine, the fan flow rate increased and the turbine would accelerate rapidly. The flatter the turbine blade angle the more serious this condition is. The turbine blades need to be rapidly adjustable or the plant requires some mechanism to halt the flow or both.

\section{Assessment and Improvements to the Free Vortex Analysis}

The experimental program thus far has shown possible improvements to the free-vortex method used in the initial turbine design:

- The use of a blade loss model.

- Inclusion of the axial kinetic energy losses in the turbine optimisation.

The constraints used in the optimisation process were also shown to be too conservative. The following will allow the total-to-static efficiency to be improved:

- Increase of the maximum IGV flow angle $\alpha_{2}$.

- Increase of the relative exit flow angle $\beta_{3}$ of the rotor.

These will be discussed in more detail at the end of the chapter along with other design improvements that stem from the detailed experimental measurements.

\subsection{DETAILED MEASUREMENTS}

The second part of the experimental program used a five-hole probe to measure pressure and velocity profiles upstream and downstream of the turbine and at one pipe diameter downstream of the diffuser exit. These measurements allow the variation of the velocity and pressure profiles across the flow passages to be seen. These allow insight into the turbine operation to be improved. They are also compared to the MTFM to asses the limitation of the inviscid flow analysis. 


\section{Choice of Measuring Points.}

The detailed measurements were taken at the two design points, (1) and (2). Even though the experimental results have already shown that higher efficiencies can be gained at other operating points, data taken at these design points will allow for good comparison with the design simulations. Figure 5.11 is the same as Figure 5.6 but with the rotor tip stagger angles used in the experiments superimposed (recall that Figure 5.6 used IGV flap angle $=0^{0}$ ). The angles of $78^{0}$ and $75.2^{0}$ are the design stagger angles for the two operating points. The low-pressure drop design point lies on $78^{0}$, while the high-pressure drop point is slightly to the left of $75.2^{0}$, they are very close to the operating points. Using these rotor setting angles, the comparison with the previous results as well as with design method is simple. In the following figures the scale of the low pressure and high pressure drop cases are kept the same to simplify comparison between the two.

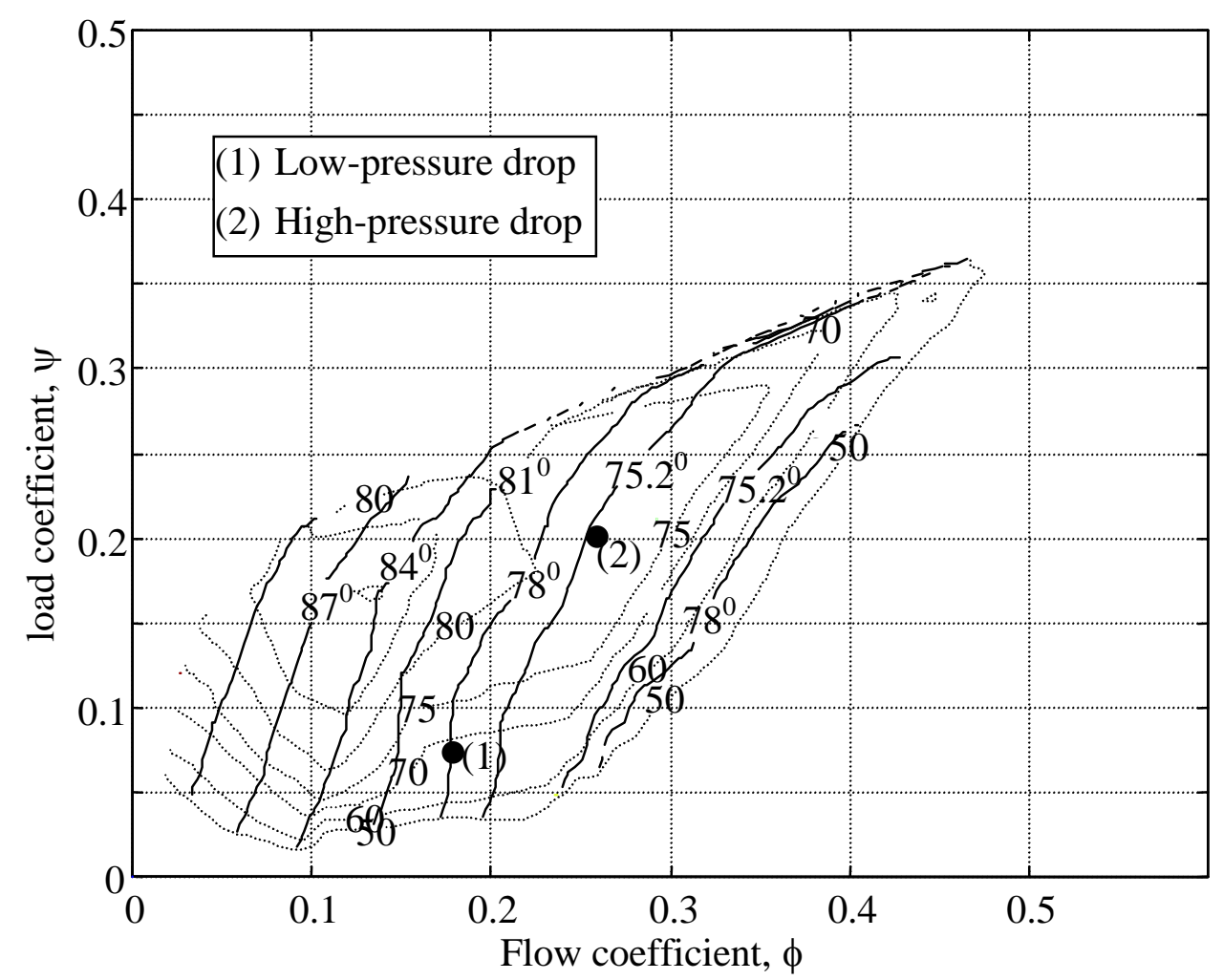

Figure 5.11. Experimentally derived total-to-static efficiencies [\%] against load, $\psi$, and flow, $\phi$, coefficients (for design IGV flap angle of $0^{0}$ ).

\section{Raw Data: Velocities}

Figure 5.12 and Figure 5.13 present the measured velocity profiles up and downstream of the rotor for cases (1) and (2) respectively. Figure 5.14 and Figure 5.15 show the corresponding total and static pressure profiles measured up and downstream of the rotor. The positions of the probe traverses are shown in Chapter 4. 
Figure 5.13 reveals some of the flow phenomena found in the solar chimney turbine. What is most notable about the flow are the skewed axial velocity profiles. The axial velocity near the turbine tip is significantly higher than at the hub. This is caused by the pre-swirl introduced by the IGV. As the swirling flow moves towards the centre the tangential flow component must accelerate in order to conserve angular momentum. Figure 5.15 shows the static pressure profile that develops just upstream of the turbine. The pressure gradient forces the flow towards the turbine tip. This shift towards the turbine tip begins within the IGV, meaning that more of the flow passes through the top of the IGV than the base. This causes even more flow through this tip region as more angular momentum is added to the flow at the IGV top than at the base, causing the pressure gradient to be steeper near the turbine tip than the hub.

This high axial velocity at the tip was predicted by the MTFM, but without the benefit of experimental results the validity of this prediction could not be verified. In the free-vortex analysis one of the underlying assumptions is that the axial flow is uniform. This is not the case and making this assumption could lead to the incorrect relative flow angles for the turbine rotor being chosen. For the present design, the free vortex analysis was used to determine the major turbine dimensions. The MTFM was used to calculate the relative flow angles.

When the turbine was designed, there was concern that the rotational speed of the full-scale turbine would be too slow, leading to difficulties in the design of a suitable generator. The rotational speed is limited by the turbine tip speed. This in turn is limited by the relative exit flow angle. As explained in more detail in earlier chapters, if the tip stagger angle becomes too flat, the losses increase significantly. In order to increase the rotational speed the pressure drop at the tip was decreased to reduce the relative exit flow angle.

It would seem that the IGVs not only reduce the amount exit whirl and lower the kinetic energy losses. They have the added benefit of increasing the axial velocity near the blade tip. This reduces the relative exit flow angle as a simple velocity triangle diagram will show. For future turbine design, the tip speed or tip pressure drop can be increased thereby increasing the turbine total-tostatic efficiency. It would also be better to base more of the turbine design on the MTFM now that it is more certain that it predicts the flow reasonably well. The MTFM simulation is evaluated in more detail later. While only case (2) has been discussed in detail the trends are similar to case (1) but with lower velocities.

Figure 5.14 shows the total and static pressure profiles before and after the turbine rotor for case (1). The inlet total pressure loss is very small, typical of accelerating flow. The design pressure distribution across the blade can be clearly seen in that the exit total pressure has a greater drop near the root than the tip. The effect of the IGV wake is small and is indicated in the figure. In all figures the boundary layers can be seen to be thin, typical of the accelerating flow through turbines. 


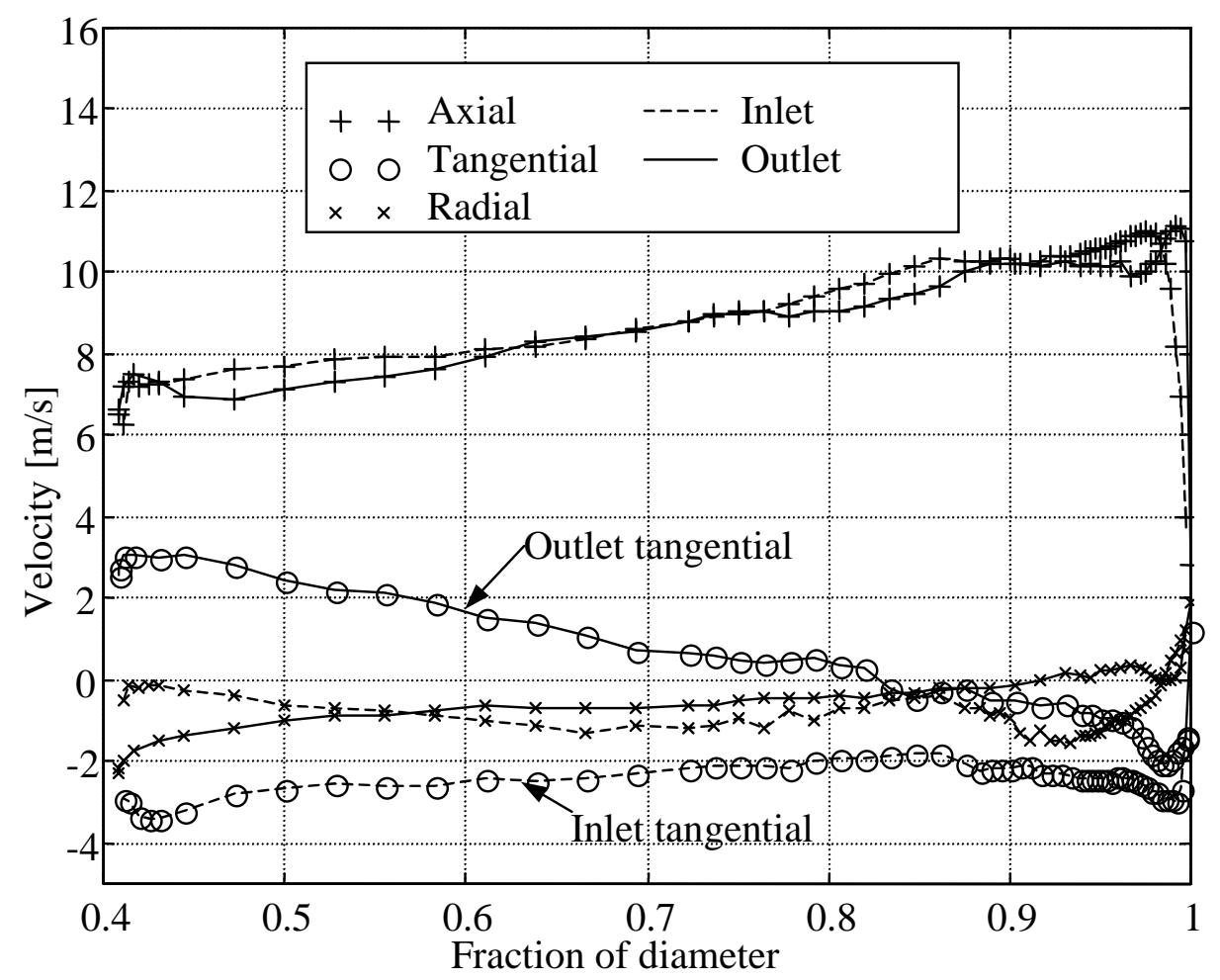

Figure 5.12. Velocity profiles for low-pressure drop (case 1).

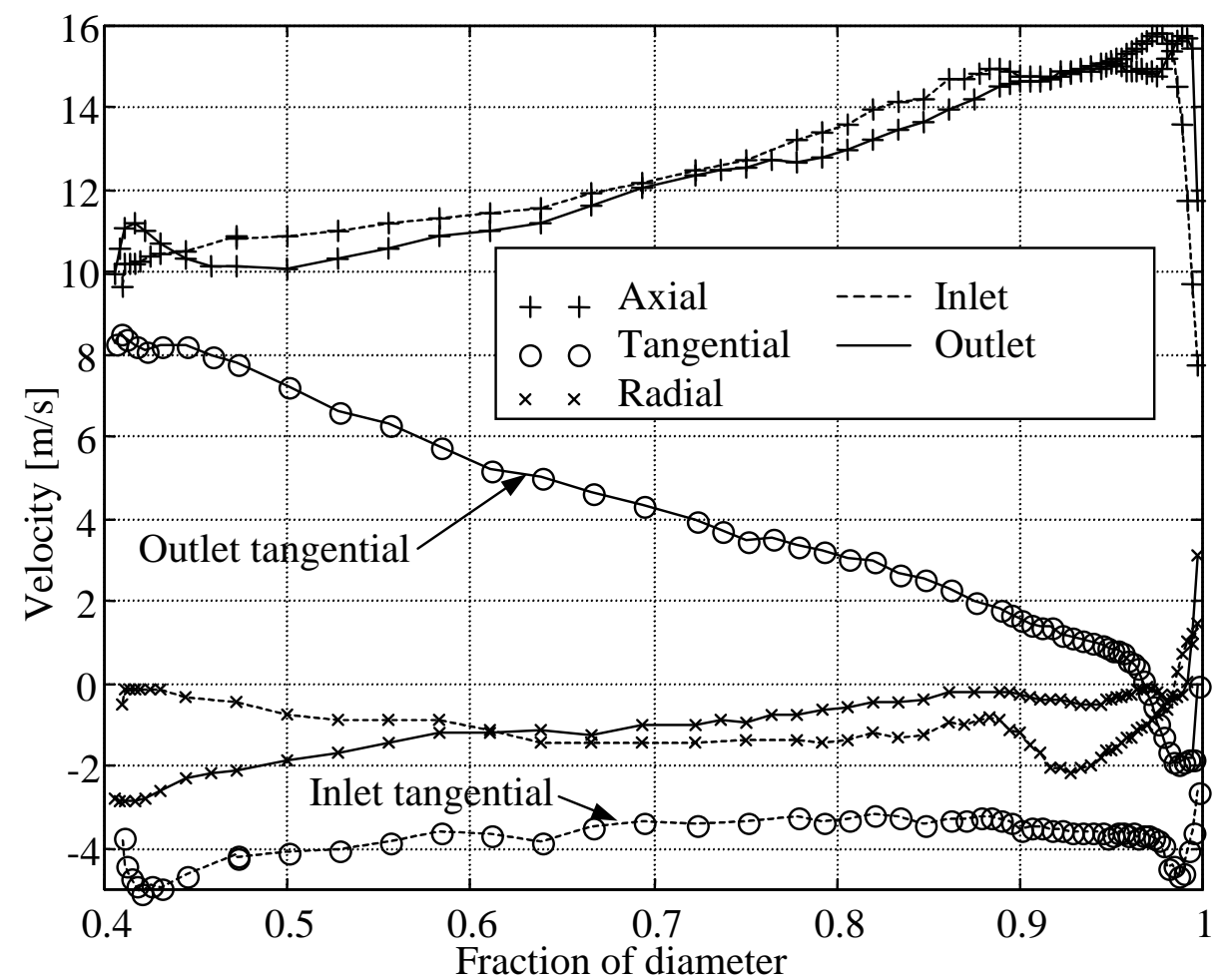

Figure 5.13. Velocity profiles for high-pressure design point (case 2). 


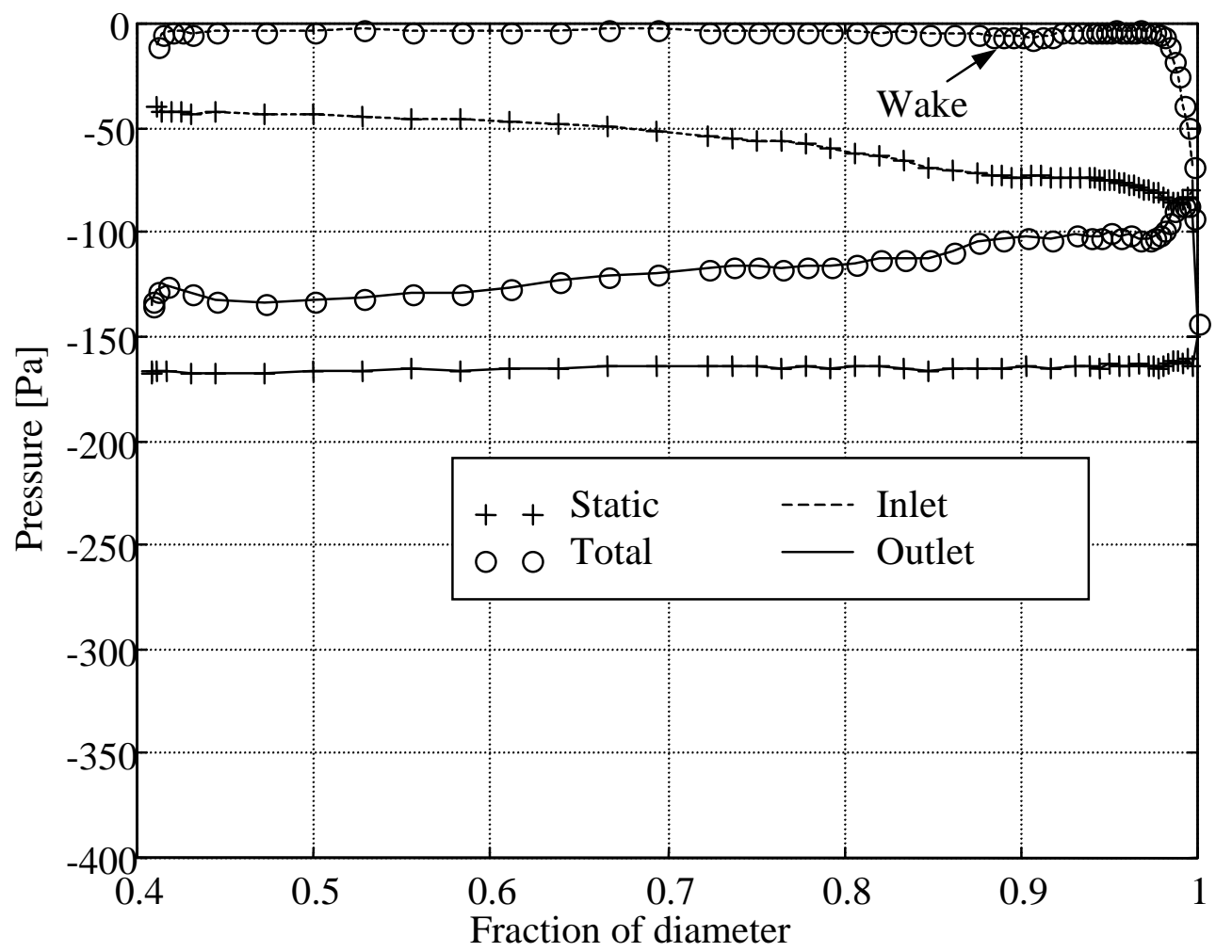

Figure 5.14. Total and static pressures for low-pressure design point (case 1).

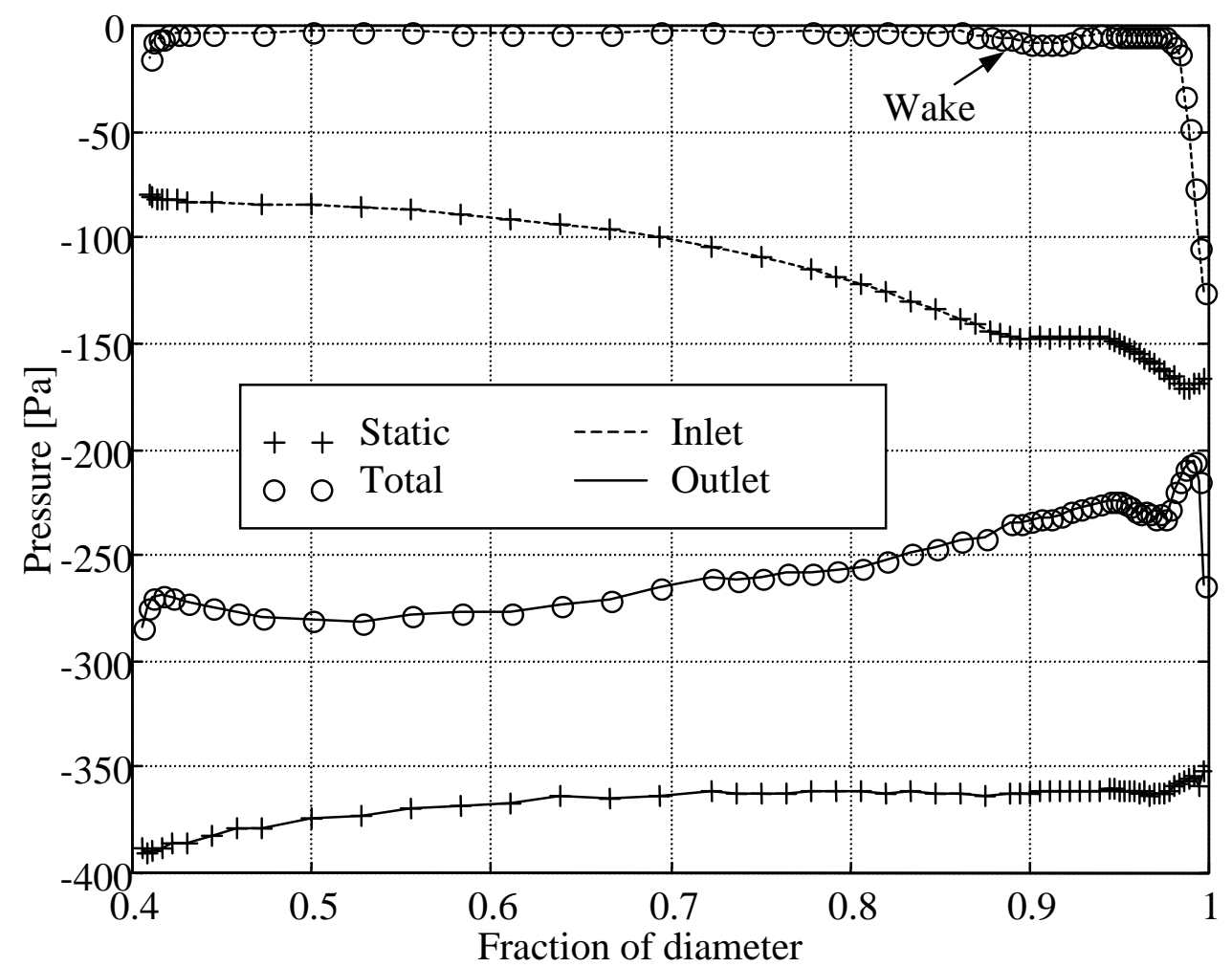

Figure 5.15. Total and static pressures for high-pressure design point (case 2). 


\section{Raw Data: Diffuser}

Measurements of the velocity and pressure profiles allow the calculation of flow-averaged total and static efficiencies to be calculated. They are more accurate than those calculated with the wall static pressures. It was not possible to measure the profiles near the centre of the pipe due to the low velocities. When the difference between the static and total pressures is very small, the calculation of the velocity components becomes inaccurate. The second problem was that the radial component became large in comparison with the axial, resulting in the flow angle being outside of the five-hole probe calibration range. The missing data from the pipe centre has little influence on the results, for two reasons. The first is that the actual pipe area missing is only $6 \%$ and $12 \%$ for the low pressure and high pressure drop cases respectively. When this is combined with the very low velocities at the pipe centre, it has a very small effect on the overall calculations.

Figure 5.17 shows the velocity profiles one diameter downstream of the diffuser. The profiles are not yet fully developed and are still very similar to the exit velocities from the turbine. The higher axial velocity at the turbine tip is beneficial to the diffuser operation as it reduces separation on the diffuser walls. As mentioned before, the axial flow velocity near the pipe centre is very small (of the order of $1 \mathrm{~m} / \mathrm{s}$ ). It was thought that there might be a region of backflow at the centre of the pipe but attempts to measure this were not successful. The total and static pressures were almost identical, meaning the backflow velocity at the centre of the pipe, if it exists, is still very small. Any backflow present was so small that it was impossible to measure using the five-hole probe. The radial velocity is still inward as the flow continues to develop.

The calculated diffuser efficiencies were very high. This is due to the favourable diffuser entrance conditions where the wall velocity is high and there is some residual swirl in the flow. Both these factors reduce the chance of flow separation at the walls. The flow deceleration in a diffuser tends to be highest nearest the walls and the high velocities in this region further aid in the high performance of the solar chimney turbine diffuser. 


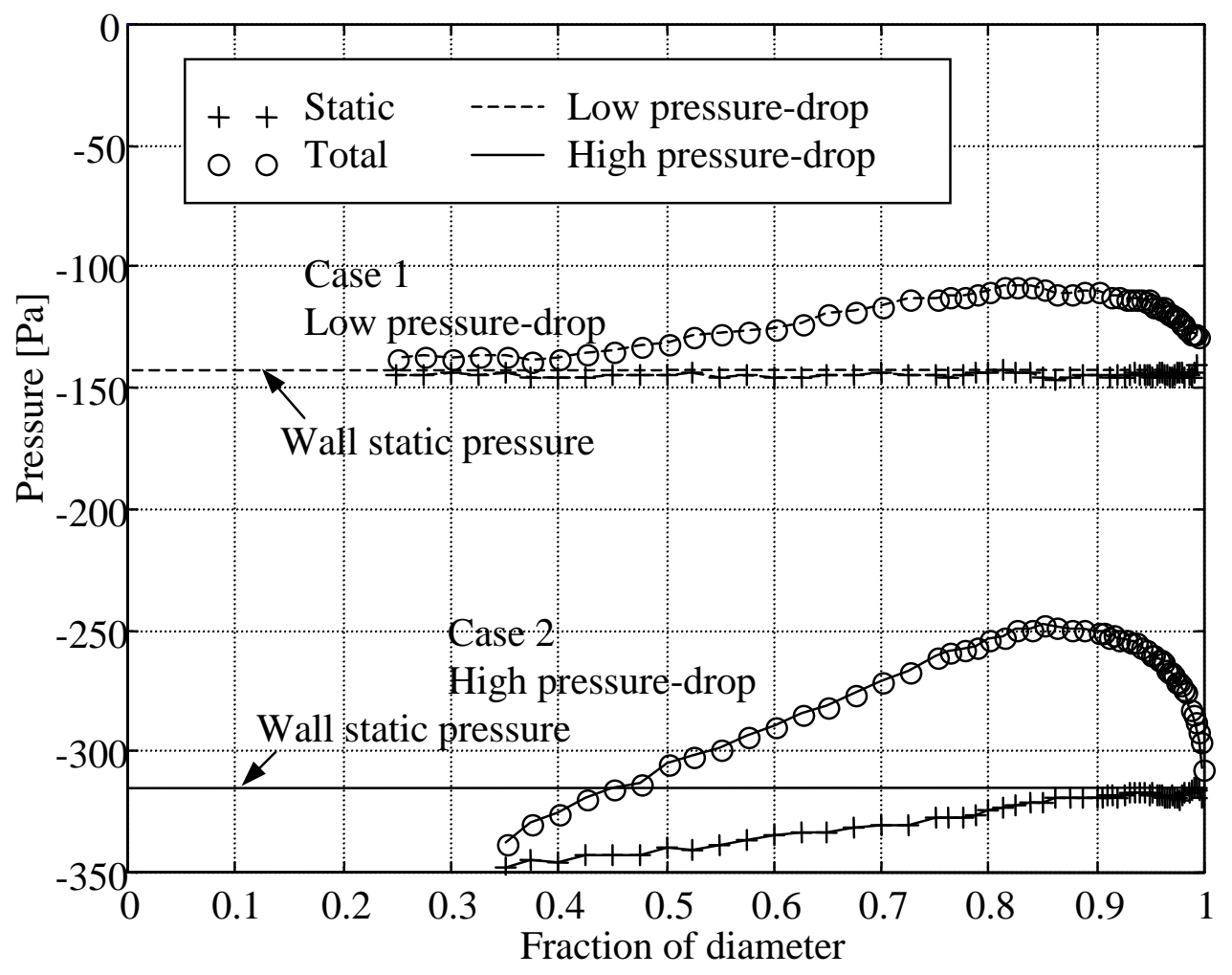

Figure 5.16. Total and static pressures one pipe diameter downstream of the diffuser.

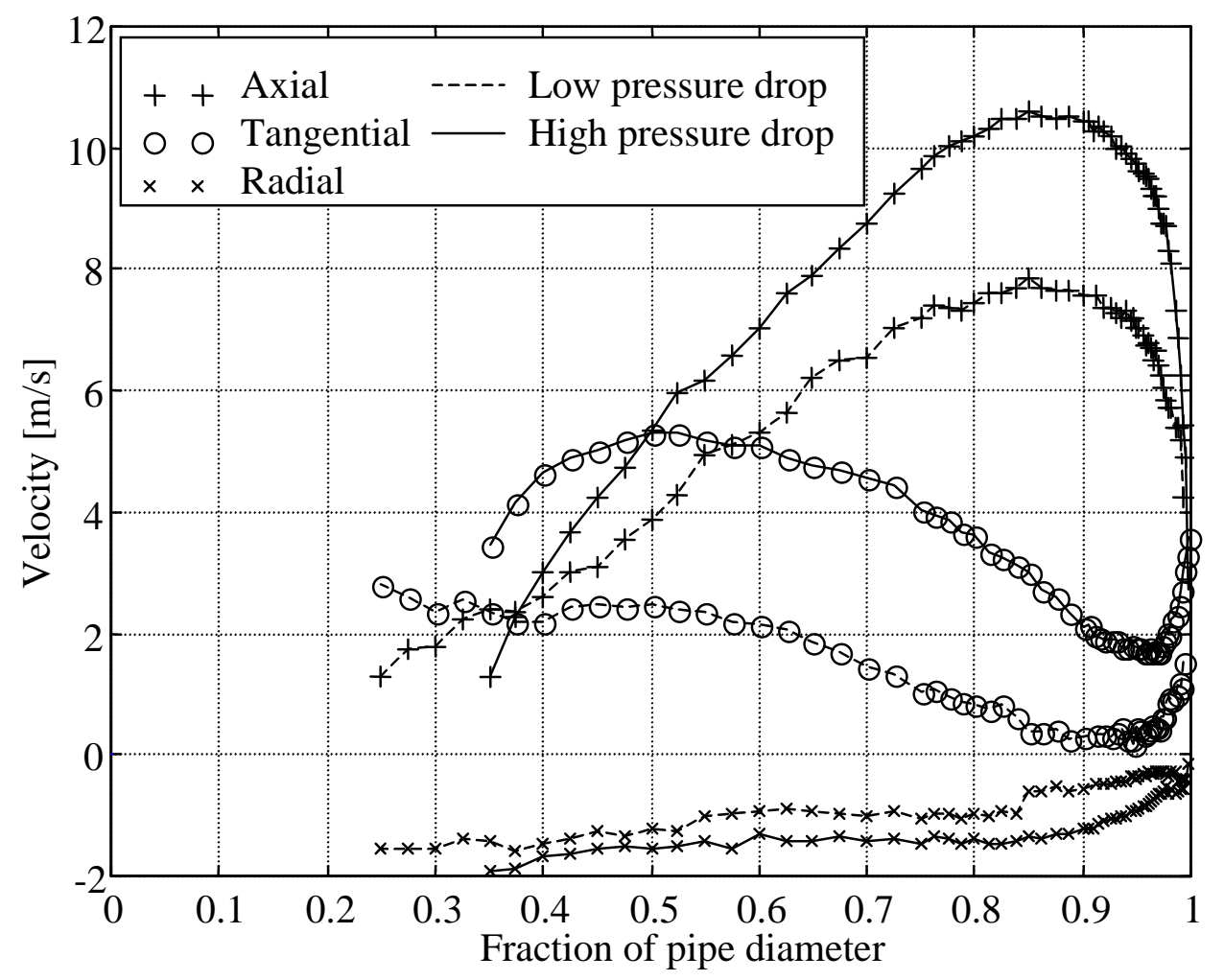

Figure 5.17. Velocities one diameter downstream of diffuser. 


\section{Volume Flow, Torque and Power Balances}

In order to check the accuracy of the profiles measured using the five-hole probe, the volume flow, momentum and power output are compared to other measurements.

The volume flow is calculated using the five-hole probe measurements by numerically integrating the volume flow across the profile. As the data points are closely spaced, the trapezium rule is used. Results from this integration are compared to the data obtained from the windmill anemometer in the table below.

Table 5.5. Comparison of volume flow rates from windmill anemometer with five-hole probe.

\begin{tabular}{|r|c|c|c|c|}
\hline & Anemometer & Rotor: Before & Rotor: After & Diffuser \\
\hline (1) Low-pressure & $2.80\left[\mathrm{~m}^{3} / \mathrm{s}\right]$ & $3.06\left[\mathrm{~m}^{3} / \mathrm{s}\right]$ & $3.01\left[\mathrm{~m}^{3} / \mathrm{s}\right]$ & $2.71\left[\mathrm{~m}^{3} / \mathrm{s}\right]$ \\
\hline Error [\%] & & 8.5 & 7.0 & 3.3 \\
\hline (2) High-pressure & $4.00\left[\mathrm{~m}^{3} / \mathrm{s}\right]$ & $4.36\left[\mathrm{~m}^{3} / \mathrm{s}\right]$ & $4.28\left[\mathrm{~m}^{3} / \mathrm{s}\right]$ & $3.57\left[\mathrm{~m}^{3} / \mathrm{s}\right]$ \\
\hline Error [\%] & & 8.3 & 6.5 & 12 \\
\hline
\end{tabular}

The integrated values and those obtained using the windmill anemometer do not show good correlation. There are two possible reasons why this may be so. The first could be due to a nonuniform velocity profile at the windmill anemometer. This is unlikely, however, as there is no reason for the flow to shift up or down in the inlet nozzle section. The main reason for the poor correlation is thought to be that the five-hole probe used was calibrated at a higher velocity than those found in the present turbine (Strohmaier 1997). Due to the small size of the probe, low Reynolds numbers affects it significantly. For case (2) the difference between the windmill anemometer and probe predictions is significant. Figure 5.16 and Figure 5.17 show that measuring the flow near the pipe centre was difficult due to very low axial flow velocities in comparison to the radial velocities. This caused the flow pitch angle relative to the probe to be out of its measuring range.

The momentum and power output can be calculated using the five-hole probe data and applying the Euler turbomachinery equation at each radial station.

$$
\begin{array}{cc}
P=\rho Q \omega\left(r_{3} C_{\theta 3}-r_{2} C_{\theta 2}\right) & \text { Power } \\
T=\rho Q\left(r_{3} C_{\theta 3}-r_{2} C_{\theta 2}\right) & \text { Torque }
\end{array}
$$

As for the case of the volume flow rate, the trapezium rule can be applied to the raw data shown in Figure 5.12 and Figure 5.13. The integrated momentum and power can be compared to the measurements obtained from the torque transducer and speed-reader. 
Table 5.6. Comparison of torque and power from torque transducer and speed-reader with five-hole probe.

\begin{tabular}{|r|c|c|c|c|}
\hline & $\begin{array}{c}\text { Transducer } \\
\text { torque }\end{array}$ & $\begin{array}{c}\text { Integrated } \\
\text { torque }\end{array}$ & $\begin{array}{c}\text { Transducer + speed- } \\
\text { reader power }\end{array}$ & $\begin{array}{c}\text { Integrated } \\
\text { power }\end{array}$ \\
\hline (1) Low-pressure & $1.96[\mathrm{Nm}]$ & $1.99[\mathrm{Nm}]$ & $243[\mathrm{~W}]$ & $266[\mathrm{~W}]$ \\
\hline Error [\%] & & 1.5 & & 8.6 \\
\hline (2) High-pressure & $7.86[\mathrm{Nm}]$ & $7.70[\mathrm{Nm}]$ & $958[\mathrm{~W}]$ & $935[\mathrm{~W}]$ \\
\hline Error [\%] & & 2.1 & & 2.5 \\
\hline
\end{tabular}

The comparison between the integrated torques and powers with those measured using the torque transducer and speed-reader is reasonable. Only for the power in case (1) is the error significant but the absolute error is small or of the order of $20 \mathrm{~W}$.

\section{Component Loss and Drag Coefficients}

The inlet and rotor loss coefficients, $\mathrm{K}_{\text {loss2 }}$ and $\mathrm{K}_{\text {loss rot, }}$ along the blade span can be calculated by using the velocity and pressure profiles. In addition, the coefficients of lift and drag, $C_{L}$ and $C_{D}$ of the rotor blades are calculated using the inlet and exit flow angles.

$\mathrm{K}_{\text {loss2 }}$, the inlet loss coefficient, includes the inlet and IGV losses. The inlet loss is the pressure difference between inlet total and atmospheric pressure and is non-dimensionalised with respect to the absolute rotor inlet velocity, $\mathrm{C}_{2}$,

$$
\mathrm{K}_{\text {loss } 2}=\frac{\mathrm{p}_{\mathrm{atm}}-\mathrm{p}_{02}}{\frac{1}{2} \rho \mathrm{C}_{2}^{2}}
$$

Rotor loss coefficients are calculated by comparing the theoretical Euler work at each station with the flow work (volume flow $\mathrm{x}$ pressure drop). The ideal or Euler pressure drop can be derived from the Euler turbomachinery equation. Assuming incompressible flow and no radial shift in the flow through the turbine, the ideal pressure drop is,

$$
\Delta \mathrm{p}_{\text {oideal }}=\rho \omega\left(\mathrm{C}_{\theta 3}-\mathrm{C}_{\theta 2}\right)
$$

The real stagnation pressure drop is measured directly,

$$
\Delta \mathrm{p}_{\text {oreal }}=\mathrm{p}_{02}-\mathrm{p}_{03}
$$

The rotor loss coefficients, $\mathrm{K}_{\text {loss rot, }}$ along the blade span are non-dimensionalised with respect to the rotor relative exit velocity $V_{3}$,

$$
\mathrm{K}_{\text {loss rot }}=\frac{\Delta \mathrm{p}_{\text {oideal }}-\Delta \mathrm{p}_{\text {o real }}}{\frac{1}{2} \rho V_{3}^{2}}
$$


Figure 5.18 shows the inlet and rotor loss coefficients across the blade span. Dealing first with the inlet loss coefficients, $\mathrm{K}_{\text {loss2 }}$, they are larger than the rotor loss coefficients. The effect of the IGV wake can be clearly seen. It is not clear why the inlet loss coefficients differ for case (1) and (2). It was found that the magnitude of the pressure drop was about the same for both cases, which means that the inlet loss coefficient for case (2) will be higher.

The rotor losses were found to be very small as was expected due to the low drag aerofoil sections used. In Figure 5.18 the loss coefficients for the rotor blades are seen to be above zero near the hub. This is impossible but was due to difficulties in measuring the very small losses over the rotor blades. The sudden peak at the blade root is thought to be due to two possible factors. The first is that there will be some radial towards the pipe centre at the turbine exit due to the spherical shape of the hub. The second reason is probably due to interference effects between the probe and the wall. A wall in close proximity to the probe tip would disturb the flow around the probe. This is evident in the way the radial velocity components head towards zero at the wall but deviate just before at a distance of approximately 0.01 of the turbine diameter. This is not possible in the case of an impermeable wall. All positive values were discarded in calculating the average value.

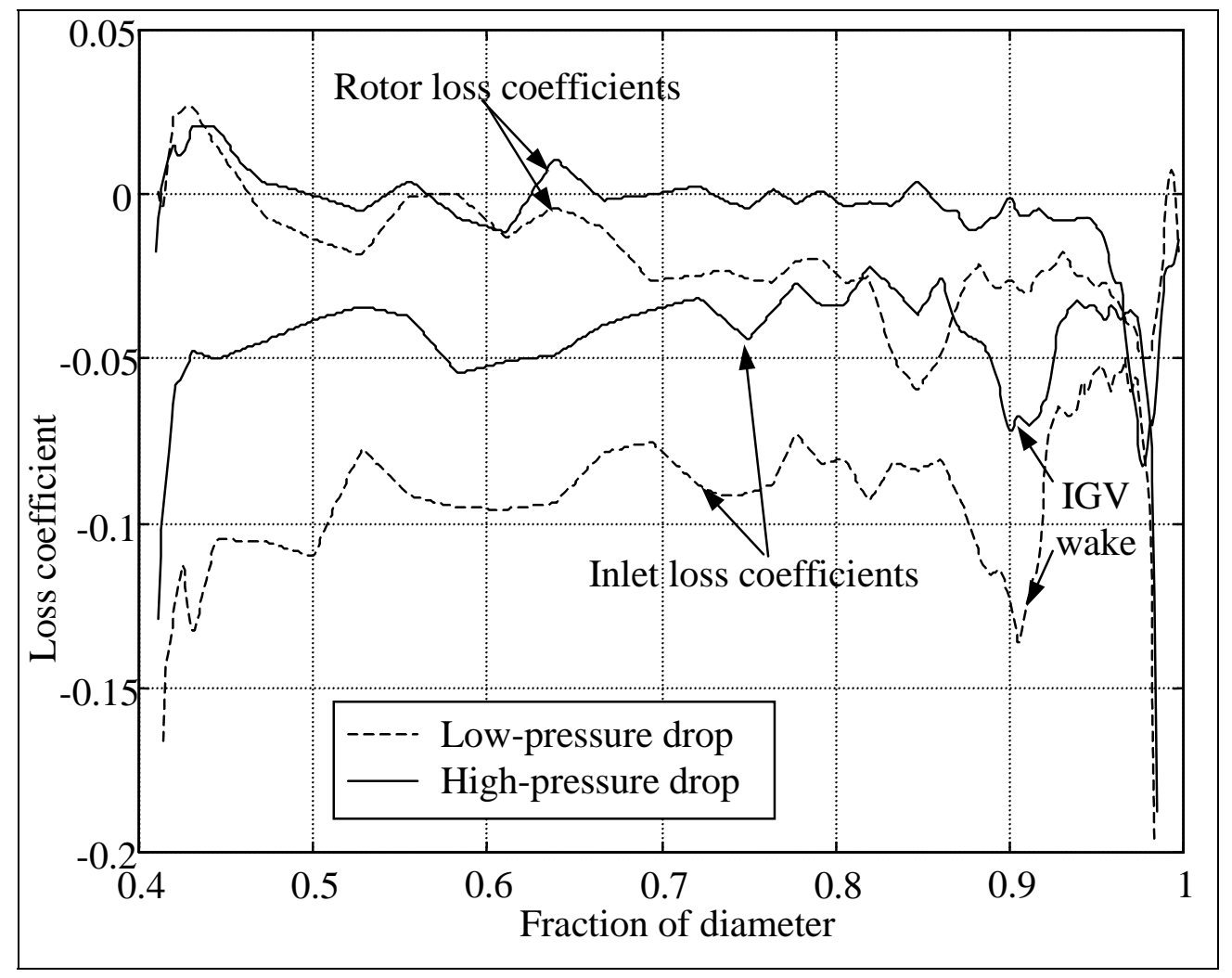

Figure 5.18. Loss coefficients for the rotor and inlet section.

The loss coefficients across the blade span are almost constant except for the low pressure rotor loss that increases slightly towards the tip. Table 5.7 shows the average values of the loss coefficients. 
Table 5.7. Average turbine loss coefficients.

\begin{tabular}{|l|l|l|}
\hline & Rotor loss coefficient & Inlet loss coefficient \\
\hline Low pressure drop & -0.021 & -0.091 \\
\hline High pressure drop & -0.0088 & -0.043 \\
\hline
\end{tabular}

In turbomachinery texts, Lewis (1996), the coefficient of drag, $C_{D}$, is often used to represent the profile loss. This is a useful term as it allows the cascade profile performance to be compared with standard aerofoil data such as in Abbot \& von Doenhoff (1959).

In the previous chapters use was made of the coefficient of drag to implement losses in the freevortex analysis. Lewis (1996) shows the relationship between the loss term, $\mathrm{K}_{\text {loss rot }}$ in equation (5.12) and the drag coefficient is,

$$
\mathrm{C}_{\mathrm{D} \infty}=\mathrm{K}_{\text {loss } \operatorname{rot}}\left(\frac{\cos \beta_{\infty}}{\cos \beta_{3}}\right)^{2}\left(\frac{\mathrm{t}}{\mathrm{l}}\right) \cos \beta_{\infty}
$$

where $\tan \beta_{\infty}=\frac{1}{2}\left(\tan \beta_{2}+\tan \beta_{3}\right)$ and the coefficient of lift for the cascade aerofoil is,

$$
\mathrm{C}_{\mathrm{L} \infty}=2\left(\frac{\mathrm{t}}{\mathrm{l}}\right)\left(\tan \left(\beta_{3}\right)-\tan \left(\beta_{2}\right)\right) \cos \beta_{\infty}-\mathrm{C}_{\mathrm{D} \infty} \tan \beta_{\infty}
$$

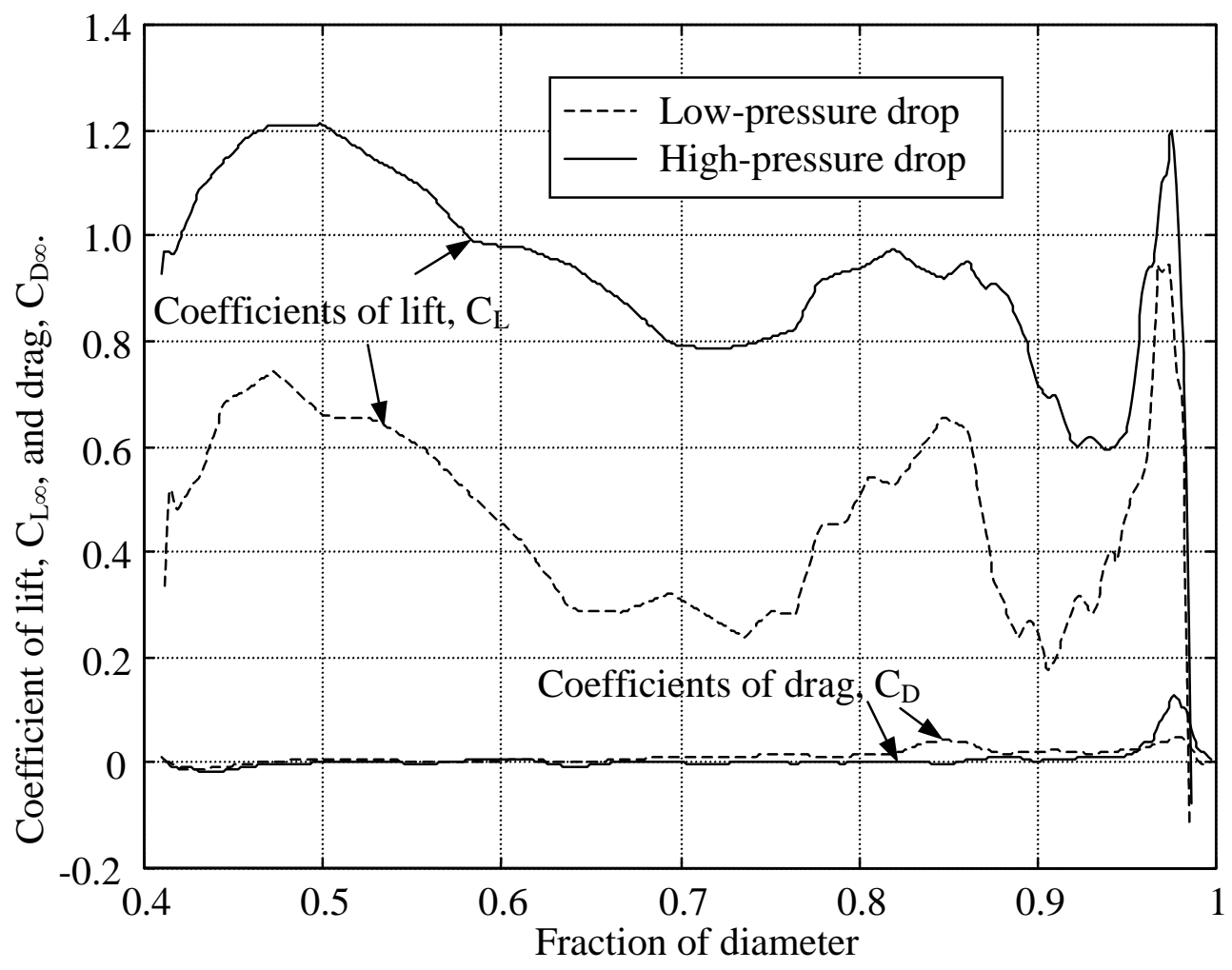

Figure 5.19. Coefficients of lift, $\mathrm{C}_{\mathrm{L} \infty}$, and drag, $\mathrm{C}_{\mathrm{D} \infty}$. 
Figure 5.19 shows the coefficients of lift and drag for the high and low pressure drop cases. The drag coefficients along the blade profile were very small and difficulty was encountered in measuring them. The average values of $C_{D \infty}$ and $C_{L \infty}$ and the ratio $C_{L \infty} / C_{D \infty}$ for each of the design cases are shown in Table 5.8. The drag coefficient values are reasonable but could be improved as coefficients as low as 0.005 are possible (Abbot \& von Doenhoff 1959). The lift coefficient values are high as is typical in a turbine where the flow is accelerating.

Table 5.8. Average turbine rotor lift and drag coefficients.

\begin{tabular}{|r|l|l|}
\hline & Low pressure drop & High pressure drop \\
\hline Coefficient of drag, $\mathrm{C}_{\mathrm{D} \infty}$ & 0.0141 & 0.0110 \\
\hline Coefficient of lift, $\mathrm{C}_{\mathrm{L} \infty}$ & 0.4708 & 0.9391 \\
\hline $\mathrm{C}_{\mathrm{L} \infty} / \mathrm{C}_{\mathrm{D} \infty}$ & 33.4 & 85.2 \\
\hline
\end{tabular}

The experimental results show that the method used to design the blade profiles was effective in minimising the losses. Recall that the blade design procedure attempted to create blades with small chords to reduce the blade size of the full-size plant. Coupled to this was an optimisation procedure that minimised the flow acceleration in an attempt to reduce the drag.

\section{Diffuser Performance}

The $\alpha$ value relating the kinetic energy of a uniform and non-uniform velocity profile for the flow downstream of the diffuser can be accurately calculated using the measured velocity profiles. The definition of $\alpha$ is given in Chapter 2. Its use here requires a numerical integration of the velocity profile across the duct. Table 5.9 shows the numerical values of $\alpha$ for the overall velocity and for the axial only. It can be see that the axial velocity is a significant part of the kinetic energy loss but the turbine total-to-static efficiency could be raised significantly by reducing the exit whirl especially for case (2). In diffusers any distortion of the inlet velocity profile is usually amplified due to the adverse pressure gradients. This is found to be the case is the present diffuser with a far greater difference between the axial velocities at the hub and tip after the diffuser than after the turbine rotor. Modification of the inlet profile may reduce this velocity distortion and in turn decreasing the exit kinetic energy further.

Table 5.9. $\alpha$ values at a) turbine exit/diffuser entrance and b) one pipe diameter downstream of the diffuser exit for total and axial velocity components.

\begin{tabular}{|c|c|c|c|c|}
\hline & \multicolumn{2}{|c|}{ Turbine exit } & \multicolumn{2}{c|}{1 diameter downstream of diffuser exit } \\
\hline & Total velocity & Axial only & Total velocity & Axial only \\
\hline Case (1) & 1.1136 & 1.0849 & 1.4292 & 1.3410 \\
\hline Case (2) & 1.2042 & 1.0874 & 1.5788 & 1.3202 \\
\hline
\end{tabular}


The diffuser pressure recovery is calculated by integration of the velocity profiles equation (5.22). Table 5.10 shows that the experimental diffuser is efficient but does not have a large area ratio. As discussed later, a longer diffuser can be expected to increase the turbine total-to-static efficiency.

Table 5.10. Diffuser efficiency and pressure recovery

\begin{tabular}{|c|c|c|c|c|}
\hline & AR & $C_{\text {pdiff ideal }}$ & $\eta_{\text {D }}[\%]$ & $C_{p \text { diff }}$ \\
\hline Case (1) & 1.47 & 0.54 & 97.3 & 0.52 \\
\hline Case (2) & 1.47 & 0.54 & 90.7 & 0.49 \\
\hline
\end{tabular}

\subsection{COMPARISON WITH DESIGN SIMULATION}

An important part of the experimental program is to verify the design methods used. To do this the experimental results are scaled up to the operational speed of the full-scale plant and compared with the results of the MTFM analysis. The scaling is performed using the standard fan-scaling laws for incompressible flow. The full-scale turbine performance is summarised as follows:

Table 5.11. Summary of full-scale turbine data.

\begin{tabular}{|c|l|l|l|}
\hline & RPM & Diameter [m] & Power [MW] \\
\hline 1) Low-pressure & 13.2 & 127.9 & 50.6 \\
\hline 2) High-pressure & 13.2 & 127.9 & 200 \\
\hline
\end{tabular}

\section{Velocity Profiles}

In Figure 5.20 the main differences between the experiments and simulation was the underprediction of the exit whirl velocity. The axial velocity profiles are also more skewed in the experimental results than in the simulations. To improve the agreement between the experimental readings and the MTFM, it was extended to include blade blockage, non-uniform work across blade rows and the effect of streamline shift in the radial cascade. The theory behind these extensions is covered in Chapter 3 but is covered briefly here. Blade blockage takes into account the physical thickness of the blade resulting in localised flow acceleration through the blade rows. In the initial simulation it was assumed that the flow turning over the blade occurred linearly. Using the results of the surface vortex analysis, the rate of turning can be accurately determined. Most of the turning through the inlet guide vanes occurs over the front of the blade, leading to the flow shifting towards the roof and turbine tip at greater rate than first predicted. Coriolis acceleration was added as it is very simple to implement but had a negligible effect on the current model due to the small size. 


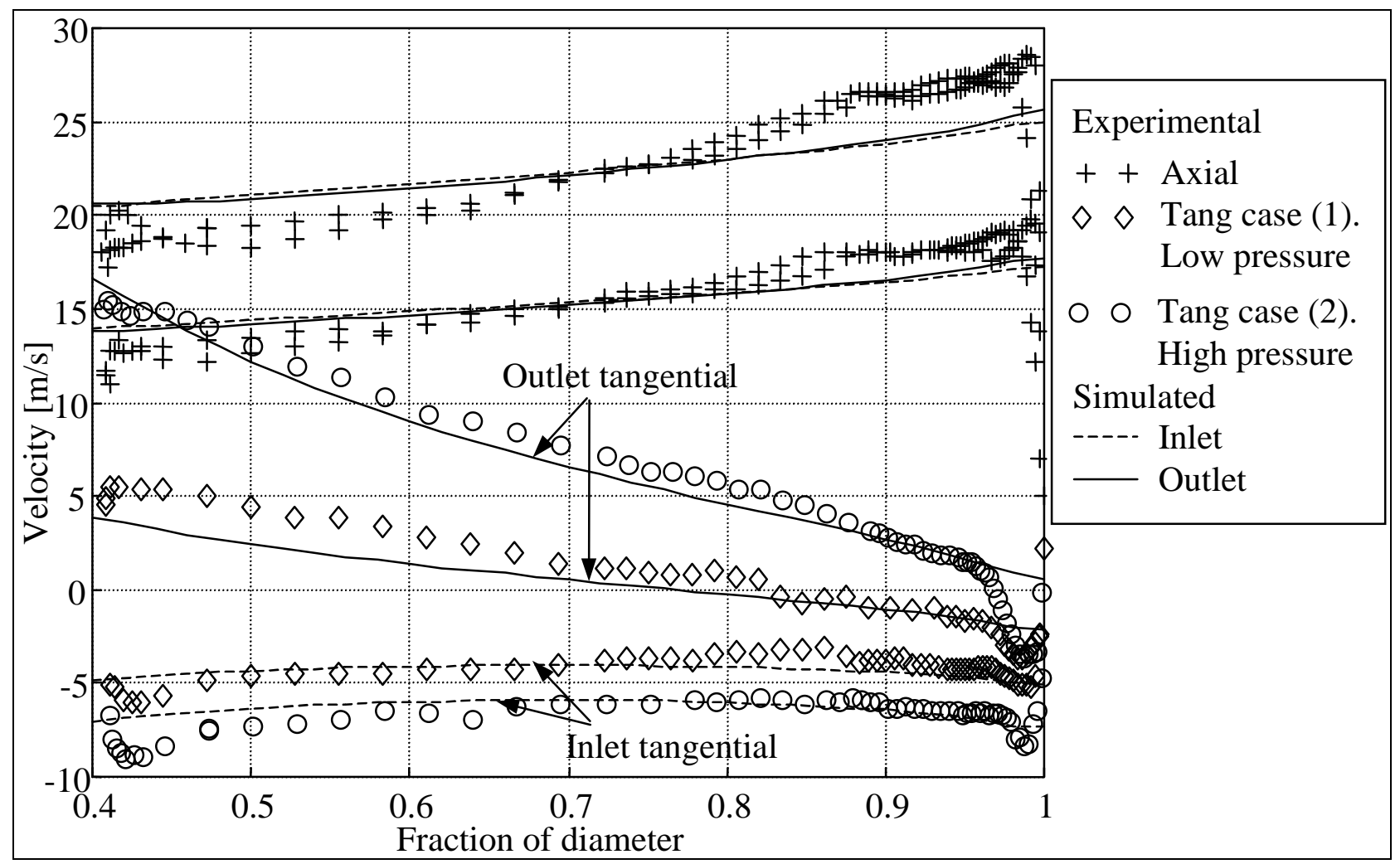

Figure 5.20. Velocity profile comparison of experimental with original MTFM results for $100 \%$ rotor total-to-total efficiency.

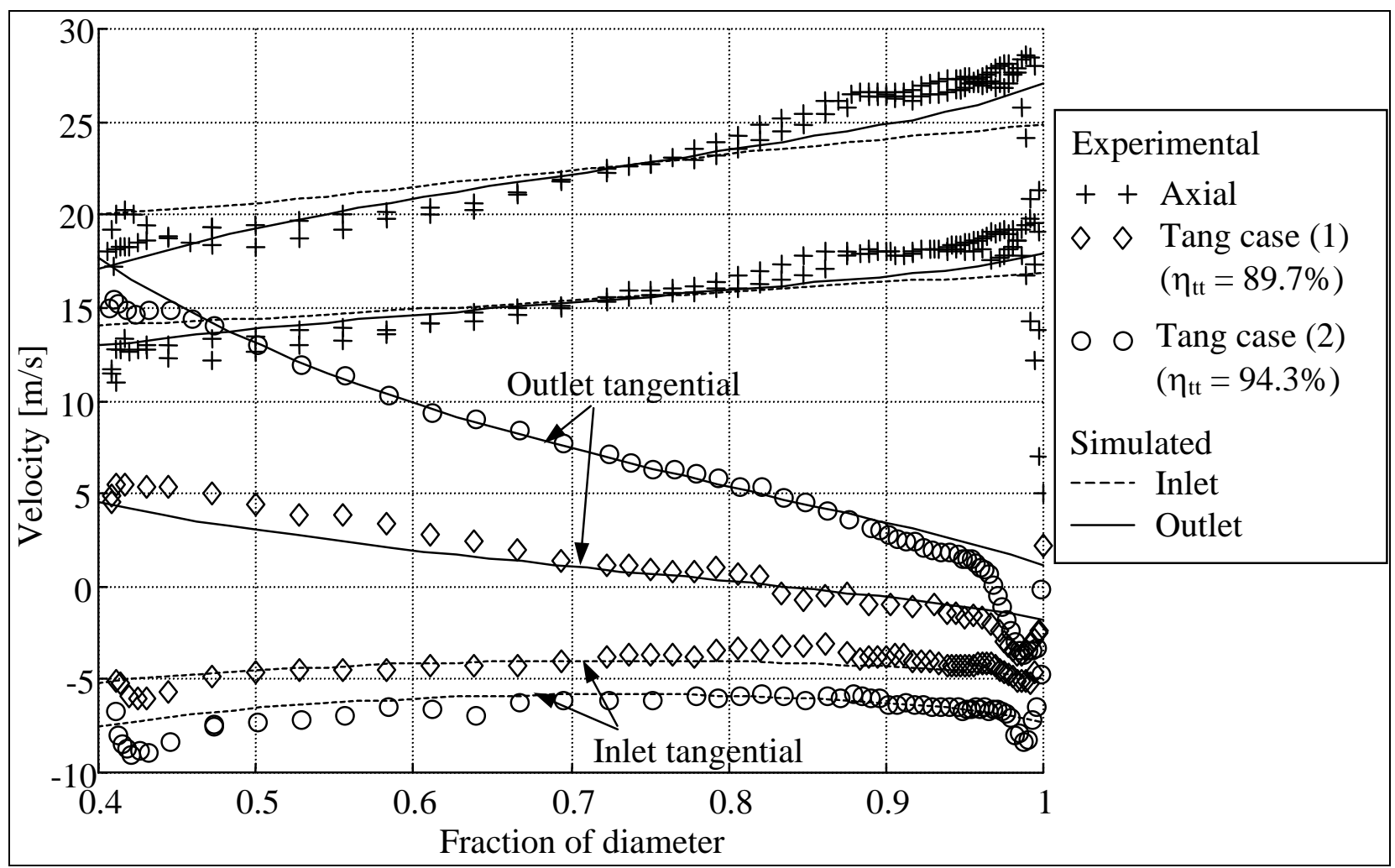

Figure 5.21. Velocity profile comparison of experimental results with MTFM results corrected for rotor efficiency. 
From Figure 5.21.onwards the results from the improved MTFM method are compared to the experimental results. The changes have the greatest effect on the turbine tip region. The prediction of exit swirl is improved by increasing the turbine temperature drop by the experimental efficiency $\Delta \mathrm{T}=\Delta \mathrm{T}_{\text {turb }} / \eta_{\text {turb }}$. This reflects the extra power required to overcome the system losses. The experimentally measured rotor total-to-total efficiencies were used. The improvement in the prediction of the whirl profiles is shown in Figure 5.21.

Figure 5.21 uses the improved MTFM simulation and shows better agreement between the experimental and theoretical axial velocity profiles than Figure 5.20. There is very good agreement at the mid-blade span but the experimental data still shows a higher axial velocity at the tip than that predicted.

\section{Flow Angles}

What is also being evaluated in this comparison is the accuracy of the blade design and manufacture. The blade performance might be slightly different to that predicted, leading to a slight change in the flow through the turbine. Figure 5.22 compares the experimental and predicted relative flow angles at the rotor inlet and exit. The overall agreement is good except in the boundary layers. Away from the boundary layers, the greatest difference between experiment and simulation occurs at the blade tip. Here the higher than predicted axial velocities reduce the relative flow angles.

Figure 5.23 compares the experimental and predicted gas deflection angles. The mid-span was used to define the change in stagger adjustment, resulting in the best agreement between the experiment and simulation occurring here. The maximum error excepting the end-wall was $1.5^{0}$.

\section{Pressures}

Figure 5.24 and Figure 5.25 compare the total and static experimental and simulated pressure profiles for the low and high pressure drop cases respectively. The pressures are all scaled to the full-size plant and the experimental pressures losses given in Table 5.7 are included in the simulation. Inclusion of the mean blade loss in the MTFM results shifts the pressure drop profiles downwards and makes the comparison with the experimental profiles better. A turbine re-design will require the inclusion of these losses and apart from a major change in its basic layout the coefficients used here should not change greatly. As with the axial velocity profiles, Figure 5.26 shows that the experimental pressure profiles are more skewed with a higher pressure-drop at the hub than predicted by the simulation. Overall prediction is acceptable. 


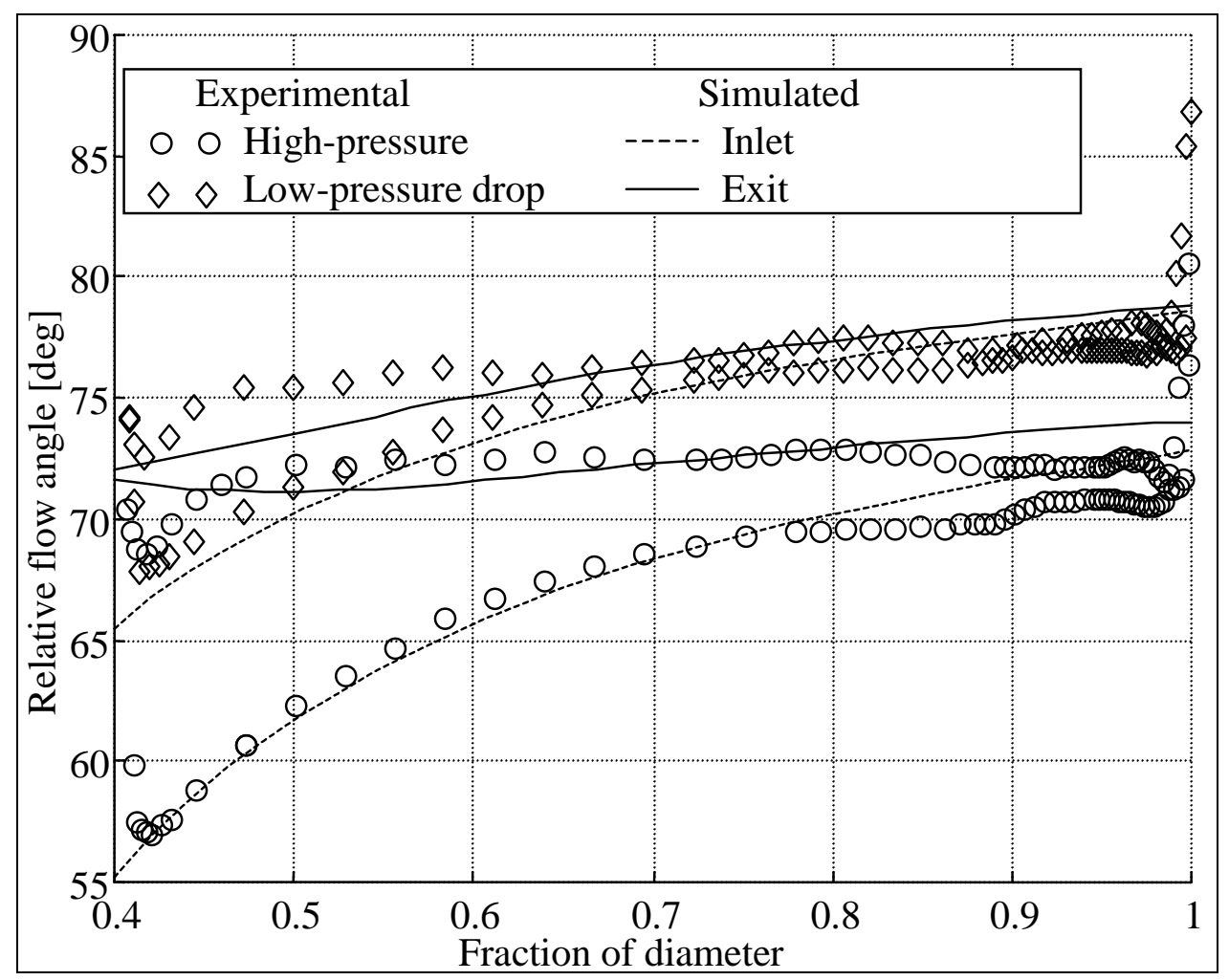

Figure 5.22. Comparison of relative rotor inlet and exit angles.

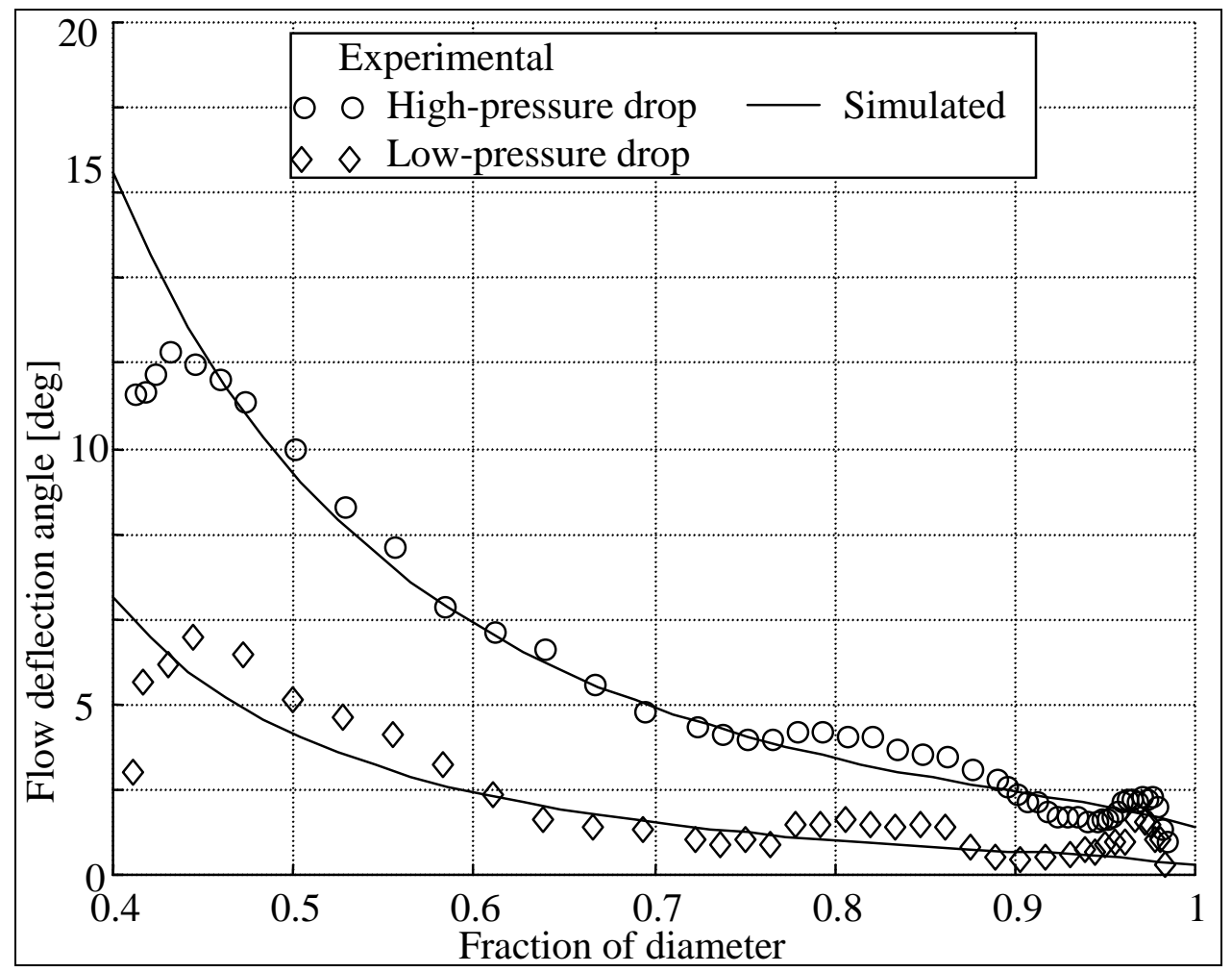

Figure 5.23. Comparison of gas deflection angles. 


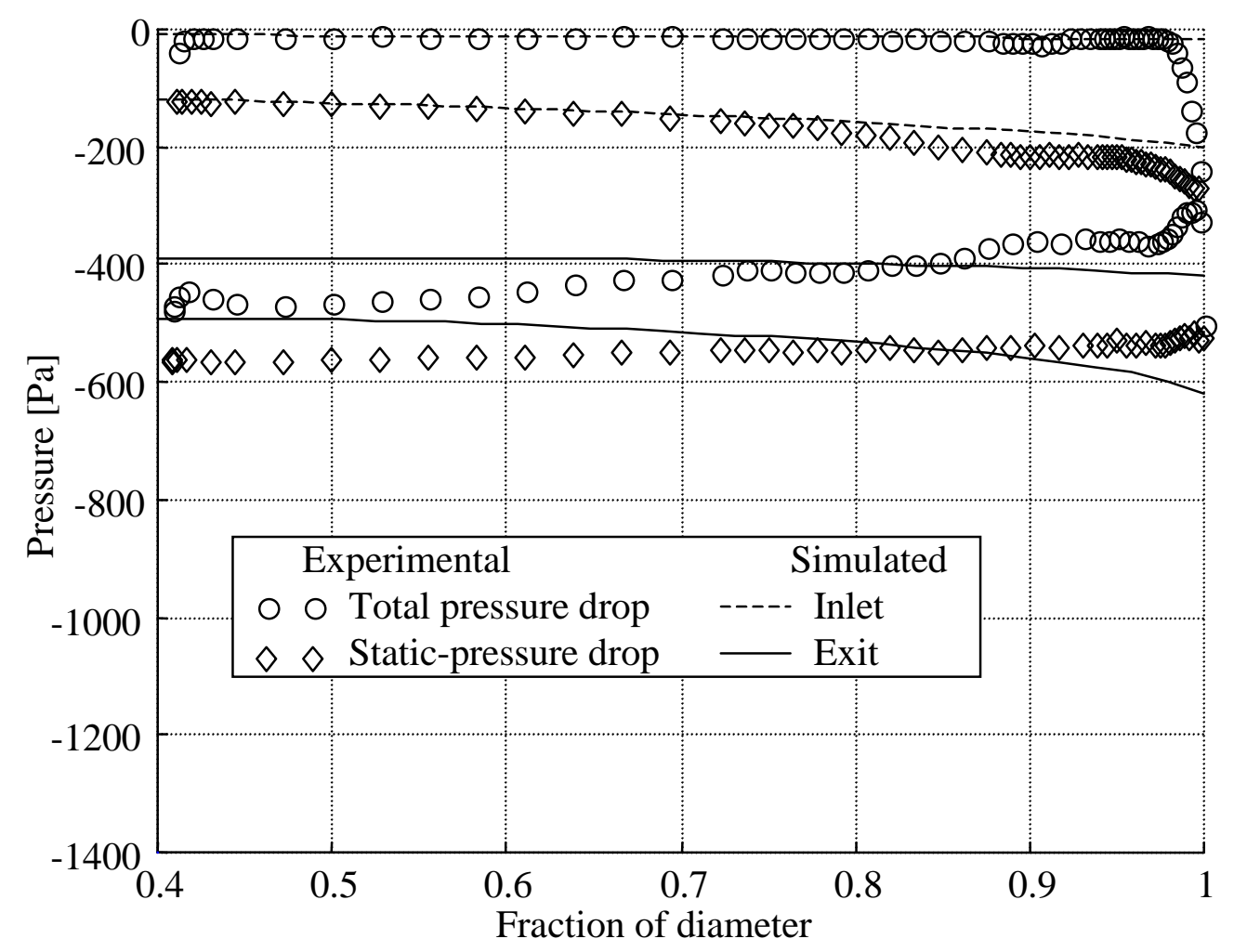

Figure 5.24. Comparison of static and total pressure profiles at turbine inlet and outlet (lowpressure drop).

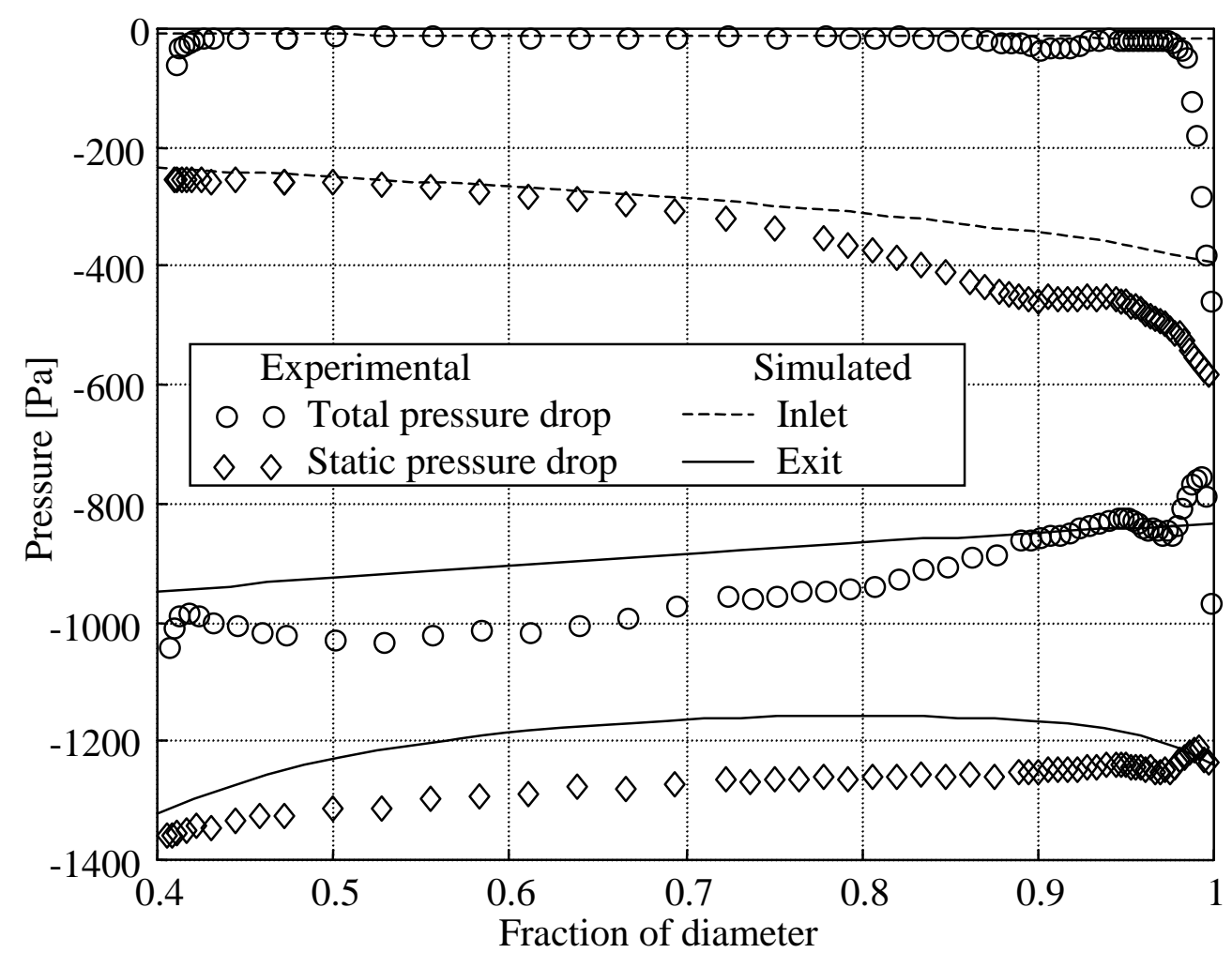

Figure 5.25. Comparison of static and total pressure profiles at turbine inlet and outlet (highpressure case). 


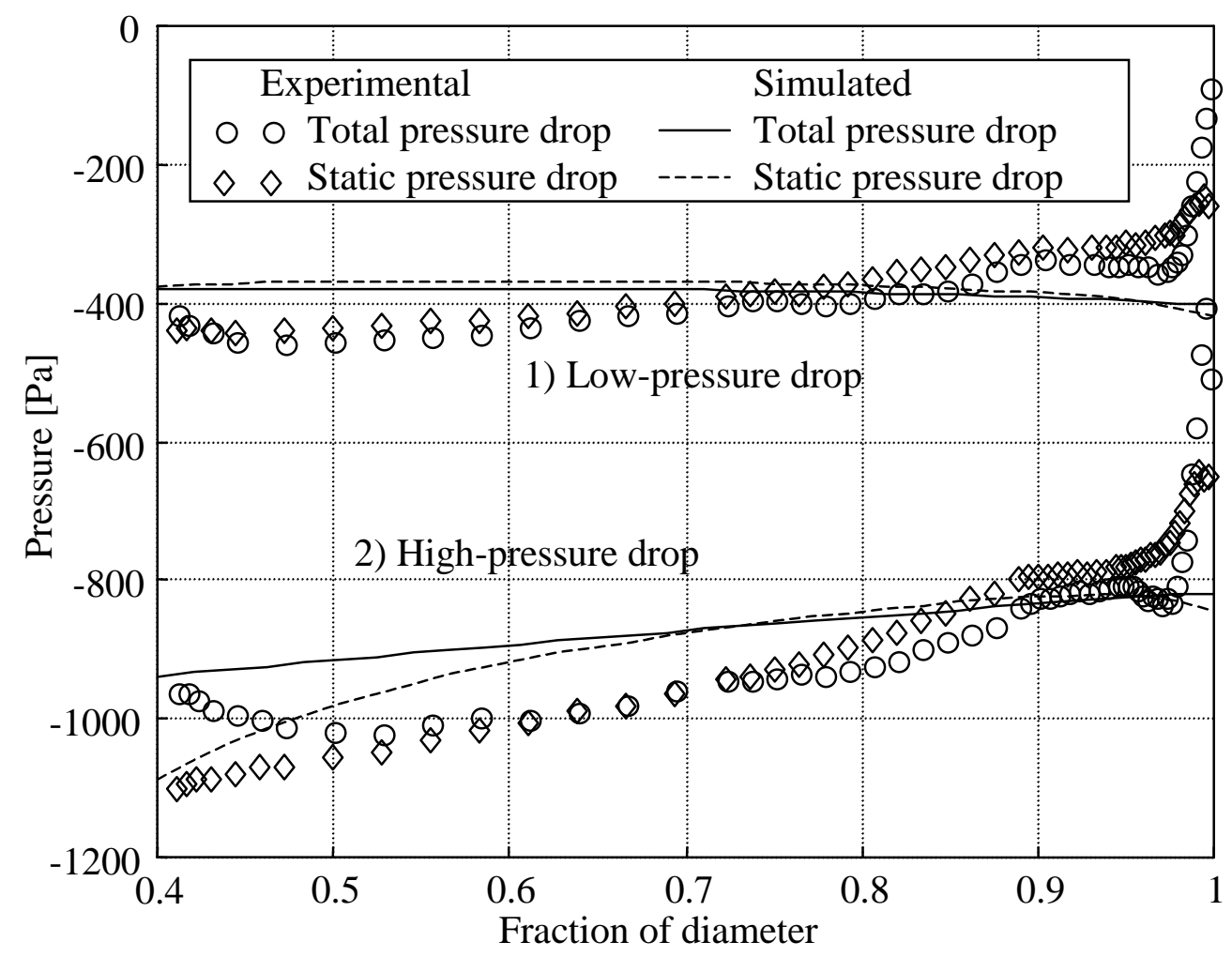

Figure 5.26. Comparison of static and total pressure drop over the turbine.

\section{Diffuser}

The flow in the diffuser is dominated by boundary layer and viscous effects, resulting in poor flow prediction when an inviscid method such as the MTFM is used. Figure 5.27 shows that the agreement between the predicted and measured velocity profiles is poor due to the viscous effect especially near the wall. The predicted pressure profiles show better agreement in Figure 5.28 than the velocity profiles. In the MTFM simulation assumed, the turbine hub continued after the turbine as discussed in Chapter 3. There is probably a large stagnation region behind the turbine hub, as it persists at the pipe centre even after the diffuser. It was mentioned that extending the turbine hub in the simulation would lead to pessimistic efficiency predictions. Investigation of the experimental results show that the results are realistic rather than pessimistic. 


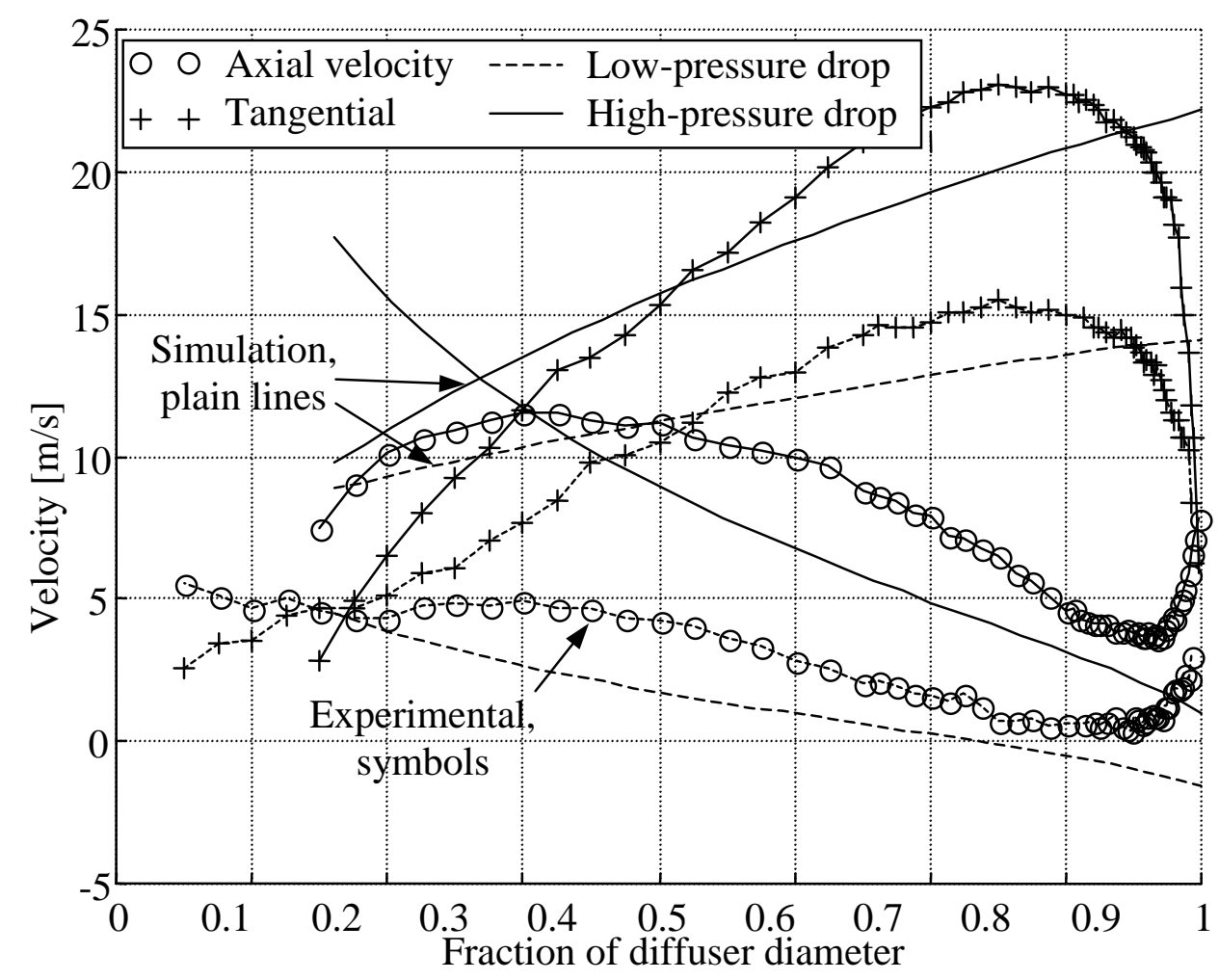

Figure 5.27. Comparison of axial and whirl velocities after the diffuser.

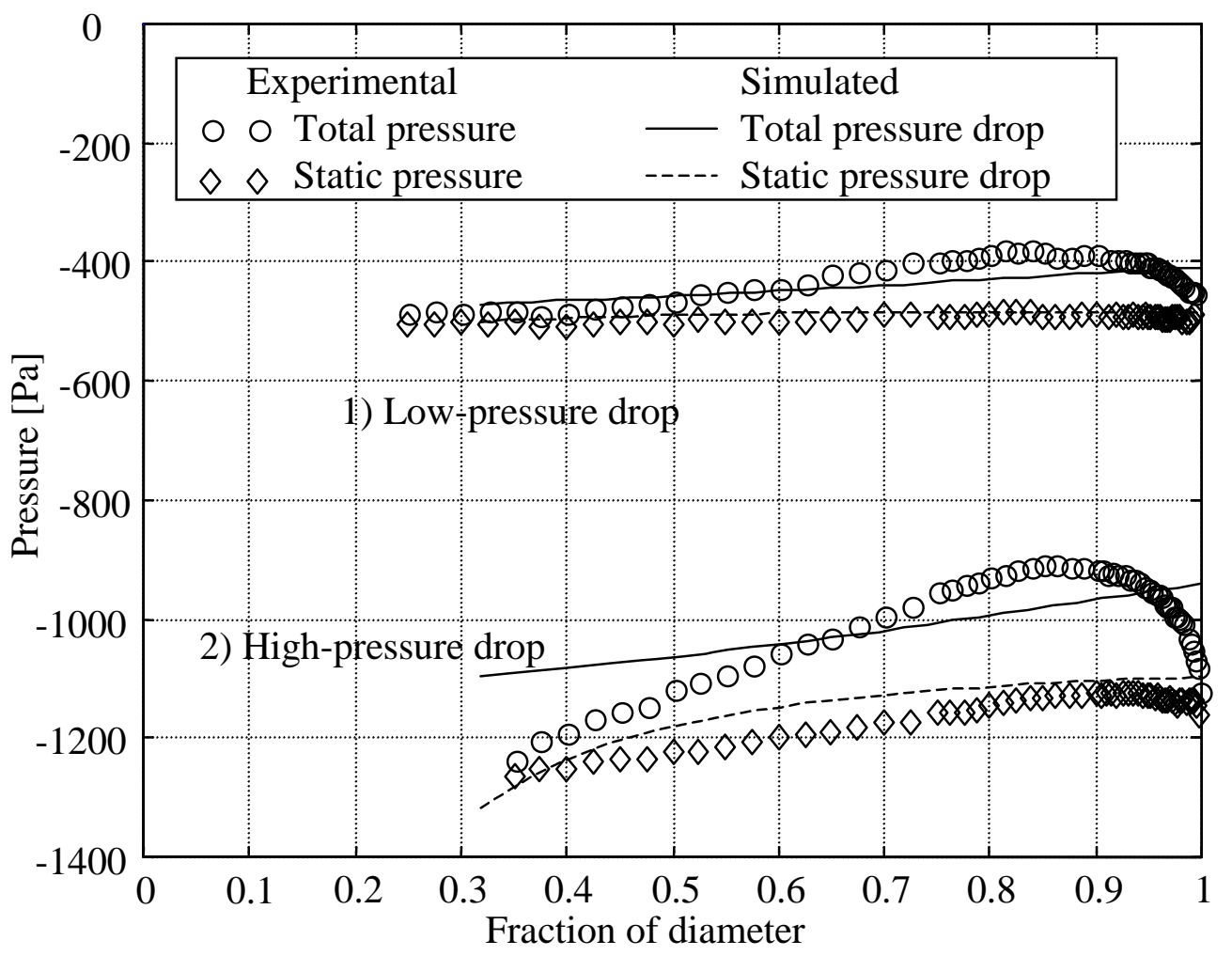

Figure 5.28. Comparison of static and total pressure profiles after the diffuser. 


\section{Efficiencies}

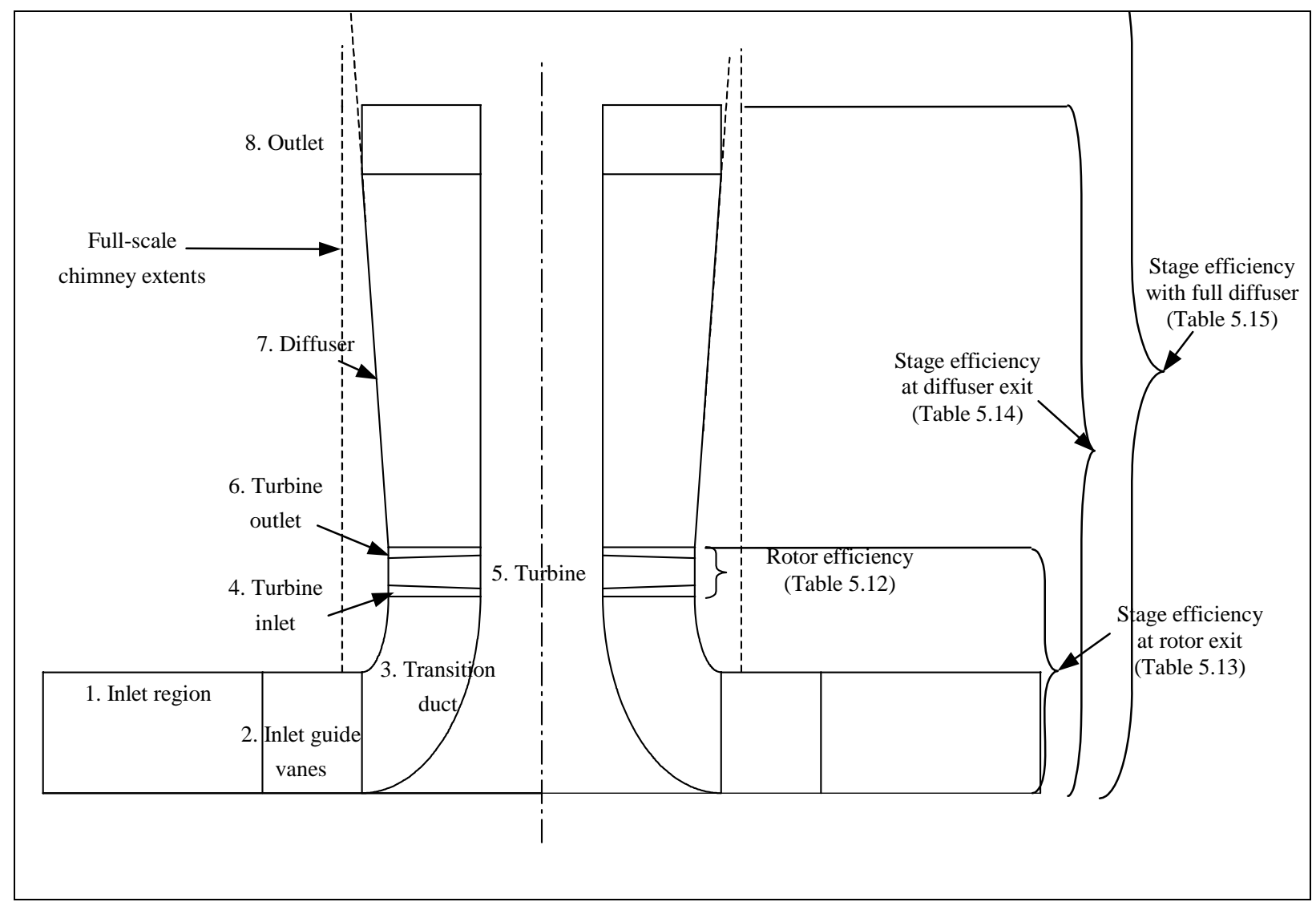

Figure 5.29. Experimental layout showing various stations used to calculate efficiency.

Using the experimental data and integrating along the velocity and pressure profiles, the turbine efficiency can be accurately calculated. It is possible to calculate both the Euler work (from the change in angular momentum) and the flow work (from the stagnation pressure drop). In the present case, as overall efficiencies were sought, the output power measured using the torque transducer and speed-reader was used as the output power. The flow work was calculated using a numerical integration across the blade. What now follows are the equations used to calculate the various efficiencies. Each of the equations applies at a certain radial station. To obtain the total value of energy extracted from the flow, a numerical integration must be performed across the rotor span or diffuser.

In all the equations presented here the expression for the ideal power $\mathrm{P}_{\text {flow }}$ is presented. The corresponding efficiency is calculated by dividing the measured power $\mathrm{P}$ with $\mathrm{P}_{\text {flow. }}$ In equations (5.15) to (5.28)

$$
\eta=\mathrm{P} / \mathrm{P}_{\text {flow }}
$$

The first experimentally calculated efficiency is the rotor total-to-total. It is assumed that the flow is purely axial. This was thought a reasonable assumption as the radial flow velocities measured were 
very small in comparison to the axial. It was only near the hub that significant radial velocities were found. The potential power $\mathrm{P}_{\text {rot tt }}$ extracted is,

$$
\mathrm{P}_{\text {rot tt }}=\mathrm{Q}\left(\mathrm{p}_{02}-\mathrm{P}_{03}\right)
$$

The rotor total-to-static efficiency is calculated by subtracting the exit static pressure from the inlet stagnation pressure and multiplying by the volume flow rate to obtain $\mathrm{P}_{\text {rot ts, }}$,

$$
\mathrm{P}_{\text {rot ts }}=\mathrm{Q}\left(\mathrm{p}_{02}-\mathrm{p}_{3}\right)
$$

The stage efficiencies are calculated by using atmospheric pressure as the reference. As has been explained, this then takes into account the losses of the inlet, IGV and duct before the rotor blades. The stage total, $\mathrm{P}_{\text {stage tt }}$ and static flow work, $\mathrm{P}_{\text {stage ts, }}$ can be calculated as follows,

$$
\begin{aligned}
& \mathrm{P}_{\text {stage tt }}=\mathrm{Q}\left(-\mathrm{p}_{03}\right) \\
& \mathrm{P}_{\text {stage ts }}=\mathrm{Q}\left(-\mathrm{p}_{3}\right)
\end{aligned}
$$

The combined efficiency of the rotor, inlet region and diffuser is also calculated using the atmospheric pressure as a reference. The combined total, $\mathrm{P}_{\text {diff } t \text {, }}$ and static flow work, $\mathrm{P}_{\text {diff } t s}$, is calculated as follows,

$$
\begin{aligned}
& \mathrm{P}_{\text {diff tt }}=\mathrm{Q}\left(-\mathrm{p}_{0 \text { diff }}\right) \\
& \mathrm{P}_{\text {diff ts }}=\mathrm{Q}\left(-\mathrm{p}_{\text {diff }}\right)
\end{aligned}
$$

The diffuser efficiency can also be determined experimentally. The diffuser efficiency is defined as the real pressure recovery coefficient, $C_{p}$ diff, over the ideal $C_{p}$ diff ideal,

$$
\eta_{\text {diff }}=\mathrm{C}_{\mathrm{p} \text { diff }} / \mathrm{C}_{\mathrm{p} \text { diff ideal }}
$$

where $C_{p}$ diff ideal is only a function of the area ratio between the turbine rotor and diffuser exit,

$$
\begin{aligned}
\mathrm{C}_{\mathrm{p} \mathrm{diff} \mathrm{ideal}} & =1-1 / \mathrm{AR}^{2} & & \text { Ideal pressure recovery } \\
\mathrm{AR} & =\mathrm{A}_{\text {diff }} / \mathrm{A}_{\text {turb }} & & \text { Area ratio } \\
\mathrm{A}_{\text {diff }} & =\frac{\pi}{4} \mathrm{D}_{\text {diff }}^{2} & & \text { Diffuser area } \\
\mathrm{A}_{\text {turb }} & =\frac{\pi}{4} \mathrm{D}_{\text {turb }}^{2}\left(1-v^{2}\right) & & \text { Turbine area }
\end{aligned}
$$

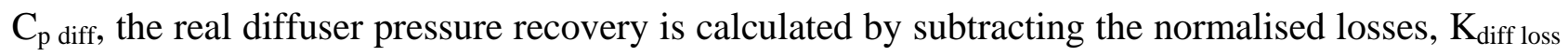
from $\mathrm{C}_{\mathrm{p} \text { diff ideal. }}$

$$
\begin{aligned}
\mathrm{C}_{\mathrm{p} \mathrm{diff}} & =\mathrm{C}_{\mathrm{pdiff} \text { ideal }}-\mathrm{K}_{\text {diff loss }} \\
\mathrm{K}_{\text {diff loss }} & =\frac{\left(\mathrm{P}_{\text {stage tt }}-\mathrm{P}_{\text {diff tt }}\right)}{\frac{1}{2} \dot{\mathrm{m}} \mathrm{C}_{3}^{2}}
\end{aligned}
$$


If using the mean turbine exit or diffuser inlet velocity $\mathrm{C}_{3}$, the equation is modified to include $\alpha_{\text {diff. }}$.

$$
\mathrm{K}_{\text {diff loss }}=\frac{\left(\mathrm{P}_{\text {stage tt }}-\mathrm{P}_{\text {diff tt }}\right)}{\alpha_{\text {diff }} \frac{1}{2} \dot{\mathrm{m}} \overline{\mathrm{C}}_{3}^{2}}
$$

Once the diffuser efficiency is known then the effect of using a longer diffuser on the turbine efficiency can be calculated. The total-to-total efficiency will increase slightly while the total-tostatic will increase significantly. The diffuser loss coefficient for the longer diffuser, $K_{\text {diff2 loss, }}$ is

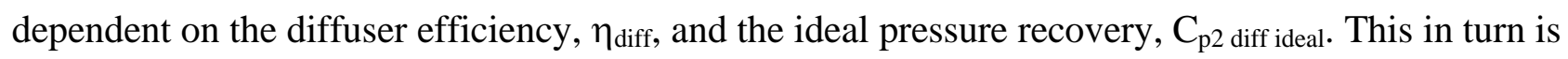
only dependent on the area ratio (equation (5.23)).

$$
\mathrm{K}_{\text {diff2 loss }}=\mathrm{C}_{\mathrm{pdiff2} \text { ideal }}\left(1-\eta_{\text {diff }}\right)
$$

The total flow work done will be the flow work at the turbine exit with the diffuser losses added,

$$
\mathrm{P}_{\text {diff2 tt }}=\mathrm{P}_{\text {rot tt }}+\frac{1}{2} \dot{\mathrm{m}} \mathrm{C}_{3}^{2} \mathrm{~K}_{\text {diff2 loss }}
$$

The static flow work done will be the static flow work at the turbine exit with the diffuser pressure recovery added. The method for calculating the pressure recovery coefficient $\mathrm{C}_{\mathrm{p} 2}$ diff can be calculated in the same way as (5.24).

$$
\mathrm{P}_{\text {diff ts }}=\mathrm{P}_{\text {rot ts }}-\frac{1}{2} \dot{\mathrm{m}} \mathrm{C}_{3}^{2} \mathrm{C}_{\mathrm{p} 2 \text { diff }}
$$

There are a number of efficiencies that are calculated as shown in Figure 5.29. Table 5.12 to Table 5.15 show the experimental efficiencies calculated, using the measured profiles and comparing them with those calculated using the free-vortex, MTFM and wall static pressures.

The following tables show the rotor efficiency (Table 5.12), stage efficiency (Table 5.13), stage with diffuser (Table 5.14) and stage efficiency with full-diffuser (Table 5.15) (refer to Figure 5.29). In each table the experimental efficiency calculated using the velocity profiles is compared to the other methods used in the design process and obtained from the wall static pressure measurements.

Table 5.12 shows the experimental total-to-total efficiency and compares total-to-static efficiencies with those calculated using the free-vortex analysis and MTFM without loss models for the rotor only. The rotor losses are small as the total-to-total efficiencies are near or over $90 \%$. The total-tototal efficiencies of the free-vortex and MTFM methods are $100 \%$ in the absence of a loss model. The comparison is done here without loss models as they were not used in the original optimisation.

Table 5.13 is similar to Table 5.12 but takes into account the losses of the inlet and IGV. This is reflected in a reduction in both the total-to-total and total-to-static efficiencies. The predicted efficiencies do not change as there are no loss models present.

Table 5.14 shows the efficiencies after the diffuser. There is only a slight reduction in the total-tototal efficiency as the diffuser efficiency was found to be very high. The total-to-static efficiencies rise by $6.6 \%$ and $8.6 \%$ for the low-pressure and high-pressure drop cases respectively. It is this 
efficiency that is most important in the solar chimney plant as the exit kinetic energy is lost at the chimney exit. The agreement between the efficiencies calculated using the wall static pressures are good for the low-pressure drop case but optimistic for the high-pressure case. This is due to the distorted static pressure profiles still present after the diffuser for the high-pressure drop case (Figure 5.28).

Table 5.12 Comparison of rotor efficiencies ref. Figure 5.29 (NL = no loss)

\begin{tabular}{|l|c|c|c|c|}
\hline & $\begin{array}{c}\text { Experimental } \\
\text { Total-to-total }\end{array}$ & $\begin{array}{l}\text { Experimental } \\
\text { Total-to-static }\end{array}$ & $\begin{array}{l}\text { MTFM (NL) } \\
\text { Total-to-static }\end{array}$ & $\begin{array}{l}\text { Free-vortex (NL) } \\
\text { Total-to-static }\end{array}$ \\
\hline Case (1) & 89.7 & 64.7 & 71.4 & 69.7 \\
\hline Case (2) & 94.3 & 67.1 & 73.2 & 73.3 \\
\hline
\end{tabular}

Table 5.13. Stage efficiency at rotor exit ref. Figure 5.29.

\begin{tabular}{|l|c|c|c|c|}
\hline & $\begin{array}{l}\text { Experimental } \\
\text { Total-to-total }\end{array}$ & $\begin{array}{l}\text { Experimental } \\
\text { Total-to-static }\end{array}$ & $\begin{array}{l}\text { MTFM (NL) } \\
\text { Total-to-static }\end{array}$ & $\begin{array}{l}\text { Free-vortex (NL) } \\
\text { Total-to-static }\end{array}$ \\
\hline Case (1) & 85.7 & 62.6 & 71.4 & 69.7 \\
\hline Case (2) & 91.9 & 65.9 & 73.2 & 73.3 \\
\hline
\end{tabular}

Table 5.14. Comparison of efficiencies after diffuser ref. Figure 5.29.

\begin{tabular}{|l|c|c|c|c|c|}
\hline & $\begin{array}{c}\text { Experimental } \\
\text { Total-to-total }\end{array}$ & $\begin{array}{l}\text { Experimental } \\
\text { Total-to-static }\end{array}$ & $\begin{array}{l}\text { MTFM (NL) } \\
\text { Total-to-static }\end{array}$ & $\begin{array}{l}\text { Free-vortex (NL) } \\
\text { Total-to-static }\end{array}$ & $\begin{array}{l}\text { Experimental } \\
\text { Wall-static }\end{array}$ \\
\hline Case (1) & 85.3 & 69.2 & 79.9 & 77.8 & 70.6 \\
\hline Case (2) & 90.2 & 74.5 & 80.4 & 80.0 & 82.8 \\
\hline
\end{tabular}

Table 5.15. Full-scale plant efficiencies with longer diffuser ref. Figure 5.29.

\begin{tabular}{|l|c|c|c|c|c|}
\hline & $\begin{array}{c}\text { Experimental } \\
\text { Total-to-total }\end{array}$ & $\begin{array}{l}\text { Experimental } \\
\text { Total-to-static }\end{array}$ & $\begin{array}{l}\text { MTFM (NL) } \\
\text { Total-to-static }\end{array}$ & $\begin{array}{l}\text { Free-vortex (NL) } \\
\text { Total-to-static }\end{array}$ & $\begin{array}{c}\text { Experimental } \\
\text { Diffuser eff. }\end{array}$ \\
\hline Case (1) & 85.2 & 77.03 & 86.5 & 81.5 & 97.3 \\
\hline Case (2) & 89.6 & 80.7 & 86.8 & 83.1 & 90.7 \\
\hline
\end{tabular}

Recalling that the full-scale plant will have a longer diffuser than the experimental model it is expected that the total-to-static efficiency will improve. Table 5.15 lists the experimental diffuser efficiencies of $97.3 \%$ and $90.7 \%$ for the low-pressure and high-pressure drop cases respectively. According to Japikse \& Baines (1997), diffuser efficiencies of up to $90 \%$ are possible. The experimental diffuser does not have a large area ratio, has a very gradual expansion and has a favourable velocity profile entering it. The calculated low-pressure diffuser efficiency of $97 \%$ is unrealistically high due to difficulties in measuring the low velocities in the diffuser for this case. With a longer diffuser total-to-static efficiencies for the two design cases of $77 \%$ and $80.7 \%$ can realistically be achieved. 


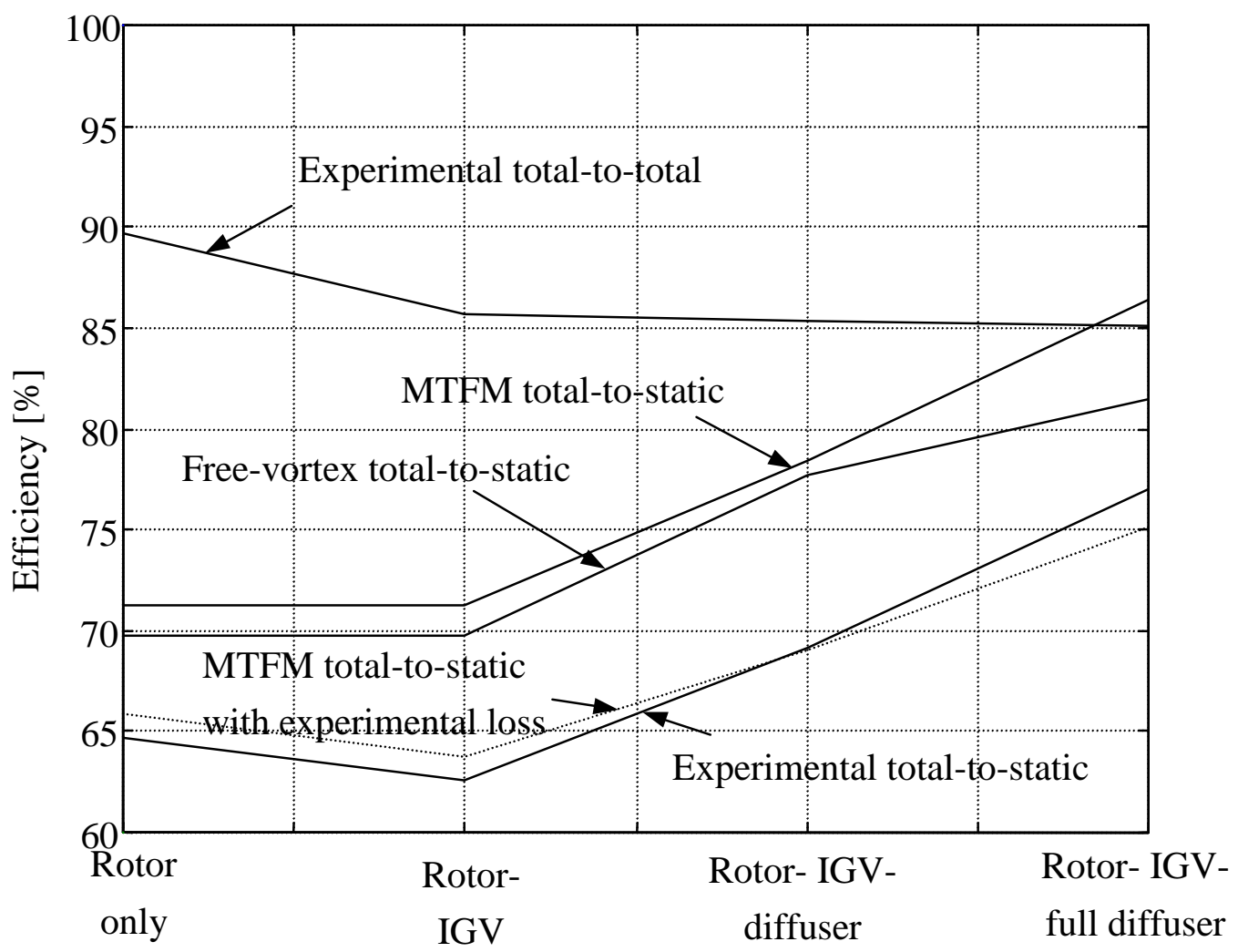

Figure 5.30. Graphical comparison of turbine efficiencies (low-pressure drop case 1).

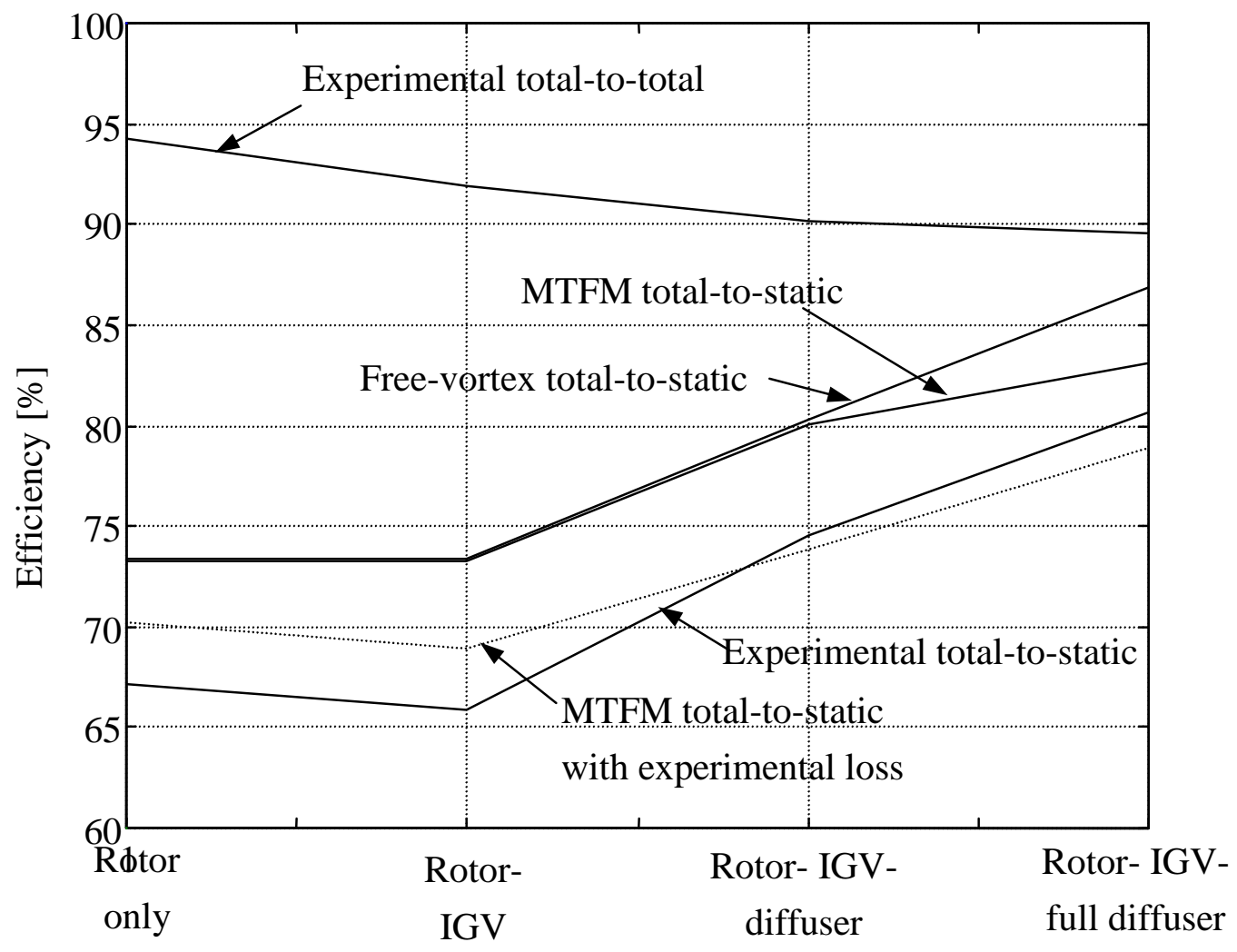

Figure 5.31. Graphical comparison of turbine efficiencies (high-pressure drop case 2). 
Figure 5.30 and Figure 5.31 attempt to present graphically the data from Table 5.12 to Table 5.15 and allow effect of each component such as the diffuser pressure recovery to be seen. They allow the matrix throughflow, MTFM, and free-vortex methods used in the design process to be compared with the experimental results. The experimental losses are subtracted from the MTFM and superimposed on the above figures. Addition of the losses obviously improves the agreement between the MTFM and the experimental results. The prediction of the rotor and rotor-IGV efficiency is within $1 \%$ for the low pressure case and $2.5 \%$ for the high-pressure case. The disagreement is due to differences in the velocity predictions (Figure 5.21).

In both cases the experimentally measured diffuser pressure recovery is higher as there is some recovery behind the turbine hub. As mentioned previously, the MTFM is inaccurate in simulating separated flows due to it being an inviscid method and so an annular diffuser was simulated. This would not affect the turbine design but only the predictions of total-to-static efficiency.

\subsection{STARTING TORQUE}

In addition to the experiments performed to determine the operating range of the turbine shown in Figure 5.5, readings were taken for a stationary turbine. For these readings the turbine shaft was fixed just below the torque transducer. Flow was drawn through the turbine and the pressure drop, flow rate and torque measured. These results are useful in determining how effective the solar chimney turbine self-starting characteristics will be. Some makes of wind turbines require the generator to be run as a motor to start them at low wind speeds. The present turbine does self-start and accelerates rapidly when it is uncoupled from the generator even for low volume flow rates in comparison to case (1). This acceleration is also important as some wind turbines have a high initial starting torque but take a long time to accelerate unaided to their operational speed (Ackermann and Söder 2000).

Only the static torque was measured and due to the ability of both the IGV and rotor blades to adjust, very high starting torques are possible. Table 5.16 shows the $\phi$ and $\psi$ values and torque compared to that found in case (1), the low pressure drop design point. As the tests were done for a stationary turbine, the design tip speed was used to non-dimensionalise the results. It can be seen that even at very low flow rates and pressure drops high torque is possible. The rotor tip stagger angle of $72.5^{\circ}$ and an IGV flap angle of $+30^{\circ}$ produced the highest starting torque for both of these cases. 
Table 5.16. Flow and load coefficient for $100 \%$ and $200 \%$ of case (1) torque at turbine start.

\begin{tabular}{|l|c|c|}
\hline & Flow coefficient $(\phi)$ & Load coefficient $(\psi)$ \\
\hline $100 \%$ of case 1 & 0.05 & 0.019 \\
\hline $200 \%$ of case 1 & 0.08 & 0.035 \\
\hline Case $(1)$ & 0.1770 & 0.0742 \\
\hline Case $(2)$ & 0.2577 & 0.2018 \\
\hline
\end{tabular}

The low rotor blade losses and high starting torque available for the solar chimney turbine mean that it should be able to accelerate to the design speed unaided. It should also be possible to keep the turbine freewheeling at the correct speed at a no load case with a small flow rate and pressure drop. This means that the plant availability is increased, as there is not a lag while the turbine accelerates to its design speed. 


\section{CHAPTER 6: CONCLUSIONS AND FUTURE RESEARCH}

The main aim of the thesis was to investigate the performance capabilities and limitations of solar chimney turbines. In the first part of the project the importance of defining and understanding the operation of the turbine was shown. The second part dealt with the design and test of the turbine.

\section{Conclusions}

1. By looking carefully at the requirements of the solar chimney it is possible to design a turbine that can be integrated with the chimney.

2. Using an air standard cycle analysis, system losses can be included and a good estimate of the plant output power for a given collector temperature rise estimated. The inclusions of a steady state and then transient collector model are important in predicting the turbine operating range.

3. A single turbine that uses the base supports of the chimney as inlet guide vanes is shown to work effectively. Using the chimney base as IGVs reduces the turbine exit kinetic energy losses while increasing the stiffness of the base supports.

4. An effective turbine design can be developed using inviscid flow analysis coupled to optimisation techniques to reduce the chord and keep the profile losses low.

5. A working scale model of the turbine demonstrated that it worked and allowed the efficiency to be measured. The experimental readings allowed improvements to be made to the simulations that lead to good agreement between the simulated predictions and experimental results.

6. A turbine was designed for three specific operating points. Case (1) and (2) had very good total-to-total efficiencies with reasonable total-to-static efficiencies. Use of the longer diffuser expected on the full-scale plant would increase the total-to-static efficiency.

Table 6.1. Summary of solar chimney turbine measured and predicted efficiencies.

\begin{tabular}{|l|c|c|c|c|}
\hline \multirow{2}{*}{} & \multicolumn{2}{|l|}{ Experimental } & \multicolumn{2}{l|}{ Long diffuser } \\
\cline { 2 - 5 } & Total-to-total & Total-to-static & Total-to-total & Total-to-static \\
\hline 1) Low-pressure & 85.3 & 69.2 & 85.2 & 77.03 \\
\hline 2) High-pressure & 90.2 & 74.5 & 89.6 & 80.7 \\
\hline
\end{tabular}

7. Case (3), intended to be used to control the plant by increasing the pressure drop, was shown not to be feasible in practice. Blade stall leads to inefficient operation.

8. The turbine is self starting with a high initial torque. 
9. The experimental program brought to light the following: adjustable rotor blades are essential to allow the plant to operate efficiently over the operating range, more inlet whirl is required to increase the total-to-static efficiency and the turbine has a very high starting torque even at low mass flow rates.

\section{Future Research}

1. The turbine needs to be redesigned without any allowance for controlling the plant power output. This should lead to an increase in turbine total-to-static efficiency. The redesign should attempt to introduce more inlet whirl before the turbine rotor to further increase the total-tostatic efficiency.

2. The flow through the inlet guide vanes and duct region into the turbine needs to be better understood. The effect of the pre-swirl introduced by the IGV needs to be investigated further as it may have other benefits such as keeping the diffuser efficiency high by having a high axial velocity near the walls at the diffuser entrance.

3. A better understanding of solar chimney power plant operation is needed. This will lead to a better understanding of the turbine requirements. Improvements to the plant simulation are needed to better define the turbine operating range.

4. A full CFD analysis of the turbine to investigate more thoroughly the flow through the plant and obtain better agreement with the experimental results. 


\section{REFERENCES}

Abbot, I.H., Von Doenhoff, A.E., (1959), Theory of Wing Sections, Dover Publications, New York.

Ackermann, T., Söder, L., (2000), Wind Energy Technology and Current Status, a Review, Renewable and Sustainable Energy Reviews, No 4, pp 315-374.

Archer, R.D., Saarlas, M., (1996), An Introduction to Aerospace Propulsion, Prentice Hall, New Jersey.

Balje, O.E., (1981), Turbomachines A guide to Design, Selection, and Theory, Wiley, New York.

Benadé, J.G., (1986), The Development of an Impulse-Type Compressor Blade Cascade, Masters Thesis, University of Stellenbosch, Stellenbosch, South Africa.

Bosman, C., Marsh, H., (1974), An Improved Method for Calculating the Flow in Turbo-Machines, Including a Consistent Loss Model, Journal of Mechanical Engineering Science, Vol. 16, no. 1, pp 25-31.

Cohen, H., Rogers, G.F.C., Saravanamuttoo, H.I.H., (1996), Gas Turbine Theory. Fourth Edition., Addison Wesley Longman Limited, England.

Davis, W.R. and Millar, D.A.J., (Oct 1975), A Comparison of the Matrix and Streamline Curvature Methods of Axial Flow Turbomachinery Analysis, From a User's Point of View, Trans ASME, Journal of Engineering for Power, pp. 549-560.

Duffy, J.A., Beckman, W.A., (1991), Solar Energy Thermal Processes, John Wiley and Sons.

Du Toit, J.H., (2000), Operation of 5-hole probe program, Private Communication.

Gannon, A.J., (1996), A Compressible Formulation of the Streamline Throughflow Method for Axial Compressors, Masters Thesis, University of Stellenbosch, Stellenbosch, South Africa.

Gannon, A.J., Von Backström, T.W., (Aug 2000), Solar Chimney Cycle Analysis with System Loss and Solar Collector Performance, ASME Journal of Solar Energy Engineering, Vol. 122, no. 3, pp 133-137.

Gannon, A.J., Von Backström, T.W., (8-10 April 2002), Controlling and Maximising Solar Chimney Power Output, $1^{\text {st }}$ Int. Conf. on Heat Transfer, Fluid Mechanics and Thermodynamics, Kruger Park, South Africa.

Granger, R.A., (1988), 'Experiments in Fluid Mechanics,' Holt, Rinehart and Winston, inc, USA. 
Greyvenstein, G.P., (December, 1981), Snelheidsdruk Numeriese Metode vir die Berekening van Tweedimensionele Elliptiese Vloei (Velocity-pressure Numerical Method for the Computation of Two-dimensional Elliptic Flow), Masters Thesis, University of Stellenbosch, Stellenbosch, South Africa.

Haaf, W., Friedrich, K., Mayr, G., Schlaich, J., (Oct 1983), Solar Chimneys: Part I: Principle and Construction of the Pilot Plant in Manzanares, International Journal of Solar Energy, Vol. 2, no. 1, pp 3-20.

Haaf, W., (Jan 1984), Solar Chimneys: Part II: Preliminary Test Results from the Manzanares Pilot Plant, International Journal of Solar Energy, Vol. 2, no. 2, pp 141-161.

Hannah, P., (1997), Windfarms of the UK - a 1997 update, Proceedings of the 1997 Nineteenth BWEA Wind Energy Conference, Heriot-Watt University, Edinburgh, UK, pp 17-22.

Harms, T.M., (1999) Private communication, University of Stellenbosch, South Africa.

Harms, T.M., (1995) A Finite Volume Method for the Analysis of Flow Fields with Complex Boundaries, Ph.D. Thesis, University of Stellenbosch, Stellenbosch, South Africa.

Harms, T.M., von Backström, T.W., and du Plessis, J.P., (1996), Simplified Control Volume FiniteElement Method, Numerical Heat Transfer, Part B, 30, pp 179-194.

Hoerner, S.F., Borst, V., (1975), Fluid-Dynamic Lift, Hoerner Fluid Dynamics, Brick Town, N.J., USA.

Holman, J.P., (1992), Heat Transfer, Seventh Edition, McGraw-Hill Book International (UK) Limited, London.

Howell, R.J., Ramesh, O.N., Hodson, H.P., Harvey, N.W., Schulte, (2001), 'High Lift and AftLoaded Profiles for Low-Pressure Turbines,' ASME Journal of Turbomachinery, Vol. 123, pp 181188.

Hunter, R. ed, (1997), Wind Energy Conversion from Theory to Practice, Proceedings of the 1997 Nineteenth BWEA Wind Energy Conference, Heriot-Watt University, Edinburgh, UK.

Incropera, F.P., De Witt, D.P., (1990), Introduction to Heat Transfer, John Wiley \& Sons, Singapore.

Japikse, D., Baines, N.C., (1997), Introduction to Turbomachinery, Concepts ETI and Oxford University Press.

Kröger, D.G., (1999-2001), Private communication, University of Stellenbosch, South Africa. 
Kröger, D.G., Blaine, D., (2001), Analysis of the Driving Potential of a Solar Chimney Power Plant, R\&D Journal, Vol 15, pp 85-94.

Kröger, D.G., Buys, J.D., (1999), Radial Flow Boundary Layer Development Analysis, $R \& D$ Journal, Vol 15, pp 95-102.

Kröger, D.G., Buys, J.D., (2001), Performance Evaluation of a Solar Chimney Power Plant, ISES 2001 Solar World Congress, Adelaide, South Australia.

Lewis, R.I., (1991), A Method for Inverse Aerofoil and Cascade Design by Surface Vorticity, ASME Paper No. 82-GT-154.

Lewis, R.I., (1991), Vortex Element Methods for Fluid Dynamic Analysis of Engineering Systems, Cambridge Press, Cambridge.

Lewis, R.I., (1996), Turbomachinery Performance Analysis, Arnold, London.

Maad, Belghith, (1994), The Intensification of the Heat Transfer in Passive Solar Systems Using Grid Generated Turbulence: Spectral Study. Renewable Energy, Vol. 4(3), pp 319-325.

MATLAB, (1996), (Programming Language), Ver. 5.0, The MathWorks Inc.

Mohamad, A.A., (1997), High Efficiency Solar Air Heater, Solar Energy, Vol 60, No. 2, pp 71-76.

Mullet, L.B., (Jan 1987), Solar Chimney - Overall Efficiency, Design and Performance, International Journal Ambient Energy, Vol. 8, no. 1, pp 35-40.

Nel, P, (1999), Private Communication, Palmiet Pump Storage Scheme, ESKOM, South Africa.

Ong, K.S., (1995), Thermal Performance of Solar Air Heaters: Mathematical Model and Solution Procedure, Solar Energy, Vol 55, pp 93-109.

Pasumarthi, N., Sherif, S.A., (1998), Experimental \& Theoretical Performance of a Demonstration Solar Chimney Model - Part I: Mathematical Model Development, International Journal of Energy Research, Vol. 22, no. 3, pp 277-288.

Pasumarthi, N., Sherif, S.A., (1997), Performance of a Demonstration Solar Chimney Model for Power Generation, Conference Proceedings of the $199735^{\text {th }}$ Heat Transfer and Fluid Mechanics Institute, California State University, Sacramento, CA, USA, pp 203-241.

Preston-Whyte, R.A., Tyson, P.D., (1989), The Atmosphere and Weather of Southern Africa, Oxford University Press, Cape Town, South Africa. 
Rao, S. S., (1996), Engineering Optimisation, Theory and Practice Third Edition, John Wiley \& Sons, New York.

Runstadler, P.W. Jr., Dolan, F.X., Dean, R.C. Jr., (1975), Diffuser Data Book, Creare, Hanover, New Hampshire.

Sayers, A. T., (1990), Hydraulic and Compressible Flow Turbomachines, McGraw-Hill, London.

Schlaich Bergermann and Partners, Civil Engineers, (1995), The Solar Chimney Transferability of Results from the Manzanares Solar Chimney Plant to Larger Scale-Plants, Stuttgart, Germany.

Schlaich, J., (1995), The Solar Chimney: Electricity from the Sun, C. Maurer, Geislingen, Germany.

Schlaich, J., (1999), Tension Structures for Solar Electricity Generation, Engineering Structures, No. 21, pp 658-668.

Schwarz, G., Knauss, H., (Feb 1981), Fluid Mechanical Layout (Design) of the Updraught Power Station Manzanares, Institute for Aerodynamic and Gas Dynamics of the University Stuttgart. (Translated from original German)

Sears, F.W., Zemansky, M.W., Young, H.D., (1987), University Physics Seventh Edition, AddisonWesley Publishing Company, USA.

Simon, M., (1976), Konzept der atmosphärischen Nutzung. In AGS/ASA-Study Energiequellen für morgen? Nichtfossile/nichtnukleare Energiequellen, Teil II: Nutzung der solaren Strahlungsenergie, pp 555-564. Umschau-Verlag.

Spera, D.A., (1995), Wind Turbine Technology Fundamental Concepts of Wind Turbine Engineering, ASME Press, New York, USA.

Stephan, K., Schaber, K., Körber, J., Tamm, M., Kim, Y.K., (1995), Expert Opinion on the Thermodynamic Modelling of Solar Chimneys, Report, University of Stuttgart, Stuttgart, Germany.

Stratford, B.S., (1959), The Prediction of Separation of the Turbulent Boundary Layer, Journal of Fluid Mechanics, Vol. 5, part I.

Strohmaier, C.K., (1997), An Evaluation of a Scale Model Medium Speed Wind Tunnel, Masters Thesis, University of Stellenbosch, Stellenbosch, South Africa.

Trieb, F., Langni^, O., Klai ^, H., (1997), "Solar Electricity Generation-a Comparative View of Technologies, Costs and Environmental Impact," Solar Energy, Vol 59, nos 1-3, pp 89-99.

Ullman, D. G., (1992), The Mechanical Design Process, McGraw Hill, Inc, Singapore. 
Von Backström, T.W. (1996-2001) Private communication, University of Stellenbosch, South Africa.

Von Backström, T.W., Bernhardt, A., Gannon, A.J., (June, 2002), Pressure Drop in Solar Power Plant Chimneys, Solar 2002, Reno, Nevada.

Von Backström, T.W., Buys, J.D., Stinnes, W.H., (1996), Minimisation of the Exit Loss of a RotorOnly Axial Fan, Engineering Optimisation, Vol. 26, pp 25-33.

Von Backström, T.W., (1998), A First Look at the Design of the Turbine for a Large Solar Chimney Power Plant, Internal report, University of Stellenbosch, Stellenbosch, South Africa.

Von Backström, T.W., Gannon, A.J., (March 2000), The Solar Chimney Air Standard Thermodynamic Cycle, R\&D Journal, Vol. 16, pp 10-16.

Von Backström, T.W., Gannon, A.J., (2000), Compressible Flow Through Solar Power Plant Chimneys, ASME Journal of Solar Energy Engineering, Vol. 122, no. 3, pp 138-145.

Von Backström, T.W., Gannon, A.J., (2001), Solar Chimney Turbine Characteristics, ISES Congress, Adelaide, Australia.

White, F.M., (1991), Viscous Fluid Flow Second Edition, McGraw-Hill, New York.

Wortmann, F.X., Scharz, G., Knauss, H., (1981), Fluid Mechanical Layout (Design) of the Updraught Power Station Manzanares (Translated from German), Institute for Aerodynamics and Gas Dynamics of the University of Stuttgart, Stuttgart. 


\section{APPENDiX A: SAMPle VAlUES FRom Solar Chimney CalCUlations.}

Table A.1 Specific power output $P_{2} *$ for solar chimney using equation (2.22). (Figure 2.4)

(Table value $=\mathrm{P}_{2} *$ x $10^{3}$ )

\begin{tabular}{|c|c|c|c|c|c|c|}
\hline $\begin{array}{l}\text { Collector } \quad \text { Temp } \\
\text { Rise } \Delta \mathrm{T}_{23}[\mathrm{~K}] . \Rightarrow\end{array}$ & & & & & & \\
\hline $\begin{array}{ll}\text { Chimney } & \text { height } \\
\Delta \mathrm{z}[\mathrm{m}] . \Downarrow & \end{array}$ & 5 & 10 & 15 & 20 & 30 & 40 \\
\hline 2000 & 1.062 & 2.124 & 3.185 & 4.247 & 6.371 & 8.494 \\
\hline 1500 & 0.796 & 1.593 & 2.389 & 3.185 & 4.778 & 6.371 \\
\hline 1000 & 0.531 & 1.062 & 1.593 & 2.124 & 3.185 & 4.247 \\
\hline 500 & 0.265 & 0.531 & 0.796 & 1.062 & 1.593 & 2.124 \\
\hline
\end{tabular}

Table A.2 Turbine pressure drop [Pa] for solar chimney using equation (2.30). (Figure 2.5)

\begin{tabular}{|l|l|l|l|l|l|l|}
\hline $\begin{array}{l}\text { Collector Temp } \\
\text { Rise } \Delta \mathrm{T}_{23}[\mathrm{~K}] . \Rightarrow\end{array}$ & \multirow{2}{*}{$\begin{array}{l}\text { Thimney height } \\
\Delta \mathrm{z}[\mathrm{m}] . \Downarrow\end{array}$} & 10 & 15 & 20 & 30 & 40 \\
\cline { 1 - 7 } 2000 & 329 & 646 & 952 & 1249 & 1813 & 2342 \\
\hline 1500 & 247 & 485 & 715 & 938 & 1362 & 1760 \\
\hline 1000 & 164 & 323 & 477 & 626 & 910 & 1176 \\
\hline 500 & 82 & 162 & 239 & 313 & 456 & 590 \\
\hline
\end{tabular}

Table A.3 Required mass flow [tonnes] for design power output of 200MW using equation (2.31). (Figure 2.6)

\begin{tabular}{|l|l|l|l|l|l|l|}
\hline $\begin{array}{l}\text { Collector Temp } \\
\text { Rise } \Delta \mathrm{T}_{23}[\mathrm{~K}] . \Rightarrow\end{array}$ & \multirow{2}{*}{$\begin{array}{l}\text { Chimney height } \\
\Delta \mathrm{z}[\mathrm{m}] . \Downarrow\end{array}$} & 10 & 15 & 20 & 30 & 40 \\
\cline { 1 - 7 } 2000 & 618 & 309 & 206 & 155 & 103 & 77 \\
\hline 1500 & 824 & 412 & 275 & 206 & 137 & 103 \\
\hline 1000 & 1236 & 618 & 412 & 309 & 206 & 154 \\
\hline 500 & 2473 & 1236 & 824 & 618 & 412 & 309 \\
\hline
\end{tabular}


Table A.4 Power / unit area $\left[\mathrm{W} / \mathrm{m}^{2}\right]$ and exit velocity $[\mathrm{m} / \mathrm{s}]$ for maximum power and choking conditions for chimney height $=1500 \mathrm{~m}, \eta_{\text {turb }}=80 \%$ and $\mathrm{k}=1$. (Equation (2.43), Figure 2.9)

\begin{tabular}{|c|c|c|c|c|c|c|}
\hline $\begin{array}{l}\text { Collector } \quad \text { Temp } \\
\text { Rise } \Delta \mathrm{T}_{23}[\mathrm{~K}] . \Rightarrow\end{array}$ & 5 & 10 & 15 & 20 & 30 & 40 \\
\hline $\mathrm{C}_{\mathrm{z} 4 \max } \quad[\mathrm{m} / \mathrm{s}]$ & 8.55 & 12.10 & 14.82 & 17.11 & 20.96 & 24.21 \\
\hline $\mathrm{P}_{\text {area max }} \quad\left[\mathrm{W} / \mathrm{m}^{2}\right]$ & 1005 & 2795 & 5053 & 7657 & 13637 & 20373 \\
\hline $\mathrm{C}_{\mathrm{z} 4 \text { choke }}[\mathrm{m} / \mathrm{s}]$ & 14.82 & 20.95 & 25.67 & 29.64 & 36.31 & 41.93 \\
\hline
\end{tabular}

Table A.5 Required Diameter [m] to obtain design power at max power condition for chimney height $=1500 \mathrm{~m}, \eta_{\text {turb }}=80 \%$ and $k=1$. (Equation (2.62), Figure 2.10)

\begin{tabular}{|l|l|l|l|l|l|l|l|}
\hline $\begin{array}{l}\text { Collector Temp } \\
\text { Rise } \Delta \mathrm{T}_{23}[\mathrm{~K}] . \Rightarrow\end{array}$ & 5 & 10 & 15 & 20 & $\mathbf{2 4 . 0 1}$ & 30 & 40 \\
\hline Diam. $[\mathrm{m}]$ & 503 & 302 & 224 & 182 & $\mathbf{1 6 0}$ & 137 & 112 \\
\hline
\end{tabular}


Table A.6 Power [MW], exit velocity [m/s], mass flow [tonnes/s] for maximum power, choking and limiting condition for chimney height $=1500 \mathrm{~m}$, diam $=160 \mathrm{~m} \eta_{\text {turb }}=80 \%, \mathrm{k}=1$ and $\alpha=$ 1.0575. (Equation (2.43), Figure 2.11, Figure 2.12, Figure 2.13)

\begin{tabular}{|c|c|c|c|c|c|c|c|}
\hline $\begin{array}{l}\text { Collector Temp } \text { Rise } \\
\Delta \mathrm{T}_{23}[\mathrm{~K}] . \Rightarrow\end{array}$ & 5 & 10 & 15 & 20 & 24.01 & 30 & 40 \\
\hline$[\mathrm{m} / \mathrm{s}]$ & 8.55 & 12.10 & 14.82 & 17.11 & 18.75 & 20.96 & 24.21 \\
\hline [tonnes/s] & 155 & 215 & 259 & 295 & 318.9 & 350 & 392 \\
\hline$[\mathrm{MW}]$ & 20.2 & 56.2 & 101.6 & 154.0 & 200 & 274.2 & 409.6 \\
\hline$\Delta \mathrm{p}_{\text {turb max }}$ & 166 & 326 & 482 & 632 & 749 & 918 & 1187 \\
\hline $\mathrm{C}_{\mathrm{z} 4 \text { choke }}$ & 14.82 & 20.95 & 25.67 & 29.64 & & 36.31 & 41.93 \\
\hline$\dot{m}_{\text {choke }}$ & 267.7 & 372.4 & 448.8 & 510.1 & & 605.7 & 678.7 \\
\hline 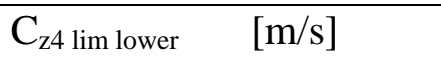 & & & & & 18.75 & 11.28 & 8.19 \\
\hline $\mathrm{C}_{\mathrm{z} 4 \mathrm{lim} \text { upper }}$ & & & & & 18.75 & 29.32 & 37.22 \\
\hline$\dot{m}_{\text {lim lower }} \quad$ [tonnes/s] & & & & & 318.9 & 188.4 & 132.7 \\
\hline$\dot{m}_{\text {lim upper }} \quad$ [tonnes/s] & & & & & 318.9 & 489.3 & 602.7 \\
\hline$\eta_{\text {lim lower }}$ & & & & & 2.60 & 3.52 & 3.75 \\
\hline$\eta_{\text {lim upper }}$ & & & & & 2.60 & 1.36 & 0.83 \\
\hline$\Delta \mathrm{p}_{\text {turb lim up }} \quad[\mathrm{Pa}]$ & & & & & 749 & 1242 & 1708 \\
\hline$\Delta \mathrm{p}_{\text {turb lim low }} \quad[\mathrm{Pa}]$ & & & & & 749 & 480 & 378 \\
\hline
\end{tabular}


Table A.7 Sample values for cycle analysis with solar collector included. Power [MW], exit velocity [m/s], mass flow [tonnes/s] for maximum power, choking and limiting condition for chimney height $=1500 \mathrm{~m}$, diam $=160 \mathrm{~m} \eta_{\text {turb }}=80 \%, k=1$ and $\alpha=1.0575$. (Figure 2.18, Figure 2.20, Figure 2.22, Figure 2.23 and Figure 2.21)

\begin{tabular}{|c|c|c|c|c|c|c|c|}
\hline \multicolumn{2}{|c|}{$\begin{array}{l}\text { Inlet Solar Radiation } \\
\mathrm{G}\left[\mathrm{W} / \mathrm{m}^{2}\right] . \Rightarrow\end{array}$} & 200 & 400 & 600 & 756.8 & 800 & 1000 \\
\hline$\Delta T_{23 \text { max }}$ & {$[\mathrm{K}]$} & 12.2 & 22.1 & 30.9 & 37.4 & 39.2 & 47.0 \\
\hline $\mathrm{C}_{\mathrm{z} 4 \max }$ & {$[\mathrm{m} / \mathrm{s}]$} & 5.75 & 7.14 & 8.12 & 8.76 & 8.92 & 9.61 \\
\hline$\dot{m}_{\max }$ & [tonnes/s] & 101.5 & 122.3 & 135.3 & 143.1 & 145.0 & 152.6 \\
\hline $\mathrm{P}_{\max }$ & [MW] & 45.7 & 100.7 & 156.0 & 200 & 212.2 & 268.6 \\
\hline$\Delta \mathrm{p}_{\text {turb max }}$ & {$[\mathrm{Pa}]$} & 559 & 985 & 1344 & 1597 & 1664 & 1953 \\
\hline$\eta_{\text {col max }}$ & [\%] & 49.8 & 54.1 & 55.9 & 56.7 & 56.9 & 57.5 \\
\hline$\eta_{\max }$ & {$[\%]$} & 3.66 & 3.69 & 3.71 & 3.72 & 3.72 & 3.72 \\
\hline$\eta_{\text {plant max }}$ & [\%] & 1.82 & 2.00 & 2.07 & 2.11 & 2.11 & 2.14 \\
\hline$\Delta T_{23 \text { choke }}$ & {$[\mathrm{K}]$} & 5.14 & 8.61 & 11.53 & & 14.2 & 16.6 \\
\hline $\mathrm{C}_{\mathrm{x} 4 \text { choke }}$ & {$[\mathrm{m} / \mathrm{s}]$} & 15.02 & 19.44 & 22.50 & & 24.95 & 27.02 \\
\hline$\dot{m}_{\text {choke }}$ & [tonnes/s] & 271.3 & 347.1 & 398.0 & & 437.4 & 470.0 \\
\hline$\eta_{\text {col choke }}$ & [\%] & 55.9 & 59.8 & 61.3 & & 62.1 & 62.5 \\
\hline$\Delta T_{23} \lim$ lower & {$[\mathrm{K}]$} & & & & 37.4 & 62.4 & 132.5 \\
\hline$\Delta \mathrm{T}_{23} \lim$ upper & {$[\mathrm{K}]$} & & & & 37.4 & 27.3 & 24.0 \\
\hline $\mathrm{C}_{\mathrm{x} 4}$ lim lower & {$[\mathrm{m} / \mathrm{s}]$} & & & & 8.76 & 5.44 & 3.03 \\
\hline $\mathrm{C}_{\mathrm{x} 4} \lim$ upper & {$[\mathrm{m} / \mathrm{s}]$} & & & & 8.76 & 12.91 & 18.8 \\
\hline$\dot{m}_{\text {lim lower }}$ & [tonnes/s] & & & & 143.1 & 82.8 & 38.7 \\
\hline$\dot{m}_{\text {lim upper }}$ & [tonnes/s] & & & & 143.1 & 217.5 & 319.0 \\
\hline$\eta_{\text {col lim lower }}$ & [\%] & & & & 56.7 & 51.7 & 41.0 \\
\hline$\eta_{\text {col lim upper }}$ & {$[\%]$} & & & & 56.7 & 59.4 & 61.4 \\
\hline$\eta_{\text {lim lower }}$ & {$[\%]$} & & & & 3.72 & 3.85 & 3.88 \\
\hline$\eta_{\text {lim upper }}$ & {$[\%]$} & & & & 3.72 & 3.36 & 2.60 \\
\hline$\eta_{\text {plant lim lower }}$ & {$[\%]$} & & & & 2.11 & 1.99 & 1.59 \\
\hline$\eta_{\text {plant lim upper }}$ & {$[\%]$} & & & & 2.11 & 1.99 & 1.59 \\
\hline$\Delta \mathrm{p}_{\text {turb lim up }}$ & {$[\mathrm{Pa}]$} & & & & 1597 & 2563 & 4565 \\
\hline$\Delta \mathrm{p}_{\text {turb lim low }}$ & {$[\mathrm{Pa}]$} & & & & 1597 & 1086 & 748 \\
\hline
\end{tabular}




\section{APPENDIX B: TABULATED EXPERIMENTAL DATA}

\section{Operating Range Data}

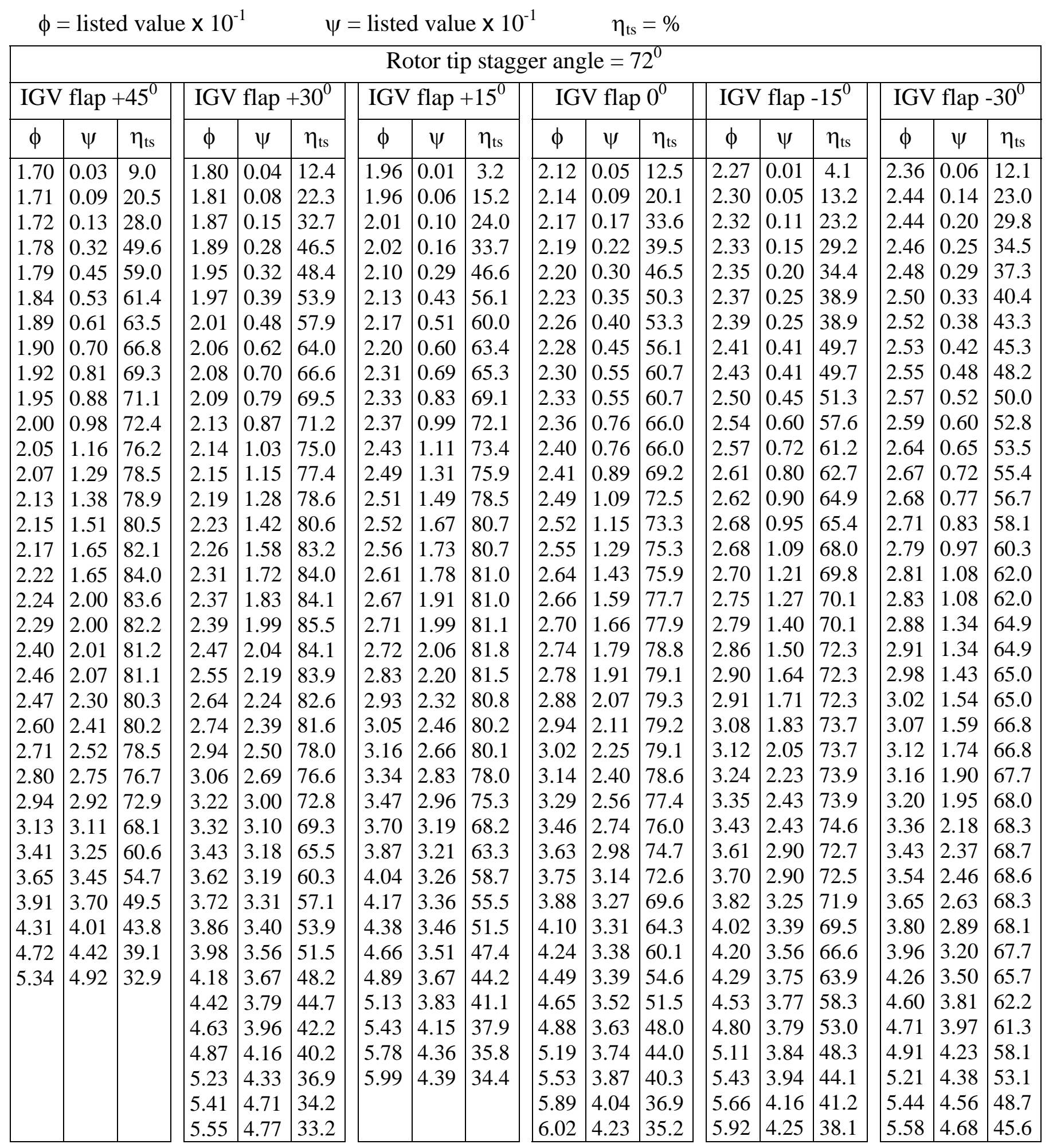




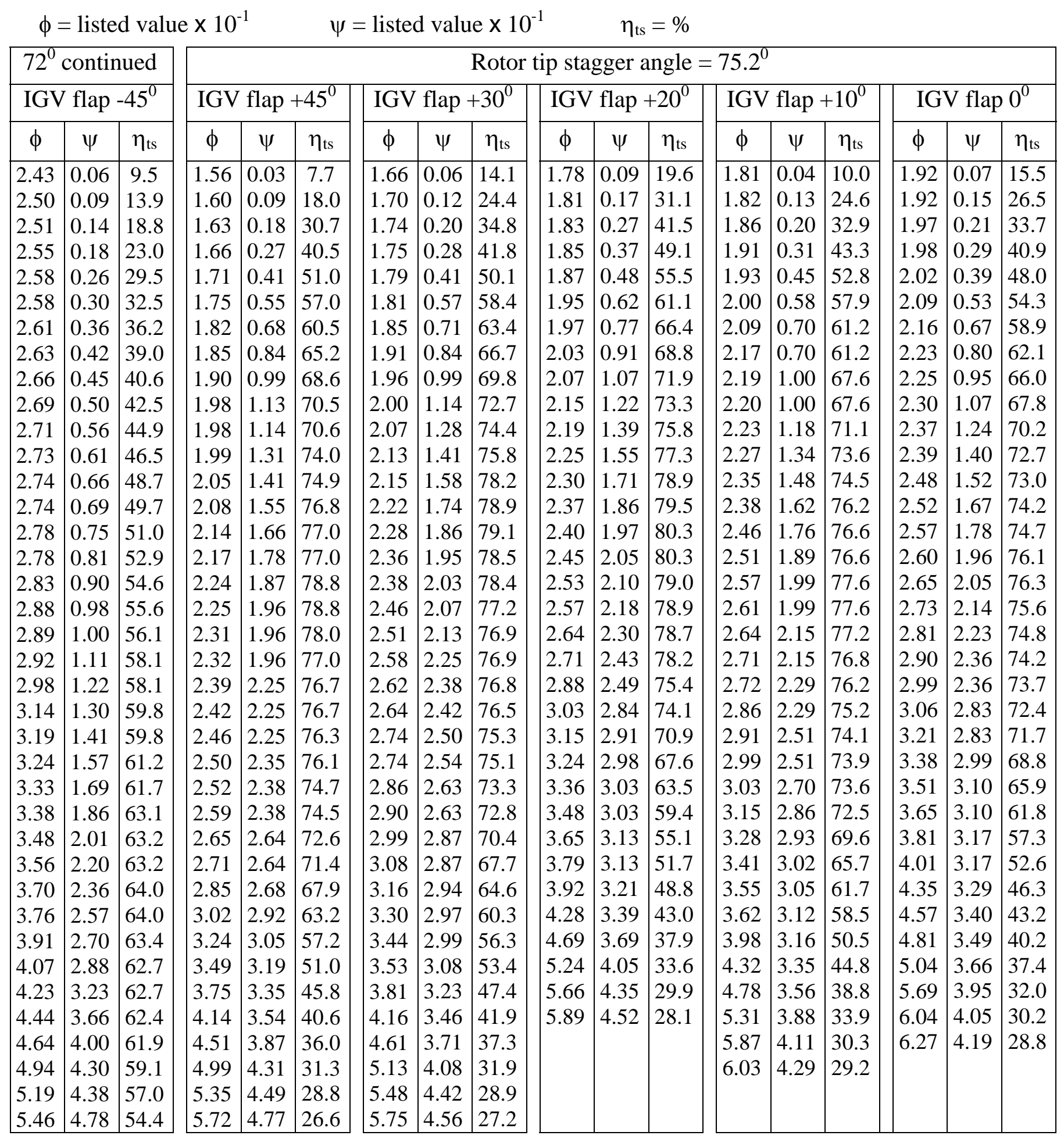


$\phi=$ listed value $\times 10^{-1} \quad \psi=$ listed value $\times 10^{-1} \quad \eta_{\mathrm{ts}}=\%$

\begin{tabular}{|c|c|c|c|c|c|c|c|c|c|c|c|c|c|c|c|c|c|}
\hline \multicolumn{12}{|c|}{$75.2^{0}$ continued } & \multicolumn{6}{|c|}{ Rotor tip stagger angle $=78^{0}$} \\
\hline \multicolumn{3}{|c|}{ IGV flap $-10^{0}$} & \multicolumn{3}{|c|}{ IGV flap $-20^{0}$} & \multicolumn{3}{|c|}{ IGV flap $-30^{0}$} & \multicolumn{3}{|c|}{ IGV flap $-45^{0}$} & \multicolumn{3}{|c|}{ IGV flap $+45^{0}$} & \multicolumn{3}{|c|}{ IGV flap $+30^{0}$} \\
\hline$\phi$ & $\psi$ & $\eta_{\text {ts }}$ & $\phi$ & $\psi$ & $\eta_{\text {ts }}$ & $\phi$ & $\psi$ & $\eta_{\mathrm{ts}}$ & $\phi$ & $\psi$ & $\eta_{\mathrm{ts}}$ & $\phi$ & $\psi$ & $\eta_{\mathrm{ts}}$ & $\phi$ & $\psi$ & $\eta_{\mathrm{ts}}$ \\
\hline 1.94 & 0.02 & 5.2 & 1.99 & 0.07 & 13.2 & 2.03 & 0.06 & 10.5 & 2.12 & 0.11 & 15.8 & 1.37 & 0.11 & 24.1 & 1.42 & 0.05 & 13.8 \\
\hline 1.99 & 0.11 & 21.1 & 2.03 & 0.12 & 20.9 & 2.07 & 0.12 & 19.8 & 2.17 & 0.18 & 23.7 & 1.42 & 0.20 & 36.8 & 1.42 & 0.11 & 25.6 \\
\hline 2.01 & 0.18 & 29.3 & 2.05 & 0.14 & 23.5 & 2.12 & 0.20 & 28.7 & 2.19 & 0.25 & 28.9 & 1.46 & 0.34 & 50.1 & 1.51 & 0.17 & 34.5 \\
\hline 2.05 & 0.25 & 36.3 & 2.11 & 0.32 & 40.0 & 2.18 & 0.30 & 36.8 & 2.23 & 0.35 & 35.5 & 1.47 & 0.43 & 57.2 & 1.52 & 0.28 & 44.8 \\
\hline 2.11 & 0.37 & 44.6 & 2.18 & 0.45 & 47.5 & 2.22 & 0.43 & 44.2 & 2.29 & 0.46 & 41.1 & 1.50 & 0.59 & 63.9 & 1.55 & 0.41 & 54.6 \\
\hline 2.14 & 0.49 & 50.8 & 2.24 & 0.59 & 53.0 & 2.25 & 0.56 & 49.8 & 2.35 & 0.58 & 45.0 & 1.54 & 0.75 & 69.1 & 1.65 & 0.54 & 59.7 \\
\hline 2.21 & 0.61 & 55.5 & 2.29 & 0.73 & 57.5 & 2.32 & 0.68 & 53.5 & 2.38 & 0.69 & 48.9 & 1.63 & 0.90 & 71.8 & 1.72 & 0.68 & 64.7 \\
\hline 2.24 & 0.75 & 60.0 & 2.33 & 0.85 & 60.5 & 2.38 & 0.80 & 56.3 & 2.44 & 0.81 & 51.7 & 1.71 & 1.05 & 73.9 & 1.73 & 0.84 & 69.9 \\
\hline 2.34 & 0.88 & 62.1 & 2.40 & 0.97 & 62.2 & 2.42 & 0.92 & 59.1 & 2.51 & 0.93 & 53.8 & 1.74 & 1.21 & 77.1 & 1.81 & 0.97 & 71.3 \\
\hline 2.39 & 1.04 & 65.1 & 2.47 & 1.09 & 63.7 & 2.47 & 1.06 & 60.8 & 2.53 & 1.06 & 56.6 & 1.76 & 1.37 & 77.1 & 1.84 & 1.14 & 75.1 \\
\hline 2.43 & 1.15 & 66.4 & 2.55 & 1.22 & 64.6 & 2.53 & 1.17 & 62.4 & 2.54 & 1.09 & 58.3 & 1.86 & 1.48 & 77.1 & 1.86 & 1.32 & 78.7 \\
\hline 2.44 & 1.30 & 69.2 & 2.57 & 1.35 & 66.7 & 2.59 & 1.29 & 63.5 & 2.58 & 1.19 & 59.6 & 1.89 & 1.64 & 77.1 & 1.89 & 1.50 & 81.0 \\
\hline 2.51 & 1.47 & 70.6 & 2.61 & 1.48 & 68.1 & 2.63 & 1.41 & 64.8 & 2.65 & 1.29 & 60.3 & 1.98 & 1.72 & 82.8 & 1.97 & 1.50 & 84.5 \\
\hline 2.58 & 1.59 & 71.0 & 2.68 & 1.62 & 68.6 & 2.67 & 1.54 & |66.1 & 2.67 & 1.38 & 61.6 & 1.99 & 1.85 & 82.8 & 1.98 & 1.97 & 83.9 \\
\hline 2.62 & 1.72 & 71.0 & 2.69 & 1.77 & 68.6 & 2.70 & 1.66 & 67.3 & 2.70 & 1.48 & 62.6 & 2.05 & 1.93 & 82.8 & 2.03 & 1.97 & 82.4 \\
\hline 2.71 & 1.83 & 72.7 & 2.77 & 1.88 & 70.8 & 2.77 & 1.78 & 67.5 & 2.78 & 1.57 & 62.7 & 2.06 & 2.05 & 82.8 & 2.11 & 2.03 & 81.7 \\
\hline 2.74 & 1.97 & 72.7 & 2.82 & 2.02 & 70.8 & 2.82 & 1.89 & 67.9 & 2.86 & 1.67 & 63.0 & 2.16 & 2.06 & 80.0 & 2.16 & 2.10 & 81.0 \\
\hline 2.78 & 2.11 & 73.4 & 2.90 & 2.15 & 70.8 & 2.89 & 2.03 & 67.9 & 2.89 & 1.81 & 64.0 & 2.21 & 2.16 & 79.9 & 2.24 & 2.13 & 81.0 \\
\hline 2.87 & 2.18 & 72.5 & 3.01 & 2.26 & 69.9 & 3.01 & 2.18 & 68.8 & 2.96 & 1.93 & 64.3 & 2.23 & 2.27 & 79.6 & 2.26 & 2.24 & 80.3 \\
\hline 2.97 & 2.30 & 71.6 & 3.10 & 2.41 & 69.5 & 3.05 & 2.37 & 68.8 & 2.98 & 2.11 & 65.8 & 2.32 & 2.36 & 77.6 & 2.32 & 2.31 & 80.3 \\
\hline 3.05 & 2.46 & 71.6 & 3.21 & 2.59 & 69.0 & 3.17 & 2.51 & 68.1 & 3.08 & 2.22 & 65.4 & 2.41 & 2.44 & 75.4 & 2.36 & 2.29 & 79.4 \\
\hline 3.18 & 2.62 & 70.5 & 3.33 & 2.71 & 68.0 & 3.31 & 2.63 & 66.6 & 3.18 & 2.36 & 65.2 & 2.46 & 2.44 & 75.1 & 2.40 & 2.41 & 79.5 \\
\hline 3.26 & 2.78 & 70.2 & 3.49 & 2.93 & 66.5 & 3.46 & 2.84 & 65.6 & 3.24 & 2.53 & 64.8 & 2.50 & 2.69 & 73.8 & 2.49 & 2.48 & 78.2 \\
\hline 3.43 & 3.00 & 68.2 & 3.60 & 3.11 & 65.6 & 3.60 & 3.07 & 64.7 & 3.30 & 2.58 & 64.4 & 2.57 & 2.69 & 72.3 & 2.50 & 2.50 & 78.2 \\
\hline 3.59 & 3.18 & 65.5 & 3.80 & 3.35 & 62.9 & 3.83 & 3.32 & 62.6 & 3.46 & 2.76 & 63.4 & 2.61 & 2.81 & 71.0 & 2.51 & 2.67 & 78.2 \\
\hline 3.76 & 3.26 & 61.8 & 3.99 & 3.45 & 59.3 & 4.05 & 3.57 & 60.0 & 3.58 & 2.91 & 62.7 & 2.72 & 2.85 & 67.6 & 2.64 & 2.72 & 75.6 \\
\hline 3.93 & 3.30 & 57.7 & 4.15 & 3.51 & 55.7 & 4.29 & 3.73 & 56.6 & 3.80 & 3.15 & 61.1 & 2.79 & 2.92 & 65.0 & 2.72 & 2.75 & 73.2 \\
\hline 4.31 & 3.34 & 48.9 & 4.60 & 3.55 & 46.9 & 4.44 & 3.91 & 53.6 & 4.04 & 3.47 & 59.2 & 2.91 & 2.95 & 61.5 & 2.83 & 2.89 & 69.1 \\
\hline 4.79 & 3.45 & 41.9 & 5.18 & 3.65 & 39.1 & 4.70 & 3.91 & 48.7 & 4.31 & 3.79 & 56.9 & 2.99 & 3.01 & 58.6 & 2.90 & 2.89 & 65.8 \\
\hline 5.33 & 3.69 & 35.7 & 5.68 & 3.89 & 33.6 & 4.99 & 4.04 & 43.9 & 4.59 & 4.19 & 54.1 & 3.08 & 3.07 & 55.5 & 3.04 & 3.03 & 61.3 \\
\hline 5.83 & 3.86 & 31.6 & 6.03 & 3.98 & 31.1 & 5.24 & 4.04 & 40.4 & 4.93 & 4.39 & 50.2 & 3.16 & 3.13 & 52.8 & 3.24 & 3.03 & 54.3 \\
\hline 6.36 & 4.12 & 28.8 & 6.28 & 4.05 & 29.4 & 5.62 & 4.07 & 36.1 & 4.99 & 4.62 & 49.2 & 3.34 & 3.14 & 49.0 & 3.32 & 3.13 & 51.9 \\
\hline & & & & & & 5.90 & 4.12 & 33.5 & 5.50 & 4.69 & 43.1 & 3.48 & 3.20 & 46.4 & 3.51 & 3.16 & 48.1 \\
\hline & & & & & & 6.27 & 4.13 & 30.9 & 5.65 & 4.69 & 41.3 & 3.62 & 3.30 & 43.8 & 3.65 & 3.24 & 45.4 \\
\hline & & & & & & & & & & & & 3.77 & 3.40 & 41.6 & 3.72 & 3.43 & 43.9 \\
\hline & & & & & & & & & & & & 3.92 & 3.54 & 39.4 & 3.95 & 3.50 & 40.8 \\
\hline & & & & & & & & & & & & 4.16 & 3.61 & 36.3 & 4.10 & 3.64 & 39.0 \\
\hline & & & & & & & & & & & & 4.38 & 3.77 & 33.7 & 4.29 & 3.79 & 36.7 \\
\hline & & & & & & & & & & & & 4.58 & 3.96 & 31.7 & 4.60 & 3.89 & 33.2 \\
\hline & & & & & & & & & & & & 4.92 & 4.09 & 29.1 & 4.76 & 4.18 & 31.3 \\
\hline & & & & & & & & & & & & 5.19 & 4.09 & 27.2 & 5.04 & 4.39 & 28.8 \\
\hline & & & & & & & & & & & & 5.26 & 4.50 & 26.7 & 5.48 & 4.53 & 26.1 \\
\hline & & & & & & & & & & & & 5.36 & 4.50 & 26.1 & 5.59 & 4.83 & 25.4 \\
\hline & & & & & & & & & & & & 5.45 & 4.59 & 25.5 & 6.08 & 5.06 & 23.2 \\
\hline & & & & & & & & & & & & 5.64 & 4.60 & 24.6 & & & \\
\hline
\end{tabular}


$\phi=$ listed value $\times 10^{-1} \quad \psi=$ listed value $\times 10^{-1} \quad \eta_{\mathrm{ts}}=\%$

\begin{tabular}{|c|c|c|c|c|c|c|c|c|c|c|c|c|c|c|c|c|c|}
\hline \multicolumn{18}{|c|}{$78^{0}$ continued } \\
\hline \multicolumn{3}{|c|}{ IGV flap $+20^{0}$} & \multicolumn{3}{|c|}{ IGV flap $+10^{0}$} & \multicolumn{3}{|c|}{ IGV flap $0^{0}$} & \multicolumn{3}{|c|}{ IGV flap $-10^{0}$} & \multicolumn{3}{|c|}{ IGV flap $-20^{0}$} & \multicolumn{3}{|c|}{ IGV flap $-30^{0}$} \\
\hline$\phi$ & $\psi$ & $\eta_{\mathrm{ts}}$ & $\phi$ & $\psi$ & $\eta_{\text {ts }}$ & $\phi$ & $\psi$ & $\eta_{\mathrm{ts}}$ & $\phi$ & $\psi$ & $\eta_{\mathrm{ts}}$ & $\phi$ & $\psi$ & $\eta_{\mathrm{ts}}$ & $\phi$ & $\psi$ & $\eta_{\mathrm{ts}}$ \\
\hline 1.42 & 0.01 & 3.6 & 1.57 & 0.05 & 14.0 & 1.57 & 0.03 & 8.8 & 1.72 & 0.06 & 13.5 & 1.82 & 0.09 & 18.9 & 1.85 & 0.10 & 19.1 \\
\hline 1.44 & 0.10 & 22.4 & 1.59 & 0.14 & 29.9 & 1.61 & 0.11 & 23.7 & 1.76 & 0.15 & 28.7 & 1.84 & 0.18 & 31.8 & 1.89 & 0.15 & 26.5 \\
\hline 1.51 & 0.22 & 40.5 & 1.65 & 0.20 & 37.4 & 1.65 & 0.18 & 35.4 & 1.83 & 0.26 & 40.6 & 1.91 & 0.26 & 39.2 & 1.95 & 0.24 & 35.9 \\
\hline 1.55 & 0.36 & 52.9 & 1.77 & 0.30 & 45.6 & 1.71 & 0.30 & 46.1 & 1.92 & 0.40 & 49.9 & 1.96 & 0.37 & 46.6 & 1.98 & 0.34 & 43.2 \\
\hline 1.58 & 0.50 & 61.4 & 1.78 & 0.45 & 56.1 & 1.74 & 0.43 & 46.1 & 1.95 & 0.52 & 55.6 & 2.02 & 0.48 & 52.2 & 2.03 & 0.47 & 49.9 \\
\hline 1.62 & 0.67 & 68.5 & 1.86 & 0.59 & 60.7 & 1.76 & 0.45 & 61.8 & 2.01 & 0.66 & 60.4 & 2.06 & 0.60 & 56.7 & 2.08 & 0.57 & 53.8 \\
\hline 1.67 & 0.83 & 68.5 & 1.88 & 0.73 & 65.3 & 1.79 & 0.56 & 61.8 & 2.05 & 0.77 & 63.4 & 2.12 & 0.75 & 60.5 & 2.13 & 0.72 & 58.2 \\
\hline 1.78 & 0.94 & 73.1 & 1.90 & 0.90 & 70.3 & 1.83 & 0.75 & 67.3 & 2.09 & 0.94 & 67.1 & 2.16 & 0.87 & 63.1 & 2.18 & 0.84 & 60.7 \\
\hline 1.79 & 0.94 & 76.3 & 1.92 & 1.07 & 74.1 & 1.94 & 0.84 & 68.6 & 2.16 & 1.05 & 68.2 & 2.22 & 1.01 & 65.5 & 2.22 & 0.98 & 63.2 \\
\hline 1.83 & 1.11 & 76.3 & 2.02 & 1.21 & 75.1 & 1.97 & 1.01 & 72.3 & 2.23 & 1.21 & 70.1 & 2.30 & 1.13 & 66.1 & 2.26 & 1.11 & 65.2 \\
\hline 1.87 & 1.30 & 79.3 & 2.09 & 1.37 & 76.4 & 2.05 & 1.16 & $\mid 73.8$ & 2.23 & 1.21 & 70.1 & 2.35 & 1.27 & 67.9 & 2.33 & 1.23 & 66.1 \\
\hline 1.97 & 1.44 & 79.5 & 2.10 & 1.56 & 79.6 & 2.11 & 1.34 & 75.3 & 2.31 & 1.47 & 72.6 & 2.40 & 1.41 & 69.0 & 2.37 & 1.38 & 66.1 \\
\hline 2.01 & 1.62 & 81.8 & 2.14 & 1.72 & 80.9 & 2.18 & 1.47 & 75.9 & 2.33 & 1.47 & 72.6 & 2.40 & 1.57 & 69.0 & 2.46 & 1.46 & 69.6 \\
\hline 2.07 & 1.76 & 82.5 & 2.16 & 1.89 & 83.1 & 2.21 & 1.62 & 77.6 & 2.41 & 1.61 & 72.8 & 2.48 & 1.68 & 73.9 & 2.46 & 1.61 & 69.6 \\
\hline 2.10 & 1.92 & 82.5 & 2.22 & 1.97 & 82.3 & 2.22 & 1.80 & 80.1 & 2.44 & 1.75 & 74.2 & 2.52 & 1.68 & 73.9 & 2.59 & 1.77 & 69.6 \\
\hline 2.16 & 2.00 & 84.8 & 2.29 & 2.06 & 81.9 & 2.28 & 1.85 & 79.2 & 2.48 & 1.88 & 74.8 & 2.53 & 2.04 & 73.6 & 2.67 & 1.82 & 71.6 \\
\hline 2.17 & 2.12 & 84.8 & 2.32 & 2.15 & 81.9 & 2.33 & 1.86 & 78.5 & 2.49 & 1.99 & 75.9 & 2.58 & 2.04 & 73.4 & 2.70 & 2.08 & 71.6 \\
\hline 2.22 & 2.19 & 84.0 & 2.35 & 2.23 & 81.7 & 2.37 & 1.98 & 78.5 & 2.56 & 2.08 & 75.5 & 2.64 & 2.04 & 72.2 & 2.83 & 2.08 & 70.9 \\
\hline 2.26 & 2.27 & 84.0 & 2.44 & 2.29 & 80.6 & 2.41 & 2.08 & 78.7 & 2.65 & 2.12 & 74.9 & 2.69 & 2.21 & 72.1 & 2.83 & 2.38 & 70.4 \\
\hline 2.34 & 2.27 & 83.3 & 2.44 & 2.32 & 80.6 & 2.44 & 2.14 & 78.7 & 2.65 & 2.32 & 73.9 & 2.74 & 2.21 & 71.9 & 2.90 & 2.38 & 70.4 \\
\hline 2.37 & 2.62 & 81.7 & 2.49 & 2.43 & 80.3 & 2.47 & 2.14 & 78.2 & 2.76 & 2.35 & 73.8 & 2.84 & 2.28 & 71.0 & 2.98 & 2.53 & 69.5 \\
\hline 2.48 & 2.62 & 81.7 & 2.61 & 2.52 & 78.7 & 2.50 & 2.25 & 77.8 & 2.77 & 2.40 & 73.7 & 2.94 & 2.43 & 70.9 & 3.09 & 2.70 & 69.1 \\
\hline 2.66 & 2.81 & 78.7 & 2.65 & 2.68 & 78.4 & 2.53 & 2.25 & 77.3 & 2.86 & 2.50 & 72.8 & 3.04 & 2.79 & 70.3 & 3.17 & 2.88 & 68.9 \\
\hline 2.80 & 3.02 & 73.9 & 2.76 & 2.76 & 76.6 & 2.61 & 2.28 & 76.1 & 2.98 & 2.61 & 71.3 & 3.11 & 2.80 & 69.7 & 3.20 & 2.93 & 68.8 \\
\hline 2.98 & 3.08 & 66.0 & 2.84 & 2.89 & 75.2 & 2.68 & 2.30 & 75.4 & 3.11 & 2.77 & 69.7 & 3.20 & 2.95 & 68.8 & 3.32 & 3.06 & 67.3 \\
\hline 3.22 & 3.11 & 57.7 & 3.01 & 2.95 & 70.6 & 2.71 & 2.46 & 74.8 & 3.21 & 2.95 & 68.4 & 3.33 & 3.07 & 67.0 & 3.56 & 3.22 & 64.0 \\
\hline 3.43 & 3.25 & 51.7 & 3.27 & 2.96 & 62.0 & 2.80 & 2.58 & 74.3 & 3.34 & 3.04 & 65.3 & 3.48 & 3.18 & 64.3 & 3.69 & 3.44 & 63.2 \\
\hline 3.76 & 3.41 & 45.5 & 3.54 & 2.98 & 53.8 & 2.91 & 2.73 & 73.0 & 3.54 & 3.04 & 59.3 & 3.80 & 3.28 & 56.8 & 3.93 & 3.50 & 58.1 \\
\hline 4.09 & 3.66 & 40.5 & 3.82 & 3.12 & 48.0 & 3.04 & 2.83 & 70.8 & 3.72 & 3.14 & 54.4 & 3.95 & |3.31 & 53.0 & 4.12 & 3.58 & 54.0 \\
\hline 4.54 & 3.95 & 35.6 & 4.16 & 3.30 & 42.5 & 3.15 & 2.99 & 68.5 & 3.95 & 3.18 & 49.7 & 4.10 & |3.31 & 49.3 & 4.23 & 3.73 & 50.8 \\
\hline 5.18 & 4.28 & 29.5 & 4.54 & 3.55 & 37.4 & 3.31 & 3.00 & 64.3 & 4.37 & 3.18 & 43.0 & 4.35 & 3.42 & 45.3 & 4.35 & 3.73 & 47.6 \\
\hline 5.28 & 4.40 & 28.6 & 5.11 & 3.82 & 32.5 & 3.38 & 3.06 & 61.3 & 4.80 & 3.37 & 37.3 & 4.52 & 3.42 & 42.1 & 4.68 & 3.85 & 42.7 \\
\hline 5.36 & 4.48 & 28.0 & 5.53 & 4.11 & 29.2 & 3.51 & 3.07 & |57.1 & 5.27 & 3.65 & 32.6 & 4.76 & 3.49 & 39.1 & 4.88 & 3.85 & 39.4 \\
\hline 5.52 & 4.50 & 27.0 & 5.72 & 4.29 & 27.7 & 3.64 & 3.08 & 53.3 & 5.75 & 3.90 & 29.6 & 5.07 & 3.54 & 35.7 & 5.23 & 3.86 & 35.7 \\
\hline 5.61 & 4.60 & 26.5 & 6.01 & 4.38 & 26.0 & 3.82 & 3.10 & 49.5 & 6.13 & 3.95 & 27.5 & 5.40 & 3.61 & 32.7 & 5.56 & 3.94 & 32.7 \\
\hline & & & & & & 4.20 & 3.23 & 43.2 & 6.35 & 4.09 & 26.2 & 5.66 & 3.78 & 30.6 & 5.77 & 4.06 & 30.8 \\
\hline & & & & & & 4.63 & 3.45 & 37.7 & & & & 6.03 & 3.84 & 28.5 & 6.10 & 4.10 & 28.6 \\
\hline & & & & & & 5.19 & 3.70 & 32.6 & & & & 6.32 & 3.97 & 27.0 & 6.53 & 4.09 & 26.4 \\
\hline & & & & & & 5.49 & 4.07 & 30.3 & & & & & & & & & \\
\hline & & & & & & 5.89 & 4.11 & 27.9 & & & & & & & & & \\
\hline & & & & & & 6.05 & 4.30 & 26.8 & & & & & & & & & \\
\hline
\end{tabular}


$\phi=$ listed value $\times 10^{-1} \quad \psi=$ listed value $\times 10^{-1} \quad \eta_{\mathrm{ts}}=\%$

\begin{tabular}{|c|c|c|c|c|c|c|c|c|c|c|c|c|c|c|c|c|c|}
\hline \multirow{2}{*}{\multicolumn{3}{|c|}{$\begin{array}{l}78^{0} \text { continued } \\
\text { IGV flap }-45^{0}\end{array}$}} & \multicolumn{15}{|c|}{ Rotor tip stagger angle $=81^{0}$} \\
\hline & & & \multicolumn{3}{|c|}{ IGV flap $+45^{0}$} & \multicolumn{3}{|c|}{ IGV flap $+30^{0}$} & \multicolumn{3}{|c|}{ IGV flap $+20^{0}$} & \multicolumn{3}{|c|}{ IGV flap $+10^{0}$} & \multicolumn{3}{|c|}{ IGV flap $0^{0}$} \\
\hline$\phi$ & $\psi$ & $\eta_{\text {ts }}$ & $\phi$ & $\psi$ & $\eta_{\text {ts }}$ & $\phi$ & $\psi$ & $\eta_{\mathrm{ts}}$ & $\phi$ & $\psi$ & $\eta_{\text {ts }}$ & $\phi$ & $\psi$ & $\eta_{\mathrm{ts}}$ & $\phi$ & $\psi$ & $\eta_{\text {ts }}$ \\
\hline 1.93 & 0.16 & 24.8 & 0.96 & 0.06 & 16.6 & 1.00 & 0.05 & 15.3 & 1.07 & 0.05 & 15.8 & 1.11 & 0.06 & 16.8 & 1.15 & 0.06 & 16.7 \\
\hline 1.94 & 0.22 & 29.9 & 1.03 & 0.12 & 31.6 & 1.06 & 0.12 & 29.8 & 1.09 & 0.10 & 27.5 & 1.15 & 0.12 & 27.9 & 1.18 & 0.13 & 29.7 \\
\hline 1.99 & 0.32 & 37.5 & 1.03 & 0.19 & 41.7 & 1.12 & 0.19 & 40.9 & 1.16 & 0.23 & 47.4 & 1.15 & 0.21 & 42.3 & 1.21 & 0.21 & 40.8 \\
\hline 2.06 & 39 & 41.0 & 1.07 & 0.30 & 54.2 & 1.14 & 0.29 & 51.7 & 1.18 & 0.34 & 56.8 & 1.20 & 0.30 & 51.3 & 1.23 & 0.31 & 50.4 \\
\hline 2.06 & 53 & 47.9 & 1.08 & 0.38 & 60.4 & 1.14 & 0.47 & 64.8 & 1.24 & 0.42 & 61.7 & 1.25 & 0.42 & 59.1 & 1.31 & 0.40 & 56.3 \\
\hline 2.09 & .66 & 52.5 & 1.12 & 0.49 & 66.4 & 1.21 & 0.60 & 69.9 & 1.29 & 0.57 & 68.5 & 1.28 & 0.58 & 67.6 & 1.33 & 0.57 & 65.5 \\
\hline 2.19 & 0.76 & 54.3 & 1.16 & 0.64 & 72.3 & 1.24 & 0.75 & |75.2 & 1.36 & 0.70 & 71.7 & 1.34 & 0.72 & 71.6 & 1.42 & 0.71 & 68.7 \\
\hline 2.25 & 0.88 & 56.8 & 1.21 & 0.81 & 77.6 & 1.30 & 0.88 & $\mid 77.1$ & 1.36 & 0.88 & 77.9 & 1.43 & 0.86 & 73.7 & 1.44 & 0.88 & 74.0 \\
\hline 2.29 & 0.88 & 56.8 & 1.26 & 0.93 & 79.5 & 1.38 & 1.02 & 78.6 & 1.42 & 1.04 & 80.0 & 1.47 & 1.02 & 77.1 & 1.47 & 1.03 & 77.1 \\
\hline 2.32 & 1.25 & 64.2 & 1.33 & 1.09 & 81.2 & 1.39 & 1.21 & 82.9 & 1.45 & 1.20 & 83.0 & 1.52 & 1.17 & 79.1 & 1.50 & 1.20 & 80.2 \\
\hline 2.35 & 1.25 & 64.2 & 1.36 & 1.26 & 84.2 & 1.45 & 1.37 & 84.2 & 1.50 & 1.42 & 85.6 & 1.55 & 1.36 & 82.1 & 1.58 & 1.34 & 80.4 \\
\hline 2.35 & 1.25 & 64.2 & 1.41 & 1.41 & 85.4 & 1.48 & 1.58 & 87.0 & 1.55 & 1.59 & 85.6 & 1.60 & 1.52 & 83.3 & 1.65 & 1.48 & 80.8 \\
\hline 2.40 & 1.46 & 66.3 & 1.45 & 1.56 & 87.5 & 1.55 & 1.71 & 87.1 & 1.62 & 1.68 & 88.5 & 1.68 & 1.63 & 82.6 & 1.67 & 1.64 & 80.8 \\
\hline 2.43 & 1.46 & 66.3 & 1.50 & 1.73 & 88.3 & 1.59 & 1.86 & 88.1 & 1.65 & 1.88 & 88.5 & 1.77 & 1.73 & 82.6 & 1.74 & 1.78 & 83.5 \\
\hline 2.43 & 1.59 & 68.0 & 1.59 & 1.81 & 86.6 & 1.66 & 1.94 & 86.8 & 1.72 & 1.94 & 86.7 & 1.84 & 1.83 & 81.3 & 1.78 & 1.91 & 83.5 \\
\hline 2.46 & 1.59 & 68.0 & 1.63 & 1.94 & 86.3 & 1.73 & 2.01 & 86.1 & 1.80 & 2.00 & 86.4 & 1.85 & 1.98 & 80.9 & 1.83 & 2.01 & 83.2 \\
\hline 2.47 & 1.83 & 69.6 & 1.70 & 1.98 & 84.6 & 1.76 & 2.11 & 85.6 & 1.82 & 2.15 & 85.0 & 1.96 & 2.01 & 80.0 & 1.88 & 2.11 & 83.0 \\
\hline 2.50 & 1.83 & 69.6 & 1.84 & 1.98 & 80.2 & 1.81 & 2.20 & 85.5 & 1.92 & 2.16 & 83.3 & 2.05 & 2.06 & 79.1 & 1.97 & 2.17 & 82.1 \\
\hline 2.60 & 1.93 & 68.9 & 1.92 & 2.03 & 78.4 & 1.87 & 2.27 & 84.4 & 1.95 & 2.27 & 83.2 & 2.06 & 2.18 & 79.1 & 1.98 & 2.29 & 81.3 \\
\hline 2.67 & 2.04 & 68.6 & 2.03 & 2.05 & 77.1 & 1.94 & 2.27 & 84.3 & 2.00 & 2.36 & 82.9 & 2.12 & 2.38 & 79.1 & 2.08 & 2.32 & 79.6 \\
\hline 2.80 & 2.27 & 68.6 & 2.05 & 2.27 & 77.1 & 1.95 & 2.47 & 82.6 & 2.10 & 2.51 & 80.8 & 2.23 & 2.39 & 76.4 & 2.15 & 2.38 & 78.5 \\
\hline 2.88 & 2.42 & 68.3 & 2.07 & 2.38 & 77.3 & 2.05 & 2.47 & 81.0 & 2.18 & 2.58 & 78.8 & 2.27 & 2.48 & 75.7 & 2.19 & 2.48 & 78.0 \\
\hline 2.98 & 2.57 & 67.9 & 2.09 & 2.38 & 77.3 & 2.10 & 2.52 & 79.8 & 2.23 & 2.62 & 77.3 & 2.41 & 2.61 & 72.0 & 2.26 & 2.55 & 76.7 \\
\hline 3.06 & 2.72 & 67.7 & 2.12 & 2.65 & 75.7 & 2.15 & 2.59 & 78.3 & 2.33 & 2.62 & 74.0 & 2.55 & 2.61 & 66.4 & 2.32 & 2.63 & 75.5 \\
\hline 3.16 & 2.86 & 67.1 & 2.15 & 2.65 & 75.5 & 2.22 & 2.63 & 76.1 & 2.54 & 2.62 & 66.0 & 2.72 & 2.64 & 59.1 & 2.45 & 2.63 & 71.6 \\
\hline 3.31 & 3.09 & 65.9 & 2.24 & 2.66 & 72.2 & 2.30 & 2.65 & 72.9 & 2.75 & 2.62 & 58.1 & 2.90 & 2.64 & 52.8 & 2.64 & 2.63 & 64.4 \\
\hline 3.50 & 3.35 & 64.1 & 2.33 & 2.66 & 67.8 & 2.41 & 2.65 & 67.0 & 2.78 & 2.62 & 54.7 & 3.13 & 2.64 & 47.3 & 2.81 & 2.73 & 57.0 \\
\hline 3.71 & 3.60 & 62.0 & 2.55 & 2.66 & 59.2 & 2.60 & 2.67 & 59.2 & 3.03 & 2.63 & 48.3 & 3.36 & 2.76 & 42.8 & 3.03 & 2.73 & 50.5 \\
\hline 4.00 & 3.84 & 58.3 & 2.69 & 2.85 & 53.4 & 2.76 & 2.67 & 53.1 & 3.30 & 2.69 & 42.9 & 3.65 & 2.89 & 38.3 & 3.30 & 2.73 & 44.9 \\
\hline 4.42 & 4.34 & 52.1 & 2.93 & 2.85 & 46.9 & 2.95 & 2.71 & 47.8 & 3.53 & 2.83 & 39.2 & 4.06 & 2.99 & 33.6 & 3.70 & 2.75 & 39.0 \\
\hline 4.77 & 4.34 & 47.0 & 3.09 & 2.85 & 43.2 & 3.22 & 2.77 & 42.4 & 3.87 & 2.97 & 34.8 & 4.37 & 3.27 & 29.8 & 3.96 & 2.94 & 35.4 \\
\hline 5.17 & 4.64 & 41.0 & 3.32 & 3.01 & 38.9 & 3.40 & 2.97 & 39.0 & 4.28 & 3.13 & 29.8 & 4.87 & 3.54 & 25.5 & 4.29 & 3.14 & 31.8 \\
\hline 5.32 & 4.64 & 37.7 & 3.70 & 3.10 & 33.4 & 3.77 & 3.08 & 33.8 & 4.90 & 3.28 & 25.0 & 5.52 & 3.74 & 21.9 & 4.76 & 3.33 & 27.6 \\
\hline \multirow[t]{4}{*}{5.70} & 4.53 & 33.6 & 3.90 & 3.41 & 30.6 & 4.18 & 3.22 & 29.2 & 5.42 & 3.63 & 21.9 & 6.10 & 4.17 & 19.2 & 5.34 & 3.58 & 23.4 \\
\hline & & & 4.55 & 3.49 & 25.5 & 4.59 & 3.50 & 25.5 & 5.98 & 4.05 & 19.3 & & & & 5.89 & 3.86 & 20.6 \\
\hline & & & 4.94 & 3.93 & 22.8 & 5.18 & 3.81 & 22.0 & & & & & & & 6.22 & 4.04 & 19.3 \\
\hline & & & 5.53 & 4.18 & 20.0 & 5.90 & 4.10 & 18.9 & & & & & & & & & \\
\hline
\end{tabular}


$\phi=$ listed value $\times 10^{-1} \quad \psi=$ listed value $\times 10^{-1} \quad \eta_{\mathrm{ts}}=\%$

\begin{tabular}{|c|c|c|c|c|c|c|c|c|c|c|c|c|c|c|c|c|c|}
\hline \multicolumn{12}{|c|}{$81^{0}$ continued } & \multicolumn{6}{|c|}{ Rotor tip stagger angle $=84^{0}$} \\
\hline \multicolumn{3}{|c|}{ IGV flap $-10^{0}$} & \multicolumn{3}{|c|}{ IGV flap $-20^{0}$} & \multicolumn{3}{|c|}{ IGV flap $-30^{0}$} & \multicolumn{3}{|c|}{ IGV flap $-45^{0}$} & \multicolumn{3}{|c|}{ IGV flap $+45^{0}$} & \multicolumn{3}{|c|}{ IGV flap $+30^{0}$} \\
\hline$\phi$ & $\psi$ & $\eta_{\mathrm{ts}}$ & $\phi$ & $\psi$ & $\eta_{\text {ts }}$ & $\phi$ & $\psi$ & $\eta_{\mathrm{ts}}$ & $\phi$ & $\psi$ & $\eta_{\mathrm{ts}}$ & $\phi$ & $\psi$ & $\eta_{\mathrm{ts}}$ & $\phi$ & $\psi$ & $\eta_{\mathrm{ts}}$ \\
\hline 1.15 & 0.07 & \begin{tabular}{|l|l}
19.5 \\
\end{tabular} & 1.22 & 0.08 & 19.6 & 1.22 & 0.02 & 5.1 & \begin{tabular}{|l|}
1.27 \\
\end{tabular} & \begin{tabular}{|l|l|} 
\\
\end{tabular} & 4.9 & \begin{tabular}{|l|l}
0.78 \\
\end{tabular} & 0.05 & 21.3 & \begin{tabular}{|l|}
0.77 \\
\end{tabular} & 0.01 & 5.0 \\
\hline 1.20 & 0.15 & 32.5 & 1.24 & 0.15 & 31.9 & 1.25 & 0.09 & 21.2 & 1.31 & 0.10 & 19.6 & 0.79 & 0.09 & 35.2 & 0.81 & 09 & 38.1 \\
\hline 1.20 & 0.24 & 44.5 & 1.29 & 0.23 & 40.6 & 1.26 & 0.17 & 33.2 & 1.35 & 0.18 & 31.4 & 0.81 & 0.16 & 52.9 & 0.84 & 16 & 53.5 \\
\hline 1.24 & 0.33 & 52.8 & 1.31 & 0.32 & 49.1 & 1.37 & 0.31 & 45.5 & 1.37 & 0.28 & 40.8 & 0.84 & 0.25 & 64.2 & 0.86 & 28 & 68.7 \\
\hline 1.28 & 0.45 & 60.5 & 1.35 & 0.44 & 56.2 & 1.41 & 0.41 & 51.9 & 1.42 & 0.40 & 48.5 & 0.85 & 0.33 & 71.5 & 91 & 41 & 76.2 \\
\hline 1.39 & 0.58 & 64.5 & 1.41 & 0.58 & 62.7 & 1.51 & 0.53 & 56.9 & 1.44 & 0.50 & 54.1 & 0.89 & 0.45 & 78.3 & 96 & 55 & 76.2 \\
\hline 1.40 & 0.75 & 70.9 & 1.48 & 0.71 & 66.0 & 1.54 & 0.66 & 61.7 & 1.51 & 0.61 & 58.1 & 0.93 & 0.60 & 83.6 & 07 & .67 & 85.3 \\
\hline 1.45 & 0.90 & 74.1 & 1.52 & 0.86 & 69.3 & 1.61 & 0.81 & 64.7 & 1.57 & 0.70 & 60.2 & 0.96 & 0.76 & 88.1 & 09 & .84 & 85.3 \\
\hline 1.51 & 1.04 & 76.0 & 1.55 & 1.01 & 72.4 & 1.65 & 0.93 & 66.7 & 1.58 & 0.82 & 64.1 & 03 & 0.89 & 88.4 & 16 & .99 & 86.1 \\
\hline 1.53 & 1.20 & 76.0 & 1.59 & 1.16 & 75.1 & 1.72 & 0.93 & 66.7 & 1.65 & 0.91 & 65.0 & 10 & 1.04 & 89.0 & 20 & 1.17 & 88.0 \\
\hline 1.62 & 1.32 & 78.9 & 1.65 & 1.29 & 75.9 & 1.73 & 1.25 & 72.0 & 1.71 & 1.01 & 66.1 & 15 & 1.20 & 89.7 & 1.23 & .37 & 90.8 \\
\hline 1.68 & 1.46 & 78.9 & 1.72 & 1.42 & 76.2 & 1.76 & 1.25 & 72.0 & 1.75 & 1.11 & 67.5 & 1.19 & 1.40 & 92.2 & 1.28 & .55 & 92.1 \\
\hline 1.71 & 1.62 & 80.9 & 1.77 & 1.56 & 77.1 & 1.81 & 1.37 & 72.9 & 1.75 & 1.30 & 71.1 & 1.25 & 1.55 & 92.4 & 1.36 & .67 & 90.6 \\
\hline 1.74 & 1.78 & 82.5 & 1.79 & 1.73 & 79.3 & 1.87 & 1.60 & 72.9 & 1.78 & 1.46 & 71.1 & 1.27 & 1.72 & 94.6 & 1.46 & 1.72 & 88.0 \\
\hline 1.86 & 1.85 & 81.7 & 1.85 & 1.84 & 79.3 & 1.94 & 1.69 & 74.8 & 1.86 & 1.55 & 74.0 & 1.34 & 1.81 & 93.3 & 1.49 & 1.84 & 87.5 \\
\hline 1.88 & 1.97 & 81.5 & 1.89 & 1.93 & 79.9 & 2.02 & 1.80 & 75.3 & 1.88 & 1.69 & 74.0 & 1.41 & 1.87 & 90.8 & 1.55 & 1.92 & 87.5 \\
\hline 1.94 & 2.06 & 80.8 & 1.95 & 2.07 & 79.6 & 2.04 & 1.93 & 75.3 & 1.89 & 1.85 & 75.8 & 1.47 & 1.95 & 89.6 & 1.58 & 2.04 & 87.5 \\
\hline 1.95 & 2.20 & 80.3 & 2.02 & 2.15 & 78.8 & 2.08 & 2.06 & 76.0 & 1.98 & 1.92 & 75.5 & 1.57 & 1.97 & 86.4 & 1.62 & 2.16 & 87.5 \\
\hline 2.03 & 2.20 & 79.8 & 2.06 & 2.26 & 78.6 & 2.11 & 2.19 & 77.2 & 2.01 & 2.06 & 74.5 & 1.60 & 2.09 & 86.2 & 1.68 & 2.22 & 85.5 \\
\hline 2.06 & 2.45 & 79.7 & 2.12 & 2.33 & 77.8 & 2.17 & 2.19 & 76.7 & 2.09 & 2.13 & 74.5 & 1.63 & 2.20 & 85.8 & 1.72 & 2.33 & 85.4 \\
\hline 2.11 & 2.45 & 78.9 & 2.21 & 2.38 & 76.1 & 2.17 & 2.08 & 73.4 & 2.17 & 2.13 & 74.3 & 1.70 & 2.25 & 83.9 & 1.80 & 2.35 & 82.4 \\
\hline 2.24 & 2.45 & 77.6 & 2.27 & 2.46 & 75.5 & 2.21 & 2.08 & 73.4 & 2.19 & 2.23 & 72.7 & 1.76 & 2.32 & 82.4 & 1.87 & 2.39 & 80.0 \\
\hline 2.24 & 2.81 & 76.2 & 2.30 & 2.59 & 75.3 & 2.24 & 2.08 & 73.4 & 2.38 & 2.23 & 69.3 & 1.82 & 2.37 & 80.5 & 1.90 & 2.47 & 79.5 \\
\hline 2.35 & 2.81 & 75.7 & 2.47 & 2.75 & 72.6 & 2.38 & 2.36 & 72.5 & 2.44 & 2.35 & 69.3 & 1.86 & 2.37 & 79.2 & 1.96 & 2.49 & 76.9 \\
\hline 2.43 & 2.88 & 73.1 & 2.66 & 2.87 & 68.0 & 2.52 & 2.36 & 71.8 & 2.48 & 2.47 & 69.3 & 1.95 & 2.47 & 75.6 & 2.04 & 2.49 & 73.0 \\
\hline 2.59 & 2.88 & 67.3 & 2.77 & 2.87 & 63.8 & 2.56 & 2.67 & 70.3 & 2.59 & 2.73 & 69.3 & 2.07 & 2.47 & 70.3 & 2.11 & 2.49 & 69.3 \\
\hline 2.80 & 2.88 & 59.1 & 3.05 & 2.95 & 54.9 & 2.66 & 2.75 & 70.0 & 2.62 & 2.89 & 69.1 & 2.12 & 2.54 & 66.5 & 2.14 & 2.49 & 66.8 \\
\hline 3.06 & 2.89 & 50.7 & 3.28 & 2.99 & 48.1 & 2.77 & 2.91 & 68.3 & 2.77 & 3.22 & 68.0 & 2.21 & 2.54 & 61.1 & 2.33 & 2.49 & 58.0 \\
\hline 3.30 & 2.89 & 45.5 & 3.56 & 2.99 & 42.6 & 2.96 & 3.00 & 63.7 & 3.06 & 3.35 & 62.5 & 2.34 & 2.52 & 54.8 & 2.41 & 2.49 & 53.5 \\
\hline 3.67 & 2.95 & 39.8 & 3.87 & 2.99 & 38.0 & 3.19 & 3.09 & 56.1 & 3.32 & 3.57 & 56.8 & 2.54 & 2.51 & 48.2 & 2.60 & 2.49 & 47.9 \\
\hline 3.96 & 3.13 & 35.9 & 4.41 & 3.38 & 32.1 & 3.43 & 3.09 & 49.0 & 3.59 & 3.57 & 50.4 & 2.69 & 2.51 & 43.9 & 2.78 & 2.49 & 43.4 \\
\hline 4.42 & 3.29 & 31.2 & 4.78 & 3.38 & 28.6 & 3.81 & 3.12 & 41.7 & 4.02 & 3.75 & 40.5 & 2.90 & 2.58 & 39.3 & 3.01 & 2.55 & 38.9 \\
\hline 4.90 & 3.56 & 27.0 & 5.36 & 3.68 & 24.5 & 4.16 & 3.12 & 36.3 & 4.34 & 3.75 & 35.2 & 3.16 & 2.64 & 34.9 & 3.34 & 2.59 & 33.6 \\
\hline 5.39 & 3.71 & 23.7 & 5.79 & 3.83 & 22.1 & 4.54 & 3.26 & 31.9 & 4.99 & 3.78 & 29.0 & 3.40 & 2.78 & 31.4 & 3.56 & 2.77 & 30.3 \\
\hline 6.03 & 4.10 & 20.4 & 6.26 & 3.94 & 20.0 & 5.10 & 3.42 & 27.3 & 5.32 & 3.84 & 26.5 & 3.76 & 2.89 & 27.6 & 3.98 & 2.87 & 26.2 \\
\hline & & & & & & 5.62 & 3.43 & 24.3 & 5.76 & 4.04 & 23.7 & 4.17 & 3.04 & 24.1 & 4.36 & 3.11 & 23.2 \\
\hline & & & & & & 6.24 & 3.59 & 21.2 & 6.20 & 4.14 & 21.6 & 4.65 & 3.28 & 21.0 & 4.98 & 3.33 & 19.7 \\
\hline & & & & & & & & & & & & $\begin{array}{l}5.18 \\
5.48\end{array}$ & 3.52 & $\begin{array}{l}18.4 \\
17.1\end{array}$ & 5.58 & 3.65 & 17.0 \\
\hline
\end{tabular}


$\phi=$ listed value $\times 10^{-1} \quad \psi=$ listed value $\times 10^{-1} \quad \eta_{\mathrm{ts}}=\%$

\section{$84^{0}$ continued}

\begin{tabular}{|c|c|c|c|c|c|c|c|c|c|c|c|c|c|c|c|c|c|}
\hline \multicolumn{3}{|c|}{ IGV flap $+20^{0}$} & \multicolumn{3}{|c|}{ IGV flap $+10^{0}$} & \multicolumn{3}{|c|}{ IGV flap $0^{0}$} & \multicolumn{3}{|c|}{ IGV flap $-10^{0}$} & \multicolumn{3}{|c|}{ IGV flap $-20^{0}$} & \multicolumn{3}{|c|}{ IGV flap $-30^{0}$} \\
\hline$\phi$ & $\psi$ & $\eta_{\mathrm{ts}}$ & $\phi$ & $\psi$ & $\eta_{\mathrm{ts}}$ & $\phi$ & $\psi$ & $\eta_{\mathrm{ts}}$ & $\phi$ & $\psi$ & $\eta_{\mathrm{ts}}$ & $\phi$ & $\psi$ & $\eta_{\mathrm{ts}}$ & $\phi$ & $\psi$ & $\eta_{\mathrm{ts}}$ \\
\hline 0.85 & 0.03 & 14.5 & \begin{tabular}{|l|}
0.88 \\
\end{tabular} & \begin{tabular}{|l|l} 
\\
\end{tabular} & 17.4 & \begin{tabular}{|l|}
0.87 \\
\end{tabular} & 0.03 & 15.2 & 0.90 & 0.07 & 29.6 & 0.87 & 0.01 & 6.8 & 0.86 & 0.02 & \begin{tabular}{|l|l}
9.8 \\
\end{tabular} \\
\hline 0.88 & 0.08 & 34.4 & 0.92 & 0.11 & 40.9 & 0.90 & 0.07 & 32.0 & 0.91 & 0.12 & 42.9 & 0.92 & 0.08 & 31.0 & 0.90 & 0.08 & 30.3 \\
\hline 0.92 & 0.16 & 50.1 & 0.94 & 0.18 & 54.4 & 0.90 & 0.12 & 47.0 & 0.94 & 0.21 & 57.3 & 0.96 & 0.16 & 48.4 & 0.94 & 0.13 & 39.5 \\
\hline 0.98 & 0.24 & 59.8 & 0.96 & 0.26 & 64.3 & 0.94 & 0.22 & 61.5 & 0.97 & 0.32 & 67.8 & 1.00 & 0.26 & 59.5 & 0.96 & 0.25 & 56.2 \\
\hline 0.99 & 0.37 & 71.1 & 1.02 & 0.37 & 71.0 & 0.96 & 0.30 & 68.6 & 1.03 & 0.46 & 74.8 & 1.04 & 0.35 & 65.9 & 1.01 & 0.35 & 64.5 \\
\hline 1.02 & 0.52 & 78.3 & 1.03 & 0.54 & 80.0 & 1.04 & 0.44 & 75.0 & 1.08 & 0.61 & 79.2 & 1.06 & 0.47 & 72.2 & 1.07 & 0.46 & 68.7 \\
\hline 1.04 & 0.69 & 84.4 & 1.09 & 0.69 & 82.9 & 1.08 & 0.58 & 79.5 & 1.13 & 0.73 & 81.0 & 1.08 & 0.61 & 77.4 & 1.09 & 0.61 & 75.7 \\
\hline 1.07 & 0.85 & 88.5 & 1.14 & 0.89 & 86.6 & 1.14 & 0.75 & 82.3 & 1.16 & 0.90 & 84.6 & 1.15 & 0.74 & 77.4 & 1.13 & 0.75 & 78.6 \\
\hline 1.11 & 1.05 & 92.1 & 1.19 & 1.02 & 87.3 & 1.19 & 0.87 & 83.4 & 1.21 & 1.04 & 85.6 & 1.24 & 0.85 & 82.1 & 1.17 & 0.85 & 80.1 \\
\hline 1.19 & 1.23 & 92.1 & 1.23 & 1.19 & 88.8 & 1.21 & 1.04 & 86.7 & 1.26 & 1.20 & 85.6 & 1.24 & 1.02 & 82.1 & 1.22 & 0.97 & 81.2 \\
\hline 1.27 & 1.36 & 91.1 & 1.27 & 1.38 & 90.6 & 1.26 & 1.22 & 86.7 & 1.35 & 1.30 & 87.1 & 1.26 & 1.17 & 84.3 & 1.27 & 1.08 & 81.7 \\
\hline 1.32 & 1.54 & 91.1 & 1.32 & 1.56 & 91.6 & 1.33 & 1.33 & 88.0 & 1.36 & 1.49 & 87.1 & 1.30 & 1.28 & 85.5 & 1.31 & 1.19 & 82.4 \\
\hline 1.35 & 1.70 & 92.8 & 1.37 & 1.69 & 91.5 & 1.36 & 1.50 & 88.0 & 1.40 & 1.61 & 87.6 & 1.35 & 1.40 & 85.5 & 1.33 & 1.35 & 84.6 \\
\hline 1.41 & 1.81 & 91.8 & 1.43 & 1.81 & 90.6 & 1.37 & 1.69 & 90.7 & 1.46 & 1.76 & 87.7 & 1.37 & 1.55 & 87.3 & 1.38 & 1.50 & 84.9 \\
\hline 1.52 & 1.83 & 87.9 & 1.48 & 1.91 & 90.2 & 1.47 & 1.78 & 88.1 & 1.51 & 1.89 & 87.5 & 1.43 & 1.67 & 86.9 & 1.47 & 1.61 & 84.8 \\
\hline 1.56 & 1.96 & 87.5 & 1.58 & 1.94 & 87.4 & 1.53 & 1.86 & 87.5 & 1.57 & 1.98 & 86.4 & 1.49 & 1.77 & 86.6 & 1.49 & 1.77 & 84.1 \\
\hline 1.63 & 2.03 & 86.7 & 1.61 & 2.07 & 86.9 & 1.57 & 2.02 & 86.7 & 1.66 & 2.05 & 84.2 & 1.54 & 1.93 & 85.9 & 1.56 & 1.88 & 83.8 \\
\hline 1.69 & 2.10 & 85.1 & 1.69 & 2.11 & 85.2 & 1.63 & 2.09 & 86.4 & 1.73 & 2.12 & 82.9 & 1.63 & 2.01 & 85.7 & 1.61 & 1.99 & 83.2 \\
\hline 1.76 & 2.16 & 83.0 & 1.74 & 2.21 & 84.4 & 1.72 & 2.12 & 83.6 & 1.77 & 2.23 & 82.8 & 1.69 & 2.13 & 84.7 & 1.68 & 2.06 & 82.8 \\
\hline 1.83 & 2.16 & 82.4 & 1.81 & 2.26 & 82.4 & 1.77 & 2.22 & 82.9 & 1.80 & 2.34 & 82.7 & 1.71 & 2.25 & 84.6 & 1.78 & 2.11 & 81.7 \\
\hline 1.83 & 2.40 & 81.5 & 1.88 & 2.45 & 81.3 & 1.86 & 2.26 & 80.4 & 1.89 & 2.38 & 80.2 & 1.80 & 2.30 & 83.6 & 1.83 & 2.34 & 81.6 \\
\hline 1.90 & 2.40 & 80.1 & 1.97 & 2.46 & 78.0 & 1.89 & 2.37 & 80.1 & 1.92 & 2.38 & 80.0 & 1.89 & 2.37 & 81.8 & 1.87 & 2.34 & 80.3 \\
\hline 1.93 & 2.47 & 78.7 & 2.02 & 2.51 & 76.1 & 1.97 & 2.40 & 77.9 & 2.06 & 2.65 & 75.2 & 1.95 & 2.45 & 80.3 & 1.89 & 2.48 & 79.5 \\
\hline 2.00 & 2.47 & 75.8 & 2.05 & 2.56 & 74.3 & 2.04 & 2.57 & 75.9 & 2.09 & 2.65 & 75.0 & 2.02 & 2.54 & 78.2 & 1.96 & 2.48 & 78.4 \\
\hline 2.00 & 2.47 & 75.5 & 2.07 & 2.56 & 72.8 & 2.20 & 2.57 & 69.7 & 2.15 & 2.65 & 72.8 & 2.08 & 2.62 & 77.4 & 2.02 & 2.55 & 77.5 \\
\hline 2.03 & 2.47 & 73.3 & 2.17 & 2.55 & 68.4 & 2.32 & 2.56 & 63.8 & 2.31 & 2.65 & 67.0 & 2.13 & 2.71 & 76.1 & 2.13 & 2.71 & 76.5 \\
\hline 2.12 & 2.47 & 68.7 & 2.26 & 2.48 & 63.8 & 2.48 & 2.45 & 55.6 & 2.37 & 2.59 & 62.7 & 2.18 & 2.71 & 74.0 & 2.20 & 2.81 & 75.2 \\
\hline 2.19 & 2.47 & 65.1 & 2.37 & 2.38 & 58.9 & 2.69 & 2.37 & 49.0 & 2.44 & 2.59 & 58.6 & 2.28 & 2.71 & 70.2 & 2.32 & 2.85 & 71.9 \\
\hline 2.28 & 2.40 & 60.8 & 2.42 & 2.38 & 56.2 & 2.91 & 2.37 & 44.1 & 2.57 & 2.49 & 53.6 & 2.35 & 2.71 & 66.9 & 2.43 & 2.85 & 68.1 \\
\hline 2.35 & 2.38 & 57.9 & 2.57 & 2.38 & 50.9 & 3.12 & 2.44 & 40.3 & 2.75 & 2.47 & 47.9 & 2.49 & 2.71 & 60.2 & 2.54 & 2.85 & 63.2 \\
\hline 2.43 & 2.36 & 54.6 & 2.75 & 2.38 & 46.5 & 3.45 & 2.47 & 35.7 & 2.90 & 2.47 & 44.3 & 2.74 & 2.63 & 51.0 & 2.69 & 2.85 & 55.7 \\
\hline 2.49 & 2.36 & 52.4 & 2.96 & 2.42 & 42.1 & 3.69 & 2.62 & 32.6 & 3.11 & 2.53 & 40.4 & 2.95 & 2.63 & 45.2 & 2.92 & 2.82 & 48.5 \\
\hline 2.52 & 2.40 & 50.9 & 3.12 & 2.54 & 39.2 & 4.05 & 2.77 & 28.5 & 3.40 & 2.59 & 36.4 & 3.17 & 2.63 & 41.1 & 3.12 & 2.82 & 43.2 \\
\hline 2.70 & 2.42 & 46.3 & 3.50 & 2.56 & 34.1 & 4.42 & 2.94 & 25.0 & 3.75 & 2.64 & 32.1 & 3.51 & 2.67 & 36.3 & 3.45 & 2.83 & 37.8 \\
\hline 2.94 & 2.44 & 41.6 & 3.95 & 2.61 & 28.8 & 5.08 & 3.08 & 20.9 & 4.09 & 2.76 & 28.5 & 3.81 & 2.77 & 32.3 & 3.79 & 2.88 & 33.2 \\
\hline 3.16 & 2.53 & 37.7 & 4.21 & 2.84 & 26.0 & 5.53 & 3.27 & 18.6 & 4.54 & 2.86 & 24.6 & 4.28 & 2.82 & 27.6 & 4.09 & 3.03 & 29.6 \\
\hline 3.46 & 2.63 & 33.1 & 4.80 & 2.95 & 21.9 & 6.21 & 3.45 & 16.1 & 5.02 & 3.06 & 21.3 & 4.81 & 2.97 & 23.5 & 4.81 & 3.03 & 24.0 \\
\hline 3.85 & 2.69 & 28.5 & 5.41 & 3.20 & 18.8 & & & & 5.70 & 3.29 & 18.1 & 5.36 & 3.22 & 20.1 & 5.34 & 3.30 & 20.6 \\
\hline 4.27 & 2.82 & 24.9 & 6.08 & 3.41 & 16.3 & & & & 6.26 & 3.45 & 16.0 & 6.04 & 3.42 & 17.0 & 5.90 & 3.44 & 17.9 \\
\hline 4.73 & 3.02 & 21.7 & & & & & & & & & & & & & 6.23 & 3.61 & 16.6 \\
\hline 5.4 & 3.24 & 18.4 & & & & & & & & & & & & & & & \\
\hline .97 & 0 & 16.3 & & & & & & & & & & & & & & & \\
\hline
\end{tabular}


$\phi=$ listed value $\times 10^{-1} \quad \psi=$ listed value $\times 10^{-1} \quad \eta_{\mathrm{ts}}=\%$

\begin{tabular}{|c|c|c|c|c|c|c|c|c|c|c|c|c|c|c|c|c|c|}
\hline \multirow{2}{*}{\multicolumn{3}{|c|}{\begin{tabular}{|c|}
$84^{0}$ continued \\
IGV flap $-45^{0}$
\end{tabular}}} & \multicolumn{15}{|c|}{ Rotor tip stagger angle $=87^{0}$} \\
\hline & & & \multicolumn{3}{|c|}{ IGV flap $+45^{0}$} & \multicolumn{3}{|c|}{ IGV flap $+30^{0}$} & \multicolumn{3}{|c|}{ IGV flap $+20^{0}$} & \multicolumn{3}{|c|}{ IGV flap $+10^{0}$} & \multicolumn{3}{|c|}{ IGV flap $0^{0}$} \\
\hline$\phi$ & $\psi$ & $\eta_{\text {ts }}$ & $\phi$ & $\psi$ & $\eta_{\text {ts }}$ & $\phi$ & $\psi$ & $\eta_{\mathrm{ts}}$ & $\phi$ & $\psi$ & $\eta_{\text {ts }}$ & $\phi$ & $\psi$ & $\eta_{\mathrm{ts}}$ & $\phi$ & $\psi$ & $\eta_{\mathrm{ts}}$ \\
\hline 0.89 & 0.01 & 3.7 & 0.46 & 0.04 & 15.2 & 0.41 & 0.06 & 19.6 & 0.47 & 0.16 & 45.9 & 0.48 & 0.11 & 30.3 & 0.48 & 0.01 & 4.7 \\
\hline 0.93 & 0.07 & 22.5 & 0.46 & 0.06 & 23.8 & 0.48 & 0.12 & 35.7 & 0.47 & 0.21 & 53.1 & 0.52 & 0.17 & 44.2 & 0.51 & 0.07 & 19.8 \\
\hline 0.93 & 0.16 & 40.0 & 0.47 & 0.15 & 45.7 & 0.52 & 0.23 & 54.7 & 0.53 & 0.35 & 68.1 & 0.58 & 0.33 & 63.1 & 0.54 & 0.13 & 32.4 \\
\hline 0.98 & 0.22 & 47.8 & 0.49 & 0.23 & 59.5 & 0.55 & 0.31 & 62.9 & 0.59 & 0.42 & 71.7 & 0.60 & 0.42 & 71.0 & 0.56 & 0.20 & 44.1 \\
\hline 1.02 & 0.29 & 54.3 & 0.53 & 0.28 & 64.0 & 0.56 & 0.45 & 75.5 & 0.65 & 0.47 & 73.0 & 0.64 & 0.56 & 77.7 & 0.57 & 0.28 & 53.2 \\
\hline 1.05 & 0.34 & 57.8 & 0.56 & 0.35 & 69.9 & 0.59 & 0.51 & 78.4 & 0.70 & 0.62 & 78.9 & 0.71 & 0.74 & 83.6 & 0.62 & 0.34 & 57.6 \\
\hline 1.06 & 0.42 & 62.8 & 0.59 & 0.38 & 71.1 & 0.64 & 0.65 & 84.8 & 0.72 & 0.79 & 85.5 & 0.75 & 0.92 & 83.6 & 0.67 & 0.47 & 66.4 \\
\hline 1.12 & 0.52 & 66.0 & 0.61 & 0.67 & 89.5 & 0.71 & 0.80 & 86.5 & 0.77 & 1.07 & 93.1 & 0.81 & 1.02 & 83.6 & 0.72 & 0.58 & 70.8 \\
\hline 1.13 & 0.66 & 72.1 & 0.65 & 0.77 & 91.0 & 0.76 & 0.96 & 89.8 & 0.86 & 1.21 & 91.2 & 0.85 & 1.21 & 83.6 & 0.75 & 0.71 & 76.7 \\
\hline 1.15 & 0.78 & 75.5 & 0.68 & 0.85 & 91.6 & 0.82 & 1.23 & 93.9 & 0.89 & 1.30 & 91.1 & 0.90 & 1.30 & 89.8 & 0.79 & 0.86 & 80.2 \\
\hline 1.18 & 0.91 & 78.2 & 0.74 & 1.00 & 92.6 & 0.86 & 1.39 & 95.0 & 0.97 & 1.47 & 90.8 & 0.98 & 1.44 & 89.8 & 0.87 & 1.09 & 84.7 \\
\hline 1.25 & 1.06 & 78.8 & 0.80 & 1.21 & 95.0 & 0.94 & 1.61 & 95.0 & 1.05 & 1.64 & 90.7 & 1.03 & 1.60 & 89.8 & 0.92 & 1.25 & 84.7 \\
\hline 1.31 & 1.17 & 78.8 & 0.83 & 1.49 & 95.0 & 0.96 & 1.74 & 95.0 & 1.09 & 1.77 & 89.9 & 1.07 & 1.79 & 91.2 & 0.99 & 1.37 & 86.1 \\
\hline 1.39 & 1.28 & 78.8 & 0.89 & 1.60 & 95.0 & 1.05 & 1.79 & 93.4 & 1.18 & 1.87 & 88.7 & 1.12 & 1.95 & 92.1 & 1.04 & 1.51 & 86.1 \\
\hline 1.49 & 1.39 & 79.3 & 0.99 & 1.71 & 95.0 & 1.13 & 1.89 & 91.7 & 1.21 & 1.95 & 88.5 & 1.19 & 1.97 & 89.4 & 1.06 & 1.71 & 89.4 \\
\hline 1.51 & 1.53 & 79.3 & 1.03 & 1.83 & 95.0 & 1.17 & 1.99 & 91.3 & 1.27 & 2.08 & 88.2 & 1.26 & 2.12 & 88.9 & 1.14 & 1.75 & 86.2 \\
\hline 1.60 & 1.61 & 79.3 & 1.07 & 1.92 & 95.0 & 1.26 & 2.03 & 88.4 & 1.32 & 2.14 & 86.8 & 1.34 & 2.15 & 85.8 & 1.24 & 1.80 & 85.3 \\
\hline 1.60 & 1.78 & 79.3 & 1.12 & 1.92 & 95.0 & 1.30 & 2.16 & 88.3 & 1.42 & 2.20 & 83.7 & 1.41 & 2.21 & 83.8 & 1.30 & 2.06 & 85.0 \\
\hline 1.67 & 1.88 & 79.3 & 1.22 & 2.17 & 91.3 & 1.37 & 2.21 & 86.0 & 1.47 & 2.20 & 81.9 & 1.51 & 2.21 & 80.9 & 1.35 & 2.17 & 82.9 \\
\hline 1.70 & 1.99 & 79.3 & 1.28 & 2.17 & 90.0 & 1.41 & 2.30 & 85.0 & 1.58 & 2.20 & 76.6 & 1.53 & 2.21 & 78.7 & 1.44 & 2.20 & 81.8 \\
\hline 1.75 & 2.10 & 79.3 & 1.34 & 2.24 & 87.6 & 1.46 & 2.37 & |83.0 & 1.61 & 2.20 & 75.6 & 1.58 & 2.21 & 77.2 & 1.50 & 2.27 & 80.2 \\
\hline 1.79 & 2.21 & 79.3 & 1.41 & 2.27 & 84.9 & 1.55 & 2.37 & $\mid 78.7$ & 1.67 & 2.20 & 72.2 & 1.61 & 2.21 & 75.6 & 1.56 & 2.27 & 78.5 \\
\hline 1.84 & 2.29 & 78.8 & 1.46 & 2.27 & 83.1 & 1.62 & 2.37 & 74.6 & 1.69 & 2.20 & 69.9 & 1.68 & 2.21 & 71.3 & 1.56 & 2.42 & 77.9 \\
\hline 1.88 & 2.38 & 78.5 & 1.56 & 2.38 & 78.4 & 1.65 & 2.36 & 72.5 & 1.78 & 2.20 & 64.9 & 1.75 & 2.21 & 66.6 & 1.66 & 2.42 & 73.9 \\
\hline 1.96 & 2.44 & 76.9 & 1.59 & 2.38 & 77.0 & 1.71 & 2.33 & 68.8 & 1.87 & 2.16 & 60.3 & 1.84 & 2.20 & 62.3 & 1.72 & 2.42 & 70.5 \\
\hline 2.04 & 2.48 & 75.1 & 1.61 & 2.38 & 75.4 & 1.78 & 2.28 & 64.5 & 1.97 & 2.08 & 54.3 & 1.92 & 2.18 & 55.8 & 1.75 & 2.42 & 67.9 \\
\hline 2.10 & 2.56 & 74.8 & 1.67 & 2.38 & 71.8 & 1.84 & 2.24 & 61.3 & 2.05 & 2.06 & 50.0 & 2.08 & 2.13 & 49.1 & 1.84 & 2.34 & 63.1 \\
\hline 2.15 & 2.65 & 74.3 & 1.73 & 2.38 & 68.4 & 1.88 & 2.21 & 58.7 & 2.15 & 2.06 & 46.5 & 2.15 & 2.13 & 46.7 & 1.92 & 2.25 & 58.6 \\
\hline 2.16 & 2.81 & 73.7 & 1.79 & 2.35 & 64.5 & 1.95 & 2.16 & 55.4 & 2.35 & 2.04 & 41.8 & 2.20 & 2.13 & 44.7 & 1.98 & 2.18 & 54.6 \\
\hline 2.28 & 2.86 & 72.2 & 1.87 & 2.31 & 59.1 & 2.02 & 2.16 & 51.9 & 2.39 & 2.04 & 40.2 & 2.42 & 2.13 & 40.0 & 2.06 & 2.12 & 51.0 \\
\hline 2.47 & 2.97 & 67.9 & 1.93 & 2.26 & 55.8 & 2.10 & 2.16 & 47.8 & 2.54 & 2.06 & 37.0 & 2.53 & 2.13 & 37.6 & 2.16 & 2.09 & 46.4 \\
\hline 2.58 & 3.20 & 65.2 & 2.06 & 2.17 & 50.9 & 2.26 & 2.16 & 43.2 & 2.64 & 2.11 & 34.8 & 2.76 & 2.18 & 33.5 & 2.36 & 2.05 & 41.6 \\
\hline 2.87 & 3.20 & 55.9 & 2.12 & 2.15 & 47.3 & 2.45 & 2.16 & 38.6 & 2.87 & 2.15 & 31.0 & 3.04 & 2.18 & 29.7 & 2.56 & 2.05 & 37.8 \\
\hline 3.13 & 3.18 & 46.5 & 2.27 & 2.15 & 42.3 & 2.59 & 2.16 & 35.3 & 3.16 & 2.18 & 27.5 & 3.29 & 2.28 & 26.4 & 2.79 & 2.07 & 34.0 \\
\hline 3.39 & 3.17 & 40.2 & 2.43 & 2.14 & 38.1 & 2.83 & 2.19 & 31.2 & 3.48 & 2.23 & 24.1 & 3.58 & 2.35 & 23.6 & 2.97 & 2.16 & 31.2 \\
\hline 3.77 & 3.15 & 34.5 & 2.63 & 2.14 & 34.2 & 3.12 & 2.20 & 27.6 & 3.78 & 2.33 & 21.4 & 3.95 & 2.46 & 20.6 & 3.26 & 2.20 & 27.5 \\
\hline 4.23 & 3.15 & 29.4 & 2.76 & 2.25 & 32.2 & 3.48 & 2.27 & 23.9 & 4.10 & 2.47 & 19.3 & 4.44 & 2.60 & 17.7 & 3.55 & 2.31 & 24.4 \\
\hline 4.71 & 3.25 & 25.4 & 2.91 & 2.25 & 29.7 & 3.65 & 2.48 & 22.0 & 4.68 & 2.59 & 16.4 & 5.02 & 2.85 & 15.3 & 3.96 & 2.36 & 21.1 \\
\hline 5.49 & 3.34 & 20.7 & 3.18 & 2.31 & 26.4 & 4.14 & 2.56 & 18.9 & 5.26 & 2.83 & 14.3 & 5.36 & 3.18 & 14.0 & 4.35 & 2.53 & 18.4 \\
\hline 6.06 & 3.58 & 17.7 & 3.46 & 2.38 & 23.7 & 4.87 & 2.65 & 15.6 & 5.50 & 3.11 & 13.3 & & & & 5.03 & 2.67 & 15.5 \\
\hline & & & 3.71 & 2.56 & 21.5 & 5.15 & 2.89 & 14.3 & & & & & & & 5.46 & 2.87 & 13.9 \\
\hline & & & 4.20 & 2.67 & 18.4 & 5.36 & 3.16 & 13.4 & & & & & & & 5.62 & 3.10 & 13.3 \\
\hline & & & 4.80 & 2.85 & 15.7 & & & & & & & & & & & & \\
\hline & & & $\begin{array}{l}5.10 \\
5.31\end{array}$ & $\begin{array}{l}3.00 \\
3.19\end{array}$ & $\begin{array}{l}14.5 \\
13.7\end{array}$ & & & & & & & & & & & & \\
\hline
\end{tabular}


$\phi=$ listed value $\times 10^{-1}$

$\psi=$ listed value $\times 10^{-1}$

$\eta_{\mathrm{ts}}=\%$

\begin{tabular}{|c|c|c|c|c|c|c|c|c|c|c|c|c|c|c|c|c|c|}
\hline \multicolumn{12}{|c|}{$87^{0}$ continued } & \multicolumn{6}{|c|}{ Rotor tip stagger angle $=90^{\circ}$} \\
\hline \multicolumn{3}{|c|}{ IGV flap $-10^{0}$} & \multicolumn{3}{|c|}{ IGV flap $-20^{0}$} & \multicolumn{3}{|c|}{ IGV flap $-30^{0}$} & \multicolumn{3}{|c|}{ IGV flap $-45^{0}$} & \multicolumn{3}{|c|}{ IGV flap $+45^{0}$} & \multicolumn{3}{|c|}{ IGV flap $+30^{0}$} \\
\hline$\phi$ & $\psi$ & $\eta_{\mathrm{ts}}$ & $\phi$ & $\psi$ & $\eta_{\text {ts }}$ & $\phi$ & $\psi$ & $\eta_{\mathrm{ts}}$ & $\phi$ & $\psi$ & $\eta_{\mathrm{ts}}$ & $\phi$ & $\psi$ & $\eta_{\mathrm{ts}}$ & $\phi$ & $\psi$ & $\eta_{\mathrm{ts}}$ \\
\hline 0.53 & 0.05 & 16.4 & 0.53 & 0.06 & 16.9 & 0.52 & 0.06 & 15.3 & 0.56 & \begin{tabular}{|l|l|}
0.09 \\
\end{tabular} & 19.2 & 0.26 & 0.23 & 33.9 & 0.25 & 0.20 & 33.8 \\
\hline 0.54 & 0.10 & 26.0 & 0.53 & 0.15 & 34.9 & 0.56 & 0.15 & |32.1 & 0.56 & 0.15 & 29.3 & 0.29 & 0.32 & 42.1 & 0.27 & 0.23 & 35.8 \\
\hline 0.56 & 0.16 & 37.2 & 0.58 & 0.24 & 45.8 & 0.57 & 0.25 & 44.5 & 0.62 & 0.23 & 39.9 & 0.31 & 0.32 & 42.3 & 0.29 & 0.28 & 41.5 \\
\hline 0.62 & 0.28 & 52.5 & 0.63 & 0.32 & 53.6 & 0.61 & 0.35 & 54.3 & 0.63 & 0.31 & 47.4 & 0.33 & 0.40 & 50.0 & 0.31 & 35 & 48.3 \\
\hline 0.66 & 0.39 & 61.2 & 0.66 & 0.44 & 62.5 & 0.65 & 0.39 & 56.2 & 0.66 & 0.40 & 54.9 & 0.36 & 0.47 & 54.0 & 0.32 & 0.43 & 54.8 \\
\hline 0.69 & 0.46 & 65.0 & 0.70 & 0.53 & 67.1 & 0.70 & 0.47 & 60.3 & 0.67 & 0.48 & 60.2 & 0.38 & 0.54 & 59.2 & 0.33 & 0.52 & 60.6 \\
\hline 0.72 & 0.58 & 71.3 & 0.72 & 0.60 & 70.0 & 0.73 & 0.56 & 64.8 & 0.72 & 0.56 & 62.9 & 0.39 & 0.65 & 65.9 & 38 & 0.58 & 62.9 \\
\hline 0.74 & 0.66 & 73.9 & 0.76 & 0.71 & 73.2 & 0.74 & 0.69 & 71.6 & 0.78 & 0.63 & 64.1 & 0.40 & 0.76 & 73.2 & 42 & 0.66 & 65.5 \\
\hline 0.77 & 0.80 & 78.8 & 0.79 & 0.89 & 79.7 & 0.79 & 0.80 & 73.7 & 0.79 & 0.76 & 69.9 & 0.42 & 0.85 & 77.4 & 43 & 0.81 & 73.9 \\
\hline 0.81 & 0.95 & 82.2 & 0.80 & 0.93 & 81.2 & 0.83 & 0.88 & 76.3 & 0.81 & 0.84 & 73.2 & 0.45 & 0.96 & 80.3 & 47 & 0.95 & 78.2 \\
\hline 0.88 & 1.09 & 82.2 & 0.90 & 1.20 & 81.2 & 0.86 & 1.00 & 78.2 & 0.86 & 1.01 & 73.2 & 0.49 & 1.08 & 83.2 & 54 & 1.08 & 79.1 \\
\hline 0.95 & 1.19 & 84.8 & 0.96 & 1.30 & 85.2 & 0.89 & 1.20 & 78.2 & 0.93 & 1.11 & 80.0 & 0.55 & 1.24 & 85.7 & 57 & 1.34 & 79.1 \\
\hline 0.99 & 1.36 & 84.8 & 1.01 & 1.45 & 85.2 & 0.97 & 1.29 & 81.6 & 0.95 & 1.28 & 80.0 & 0.58 & 1.44 & 90.4 & 65 & 1.47 & 90.5 \\
\hline 1.03 & 1.49 & 85.3 & 1.05 & 1.63 & 87.3 & 1.05 & 1.39 & 83.8 & 1.00 & 1.42 & 80.9 & 0.67 & 1.51 & 90.0 & 70 & 1.64 & 90.5 \\
\hline 1.05 & 1.66 & 85.3 & 1.07 & 1.80 & 89.7 & 1.06 & 1.58 & 83.8 & 1.05 & 1.56 & 80.9 & 0.73 & 1.66 & 89.9 & 0.78 & 1.73 & 89.4 \\
\hline 1.11 & 1.79 & 88.9 & 1.12 & 1.94 & 90.2 & 1.10 & 1.73 & 83.8 & 1.15 & 1.63 & 80.7 & 0.79 & 1.77 & 88.7 & 0.84 & 1.84 & 88.9 \\
\hline 1.16 & 1.92 & 88.9 & 1.22 & 1.98 & 87.4 & 1.17 & 1.84 & 86.0 & 1.20 & 1.74 & 80.7 & 0.86 & 1.85 & 88.6 & 0.92 & 1.92 & 87.2 \\
\hline 1.23 & 2.01 & 87.6 & 1.26 & 2.11 & 86.3 & 1.20 & 1.98 & 86.0 & 1.24 & 1.88 & 80.7 & 0.92 & 1.96 & 87.8 & 0.97 & 2.02 & 87.0 \\
\hline 1.28 & 2.15 & 87.5 & 1.37 & 2.25 & 85.4 & 1.29 & 2.03 & 83.1 & 1.27 & 2.04 & 82.3 & 1.00 & 2.06 & 86.4 & 1.08 & 2.07 & 82.7 \\
\hline 1.38 & 2.18 & 84.0 & 1.47 & 2.27 & 82.0 & 1.35 & 2.13 & 82.9 & 1.33 & 2.11 & 81.5 & 1.08 & 2.19 & 84.7 & 1.19 & 2.18 & 78.6 \\
\hline 1.46 & 2.23 & 81.4 & 1.55 & 2.34 & 79.9 & 1.40 & 2.23 & 82.5 & 1.42 & 2.31 & 81.3 & 1.15 & 2.29 & 81.8 & 1.25 & 2.18 & 74.8 \\
\hline 1.57 & 2.37 & 78.5 & 1.60 & 2.40 & 78.7 & 1.50 & 2.23 & 81.2 & 1.51 & 2.32 & 78.6 & 1.24 & 2.29 & 76.4 & 1.33 & 2.17 & 68.7 \\
\hline 1.60 & 2.37 & 77.5 & 1.66 & 2.40 & 77.1 & 1.51 & 2.45 & 79.2 & 1.54 & 2.44 & 78.4 & 1.34 & 2.23 & 69.7 & 1.41 & 2.08 & 63.1 \\
\hline 1.70 & 2.44 & 72.9 & 1.75 & 2.40 & 72.8 & 1.57 & 2.45 & 78.5 & 1.58 & 2.54 & 78.0 & 1.43 & 2.13 & 63.3 & 1.44 & 2.04 & 60.9 \\
\hline 1.76 & 2.44 & 69.6 & 1.80 & 2.40 & 69.3 & 1.62 & 2.51 & 77.5 & 1.64 & 2.61 & 76.9 & 1.46 & 2.10 & 59.0 & 1.48 & 1.96 & 56.7 \\
\hline 1.82 & 2.43 & 65.1 & 1.87 & 2.40 & 65.3 & 1.66 & 2.59 & 76.8 & 1.66 & 2.61 & 76.9 & 1.56 & 1.98 & 53.6 & 1.55 & 1.89 & 51.8 \\
\hline 1.91 & 2.36 & 60.4 & 1.94 & 2.40 & 59.6 & 1.71 & 2.66 & 75.5 & 1.77 & 2.76 & 72.9 & 1.69 & 1.85 & 47.5 & 1.63 & 1.81 & 47.7 \\
\hline 1.99 & 2.27 & 56.0 & 2.10 & 2.31 & 51.6 & 1.82 & 2.66 & 71.2 & 1.83 & 2.76 & 71.2 & 1.74 & 1.85 & 44.0 & 1.71 & 1.77 & 44.4 \\
\hline 2.04 & 2.22 & 52.6 & 2.30 & 2.19 & 44.4 & 1.91 & 2.65 & 66.5 & 1.90 & 2.79 & 68.9 & 1.87 & 1.78 & 39.8 & 1.80 & 1.77 & 41.2 \\
\hline 2.20 & 2.13 & 45.8 & 2.50 & 2.18 & 39.5 & 2.02 & 2.62 & 58.7 & 2.09 & 2.79 & 61.9 & 1.94 & 1.78 & 37.0 & 1.88 & 1.76 & 38.6 \\
\hline 2.34 & 2.13 & 41.9 & 2.76 & 2.18 & 34.9 & 2.17 & 2.49 & 51.1 & 2.25 & 2.77 & 53.7 & 2.07 & 1.76 & 33.8 & 1.97 & 1.76 & 35.9 \\
\hline 2.52 & 2.13 & 38.1 & 2.98 & 2.18 & 31.8 & 2.34 & 2.40 & 44.9 & 2.45 & 2.66 & 45.2 & 2.23 & 1.75 & 30.2 & 2.11 & 1.76 & 32.8 \\
\hline 2.76 & 2.14 & 34.3 & 3.24 & 2.29 & 28.7 & 2.53 & 2.35 & 39.8 & 2.66 & 2.63 & 39.3 & 2.34 & 1.75 & 28.2 & 2.21 & 1.76 & 30.5 \\
\hline 2.95 & 2.23 & 31.4 & 3.59 & 2.32 & 24.9 & 2.78 & 2.35 & 35.0 & 2.87 & 2.63 & 35.0 & 2.59 & 1.75 & 24.8 & 2.43 & 1.76 & 27.0 \\
\hline 3.27 & 2.27 & 27.6 & 3.92 & 2.43 & 21.8 & 3.05 & 2.35 & 31.1 & 3.28 & 2.63 & 29.3 & 2.89 & 1.84 & 21.7 & 2.53 & 1.76 & 25.4 \\
\hline 3.67 & 2.29 & 23.8 & 4.46 & 2.49 & 18.5 & 3.37 & 2.38 & 27.2 & 3.50 & 2.63 & 26.4 & 3.02 & 1.84 & 20.2 & 2.85 & 1.76 & 21.9 \\
\hline 4.03 & 2.39 & 20.8 & 5.09 & 2.65 & 15.6 & 3.70 & 2.44 & 23.6 & 4.03 & 2.63 & 21.8 & 3.33 & 1.89 & 17.8 & 3.15 & 1.76 & 19.2 \\
\hline 4.46 & 2.54 & 18.1 & 5.51 & 2.85 & 13.8 & 4.08 & 2.55 & 20.6 & 4.51 & 2.63 & 18.6 & 3.73 & 1.93 & 15.4 & 3.42 & 1.82 & 17.2 \\
\hline 5.05 & 2.71 & 15.5 & 5.61 & 3.11 & 13.4 & 4.66 & 2.67 & 17.3 & 5.11 & 2.77 & 15.6 & 4.25 & 1.98 & 13.2 & 3.85 & 1.86 & 14.8 \\
\hline 5.53 & 2.88 & 13.7 & & & & 5.09 & 2.79 & 15.4 & 5.41 & 2.92 & 14.3 & 4.83 & 2.11 & 11.3 & 4.29 & 1.95 & 12.9 \\
\hline 5.84 & 3.04 & 12.7 & & & & 5.45 & 3.23 & 13.7 & 5.84 & 3.11 & 12.8 & 5.27 & 2.24 & 10.2 & 4.95 & 2.05 & 10.9 \\
\hline & & & & & & & & & & & & 5.66 & 2.31 & 9.3 & 5.58 & 2.28 & 9.3 \\
\hline
\end{tabular}


$\phi=$ listed value $\times 10^{-1}$

$\psi=$ listed value $\times 10^{-1}$

$\eta_{\mathrm{ts}}=\%$

$90^{0}$ continued

\begin{tabular}{|c|c|c|c|c|c|c|c|c|c|c|c|c|c|c|c|c|c|}
\hline \multicolumn{3}{|c|}{ IGV flap $+15^{0}$} & \multicolumn{3}{|c|}{ IGV flap $0^{0}$} & \multicolumn{3}{|c|}{ IGV flap $-15^{0}$} & \multicolumn{3}{|c|}{ IGV flap $-30^{0}$} & \multicolumn{3}{|c|}{ IGV flap $-45^{0}$} & \multicolumn{3}{|c|}{$-45^{0}$ continued } \\
\hline$\phi$ & $\psi$ & $\eta_{\mathrm{ts}}$ & $\phi$ & $\psi$ & $\eta_{\mathrm{ts}}$ & $\phi$ & $\psi$ & $\eta_{\mathrm{ts}}$ & $\phi$ & $\psi$ & $\eta_{\mathrm{ts}}$ & $\phi$ & $\psi$ & $\eta_{\mathrm{ts}}$ & $\phi$ & $\psi$ & $\eta_{\mathrm{ts}}$ \\
\hline 0.26 & 0.14 & 22.2 & 0.26 & 0.05 & 7.6 & 0.24 & $\mid 0.07$ & 11.2 & 0.28 & 0.16 & 21.9 & 0.26 & \begin{tabular}{|l|}
0.09 \\
\end{tabular} & 13.0 & 2.61 & 1.97 & 25.2 \\
\hline 0.29 & 0.18 & 26.9 & 0.27 & 0.10 & 14.7 & 0.28 & 0.10 & 15.9 & 0.33 & 0.25 & 31.0 & 0.27 & 0.17 & 22.9 & 2.93 & 1.95 & 21.5 \\
\hline 0.31 & 0.22 & 31.3 & 0.28 & 0.16 & 22.4 & 0.30 & 0.21 & 28.5 & 0.34 & 0.33 & 37.8 & 0.29 & 0.21 & 27.9 & 3.31 & 1.92 & 18.2 \\
\hline 0.31 & 0.31 & 40.9 & 0.28 & 0.23 & 30.7 & 0.31 & 0.28 & 35.3 & 0.34 & 0.43 & 45.7 & 0.31 & 0.27 & 33.0 & 3.70 & 1.92 & 15.7 \\
\hline 0.31 & 0.40 & 49.8 & 0.30 & 0.31 & 38.4 & 0.34 & 0.35 & 41.6 & 0.38 & 0.48 & 48.7 & 0.34 & 0.32 & 37.7 & 4.19 & 1.97 & 13.3 \\
\hline 0.35 & 0.46 & 52.7 & 0.31 & 0.41 & 47.5 & 0.35 & 0.44 & 48.5 & 0.39 & 0.58 & 55.0 & 0.35 & 0.40 & 43.6 & 4.82 & 2.05 & $\mid 11.1$ \\
\hline 0.37 & 0.56 & 58.8 & 0.34 & 0.56 & 57.4 & 0.38 & 0.48 & 50.2 & 0.41 & 0.66 & 59.2 & 0.36 & 0.49 & 50.2 & 5.38 & 2.14 & 9.6 \\
\hline 0.39 & 0.65 & 64.2 & 0.38 & 0.64 & 61.3 & 0.40 & 0.58 & 55.9 & 0.45 & 0.77 & 63.9 & 0.41 & 0.67 & 59.8 & 5.79 & 2.22 & 8.7 \\
\hline 0.43 & 0.70 & 64.6 & 0.40 & 0.73 & 65.5 & 0.43 & 0.70 & 62.1 & 0.48 & 0.82 & 65.2 & 0.45 & 0.71 & 60.4 & & & \\
\hline 0.47 & 0.85 & 70.3 & 0.42 & 0.81 & 68.7 & 0.46 & 0.80 & 62.1 & 0.50 & 0.95 & 70.2 & 0.47 & 0.80 & 63.8 & & & \\
\hline 0.49 & 0.92 & 72.9 & 0.45 & 0.92 & 73.1 & 0.53 & 0.86 & 72.0 & 0.52 & 1.04 & 72.9 & 0.48 & 0.90 & 67.7 & & & \\
\hline 0.53 & 1.04 & 76.9 & 0.48 & 0.99 & 75.6 & 0.53 & 1.00 & 72.0 & 0.55 & 1.11 & 74.0 & 0.52 & 0.94 & 68.2 & & & \\
\hline 0.56 & 1.17 & 80.6 & 0.54 & 1.11 & 77.0 & 0.59 & 1.07 & $\mid 72.1$ & 0.59 & 1.17 & 74.4 & 0.53 & 1.02 & 70.5 & & & \\
\hline 0.57 & 1.35 & 80.6 & 0.58 & 1.26 & 80.5 & 0.62 & 1.30 & 79.2 & 0.63 & 1.22 & 74.4 & 0.55 & 1.13 & 70.5 & & & \\
\hline 0.62 & 1.38 & 87.3 & 0.61 & 1.44 & 80.5 & 0.68 & 1.44 & 81.1 & 0.66 & 1.27 & 74.4 & 0.61 & 1.17 & 72.9 & & & \\
\hline 0.64 & 1.48 & 87.3 & 0.70 & 1.50 & 80.5 & 0.72 & 1.63 & 84.9 & 0.68 & 1.40 & 80.0 & 0.64 & 1.23 & 72.9 & & & \\
\hline 0.67 & 1.56 & 87.3 & 0.73 & 1.68 & 86.4 & 0.81 & 1.71 & 83.1 & 0.73 & 1.44 & 80.0 & 0.65 & 1.39 & 72.9 & & & \\
\hline 0.71 & 1.61 & 87.5 & 0.81 & 1.77 & 86.4 & 0.89 & 1.80 & 81.8 & 0.74 & 1.55 & 80.0 & 0.71 & 1.44 & 79.3 & & & \\
\hline 0.76 & 1.64 & 88.4 & 0.86 & 1.88 & 86.4 & 0.98 & 1.86 & 80.6 & 0.75 & 1.66 & 83.0 & 0.73 & 1.54 & 79.3 & & & \\
\hline 0.77 & 1.75 & 88.4 & 0.91 & 2.03 & 87.8 & 1.05 & 1.95 & $\mid 79.7$ & 0.80 & 1.67 & 82.4 & 0.78 & 1.61 & 79.2 & & & \\
\hline 0.83 & 1.86 & 88.0 & 1.01 & 2.07 & 83.8 & 1.10 & 2.15 & 78.9 & 0.86 & 1.70 & 82.4 & 0.84 & 1.65 & 78.8 & & & \\
\hline 0.90 & 1.97 & 87.5 & 1.11 & 2.13 & 80.2 & 1.22 & 2.23 & 76.6 & 0.88 & 1.86 & 82.4 & 0.87 & 1.70 & 78.6 & & & \\
\hline 0.98 & 2.03 & 84.6 & 1.16 & 2.23 & 78.6 & 1.32 & 2.28 & $\mid 71.7$ & 0.92 & 1.93 & 82.4 & 0.89 & 1.78 & 78.6 & & & \\
\hline 1.06 & 2.08 & 82.0 & 1.25 & 2.23 & 73.6 & 1.43 & 2.21 & 63.5 & 1.01 & 1.94 & 79.0 & 0.92 & 1.83 & 78.4 & & & \\
\hline 1.14 & 2.21 & 80.1 & 1.34 & 2.19 & 68.2 & 1.51 & 2.10 & 57.2 & 1.09 & 2.00 & 77.3 & 0.96 & 1.88 & 78.4 & & & \\
\hline 1.21 & 2.21 & 76.4 & 1.39 & 2.16 & 64.1 & 1.61 & 1.95 & 50.4 & 1.14 & 2.12 & 77.2 & 0.99 & 1.92 & 77.9 & & & \\
\hline 1.31 & 2.20 & 69.3 & 1.50 & 1.99 & 55.7 & 1.67 & 1.85 & 45.4 & 1.22 & 2.17 & 74.9 & 1.03 & 1.92 & 77.9 & & & \\
\hline 1.34 & 2.16 & 64.2 & 1.62 & 1.80 & 47.9 & 1.81 & 1.80 & 39.8 & 1.31 & 2.22 & 71.9 & 1.07 & 2.15 & 77.7 & & & \\
\hline 1.43 & 2.00 & 57.6 & 1.75 & 1.67 & 41.2 & 1.94 & 1.80 & 35.7 & 1.40 & 2.27 & 68.8 & 1.08 & 2.15 & 77.7 & & & \\
\hline 1.52 & 1.87 & 51.7 & 1.85 & 1.63 & 38.0 & 2.12 & 1.80 & 31.8 & 1.43 & 2.40 & 67.0 & 1.12 & 2.18 & 77.6 & & & \\
\hline 1.61 & 1.76 & 46.3 & 1.95 & 1.62 & 35.4 & 2.25 & 1.80 & 29.3 & 1.58 & 2.21 & 58.3 & 1.14 & 2.18 & 76.4 & & & \\
\hline 1.70 & 1.70 & 42.3 & 2.16 & 1.59 & 31.2 & 2.48 & 1.80 & 26.1 & 1.65 & 2.13 & 52.4 & 1.19 & 2.35 & 76.2 & & & \\
\hline 1.80 & 1.68 & 39.7 & 2.43 & 1.59 & 27.1 & 2.62 & 1.80 & 24.2 & 1.69 & 2.08 & 49.0 & 1.21 & 2.35 & 75.9 & & & \\
\hline 1.98 & 1.68 & 35.0 & 2.63 & 1.59 & 24.5 & 2.92 & 1.80 & 21.3 & 1.74 & 2.01 & 45.1 & 1.28 & 2.40 & 75.2 & & & \\
\hline 2.09 & 1.68 & 32.8 & 2.85 & 1.62 & 22.1 & 3.10 & 1.80 & 19.7 & 1.83 & 1.93 & 40.8 & 1.28 & 2.55 & 74.2 & & & \\
\hline 2.26 & 1.68 & 29.8 & 3.15 & 1.65 & 19.4 & 3.44 & 1.81 & $\mid 17.1$ & 1.95 & 1.86 & 36.7 & 1.39 & 2.50 & 70.4 & & & \\
\hline 2.57 & 1.64 & 25.6 & 3.33 & 1.65 & 17.7 & 3.83 & 1.86 & 14.7 & 2.07 & 1.83 & 33.4 & 1.48 & 2.45 & 66.0 & & & \\
\hline 2.82 & 1.64 & 22.7 & 3.59 & 1.70 & 16.3 & 4.41 & 1.89 & 12.3 & 2.19 & 1.80 & 30.8 & 1.58 & 2.45 & 61.4 & & & \\
\hline 3.03 & 1.69 & 20.5 & 4.07 & 1.70 & 13.8 & 4.99 & 2.02 & 10.7 & 2.39 & 1.77 & 27.6 & 1.68 & 2.37 & 54.7 & & & \\
\hline 3.23 & 1.78 & 18.6 & 4.51 & 1.83 & 12.0 & 5.55 & 2.05 & 9.4 & 2.54 & 1.77 & 25.4 & 1.74 & 2.31 & 49.7 & & & \\
\hline 3.63 & 1.80 & 16.0 & 5.01 & 1.98 & 10.6 & 5.85 & 2.10 & 8.8 & 2.74 & 1.77 & 23.0 & 1.80 & 2.25 & 46.0 & & & \\
\hline 3.96 & 1.92 & 14.3 & 5.46 & 1.98 & 9.5 & & & & 2.96 & 1.77 & 21.0 & 1.88 & 2.18 & 41.6 & & & \\
\hline 4.51 & 2.02 & 12.2 & 6.01 & 2.13 & 8.5 & & & & 3.12 & 1.80 & 19.4 & 2.00 & 2.09 & 38.1 & & & \\
\hline 4.99 & 2.19 & 10.8 & & & & & & & 3.38 & 1.80 & 17.6 & 2.07 & 2.06 & 35.8 & & & \\
\hline 5.44 & 2.37 & 9.6 & & & & & & & 3.69 & 1.80 & 15.5 & 2.26 & 2.01 & 31.5 & & & \\
\hline & & & & & & & & & 4.16 & 1.83 & 13.2 & 2.38 & 1.98 & 28.6 & & & \\
\hline & & & & & & & & & 4.71 & 1.90 & 11.3 & & & & & & \\
\hline & & & & & & & & & 5.45 & 2.01 & 9.5 & & & & & & \\
\hline
\end{tabular}


$\phi=$ listed value $\times 10^{-1} \quad \psi=$ listed value $\times 10^{-1} \quad \eta_{\mathrm{ts}}=\%$

Rotor tip stagger angle $=93^{0}$

\begin{tabular}{|c|c|c|c|c|c|c|c|c|c|c|c|c|c|c|c|c|c|}
\hline \multicolumn{3}{|c|}{ IGV flap $+45^{0}$} & \multicolumn{3}{|c|}{ IGV flap $+30^{0}$} & \multicolumn{3}{|c|}{ IGV flap $+15^{0}$} & \multicolumn{3}{|c|}{ IGV flap $0^{0}$} & \multicolumn{3}{|c|}{ IGV flap $-15^{0}$} & \multicolumn{3}{|c|}{ IGV flap $-30^{0}$} \\
\hline$\phi$ & $\psi$ & $\eta_{\mathrm{ts}}$ & $\phi$ & $\psi$ & $\eta_{\mathrm{ts}}$ & $\phi$ & $\psi$ & $\eta_{\mathrm{ts}}$ & $\phi$ & $\psi$ & $\eta_{\mathrm{ts}}$ & $\phi$ & $\psi$ & $\eta_{\mathrm{ts}}$ & $\phi$ & $\psi$ & $\eta_{\mathrm{ts}}$ \\
\hline 0.12 & 0.28 & 25.1 & 0.13 & \begin{tabular}{|l|}
0.27 \\
\end{tabular} & 26.0 & \begin{tabular}{|l|}
0.11 \\
\end{tabular} & 0.23 & 22.4 & \begin{tabular}{|l}
0.12 \\
\end{tabular} & 0.39 & 36.1 & \begin{tabular}{|l|}
0.11 \\
\end{tabular} & 0.30 & 27.9 & 0.08 & 0.05 & \begin{tabular}{|l|}
4.9 \\
\end{tabular} \\
\hline 0.12 & 0.53 & 44.6 & 0.14 & 0.45 & 40.5 & 0.12 & 0.42 & 37.3 & 0.13 & 0.47 & 41.9 & 0.13 & 0.43 & 37.9 & 0.09 & 0.11 & 10.2 \\
\hline 0.16 & 0.55 & 44.8 & 0.16 & 0.67 & 54.6 & 0.13 & 0.52 & 44.7 & 0.14 & 0.52 & 45.1 & 0.14 & 0.55 & 45.6 & 0.10 & 0.29 & 26.3 \\
\hline 0.18 & 0.63 & 48.7 & 0.20 & 0.75 & 56.9 & 0.14 & 0.68 & 44.7 & 0.15 & 0.58 & 48.5 & 0.17 & 0.62 & 49.9 & 0.13 & 0.42 & 35.9 \\
\hline 0.19 & 0.77 & 57.5 & 0.22 & 0.77 & 56.9 & 0.18 & 0.73 & 64.7 & 0.17 & 0.77 & 60.2 & 0.17 & 0.77 & 57.9 & 0.17 & 0.63 & 49.0 \\
\hline 0.21 & 0.86 & 61.1 & 0.23 & | 0.87 & 61.1 & 0.19 & 0.90 & 64.7 & 0.18 & 0.89 & 65.5 & 0.20 & 0.87 & 61.8 & 0.19 & 0.75 & 55.1 \\
\hline 0.22 & 0.93 & 64.4 & 0.24 & 1.06 & 61.1 & 0.22 & 0.90 & 64.7 & 0.21 & 1.01 & 70.0 & 0.22 & 1.02 & 67.2 & 0.19 & 0.94 & 65.4 \\
\hline 0.23 & 0.93 & 64.4 & 0.28 & 1.08 & 80.5 & 0.24 & 1.29 & 74.5 & 0.24 & 1.09 & 70.9 & 0.25 & 1.13 & 70.4 & 0.21 & 1.06 & 65.4 \\
\hline 0.24 & 1.24 & 75.9 & 0.32 & 1.08 & 80.5 & 0.33 & 1.29 & 74.5 & 0.28 & 1.27 & 75.9 & 0.27 & 1.23 & 73.9 & 0.25 & 1.06 & 68.2 \\
\hline 0.28 & 1.24 & 75.9 & 0.42 & 1.48 & 79.4 & 0.39 & 1.42 & 76.6 & 0.29 & 1.42 & 75.9 & 0.31 & 1.32 & 75.7 & 0.29 & 1.31 & 72.9 \\
\hline 0.31 & 1.38 & 75.9 & 0.45 & 1.48 & 79.4 & 0.45 & 1.56 & 78.6 & 0.36 & 1.49 & 80.8 & 0.33 & 1.46 & 79.6 & 0.33 & 1.31 & 72.9 \\
\hline 0.38 & 1.43 & 78.8 & 0.53 & 1.55 & 79.4 & 0.49 & 1.61 & 78.6 & 0.43 & 1.60 & 81.1 & 0.37 & 1.53 & 80.0 & 0.35 & 1.31 & 76.4 \\
\hline 0.45 & 1.46 & 80.6 & 0.57 & 1.71 & 79.4 & 0.57 & 1.69 & 78.3 & 0.49 & 1.72 & 81.1 & 0.41 & 1.63 & 81.6 & 0.40 & 1.41 & 72.8 \\
\hline 0.45 & 1.57 & 80.6 & 0.62 & 1.83 & 79.4 & 0.62 & 1.82 & 78.2 & 0.53 & 1.80 & 82.2 & 0.44 & 1.69 & 81.7 & 0.45 & 1.50 & 72.7 \\
\hline 0.50 & 1.76 & 84.8 & 0.70 & 1.87 & 77.4 & 0.68 & 1.93 & 77.4 & 0.61 & 1.89 & 80.8 & 0.50 & 1.70 & 79.7 & 0.47 & 1.50 & 72.7 \\
\hline 0.59 & 1.79 & 82.8 & 0.78 & 1.88 & 73.5 & 0.76 & 1.95 & 74.5 & 0.68 & 1.96 & 79.1 & 0.55 & 1.72 & 77.2 & 0.51 & 1.57 & 73.0 \\
\hline 0.62 & 1.88 & 81.4 & 0.86 & 1.87 & 69.4 & 0.80 & 1.99 & 72.6 & 0.77 & 1.93 & 73.5 & 0.61 & 1.80 & 77.1 & 0.54 & 1.57 & 73.0 \\
\hline 0.69 & 1.92 & 80.3 & 0.92 & 1.86 & 65.0 & 0.86 & 1.99 & 68.2 & 0.86 & 1.91 & 68.3 & 0.66 & 1.81 & 75.9 & 0.60 & 1.74 & 73.0 \\
\hline 0.74 & 1.92 & 78.8 & 0.99 & 1.79 & 60.0 & 0.94 & 1.87 & 61.2 & 0.94 & 1.87 & 62.8 & 0.70 & 1.92 & 74.9 & 0.62 & 1.74 & 73.0 \\
\hline 0.82 & 2.01 & 73.8 & 1.06 & 1.69 & 54.2 & 1.02 & 1.71 & 53.5 & 1.00 & 1.83 & 58.3 & 0.75 & 1.94 & 73.5 & 0.67 & 1.80 & 73.0 \\
\hline 0.86 & 2.01 & 71.5 & 1.13 & 1.56 & 48.0 & 1.09 & 1.58 & 47.6 & 1.05 & 1.76 & 53.5 & 0.84 & 1.94 & 69.2 & 0.69 & 1.90 & 73.0 \\
\hline 0.95 & 1.92 & 65.1 & 1.18 & 1.47 & 43.5 & 1.17 & 1.42 & 41.1 & 1.13 & 1.60 & 47.0 & 0.93 & 1.94 & 64.4 & 0.74 & 1.94 & 71.7 \\
\hline 1.02 & 1.84 & 59.6 & 1.25 & 1.35 & 38.5 & 1.27 & 1.27 & 34.6 & 1.19 & 1.46 & 40.2 & 0.96 & 1.94 & 61.4 & 0.85 & 1.99 & 69.1 \\
\hline 1.07 & 1.75 & 54.6 & 1.30 & 1.30 & 34.9 & 1.37 & 1.21 & 30.2 & 1.24 & 1.36 & 36.1 & 1.04 & 1.83 & 54.3 & 0.90 & 2.00 & 66.4 \\
\hline 1.13 & 1.65 & 49.7 & 1.44 & 1.21 & 30.2 & 1.52 & 1.14 & 26.3 & 1.31 & 1.29 & 32.3 & 1.11 & 1.71 & 48.7 & 0.96 & 2.00 & 63.1 \\
\hline 1.23 & 1.49 & 43.5 & 1.56 & 1.17 & 27.3 & 1.64 & 1.11 & 23.9 & 1.40 & 1.23 & 29.1 & 1.15 & 1.62 & 44.7 & 1.02 & 1.99 & 59.2 \\
\hline 1.30 & 1.39 & 38.1 & 1.66 & 1.16 & 25.3 & 1.77 & 1.09 & 21.7 & 1.48 & 1.20 & 26.6 & 1.23 & 1.46 & 38.5 & 1.12 & 1.83 & 51.1 \\
\hline 1.43 & 1.29 & 33.0 & 1.77 & 1.16 & 23.4 & 2.03 & 1.08 & 18.6 & 1.61 & 1.13 & 24.1 & 1.33 & 1.31 & 32.1 & 1.18 & 1.71 & 45.3 \\
\hline 1.55 & 1.23 & 29.4 & 1.93 & 1.13 & 21.0 & 2.28 & 1.07 & 16.2 & 1.74 & 1.12 & 21.9 & 1.43 & 1.23 & 28.3 & 1.21 & 1.66 & 42.5 \\
\hline 1.67 & 1.18 & 26.3 & 2.10 & 1.13 & 18.9 & 2.51 & 1.06 & 14.3 & 1.94 & 1.08 & 19.2 & 1.60 & 1.12 & 24.3 & 1.28 & 1.52 & 37.6 \\
\hline 1.77 & 1.18 & 24.1 & 2.29 & 1.13 & 16.9 & 2.76 & 1.06 & 12.6 & 2.19 & 1.05 & 16.5 & 1.75 & 1.08 & 21.5 & 1.39 & 1.38 & 32.8 \\
\hline 1.92 & 1.15 & 21.8 & 2.45 & 1.13 & 15.4 & 3.03 & 1.05 & 11.1 & 2.44 & 1.05 & 14.6 & 1.91 & 1.06 & 19.0 & 1.55 & 1.23 & 26.9 \\
\hline 2.08 & 1.14 & 19.6 & 2.70 & 1.13 & 13.5 & 3.39 & 1.05 & 9.6 & 2.74 & 1.05 & 12.7 & 2.11 & 1.04 & 16.8 & 1.62 & 1.20 & 24.0 \\
\hline 2.27 & 1.12 & 17.5 & 3.06 & 1.13 & 11.5 & 3.59 & 1.08 & 8.8 & 3.03 & 1.05 & 11.0 & 2.34 & 1.02 & 14.8 & 1.76 & 1.15 & 21.2 \\
\hline 2.54 & 1.11 & 15.4 & 3.43 & 1.13 & 9.8 & 4.03 & 1.11 & 7.6 & 3.29 & 1.08 & 9.8 & 2.63 & 1.02 & 12.9 & 1.87 & 1.11 & 19.5 \\
\hline 2.72 & 1.11 & 13.9 & 3.73 & 1.13 & 8.8 & 4.54 & 1.16 & 6.5 & 3.60 & 1.08 & 8.7 & 2.83 & 1.02 & 11.7 & 1.97 & 1.11 & 17.8 \\
\hline 3.00 & 1.11 & 12.3 & 4.26 & 1.14 & 7.5 & 5.10 & 1.27 & 5.7 & 4.35 & 1.15 & 6.9 & 3.22 & 1.08 & 10.0 & 2.29 & 1.07 & 14.9 \\
\hline 3.42 & 1.17 & 10.4 & 5.01 & 1.17 & 6.3 & 5.66 & 1.27 & 5.1 & 5.00 & 1.15 & 5.9 & 3.62 & 1.08 & 8.5 & 2.44 & 1.06 & 13.5 \\
\hline 3.62 & 1.17 & 9.5 & 5.20 & 1.28 & 6.0 & & & & 5.38 & 1.20 & 5.4 & 4.24 & 1.11 & 7.0 & 2.80 & 1.06 & 11.6 \\
\hline 4.20 & 1.17 & 8.0 & 5.77 & 1.35 & 5.3 & & & & 5.75 & 1.30 & 4.9 & 4.66 & 1.14 & 6.1 & 3.00 & 1.06 & 10.5 \\
\hline 4.72 & 1.26 & 7.0 & & & & & & & & & & 5.06 & 1.17 & 5.5 & 3.46 & 1.06 & 8.7 \\
\hline 5.20 & 1.29 & 6.3 & & & & & & & & & & 5.48 & 1.22 & 4.9 & 3.87 & 1.06 & 7.5 \\
\hline 5.95 & 1.37 & 5.5 & & & & & & & & & & 5.92 & 1.26 & 4.5 & 4.40 & 1.12 & 6.4 \\
\hline & & & & & & & & & & & & & & & 5.03 & 1.16 & 5.4 \\
\hline & & & & & & & & & & & & & & & 5.43 & 1.26 & 4.9 \\
\hline
\end{tabular}


$\phi=$ listed value $\times 10^{-1} \quad \psi=$ listed value $\times 10^{-1} \quad \eta_{\mathrm{ts}}=\%$

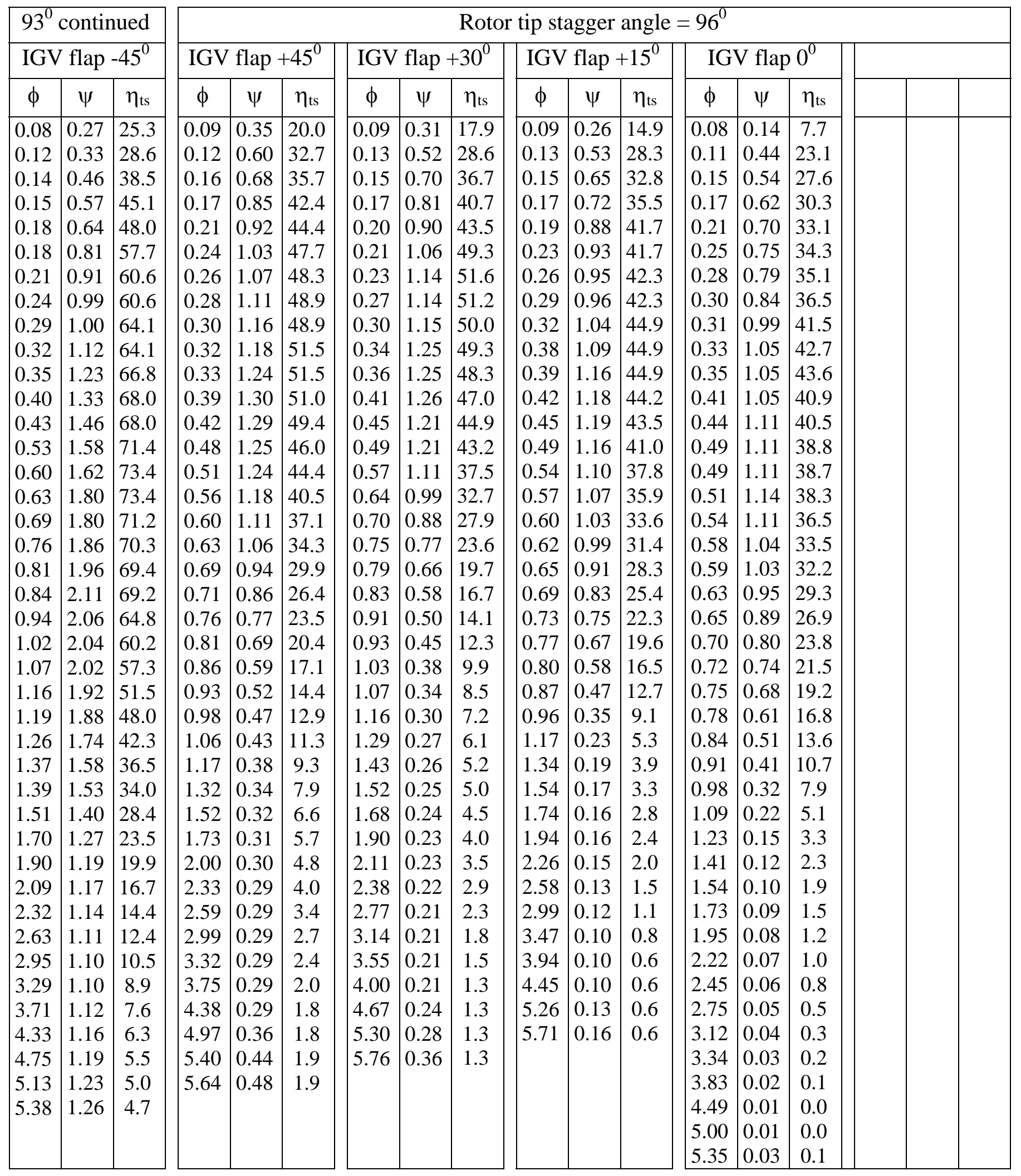


$\phi=$ listed value $\times 10^{-1}$

$\psi=$ listed value $\times 10^{-1}$

$\eta_{\mathrm{ts}}=\%$

\begin{tabular}{|c|c|c|c|c|c|c|c|c|c|c|c|}
\hline \multicolumn{3}{|c|}{ IGV flap $-15^{0}$} & \multicolumn{3}{|c|}{$-15^{0}$ continued } & \multicolumn{3}{|c|}{ IGV flap $-30^{0}$} & \multicolumn{3}{|c|}{ IGV flap $-45^{0}$} \\
\hline$\phi$ & $\psi$ & $\eta_{\mathrm{ts}}$ & $\phi$ & $\psi$ & $\eta_{\mathrm{ts}}$ & $\phi$ & $\psi$ & $\eta_{\mathrm{ts}}$ & $\phi$ & $\psi$ & $\eta_{\mathrm{ts}}$ \\
\hline 0.14 & $\mid 0.23$ & $\mid 12.4$ & 3.20 & -0.01 & -0.1 & \begin{tabular}{|l|l|}
0.14 \\
\end{tabular} & \begin{tabular}{|l|l} 
\\
\end{tabular} & 13.0 & \begin{tabular}{|l|l|}
0.14 \\
\end{tabular} & \begin{tabular}{|l|l} 
\\
\end{tabular} .33 & 17.0 \\
\hline 0.15 & 0.30 & 15.9 & 3.44 & -0.02 & -0.2 & 0.16 & 0.38 & 19.0 & 0.16 & 0.39 & 19.7 \\
\hline 0.17 & $\mid 0.37$ & 18.9 & 3.77 & -0.04 & -0.3 & 0.19 & 0.50 & 24.4 & 0.17 & 0.39 & 19.7 \\
\hline 0.17 & 0.52 & 25.8 & 4.13 & -0.05 & -0.3 & 0.20 & 0.66 & 30.9 & 0.18 & 0.59 & 19.7 \\
\hline 0.20 & 0.57 & 27.5 & 4.63 & -0.06 & -0.3 & 0.23 & 0.71 & 32.3 & 0.24 & 0.59 & 34.2 \\
\hline 0.23 & 0.65 & 30.2 & 5.41 & -0.06 & -0.2 & 0.24 & 0.78 & 34.6 & 0.24 & 0.76 & 34.2 \\
\hline 0.24 & 0.72 & 32.9 & 5.71 & -0.05 & -0.2 & 0.24 & 0.78 & 34.6 & 0.26 & 0.76 & 34.2 \\
\hline 0.27 & 0.75 & 33.5 & & & & 0.29 & 1.03 & 34.6 & 0.27 & 0.98 & 40.1 \\
\hline 0.27 & 0.75 & 33.5 & & & & 0.31 & 1.03 & 42.2 & 0.32 & 0.98 & 40.1 \\
\hline 0.30 & 0.87 & 37.0 & & & & 0.35 & 1.06 & 42.2 & 0.35 & 1.03 & 40.8 \\
\hline 0.32 & 0.87 & 37.0 & & & & 0.38 & 1.06 & 42.2 & 0.38 & 1.09 & 41.9 \\
\hline 0.36 & 0.93 & 38.1 & & & & 0.42 & 1.06 & 41.1 & 0.39 & 1.09 & 43.5 \\
\hline 0.38 & & 39.7 & & & & 0.44 & 1.16 & 40.0 & 0.44 & 1.14 & 41.1 \\
\hline 0.40 & 1.04 & 40.3 & & & & 0.49 & 1.16 & 38.8 & 0.48 & 1.14 & 40.3 \\
\hline 0.43 & & & & & & 0.50 & 1.16 & 38.6 & 0.49 & 1.19 & 39.9 \\
\hline 0.45 & 1.05 & 39.3 & & & & 0.57 & 1.05 & 34.3 & 51 & 1.21 & 39.8 \\
\hline 0.49 & 1.07 & .6 & & & & 0.60 & 1.03 & 32.6 & .56 & 1.17 & 36.3 \\
\hline 0.52 & 1.07 & |36.7 & & & & 0.63 & 1.00 & 30.8 & 0.56 & 1.13 & 36.3 \\
\hline 0.56 & 1.04 & 34 & & & & 0.64 & 0.97 & 29.3 & 63 & 1.06 & 31.8 \\
\hline 0.60 & 1.00 & 3 & & & & 0.65 & 0.96 & 28.5 & 0.66 & 1.02 & 29.9 \\
\hline 0.62 & 0.98 & 31.2 & & & & 0.67 & 0.91 & 26.4 & 74 & 0.88 & 24.7 \\
\hline 0.65 & & & & & & & 0.86 & 24.5 & .80 & 0.77 & 20.8 \\
\hline 0.69 & 0.86 & 2 & & & & 0.73 & 0.80 & 22.4 & 0.84 & 0.68 & 17.7 \\
\hline 0.73 & & 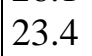 & & & & 0.79 & 0.69 & 18.9 & 89 & 0.59 & 14.9 \\
\hline 0.75 & 0.74 & 21.4 & & & & 0.84 & 0.59 & 15.6 & 0.96 & 0.50 & 12.3 \\
\hline 0.79 & 0.69 & 19.9 & & & & 0.92 & 0.46 & 11.5 & .06 & 0.34 & 7.7 \\
\hline 0.80 & 5 & & & & & & 0.3 & 9.3 & 5 & 0. & 6.1 \\
\hline 0.85 & 0.57 & 15.6 & & & & 1.02 & 0.30 & 7.1 & 16 & 0.24 & 4.9 \\
\hline 0.88 & & & & & & & 0.23 & 5.1 & 28 & 0.18 & 3.6 \\
\hline 0.94 & 0.44 & 11.5 & & & & 1.21 & 0.18 & 3.8 & 1.34 & 0.15 & 2.9 \\
\hline 0.99 & 0.36 & $\mid 9.2$ & & & & 1.29 & 0.15 & 3.0 & .43 & 0.12 & 2.2 \\
\hline 1.07 & 7 & 6.6 & & & & 1.30 & 0.13 & 2.7 & .57 & 0.07 & 1.2 \\
\hline 1.13 & 0.23 & 5.4 & & & & 1 & 0.07 & 1.2 & 75 & 0.03 & 0.5 \\
\hline 1.18 & 0 & 4.5 & & & & 1.64 & 0.05 & 0.8 & .90 & 0.01 & 0.1 \\
\hline 1.32 & 0.13 & 2.8 & & & & 1.75 & 0.03 & 0.5 & 16 & -0.01 & -0.1 \\
\hline 1.40 & & $\mid 1.9$ & & & & & 0.02 & $\mid$\begin{tabular}{|l|l} 
\\
\end{tabular} & 2.35 & -0.02 & -0.2 \\
\hline 1.54 & 8 & 1.5 & & & & 3 & 0.00 & 0.0 & 2.68 & -0. & -0.3 \\
\hline 1.68 & & $\mid \begin{array}{l}1.0 \\
\end{array}$ & & & & & & $\mid-0.1$ & 83 & -0.05 & -0.4 \\
\hline 1.79 & 4 & $\mid$\begin{tabular}{|l}
$\mid$ \\
\end{tabular} & & & & 19 & -0.02 & $\mid-0.2$ & 3.23 & -0.07 & -0.5 \\
\hline 1.96 & & 0 & & & & 2.87 & -0.03 & -0.3 & 3.61 & -0.09 & -0.5 \\
\hline 2.09 & 0.02 & 0.3 & & & & 3.15 & -0.04 & -0.4 & 4.16 & -0.10 & -0.5 \\
\hline 2.30 & 0.02 & 0. & & & & 3.58 & -0.07 & -0.4 & 4.85 & -0.11 & -0.5 \\
\hline 2.53 & 0.01 & $\mid$\begin{tabular}{|l}
$\mid$ \\
\end{tabular} & & & & 3.84 & -0.08 & -0.4 & 5.29 & -0.14 & -0.5 \\
\hline 2.88 & 0.00 & 0.0 & & & & & -0.0 & 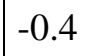 & & & \\
\hline & & & & & & 9 & -0.10 & -0 & & & \\
\hline & & & & & & & -0.10 & -0.4 & & & \\
\hline & & & & & & 67 & -0.11 & -0. & & & \\
\hline
\end{tabular}




\section{Profile Data}

Data from measured velocity profiles scaled to full-size plant as presented in thesis.

$\mathrm{D} / \mathrm{D}_{\text {turb }}$ : Fraction of outer diameter [\%] $\quad \mathrm{Cr} \mathrm{C}_{\theta} \mathrm{C}_{\mathrm{z}}$ : Radial, tangential \& axial velocities [m/s] $\mathrm{p}_{\mathrm{s}} \mathrm{p}_{\mathrm{o}}$ : Static and total pressure $[\mathrm{Pa}]$

Case (1) Turbine velocity profiles.

\begin{tabular}{|c|c|c|c|c|c|c|c|c|c|c|c|}
\hline \multicolumn{6}{|c|}{ Upstream (before) } & \multicolumn{6}{|c|}{ Downstream (after) } \\
\hline $\mathrm{D} / \mathrm{D}_{\text {turb }}$ & $\mathrm{C}_{\mathrm{r}}$ & $\mathrm{C}_{\theta}$ & $\mathrm{C}_{\mathrm{z}}$ & $p_{s}$ & $\mathrm{p}_{\mathrm{o}}$ & $\mathrm{D} / \mathrm{D}_{\text {turb }}$ & $\mathrm{C}_{\mathrm{r}}$ & $\mathrm{C}_{\theta}$ & $\mathrm{C}_{\mathrm{z}}$ & $\mathrm{p}_{\mathrm{s}}$ & $\mathrm{p}_{\mathrm{o}}$ \\
\hline 99.7 & 1.28 & .41 & 7.02 & -271 & -238 & 100 & 3.32 & 2.16 & 4.99 & -524 & -502 \\
\hline 99.4 & 1.65 & 4.65 & 12.15 & -271 & -172 & 99.7 & 2.20 & -2.55 & 19.05 & -531 & -326 \\
\hline 99.2 & 1.20 & -5.18 & 14.32 & -268 & -134 & 99.4 & 0.49 & -2.88 & 19.58 & -518 & -304 \\
\hline 98.9 & 0.85 & -5.12 & 16.73 & -261 & -85 & 99.2 & 0.04 & -3.08 & 19.78 & -525 & -307 \\
\hline 98.6 & 0.27 & -5.12 & 17.78 & -257 & -60 & 98.9 & 0.00 & -3.42 & 19.47 & -521 & -307 \\
\hline 98.3 & -0.20 & -5.07 & 18.67 & -250 & -36 & 98.6 & 0.00 & -3.65 & 19.18 & -524 & -316 \\
\hline 98.1 & -0.66 & -4.80 & 19.15 & -243 & -20 & 98.3 & -0.01 & -3.65 & 18.61 & -530 & -333 \\
\hline 97.8 & -0.86 & -4.74 & 9.02 & -237 & -17 & 98.1 & 0.11 & -3.40 & 18.22 & -534 & -346 \\
\hline 97.5 & -1.02 & -4.57 & 9.19 & -236 & -13 & 97.8 & 0.22 & -3.19 & 18.11 & -539 & -354 \\
\hline 97.2 & -1.19 & -4.47 & .12 & -233 & -11 & 97.5 & 0.36 & -2.91 & 17.82 & -536 & -358 \\
\hline 96.9 & -1.26 & -4.29 & 03 & -232 & -13 & 97.2 & 0.57 & -2.37 & 17.70 & -537 & -363 \\
\hline 96.7 & -1.50 & -4.17 & 5 & -229 & -10 & 96.7 & 0.65 & -1.98 & 17.60 & -536 & -364 \\
\hline 96.4 & -1.61 & -4.18 & 3.84 & -226 & -11 & 96.1 & 0.57 & -1.72 & 18.19 & -538 & -355 \\
\hline 96.1 & -1.73 & .16 & 18.79 & -225 & -1 & 056 & 0.46 & -1.61 & 18.03 & -537 & -357 \\
\hline 95.8 & $\mid-1.78$ & -4.08 & 18.67 & -223 & -1 & 050 & 0.43 & -1.67 & 18.04 & -531 & -352 \\
\hline 95.6 & -1.84 & -4.30 & 18.57 & -221 & 1 & 0 & 0.09 & -1.42 & 18.16 & -539 & -358 \\
\hline 95.3 & -1.97 & .27 & 18.52 & -219 & 1 & 020 & 0.18 & -1.42 & 18.00 & -536 & -358 \\
\hline 95.0 & -2.29 & -4.21 & 18.48 & -219 & -1 & 021 & 0.30 & -0.95 & 18.20 & -536 & -354 \\
\hline 94.7 & -2.25 & -4.21 & 18.44 & -219 & -1 & 017 & -0.04 & -1.13 & 18.00 & -540 & -362 \\
\hline 94.4 & -2.34 & -4.21 & 18.34 & -218 & -12 & 003 & -0.20 & -0.95 & 18.08 & -537 & -358 \\
\hline 94.2 & -2.39 & -4.25 & 18.33 & -217 & -11 & 88.9 & -0.35 & -0.91 & 18.10 & -541 & -362 \\
\hline 93.9 & -2.42 & -4.23 & 18.23 & -217 & -13 & 87.5 & -0.31 & -0.39 & 17.81 & -542 & -368 \\
\hline 93.3 & -2.74 & -4.13 & 18.16 & -216 & -1 & 86.1 & -0.39 & -0.51 & 17.10 & -545 & -385 \\
\hline 92.8 & -2.62 & -4.01 & 18.18 & -214 & -12 & 84.7 & -0.57 & -0.75 & 16.76 & -550 & -396 \\
\hline 92.2 & -2.62 & -4.00 & 18.15 & -216 & -14 & 83.3 & -0.56 & -0.34 & 16.56 & -546 & -396 \\
\hline 91.7 & -2.19 & -4.05 & 7.95 & -217 & -21 & 81.9 & -0.78 & 0.52 & 16.27 & -543 & -398 \\
\hline 91.1 & -2.59 & -3.69 & 17.83 & -215 & -22 & 80.6 & -0.64 & 0.69 & 16.04 & -546 & -405 \\
\hline 90.6 & -2.23 & -3.66 & 17.78 & -216 & -25 & 79.2 & -0.77 & 0.99 & 16.00 & -549 & -409 \\
\hline 90.0 & -1.66 & -3.79 & 18.01 & -218 & -22 & 77.8 & -0.78 & 0.82 & 15.77 & -547 & -411 \\
\hline 89.4 & $\mid-1.43$ & -3.74 & 18.07 & -217 & -2 & 76. & -0.82 & 0.79 & 16.00 & -552 & -411 \\
\hline 88.9 & $\mid-1.49$ & -3.80 & 17.96 & -215 & -2 & 75.0 & -0.91 & 0.89 & 15.90 & -547 & -408 \\
\hline 88.3 & $\mid-1.21$ & -3.92 & |17.97 & -215 & -2 & 73.6 & -1.11 & 1.09 & 15.89 & -548 & -409 \\
\hline 87.5 & $\mid-1.20$ & -3.54 & 17.98 & -211 & $-1 \varepsilon$ & 72.2 & -1.16 & 1.15 & 15.60 & -548 & -413 \\
\hline 86.1 & $\mid-0.71$ & -3.09 & 18.05 & -207 & -15 & 69.4 & -1.20 & 1.34 & 15.15 & -550 & -423 \\
\hline 84.7 & $\mid-0.79$ & -3.16 & 17.77 & -202 & -15 & 66.7 & -1.27 & 1.92 & 14.96 & -550 & -425 \\
\hline 83.3 & $\mid-0.89$ & -3.23 & 17.38 & -193 & -14 & 63.9 & -1.18 & 2.44 & 14.74 & -556 & -433 \\
\hline 81.9 & -1.24 & -3.39 & 16.92 & -187 & -15 & 61.1 & -1.11 & 2.73 & 14.11 & -557 & -444 \\
\hline 80.6 & $\mid-1.22$ & -3.36 & 16.75 & -181 & -13 & 58.3 & -1.29 & 3.37 & 13.53 & -560 & -453 \\
\hline 79.2 & $\mid-1.78$ & -3.49 & 16.42 & -176 & -12 & 55.6 & -1.50 & 3.81 & 13.19 & -560 & -456 \\
\hline 77.8 & $\mid-1.35$ & -3.80 & 16.16 & -170 & -11 & 52.8 & -1.50 & 3.88 & 12.96 & -562 & -461 \\
\hline 7 & -2.08 & -3.67 & 5.81 & -166 & -13 & 50.0 & -1.76 & 4.39 & 12.67 & -564 & -464 \\
\hline 75.0 & 1.59 & -3.68 & .74 & -164 & -13 & 47.2 & -2.08 & 5.05 & 12.21 & -568 & -470 \\
\hline
\end{tabular}




\begin{tabular}{|l|l|l|l|l|l|l|l|l|l|l|l|}
\hline 73.6 & -1.97 & -3.69 & 15.53 & -161 & -13 & 44.4 & -2.38 & 5.40 & 12.33 & -567 & -465 \\
72.2 & -2.07 & -3.78 & 15.31 & -157 & -12 & 43.1 & -2.62 & 5.37 & 12.96 & -565 & -454 \\
69.4 & -1.98 & -3.96 & 15.03 & -150 & -10 & 41.7 & -3.09 & 5.45 & 13.35 & -561 & -442 \\
66.7 & -2.33 & -4.21 & 14.61 & -145 & -10 & 41.1 & -3.50 & 5.43 & 12.71 & -563 & -451 \\
63.9 & -1.98 & -4.30 & 14.33 & -142 & -11 & 40.8 & -3.88 & 4.92 & 11.73 & -564 & -468 \\
61.1 & -1.75 & -4.21 & 14.16 & -138 & -11 & 40.8 & -4.03 & 4.57 & 11.52 & -568 & -475 \\
58.3 & -1.49 & -4.51 & 13.85 & -134 & -11 & & & & & & \\
55.6 & -1.30 & -4.50 & 13.87 & -133 & -11 & & & & & & \\
52.8 & -1.17 & -4.48 & 13.80 & -130 & -9 & & & & & & \\
50.0 & -1.05 & -4.62 & 13.42 & -128 & -12 & & & & & & \\
47.2 & -0.70 & -4.84 & 13.28 & -126 & -11 & & & & & & \\
44.4 & -0.49 & -5.60 & 12.93 & -125 & -11 & & & & & & \\
43.1 & -0.28 & -5.96 & 12.70 & -127 & -14 & & & & & & \\
42.5 & -0.28 & -5.98 & 12.70 & -125 & -12 & & & & & & \\
41.9 & -0.30 & -5.86 & 12.60 & -124 & -14 & & & & & & \\
41.4 & -0.22 & -5.22 & 12.79 & -125 & -15 & & & & & & \\
41.1 & -0.90 & -5.09 & 10.97 & -122 & -37 & & & & & & \\
\hline
\end{tabular}

$\mathrm{D} / \mathrm{D}_{\text {turb }}$ : Fraction of outer diameter [\%] $\quad \mathrm{Cr} \mathrm{C}_{\theta} \mathrm{C}_{\mathrm{z}}$ : Radial, tangential \& axial velocities [m/s] $\mathrm{p}_{\mathrm{s}} \mathrm{p}_{\mathrm{o}}$ : Static and total pressure $[\mathrm{Pa}]$

Case (2) Turbine velocity profiles.

\begin{tabular}{|c|c|c|c|c|c|c|c|c|c|c|c|}
\hline \multicolumn{6}{|c|}{ Upstream (before) } & \multicolumn{6}{|c|}{ Downstream (after) } \\
\hline $\mathrm{D} / \mathrm{D}_{\text {turb }}$ & $\mathrm{C}_{\mathrm{r}}$ & $\mathrm{C}_{\theta}$ & $\mathrm{C}_{\mathrm{z}}$ & $\mathrm{p}_{\mathrm{s}}$ & $\mathrm{p}_{\mathrm{o}}$ & $\mathrm{D} / \mathrm{D}_{\text {turb }}$ & $\mathrm{C}_{\mathrm{r}}$ & $\mathrm{C}_{\theta}$ & $\mathrm{C}_{\mathrm{z}}$ & $\mathrm{p}_{\mathrm{s}}$ & $\mathrm{p}_{0}$ \\
\hline 99.7 & 2.59 & .65 & 3.83 & -583 & -456 & 9 & 5.64 & 0.05 & 21.34 & -1235 & -964 \\
\hline 99.4 & 1.74 & & .30 & 77 & & & 19 & 3.21 & 28.04 & & 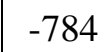 \\
\hline 99.2 & 1.79 & 12 & 0.91 & 64 & & 99 & 04 & 3.28 & 28.46 & 1209 & -752 \\
\hline 98.9 & & 13 & 1.19 & 54 & & 98 & 41 & 3.36 & 28.56 & 15 & 755 \\
\hline 98.6 & & & .83 & & & 98 & & .53 & 28.37 & 19 & 764 \\
\hline 98.3 & & & & & & 98 & & .34 & 27.90 & & 786 \\
\hline 98.1 & & & & & & & 77 & 2.90 & 27.57 & & 303 \\
\hline 97.8 & & & 6 & & & & & 2.30 & 27.10 & & 332 \\
\hline 97.5 & & & 4 & & & & & 1.67 & 26.80 & & \\
\hline 97.2 & & & 3 & & & & & 0.94 & 27.03 & & 441 \\
\hline 96.9 & & & 77 & & & & 12 & -0.41 & 26.86 & & 350 \\
\hline 96.7 & & & 7.71 & & & & & 0.19 & 27.15 & & 442 \\
\hline 96.4 & & 58 & 2 & & & & & 0.78 & 27.04 & & 838 \\
\hline 96.1 & & 34 & 16 & & & & & 1.00 & 27.00 & & -841 \\
\hline 95.8 & 7 & 45 & 7.30 & & & & & 1.14 & 27.04 & & -837 \\
\hline 95.6 & 2 & 40 & 7.10 & & & & & 1.41 & 27.21 & & -830 \\
\hline 95.3 & 75 & 43 & 02 & & & & & 1.57 & 27.41 & & -82 \\
\hline 95.0 & 85 & & 6.87 & & & & & 1.60 & 27.45 & & -821 \\
\hline 4.7 & & & 76 & & & & & 1.62 & 27.35 & & -822 \\
\hline 4 & 6 & & 26.68 & & & & & 1.81 & 27.34 & & -820 \\
\hline & & & 7 & & & & & 1.93 & 27.28 & & 2 \\
\hline & & & 26.53 & & & & & 1.97 & 27.16 & & -829 \\
\hline 92.8 & 2 & & 14 & & & & & 2.12 & 27.04 & & -833 \\
\hline & 263 & & 26.31 & & -2 & & & 2.20 & 26.98 & & -838 \\
\hline & 260 & & 26.15 & & -2 & & & 2.54 & 26.71 & & -846 \\
\hline & -2.93 & & 26.31 & & & & & 2.56 & 26.58 & & -851 \\
\hline & -2.62 & -6.21 & 26.26 & & -2 & & & 2.64 & 26.54 & & -852 \\
\hline & -2.07 & -6.27 & 26.34 & & -3 & 90 & & 2.90 & 26.53 & & -855 \\
\hline 89.4 & -1.99 & -5.93 & 26.56 & -456 & -26 & 89.4 & -0.36 & 3.11 & 26.42 & -1251 & -85 \\
\hline
\end{tabular}




\begin{tabular}{|c|c|c|c|c|c|c|c|c|c|c|c|}
\hline 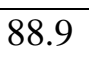 & 51 & .84 & 65 & -453 & 21 & 88.9 & -0.32 & 3.28 & 26.32 & -1251 & -860 \\
\hline 883 & & & & & & & & & & & \\
\hline 87. & & & 6 & & & & & & & & \\
\hline 86.9 & & & 5 & & & 84.7 & & 71 & 83 & & 03 \\
\hline 5.1 & & 80 & 5 & & & 83.3 & & .90 & 49 & & 09 \\
\hline 84.7 & & 00 & 38 & & & 8 & & .47 & 01 & & 24 \\
\hline .3 & & .77 & .16 & & & 0.6 & & .54 & 55 & 51 & 34 \\
\hline .99 & & 64 & 1.84 & & & 9.2 & & .93 & 25 & 63 & 42 \\
\hline .6 & & 78 & 25 & & & 7.8 & & 18 & 01 & 262 & 445 \\
\hline .2 & & & 88 & & & & & 44 & 08 & 66 & 4 \\
\hline 7.8 & & 78 & 55 & & & & & 39 & 75 & 265 & 53 \\
\hline 75.0 & & & 72 & & & 6 & & .78 & 64 & 268 & 56 \\
\hline 72.2 & & .00 & & & & 2.2 & & 7.20 & 43 & 263 & 5 \\
\hline 4 & & 98 & & & & 9.4 & & 7.86 & & 271 & 968 \\
\hline .7 & & 17 & & & & 6.7 & & 8.49 & & 80 & 99 \\
\hline & & 80 & & & & 3.9 & & 9.15 & & 78 & 00 \\
\hline & & 50 & & & & .1 & & 9.46 & & 89 & 1 \\
\hline 58.3 & & 41 & & & & .3 & & 10.44 & & 92 & 01 \\
\hline 6 & & 83 & & & & 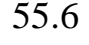 & & & & 99 & 01 \\
\hline & & & & & & & & & & 13 & 3 \\
\hline 5 & & & & & & & & & & 16 & 32 \\
\hline & & & & & & & & & & 28 & 02 \\
\hline 47 & & & & & & & & & & 28 & 1 \\
\hline & & & & & & & & & 80 & 35 & 003 \\
\hline & & & & & & & & & & 45 & 97 \\
\hline & & & & & & & & 14.71 & 01 & 42 & -985 \\
\hline & & & & & & & & 14.94 & 32 & -1350 & -981 \\
\hline 41. & & 64 & & & & & & 15.30 & 04 & -1355 & 987 \\
\hline 4 & 27 & 34 & & & & 40 & & 15.52 & 26 & -1360 & 004 \\
\hline 41.0 & 23 & -7.84 & 3.15 & -254 & -2 & 40.6 & -5.09 & 15.11 & 18.04 & -1359 & -1037 \\
\hline 40.9 & -0.85 & -6.65 & 7.16 & -253 & 27 & & & & & & \\
\hline
\end{tabular}

$\mathrm{D} / \mathrm{D}_{\text {turb }}$ : Fraction of outer diameter [\%]

Cr $\mathrm{C}_{\theta} \mathrm{C}_{\mathrm{z}}$ : Radial, tangential \& axial velocities $[\mathrm{m} / \mathrm{s}]$ $\mathrm{p}_{\mathrm{s}} \mathrm{p}_{\mathrm{o}}$ : Static and total pressure $[\mathrm{Pa}]$

Case (1\&2) Diffuser velocity profiles.

\begin{tabular}{|c|c|c|c|l|l|l|l|l|l|l|l|}
\hline \multicolumn{10}{l|}{ Case (1) } \\
\hline $\mathrm{D} / \mathrm{D}_{\text {diff }}$ & $\mathrm{C}_{\mathrm{r}}$ & $\mathrm{C}_{\theta}$ & $\mathrm{C}_{\mathrm{z}}$ & $\mathrm{p}_{\mathrm{s}}$ & $\mathrm{p}_{\mathrm{o}}$ & $\mathrm{D} / \mathrm{D}_{\text {diff }}$ & $\mathrm{C}_{\mathrm{r}}$ & $\mathrm{C}_{\theta}$ & $\mathrm{C}_{\mathrm{z}}$ & $\mathrm{p}_{\mathrm{s}}$ & $\mathrm{p}_{\mathrm{o}}$ \\
\hline 99.3 & -0.81 & 3.01 & 8.35 & -488 & -452 & 99.8 & -0.32 & 7.84 & 6.26 & -1161 & -1122 \\
99.0 & -0.82 & 2.19 & 10.22 & -497 & -447 & 99.5 & -0.86 & 7.18 & 10.66 & -1144 & -1080 \\
98.8 & -0.85 & 2.37 & 10.67 & -501 & -446 & 99.3 & -1.22 & 6.65 & 11.79 & -1138 & -1066 \\
98.5 & -0.56 & 1.96 & 10.65 & -500 & -446 & 99.0 & -1.24 & 5.95 & 13.62 & -1136 & -1050 \\
98.3 & -0.56 & 1.84 & 11.25 & -500 & -441 & 98.8 & -1.28 & 5.41 & 14.96 & -1137 & -1039 \\
98.0 & -0.67 & 1.86 & 11.31 & -495 & -435 & 98.5 & -1.37 & 5.07 & 15.97 & -1140 & -1031 \\
97.8 & -0.60 & 1.74 & 11.57 & -494 & -432 & 98.3 & -1.26 & 4.83 & 17.66 & -1137 & -1007 \\
97.5 & -0.52 & 1.21 & 11.94 & -493 & -428 & 98.0 & -1.37 & 4.36 & 18.12 & -1138 & -1003 \\
97.3 & -0.56 & 1.21 & 12.33 & -494 & -424 & 97.8 & -1.15 & 4.41 & 19.01 & -1144 & -996 \\
97.0 & -0.56 & 0.84 & 12.66 & -497 & -424 & 97.5 & -1.32 & 4.17 & 19.10 & -1140 & -992 \\
96.8 & -0.54 & 0.93 & 13.18 & -498 & -419 & 97.3 & -1.42 & 3.94 & 19.09 & -1139 & -992 \\
96.5 & -0.61 & 0.93 & 12.85 & -495 & -420 & 97.0 & -1.40 & 3.72 & 19.63 & -1134 & -979 \\
96.3 & -0.61 & 1.00 & 13.27 & -495 & -415 & 96.8 & -1.45 & 3.67 & 20.02 & -1138 & -977 \\
96.0 & -0.54 & 0.87 & 13.42 & -491 & -409 & 96.5 & -1.58 & 3.80 & 20.02 & -1139 & -978 \\
95.8 & -0.72 & 0.73 & 13.34 & -491 & -410 & 96.3 & -1.66 & 3.72 & 20.32 & -1138 & -972 \\
\hline
\end{tabular}




\begin{tabular}{|c|c|c|c|c|c|c|c|c|c|c|c|}
\hline 95.5 & \begin{tabular}{|l|}
-0.66 \\
\end{tabular} & 0.65 & 13.62 & -492 & -407 & 96.0 & \begin{tabular}{|l|}
-1.73 \\
\end{tabular} & \begin{tabular}{|l|}
3.87 \\
\end{tabular} & 20.65 & -1132 & -960 \\
\hline 95.3 & -0.67 & 0.80 & 13.87 & -494 & -406 & 95.8 & -1.83 & 3.71 & 20.75 & -1130 & -957 \\
\hline 95.0 & -0.77 & 0.92 & 13.84 & -492 & -405 & 95.5 & -1.89 & 3.68 & 20.85 & -1133 & -958 \\
\hline 94.8 & -0.70 & 0.39 & 14.18 & -490 & -398 & 95.3 & -1.94 & 3.89 & 20.95 & -1128 & -951 \\
\hline 94.5 & -0.74 & 0.48 & 14.16 & -491 & -400 & 95.0 & -2.00 & 3.84 & 21.26 & -1131 & -949 \\
\hline 94.0 & -0.83 & 0.55 & 14.41 & -492 & -397 & 94.5 & -2.07 & 3.93 & 21.41 & -1127 & -942 \\
\hline 93.5 & -0.85 & 0.92 & 14.19 & -488 & -396 & 94.0 & -2.18 & 3.88 & 21.59 & -1125 & -937 \\
\hline 93.0 & -0.85 & 0.75 & 14.35 & -492 & -398 & 93.5 & -2.25 & 3.89 & 21.84 & -1123 & -931 \\
\hline 92.5 & -0.92 & 0.57 & 14.57 & -492 & -396 & 93.0 & -2.30 & 4.14 & 21.76 & -1125 & -933 \\
\hline 92.0 & -0.98 & 0.68 & 14.49 & -490 & -394 & 92.5 & -2.34 & 4.16 & 22.20 & -1125 & -926 \\
\hline 91.3 & -0.98 & 0.65 & 14.92 & -494 & -392 & 92.0 & -2.39 & 4.16 & 22.38 & -1128 & -926 \\
\hline 90.0 & -1.07 & 0.59 & 14.97 & -488 & -386 & 91.5 & -2.45 & 4.21 & 22.55 & -1127 & -921 \\
\hline 88.8 & -1.16 & 0.55 & 15.11 & -490 & -386 & 91.0 & -2.63 & 4.36 & 22.41 & -1127 & -922 \\
\hline 87.5 & -1.07 & 0.84 & 15.10 & -493 & -389 & 90.5 & -2.62 & 4.69 & 22.73 & -1127 & -917 \\
\hline 86.3 & -1.20 & 0.74 & 15.21 & -495 & -389 & 90.0 & -2.66 & 4.60 & 22.72 & -1126 & -916 \\
\hline 85.0 & -1.21 & 0.75 & 15.51 & -492 & -383 & 88.8 & -2.85 & 5.08 & 22.94 & -1127 & -911 \\
\hline 83.8 & $\mid-1.90$ & 1.25 & 15.19 & -486 & -380 & 87.5 & -2.80 & 5.63 & 22.82 & -1129 & -912 \\
\hline 82.5 & $\mid-1.87$ & 1.66 & 15.06 & -486 & -381 & 86.3 & -3.00 & 5.90 & 22.96 & -1128 & -908 \\
\hline 81.3 & -2.03 & 1.46 & 15.02 & -484 & -379 & 85.0 & -2.93 & 6.55 & 23.07 & -1132 & -906 \\
\hline 80.0 & $\mid-1.91$ & 1.63 & 14.69 & -487 & -387 & 83.8 & -3.11 & 6.78 & 22.86 & -1135 & -912 \\
\hline 78.8 & -2.05 & 1.71 & 14.49 & -488 & -390 & 82.5 & -3.18 & 7.13 & 22.83 & -1139 & -914 \\
\hline 77.5 & $\mid-1.93$ & 1.92 & 14.51 & -491 & -392 & 81.3 & -3.23 & 7.23 & 22.46 & -1142 & -923 \\
\hline 76.3 & $\mid-1.93$ & 2.12 & 14.65 & -494 & -393 & 80.0 & -2.98 & 7.93 & 22.24 & -1147 & -928 \\
\hline 75.0 & -2.07 & 2.07 & 14.25 & -492 & -397 & 78.8 & -3.15 & 8.00 & 22.00 & -1153 & -937 \\
\hline 72.5 & $\mid-1.82$ & 2.58 & 13.86 & -490 & -399 & 77.5 & -3.06 & 8.47 & 21.84 & -1157 & -942 \\
\hline 70.0 & -2.00 & 2.84 & 12.94 & -490 & -409 & 76.3 & -2.96 & 8.59 & 21.49 & -1158 & -948 \\
\hline 67.5 & $\mid-1.93$ & 3.36 & 12.81 & -495 & -414 & 75.0 & -3.16 & 8.77 & 21.08 & -1159 & -954 \\
\hline 65.0 & $\mid-1.83$ & 3.63 & 12.23 & -496 & -421 & 72.5 & -3.00 & 9.69 & 20.15 & -1173 & -977 \\
\hline 62.5 & $\mid-1.79$ & 4.04 & 11.15 & -500 & -435 & 70.0 & -3.13 & 9.99 & 19.08 & -1176 & -993 \\
\hline 60.0 & $\mid-1.87$ & 4.20 & 10.52 & -501 & -442 & 67.5 & -2.93 & 10.18 & 18.19 & -1182 & -1010 \\
\hline 57.5 & -1.95 & 4.35 & 10.07 & -500 & -443 & 65.0 & -3.15 & 10.40 & 17.19 & -1189 & -1029 \\
\hline 55.0 & -1.97 & 4.66 & 9.79 & -501 & -446 & 62.5 & -3.08 & 10.69 & 16.58 & -1193 & -1039 \\
\hline 52.5 & -2.46 & 4.72 & 8.46 & -497 & -452 & 60.0 & -2.81 & 11.16 & 15.35 & -1199 & -1057 \\
\hline 50.0 & -2.40 & 4.91 & 7.67 & -503 & -463 & 57.5 & -3.36 & 11.12 & 14.30 & -1206 & -1075 \\
\hline 47.5 & -2.63 & 4.80 & 7.02 & -502 & -466 & 55.0 & -3.12 & 11.27 & 13.47 & -1216 & -1093 \\
\hline 45.0 & -2.47 & 4.85 & 6.08 & -502 & -472 & 52.5 & -3.28 & 11.54 & 13.00 & -1224 & -1103 \\
\hline 42.5 & -2.72 & 4.77 & 5.90 & -507 & -478 & 50.0 & -3.41 & 11.53 & 11.64 & -1224 & -1116 \\
\hline 40.0 & -2.92 & 4.33 & 5.13 & -509 & -485 & 47.5 & -3.25 & 11.28 & 10.31 & -1238 & -1143 \\
\hline 37.5 & -3.16 & 4.32 & 4.64 & -510 & -487 & 45.0 & -3.39 & 10.91 & 9.21 & -1237 & -1154 \\
\hline 35.0 & -2.84 & 4.61 & 4.70 & -503 & -480 & 42.5 & -3.54 & 10.67 & 8.03 & -1241 & -1167 \\
\hline 32.5 & -2.71 & 5.06 & 4.39 & -504 & -480 & 40.0 & -3.68 & 10.10 & 6.54 & -1253 & -1191 \\
\hline 30.0 & -3.06 & 4.64 & 3.54 & -503 & -483 & 37.5 & -4.05 & 9.05 & 4.95 & -1252 & -1205 \\
\hline 27.5 & -3.05 & 5.14 & 3.43 & -504 & -482 & 35.0 & -4.21 & 7.54 & 2.79 & -1266 & -1234 \\
\hline 25.0 & -3.05 & 5.54 & 2.57 & -506 & -485 & & & & & & \\
\hline
\end{tabular}




\section{APPENDiX C: TURBINE EFFICIENCY SAMPle CALCULATION.}

In the current appendix a sample calculation to calculate the turbine total-to-static efficiency is presented. Table 5.3 and Table 5.4 are repeated here for clarity. They show the measured total-tostatic efficiencies for design cases (1) and (2).

Table 5.3. Experimental total-to-static efficiencies measured at design points.

\begin{tabular}{|c|c|c|c|}
\hline & Flow coefficient $(\phi)$ & Load coefficient $(\psi)$ & Efficiency. (\%) \\
\hline 1) Low press. & 0.1770 & 0.0742 & 70.6 \\
\hline 2) High press. & 0.2577 & 0.2018 & 82.8 \\
\hline
\end{tabular}

Table 5.4. Comparison of experimental and predicted total-to-static efficiencies at the design points.

\begin{tabular}{|l|c|c|c|c|}
\hline & $\begin{array}{c}\text { Figure 5.5 } \\
\text { Experimental }\end{array}$ & $\begin{array}{c}\text { Figure 5.6 } \\
\text { Experimental 22.5 }\end{array}$ & $\begin{array}{c}\text { Figure 5.7 } \\
\text { Simulated ideal }\end{array}$ & $\begin{array}{c}\text { Figure 5.8 } \\
\text { Simulated loss model }\end{array}$ \\
\hline 1) Low Press & 70.6 & 69.0 & 81.9 & 66.6 \\
\hline 2) High Press & 82.8 & 78.2 & 83.3 & 74.9 \\
\hline
\end{tabular}

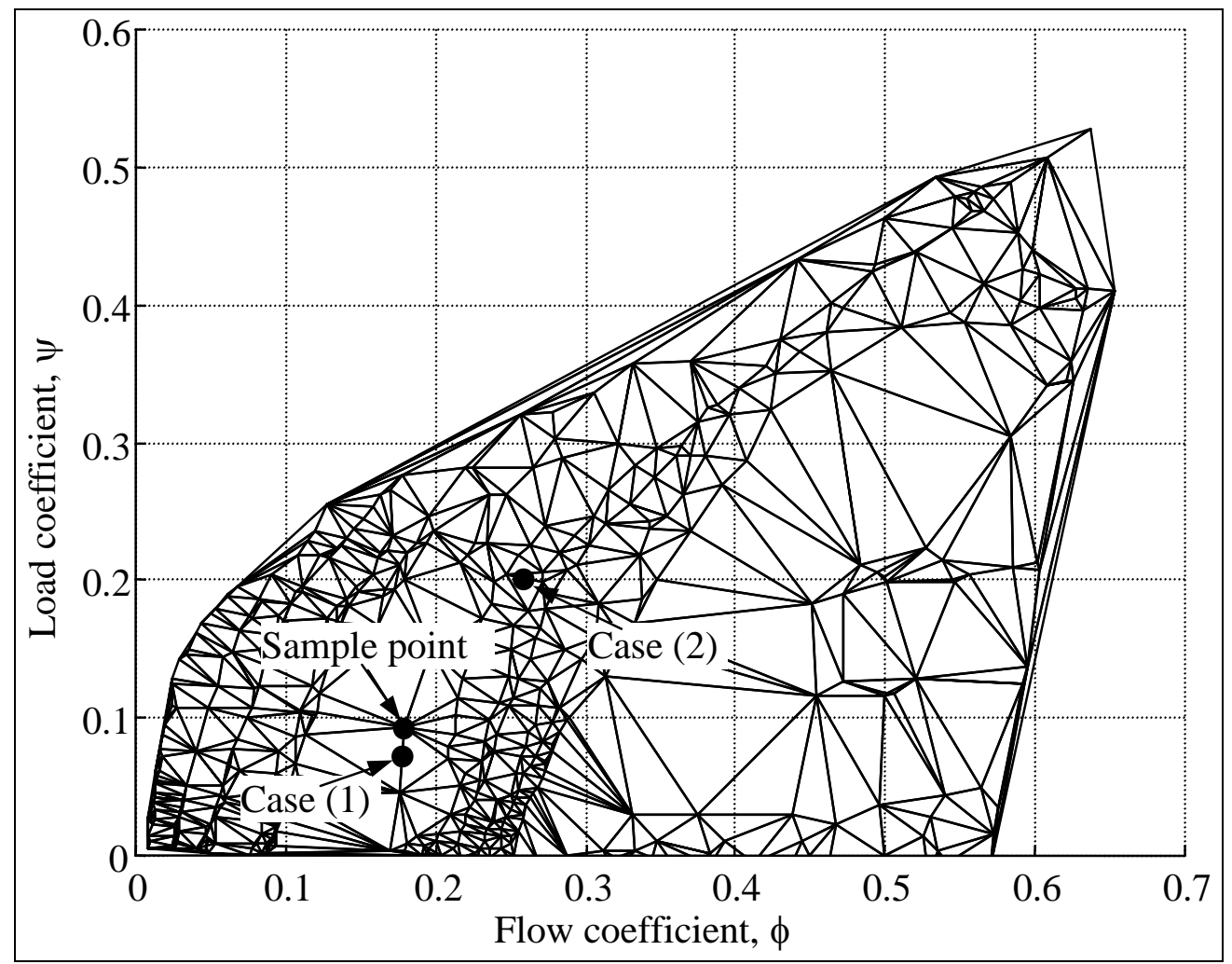

Figure C.1. Source data for (Insert cross reference) figure 5.5.

The efficiencies for the flow $(\phi)$ and load $(\psi)$ coefficients were interpolated from measured points closest to the design values. The MATLAB function griddata.m is used for interpolation on non- 
uniform grids. Figure C.1 shows the underlying data point that Figure 5.5 and Figure 5.6 are derived from. Recall that only the highest efficiency points are used. Each intersection of lines represents a data point. The sample calculation point is superimposed on this figure. In the relevant column of Appendix B it appears in bold. The measured data for the sample point is presented in Table C.1.

Table C.1. Numerical values of data for sample calculation point.

\begin{tabular}{|r|c|l|l|}
\hline Quantity & Symbol & Unit & Value \\
\hline Tip stagger angle & $\beta_{\text {3tip }}$ & deg & $78^{0}$ \\
\hline IGV flap angle & - & deg & $+20^{0}$ \\
\hline Turbine diameter & $\mathrm{D}_{\text {turb }}$ & $\mathrm{m}$ & 0.72 \\
\hline Hub-tip ratio & $\mathrm{v}$ & - & 0.4 \\
\hline Hub diameter & $\mathrm{D}_{\text {hub }}$ & $\mathrm{m}$ & 0.288 \\
\hline Density & $\rho$ & $\mathrm{kg} / \mathrm{m}^{3}$ & 1.1613 \\
\hline Specific heat capacity & $\mathrm{C}_{\mathrm{p}}$ & $\mathrm{J} / \mathrm{kg} . \mathrm{K}$ & 1005 \\
\hline Torque & $\mathrm{M}_{\text {turb }}$ & $\mathrm{Nm}$ & 2.245 \\
\hline Rotational speed & $\mathrm{RPM}$ & $\mathrm{s}^{-1}$ & 1150.5 \\
\hline & $\omega$ & $\mathrm{rad} . \mathrm{s}^{-1}$ & 120.48 \\
\hline Volume flow rate & $\mathrm{Q}$ & $\mathrm{m}^{3} / \mathrm{s}$ & 2.644 \\
\hline Wall static pressure & $\Delta \mathrm{p}_{\text {turb }}$ & $\mathrm{Pa}$ & 139.87 \\
\hline
\end{tabular}

\section{Flow Coefficient $(\phi)$}

The non-dimensional flow coefficient is defined in equation (5.2)

$$
\phi=\frac{\mathrm{C}_{\mathrm{z}}}{\mathrm{U}_{\text {tip }}}
$$

$\mathrm{C}_{\mathrm{z}}$ is the axial velocity through the turbine rotor area.

$$
\begin{aligned}
C_{z} & =\frac{Q}{A_{R}}=\frac{Q}{\frac{\pi}{4}\left(D_{\text {turb }}^{2}-D_{\text {hub }}^{2}\right)} \\
& =\frac{2.644}{\frac{\pi}{4}\left(0.72^{2}-0.288^{2}\right)} \\
& =7.731 \mathrm{~m} / \mathrm{s}
\end{aligned}
$$

$\mathrm{U}_{\text {tip }}$ is the turbine rotational speed at the tip,

$$
\begin{aligned}
\mathrm{U}_{\text {tip }} & =\omega \frac{\mathrm{D}}{2} \\
& =120.48 \frac{0.72}{2} \\
& =43.37 \mathrm{~m} / \mathrm{s}
\end{aligned}
$$


The flow coefficient is then

$$
\begin{aligned}
\phi & =\frac{7.731}{43.37} \\
& =\underline{0.178}
\end{aligned}
$$

\section{Load Coefficient $(\psi)$}

The non-dimensional load coefficient is defined in equation (5.3)

$$
\psi=\frac{2 \mathrm{c}_{\mathrm{p}} \Delta \mathrm{T}_{\text {turb }}}{\mathrm{U}_{\text {tip }}^{2}}
$$

The relationship between the power and total temperature is,

$$
\mathrm{P}=\dot{\mathrm{mc}}_{\mathrm{p}} \Delta \mathrm{T}_{\text {turb }}
$$

The power is the product of torque $\mathrm{M}_{\text {turb }}$ and rotational speed $\omega, \mathrm{P}=\mathrm{M}_{\text {turb }} \omega$ and mass flow $\dot{\mathrm{m}}=\rho \mathrm{Q}$. The load coefficient is

$$
\begin{aligned}
\psi & =\frac{2 \mathrm{P}}{\rho \mathrm{QU}_{\text {tip }}^{2}}=\frac{2 \mathrm{M}_{\text {turb }} \omega}{\rho \mathrm{QU}_{\text {tip }}^{2}} \\
& =\frac{2 \times 2.245 \times 120.48}{1.1613 \times 2.644 \times\left(120.48 \times \frac{0.72}{2}\right)^{2}} \\
& =\underline{0.94}
\end{aligned}
$$

\section{Turbine Total-to-Static Efficiency}

The total-to-static efficiency is calculated using (4.2)

$$
\begin{aligned}
\eta_{\text {turb ts }} & =\frac{\mathrm{P}_{\text {turb }}}{\mathrm{P}_{\text {ideal ts }}} \\
& =\frac{\omega \mathrm{M}_{\text {turb }}}{\mathrm{Q} \Delta \mathrm{p}_{\text {turb ts }}} \\
& =\frac{120.48 \times 2.245}{2.644 \times 139.87} \\
& =\underline{73.1 \%}
\end{aligned}
$$

\section{Application to Full-Scale Plant}

In applying the experimental data to full-scale plants that are not of the size dealt with in this work (ie 200MW, 1500m) it is suggested that the turbine dimensions relative to the chimney and tip speed as laid out in Table 3.5 are used. The volume flow rate and pressure drop will be available from the simulation. This is enough information to calculate the flow $(\phi)$ and load $(\psi)$ coefficients. Figure 5.5 or Appendix B can then used to find the turbine total-to-static efficiency. 


\section{Appendix D: Profile Data Calculations.}

In the current appendix the intermediate steps required to calculate the efficiencies in Table 5.12 to Table 5.15. Table 5.15 has perhaps the most useful data for use in the modelling of solar chimney power plant as it covers the expected range of operating conditions of the turbine. For the lowest predicted power settings the total-to-total and total-to-static efficiency values for case (1) can be used. For the highest power setting case (2) can be used. Intermediate values can be linearly interpolated against the power output.

In all the calculations of efficiency the shaft power $\mathrm{P}$ measured is divided by the power removed from the flow $\mathrm{P}_{\text {flow. }}$

$$
\eta=P / P_{\text {flow }}
$$

The steps used to calculate the values presented in Table 5.14 and Table 5.15 will be presented. The shaft power is measured directly from the turbine shaft. The fluid power requires a numerical integration to be performed using the measured profile data. The incremental value of power removed from the flow is obtained from the following

$$
\begin{aligned}
\mathrm{dP}_{0 \text { diff }} & =\mathrm{dQ} \times \Delta \mathrm{p}_{0} \\
\mathrm{dP}_{\text {diff }} & =\mathrm{dQ} \times \Delta \mathrm{p}
\end{aligned}
$$

The pressures $\Delta \mathrm{p}_{0}$ and $\Delta \mathrm{p}$ are measured directly at each station using the 5-hole probe. The incremental volume flow rate $\mathrm{dQ}$ is the amount of flow that passes between each measuring station and is the product of axial velocity $\mathrm{C}_{\mathrm{z}}$ and incremental area $\mathrm{dA}$. The raw data is listed in Appendix B.

$$
\mathrm{dQ}=\mathrm{C}_{\mathrm{z}} \times \mathrm{dA}=\mathrm{C}_{\mathrm{z}} \times 2 \pi \mathrm{rdr}
$$

The first two columns of Table 5.14 are expanded to include the measured shaft powers and integrated flow power

Table D.1 Experimental turbine performance details.

\begin{tabular}{|l|l|l|c|c|}
\hline & & & $\begin{array}{c}\text { Experimental } \\
\text { total-to-total }\end{array}$ & $\begin{array}{c}\text { Experimental } \\
\text { total-to-static }\end{array}$ \\
\hline Case (1) & Shaft power & $\mathrm{P}$ & $265.7 \mathrm{~W}$ & $265.7 \mathrm{~W}$ \\
\hline & Flow power & $\mathrm{P}_{\text {flow }}$ & $311.5 \mathrm{~W}$ & $384.1 \mathrm{~W}$ \\
\hline & Efficiency & & 85.3 & 69.2 \\
\hline Case (2) & Shaft power & $\mathrm{P}$ & $934.8 \mathrm{~W}$ & $934.8 \mathrm{~W}$ \\
\hline & Flow power & $\mathrm{P}_{\text {flow }}$ & $1036.8 \mathrm{~W}$ & $1255.2 \mathrm{~W}$ \\
\hline & Efficiency & & 90.2 & 74.5 \\
\hline
\end{tabular}


Table 5.15 is derived from Table 5.14 using the diffuser data presented in Table 5.10. Due to the conservative diffuser design with a small expansion angle it was assumed that the diffuser efficiency would be the same for a slightly longer diffuser. The first two columns of Table 5.15 are expanded and the predicted flow powers with the longer diffuser inserted.

Table D.2 Full-scale turbine performance details.

\begin{tabular}{|l|l|l|c|c|}
\hline & & & $\begin{array}{c}\text { Experimental } \\
\text { total-to-total }\end{array}$ & $\begin{array}{c}\text { Experimental } \\
\text { total-to-static }\end{array}$ \\
\hline Case (1) & Shaft power & $\mathrm{P}$ & 265.7 & 265.7 \\
\hline & Flow power & $\mathrm{P}_{\text {flow }}$ & 312.0 & 345.0 \\
\hline & Efficiency & & 85.2 & 77.0 \\
\hline Case (2) & Shaft power & $\mathrm{P}$ & 934.8 & 934.8 \\
\hline & Flow power & $\mathrm{P}_{\text {flow }}$ & 1043.3 & 1158.8 \\
\hline & Efficiency & & 89.6 & 80.7 \\
\hline
\end{tabular}

\section{Diffuser Performance}

The calculation of the diffuser performance requires a numerical integration at diffuser entry and exit. This allows the losses and dynamic pressure recovery to be calculated accurately. The two parameter that are required are the diffuser efficiency $\eta_{\text {diff }}$ and the diffuser pressure recovery

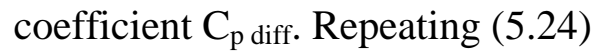

$$
\begin{aligned}
\mathrm{C}_{\mathrm{p} \mathrm{diff}} & =\mathrm{C}_{\mathrm{pdiff} \text { ideal }}-\mathrm{K}_{\text {diff loss }} \\
\mathrm{K}_{\text {diff loss }} & =\frac{\left(\mathrm{P}_{\text {stage tt }}-\mathrm{P}_{\text {diff tt }}\right)}{\frac{1}{2} \dot{\mathrm{m}} \mathrm{C}_{3}^{2}}
\end{aligned}
$$

All three terms $\mathrm{P}_{\text {stage tt }}$ (diffuser entry power based on total pressure), $\mathrm{P}_{\text {diff tt }}$ (diffuser exit based on total pressure) and the denominator term $\frac{1}{2} \dot{m C}_{3}^{2}$ require the integration of the velocity and pressure profiles to determine the total value. $\mathrm{C}_{\text {pdiff ideal }}$ is based on the area ratio between the diffuser entry and exit AR. The experimental performance details are listed in the following table.

Table D.3 Experimental diffuser performance details.

\begin{tabular}{|l|l|l|l|l|l|l|l|}
\hline & $\mathrm{P}_{\text {stage tt }}$ & $\mathrm{P}_{\text {diff tt }}$ & $\frac{1}{2} \dot{\mathrm{mC}}_{3}^{2}$ & $\mathrm{~K}_{\text {diff loss }}$ & $\mathrm{C}_{\text {pdiff ideal }}$ & $\mathrm{C}_{\text {pdiff }}$ & $\eta_{\text {diff }}$ \\
\hline Case (1) & $309.9 \mathrm{~W}$ & $311.5 \mathrm{~W}$ & $114.5 \mathrm{~W}$ & 0.0141 & 0.5371 & 0.523 & $97.3 \%$ \\
\hline Case (2) & $1016.7 \mathrm{~W}$ & $1036.8 \mathrm{~W}$ & $401.5 \mathrm{~W}$ & 0.0499 & 0.5371 & 0.487 & $90.7 \%$ \\
\hline
\end{tabular}


The properties of the full-scale diffuser and the efficiencies listed in Table D.2 can now be calculated.

Table D.4 Full-scale diffuser performance details.

\begin{tabular}{|l|c|c|c|c|c|}
\hline & $\frac{1}{2} \dot{\mathrm{m}} \mathrm{C}_{3}^{2}$ & $\mathrm{~K}_{\text {diff2 loss }}$ & $\mathrm{C}_{\text {pdiff2 ideal }}$ & $\mathrm{C}_{\text {pdiff2 }}$ & $\eta_{\text {diff }}$ \\
\hline Case (1) & $114.5 \mathrm{~W}$ & 0.0186 & 0.7123 & 0.6936 & $97.3 \%$ \\
\hline Case (2) & $401.5 \mathrm{~W}$ & 0.0662 & 0.7123 & 0.6461 & $90.7 \%$ \\
\hline
\end{tabular}

The values used to calculate the full-scale turbine efficiencies in Table D.2 can now be determined using eqn (5.27) and (5.28).

$$
\begin{aligned}
& \mathrm{P}_{\text {diff2 tt }}=\mathrm{P}_{\text {rot tt }}+\frac{1}{2} \dot{\mathrm{m}} \mathrm{C}_{3}^{2} \mathrm{~K}_{\text {diff2 loss }} \\
& \mathrm{P}_{\text {diff2 ts }}=\mathrm{P}_{\text {rot ts }}-\frac{1}{2} \dot{\mathrm{m}} \mathrm{C}_{3}^{2} \mathrm{C}_{\mathrm{p} 2 \text { diff }}
\end{aligned}
$$

Table D.5 Turbine flow power $\mathbf{P}_{\text {flow }}$ calculation values.

\begin{tabular}{|l|l|l|c|c|}
\hline & & & $\begin{array}{c}\text { Experimental } \\
\text { total-to-total }\end{array}$ & $\begin{array}{c}\text { Experimental } \\
\text { total-to-static }\end{array}$ \\
\hline Case (1) & Shaft power & $\mathrm{P}$ & $265.7 \mathrm{~W}$ & 265.7 \\
\hline & Turbine exit & $\mathrm{P}_{\text {rot tt }}$ & $309.9 \mathrm{~W}$ & $424.4 \mathrm{~W}$ \\
\hline & $\frac{1}{2} \dot{\mathrm{mC}}_{3}^{2} \mathrm{~K}_{\text {diff2 loss }}$ & & $2.13 \mathrm{~W}$ & \\
\hline & $\frac{1}{2} \dot{\mathrm{mC}}_{3}^{2} \mathrm{C}_{\mathrm{p} 2 \text { diff }}$ & & & $79.4 \mathrm{~W}$ \\
\hline & Flow power & $\mathrm{P}_{\text {flow }}$ & 312.0 & 345.0 \\
\hline & Efficiency & & 85.2 & 77.0 \\
\hline Case (2) & Shaft power & $\mathrm{P}$ & $934.8 \mathrm{~W}$ & $934.8 \mathrm{~W}$ \\
\hline & Turbine exit & $\mathrm{P}_{\text {rot tt }}$ & $1016.7 \mathrm{~W}$ & $1418.2 \mathrm{~W}$ \\
\hline & $\frac{1}{2} \dot{\mathrm{mC}}_{3}^{2} \mathrm{~K}_{\text {diff2 loss }}$ & & $26.8 \mathrm{~W}$ & \\
\hline & $\frac{1}{2} \dot{\mathrm{mC}}_{3}^{2} \mathrm{C}_{\mathrm{p} 2 \text { diff }}$ & & & $259.4 \mathrm{~W}$ \\
\hline & Flow power & $\mathrm{P}_{\text {flow }}$ & $1043.3 \mathrm{~W}$ & $1158.8 \mathrm{~W}$ \\
\hline & Efficiency & & 89.6 & 80.7 \\
\hline
\end{tabular}

